\title{
Hydrogeochemical Investigations of Historic Mining Districts, Central Western Slope of Colorado, Including Influence on Surface-Water Quality
}

By J. Thomas Nash

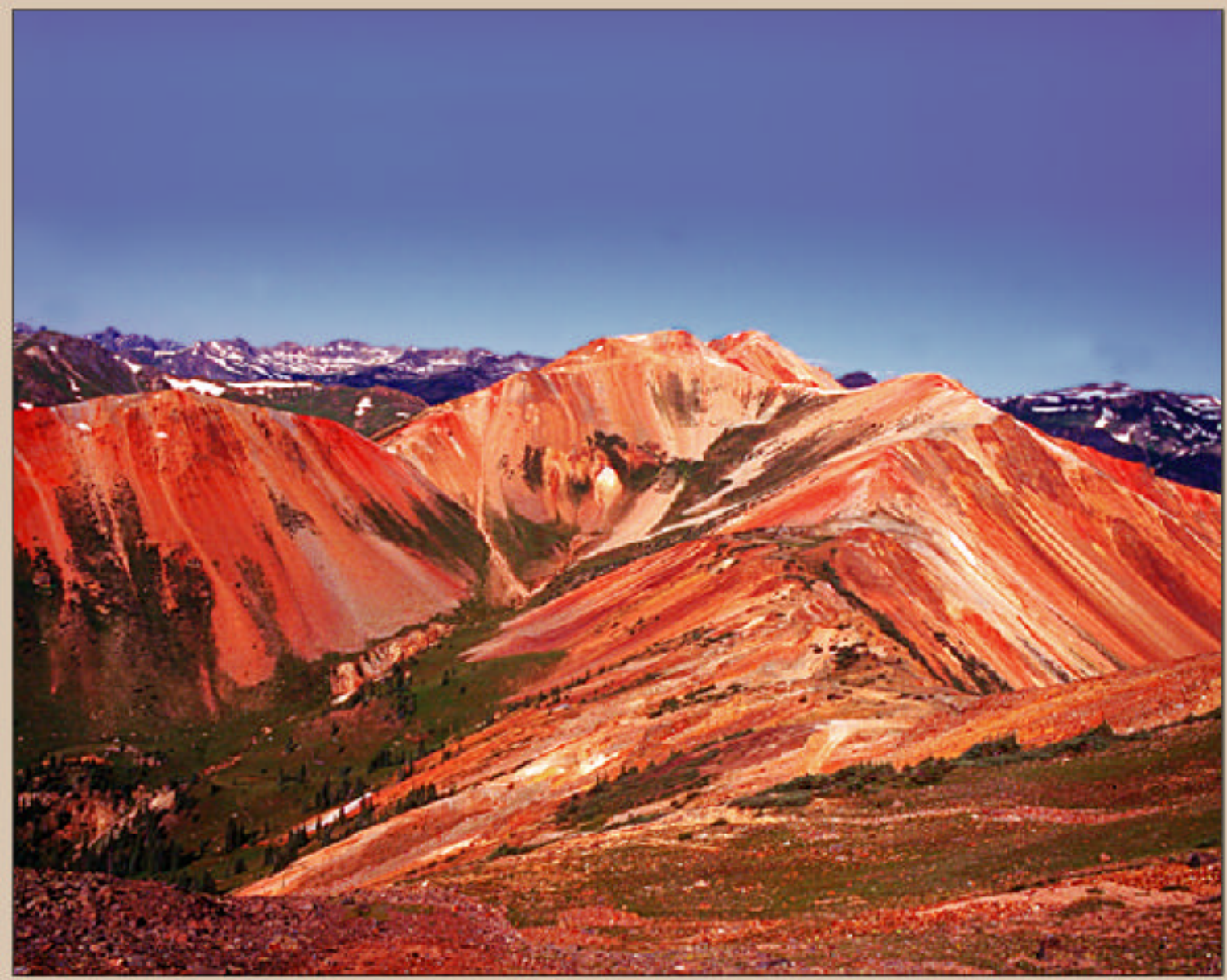

USGS Digital Data Series

DDS-73

Version 1.02002

U.S. Department of the Interior

U.S. Geological Survey 


\title{
Hydrogeochemical Investigations of Historic Mining Districts, Central Western Slope of Colorado, Including Influence on Surface-Water Quality
}

\author{
By J. Thomas Nash
}

U.S. Geological Survey Digital Data Series DDS-73 


\title{
U.S. Department of the Interior
}

\author{
Gale A. Norton, Secretary
}

\section{U.S. Geological Survey \\ Charles G. Groat, Director}

February 2002, version 1.0

For sale by U.S. Geological Survey, Information Services

Box 25286, Denver Federal Center

Denver, C0 80225

For more information about the USGS and its products:

Telephone: 1-888-ASK-USGS

World Wide Web: http://www.usgs.gov/

This publication is also available online at:

http://geology.cr.usgs.gov/pub/dds/dds-073/

Any use of trade, product, or firm names in this publication

is for descriptive purposes only and does not

imply endorsement by the U.S. Government 


\section{Contents}

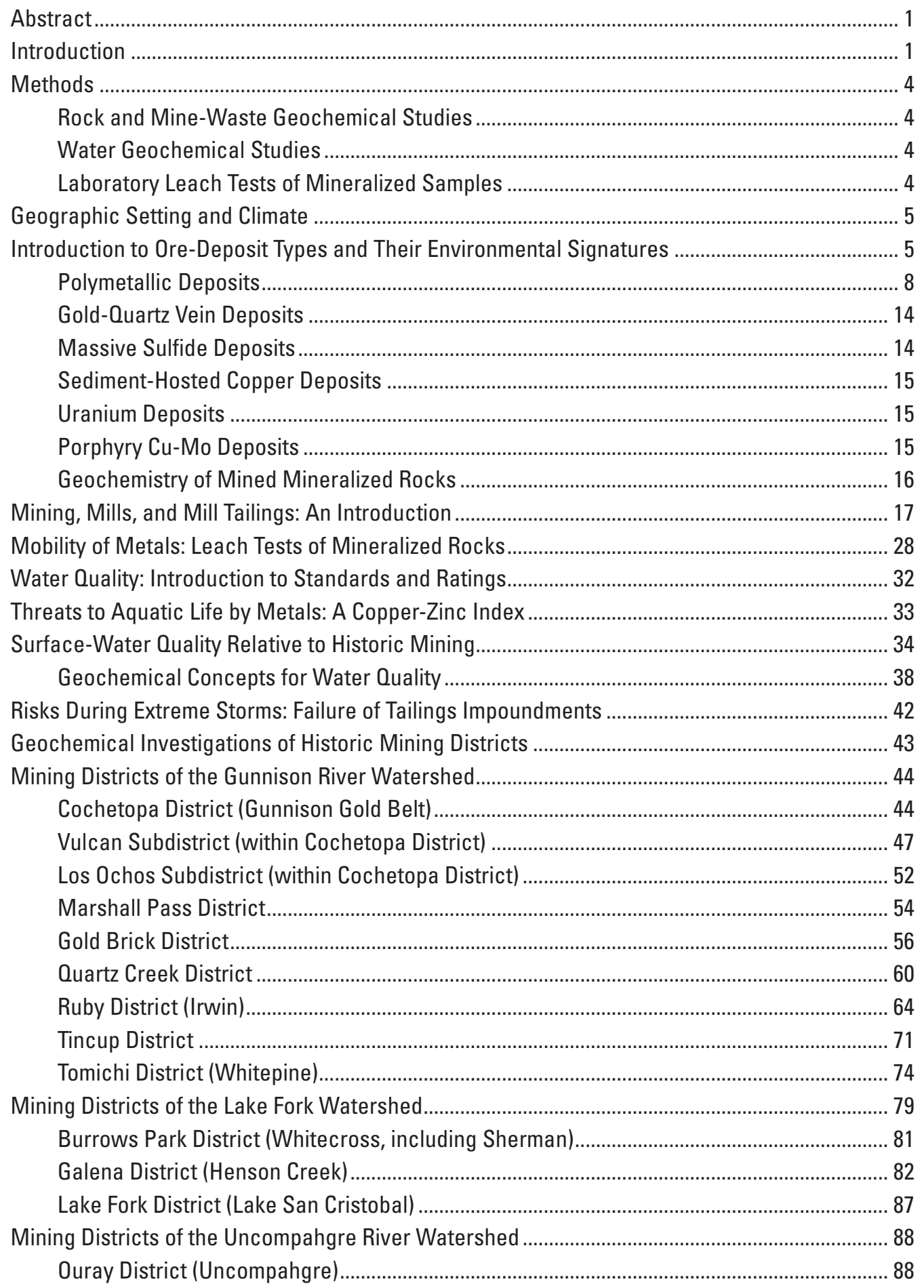




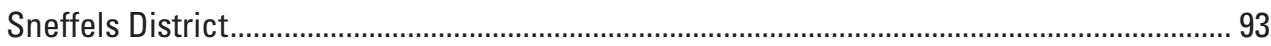

Mineral Point District (including Upper Uncompahgre)................................................... 100

Red Mountain District ................................................................................................... 103

Mining Districts of the Upper San Miguel River Watershed .................................................... 117

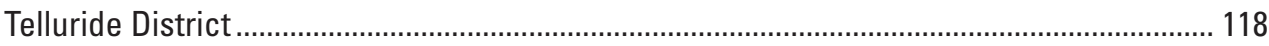

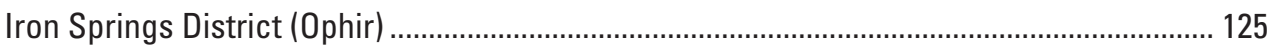

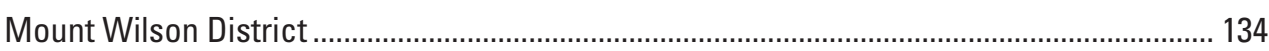

Placerville District (Lower San Miguel) ........................................................................... 135

Mining Districts of the Lower San Miguel River Watershed ........................................................ 135

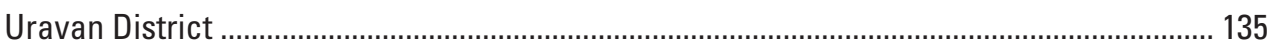

Cashin District (La Sal Creek) ............................................................................................. 139

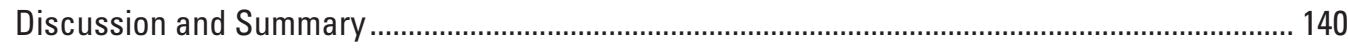

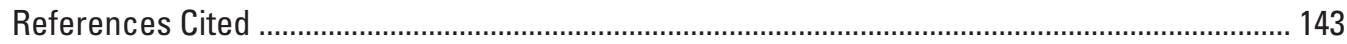

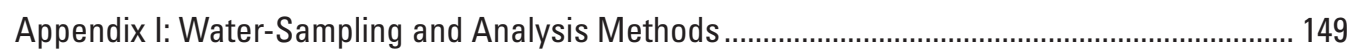

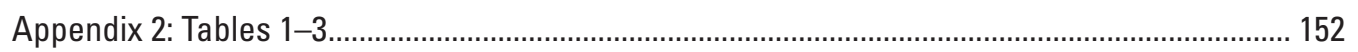

\section{Figures}

1. Location of mining districts studied, central Western Slope, Colorado .............................. 3

2. Composition of mined materials by deposit type, central Western Slope,

Colorado

3. Composition of mineralized rocks by sample type, central Western Slope,

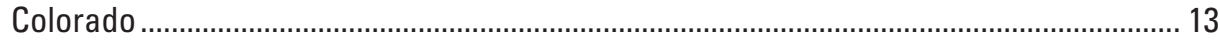

4. Location of mill tailings and mill sites, central Western Slope, Colorado ........................ 17

5. Compositions of leachate solutions by deposit type ...................................................... 29

6. Compositions of leachates from mineralized rocks as a function of $\mathrm{pH}$...........................30

7. Copper-zinc index (CZI) values for surface waters, central Western

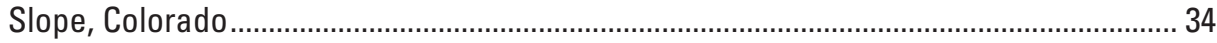

8. Comparison of mine drainage and stream water compositions, central Western Slope, Colorado

9. Compositions of surface waters, central Western Slope, Colorado,

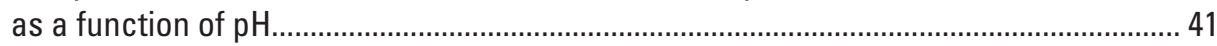

10. Location of studied mining districts in the Gunnison River watershed ............................. 45

11. Features of part of the Cochetopa mining district, showing sample localities................ 46

12. Features of the Vulcan mine area, showing sample localities ......................................... 48

13. Features of part of the Marshall Pass mining district, showing major

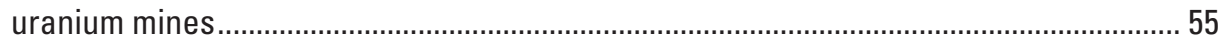

14. Features of the Gold Brick mining district, showing sample localities ........................... 57

15. Features of the Tincup and northern part of the Quartz Creek mining districts, showing sample localities.

16. Features of the northeast part of the Ruby mining district, showing sample localities. 
17. Sample sites and water compositions in the Elk Creek watershed near the Standard mine. 70

18. Features of the Tomichi mining district, showing sample localities . .75

19. Features of the Galena, Lake Fork, and Burrows Park mining districts,

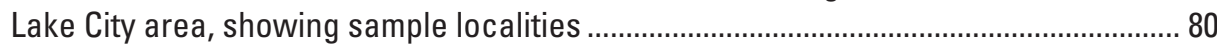

20. Location of mining districts in the San Juan Mountains region ........................................ 81

21. Features of the Ouray mining district, showing sample localities .................................... 89

22. Features of the Sneffels mining district, showing sample localities .............................. 94

23. Features of part of the Mineral Point mining district, showing sample localities ......... 101

24. Features of the Red Mountain mining district, showing sample localities.................... 104

25. Features of part of the Telluride mining district, east of Telluride, showing sample localities.

26. Features of the Iron Springs mining district, near Ophir, showing sample localities

27. Location of streams, central Western Slope, Colorado, that have degraded water quality . 141

28. Analytical error bars for various metal concentrations in water samples 151

\section{Photographs}

1. The Dolores River at Slickrock creates an oasis for plants and wildlife and is in sharp contrast to the dry hills nearby

2. Cedar-pinion forests are characteristic of mesas in the Uravan district of western Colorado

3. The Cochetopa district is typical of sagebrush semiarid climate of the hills south of Gunnison

4. The Marshall Pass uranium mining area, at about $11,000 \mathrm{ft}$ elevation, receives more than 200 inches of snow.

5. The Hough mine, Palmetto basin, is above treeline at an elevation of about $12,000 \mathrm{ft}$

6. Slag is a black, vitreous rock-like substance produced when ores are melted during smelting

7. Mill tailings, produced when mills grind ore, are fine sandy materials that resemble beach sands

8. The National Belle mine, Red Mountain district, produced rich silver ore from a small breccia pipe within this hill

9. This picturesque mill on the Crystal River is typical of early mills that utilized water power and discharged mill tailings directly into the river

10. Tomboy, east and above Telluride, was the site of at least six mills that served medium-sized mines that converged on this one relatively flat area at $11,000 \mathrm{ft}$ elevation.

11. The Akron mine and mill near Whitepine was active in the 1940's-50's 
12. Mining of uranium-vanadium in the Uravan district, from the 1940s through the 1970's, was typically from small- to medium-sized mines that brought relatively small volumes of waste to the surface............................................................... 28

13. Small mines and mills are typical of the Cochetopa district ............................................. 47

14. The Vulcan mine and nearby Good Hope mine created dumps of moderate size but very high sulfide content.................................................................. 49

15. North of the Vulcan mine and dump is this zone devoid of vegetation, termed a "kill zone"

16. The remains of the smelter at the Vulcan mine are barely identifiable, but the black vitreous material to the left is unmistakably slag

17. Vulcan mine dump, rich in sulfide minerals, develops this white crust of sulfate minerals that is very reactive in water..

18. This popcorn texture is created as sulfate crusts grow on pyritic waste rocks of the Vulcan mine dump... 51

19. Uranium mines of the Los Ochos district are chiefly along an east-west fault zone in the dry hills of the Los Ochos district .. .53

20. Uranium of the Los 0 chos district are rich in pyrite, causing them to turn red and yellow on the surface as they weather.

21. The Pitch uranium deposit formed along a major fault that placed gray shales and limestones against Precambrian granite.....

22. The Raymond mill on Gold Creek, Gold Brick district, had 40 stamps and was active before 1915

23. The Gold Link mill was a large and impressive structure in 1915 .

24. Tailings from the Gold Link mill were placed in gullies east of the mill ........................... 59

25. Tailings from the Raymond mill were sluiced to the east about $1 / 2 \mathrm{mi}$ and placed on a bench above Gold Creek 59

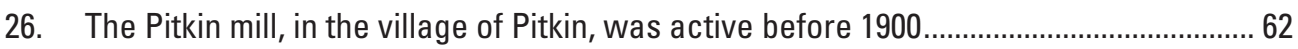

27. The Roosevelt mill, about 2 mi south of Pitkin, is remarkably intact........................................ 62

28. Altered rocks at Cumberland Pass formed in association with a diorite intrusion that also created several molybdenum-tungsten vein deposits..........................63

29. The Bon Ton mine was a modest producer of molybdenum during World War I............ 63

30. Mine drainage from the Bon Ton mine is acidic and makes tell-tale red colors .............. 64

31. An old mine and waste dump in upper Elk basin creates acidic mine drainage ............. 67

32. The Standard mine, from the 1950's, is relatively intact, and no reclamation work has been done

33. Drainage from the Standard mine tunnel reacts with waste dump rocks, gaining more metals and acid, then runs into Elk Creek

34. Only the foundation remains from the Standard mine mill, and the tailings pond is intact to the south

35. The Standard mine tailings pond collects runoff from waste dumps

36. The red material is a variety of ferricrete; the iron was deposited from acidic drainage from the Keystone vein

37. Tailings from the mill south of Tincup have been dug into, possibly to reprocess the tailings 
38. The stamp mill south of Tincup is now a pile of beams ................................................... 72

39. The mill at the Blistered Horn silver mine is in relatively good condition .......................... 73

40. The Tomichi smelter, southwest of Whitepine, was part way up the hillside and slag was poured out onto the flat area below ............................................ 76

41. The Akron mill on the south edge of Whitepine was productive through 1956 .............. 76

42. The Akron mine tunnel served both for rail haulage and mine dewatering .................... 77

43. This tailings impound is 200 yards south of the Akron mill ............................................ 77

44. This view is from the south tailings impoundment to the Akron millsite

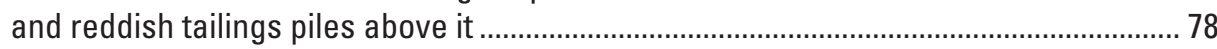

45. This small tailings pond was built across Tomichi Creek before 1900............................. 78

46. These gray sands are mill tailings that were eroded and carried south along Tomichi Creek

47. Mill tailings from the Hidden Treasure mill are on the south bank of Henson Creek about 3 mi west of Lake City... 83

48. The Ute-Ulay mine was the largest in the lower Galena district........................................ 83

49. Tailings and mine waste at the Ute-Ulay mine are stacked on a narrow bench very close to Henson Creek

50. The large dumps from the Hough mine are rich in sulfide minerals and pose problems for the alpine ecology

51. The Palmetto mill at treeline in the headwaters of Henson Creek processed ore from several mines in the Engineer Pass area

52. This brick smokestack is the remains of the Lee smelter, located southwest of Capitol City...

53. The Empire Chief mill is a popular tourist stop on the Engineer Pass road, southwest of Capitol City... 86

54. This small stamp mill on Dexter Creek probably operated before 1900 ........................... 90

55. This stone and concrete foundation probably was for a mill located just below the old railroad near Corbett Creek about 4 mi north of Ouray

56. The Silvershield mill processed ore carried in by a tram from mines above the red rock cliffs north of Ouray.

57. Tailings from the Silvershield mill are about 100 yards west of the Uncompahgre River.

58. The Banner mill site, 2 mi north of Ouray, was developed for housing in the 1970's, but the tailings were left in place.

59. The Camp Bird mine was the major producer in the Sneffels district and created large waste and tailings piles along Canyon Creek.

60. The Atlas stamp mill was a significant producer in the early years of mining in the Sneffels district

61. Mill tailings from the Atlas mill were placed along and in Sneffels Creek with no concern for their impact on water quality prior to regulations against this practice, which were enacted in 1935

62. These cement pieces of a foundation are all that remains of the

Camp Bird 3-level mill . 
63. The Camp Bird 3-level mill produced this small volume of tailings before problems with avalanches forced the construction of a new mill on the 14th level near Canyon Creek

64. These eroded gray tailings are from a small mill, ca. 1900, on Imogene Creek between the Camp Bird 3- and 14-level mills

65. Overbank deposits of tailings $c$ an be seen at several places along Imogene and Canyon Creeks

66. The Hidden Treasure mine is one of the few in the Sneffels district that produces acidic mine drainage ...

67. The Hidden Treasure mill was only 100 yards north of the Camp Bird 3-level mill .....

68. The San Juan Chief mine created a series of workings up this hill, following a major vein.

69. The Red Mountain complex of highly altered rocks and associated mineral deposits are at the geographic center of three watersheds that are among the most contaminated by acid and metals in all of Colorado

70. This colorful, silicified knob encloses the National Belle pipe deposit 105

71. Brown iron deposits (ferricrete) guided prospectors to the Guston and other deposits in the Red Mountain district.

72. This ferricrete deposit formed at the site of the Hudson deposit, Red Mountain district, just like the larger one at Guston

73. The Genessee mine, at the base of Red Mountain Number 2 and 3, produced a large dump of pyritic waste rock

74. The highly sulfidic dump of the Genessee mine is slowly releasing acid and metals

75. Acidic waters from the Guston mine and dump have killed vegetation below it

76. Red Mountain Creek is highly degraded by many sources ............................................. 108

77. Tailings contribute to problems along Red Mountain Creek ........................................... 109

78. Seepage from mill tailings is acidic and as high in metals as mine drainage ................ 109

79. The Larson Brothers mine tunnel is one of the few on the west side of the Red Mountain district that creates acidic drainage

80. Tailings impoundments of the Idarado mill have been reclaimed and concrete gutters put in place to control drainage.

81. The Idarado mine complex at the portal to the Treasury tunnel is near the summit of Red Mountain Pass

82. The quality of Red Mountain Creek improves as it mixes with other streams, especially those coming in from the west..

83. The Vernon mine in the headwaters of Gray Copper Gulch Creek is a concern because the highly sulfidic waste and mine drainage are degrading the creek

84. The Vernon mine on Gray Copper Gulch Creek creates several problems

85. The headwaters of Gray Copper Gulch Creek received contaminated waters from several sources, including small mine dumps 
86. The highly altered rocks on Red Mountain No. 3 on the west side of Gray Copper Gulch are a likely source of much of the acid and metals in the creek. 113

87. Corkscrew Gulch Creek on the flank of Red Mountain No. 1 is highly degraded for a watershed containing only a few small mines.

88. These are the sparse remains of a pre-1900 stamp mill in Savage basin, east of Telluride.

89. Tomboy basin above Telluride was the site of several large mills between 1890 and 1910, before ore was carried down the mountain to Pandora in tunnels or trams.

90. Four or more mills at Tomboy were located at the west edge of the upland basin at the edge of the cliffs

91. Mills require strong foundations for the heavy equipment, and often that is all that remains after a fire.

92. These ruins are another mill southwest of those shown in photograph 90 122

93. The Japan mill was a medium-sized producer in Tomboy basin through about 1910 .

94. The Argentine mine and mill complex in Tomboy basin was one of the large producers in that area from about 1890 to 1920

95. This view of Marshall Creek, west of the junction with Savage Creek, shows the rugged character of the canyon below Tomboy.

96. Mill tailings are in and along the San Miguel River at Society Turn, about 4 mi west of Telluride

97. Tailings from the Carbonero mill were placed close to the Howard Fork and are eroded by smaller side streams

98. View of the Carbonero tailings from the east edge of Ophir 127

99. Tailings from the Carribeau mill were placed on a flat area on the south side of the Howard Fork

100. Drainage from the Carribeau mine has high flow and carries fairly high concentrations of iron and other metals

101. Tailings from the Carribeau mill are flooded by mine drainage from the adit ............... 129

102. This view of flooded Carribeau mill tailings shows the influence of active beaver dams

103. Tailings from the Silver Bell mill were stacked high above the Howard Fork ............... 130

104. Large iron deposits (ferricrete) formed from iron-rich springs after glaciers carved the Ophir valley 130

105. Iron was mined at Iron Springs east of Ophir from this cut that shows the layering in the iron deposit.

106. Artesian flow of water carries high concentrations of iron and other metals from a drill hole made for metal exploration in the 1960's(?)

107. Uranium mines of the Uravan district commonly tunneled into rim sandstones on steep canyon walls, as here on the edge of Atkinson Mesa.

108. Mine waste in mesa terrain is subject to erosion is storm events 136

109. Widespread mining across the Uravan district created thousands of small waste dumps like this one at the Cougar mine near Slickrock. 
110. The ore-buying station at Poverty Flat, near Slickrock, was thoroughly reclaimed in the 1990's.

111. The Cashin mine, located in the meandering canyon of La Sal Creek, had underground workings to the right of the buildings 139

\section{Tables}

[Tables 1-3, because of their length, appear in Appendix 2 at the end of this report]

1. Descriptions and locations of sample sites and analyzed samples ................................ 153

2. Chemical analyses of mineralized rock samples ........................................................ 162

3. Analytical results for selected elements in surface-water samples .............................. 165

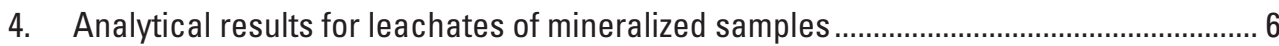

5. Simple classification of mineral deposits for environmental assessment, CWS, Colorado 11

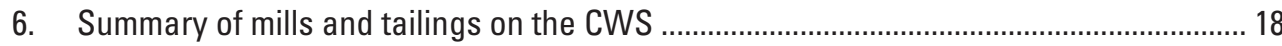

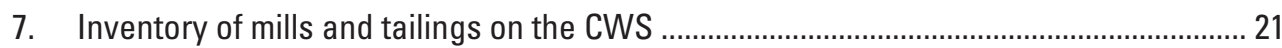

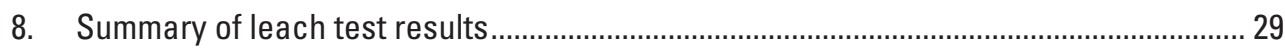

9. Colorado basic stream standards for metals: class-1 aquatic life.................................... 32

10. Copper-zinc indices (CZI) for surface-water samples from the CWS ..............................35

11. Summary of analyses of surface-water samples on the CWS ...................................... 39

12. Summary of surface-water compositions, Red Mountain district ................................... 115

13. Summary of persistent zinc concentrations in near-neutral surface waters ................ 142

14. Elements determined in water and leachate samples by ICP-MS .................................. 150

\section{Data Files}

[Data files, in Excel spreadsheet format (.xls), are stored in the "Data" folder at the root directory of this CD-ROM. Files with .csv extensions are "comma separated value" files. Filenames and brief descriptions of the files are provided below]

1. NSAMDL.xls Descriptions and locations of sample sites

2. NSAMDL.cSV

3. NGMR.xls Chemical analyses of mineralized rock samples

4. NGMR.csv

5. NGWC.xls

Analytical results for surface-water samples 1997-98

6. NGWC.csv

7. NGWC9.xls Analytical results for surface-water samples 1999

8. NGWC9.csv

9. NGLC.xls

$$
\text { Analytical results for leachates of mineralized samples }
$$


10. NGLC.csv

11. NGMT.xls Inventory of mills and tailings on the CWS

12. NGMT.csv

13. NGCZI.xls

Copper-zinc indices for surface waters

14. NGCZI.csv 


\title{
Hydrogeochemical Investigations of Historic Mining Districts, Central Western Slope of Colorado, Including Influence on Surface-Water Quality
}

\author{
By J. Thomas Nash
}

\section{Abstract}

Reconnaissance studies of mining districts in the central part of the Western Slope (CWS) of Colorado were undertaken to characterize mined materials, mine drainage, and the influence of historic mining on nearby streams. The geochemical studies included sampling and chemical analysis of mine dumps, mill tailings, mine drainage, and surface waters. Descriptions and interpretations are based on more than 400 new geochemical analyses. Area geology, mining history, and geochemical methods are reviewed in relatively nontechnical style to provide a background for the descriptions of mining districts.

These geochemical investigations show that contamination from historic mines is generally less than that asserted by some or suggested by geochemical models. Most of the studied mining districts have at least a few sources of contaminants, but, in the majority of cases, the metals and acidity of concern are naturally mitigated to acceptable levels within about 2 or $3 \mathrm{mi}$ of the sources. For these self-mitigating areas, the major concern is zinc and cadmium concentrations that are at or somewhat higher than $(1 \times$ to $2 \times)$ the chronic exposure standard for aquatic life. A few districts having a combination of stronger sources and limited buffering capacity have greater impacts on water quality, and presumably on aquatic health, beyond the area of mining and downstream for distances of about 3 to $10 \mathrm{mi}$. In these headwater streams, metals such as $\mathrm{Al}, \mathrm{Cu}, \mathrm{Fe}, \mathrm{Mn}$, and $\mathrm{Pb}$, in addition to $\mathrm{Zn}$ and $\mathrm{Cd}$, tend to be above standards for aquatic life. In such situations, land managers and owners need to consider remediation methods that will reduce the release of metals and acid in miningrelated sources. Several areas have unmined sources of acid and metals that complicate mitigation planning.

In this study of 22 mining districts, only one poses a significant threat to watershed health - the Red Mountain district in the headwaters of the Uncompahgre River watershed. Red Mountain Creek in the Red Mountain district appears to be the most contaminated of those studied here; there are indications of numerous sources in mines, dumps, mill tailings, and in the highly altered unmined rocks of Red Mountain. Several other watersheds have mining-impacted waters of less magnitude or extent. Short reaches of streams show relatively low-level effects of historic mining: the Howard Fork of the San Miguel River, Elk Creek above Coal Creek (west of Crested Butte), Canyon Creek above Ouray, uppermost Henson Creek, and uppermost Uncompahgre River. The problems in these reaches involve acidic mine drainage before it can be buffered by natural processes and elevated $\mathrm{Zn}, \mathrm{Cd}$, and $\mathrm{Cu}$ concentrations. More study of these waters is needed, and ways to reduce acidity and metals concentrations to levels that will sustain or improve aquatic life communities must be found.

Tailings are identified here as important contributors of contamination in many mining areas. Also, many tailings impoundments appear to be at risk of catastrophic failure in an extreme storm. Most of the damage from a tailings failure cannot be reversed once the tailings are in a stream because cleanup is costly and likely to damage riparian habitat. Many tailings impoundments are collapsing after 50 or more years of neglect, and some are located in flood plains where they are at risk in a major storm. Locally focused flash floods are fairly common in Colorado, especially in the summer, and are capable of moving millions of tons of earth materials. A case can be made that failure of a tailings impoundment in a storm event can cause far more intensive and extensive damage to a watershed than the more predictable effects of acid mine drainage. Further study of tailings stability in extreme storms is needed to make an appropriate risk analysis.

\section{Introduction}

More than 130 years of mining on the Western Slope of Colorado has left a legacy of trails, ruins, and waste piles that are viewed with concern or affection by residents, visitors, and Federal land managing agencies (FLMA's). Newspapers and journals contain divergent descriptions of the effects of historic mining that range from prophesy of doom to naive assertions that there are no problems. The truth, if it can be found, is somewhere in between: the effects of historic mining on surface-water quality and biologic habitat are enor- 
Hydrogeochemical Investigations of Historic Mining Districts, Central Western Slope of Colorado

mously variable from site to site and area to area. Predictions of mining impacts based on scientific measures of geology, ore character, mining style, climate, and other parameters are improving but tend to lack the specificity required by FLMA's to plan restoration activities. This investigation was undertaken to provide, most importantly, new descriptive data and, secondly, some interim interpretations and suggestions. Data and observations in this study are not perfect but should hold up under scrutiny; the interpretations are subject to state-of-the-art understanding and should be viewed as concepts, not facts.

Mining on the central Western Slope (CWS) of Colorado played a major role in the settlement and growth of the region, starting unofficially a few years before treaties with the Native Americans in the 1860's. Mining and related service industries brought millions of dollars of new wealth to dozens of communities. Mining started with few Federal regulations, and those that existed in the first 50 years tended to have the intent of encouraging new mining activity. Handling of mine wastes does not appear to have been regulated significantly until 1935, when an Executive Order stipulated that mill tailings could not be dumped into streams. Concerns today about contamination from historic mines are valid but should be tempered with an understanding of the fact that few or no regulations existed for the first 75 years of mining on the CWS.

Environmental assessment of watersheds and mine sites can be viewed as rigorous science or evolving art. I will not debate this issue here, but the reader needs to understand (1) that some aspects of geochemistry may not be quite as they appear and (2) that highly precise measurements by a skilled scientist may not be reproducible or appropriate to the perceived problem. The author brings more than 30 years of geologic and geochemical experience to this study but just a few years of work on some techniques such as water chemistry. The field observations, sampling, and interpretations in this study are based much more on geology than on protocols used by regulators and health scientists. Readers and FLMA's need to understand that my observations at a site are likely to be quite different from those of a skilled person with Colorado Department of Health; it is possible that while our data or observations might differ, both could be correct in their context. The results and interpretations reported here are reconnaissance in nature, designed to identify and rank the major problems on the CWS, and are given with the caveat that further detailed studies by more precise methods will be required to confirm and quantify chemical conditions.

Scale is a major operational problem for geo-environmental studies. Focus and detail are required to evaluate point sources and sites. In some situations a scientist may need to spend days characterizing water from one pipe or the waste on an acre site. At the other extreme is the need to understand numerous kinds of mines and geologic settings across more than 10,000,000 acres of land on the CWS, with many kinds of ownership, to begin to comprehend trends in geochemical data. In an attempt to deal with issues of scale, discussions in this report will start with watershed scale of the four major rivers and will shift to site scale for limited discussion of problem sites. Because my fieldwork was reconnaissance in nature, I generally did not have detailed maps or knowledge of land status, thus cannot stipulate for most sites whether the status is private, public, or mixed. The study area on the CWS and the districts that were investigated and sampled for geochemical analysis are shown on figure 1 .

Work in the field was guided by information in the literature, in digital databases, and on published 1:24,000-scale U.S. Geological Survey (USGS) topographic maps that show major mining operations. The goal in most districts was to visit representative and substantial mines and mill tailings to characterize the district ${ }^{1}$ and observe and sample the most likely sources of contamination. While in the field, the next criterion was accessibility - both to save time and with an eye on potential for reclamation (experience elsewhere suggests that reclamation is less likely to happen if new roads must be constructed). And, finally, hands-on work and sampling was influenced by visible indications of land status. If mine sites were posted as private, or fenced and gated, these sites were not examined. If there was no diligence (signage or fences), and if there were indications of substantial traffic by the public, then I would go on the property and collect samples for chemical analysis. For water characterization, an attempt was made to sample streams above, immediately below, and a mile or two below the major mines or mills.

This study was made in parallel with geologic and mineral resources studies of the same area by colleagues in the USGS (Bankey, in press). Studies of area geology (Day and Bove, in press), mineral deposits (Wilson and Spanski, in press), and geochemistry (Smith, in press) were accomplished in that program.

Acknowledgments.-This study has benefited from the support and suggestions of many people in the USGS, U.S. Forest Service (USFS), and U.S. Bureau of Land Management (BLM). The broad goals of geo-environmental investigations and the need for more information on the CWS were defined by Geoff Plumlee, USGS. I appreciate the support given by my supervisors for experiments with reconnaissance methods and new ways to present results. Colleagues on the CWS mineral assessment project have shared their knowledge and have provided preliminary maps. Staff scientists with the USFS and BLM in district offices have suggested sites and areas in need of study. I thank in particular Barbara Hite, BLM, and Daryl Gusey, USFS, for numerous suggestions and feedback as this work evolved. Preliminary drafts of manuscripts were reviewed by Bob Eppinger, Harley King, Bill Miller, and Brad Van Gosen, who offered numerous helpful suggestions. Errors of fact or interpretation that remain are the responsibility of the author.

\footnotetext{
1 District is used informally in this report, chiefly for geography and history rather than for legal implications. Names applied to districts can vary, and I have attempted to use the most common one. In a companion study of mineral resources of this study area, my colleagues Wilson and Spanski (in press) use the term "mining area," which is appropriate but not in general usage.
} 


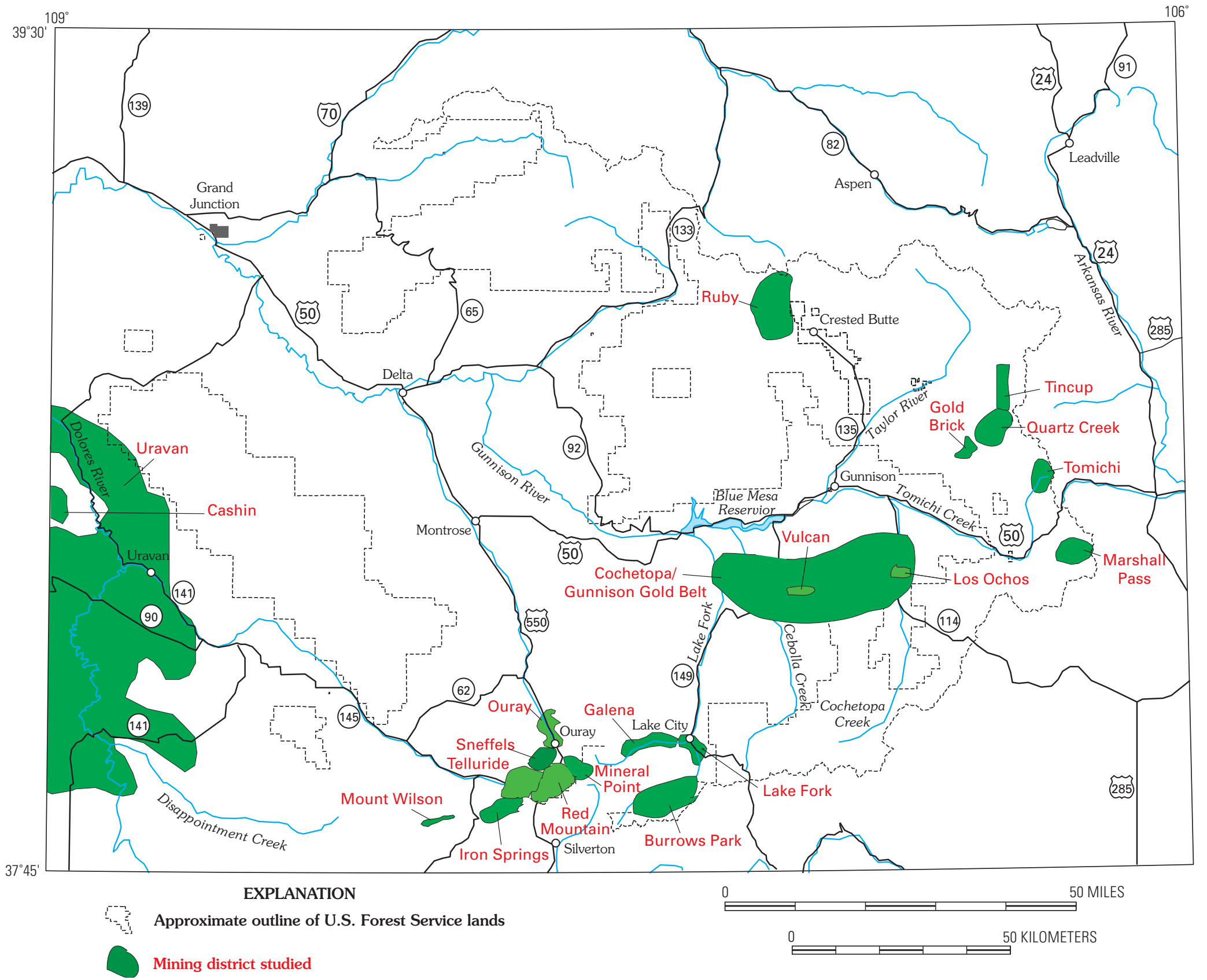

Figure 1. Location of mining districts studied, central Western Slope, Colorado. 
Hydrogeochemical Investigations of Historic Mining Districts, Central Western Slope of Colorado

\section{Methods}

Fieldwork for this study was conducted in June, July, and September of 1997, July and August of 1998, and in September of 1999. Many of the districts were visited more than once as the goals of the project evolved or to seek better water samples earlier in the year. Sites visited were determined by published information, USGS records in the Mineral Resources Data System (MRDS), information on USGS 1:24,000-scale topographic maps, by suggestions from BLM and USFS colleagues, and by access. Sample localities were recorded on standard 1:24,000-scale topographic maps and measured onsite with a conventional GPS (global positioning system) instrument having an accuracy of about $\pm 200 \mathrm{ft}$ based on tests at known sites; the GPS unit was run under standard WGS84 for datum and spheroid. Sample sites and analyzed samples are briefly described, and locations given, in table $1^{2}$.

\section{Rock and Mine-Waste Geochemical Studies}

Mineralized samples were collected from mine dumps, outcrops, mill tailings, and smelter sites. In most cases the intent was to collect a representative sample, but for some sites a special sample was collected to determine a special property. The most commonly employed sampling method for dumps and tailings involved the collection of numerous small portions at 10 to 30 sites, then processing those subsamples through a 2-mm stainless steel sieve to derive a composite sample weighing about 2 to 4 pounds.

Rock samples were crushed and pulverized for analysis by the USGS and analyzed by a commercial laboratory. In one method, the rocks are dissolved in a mixture of four acids, then the concentration of 35 major and trace elements are determined using ICP-AES (inductively coupled plasmaatomic emission spectroscopy); this is considered a total analysis because the strong acids dissolve all but the very refractory minerals such as zircon. Mineralized samples were also analyzed by a method that employs weaker acids to dissolve most minerals, then uses an organic reagent to collect 11 metals of interest (Ag, As, $\mathrm{Au}, \mathrm{Bi}, \mathrm{Cd}, \mathrm{Co}, \mathrm{Cu}, \mathrm{Mo}, \mathrm{Pb}, \mathrm{Sb}$, and $\mathrm{Zn}$ ); analysis is by ICP-AES. This second analytical method has lower levels of determination and works well for some metallic elements. Quality-assurance monitoring showed that the deviation of the reported values from correct ones is less than 5 percent for most elements.

Analytical results for mineralized rock samples (dumps, tailings, altered rocks) are in table $2^{2}$; these results are also available in the Data folder at the root directory of this CD-ROM as the original spreadsheet file (NGMR.xls and NGMR.csv).

\section{Water Geochemical Studies}

Water compositions were investigated by a reconnais- sance sampling method when water was observed at mine portals, seeping from mine dumps or tailings, or where streams were found near mining operations. The volume of flow was estimated at each site but is very approximate. Notes were taken on the nature of any mineral coatings related to the water, if any, because these often are an indicator of degraded water quality. However, in the case of highly acidic waters, there is no immediate precipitate; thus, the waters have the appearance of good quality, neutral waters.

Methods used to measure $\mathrm{pH}$, conductivity, and sample waters in the filed are described in Appendix 1. The routine method for sample collection in plastic bottles is not effective for mercury because it becomes adsorbed on the container and is not available for analysis (J. Gray, oral commun., 1998). In 1998, some representative samples were collected in special glass bottles containing a chromic acid preservative for $\mathrm{Hg}$, courtesy of Jim Crock, USGS. The filtered and acidified water samples were analyzed by a commercial laboratory in Denver, Colo., using ICP-MS. The elements determined by this method and quality control are discussed in Appendix 1.

Analytical results for water samples collected and analyzed for this study are in table $3^{2}$; the results are also included in the Data folder at the root directory of this CD-ROM in spreadsheet form as NGWC.xls and NGWC.csv. Results for 30 samples collected in 1999 are shown at the end of table 3 but appear in a separate file in the Data folder at the root directory of this CD-ROM (NGWC9.xls, NGWC9.csv).

\section{Laboratory Leach Tests of Mineralized Samples}

Solid materials from dumps, mill tailings, outcrops, and slag piles have been processed in the laboratory by a passive leach method (Fey and others, 2000a) that provides a measure of reactions in nature, such as during storm events. Quite simply, $100 \mathrm{~g}$ of rock sample was placed in a beaker with $2,000 \mathrm{~mL}$ of deionized water, stirred slightly, and an initial $\mathrm{pH}$ measured. After about 20 hours, the upper part of the liquid was stirred slightly to mix the leachate solution. At 24 hours, the $\mathrm{pH}$ and conductivity of the leachate were measured and a 60-mL aliquot was taken with a syringe and passed through a $0.45-\mu \mathrm{m}$ filter, just as was done for water samples in the field. The leachate samples were acidified with 5 drops of 1:1 ultrapure $\mathrm{HNO}_{3}$ to stabilize metals ${ }^{3}$ in the solution-chiefly to minimize adsorption on the plastic bottle or formation of precipitates. Results from this method closely resemble those from EPA method 1312 that tumbles the sample (Fey and others, 2000a). For this project, the leachate was sent to a

\footnotetext{
2 Tables 1-3, because of their length, appear in Appendix 2 at the end of this report. Appendix 2 begins on page 152.

3 The term "metal" will be used in a broad sense in many places in this report to include metaloids and nonmetals, such as As and Se; this broad context will not consider speciation such as As ${ }^{I I I}$ and $A s^{V}$, or oxidation state, as in the case of oxyanions $\left(\mathrm{MoO}_{4}\right)$.
} 
commercial laboratory and analyzed by ICP-MS in the same manner as water samples. This analytical method is well suited for this task because the leachates are highly varied in composition and ICP-MS provides results for elemental concentrations that span the range from less than 0.1 to $1,000,000$ $\mathrm{ppb}$ (parts per billion, the same as microgram/liter except at very high concentration); the more concentrated solutions are diluted 1:10 to 1:50 to stay within the calibration range. Anions are not determined by ICP-MS; no supplemental analyses for them were made because the dominant anion is expected to be sulfate. Quality-control tests using replicate samples and standard waters show that the results have a precision and accuracy of about \pm 10 percent for most elements, which is well within the sampling error and probable seasonal variability of waters in this study. These analyses are effective for the description and ranking of samples or sites.

For most rock samples, no sample preparation was done for these tests. The majority of dump samples were sieved in the field to pass through a 2-mm sieve. Mill tailings are, by their nature, ground materials of fine sand size, thus they did not require grinding or sieving. A special type of dump or tailing sample, efflorescent crusts, was picked in the field and cleaned somewhat in the laboratory to provide material for leaching that was more than 50 percent crust. The samples were dry and friable when weighed and placed in the reaction vessel.

The behavior of samples during the 24-hour tests was variable. For many samples, especially those with abundant iron oxides or jarosite (rusty-appearing minerals), the $\mathrm{pH}$ dropped to below 3.5 in just a few minutes. In some samples, the $\mathrm{pH}$ evolved to lower values during the 24 hours, and in a smaller number of samples ( 20 percent), the $\mathrm{pH}$ rose a few tenths of a unit, reflecting acid consumption in reactions with minerals in those samples. Most of the materials created orange- to pale-yellow-colored water; a few were light gray and some introduced little color. Suspended material in the leachates tended to settle in about 10 hours, and these were easy to filter. A small percentage of samples created milky solutions that did not clear overnight, and some of these were difficult to filter because clay- or colloid-sized material clogged the filter. Elements in the solution for analysis, which passed through the $0.45-\mu \mathrm{m}$ filter, are commonly termed "dissolved" in the literature (as opposed to "colloidal" or "total").

Analytical results for the leachate solutions and the leachate $\mathrm{pH}$ are in table 4; these results are also in the Data folder at the root directory of this CD-ROM in spreadsheet format (NGLC.xls, NGLC.csv).

\section{Geographic Setting and Climate}

The study area spans diverse ecoregions from the alpine zone at the Continental Divide to pinyon-juniper or sagebrush-vegetated semidesert of the Colorado Plateau at the Utah border (Bailey, 1998). The climate of mining areas varies both across the study area and locally. Because water is the key issue in this study, and because water drives most of the chemical reactions that create acid or mobilize metals, annual precipitation and style of precipitation are important factors in the geo-environmental setting of ore deposits (Plumlee, 1999). Climate records (Benci and McKee, 1977; Colorado Climate Center, 1984) provide helpful information, but one must remember that local conditions on a mountain can be much more severe than at the nearest weather station.

Precipitation in the CWS ranges from less than 10 inches per year at low elevations on the western side to more than 50 inches above 11,000 ft elevation. Many of the major mining districts receive snow for 10 months of the year, and avalanches were major threats to the early miners who lived near the early mines. The high mountains also tend to get major rainfall in the late summer from storms originating to the southwest; these storms can dump several inches of rain in less than 24 hours and create flash floods (Follansbee and Sawyer, 1948). Precipitation and evaporation vary locally on sunny southern slopes and dark north-facing slopes; this appears to be a significant factor in detail, such as observed in mine drainages of the Silverton area (Nash, 1999a) but will not be considered further here. Just as changes in vegetation are easily seen as a function of climate, one can see the higher volume of mine runoff and adit drainage in snowy or wet mountain settings that contrast with relatively dry settings, as in the Cochetopa district (fig. 1), where adits are dry and crusts of sulfate minerals form when waters evaporate on mine dumps. The kinds and magnitudes of mining-related geochemical problems are predictably related to climate: mine drainage is most voluminous and often most degraded at wet, high elevation sites; seasonal or episodic flash floods or flushes tend to be more significant at dryer, lower elevations. These associations will be discussed in later sections.

Many books chronicle the history of mining camps in Colorado and provide useful information on the mining districts described here. Some of the books, for example by Benham (1980), Smith (1994), and Southworth (1997), also provide prints of historic photographs that clearly show the rugged conditions and simple structures of the early mines and associated communities.

\section{Introduction to Ore-Deposit Types and Their Environmental Signatures}

Many types of ore deposits have been located or mined in the CWS over the past 130 years. Economic geologists utilize numerous classification systems to compare and summarize geologic and economic attributes of deposits (Lindgren, 1933; Cox and Singer, 1986; Guilbert and Park, 1986). In the resource assessment of the CWS, more than 25 mineraldeposit types were considered (Spanski and Bankey, in press; Wilson and Spanski, in press). These classifications subdivide 
[Samples passively leached for 24 hours in deionized water; leachate filtered $(<0.45 \mu \mathrm{m})$ and acidified; solution analyzed by ICP-MS in commercial laboratory. Sample type: third character in sample ID indicates type: D, dump; T tailing; R, rock (unmined); C, crust on dump or tailing sample. Values are reported in parts per billion; <, not detected at value shown; number of figures reported by spreadsheet may not be significant; nd, not determined. Districts: CO, Cochetopa; GB, Gold Brick; GN, Galena; IS, Iron Spring; LO, Los Ochos; MP, Mineral Point;OU, Ouray; QC, Quartz, Creek; RB, Ruby; RM, Red Mountain; SN, Sneffels; TE. Telluride; TM, Tomichi; TC, Tincup; UV, Uravan. Deposit type: BP, breccia pipe; FE, fericrete; PMR, polymetallic replacement; PMV, polymetallic vein; SC, sediment-hosted copper; SK, skarn; SU, sandstone uranium; TV, volcanic-hosted vein; VMS, volcanogenic massive sulfide]

\begin{tabular}{|c|c|c|c|c|c|c|c|c|c|c|c|c|c|c|c|c|c|}
\hline Sample ID & District & $\begin{array}{l}\text { Deposit } \\
\text { type }\end{array}$ & $\mathrm{pH}$ & $\begin{array}{c}\text { Conductivity } \\
(\mu \mathrm{S} / \mathrm{cm})\end{array}$ & $\begin{array}{c}\mathrm{Al} \\
(\mathrm{ppb})\end{array}$ & $\begin{array}{c}\text { As } \\
\text { (ppb) }\end{array}$ & $\begin{array}{c}\mathrm{Cd} \\
(\mathrm{ppb})\end{array}$ & $\begin{array}{c}\mathrm{Cu} \\
(\mathrm{ppb})\end{array}$ & $\begin{array}{c}\mathrm{Fe} \\
(\mathrm{ppb})\end{array}$ & $\begin{array}{c}\mathrm{Mg} \\
(\mathrm{ppb})\end{array}$ & $\begin{array}{l}\mathrm{Mn} \\
\text { (ppb) }\end{array}$ & $\begin{array}{l}\text { Mo } \\
\text { (ppb) }\end{array}$ & $\begin{array}{c}\mathrm{Pb} \\
(\mathrm{ppb})\end{array}$ & $\begin{array}{c}\mathrm{Sb} \\
(\mathrm{ppb})\end{array}$ & $\begin{array}{c}\mathrm{Se} \\
(\mathrm{ppb})\end{array}$ & $\underset{(p p b)}{U}$ & $\begin{array}{c}\mathrm{Zn} \\
(\mathrm{ppb})\end{array}$ \\
\hline NGCL223 & & & & & & & & & & & & & & & & & \\
\hline NGCL225 & $\mathrm{CO}$ & VMS & 3.3 & nd & $1,537,171.9$ & 21.4 & $1,494.5$ & $70,581.8$ & 60,976 & $3,064,039$ & $275,626.5$ & 4.4 & 8.0 & 0.2 & 360.5 & 29.7 & $322,773.8$ \\
\hline NGCL227 & $\mathrm{CO}$ & VMS & 2.1 & nd & $299,391.8$ & $12,507.1$ & $1,212.4$ & $9,584.0$ & $1,908,102$ & 191,911 & $1,745.2$ & 950.3 & $1,781.3$ & 2.9 & $27,422.3$ & 13.8 & $350,125.8$ \\
\hline NGCL228C & $\mathrm{CO}$ & VMS & 1.9 & $>2,000$ & $308,410.4$ & 233.6 & $3,777.1$ & $222,516.2$ & $1,878,930$ & 315,452 & $4,723.1$ & $1,277.2$ & 22.3 & 0.9 & 905.6 & 47.0 & $934,449.8$ \\
\hline NGCL504 & $\mathrm{CO}$ & VMS & 2.3 & $>2,000$ & $429,021.9$ & 346.4 & $3,734.2$ & $446,646.8$ & $2,577,782$ & 356,969 & $4,352.8$ & 55.7 & 201.6 & $<0.1$ & 602.4 & 40.3 & $962,023.5$ \\
\hline NGDL101 & UV & SU & 7.1 & nd & 4.9 & 19.3 & 0.1 & 1.3 & 1,344 & 1,018 & 0.7 & 11.3 & 0.3 & 0.4 & 32.3 & 0.2 & 2.8 \\
\hline NGDL102 & UV & SU & 7.2 & nd & 6.4 & 23.5 & 0.1 & 0.6 & 1,469 & 2,153 & 0.8 & 18.8 & 0.1 & 0.4 & 81.0 & 0.1 & 2.0 \\
\hline NGDL108 & UV & SU & 8.1 & nd & 69.2 & 8.9 & 0.1 & 6.7 & 48 & 763 & 1.7 & 4.6 & 3.4 & 0.2 & 4.7 & 1.1 & 1.8 \\
\hline NGDL109 & UV & SU & 8.2 & nd & 343.5 & 18.1 & 0.2 & 3.7 & 109 & 434 & 0.6 & 32.4 & 0.6 & 0.2 & 228.7 & 2.4 & 1.2 \\
\hline NGDL110 & UV & SU & 7.4 & 200 & 30.8 & 24.6 & 9.9 & 1.5 & 167 & 822 & 0.7 & $1,977.5$ & 0.5 & 1.8 & 79.5 & 0.7 & 2.7 \\
\hline NGDL111 & UV & SU & 7.2 & 330 & 19.2 & 13.6 & 2.4 & 1.2 & 232 & 1,878 & 7.8 & 797.4 & 0.5 & 1.7 & 706.6 & 1.0 & 5.1 \\
\hline NGDL112 & UV & SU & 6.0 & nd & 44.9 & 22.3 & 1.0 & 1.6 & 28 & 534 & 0.2 & 198.0 & 0.4 & 0.7 & 4.8 & 7.9 & 0.3 \\
\hline NGDL113 & UV & SU & 7.6 & 40 & 70.3 & 64.5 & 0.5 & 1.4 & 97 & 773 & 1.2 & 144.1 & 1.2 & 0.9 & 4.2 & 3.7 & 4.5 \\
\hline NGDL114 & UV & SU & 5.0 & nd & 11.9 & 2.1 & 0.1 & 1.1 & 1,366 & 432 & 1.0 & 11.6 & 0.1 & 0.1 & 16.0 & 0.7 & 2.5 \\
\hline NGDL116 & UV & SU & 6.2 & nd & 67.5 & 10.2 & 0.1 & 1.2 & 24 & 591 & 0.2 & 18.9 & 0.3 & 0.3 & 8.5 & 3.0 & 0.3 \\
\hline NGDL118 & UV & SU & 6.6 & nd & 104.2 & 32.4 & 0.1 & 0.7 & 49 & 1,339 & 0.6 & 10.8 & 0.7 & 0.4 & 18.8 & 3.7 & 2.9 \\
\hline NGDL201 & TM & PMR & 7.1 & nd & 80.6 & 1.6 & 0.2 & 9.8 & 1,843 & 599 & 75.8 & 4.8 & 595.6 & 0.2 & -0.2 & 0.3 & 486.2 \\
\hline NGDL204 & $\mathrm{TM}$ & PMR & 4.5 & nd & 6.7 & 1.3 & $1,305.8$ & 13.0 & 3,649 & 703,424 & $121,467.9$ & 5.6 & 485.8 & 0.2 & 40.8 & 11.5 & $130,908.6$ \\
\hline NGDL206 & $\mathrm{TM}$ & PMR & 5.7 & nd & 2.4 & 0.2 & 33.4 & 4.8 & 1,355 & 4,218 & 759.0 & 0.8 & 8.7 & 0.1 & 4.6 & 0.1 & $2,468.9$ \\
\hline NGDL207 & $\mathrm{TM}$ & PMR & 7.9 & nd & 6.2 & 2.7 & 8.3 & 2.9 & 24 & 541 & 2.7 & 1.6 & 18.1 & 1.3 & 0.2 & 0.0 & 37.5 \\
\hline NGDL209 & $\mathrm{TM}$ & PMR & 7.4 & nd & 6.7 & 0.5 & 1.5 & 1.4 & 59 & 2,073 & 1.5 & 2.8 & 16.4 & 0.2 & 6.7 & 0.0 & 17.9 \\
\hline NGDL213 & $\mathrm{TM}$ & PMR & 5.3 & nd & 2.6 & 0.1 & 102.0 & 4.3 & 1,434 & 17,712 & $2,367.0$ & 0.6 & 777.5 & 0.3 & 2.5 & 0.1 & $6,259.7$ \\
\hline NGDL217 & $\mathrm{CO}$ & VMS & 3.5 & nd & $15,228.3$ & 1.4 & 435.8 & $50,143.1$ & 1,210 & 34,359 & $2,375.3$ & 3.3 & 1.7 & 0.1 & 80.8 & 21.2 & $83,820.1$ \\
\hline NGDL223D & $\mathrm{CO}$ & VMS & 3.8 & nd & $9,986.4$ & 0.2 & 33.3 & $141,013.7$ & 572 & 267,471 & $7,781.5$ & 0.8 & 6.6 & 0.0 & 53.3 & 4.9 & $4,588.7$ \\
\hline NGDL224 & $\mathrm{CO}$ & VMS & 3.6 & nd & $20,043.8$ & 1.8 & 171.8 & $6,410.7$ & 11,214 & 52,791 & 858.3 & 1.5 & 0.8 & 0.0 & 66.7 & 2.3 & $35,534.4$ \\
\hline NGDL226 & $\mathrm{CO}$ & VMS & 2.5 & nd & $9,726.7$ & 53.7 & 35.1 & $1,962.7$ & 100,278 & 8,518 & 111.1 & 6.2 & 17.5 & 0.1 & 81.4 & 1.3 & $8,873.9$ \\
\hline NGDL229 & $\mathrm{CO}$ & VMS & 3.4 & nd & 595.9 & 0.6 & 9.5 & 920.2 & 4,358 & 504 & 15.9 & 0.5 & 4.3 & 0.0 & 5.0 & 0.4 & $2,602.3$ \\
\hline NGDL231 & $\mathrm{CO}$ & VMS & 3.3 & $>2,000$ & $68,449.0$ & 10.6 & 166.7 & $9,541.5$ & 10,971 & 122,412 & $4,387.1$ & 61.6 & 0.5 & 0.1 & 101.3 & 11.2 & $30,477.7$ \\
\hline NGDL235 & GB & PMV & 5.9 & nd & 45.5 & 2.4 & 0.4 & 7.4 & 47 & 344 & 6.9 & 0.4 & 2.1 & 0.2 & 0.3 & 0.0 & 17.9 \\
\hline NGDL240 & $\mathrm{TC}$ & PMR & 6.2 & nd & 34.8 & 1.6 & 0.0 & 2.8 & 49 & 808 & 4.7 & 0.8 & 3.0 & 0.8 & 0.2 & 0.0 & 0.8 \\
\hline NGDL241 & $\mathrm{TC}$ & PMR & 5.6 & nd & 15.8 & 4.6 & 0.2 & 24.4 & 54 & 502 & 10.8 & 1.6 & 17.5 & 1.7 & 0.2 & 0.0 & 20.1 \\
\hline NGDL242 & $\mathrm{TC}$ & PMR & 4.3 & 60 & 312.5 & 0.5 & 0.2 & 16.4 & 2,006 & 86 & 264.9 & 0.8 & 239.8 & 1.7 & 0.2 & 0.2 & 64.0 \\
\hline NGDL250 & LO & VU & 3.5 & nd & $4,432.5$ & 12.9 & 3.7 & 94.0 & 178 & 1,141 & 109.6 & 0.6 & 2.6 & 0.6 & 0.4 & 253.3 & 286.8 \\
\hline NGDL251 & LO & VU & 3.5 & nd & $9,006.0$ & 4.1 & 1.5 & 138.9 & 3,384 & 912 & 122.4 & 1.5 & 3.3 & 0.2 & 9.8 & 405.9 & 218.8 \\
\hline NGDL252 & LO & VU & 3.1 & nd & $5,670.2$ & 11.3 & 3.5 & 23.9 & 1,607 & 564 & 21.3 & 1.5 & 0.7 & 0.2 & 3.2 & 901.1 & 80.9 \\
\hline NGDL253 & LO & VU & 5.4 & nd & 57.1 & 16.4 & 0.1 & 3.9 & 21 & 88 & 1.4 & 10.0 & 0.8 & 1.6 & 0.2 & 7.4 & 0.9 \\
\hline NGDL255 & LO & VU & 7.7 & nd & 10.9 & 2.0 & 0.1 & 41.5 & 16 & 65 & 5.9 & 1.6 & 0.6 & 0.7 & 0.6 & 9.4 & 11.7 \\
\hline NGDL255 & $\mathrm{LO}$ & VU & 4.2 & nd & 13.9 & 2.0 & 0.1 & 40.3 & 16 & 63 & 6.2 & 1.0 & 0.3 & 0.7 & 0.9 & 10.4 & 10.4 \\
\hline NGDL256 & LO & $\mathrm{VU}$ & 3.1 & nd & $4,390.0$ & 8.7 & 32.2 & 136.3 & 971 & 1,536 & 352.0 & 9.2 & 1.7 & 3.0 & 2.6 & $3,402.7$ & $1,120.1$ \\
\hline NGDL261 & $\mathrm{CO}$ & VMS & 4.8 & nd & 7.5 & 9.3 & 544.9 & 659.8 & 611 & 17,194 & $2,959.7$ & 1.5 & 0.7 & 0.2 & 12.1 & 1.1 & $148,329.6$ \\
\hline NGDL262 & $\mathrm{CO}$ & VMS & 4.2 & nd & 813.2 & 112.0 & 487.8 & $33,287.7$ & 489 & 62,698 & $10,074.0$ & 0.9 & 7.3 & 1.0 & 54.8 & 7.2 & $139,927.7$ \\
\hline NGDL265 & $\mathrm{QC}$ & PMV & 3.7 & nd & 48.2 & 0.4 & 0.3 & 50.8 & 62 & 73 & 9.5 & 20.0 & 0.4 & 0.1 & 0.7 & 6.8 & 34.8 \\
\hline NGDL270 & $\mathrm{QC}$ & PMV & 3.5 & nd & $1,553.7$ & 17.6 & 25.5 & $4,671.2$ & 1,959 & 512 & 51.9 & 13.1 & 8.0 & 17.2 & 1.8 & 1.9 & $1,919.2$ \\
\hline NGDL274 & $\mathrm{TC}$ & PMR & 5.7 & nd & 8.3 & 0.6 & 2.1 & 3.0 & 109 & 2,117 & 79.5 & 4.9 & 0.9 & 0.4 & 1.9 & 1.0 & 39.0 \\
\hline
\end{tabular}


Table 4. Analytical results by ICP-MS for selected elements in leachate solutions, CWS, Colorado-Continued.

\begin{tabular}{|c|c|c|c|c|c|c|c|c|c|c|c|c|c|c|c|c|c|}
\hline Sample ID & District & $\begin{array}{l}\text { Deposit } \\
\text { type }\end{array}$ & $\mathrm{pH}$ & $\begin{array}{l}\text { Conductivity } \\
(\mu \mathrm{S} / \mathrm{cm})\end{array}$ & $\begin{array}{c}\mathrm{Al} \\
(\mathrm{ppb})\end{array}$ & $\begin{array}{l}\text { As } \\
\text { (ppb) }\end{array}$ & $\begin{array}{c}\mathrm{Cd} \\
(\mathrm{ppb})\end{array}$ & $\begin{array}{c}\mathrm{Cu} \\
(\mathrm{ppb})\end{array}$ & $\begin{array}{c}\mathrm{Fe} \\
(\mathrm{ppb})\end{array}$ & $\begin{array}{c}\mathrm{Mg} \\
(\mathrm{ppb})\end{array}$ & $\begin{array}{l}\mathrm{Mn} \\
(\mathrm{ppb})\end{array}$ & $\begin{array}{l}\text { Mo } \\
\text { (ppb) }\end{array}$ & $\begin{array}{c}\mathrm{Pb} \\
(\mathrm{ppb})\end{array}$ & $\begin{array}{c}\mathrm{Sb} \\
(\mathrm{ppb})\end{array}$ & $\begin{array}{l}\mathrm{Se} \\
(\mathrm{ppb})\end{array}$ & $\underset{(p p b)}{U}$ & $\begin{array}{l}\mathrm{Zn} \\
(\mathrm{ppb})\end{array}$ \\
\hline NGDL280 & TC & PMR & 4.1 & nd & 112.9 & 0.3 & 6.7 & 681.5 & 61 & 499 & 38.2 & 12.5 & 120.4 & 1.9 & 0.3 & 3.1 & 574.0 \\
\hline NGDL288 & GN & TV & 2.9 & nd & $10,606.7$ & 6.1 & 134.8 & $2,050.8$ & 16,930 & 9,902 & $3,315.2$ & 22.0 & 12.7 & 0.3 & 2.5 & 4.0 & $19,465.1$ \\
\hline NGDL303 & OU & PMR & 4.1 & nd & 13.4 & 0.2 & 1.3 & 37.9 & 32 & 303 & 296.2 & 1.8 & 35.2 & 0.4 & 0.4 & 0.5 & 176.3 \\
\hline NGDL321 & IS & TV & 3.1 & 850 & 582.7 & 0.6 & 3.8 & 377.2 & 1,520 & 716 & $1,447.9$ & 112.9 & 11.1 & 0.2 & 1.0 & 2.4 & 624.0 \\
\hline NGDL322 & IS & TV & 3.1 & 590 & 775.0 & 0.3 & 6.5 & 333.2 & 2,042 & 811 & 411.6 & 1.3 & 44.2 & 1.8 & 0.3 & 0.5 & $1,098.3$ \\
\hline NGDL327 & $\mathrm{RM}$ & $\mathrm{BP}$ & 2.7 & nd & $2,334.8$ & 22.2 & 14.1 & $2,534.4$ & 16,796 & 1,281 & 112.2 & 0.8 & $5,654.0$ & 8.3 & 2.8 & 0.5 & $1,479.5$ \\
\hline NGDL329 & RM & BP & 2.9 & 760 & $1,023.7$ & 0.8 & 0.4 & 475.5 & 5,253 & 784 & 51.4 & 33.0 & 4.6 & 0.2 & 0.4 & 0.5 & 83.4 \\
\hline NGDL330 & $\mathrm{RM}$ & BP & 2.7 & 1,550 & 337.1 & 110.5 & 1.1 & 994.0 & 14,229 & 322 & 7.7 & 1.2 & $2,650.0$ & 102.5 & 5.3 & 2.4 & 299.9 \\
\hline NGDL331 & RM & BP & 3.1 & nd & 474.9 & 1.6 & 1.4 & 250.4 & 688 & 415 & 73.8 & 0.7 & $3,867.4$ & 2.6 & 0.5 & 0.4 & 286.3 \\
\hline NGDL332 & $\mathrm{RM}$ & BP & 3.4 & 160 & 76.5 & 2.2 & 1.1 & 396.5 & 69 & 470 & 40.7 & 0.8 & $1,708.3$ & 1.0 & 0.2 & 0.1 & 361.0 \\
\hline NGDL339 & $\mathrm{RM}$ & BP & 3.2 & 390 & 111.8 & 0.4 & 0.4 & 74.4 & 1,279 & 280 & 51.1 & 15.9 & 297.8 & 0.4 & 0.3 & 0.1 & 187.5 \\
\hline NGDL341 & $\mathrm{RM}$ & BP & 2.5 & nd & $3,512.9$ & 34.0 & 14.6 & 618.2 & 37,143 & 1,487 & 58.8 & 2.7 & $4,684.8$ & 3.9 & 4.6 & 2.4 & $3,394.5$ \\
\hline NGDL350 & $\mathrm{RM}$ & TV & 2.7 & nd & $2,337.1$ & 0.2 & 31.8 & 119.8 & 6,501 & 1,278 & 633.9 & 0.7 & 2.6 & 0.0 & 1.8 & 0.4 & $6,399.7$ \\
\hline NGDL351 & RM & TV & 3.0 & 1,420 & 442.6 & 0.4 & 95.0 & 150.9 & 3,919 & 500 & 564.1 & 1.7 & 731.8 & 0.1 & 3.1 & 0.2 & $12,012.9$ \\
\hline NGDL352 & $\mathrm{RM}$ & TV & 3.7 & 90 & 49.5 & $<0.1$ & 3.7 & 7.7 & 23 & 194 & 139.4 & 1.3 & 2.8 & 0.1 & 0.2 & 0.1 & 900.9 \\
\hline NGDL355 & RM & BP & 3.1 & nd & 705.9 & 0.3 & 0.7 & 66.6 & 2,604 & 313 & 46.0 & 0.4 & $5,015.0$ & 0.7 & 0.7 & 0.7 & 145.6 \\
\hline NGDL358 & $\mathrm{RM}$ & BP & 3.7 & nd & 9.9 & 1.0 & 0.0 & 4.0 & 64 & 8 & 1.1 & 0.3 & 46.2 & 0.6 & 0.2 & 0.0 & 9.5 \\
\hline NGDL361 & $\mathrm{RM}$ & BP & 2.6 & nd & 739.1 & 37.3 & 1.1 & $1,608.0$ & 22,392 & 226 & 18.6 & 0.6 & $1,411.9$ & 23.3 & 3.8 & 1.3 & 290.4 \\
\hline NGDL366 & $\mathrm{RM}$ & TV & 3.4 & nd & 10.6 & 2.7 & 0.4 & 59.0 & 230 & 25 & 3.2 & 0.3 & $2,153.4$ & 5.3 & 0.4 & 0.0 & 64.6 \\
\hline NGDL372 & SN & $\mathrm{TV}$ & 5.5 & 70 & 8.1 & 0.1 & 0.2 & 0.6 & 84 & 993 & 20.4 & 10.5 & 2.6 & 0.2 & 0.5 & 0.0 & 19.6 \\
\hline NGDL379 & MP & TV & 3.4 & 420 & $1,276.9$ & 0.1 & 38.8 & 278.1 & 240 & 1,317 & $2,134.7$ & 1.6 & 242.3 & 0.2 & 0.6 & 0.1 & $3,542.4$ \\
\hline NGDL381 & MP & TV & 3.4 & nd & $3,395.8$ & 0.5 & 16.8 & $1,044.4$ & 795 & 860 & $1,011.6$ & 0.3 & 559.0 & 0.9 & 0.8 & 1.5 & $2,077.0$ \\
\hline NGDL389 & $\mathrm{TE}$ & TV & 4.0 & nd & 260.7 & 0.2 & 123.3 & 648.9 & 284 & 604 & 4305.3 & 1.0 & 3769.1 & 0.3 & $<2$ & 0.5 & 27115.1 \\
\hline NGDL390 & $\mathrm{TE}$ & TV & 3.6 & nd & 106.6 & 2.2 & 7.2 & 61.6 & 165 & 291 & 714.3 & 0.6 & 122.7 & 0.5 & $<2$ & 0.2 & 1658.9 \\
\hline NGDL391 & $\mathrm{TE}$ & TV & 2.8 & 940 & 757.9 & 33.5 & 3.2 & 127.8 & 8,626 & 180 & 87.8 & 3.2 & 205.3 & 1.0 & 0.2 & 0.1 & 643.2 \\
\hline NGDL395 & GN & TV & 2.7 & nd & $10,469.8$ & 79.4 & 176.2 & $1,288.7$ & 12,924 & 4,312 & $5,525.8$ & 5.0 & 3.4 & 1.4 & 4.4 & 3.3 & $21,480.7$ \\
\hline NGDL507 & $\mathrm{RB}$ & TV & 3.3 & 334 & 865.2 & 30.8 & 215.1 & $1,553.0$ & 9,405 & 1,994 & $10,973.0$ & 2.1 & $2,607.5$ & 0.1 & 27.4 & 0.5 & $28,313.8$ \\
\hline NGDL524 & $\mathrm{RB}$ & TV & 3.2 & 441 & 771.6 & 5.1 & 283.5 & $1,035.5$ & 879 & 1,117 & $17,614.2$ & $<0.05$ & $3,863.3$ & 0.2 & 12.3 & 3.4 & $39,459.7$ \\
\hline NGDL525 & $\mathrm{RB}$ & TV & 2.9 & 652 & 905.5 & 2.0 & 33.7 & 241.7 & 4,559 & 404 & 566.7 & $<0.05$ & $1,182.8$ & 0.1 & 1.6 & 0.3 & $2,443.0$ \\
\hline NGDL528 & $\mathrm{RB}$ & TV & 3.2 & 227 & 19.1 & 0.3 & 4.2 & 32.6 & 116 & 115 & 235.9 & $<0.05$ & $1,086.7$ & 0.0 & 0.7 & 0.0 & 366.9 \\
\hline NGDL556 & RB & TV & 3.0 & 485 & $2,139.3$ & 1.5 & 39.4 & 379.7 & 5,452 & 723 & $4,154.2$ & $<0.05$ & $5,305.8$ & 0.4 & 3.9 & 0.8 & $5,988.2$ \\
\hline NGDL585 & $\mathrm{RM}$ & TV & 2.7 & 903 & $1,643.5$ & 143.0 & 2.2 & $1,392.5$ & 35,098 & 551 & 53.5 & 5.6 & 624.7 & 4.6 & 5.1 & 0.2 & 381.0 \\
\hline NGDL591 & $\mathrm{RM}$ & TV & 2.9 & 525 & $1,807.4$ & 9.1 & 10.3 & $1,973.1$ & 23,966 & 2,845 & 133.4 & $<0.05$ & $2,579.6$ & 4.3 & 0.2 & 0.9 & $2,667.3$ \\
\hline NGDL598 & $\mathrm{RM}$ & TV & 3.1 & 209 & 501.5 & 2.0 & 13.6 & 638.5 & 531 & 625 & 174.5 & 0.3 & $2,164.8$ & 3.8 & 0.3 & 1.4 & $1,748.6$ \\
\hline NGDL606 & RM & TV & 3.0 & 325 & $1,144.0$ & 0.4 & 1.4 & 111.5 & 4,072 & 212 & 93.1 & 3.0 & 112.5 & 0.7 & 0.3 & 0.1 & 211.9 \\
\hline NGDL661 & $\mathrm{TE}$ & TV & 3.1 & 390 & 539.3 & 0.4 & 53.0 & 801.3 & 861 & 222 & $1,307.0$ & 0.4 & 47.4 & 0.3 & 0.9 & 1.3 & $7,168.7$ \\
\hline NGDL679 & SN & TV & 3.1 & 762 & $14,474.6$ & 1.8 & 29.0 & 113.6 & 4,615 & 11,714 & $15,488.4$ & $<0.05$ & 16.3 & 0.1 & 2.5 & 0.9 & $4,818.6$ \\
\hline NGGL201 & $\mathrm{TM}$ & PMR & 3.1 & nd & 80.6 & 1.6 & 0.2 & 9.8 & 1,843 & 599 & 75.8 & 4.8 & 595.6 & 0.2 & 0.2 & 0.3 & 486.2 \\
\hline NGRL311 & IS & FC & 3.8 & 60 & 252.1 & $<0.1$ & 1.8 & 95.4 & 4 & 64 & 22.9 & 1.3 & 2.8 & 0.1 & 0.2 & 0.4 & 78.6 \\
\hline NGRL326 & $\mathrm{RM}$ & BP & 4.6 & 30 & 8.9 & 2.2 & 0.1 & 3.7 & 98 & 116 & 7.8 & 0.4 & 4.9 & 0.7 & 0.2 & 0.0 & 23.6 \\
\hline NGRL329 & $\mathrm{RM}$ & BP & 4.3 & 60 & 362.8 & 0.4 & 0.2 & 12.1 & 2,112 & 79 & 247.4 & 0.7 & 256.9 & 1.2 & 0.2 & 0.2 & 74.6 \\
\hline NGRL617 & IS & $\mathrm{FC}$ & 3.5 & 49 & 188.6 & $<0.1$ & 1.6 & 65.9 & 20 & 22 & 4.3 & 0.1 & 12.5 & 0.0 & 0.2 & 0.4 & 50.3 \\
\hline NGRL682 & SN & TV & 4.9 & 29 & 37.5 & 0.6 & 0.2 & 3.8 & 56 & 127 & 33.1 & 4.7 & 1.2 & 0.2 & 0.2 & 0.0 & 16.2 \\
\hline NGTL203 & TM & PMR & 5.4 & nd & 1.7 & 0.2 & 90.4 & 1.0 & 1,939 & 271,529 & $16,299.3$ & 1.1 & 46.8 & 0.1 & 1.7 & 0.1 & $2,908.5$ \\
\hline NGTL212 & $\mathrm{TM}$ & PMR & 5.9 & nd & 30.5 & 0.1 & 43.5 & 2.7 & 953 & 67,029 & 367.9 & 1.1 & 21.1 & 0.1 & 2.7 & 0.1 & 515.0 \\
\hline NGTL237 & GB & PMV & 4.2 & nd & 23.3 & 32.6 & 36.0 & 174.1 & 1,324 & 930 & 528.5 & 3.6 & $3,386.4$ & 0.1 & 73.5 & 0.1 & $5,807.4$ \\
\hline NGTL238 & GB & PMV & 3.4 & nd & 365.5 & 13.4 & 79.2 & 152.8 & 601 & 1,391 & 480.3 & 1.6 & $2,247.5$ & 0.4 & 22.2 & 18.2 & $4,352.2$ \\
\hline NGTL239 & GB & PMV & 5.1 & nd & 23.5 & 0.3 & 1.1 & 9.1 & 30 & 313 & 27.2 & 0.4 & 35.8 & 0.1 & 0.2 & 0.0 & 39.1 \\
\hline NGTL272 & $\mathrm{TC}$ & PMR & 4.3 & nd & 159.7 & 0.3 & 213.8 & $2,010.2$ & 97 & 348 & 49.0 & 1.4 & $61,122.1$ & 0.2 & 0.3 & 0.1 & $1,907.0$ \\
\hline
\end{tabular}




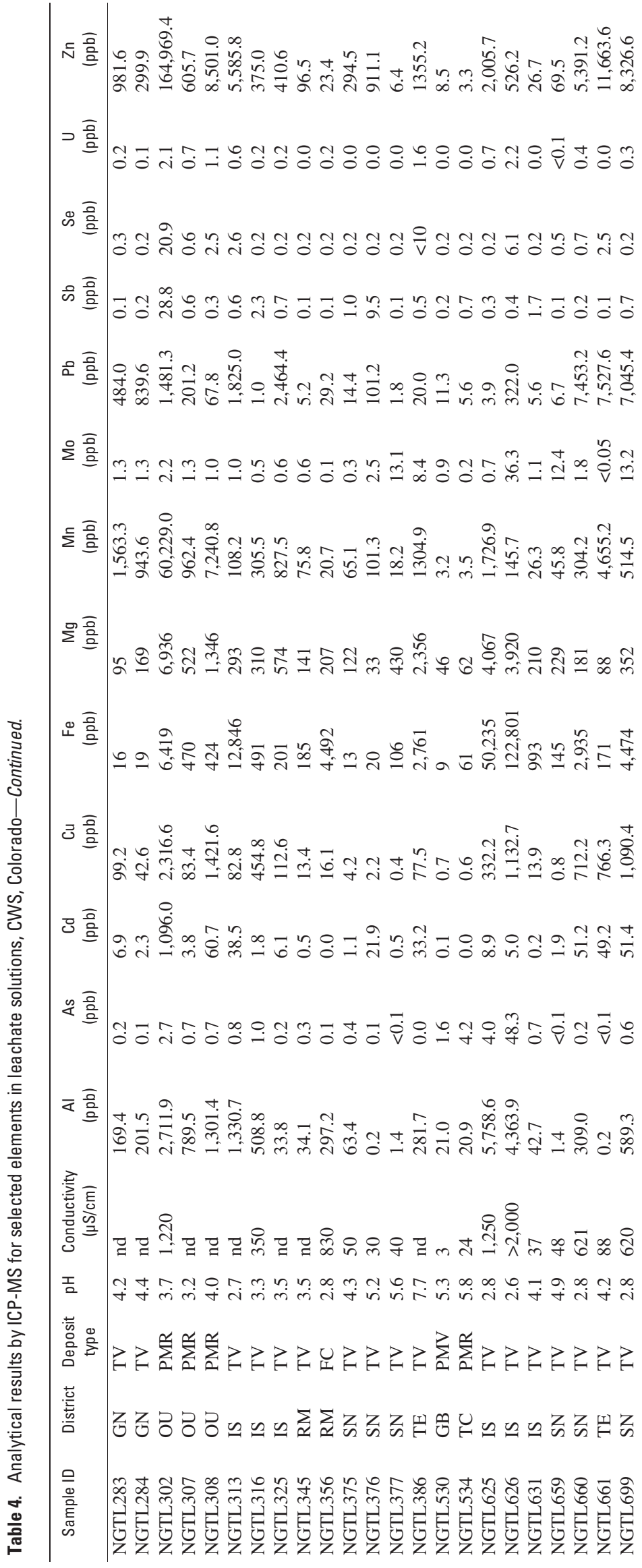

deposits according to finer points of geologic age, ore genesis, mineralogy, and additional attributes necessary to properly evaluate the economic potential (likelihood of occurrence, ore grade, ore tonnage, and ore metallurgy) of a geologic terrain or prospect. These specialized classifications include much detail that is not necessary for a general understanding of mine-related geochemical impact on the environment. A much simpler system is used here (table 5) that emphasizes fundamental geology (host-rock lithology) and ore composition, both of which play major roles in the generation and dispersion of acidic waters and metal contamination. Compositions of mined and mineralized rock samples are summarized in figures 2 and 3 .

\section{Polymetallic Deposits}

Many of the mines and prospects in the CWS are of the polymetallic type in which silver was the commodity of prime interest in the past. These deposits also carry substantial amounts of base-metal sulfide minerals, allowing some of the mines to recover copper, lead, or zinc. Gold was a byproduct in some. Many of the classic original mines of the region worked ores of this type. Although the polymetallic character, produced by abundant base-metal sulfide minerals with important traces of silver and gold, is generally similar among this group, economic geologists will recognize that there are many differences within this group. Some examples of this type are small, simple veins filled by quartz and sulfide minerals, formerly amenable to mining by small underground operations, as in the Ruby and Galena districts. Some were larger and deeper vein systems, as at the Idarado or Camp Bird mines of the Red Mountain and Sneffels districts. Some are vein and replacement zones in limestone, as at the Tincup or Tomichi districts. Some of the systems formed close to intrusive stocks and are rich in molybdenum and tungsten, as in the Quartz Creek or Eureka districts.

Polymetallic deposits have the potential to create acidic mine or rock drainage from the high amounts of sulfide minerals, and they have the potential to release many toxic heavy metals in those acidic waters (Plumlee and others, 1995a, 1995b; Plumlee and others, 1999). However, in this study and in ongoing research for the Animas watershed near Silverton (Nash, 1999a, 1999b), it is clear that geologic setting can be even more important than deposit mineralogy in the source and fate of metals and acid waters: host rocks can effectively mitigate locally acidic mine drainage at the mine site or close to it. Thus, the polymetallic group of deposits in the 


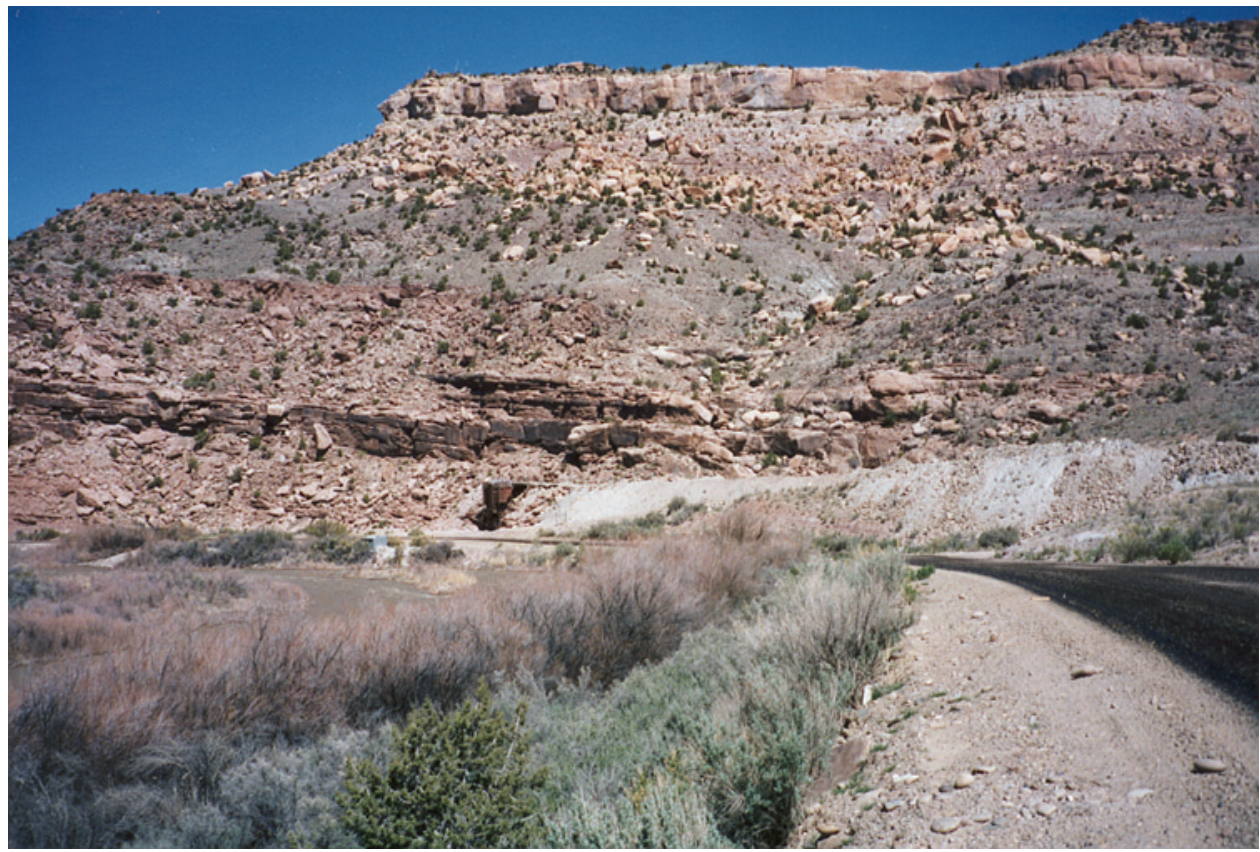

Photograph 1. The Dolores River at Slickrock creates an oasis for plants and wildlife and is in sharp contrast to the dry hills nearby. Uranium mine dumps are close to the riparian zone in many places. There is no mine drainage in this area, but rare extreme storm events create runoff and can erode mine dumps.

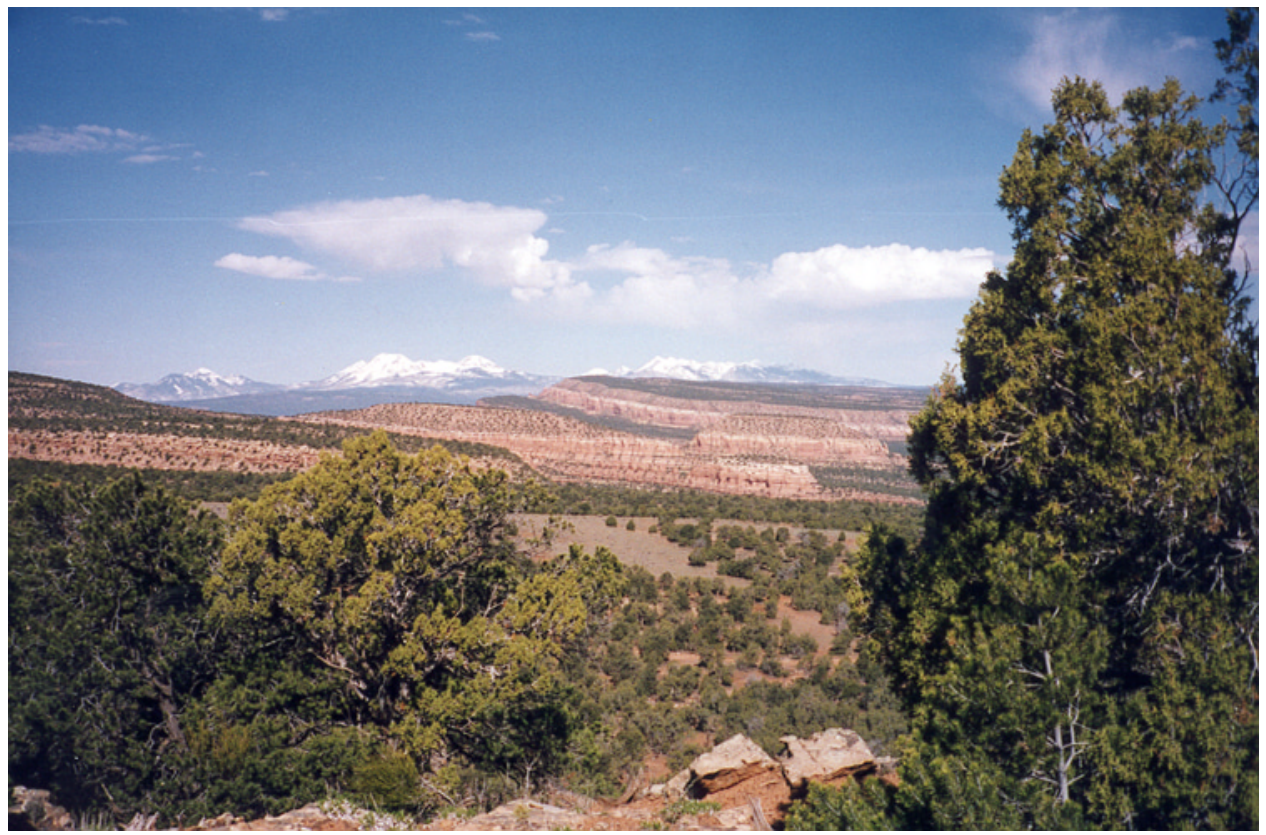

Photograph 2. Cedar-pinyon forests are characteristic of mesas in the Uravan district of western Colorado. This vegetation indicates that somewhat higher precipitation falls on the mesas relative to the lower valleys. 


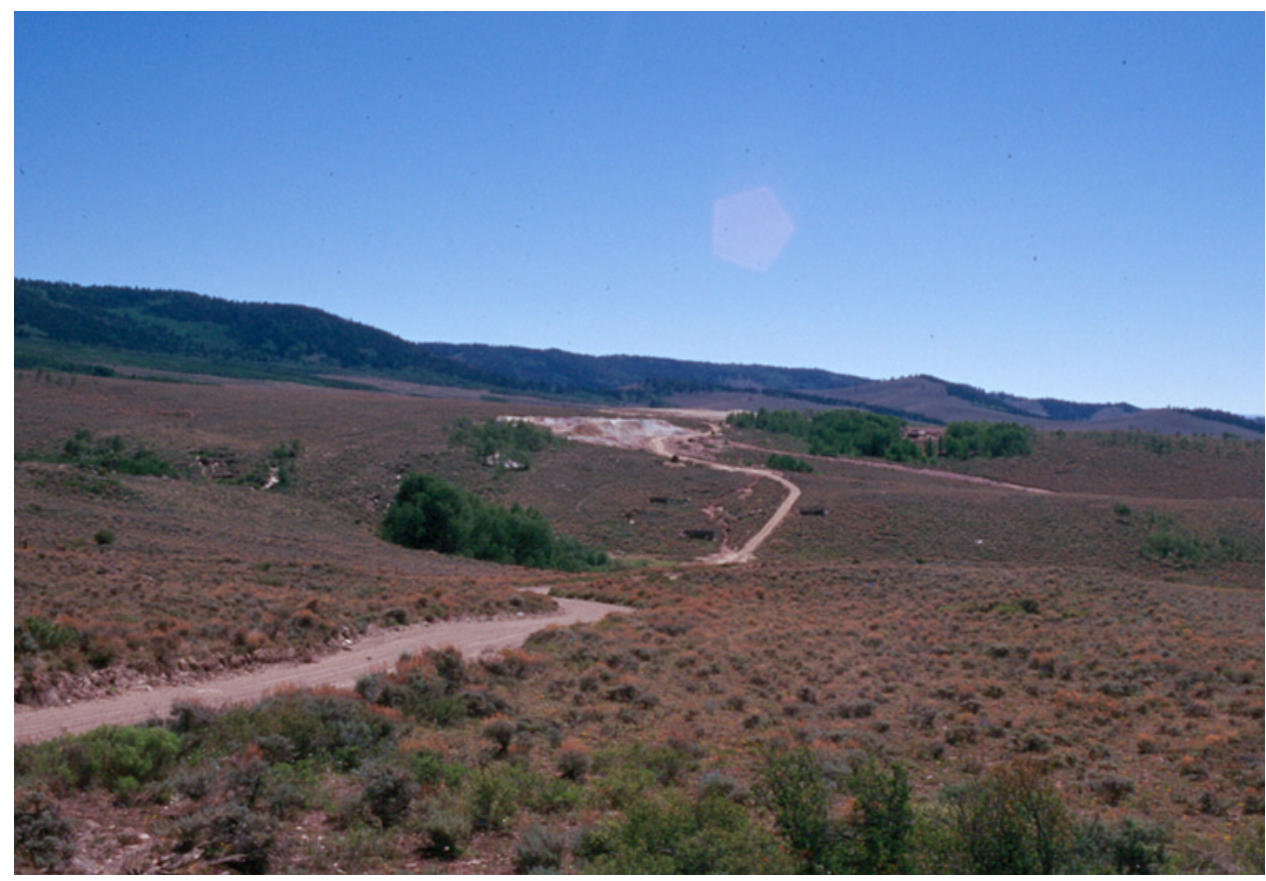

Photograph 3. The Cochetopa district is typical of sagebrush semiarid climate of the hills south of Gunnison at an elevation of about $9,000 \mathrm{ft}$.

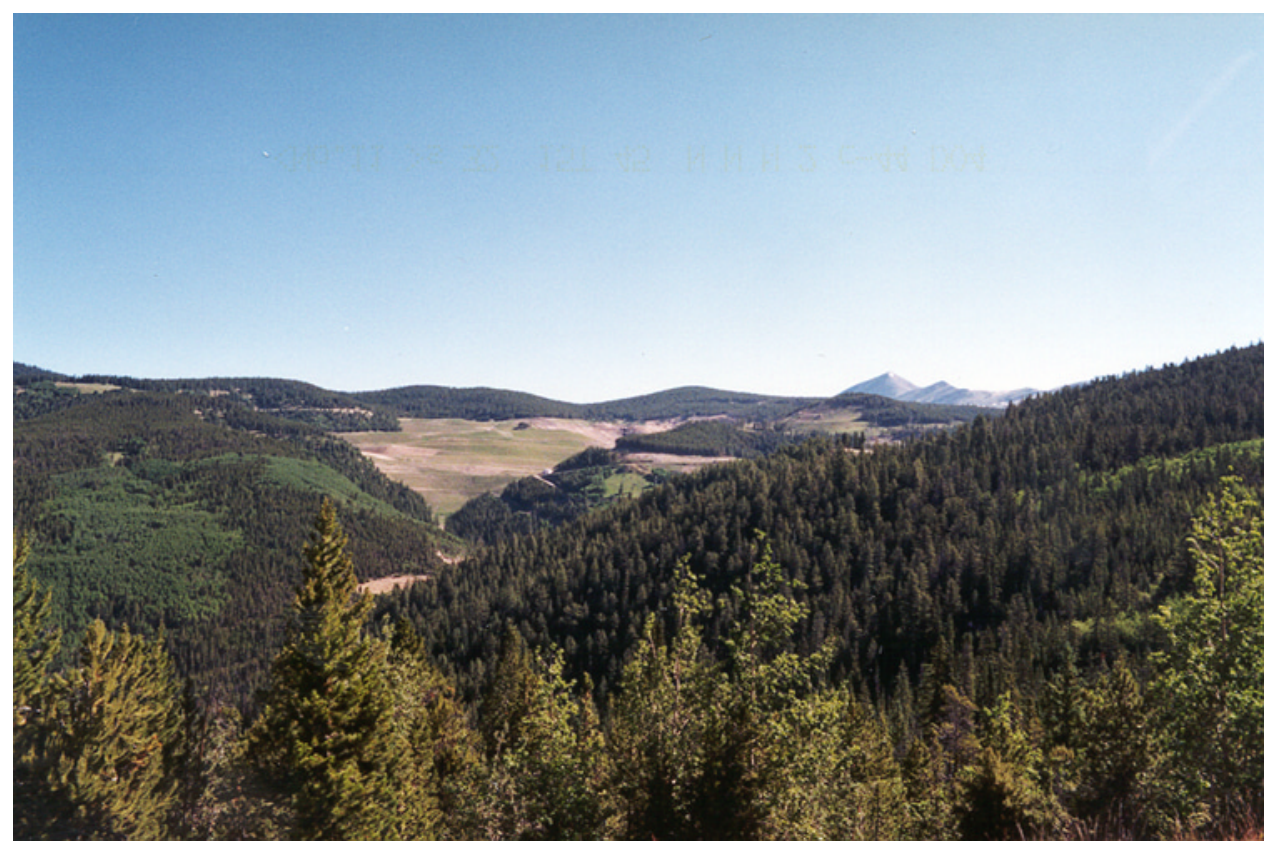

Photograph 4. The Marshall Pass uranium mining area at about $11,000 \mathrm{ft}$ elevation receives more than 200 inches of snow. Reclamation work has been done on waste dumps and the open pit of the Pitch mine, in the distance. The high amount of precipitation here, relative to other mining areas on the CWS, helps revegetation but creates problems in the pit lake and mine drainage that do not occur in other, drier uranium mining areas. 
Table 5. Simple classification of mineral deposits for environmental assessment, CWS, Colorado.

\begin{tabular}{|c|c|c|c|c|}
\hline Geologic setting & Ore controls & Mineralogy & Geochemistry & Examples \\
\hline \multicolumn{5}{|c|}{ 1. Polymetallic replacement, veins, or breccia pipe } \\
\hline $\begin{array}{l}\text { A. In altered igneous, } \\
\text { sedimentary rocks, } \\
\text { limestone }\end{array}$ & $\begin{array}{l}\text { Faults, veins, } \\
\text { replacements }\end{array}$ & $\begin{array}{l}\text { Multiple sulfides: pyrite, } \\
\text { chalcopyrite, galena, } \\
\text { sphalerite, Ag-minerals, } \\
\text { arsenopyrite. }\end{array}$ & $\begin{array}{l}\text { High } \mathrm{Ag}, \mathrm{As}, \mathrm{Cd}, \mathrm{Cu} \\
\mathrm{Mo}, \mathrm{Pb}, \mathrm{Zn} \text {, with } \mathrm{Au} \text {, } \\
\mathrm{Se}, \mathrm{Te}\end{array}$ & Whitepine; Tincup \\
\hline $\begin{array}{l}\text { B. In volcanic } \\
\text { rocks }\end{array}$ & $\begin{array}{l}\text { Faults, veins, } \\
\text { caldera margin }\end{array}$ & As above & As above & Idarado, Camp Bird \\
\hline C. In breccia pipe & $\begin{array}{l}\text { Pipes, small } \\
\text { intrusions }\end{array}$ & As above, silica & As above, high As & $\begin{array}{l}\text { National Belle, } \\
\text { Guston }\end{array}$ \\
\hline \multicolumn{5}{|c|}{ 2. Gold-quartz vein } \\
\hline $\begin{array}{l}\text { Tertiary volcanic } \\
\text { rocks, some in } \\
\text { pre-Tertiary rocks }\end{array}$ & $\begin{array}{l}\text { faults, } \\
\text { breccias }\end{array}$ & $\begin{array}{l}\text { Low sulfide content, } \\
\text { low galena, sphalerite, } \\
\text { high adularia }\end{array}$ & $\begin{array}{l}\text { High As, Sb, Hg, Ag, } \\
\mathrm{Au}, \mathrm{Se}, \mathrm{Te}, \pm \mathrm{Cu}, \mathrm{Pb} \text {, } \\
\mathrm{Zn}\end{array}$ & $\begin{array}{l}\text { Late-stage veins } \\
\text { Idarado, Camp Bird, } \\
\text { few mined alone }\end{array}$ \\
\hline \multicolumn{5}{|c|}{ 3. Massive sulfide } \\
\hline $\begin{array}{l}\text { Metavolcanic } \\
\text { terrane }\end{array}$ & $\begin{array}{l}\text { Bedded layers, } \\
\text { shears }\end{array}$ & $\begin{array}{l}\text { Very high pyrite, } \mathrm{Cu}- \\
\text { sulfides, sphalerite, }\end{array}$ & $\begin{array}{l}\mathrm{Cu}, \mathrm{Cd}, \mathrm{Fe}, \\
\mathrm{Zn}, \pm \mathrm{Pb}, \mathrm{As}, \mathrm{Sb}, \mathrm{Ag}\end{array}$ & Vulcan, Cochetopa \\
\hline \multicolumn{5}{|c|}{ 4. Sedimentary-hosted copper } \\
\hline $\begin{array}{l}\text { Sandstones, esp. } \\
\text { redbed sequences }\end{array}$ & Faults, bedding & $\begin{array}{l}\text { Cu-sulfide, } \mathrm{Cu} \text {-oxides } \\
\text { low sulfide content }\end{array}$ & $\begin{array}{l}\text { High } \mathrm{Cu}, \mathrm{Ag} ? \\
\text { Local U-V? }\end{array}$ & Cashin \\
\hline \multicolumn{5}{|c|}{ 5. Vein uranium } \\
\hline $\begin{array}{l}\text { Igneous, } \\
\text { sedimentary; } \\
\text { or volcanic rocks }\end{array}$ & $\begin{array}{l}\text { Faults, veins } \\
\text { igneous contacts }\end{array}$ & $\begin{array}{l}\text { High pyrite, uraninite, } \\
\text { autunite, iron oxides, }\end{array}$ & $\begin{array}{l}\text { High } \mathrm{U}, \text { radium, } \\
\pm \mathrm{As}, \mathrm{Cu}, \mathrm{Pb}, \mathrm{Se}, \mathrm{Zn} \\
\pm \mathrm{Cu}-\mathrm{Pb}-\mathrm{Zn} \text { sulfides }\end{array}$ & $\begin{array}{l}\text { Pitch mine, } \\
\text { Los Ochos }\end{array}$ \\
\hline \multicolumn{5}{|c|}{ 6. Sandstone uranium } \\
\hline $\begin{array}{l}\text { Sandstone, } \\
\text { shale }\end{array}$ & $\begin{array}{l}\text { Bedding } \\
\text { faults, organics }\end{array}$ & $\begin{array}{l}\text { Uraninite, carnotite } \\
\text { autunite, pyrite }\end{array}$ & $\begin{array}{l}\text { High } \mathrm{U}, \mathrm{V}, \mathrm{Se}, \mathrm{Mo} \text {, } \\
\text { radium }\end{array}$ & Uravan district \\
\hline \multicolumn{5}{|c|}{ 7. Porphyry Cu-Mo } \\
\hline $\begin{array}{l}\text { Igneous stocks, } \\
\text { intruded sediments }\end{array}$ & $\begin{array}{l}\text { Contacts, veins } \\
\text { stockworks }\end{array}$ & $\begin{array}{l}\text { High pyrite, } \mathrm{Cu} \text {-sulfides } \\
\text { molybdenite, } \mathrm{Cu} \text {-oxides } \\
\pm \text { fluorite }\end{array}$ & $\begin{array}{l}\mathrm{As}, \mathrm{Cu}, \mathrm{F}, \mathrm{Mo}, \mathrm{S} \\
\mathrm{Pb}, \mathrm{U}, \mathrm{Zn}\end{array}$ & $\begin{array}{l}\text { Mount } \\
\text { Emmons }\end{array}$ \\
\hline
\end{tabular}



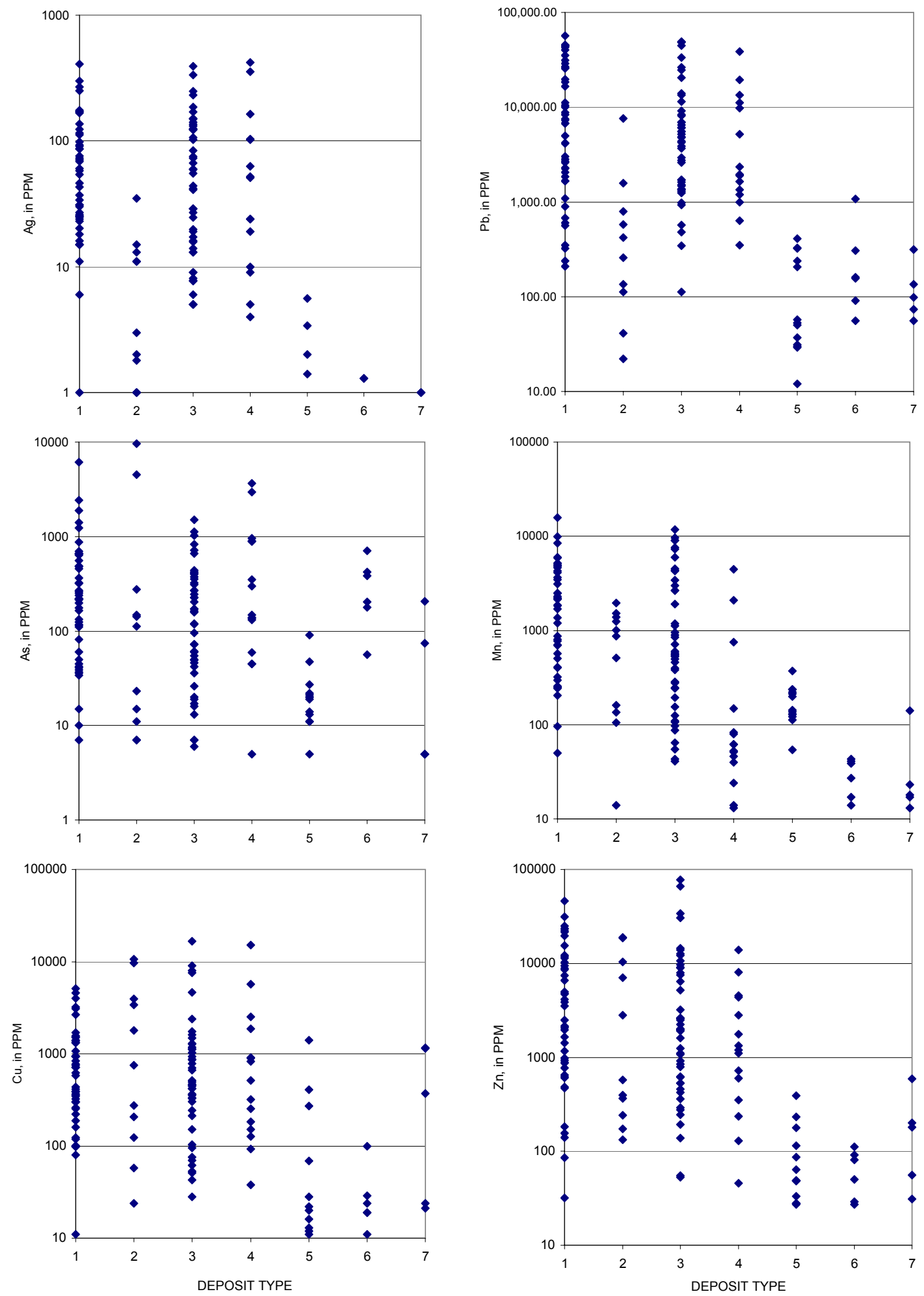

Figure 2. Composition of mined materials by deposit type, central Western Slope, Colorado. Deposit-type codes: 1, polymetallic vein and replacement; 2 , volcanogenic massive sulfide; 3 , polymetallic veins in volcanic rocks; 4 , breccia pipe; 5 , sandstone uranium; 6 , vein uranium; 7 , ferricrete. 

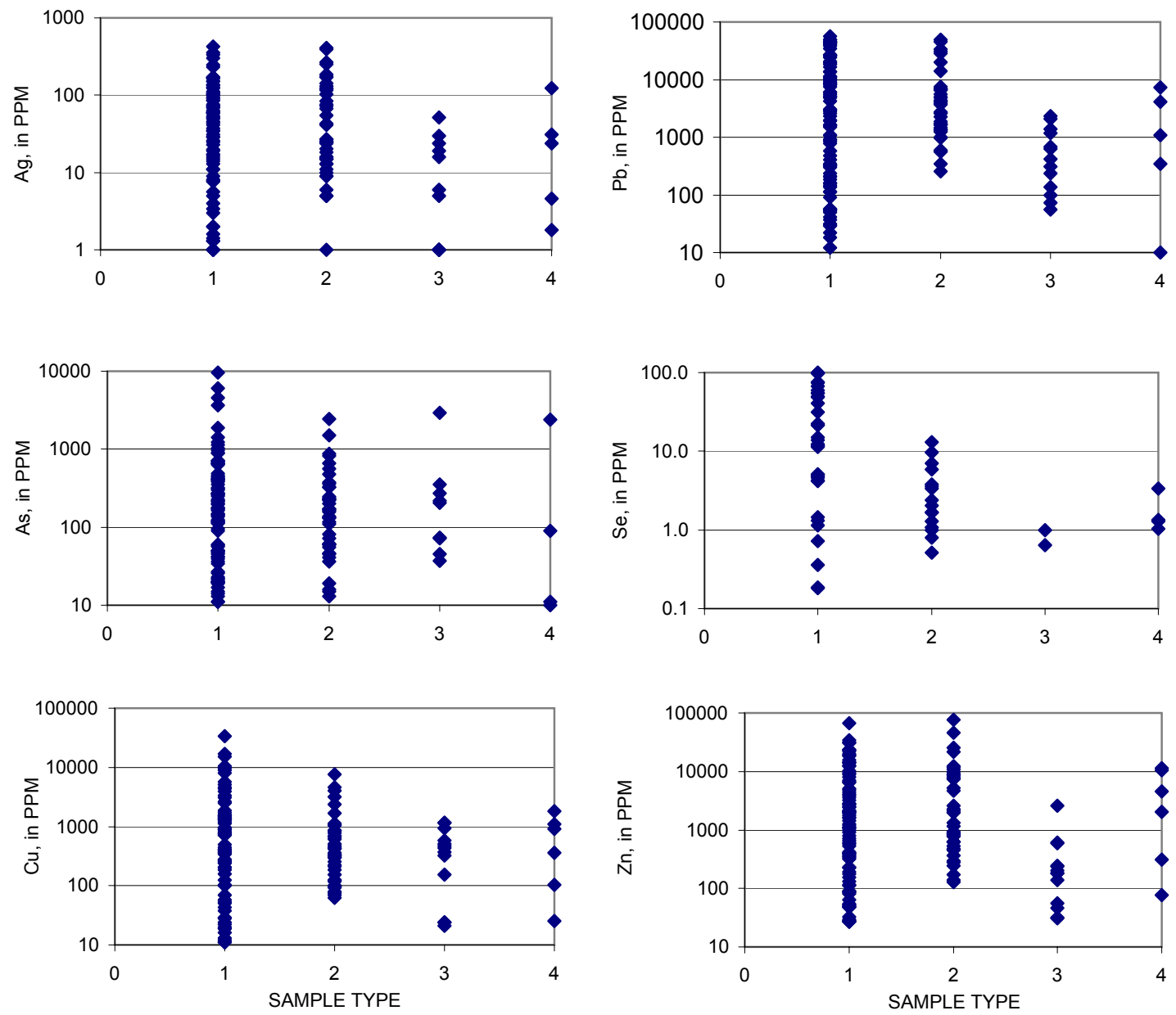

Figure 3. Composition of mineralized rocks by sample type, central Western Slope, Colorado. Sample types: 1, mine dump; 2 , mill tailing; 3 , unmined altered rock; 4 , smelter slag.

CWS can be subdivided according to host rocks and wallrock alteration into five classes: (1) volcanic-hosted, highly altered red rocks; (2) volcanic-hosted breccia pipes, silicified rocks; (3) volcanic-hosted, weakly altered green rocks; (4) carbonate-hosted, in sedimentary rocks with high acid-neutralizing capacity (ANC); and (5) other settings with low ANC, such as granite and quartzite. Deposits of types 2 and 3 have natural capacities to mitigate harmful effects by neutralizing acid and causing reactions that reduce metal concentrations (Smith and others, 1994; Plumlee and others, 1999). Deposits of type 4 tend to neither enhance nor mitigate acid generation and metal transport, whereas the setting of type 1 tends to enhance problems because they contribute acid and metal sources in unmined altered rocks and the unmined wallrocks have little or no ANC (alteration processes previously destroyed minerals capable of consuming acid). Because the polymetallic deposits are so numerous and important in the CWS, discussion of the four types is expanded below.

Volcanic-hosted deposits in highly altered red rocks.The prototype area is Red Mountain Pass where several mountains of bright red volcanic rocks help create some of the worst metal contamination in Colorado from both mined and unmined sources (Plumlee and others, 1995c; Plumlee and others, 1999). Mine drainage from portals, and natural springs in unmined rocks in this large area, commonly have $\mathrm{pH}$ values below 3 , and metal concentrations are commonly 10 to 1,000 times greater than water-quality standards. The Summitville mine in the eastern San Juan Mountains is in this class (Plumlee and others, 1999) and is now famous for creating terrible water contamination because of inadequate mining and milling methods (King, 1995). Some examples of this class contain relatively rare minerals such as alunite (aluminum sulfate) that can help contribute acid, but the chief problems are widespread pyrite in altered rocks and the low ANC of altered host rocks (Plumlee and others, 1999). Economic geologists term this alteration "acid sulfate type" for the sulfate minerals and the acid hydrothermal conditions of formation, not the acid mine drainage. This alteration, shown by the bright red iron oxide minerals that form during weathering, and the associated polymetallic deposits tend to form in volcanic centers and along caldera margins, but they can form in other settings, as in the small intrusion at Calico Peak, west of Rico, Colo. For 
Hydrogeochemical Investigations of Historic Mining Districts, Central Western Slope of Colorado

the CWS, examples are the Red Mountains of Red Mountain district and Red Mountain of the Galena district near Lake City.

Volcanic-hosted breccia pipe deposits in silicified rocks.-Breccia pipe deposits rich in silver were the first bonanza ores on the CWS and were aggressively mined in the Red Mountain district through the mid-1890's. These pipe deposits are associated with the red, acid-sulfate alteration zones but are distinguished here because these deposits are mined in many places, are characterized by intense silicification of brecciated and leached volcanic rocks, and are known to be rich in arsenic-bearing ore minerals (Burbank and Luedke, 1968; Burbank and others, 1972; Fisher and Leidy, 1972). The silica alteration zones have very low ANC, thus the pyrite-rich mine workings and waste materials create very acidic drainage, commonly below $\mathrm{pH}$ 2.5.

Volcanic-hosted deposits in weakly altered green rocks.Large areas of San Juan volcanic rocks are green from pervasive alteration called "propylitic" in which minerals such as chlorite, epidote, and calcite are added by hydrothermal fluids (heated waters, such as steam) (Burbank, 1960; Burbank and Luedke, 1961). Calcite and other carbonate minerals are well known for their high ANC, but even the chlorite in these green rocks has similar ANC according to recent research (Desborough and others, 1998). The beneficial properties of green alteration has been recognized (Smith and others, 1994), and this study demonstrates the wide distribution and importance of the green alteration in volcanic rocks. In some detail, pH's near 7 rather than 8 suggest that the buffering may be produced by chlorite rather than calcite; water in equilibrium with calcite should have a $\mathrm{pH}$ of about 8.1. The importance of these rocks is well shown in the Red Mountain district, where the eastern side, in red rocks of the Red Mountains, has numerous sources of highly acidic, metal-rich waters, whereas the western side, in green rocks, is characterized by circum-neutral waters. Equally dramatic, the extensive mining and milling in the Sneffels district creates relatively minor contamination in Canyon Creek, whereas similar practices in the Red Mountain Creek area have contributed highly polluted waters that overwhelm natural attenuation processes. Fortunately for the CWS, type-3 green alteration is present in more mining districts than the red type 1.

Carbonate-hosted deposits.-Polymetallic sulfide deposits were important as veins and replacements in pre-Tertiary sedimentary rocks in the Ouray, Tomichi, and other districts on the CWS, and these are characterized by relatively minor water contamination. The $\mathrm{pH}$ of surface waters is neutral to slightly alkaline, and the metals of concern are $\mathrm{Cd}$ and $\mathrm{Zn}$. The beneficial effects of carbonate rocks are well known (Smith and others, 1994; Plumlee and others, 1999) and more easily predicted from geologic maps and mine descriptions than the similar effects of green volcanic rocks. However, the near-neutral $\mathrm{pH}$ environment of carbonate rocks can pose a distinct set of problems relative to the better known acidic mine waters, including the transport of $\mathrm{Mn}, \mathrm{Mo}, \mathrm{Se}, \mathrm{As}$, and $\mathrm{U}$ in addition to Cd-Zn (Smith and others, 1994). Further, neutralization processes on acidic waters that precipitate many toxic metals do not occur in these drainages.

Other settings.-Polymetallic veins also occur in siliceous sedimentary, igneous, and metamorphic rocks that lack significant ANC. Veins in Precambrian granite gneiss near Cumberland Pass create local, small volumes of acidic mine drainage. If the deposits were large, there would be potential for major impact because silicate minerals in host rocks and alluvium are not capable of neutralizing the acidic waters. Deposits in these settings were mined in the Gold Brick and Quartz Creek districts, but the deposits of this type are neither large nor common on the CWS and do not appear to pose significant problems.

\section{Gold-Quartz Vein Deposits}

Veins in which gold is the chief or only commodity of value have been mined in many parts of the Western United States, and some have been mined on the CWS. Because of the high unit value of gold in recent years, deposits containing only trace concentrations of gold (about 2 parts per million) have been mined and continue to be mined in Nevada. In many of these Nevada deposits, the concentrations of sulfide minerals and base metals is very low, which means they pose few environmental hazards (Nash and Trudel, 1996); most large mines in Nevada are in sedimentary rocks with high ANC, and acidic drainage is rarely a problem (Hofstra and others, 1995). On the CWS, gold-quartz-adularia veins are well known and have contributed important Au values to many mines. These veins occur along polymetallic veins, but they formed at a slightly different stage, and, generally, both vein types are mined together. In the 1980's the possibility of selectively mining gold veins in the upper part of the Camp Bird mine was investigated. The waste products from gold-bearing veins are nearly always commingled with waste from polymetallic veins. In this study, it was not possible to collect samples of mine waste or mine drainage to characterize this type of deposit, thus gold veins will not be considered further as a separate ore type in this investigation.

\section{Massive Sulfide Deposits}

These deposits are extremely rich in pyrite and can be mined for their content of sulfur, copper, zinc, or byproduct silver and gold. The deposits occur as layers or lenses of pyritic rock that contain high to very high concentrations of $\mathrm{As}, \mathrm{Cd}, \mathrm{Cu}, \mathrm{Fe}, \mathrm{Pb}, \mathrm{Zn}$, and other metals (Day and others, in press). Their chemical and mineralogical composition is similar to polymetallic deposits. The rock units containing these sulfide deposits on CWS have been metamorphosed, but their bulk composition has not changed greatly and the newly formed minerals contribute to a generally high ANC that is similar to the green-altered volcanic rocks described above.

The high concentrations of pyrite and base-metal sulfide 
minerals in massive sulfide deposits can produce extremely acidic drainage, as in the Shasta district, California (Alpers and Nordstrom, 1991; Taylor and others, 1995; Plumlee and others, 1999). The combination of bad mining practices and wet climate can cause severe environmental degradation, but not all massive sulfides have this impact. The combination of dry climate and deep oxidation in the Basin and Range Province of Arizona and Nevada greatly reduces the tendency for acid generation (Nash and others, 1996). Also, the green propylitic alteration of basaltic host rocks, common in this deposit type, introduces calcite and chlorite that provide high ANC. Water draining from many massive sulfide deposits is neutral to weakly alkaline, caused by the beneficial effects of altered wallrocks. Despite geologic differences, massive sulfide deposits have many geochemical features in common with polymetallic deposits: ore compositions alone would suggest huge problems, but natural mitigation by wallrocks can greatly reduce risks in some areas.

Massive sulfide deposits were mined in the Cochetopa district south of Gunnison (fig. 10). Compared to deposits in Arizona and California, the known deposits are small; the largest mined deposit was the Vulcan-Good Hope (Hartley, 1983). As will be discussed later, the relatively dry climate of this mining area may be a big factor in minimizing contamination from mines in the Cochetopa district.

\section{Sediment-Hosted Copper Deposits}

More than 100 years ago, vein-like deposits rich in copper and silver were located in red-bed sandstones on the Colorado Plateau (Fischer, 1936) in what would later become uraniumvanadium country. The copper-silver deposits were mined intermittently through 1945 by relatively small mining operations that are often forgotten in the shadow of the more famous mines of the San Juans. However, new geologic information indicates that the copper minerals are not confined to fracture zones, but are broadly disseminated in a much larger volume of rock (Hahn and Thorson, 1995). Advances in metallurgical methods suggest that new, environmentally friendly methods can be used to good advantage on these mineralogically simple ores. Consequently, a permit has been granted for mining this variety of ore in the Lisbon Valley, Utah, a short distance west of the Colorado border, and preliminary drilling results suggest a similar mine may be viable at the site of the former Cashin mine in western Montrose County (fig. 1) (Hahn and Thorson, 1995).

These copper deposits, with byproduct silver, are now believed to form in structures related to the salt anticlines of western Colorado-eastern Utah. These structures are fairly well known, but because the copper deposit model is so new, few of the structures in Colorado have been tested for metals. The potential for these deposits is not well known, but several of these relatively small deposits could exist on the Western Slope. Environmentally, the metals of concern are $\mathrm{Cu}$ and $\mathrm{Ag}$, with relatively small amounts of sulfur and arsenic. Acidic waters are not likely to be generated or persist in the calcitebearing sandstone host rocks.

\section{Uranium Deposits}

Many kinds of uranium deposits in plutonic, volcanic, and sedimentary rocks have been identified in Colorado. The behavior of uranium and associated metals is quite different among the various types of uranium deposits, but many of the problem metals tend to be mobile in either highly acidic or alkaline waters. Mitigation of the metals is technically different for acidic versus alkaline conditions.

On the CWS, three types of uranium deposits have been mined: (1) uranium-vanadium deposits in sandstone that are widespread and famous in the Uravan district, westernmost Colorado-Utah (also called Uravan mineral belt) (Chenoweth, 1981); (2) veins of uranium in sandstone in the Los Ochos subdistrict of the Cochetopa district, southeast of Gunnison (McCulla, 1980); and (3) vein-like and breccia-filling ores in dolomite and adjacent sedimentary rocks at the Pitch mine, Marshall Pass district (Nash, 1988) (fig. 1). Other minor occurrences of uranium have been prospected and mined in small operations at other scattered sites that will not be considered here. Examples of sandstone-type and vein-type uranium deposits were examined briefly for this study.

The environmental geochemistry of uranium mines is more complex than for other metal mines because radiation and decay products of uranium, such as radon and radium, also are involved. Regulatory standards are established for uranium and radionuclides in water and in mill tailings but have not been set for dump waste rocks (CWQCC, 2000). Standards for released water and base metals are the same as those for metal mines. Mine reclamation generally focuses on exposure to radiation rather than chemical concentrations of uranium, but release of uranium and radionuclides to surface and ground waters also is a concern and water-quality standards must be met (CWQCC, 2000).

\section{Porphyry Cu-Mo Deposits}

Deposits of copper and molybdenum, associated with shallow-level igneous stocks having characteristic porphyritic texture (large crystals mixed with very fine ones), are the major source of $\mathrm{Cu}$ and $\mathrm{Mo}$ in the United States and the world. Mines range in size from large to huge (as at Climax, Colo.), and commonly are open-pit operations. The geologic features with potential environmental implications include (1) large tonnages of rocks with high sulfide and base-metal concentrations; (2) igneous rocks and alteration zones that can have low to moderate acid-neutralizing capacity; and (3) high fracture permeability that promotes percolation of surface waters and encourages acid-generating and metal-liberating reactions. The environmental aspects of porphyry deposits are reviewed elsewhere (Cox and others, 1995; Ludington and others, 1995; 


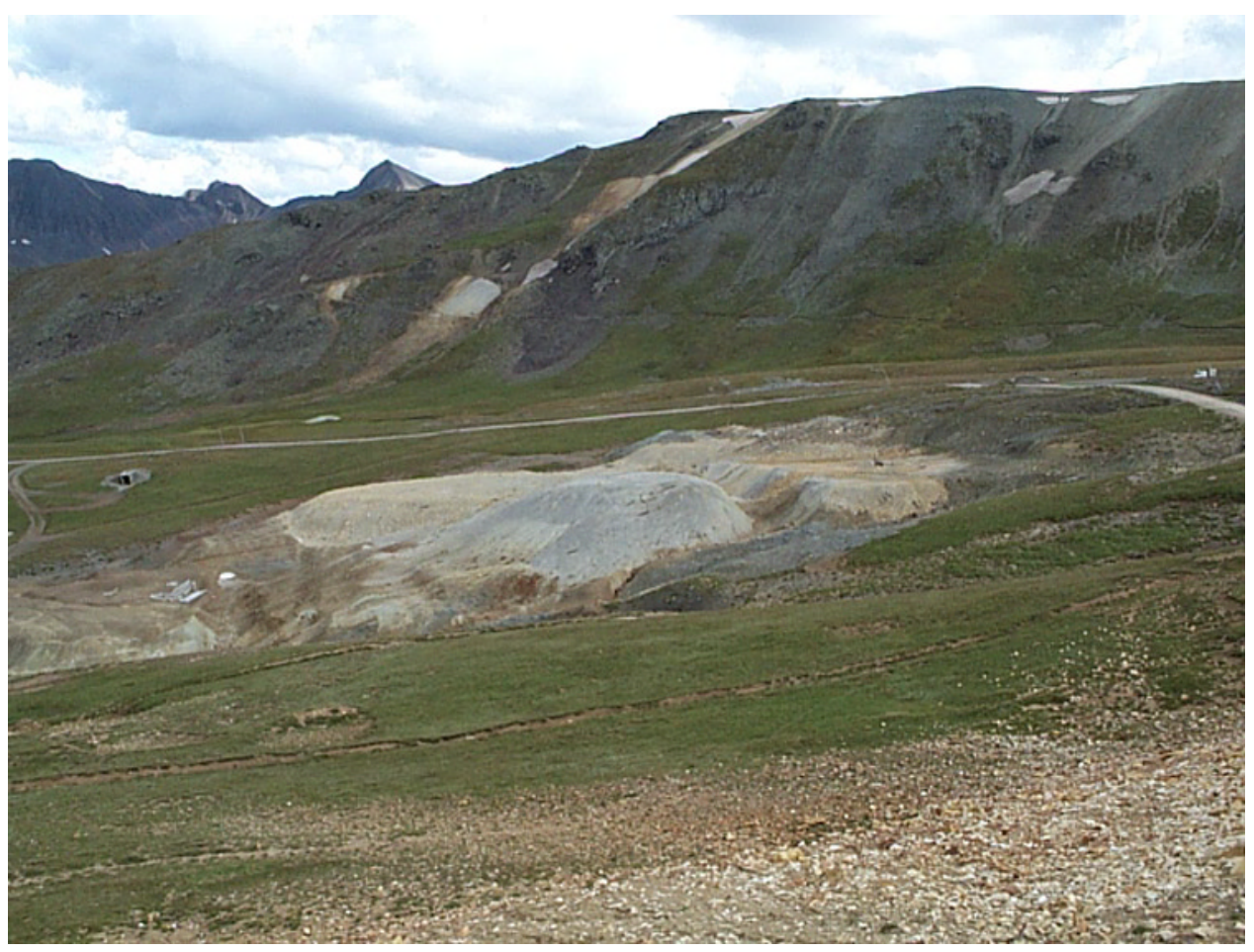

Photograph 5. The Hough mine, Palmetto basin, is above treeline at an elevation of about $12,000 \mathrm{ft}$. High precipitation, mostly as snow, and short growing season complicate the environmental effects of mines in this setting. Mine adits and mine-dump runoff tend to be problems in this environment.

Plumlee and others, 1999). Metals of major concern include As, $\mathrm{Cd}, \mathrm{Cu}, \mathrm{Fe}, \mathrm{Mo}, \mathrm{Pb}, \mathrm{S}, \mathrm{Sb}, \mathrm{Te}$, and $\mathrm{Zn}$ from the $\mathrm{Cu}-\mathrm{Mo}$ deposits. The Mo-rich variety (termed "Climax-type") have the potential to produce acidic waters that are rich in $\mathrm{F}$ and $\mathrm{U}$.

No porphyry-type deposits have been mined on the CWS, but several have been identified. The most thoroughly studied is the Mt. Emmons molybdenum deposit near Crested Butte (fig. 10 and fig. 16) (Thomas and Galley, 1982), for which economic feasibility and engineering studies have been made. I did not attempt to sample the molybdenum systems near Crested Butte because AMAX has studied the mineralized rocks and drainage waters for many years and is working closely with the State and USFS on both historic and possible future mine sites. Geologically, the Mt. Emmons deposit differs from mined deposits of the "Climax type" in that sedimentary rocks host the ore. Based on observations on other sulfide deposits in sedimentary rocks (Plumlee and Nash, 1995; Plumlee and others, 1999), we can predict that sedimentary rocks will provide more ANC than granitic host rocks, thus environmental degradation may be less.

\section{Geochemistry of Mined Mineralized Rocks}

The chemical composition of mined rocks in 149 dump and tailings samples collected in this study show a wide range in element concentrations, generally consistent with the introductory descriptions of deposit types above. Some unexpected trends are evident in the new chemical data, however, that merit discussion. Although the number of samples collected is relatively small, the same methods and philosophy of sampling were used by one person, the author, so these results may be consistent and representative of the spectrum of compositions in the study area. Only a few of the samples were collected as "high grade" (atypical of the mine dump). The unexpected trends, shown in figure 2, is that the four major types of metal deposits (sediment-hosted polymetallic vein and replacement, volcanogenic massive sulfide, volcanic-hosted veins, and breccia pipes) have generally similar ranges in metal concentrations. Only the uranium vein and sandstone deposits are clearly distinct for their lower metal concentrations (which is not surprising). The polymetallic character, with substantial enrichment in many base metals, is evident for the four major deposit types. For this analysis, polymetallic vein and polymetallic replacement deposits in pre-Tertiary sedimentary rocks were lumped together because, in most districts, the two ore types are found within a mine and are mixed on the dumps and tailings piles. The limited data suggest that there are a few distinctions, such as lower concentrations of $\mathrm{Ag}, \mathrm{Pb}$, and $\mathrm{Mn}$ in massive sulfide deposits relative to the other three, and $U$ is clearly lower in the metallic deposits.

One inference from this chemical information is that the four major metallic deposit types on the CWS can be expected to have generally similar high concentrations of base metals in their mine waste. The important differences between deposit types is the lithology and chemical properties (ANC) of the 
host rocks. There probably is more variability in metal concentrations from dump to dump, or tailings to tailings, than between the deposit types. Any of these deposit types must be considered to potentially have sufficient $\mathrm{Ag}, \mathrm{Cu}, \mathrm{Fe}, \mathrm{Mn}$, $\mathrm{Pb}$, or $\mathrm{Zn}$ to pose problems. This generalization also applies to the non-ore metalloids ( $\mathrm{As}, \mathrm{Bi}$, or $\mathrm{Se}$ ) associated with these ore types. There are two inferences from this generalization: (1) chemical compositions of mined waste probably need to be checked because one cannot simply assume that metal concentrations are sufficiently low to be unimportant in any of the deposit types, although some can be relatively low if most of the waste on the pile comes from workings in weakly altered rocks that were mined to access ore zones; and (2) even if these deposit types have similar ranges in metal concentrations, the mobility of the metals may be highly variable as a function of other geologic properties of the deposit type (chiefly wallrock composition and alteration mineralogy). Stated in different words, concentrations of potentially toxic metals (such as $\mathrm{Pb}$ or $\mathrm{Zn}$ ) may be high in waste from any of these deposit types, but the impact of the metals on the environment is determined by processes that control the mobility of the metals. Leach tests, described earlier, are a simple quantitative measure of those metal mobilities.

\section{Mining, Mills, and Mill Tailings: An Introduction}

Mills and smelters were an essential part of the mining infrastructure on the Western Slope and played a large part in

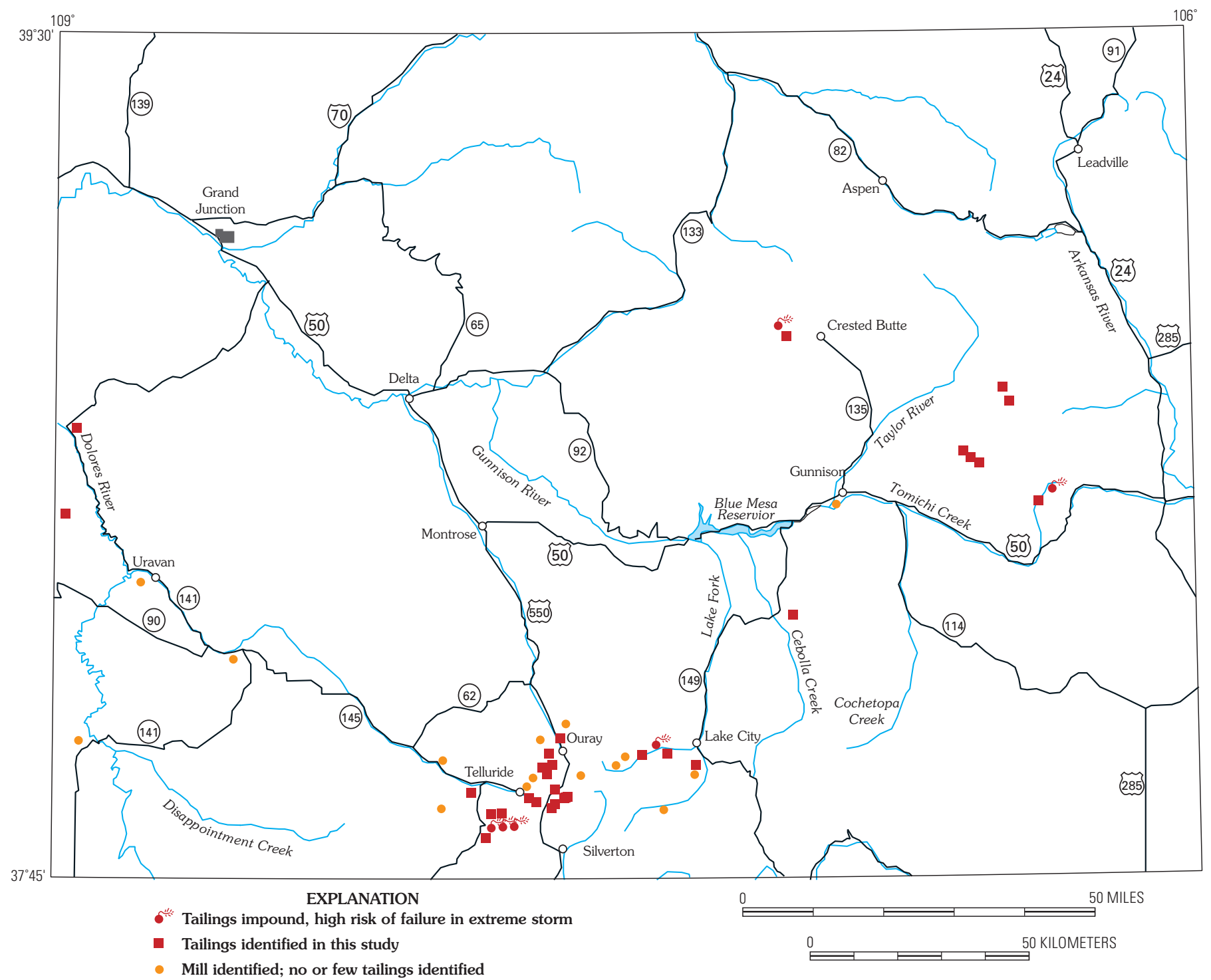

Figure 4. Location of mill tailings and mill sites, central Western Slope, Colorado. Not all sites can be shown at this scale. Mills near Silverton not shown. 
Table 6. Summary of mills, tailings, and smelters, CWS, Colorado.

[The "Mill sites" column includes several sites that contained tailings but no obvious mill building. The "Tailings sites" column does not include the many sites that have no visible tailings near confirmed structures. At more than half of the tailings sites, it appears that major amounts (50 percent or more?) have been lost to erosion. Approximately 12 of the tailings impoundments have been reclaimed]

\begin{tabular}{|c|c|c|c|}
\hline District & Mill sites & Tailings sites & Smelter sites \\
\hline \multicolumn{4}{|c|}{ Lake Fork of Gunnison watershed } \\
\hline Burrows Park & 1 & 0 & 0 \\
\hline Galena & 6 & 5 & 3 \\
\hline Lake Fork & 2 & 1 & 1 \\
\hline \multicolumn{4}{|c|}{ Gunnison River watershed } \\
\hline Cochetopa & 1 & 0 & 1 \\
\hline Gold Brick & 4 & 3 & 0 \\
\hline Quartz Creek & 2 & 0 & 0 \\
\hline Ruby & 3 & 2 & 0 \\
\hline Tincup & 2 & 2 & 1 \\
\hline Tomichi & 1 & 2 & 1 \\
\hline \multicolumn{4}{|c|}{ Uncompahgre River watershed } \\
\hline Ouray & 3 & 3 & 0 \\
\hline Red Mountain & 6 & 6 & 1 \\
\hline Sneffels & 8 & 7 & 0 \\
\hline \multicolumn{4}{|c|}{ San Miguel River watershed } \\
\hline Cashin & 1 & 1 & 0 \\
\hline Mount Wilson & 1 & 0 & 0 \\
\hline Iron Springs & 7 & 7 & 0 \\
\hline Placerville & 1 & 1 & 0 \\
\hline Telluride & 16 & 8 & 0 \\
\hline \multicolumn{4}{|c|}{ Uranium mills } \\
\hline & 5 & 5 & 0 \\
\hline TOTAL & 70 & 53 & 8 \\
\hline
\end{tabular}

the success or failure of mining ventures. The legacy of mill processing of ores is numerous small to very large piles or "impoundments" of finely ground mineralized rock known as tailings. The technology of mills and smelters, and the location of mills, smelters, and tailings are poorly documented in the literature but were emphasized in this investigation because this class of mine-related waste has not received adequate inventory and study for its role in contamination of waters. Significantly, only a few percent of historic mills and smelters are listed in electronic databases of the Department of Interior, such as MRDS and MAS/MILS (McFaul and others, 2000). Also, electronic search of literature tends to identify chiefly uranium mill tailings because they have been the subject of much litigation and reclamation effort over the past 20 years.
On-site and literature search in this project has identified 8 smelter sites, 70 probable mill sites, and 53 tailings sites (summarized in table 6; descriptions are in table 7); the discrepancy between number of mill sites and tailings sites reflects the total loss of visible tailings from many of the older mills. Locations of most mill tailings and mill sites are shown on figure 4. Presumably there are many more mills and tailings sites hidden in obscure places or beyond access on private property. Most mills are on private property (patented mill claims), but in many mining areas tailings may have been placed on public lands, presenting a mixed-ownership problem that complicates reclamation by Federal lands managers.

What is a mill and why are tailings a concern today? Metals of value were tucked away inside rocks and veins and had to be removed before they were refined (smelted) for use. Depending upon the kind of ore, 80 to more than 99 percent of the ore was actually of no value and had to be separated from the gold, galena, sphalerite, or other ore minerals. The first stage was grinding, generally to a size finer than beach sand. Then a variety of ingenious physical and chemical methods were applied to concentrate the minerals by jigs, tables, amalgamation, and floatation. For some ores, cyanide was used to dissolve the metal of interest; cyanide is highly toxic when fresh, but generally degrades with exposure to air (Smith and Mudder, 1999) and is not a problem in the historic wastes investigated here. After going through several stages, involving tanks and pumps and thickeners, one or more concentrates were created, and a great deal more fine sand (tailings) were pumped out as a slurry (similar to wet concrete) and disposed of. The easiest way to dispose of tailings was to pour or pump them into a nearby stream; in the 1930's "ponds" were devised to collect the solids while also allowing the water and chemicals to be recycled through the mill.

Mill tailings are a class of mining-related waste that pose special problems for the environment. First, mills and tailings nearly always were located near streams. Early mills used water power, and all mills required large amounts of water for ore processing. Second, gravity was utilized in mills: crushed rock and tailings were processed downward, and tailings were always placed below the mill (which generally was below the mine), and that typically means the tailings were placed in flood plains and riparian zones. Third, the fine grain size of tailings tends to make the constituents more reactive than in coarse mine waste or in unmined rocks. As a rule of thumb, there is much more interaction of tailings with water than there is for other kinds of mine waste. Also, fine tailings are easily eroded during storm events, such as flash floods, and are much more likely to interact with floodwaters than other kinds of mine waste. Mill tailings are not a de facto threat to water and the natural environment, but they should be a high priority for evaluation. The character of mill tailings is currently difficult to assess from the desktop, but there is one good predictor: large mines required large mills that produced large tonnages of tailings (often more than a million tons), and these are more likely to be a significant problem than a 100-ton pile. 
Milling and associated waste evolved dramatically from 1875 to the present. Three general stages can be highlighted.

1. Early (1875 to about 1900): Many of the bonanza ores could not be treated by mills close to the mines because milling technology was crude. Thus mining was highly selective and created very rich products that were sent directly to smelters for recovery of valuable metals. The early mines often had very narrow workings for selective mining, and the ore typically was hand sorted, both inside the mine and outside, to create "direct shipping" ore for the smelters. On the CWS, some small smelters were built to serve early mines near Lake City, Red Mountain, Silverton, Tincup, and Whitepine, but these generally were ineffective and failed. Large smelters in Denver, Pueblo, and Durango were capable of efficient recovery of silver and gold, but transportation was difficult and costly, which created the demand for railroads on the Western Slope. The combination of greatly diminished silver prices between 1891-95 and depletion of rich "bonanza" ores close to the surface forced closure of many of the early mines that created much wealth in a short period of time. The consequences today:

- Relatively small amounts of surface disturbance and limited subsurface workings because the mining was very selective and nearly always underground,

- Few mills and small volumes of tailings,

- Small, generally unreactive slag piles created by local smelters.

2. Middle stage (about 1900 to 1935): This was an era of consolidation of small mines into fewer major mines. The mines were served by deep tunnels and long aerial tramways and were supported by mills with new technology capable of separating and concentrating gold, silver-lead, copper, and zinc. The ores mined from

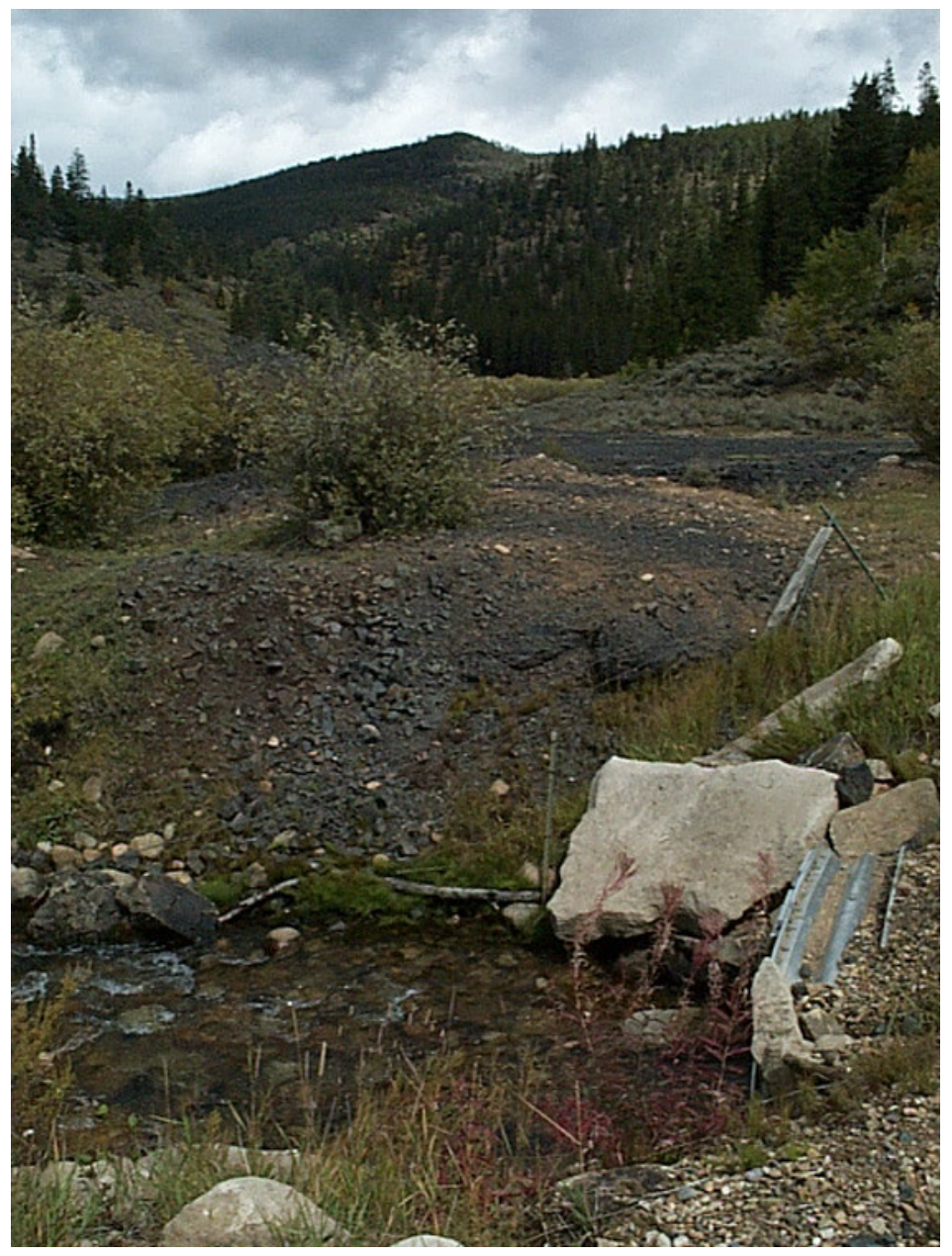

Photograph 6. Slag is a black, vitreous rock-like substance produced when ores are melted during smelting. The slag shown here was poured close to Tomichi Creek but probably does not contaminate the water because it is relatively unreactive. 
large veins, as at Camp Bird, Idarado, and Sunnyside mines, were described as "low grade" because they contained much lower concentrations of ore metals than the early bonanza ores. The mining was possible chiefly because of the new milling technology (Burbank and Luedke, 1969). During this era, larger tonnages were mined per day, producing larger waste dumps outside the mines and much larger tonnages of mill tailings. The consequences today:

- Large mine dumps that can produce significant amounts of acid and metals in drainage waters,

- Tailings that tend to be either unconfined (released directly into flowing streams) or poorly confined. Most of the mills dating to this era have only a fraction of their tailings nearby - the majority were never impounded or were lost when impoundments failed in major storm events. The impact of these tailings practices is poorly documented, but there is indirect evidence (described later) and direct measurements (Vincent and others, 1999), that sulfide minerals from tailings now lie in and along streams miles from the mill, slowly releasing base metals to surface waters.

3. Modern stage (post-1935): An Executive order in 1935 placed new regulations on the discharge of tailings into streams. This order enforced new practices developed by major mining companies in Colorado and elsewhere to confine mill tailings in "ponds" and thereby keep the mill sands, slimes, and waters out of nearby streams. Milling technology continued to improve on recovery methods, but this was not a major change on impact to the environment. Improvements to smelters greatly reduced the output of noxious fumes, but this was not a factor on the Western Slope because the smelters were located elsewhere. After World War II, consolidation tended to create a few very large mines in the major districts, and these mines moved thousands of tons of ore a day. Mass mining by open-pit methods was advanced elsewhere but was tried at only a few small pits on the Western Slope; thus, that technology is not a factor on the CWS as it is in Arizona, Nevada, and other Western States. The consequences today:

- Impoundments of tailings, combined with mills of larger throughput, produced large piles of tailings

- Some tailings impoundments were either inadequately designed or were in the eye of a major flash flood and failed catastrophically.

All tailings impoundments on the CWS lack an impermeable base; thus, water that percolates through the tailings cannot be controlled before it infiltrates ground water. Reclamation of the tailings at the Keystone mill, west of Crested Butte, required a major effort to place a plastic barrier on the downslope edge of the tailings to control drainage. Reclamation of other large tailings impoundments, for example in the Telluride and Red Mountain areas, has not addressed this issue. Risk of catastrophic failure of tailings impoundments has been considered for uranium tailings, but I am not aware of such analysis applied to historic metallic mineral tailings and their management.

Text continues on page 28

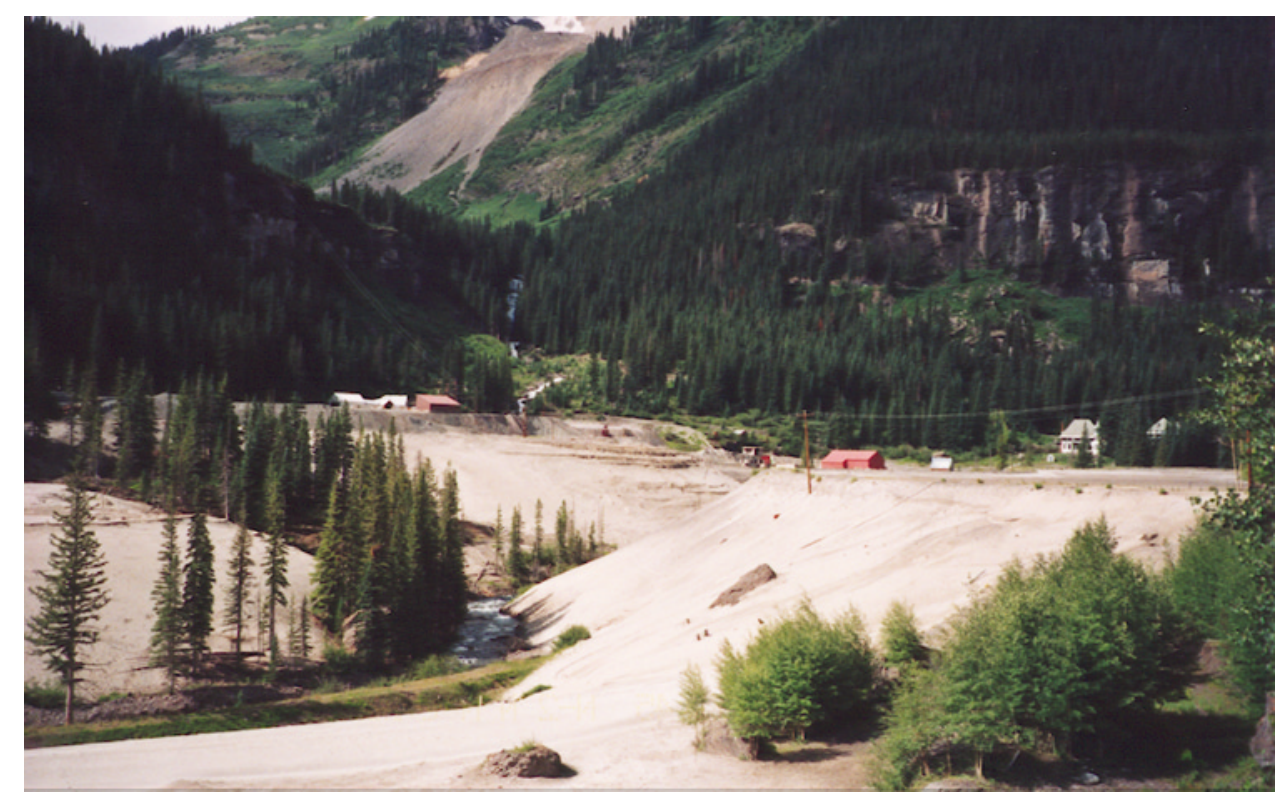

Photograph 7. Mill tailings, produced when mills grind ore, are fine sandy materials that resemble beach sands. In many situations, as shown here at the Camp Bird mine, the tailings are close to streams and are easily eroded during storm events. 
Table 7. Inventory of mill and tailings sites, CWS, Colorado.

[Name: Informal name from local feature, such as a mine; not reliable or legally correct. Mill: Y, yes, features highly suggestive of mill structure; ?, uncertain. Tailings: amount of tailings observed or reported: S, small (<100 tons); M, medium; L, large; VL, very large ( $>1$ million tons); N, none; Nx, tails eroded. ESRisk: risk of failure in extreme storm; L, low, M, medium, $\mathrm{H}$, high]

\begin{tabular}{|c|c|c|c|c|c|c|c|c|}
\hline TN-ID & Name & District & Mill & Tailings & $\begin{array}{c}\text { Lat (N.) } \\
\text { (degrees) }\end{array}$ & $\begin{array}{l}\text { Long (W.) } \\
\text { (degrees) }\end{array}$ & ESRisk & Description \\
\hline \multicolumn{9}{|c|}{ Smelter sites } \\
\hline NGG201 & Tomichi & Tomichi & $\mathrm{Y}$ & S & 38.5169 & 106.4233 & $\mathrm{~L}$ & $\begin{array}{l}\text { Smelter site, with small area of slag } \\
\text { pours }\end{array}$ \\
\hline NGG454 & $\begin{array}{l}\text { Lee Smelter } \\
\text { Galena? }\end{array}$ & Henson & $\mathrm{Y}$ & $\mathrm{Nx}$ & 37.9789 & 107.4975 & $\mathrm{~L}$ & $\begin{array}{l}\text { Smelter stack by Henson Creek; no } \\
\text { visible slag }\end{array}$ \\
\hline NGG501 & Vulcan & Cochetopa & $\mathrm{Y}$ & S & 38.3453 & 107.0058 & $\mathrm{~L}$ & $\begin{array}{l}\text { Some equipment, small mounds of } \\
\text { slag }\end{array}$ \\
\hline NGG684 & Saratoga & Red Mtn & $\mathrm{Y}$ & S & 37.9461 & 107.6636 & $\mathrm{~L}$ & $\begin{array}{l}\text { Saratoga smelter structure sparse, good } \\
\text { piles of slag }\end{array}$ \\
\hline NGG992 & Lee smelter & Henson & $?$ & $\mathrm{Nx}$ & 38.0083 & 107.4500 & $\mathrm{~L}$ & $\begin{array}{l}\text { Possible foundation at Smelter Creek } \\
\text { jct with Henson Crk; no slag seen }\end{array}$ \\
\hline NGG965 & Crooke & Galena & Y? & S? & 38.0139 & 107.3111 & $\mathrm{~L}$ & $\begin{array}{l}\text { Likely location of smelter on Lake } \\
\text { Fork by falls }\end{array}$ \\
\hline \multicolumn{9}{|c|}{ Mill sites } \\
\hline NGM102 & Cashin & Cashin & $\mathrm{Y}$ & $\mathrm{S}$ & 38.3111 & 108.9508 & M & $\begin{array}{l}\text { Wooden mill standing, tails along } \\
\text { creek }\end{array}$ \\
\hline NGM202 & Akron & Tomichi & $\mathrm{Y}$ & $\mathrm{L}$ & 38.5381 & 106.3961 & $\mathrm{H}$ & $\begin{array}{l}\text { Large concrete foundation, } 2 \\
\text { impounds }\end{array}$ \\
\hline NGM219 & Lot mine & Powderhorn & $\mathrm{Y}$ & S & 38.3194 & 107.0917 & $\mathrm{~L}$ & $\begin{array}{l}\text { Small mill standing, small tails, dry } \\
\text { and ok }\end{array}$ \\
\hline NGM232 & Carter & Gold Brick & $\mathrm{Y}$ & M & 38.6128 & 106.5944 & $\mathrm{H}$ & $\begin{array}{l}\text { Big wooden mill ruins, jigg tables; } \\
\text { tails far to east }\end{array}$ \\
\hline NGM233 & Raymond & Gold Brick & $\mathrm{Y}$ & M & 38.6208 & 106.5861 & $\mathrm{H}$ & $\begin{array}{l}\text { Big mill ruins, stone foundation; tails } \\
\text { far to east }\end{array}$ \\
\hline NGM234 & Gold Link & Gold Brick & $\mathrm{Y}$ & M? & 38.6306 & 106.5789 & $\mathrm{H}$ & Big mill ruins ; tails to east \\
\hline NGM281 & Hidden Treasure & Galena & $\mathrm{Y}$ & $\mathrm{S}$ & 38.0189 & 107.3594 & M & Concrete foundation parts; \\
\hline NGM282 & Ute-Ulay & Galena & Y & M & 38.0172 & 107.3486 & $\mathrm{H}$ & $\begin{array}{l}\text { Concrete foundation; several tails } \\
\text { impounds near Henson Creek; } \\
\text { unstable }\end{array}$ \\
\hline NGM286 & Pelican? & Galena & $\mathrm{Y}$ & NX & 38.0200 & 107.3350 & $\mathrm{~N}$ & Big concrete foundation; no tails \\
\hline NGM960 & Capitol City & Galena & $\mathrm{Y}$ & $\mathrm{Nx}$ & 37.9958 & 107.4819 & $\mathrm{~L}$ & $\begin{array}{l}\text { Mill site reported by B. Hite, BLM } \\
\text { (1999) }\end{array}$ \\
\hline NGM302 & Bachelor & Ouray & $\mathrm{Y}$ & $\mathrm{S}$ & 38.0622 & 107.6622 & $\mathrm{~L}$ & $\begin{array}{l}\text { Wood bldg collapsed, steel machinery; } \\
\text { small tails dry above Dexter Crk }\end{array}$ \\
\hline NGM306 & Banner & Ouray & Y? & Mx & 38.0542 & 107.6875 & $\mathrm{~L}$ & $\begin{array}{l}\text { Mill removed, site sold for homes, tails } \\
\text { in place beneath homes }\end{array}$ \\
\hline NGM308 & Silvershield & Ouray & $\mathrm{Y}$ & Sx & 38.0408 & 107.6831 & $\mathrm{~L}$ & $\begin{array}{l}\text { Tin building stands west of river, tram } \\
\text { cable; ocher tails nearby }\end{array}$ \\
\hline NGM323 & Alta & Iron Springs & $\mathrm{Y}$ & $\mathrm{L} ?$ & 37.8850 & 107.8544 & $\mathrm{~L}$ & $\begin{array}{l}\text { Mill burned; foundation clear; tails in } \\
\text { two ponds to west T323 and T325 }\end{array}$ \\
\hline NGM362 & Silver Queen & Red Mtn & $?$ & M? & 37.9167 & 107.6864 & $\mathrm{~L}$ & $\begin{array}{l}\text { No structure evident at lower level } \\
\text { dumps; tails to north must have } \\
\text { mill }\end{array}$ \\
\hline NGM372 & Camp Bird & Sneffels & $\mathrm{y}$ & VL & 37.9706 & 107.7272 & $\mathrm{H}$ & $\begin{array}{l}\text { Concrete foundation for mill stands } \\
\text { near 14th level portal; large tails to } \\
\text { SE }\end{array}$ \\
\hline NGM375 & Atlas & Sneffels & Y & M & 37.9756 & 107.7578 & $\mathrm{H}$ & $\begin{array}{l}\text { Wooden structure, metal parts, on } \\
\text { slope above tailings }\end{array}$ \\
\hline NGM386 & Society & Telluride & $?$ & M & 37.9478 & 107.8722 & M & $\begin{array}{l}\text { No structure evident; located near old } \\
\text { railroad to south; tails along river }\end{array}$ \\
\hline
\end{tabular}


Table 7. Inventory of mill and tailings sites, CWS, Colorado-Continued.

\begin{tabular}{|c|c|c|c|c|c|c|c|c|}
\hline TN-ID & Name & District & Mill & Tailings & $\begin{array}{l}\text { Lat (N.) } \\
\text { (degrees) }\end{array}$ & $\begin{array}{l}\text { Long (W.) } \\
\text { (degrees) }\end{array}$ & ESRisk & Description \\
\hline \multicolumn{9}{|c|}{ Mill sites-Continued } \\
\hline NGM396 & Palmetto & Henson & Y & $\mathrm{Nx}$ & 37.9822 & 107.5592 & $\mathrm{~N}$ & $\begin{array}{l}\text { Small stamp mill below Palmetto } \\
\text { Gulch }\end{array}$ \\
\hline NGM397 & Empire Chief & Henson & $\mathrm{Y}$ & $\mathrm{Nx}$ & 37.9744 & 107.5222 & $\mathrm{~N}$ & $\begin{array}{l}\text { Wooden mill } 4 \text { mi west Capitol City; } \\
\text { no tails }\end{array}$ \\
\hline NGM526 & Standard & Ruby & $\mathrm{Y}$ & $\mathrm{S}$ & 38.8786 & 107.0750 & $\mathrm{H}$ & $\begin{array}{l}\text { Cement foundation next to mine } \\
\text { buildings, tails to south }\end{array}$ \\
\hline NGM534 & Tincup & Tincup & $\mathrm{Y}$ & $\mathrm{S}$ & 38.7278 & 106.4972 & $\mathrm{~L}$ & $\begin{array}{l}\text { Wooden structure collapsed; tram to } \\
\text { SE, tails to NW }\end{array}$ \\
\hline NGM539 & Golden Fleece? & Lake Fork & Y? & Sx? & 37.9811 & 107.2906 & $\mathrm{~N}$ & $\begin{array}{l}\text { Concrete structure by lake; tails in } \\
\text { lake?; cited by Irving and Bancroft, } \\
1911\end{array}$ \\
\hline NGM542 & Sherman & Burrows Park & $\mathrm{Y}$ & $\mathrm{Nx}$ & 37.9022 & 107.4261 & $\mathrm{~N}$ & $\begin{array}{l}\text { Stone foundation; cited by Irving and } \\
\text { Bancroft; no tails; waterpowered } \\
\text { mill? }\end{array}$ \\
\hline NGM606 & Mountain Silver & Red Mtn & $\mathrm{Y}$ & Sx? & 0.9328 & 107.6611 & $\mathrm{~N}$ & $\begin{array}{l}\text { Small mill, metal parts; near adit, no } \\
\text { tails found }\end{array}$ \\
\hline NGM619 & Carbonero & Iron Springs & $\mathrm{Y}$ & M & 37.8583 & 107.8194 & $\mathrm{H}$ & $\begin{array}{l}\text { Mill site shown on old topo, nothing } \\
\text { visible in trees, some spilled tails }\end{array}$ \\
\hline NGM625 & Silver Bell & Iron Springs & $\mathrm{Y}$ & M & 37.8600 & 107.8689 & $\mathrm{H}$ & $\begin{array}{l}\text { Building intact by old railroad loop, } \\
\text { tails to west }\end{array}$ \\
\hline NGM628 & Caribeau & Iron Springs & $\mathrm{Y}$ & M & 37.8575 & 107.8483 & $\mathrm{H}$ & $\begin{array}{l}\text { Some concrete forms, no structures } \\
\text { near mine portal, abundant tailings }\end{array}$ \\
\hline NGM636 & Blistered Horn & Tincup & $\mathrm{Y}$ & Sx & 38.7028 & 106.4917 & $\mathrm{~N}$ & $\begin{array}{l}\text { Wooden structure collapsed, a few } \\
\text { tails at foundation and south }\end{array}$ \\
\hline NGM638 & Gold King & Iron Springs & $\mathrm{Y}$ & $S$ & 37.8794 & 107.8478 & $\mathrm{~L}$ & $\begin{array}{l}\text { Collapsed building some steel parts; } \\
\text { cited in lierature, location on map }\end{array}$ \\
\hline NGM649 & San Juan & Mineral Point & $\mathrm{Y}$ & $\mathrm{S}$ & 37.9606 & 107.5925 & $\mathrm{~N}$ & $\begin{array}{l}\text { Wooden mill and tanks standing; some } \\
\text { red tails in foundation }\end{array}$ \\
\hline NGM659 & Richmond & Sneffels & $\mathrm{Y}$ & M & 37.9525 & 107.7264 & $\mathrm{H}$ & $\begin{array}{l}\text { Richmond basin, mill by falls, medium } \\
\text { volume of gray tails in Imogene } \\
\text { Creek }\end{array}$ \\
\hline NGM660 & Camp Bird 3 & Sneffels & $\mathrm{Y}$ & S & 37.9488 & 107.7312 & $\mathrm{~L}$ & $\begin{array}{c}\text { Concrete foundation at upper adit level } \\
\text { of Camp Bird; tails in basin/creek }\end{array}$ \\
\hline NGM661 & Savage & Telluride & $\mathrm{Y}$ & S & 37.9331 & 107.7456 & M & $\begin{array}{l}\text { Small stamp mill remains, jig tables; } \\
\text { tails to southwest }\end{array}$ \\
\hline NGM663 & Tomboy-1 & Telluride & $\mathrm{Y}$ & $S$ & 37.9339 & 107.7525 & M & $\begin{array}{l}\text { Wooden structure, tails nearby, SE of } \\
\text { main Tomboy mills; Argentine? }\end{array}$ \\
\hline NGM665 & Tomboy-2 & Telluride & $\mathrm{Y}$ & $\mathrm{S}$ & 37.9356 & 107.7542 & M & $\begin{array}{l}\text { Foundation and metal, many tails } \\
\text { nearby }\end{array}$ \\
\hline NGM666 & Tomboy-3 & Telluride & $\mathrm{Y}$ & $\mathrm{Nx}$ & 37.9375 & 107.7572 & $\mathrm{~N}$ & $\begin{array}{l}\text { Burned remains of mill with } 60 \text { or } \\
\text { more stamps, largest at Tomboy; } \\
\text { no tails }\end{array}$ \\
\hline NGM667 & Tomboy-4 & Telluride & $\mathrm{Y}$ & $\mathrm{Nx}$ & 37.9372 & 107.7581 & $\mathrm{~N}$ & $\begin{array}{l}\text { Burned, just west of large mill, close } \\
\text { to creek, used water power?, no } \\
\text { tails }\end{array}$ \\
\hline NGM668 & Tomboy-5 & Telluride & $\mathrm{Y}$ & Sx & 37.9381 & 107.7583 & $\mathrm{~N}$ & $\begin{array}{l}\text { SW corner of cluster, burned; concrete } \\
\text { foundation; a few tails in } \\
\text { foundation }\end{array}$ \\
\hline NGM669 & Tomboy-6 & Telluride & $\mathrm{Y}$ & $\mathrm{Nx}$ & 37.9372 & 107.7592 & $\mathrm{~N}$ & $\begin{array}{l}\text { Wood and cement piers remain, } \mathrm{SW} \text { of } \\
\text { all; no tails }\end{array}$ \\
\hline NGM670 & Japan? & Telluride & Y & $\mathrm{Nx}$ & 37.9378 & 107.7547 & $\mathrm{~N}$ & Wood remains, with jig tables, no tails \\
\hline NGM674 & Liberty Bell & Telluride & $\mathrm{Y}$ & $\mathrm{Nx}$ & 37.9342 & 107.7967 & $\mathrm{~L}$ & $\begin{array}{l}\text { Stone foundation only, mentioned in } \\
\text { literature; tails in river? }\end{array}$ \\
\hline
\end{tabular}


Table 7. Inventory of mill and tailings sites, CWS, Colorado-Continued.

\begin{tabular}{|c|c|c|c|c|c|c|c|c|}
\hline TN-ID & Name & District & Mill & Tailings & $\begin{array}{l}\text { Lat (N.) } \\
\text { (degrees) }\end{array}$ & $\begin{array}{l}\text { Long }(W .) \\
\text { (degrees) }\end{array}$ & ESRisk & Description \\
\hline \multicolumn{9}{|c|}{ Mill sites-Continued } \\
\hline NGM675 & unknown & Telluride & $\mathrm{Y} ?$ & $\mathrm{Nx}$ & 37.9336 & 107.7944 & $\mathrm{~N}$ & $\begin{array}{l}\text { Foundation resembles mill, no tailings } \\
\text { evident }\end{array}$ \\
\hline NGM679 & Eclipse & Sneffels & $\mathrm{Y}$ & $S$ & 37.9764 & 107.7472 & $\mathrm{~L}$ & $\begin{array}{l}\text { Small wooden mill, small tailings to } \\
\text { SW }\end{array}$ \\
\hline NGM687 & Silver Pick & Mt. Wilson & $\mathrm{Y}$ & $\mathrm{Nx}$ & 37.8733 & 108.0050 & $\mathrm{~N}$ & $\begin{array}{l}\text { Remains of mill at treeline, private } \\
\text { cabin site; no tails seen }\end{array}$ \\
\hline NGM854 & Whitepine & Tomichi & $\mathrm{Y}$ & Sx & 38.5338 & 106.3992 & $\mathrm{H}$ & $\begin{array}{l}\text { Stone foundation, not much else; small } \\
\text { tails pond to south was breached }\end{array}$ \\
\hline NGM870 & Hidden Treasure & Sneffels & $\mathrm{Y}$ & Sx & 37.9499 & 107.7806 & M & $\begin{array}{l}\text { Mill burned, some steel, tram cable, } \\
\text { foundation, tails }\end{array}$ \\
\hline NGM964 & Gateway & Uravan & $\mathrm{Y}$ & S & 38.6750 & 108.9722 & $\mathrm{~L}$ & $\begin{array}{l}\text { Reported in literature; observed by } \\
\text { BLM }\end{array}$ \\
\hline NGM967 & unknown & Ouray & $\mathrm{Y}$ & $\mathrm{S}$ & 38.0589 & 107.6900 & $\mathrm{~N}$ & Recent custom mill, small capacity \\
\hline NGM968 & unknown & Ouray & $\mathrm{Y}$ & $\mathrm{Nx}$ & 38.0539 & 107.6906 & $\mathrm{~N}$ & $\begin{array}{l}\text { Cement foundation, two ages of } \\
\text { building? on railroad ; no tailings } \\
\text { visible }\end{array}$ \\
\hline NGM969 & San Bernardo & Iron Springs & $\mathrm{Y}$ & $\mathrm{Mx}$ & 37.8581 & 107.8844 & $\mathrm{~L}$ & $\begin{array}{l}\text { Wooden mill stands at end of tram to } \\
\text { mine; tailings to NW above creek }\end{array}$ \\
\hline NGM970 & Keystone & Ruby & $\mathrm{Y}$ & $\mathrm{L}$ & 38.8683 & 107.0361 & $\mathrm{~N}$ & $\begin{array}{l}\text { Mill near lowest adit, concrete } \\
\text { remains; tails to SE; reclaimed }\end{array}$ \\
\hline NGM975 & Barstow & Red Mtn & $\mathrm{Y}$ & M? & 37.9208 & 107.7111 & $\mathrm{~N}$ & $\begin{array}{l}\text { Reported by Smith (1994); location } \\
\text { approximated; tails not known }\end{array}$ \\
\hline NGM976 & Tobasco & Burrows Park & $\mathrm{Y}$ & Sx? & 37.9344 & 107.5100 & $\mathrm{~N}$ & Reported by BLM Alpine Loop guide \\
\hline NGM976 & unknown & Quartz Creek & $?$ & $\mathrm{Sx} ?$ & 38.6111 & 106.4917 & $\mathrm{~N}$ & $\begin{array}{l}\text { Reported by Hill (1909), not located, } \\
\text { presumed to be small }\end{array}$ \\
\hline NGM977 & Vancorum & Uravan & $\mathrm{Y}$ & $\mathrm{R}$ & 38.2208 & 108.6000 & $\mathrm{~N}$ & $\begin{array}{l}\text { Older vanadium mill; reclaimed; site } \\
\text { from map }\end{array}$ \\
\hline NGM978 & Slick Rock? & Slick Rock & $\mathrm{Y} ?$ & $\mathrm{R}$ & 38.0361 & 108.9083 & $\mathrm{~N}$ & $\begin{array}{l}\text { Slick Rock mill or buying station; } \\
\text { reclaimed '90's; site from map }\end{array}$ \\
\hline NGM979 & Uravan U & Uravan & $\mathrm{Y}$ & $\mathrm{R}$ & 38.3667 & 108.7361 & $\mathrm{~N}$ & $\begin{array}{l}\text { Uravan mill complex; reclaimed ' } 90 \text { 's; } \\
\text { site from map }\end{array}$ \\
\hline NGM981 & Ohio City & Gold Brick & $?$ & $\mathrm{Nx}$ & 38.5639 & 106.5972 & $\mathrm{~N}$ & $\begin{array}{l}\text { Small early mill in literature; not } \\
\text { visible; no tails? }\end{array}$ \\
\hline NGM982 & Gunnison U & na & $\mathrm{Y}$ & $\mathrm{R}$ & 38.5250 & 106.9444 & $\mathrm{~N}$ & $\begin{array}{l}\text { Uranium mill for Los Ochos and other } \\
\text { U mines; reclaimed in '90's }\end{array}$ \\
\hline NGM990 & Idarado & Red Mtn & $\mathrm{Y}$ & VL & 37.9128 & 107.7014 & $\mathrm{~L}$ & $\begin{array}{l}\text { Mill buildings east of Treasury tunnel; } \\
\text { tailings to north }\end{array}$ \\
\hline NGM993 & Pandora & Telluride & $\mathrm{Y}$ & VL & 37.9306 & 107.7778 & $\mathrm{~L}$ & $\begin{array}{l}\text { Large modern mill from Treasury } \\
\text { tunnel (Idarado) }\end{array}$ \\
\hline NGM996 & Revenue & Sneffels & $\mathrm{Y}$ & $\mathrm{Mx} ?$ & 37.9750 & 107.7494 & $\mathrm{~L}$ & $\begin{array}{l}\text { Mill loc from map; described in } \\
\text { literature; tails covered by waste } \\
\text { rock? }\end{array}$ \\
\hline NGM997 & Contention & Lake Fork & $\mathrm{Y}$ & SX & 37.9819 & 107.2917 & M & $\begin{array}{l}\text { Mill by Crystobal Lake, tails below } \\
\text { along Lake Fork }\end{array}$ \\
\hline NGM998 & Pitkin & Qtz Creek & $\mathrm{Y}$ & NX & 38.6072 & 106.5222 & $\mathrm{~N}$ & $\begin{array}{l}\text { Stone foundation, } \mathrm{W} \text { side of Pitkin } \\
\text { village; tails in wetland to SE? }\end{array}$ \\
\hline NGM999 & Roosevelt & Qtz Creek & $\mathrm{Y}$ & NX & 38.5772 & 106.5467 & $\mathrm{~N}$ & $\begin{array}{l}\text { Wooden mill with stamps, east side } \\
\text { Qtz Creek; no tails; never } \\
\text { operated? }\end{array}$ \\
\hline \multicolumn{9}{|c|}{ Tailings sites } \\
\hline NGT102 & Cashin & Cashin & $\mathrm{Y}$ & $\mathrm{S}$ & 38.3106 & 108.9508 & $\mathrm{M} ?$ & Light colored sandy tails along creek \\
\hline NGT202 & Akron & Tomichi & $\mathrm{Y}$ & M & 38.5378 & 106.3956 & $\mathrm{H}$ & $\begin{array}{l}\text { Gray tailings, southern impound, with } \\
\text { pond }\end{array}$ \\
\hline
\end{tabular}


Table 7. Inventory of mill and tailings sites, CWS, Colorado-Continued.

\begin{tabular}{|c|c|c|c|c|c|c|c|c|}
\hline TN-ID & Name & District & Mill & Tailings & $\begin{array}{l}\text { Lat (N.) } \\
\text { (degrees) }\end{array}$ & $\begin{array}{l}\text { Long (W.) } \\
\text { (degrees) }\end{array}$ & ESRisk & Description \\
\hline \multicolumn{9}{|c|}{ Tailings sites-Continued } \\
\hline NGT212 & Akron & Tomichi & $\mathrm{Y}$ & M & 38.5383 & 106.3953 & $\mathrm{H}$ & $\begin{array}{l}\text { Ocher tails, heaped above mill, mixed } \\
\text { with waste rocks }\end{array}$ \\
\hline NGT216 & Whitepine & Tomichi & $?$ & $\mathrm{~S}$ & 38.5247 & 106.4122 & $\mathrm{H}$ & $\begin{array}{l}\text { Tails in Tomichi Creek; source likely } \\
\text { was NMG856 south of Akron mill. }\end{array}$ \\
\hline NGT237 & Gold Link & Gold Brick & $\mathrm{Y}$ & M & 38.6294 & 106.5811 & $\mathrm{H}$ & Shoestring impound east of mill, ocher \\
\hline NGT238 & Raymond & Gold Brick & $\mathrm{Y}$ & M & 38.6161 & 106.5892 & $\mathrm{H}$ & $\begin{array}{l}\text { Shoestring impound east of mill, south } \\
\text { and close to creek }\end{array}$ \\
\hline NGT239 & Carter & Gold Brick & $\mathrm{Y}$ & M & 38.6111 & 106.5972 & $\mathrm{H}$ & $\begin{array}{l}\text { Shoestring impound east of mill, north } \\
\text { of creek }\end{array}$ \\
\hline NGT281 & Hidden Treasure & Galena & $\mathrm{Y}$ & $S$ & 38.0183 & 107.3578 & $\mathrm{H}$ & $\begin{array}{l}\text { Small tails on } \mathrm{S} \text { side Henson Crk; mill } \\
\text { to } \mathrm{N} \text { between rd and creek }\end{array}$ \\
\hline NGT282 & Ute-Ulay & Galena & Y & M & 38.0172 & 107.3819 & $\mathrm{H}$ & $\begin{array}{l}\text { Several tails impounds near Henson } \\
\text { Creek; unstable }\end{array}$ \\
\hline NGT306 & Banner & Ouray & $\mathrm{Y} ?$ & $\mathrm{Mx}$ & 38.0542 & 107.6875 & $\mathrm{~L}$ & $\begin{array}{l}\text { Tailings under housing development, } \\
\text { on bench above Unc. River }\end{array}$ \\
\hline NGT308 & Silvershield & Ouray & $\mathrm{Y}$ & Sx & 38.0408 & 107.6828 & $\mathrm{~L}$ & Ocher tails on west side of Unc. River \\
\hline NGT313 & Carbonero & Iron $\mathrm{Spr}$ & Y? & M-L & 37.8558 & 107.8214 & $\mathrm{H}$ & Ocher tails in wet area by Howard Fk \\
\hline NGT315 & Carribeau & Iron Spr & $\mathrm{Y} ?$ & $\mathrm{~L}$ & 37.8575 & 107.8492 & $\mathrm{H}$ & $\begin{array}{l}\text { Large area ocheer tails on bench near } \\
\text { Howard Fk }\end{array}$ \\
\hline NGT323 & Alta & Iron Springs & $\mathrm{Y}$ & $S$ & 37.8850 & 107.8556 & $\mathrm{~L}$ & Smaller of two impounds \\
\hline NGT325 & Alta & Iron Springs & $\mathrm{Y}$ & $\mathrm{L}$ & 37.8856 & 107.8572 & $\mathrm{~L}$ & $\begin{array}{l}\text { Larger area on flat west of mine; } \\
\text { reclaimed } 1997 \text { but tails still in } \\
\text { place }\end{array}$ \\
\hline NGT343 & Idarado & Red Mtn & $\mathrm{Y}$ & $\mathrm{S}$ & 37.9183 & 107.8306 & M? & $\begin{array}{l}\text { One of smaller tails ponds, by Red } \\
\text { Mtn creek, not reclaimed }\end{array}$ \\
\hline NGT345 & Idarado & Red mtn & Y & VL & 37.9183 & 107.6994 & $\mathrm{~L}$ & Large tail impound, mid-north \\
\hline NGT362 & Silver Queen & Red Mtn & $?$ & Sx & 37.9178 & 107.6867 & $\mathrm{~L}$ & $\begin{array}{l}\text { Small imound below dump, possibly } \\
\text { extends under dump, partly } \\
\text { reclaimed? }\end{array}$ \\
\hline NGT364 & $\begin{array}{l}\text { Silver QueenRed } \\
\text { Mtn }\end{array}$ & & $?$ & $\mathrm{Mx}$ & 37.9297 & 107.6864 & $\mathrm{~L}$ & Larger unconfined slosh of tails \\
\hline NGT345 & Idarado & Red Mtn & $\mathrm{Y}$ & M & 37.9185 & 107.6855 & $\mathrm{H}$ & $\begin{array}{l}\text { Unconfined tails in channel of Red } \\
\text { Mountain Creek, older stage or } \\
\text { spill? }\end{array}$ \\
\hline NGT964 & Idarado 3 & Red Mtn & Y & M & 37.9165 & 107.7010 & $\mathrm{~L}$ & $\begin{array}{l}\text { Confined and reclaimed tailings near } \\
\text { US550; pond } 3\end{array}$ \\
\hline NGT370 & Idarado 4 & Red Mtn & $\mathrm{Y}$ & VL & 37.9375 & 107.6722 & $\mathrm{~L}$ & $\begin{array}{l}\text { Huge reclaimed tails, northernmost } \\
\text { from Idarado; pond } 4\end{array}$ \\
\hline NGT963 & Idarado 2 & Red Mtn & $\mathrm{Y}$ & M & 37.9219 & 107.6994 & $\mathrm{~L}$ & Reclaimed tails, pond 2 \\
\hline NGT962 & Idarado 1 & Red Mtn & $\mathrm{Y}$ & $\mathrm{S}$ & 37.9189 & 107.6989 & $\mathrm{~L}$ & Reclaimed tails, pond 1 \\
\hline NGT841 & Idarado & Red Mtn & Y & Sx & 37.9192 & 107.6972 & $\mathrm{H}$ & $\begin{array}{l}\text { Fluvial tails as overbank sediments } \\
\text { along Red Mtn. Crk }\end{array}$ \\
\hline NGT372 & Camp Bird & Sneffels & $\mathrm{Y}$ & VL & 37.9736 & 107.7236 & $\mathrm{H}$ & $\begin{array}{l}\text { Very large impound along Canyon } \\
\text { creek }\end{array}$ \\
\hline NGT375 & Atlas & Sneffels & $\mathrm{Y}$ & M & 37.9761 & 107.7569 & $\mathrm{H}$ & $\begin{array}{l}\text { Gray and ocher tails along creek; mill } \\
\text { to west }\end{array}$ \\
\hline NGT386 & Society & Telluride & $?$ & M & 37.9478 & 107.8722 & M? & $\begin{array}{l}\text { Surprising area of tails on bank near } \\
\text { San Miguel River }\end{array}$ \\
\hline NGT526 & Standard & Ruby & Y & $\mathrm{S}$ & 38.8781 & 107.0750 & $\mathrm{H}$ & $\begin{array}{l}\text { Ocher tails, impound and pond intact, } \\
\text { next to Elk Crk. }\end{array}$ \\
\hline NGT534 & Tincup & Tincup & Y & $\mathrm{S}$ & 38.7281 & 106.4978 & $\mathrm{~L}$ & $\begin{array}{l}\text { Brown coarse tails, some recently } \\
\text { removed; mill to SE }\end{array}$ \\
\hline
\end{tabular}


Table 7. Inventory of mill and tailings sites, CWS, Colorado-Continued.

\begin{tabular}{|c|c|c|c|c|c|c|c|c|}
\hline TN-ID & Name & District & Mill & Tailings & $\begin{array}{c}\text { Lat (N.) } \\
\text { (degrees) }\end{array}$ & $\begin{array}{l}\text { Long (W.) } \\
\text { (degrees) }\end{array}$ & ESRisk & Description \\
\hline \multicolumn{9}{|c|}{ Tailings sites-Continued } \\
\hline NGT538 & Contention & Lake Fork & Y? & $\mathrm{S}$ & 37.9867 & 107.2931 & M? & $\begin{array}{l}\text { Yellowish tails in draw next to Lake } \\
\text { Fork Crk outlet; mill above to } \\
\text { west? }\end{array}$ \\
\hline NGT625 & Silver Bell & Iron Springs & $\mathrm{Y}$ & M & 37.8617 & 107.8733 & $\mathrm{H}$ & $\begin{array}{l}\text { Ocher tails piled high above Howard } \\
\text { Fork, west of highway }\end{array}$ \\
\hline NGT628 & Caribeauu & Iron Springs & $\mathrm{Y}$ & M & 37.8581 & 107.8492 & $\mathrm{H}$ & $\begin{array}{l}\text { Ocher tails on south side of Howard } \\
\text { Fork, wet from mine drainage }\end{array}$ \\
\hline NGT638 & Gold King & Iron Springs & Y & $\mathrm{S}$ & 37.8803 & 107.8494 & $\mathrm{~L}$ & Tan sandy tails west of mill \\
\hline NGT649 & San Juan & Mineral Point & $\mathrm{Y}$ & $\mathrm{S}$ & 37.9608 & 107.5925 & $\mathrm{~L}$ & $\begin{array}{l}\text { Small amount of red tails (roasted) at } \\
\text { foundation; low production or in } \\
\text { creek? }\end{array}$ \\
\hline NGT660 & Hidden Treasure & Sneffels & $\mathrm{Y}$ & $\mathrm{S}$ & 37.9500 & 107.7303 & M & $\begin{array}{l}\text { Ocher tails poured down slope into } \\
\text { small creek and wetland from } \\
\text { NGM870 }\end{array}$ \\
\hline NGT661 & Savage & Telluride & $\mathrm{Y}$ & $\mathrm{S}$ & 37.9328 & 107.7461 & $\mathrm{~L}$ & $\begin{array}{l}\text { Small stamp mill remains; tails to } \\
\text { southwest }\end{array}$ \\
\hline NGT679 & Eclipse & Sneffels & $\mathrm{Y}$ & $\mathrm{S}$ & 37.9761 & 107.7475 & $\mathrm{~L}$ & Tails about 1 acre, grass growing \\
\hline NGT868 & Camp Bird 3 & Sneffels & $\mathrm{Y}$ & $\mathrm{Sx}$ & 37.9482 & 107.7306 & $\mathrm{~L}$ & $\begin{array}{l}\text { Tails east of mill, some probably } \\
\text { eroded; covered by } 1970 \text { 's waste } \\
\text { dumps }\end{array}$ \\
\hline NGT867 & X-Richmond & Sneffels & $\mathrm{Y}$ & Sx & 37.9574 & 107.7242 & $\mathrm{H}$ & $\begin{array}{l}\text { Fluvial tails as overbank sediments } \\
\text { along Imogene Creek, below } \\
\text { Richmond }\end{array}$ \\
\hline NGT986 & Pandora & Telluride & $\mathrm{Y}$ & VL & 37.9319 & 107.7833 & $\mathrm{~L}$ & $\begin{array}{l}\text { Very large impound W of mill, S side } \\
\text { San Miguel River }\end{array}$ \\
\hline NGT987 & Sutton & RedMtn? & $\mathrm{Y}$ & $\mathrm{Nx}$ & 37.9989 & 107.6583 & $\mathrm{~N}$ & $\begin{array}{l}\text { In Canyon, end of tramway; from map; } \\
\text { no tails }\end{array}$ \\
\hline NGT988 & Idarado & Red Mtn & $\mathrm{Y}$ & $\mathrm{L}$ & 37.9236 & 107.6931 & $\mathrm{~L}$ & $\begin{array}{l}\text { Mid-north in Red Mtn Creek valley, } \\
\text { from map }\end{array}$ \\
\hline NGT982 & Gunnison $\mathrm{U}$ & na & $\mathrm{Y}$ & $\mathrm{R}$ & 38.5250 & 106.9444 & $\mathrm{~N}$ & $\begin{array}{l}\text { Tails reclaimed--removed and placed } \\
\text { elswhere }\end{array}$ \\
\hline NGT979 & Uravan U & Uravan & Y & $\mathrm{R}$ & 38.3667 & 108.7361 & $\mathrm{~N}$ & $\begin{array}{l}\text { Tailings reclaimed by Superfund } \\
\text { action in late 1990's }\end{array}$ \\
\hline NGT977 & Vancorum & Uravan & $\mathrm{Y}$ & $\mathrm{R}$ & 38.2208 & 108.6000 & $\mathrm{~N}$ & Tailings and other waste reclaimed \\
\hline NGT978 & Slick Rock? & Slick Rock & Y? & $\mathrm{R}$ & 38.0361 & 108.9083 & $\mathrm{~N}$ & $\begin{array}{l}\text { Radioactive waste reclaimed in } 1990 \text { 's } \\
\text { by UMTRA }\end{array}$ \\
\hline
\end{tabular}




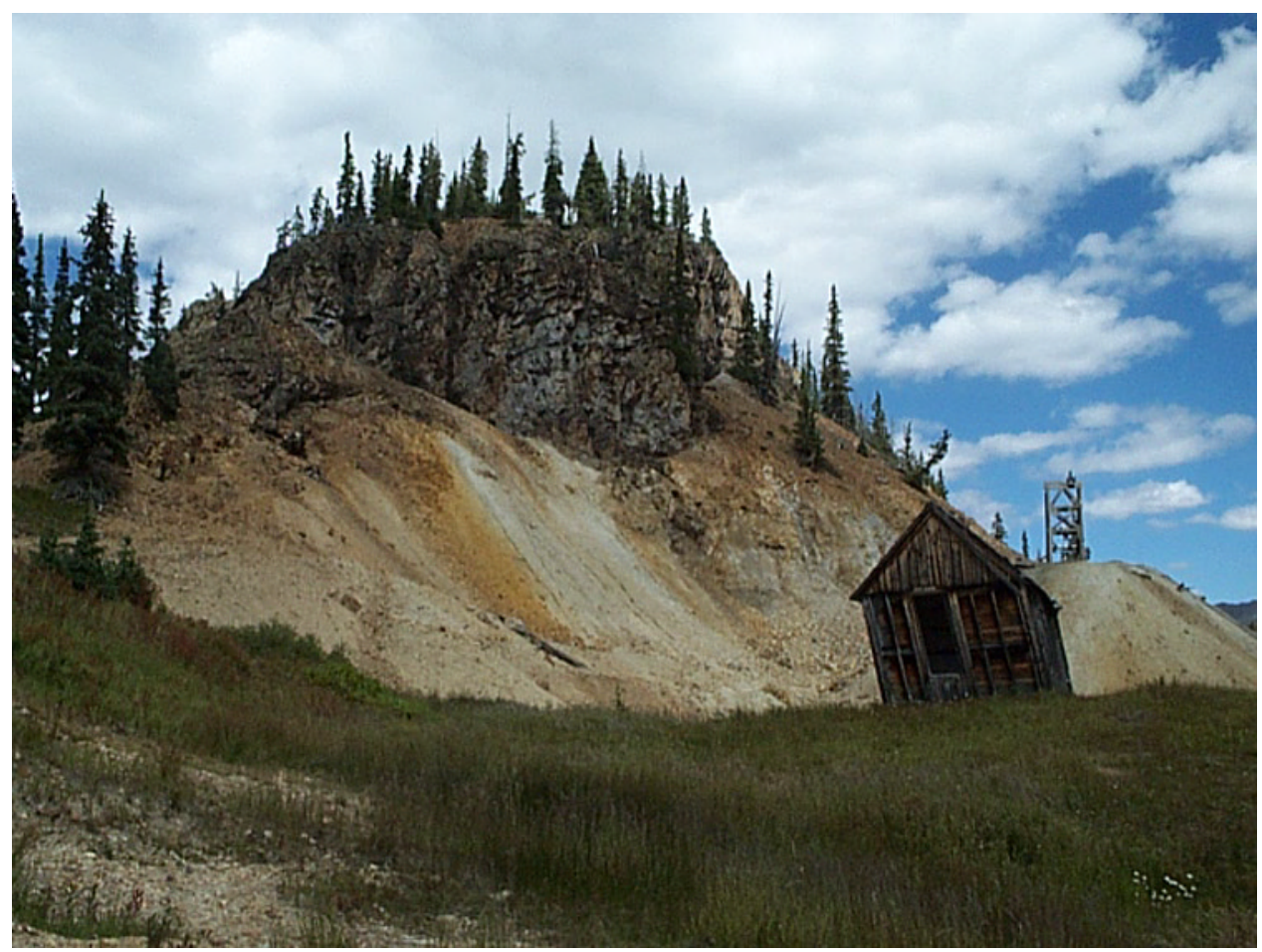

Photograph 8. The National Belle mine, Red Mountain district, produced rich silver ore from a small breccia pipe within this hill; the mine dump is relatively small (right side) for the value of ore produced prior to 1900. The rusty rocks on the left side are natural exposures.

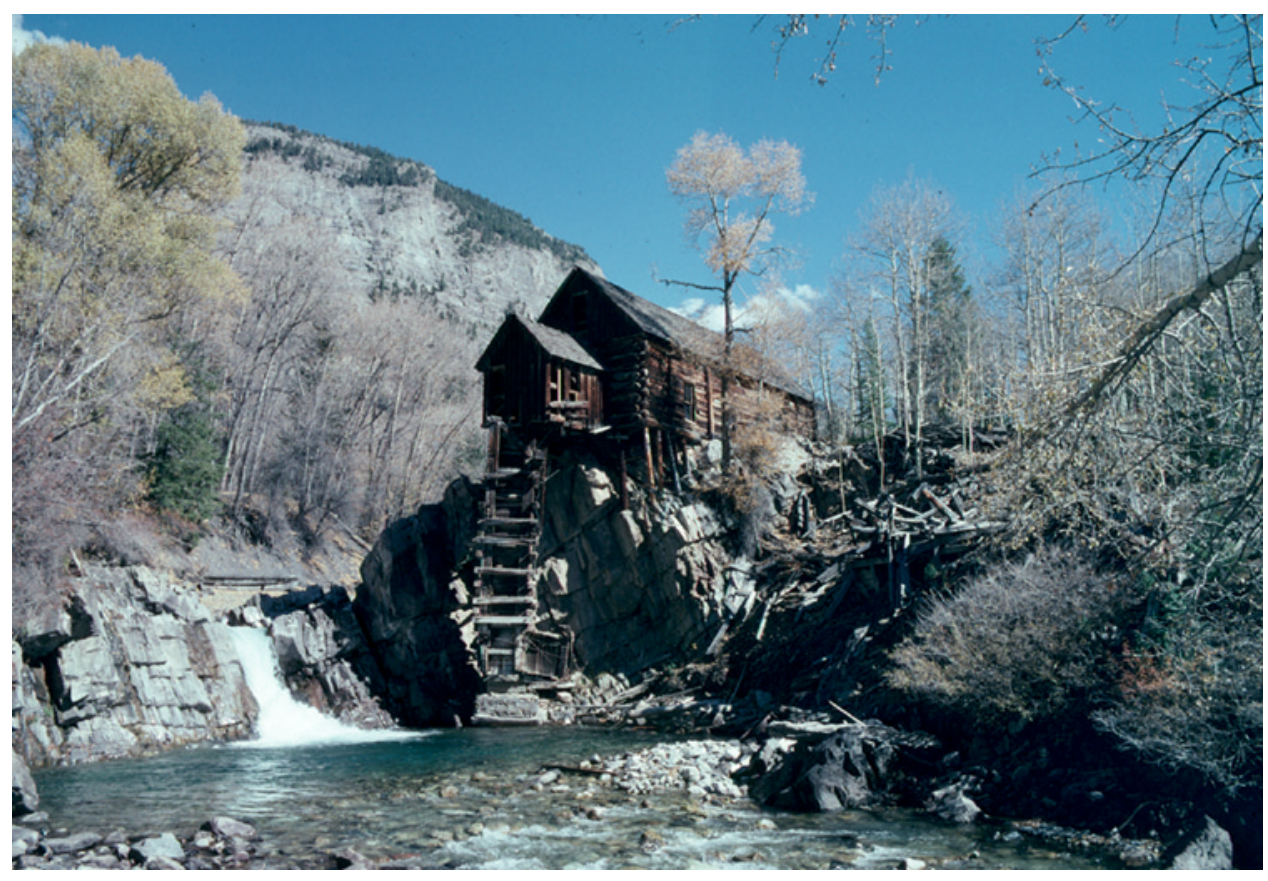

Photograph 9. This picturesque mill on the Crystal River is typical of early mills that utilized water power and discharged mill tailings directly into the river. 


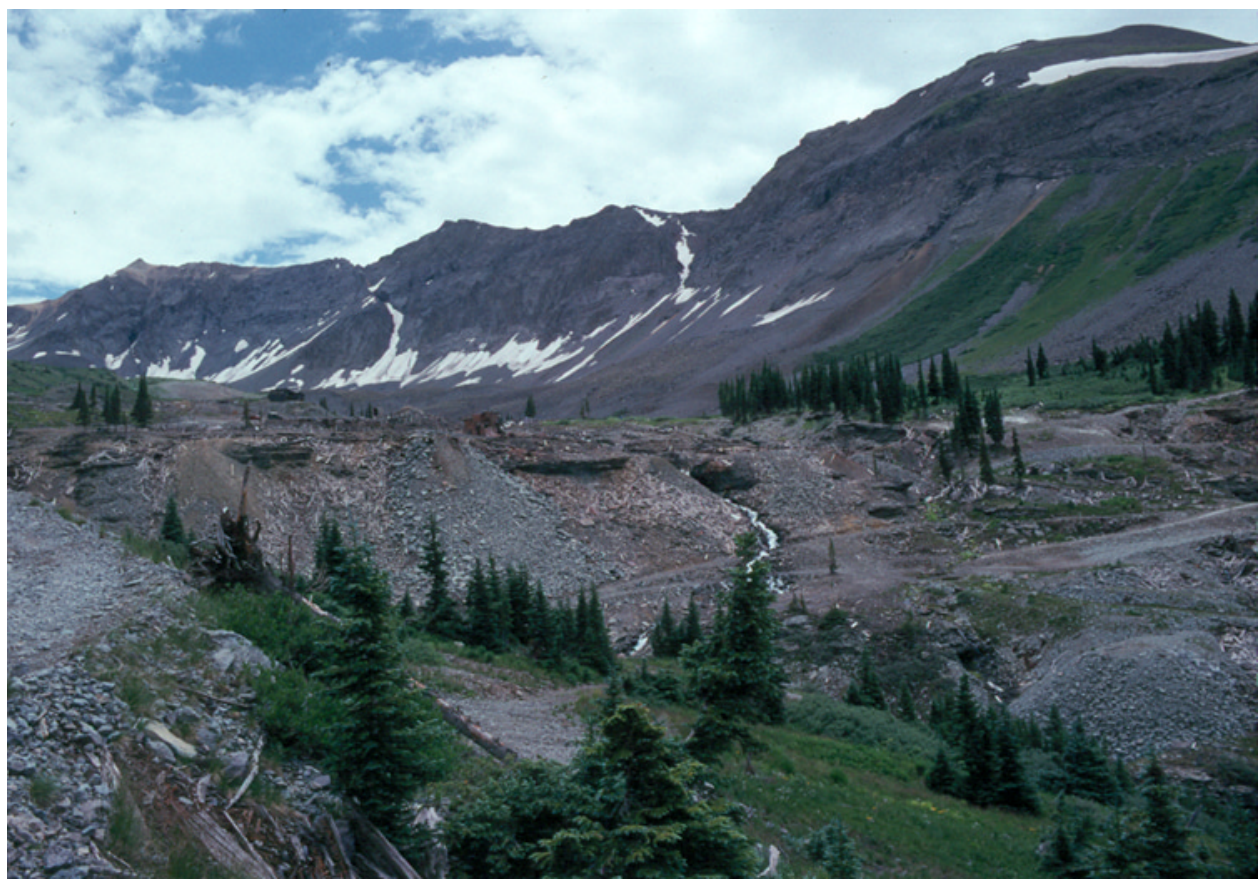

Photograph 10. Tomboy, east and above Telluride, was the site of at least six mills that served medium-sized mines that converged on this one relatively flat area at 11,000 ft elevation. These mills were active from about 1890 to 1930 and were phased out after difficulties with fires and avalanches in favor of mills at lower elevations that received ores from long tunnels and tramways. Almost no tailings were impounded at Tomboy-because there was no space and no regulations, the tailings were allowed to wash away in Savage and Marshall Creeks.

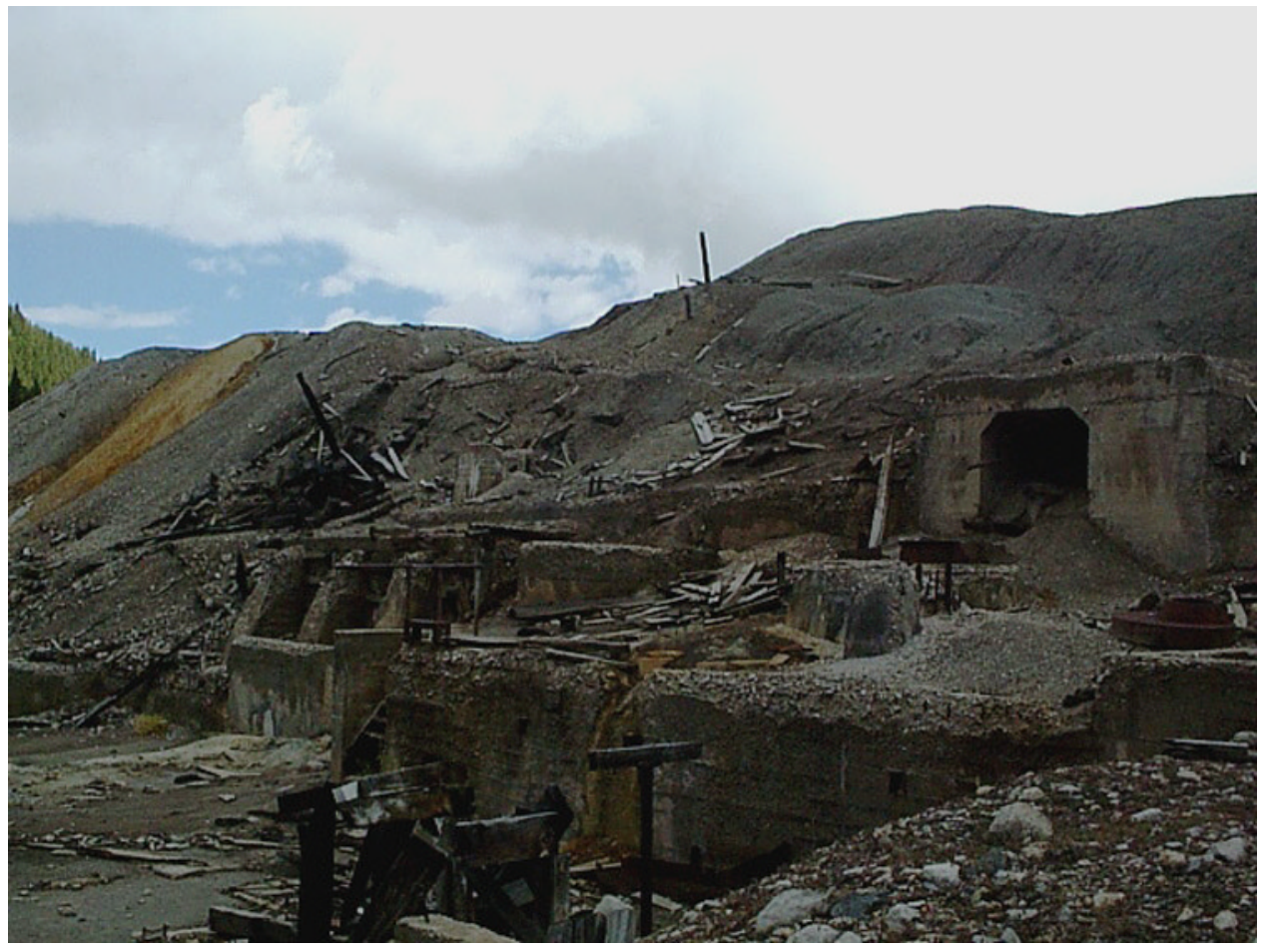

Photograph 11. The Akron mine and mill near Whitepine was active in the 1940's-50's. The large piles of waste rocks and mill tailings were created as ore and waste were hauled out of a tunnel that was driven to the east (right) into several previously mined deposits. 


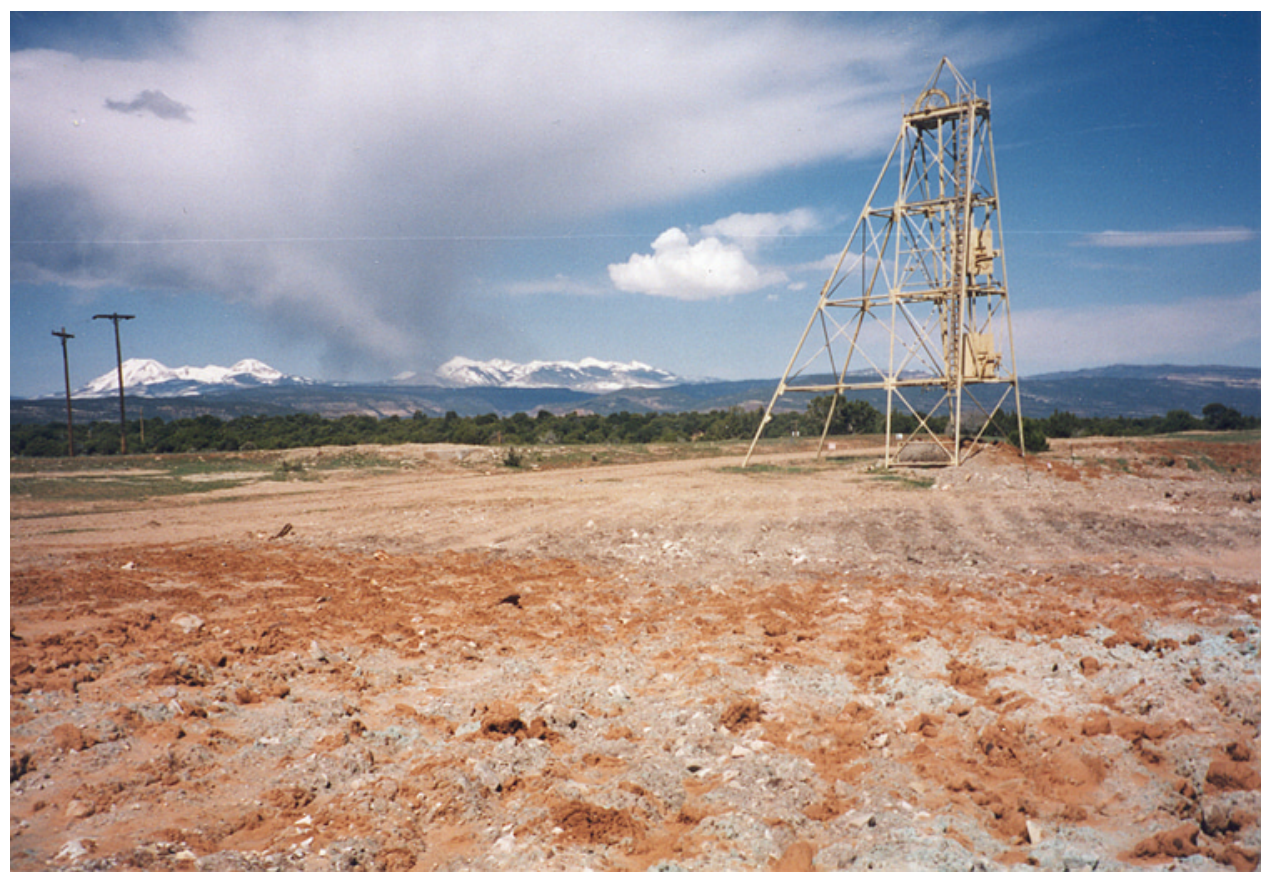

Photograph 12. Mining of uranium-vanadium in the Uravan district, from the 1940's through the 1970's, was typically from small- to medium-sized mines (shaft and adits) that brought relatively small volumes of waste to the surface. They left no tailings onsite because ore was hauled to a central mill. The Golden Cycle mine site was reclaimed in 1997.

\section{Mobility of Metals: Leach Tests of Mineralized Rocks}

The soluble or mobile portion of metals in rocks is the part that has the potential to degrade surface waters and harm aquatic life; thus, it is of more interest than the total metal concentrations described earlier. To better understand the mobility of metals, laboratory tests were undertaken to simulate reactions in mined waste materials. Solid materials from dumps, mill tailings, outcrops, and slag piles-all of which can be termed rocks - have been processed in the laboratory by a passive leach method that provides an approximate measure of reactions in nature, such as during storm events. Results of the leach tests, summarized in table 8 , span a wide range of concentrations that will be described here and discussed in more detail in later sections on the mining districts.

Leach tests demonstrate great variability in the amount of metal that is soluble in dump and tailings materials; the variability is large from element to element, and from site to site. The results for 116 samples are listed in table 4, summarized in table 9 , and shown in figures 5 and 6 . The behavior of samples from the CWS are generally similar to trends for more than 150 samples from the Silverton area (Fey and others, 2000a; Fey and others, 2000b) and also from Montana and Nevada (Nash, 2000b). There are many mineralogic and geologic factors that control the water-rock reactions, which are complex in detail. At the most general level, results for dump, tailings, and altered-rock samples show that leachate metal concentrations generally are highest in dump samples and lowest in altered-rock samples. If one looks more closely, there are large differences even among a group of materials: some tailings samples in a district can release much higher metal concentrations than others (such as the common case of tailings from old, inefficient stamp mills compared to those from modern mills). There also is a wide range in results plotted by deposit type (fig. 5), suggesting that there is almost as much variability within materials from a deposit type as there is between deposit types. This variability is not unexpected to a geologist, but probably is perplexing to a manager who wants to see a clear and predictable pattern to the behavior of metals. From my experience with hundreds of dumps and several hundred tailings impoundments, predictions are a poor substitute for hands-on sampling and testing of materials.

Results of these laboratory leach tests confirm what others are finding (Fey and others, 2000a): release and mobility of metals is strongly correlated to acidity generated by the materials. Nearly all metals of concern have higher concentrations and higher mobility in acidic solutions. The increase is nearly linear from $\mathrm{pH} 5$ to $\mathrm{pH} 2$. The principles underlying this relationship are the same as for mine drainage and stream waters, which are discussed elsewhere in this report and treated in much more chemical detail by others (Drever, 1997; Langmuir, 1997; Nordstrom and Alpers, 1999). The following 
Table 8. Statistical summary of some selected features of leachate and mine drainage chemistry, central Western Slope, Colorado.

[Chemical data from ICP-MS analyses, reported in parts per billion; $\mathrm{pH}$ in standard units]

\begin{tabular}{|c|c|c|c|c|c|c|}
\hline & \multicolumn{2}{|c|}{ Dump leachates $(n=76)$} & \multicolumn{2}{|c|}{ Tailings leachates $(n=28)$} & \multicolumn{2}{|c|}{ Mine drainage $(\mathrm{n}=62)$} \\
\hline & Median & Maximum & Median & Maximum & Median & Maximum \\
\hline $\mathrm{pH}$ & 3.5 & $2.5^{*}$ & 4.1 & $2.6^{*}$ & 4.5 & $2.0^{*}$ \\
\hline $\mathrm{Al}$ & 344 & 68,450 & 43.7 & 5,760 & 493 & 470,000 \\
\hline As & 1.8 & 143 & 0.4 & 48.3 & 3.5 & 1,000 \\
\hline $\mathrm{Cd}$ & 3.7 & 1,310 & 6.1 & 1,100 & 7.0 & 165 \\
\hline $\mathrm{Cu}$ & 112 & 141,000 & 82.8 & 2,320 & 139 & 28,000 \\
\hline $\mathrm{Fe}$ & 880 & 100,300 & 469 & 122,800 & 5,400 & $1,500,000$ \\
\hline Mn & 87.8 & 121,450 & 146 & 60,230 & 1,200 & 57,800 \\
\hline Mo & 1.6 & 1,980 & 1.1 & 36.3 & 1.7 & 174 \\
\hline $\mathrm{Pb}$ & 46.8 & 61,100 & 12.7 & 5,650 & 28.9 & 1,374 \\
\hline $\mathrm{Sb}$ & 0.4 & 103 & 0.3 & 28.8 & 0.4 & 13 \\
\hline $\mathrm{Se}$ & 2.2 & 707 & 0.2 & 73.5 & 1.2 & 194 \\
\hline $\mathrm{U}$ & 0.8 & 3,400 & 0.1 & 18.2 & 1.1 & 555 \\
\hline $\mathrm{Zn}$ & 361 & 148,300 & 526 & 164,900 & 611 & 21,500 \\
\hline
\end{tabular}

* for $\mathrm{pH}$, maximum acidity is minimum $\mathrm{pH}$ value.
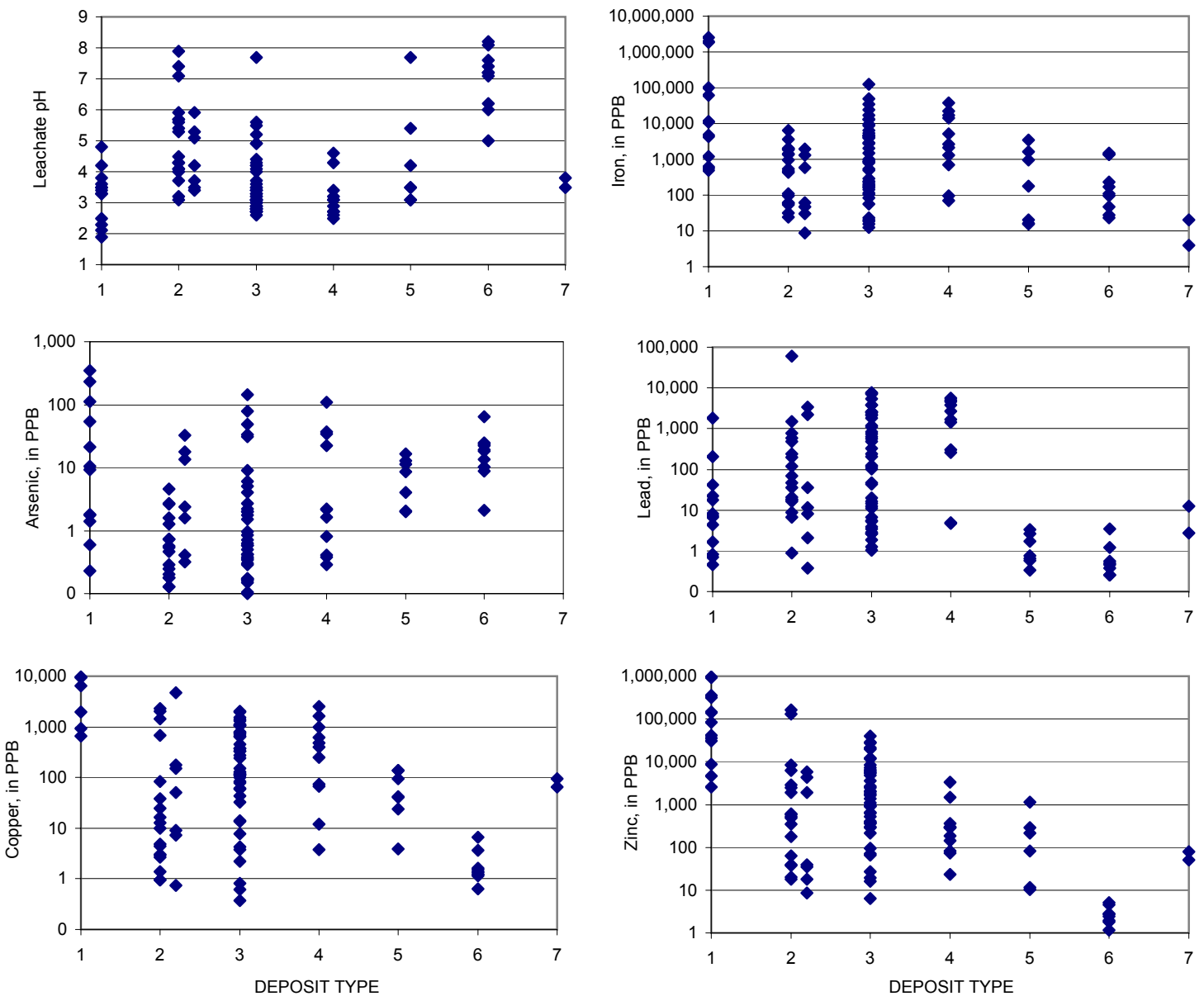

Figure 5. Compositions of leachate solutions by deposit type. Deposit-type codes: 1, massive sulfide; 2 , polymetallic vein and replacement; 3 , volcanic-hosted polymetallic vein; 4, breccia pipe; 5, sandstone uranium; 6, vein uranium; 7 , ferricrete. 

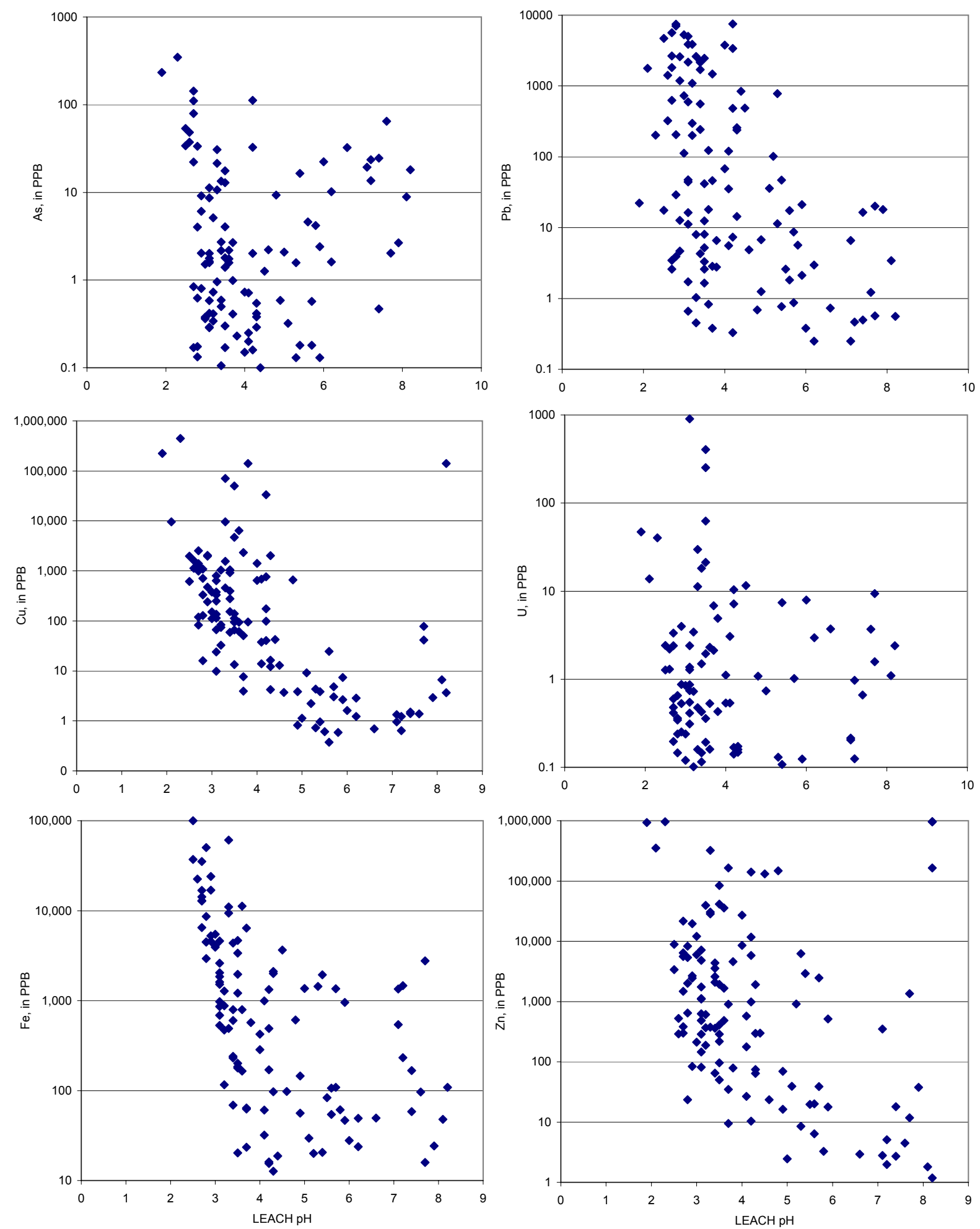

Figure 6. Compositions of leachates from mineralized rocks as a function of $\mathrm{pH}$. 
summary comments by element are intended to provide some guidance for the potentially toxic metals expected in metal mining areas of the CWS. However, note that the leach test results are an approximate measure of the potential for the material to release that element, but this may not occur in the real world because of lack of moisture, low permeability, or other factors. Further, the leachate concentrations can only be qualitatively judged relative to regulatory standards for surface waters, a complex topic that will be discussed in the next section of this report. Because the water-quality standard values range from less than $1 \mathrm{ppb}$ to more than $1,000 \mathrm{ppb}$, for various elements and various beneficial uses, the values mentioned below are provided to suggest an order of magnitude and may not be appropriate for all sites.

Aluminum.-Many samples yielded high Al concentrations if the leachate $\mathrm{pH}$ was below 4; the median ${ }^{4}$ value was $260 \mathrm{ppb}$. The toxicity standard for aquatic life (chronic exposure) is $100 \mathrm{ppb} \mathrm{Al}$ (CWQCC, 2000; discussed later). This tendency for acid mine drainage to carry high amounts of $\mathrm{Al}$ is potentially significant to fish habitat.

Arsenic.-Mobility of As is not as acid-dependent as true metals, and only 5 percent of the CWS samples released more than $100 \mathrm{ppb}$ (figs. 5, 6). The median was $1.6 \mathrm{ppb}$. Mined materials on the CWS tend to release relatively low amounts of As, which is good news because higher amounts were expected from predominant polymetallic ores.

Cadmium.-This metal is toxic at low concentrations and is commonly released in significant concentrations if the leachate $\mathrm{pH}$ is below 5. Nearly half the samples released more than $10 \mathrm{ppb} \mathrm{Cd}$. Mined materials in the study area have the potential to release environmentally significant amounts of $\mathrm{Cd}$.

Copper.-This element is toxic to aquatic life (but not humans) at relatively low concentrations (about 5-10 ppb). Mined materials in the study area have a tendency to release significant amounts if the $\mathrm{pH}$ is below about 5 (fig. 6). More than half of the samples tested released more than $100 \mathrm{ppb}$ (which is about 20 times higher than the aquatic life toxicity standard). Copper and $\mathrm{Zn}$ are potentially the biggest threats to fisheries on the CWS and, for this reason, will be discussed in several subsequent sections.

Iron.- This element is mobile in acidic waters (fig. 6) and is commonly released in high concentrations from the materials tested ( 22 percent yielded $>5,000 \mathrm{ppb}$, which is five times the aquatic life standard). This is consistent with the common occurrence of high Fe in surface waters of the study area and red-ocher precipitates on stream beds.

Manganese.-The behavior of $\mathrm{Mn}$ is theoretically similar to that of Fe, but in this study it is not as problematic. Although

\footnotetext{
${ }^{4}$ Median values are reported here for several reasons: the number of samples is relatively small, the statistical validity of these calculations is not be considered to be rigorous, and the data values generally do not have a normal distribution (the presence of extremely high values skews the distribution and creates an average that is much higher than the median).
}

some samples released high concentrations, only 9 percent were above 5,000 ppb. Manganese appears to be a concern chiefly for drinking-water supplies, for which the standard is $50 \mathrm{ppb}$, whereas $\mathrm{Mn}$ is not a threat to aquatic life until concentrations of about 1,000 ppb are reached (CWQCC, 2000).

Molybdenum.-Molybdenum is a common trace metal in the ores studied, but it appears to be less mobile than other metals. Only 6 percent of leached samples released more than $100 \mathrm{ppb}$ (the aquatic life toxicity standard is $19 \mathrm{ppb}$ ). This is an element that is relatively mobile under alkaline conditions as the oxyanion molybdate, as shown in leach tests on sandstonetype uranium ores.

Lead.-Although lead is abundant in many of the ores, it generally is not as mobile as associated $\mathrm{Cd}, \mathrm{Cu}$, or $\mathrm{Zn}$. Leach tests show that a third of the samples released more than 250 $\mathrm{ppb}$ at $\mathrm{pH}<5$ (fig. 6); the aquatic life standard is about $25 \mathrm{ppb}$. This suggests that $\mathrm{Pb}$ is potentially a significant problem, but it is less common than some other base metals.

Antimony.-Antimony is not listed among the metals that are toxic to aquatic life, but it is of concern to human health. Thus, there is a drinking-water standard of $146 \mathrm{ppb}$. This element is enriched in many of the ores but does not appear to be very mobile. Only one sample released more than $100 \mathrm{ppb}$. Because the toxicity threshold is relatively high, and the leach tests suggest that it is not highly mobile, there seems to be low potential for problems from $\mathrm{Sb}$.

Selenium.-This metaloid is toxic to aquatic life at relatively low concentrations (5-10 ppb) and is relatively mobile; thus, it may pose problems despite its trace-level concentrations in most ore types. Leach tests show that 16 percent of the CWS samples release more than $25 \mathrm{ppb}$ Se. Selenium is mobile as the oxyanion $\mathrm{SeO}_{4}$ at neutral-alkaline $\mathrm{pH}$; thus its behavior is distinct from most of the true metals. Compositions and $\mathrm{pH}$ values favorable for Se mobility are most common in sandstone-type uranium ores, but locally it may be significant at massive sulfide deposits such as Vulcan in the Cochetopa district.

Uranium.-This element is mobile under both acidic and alkaline conditions, which explains why it can be mobilized from many ore wastes, even ores like those of the massive sulfide type that do not contain much U. Potentially significant levels of uranium were observed in only 4 leachates with $\mathrm{pH}$ $<5$ (fig 6). The water-quality standard for $U$ is relatively high (44 ppb, converted from the standard of 30 picocuries). The median value for all samples was relatively low at $0.5 \mathrm{ppb}$.

Zinc.-This element is enriched in many ore types and is probably the most mobile and concentrated toxic metal relative to its standard of about $50 \mathrm{ppb}$ for aquatic life. Zinc is not generally a risk for human health. Slightly more than half of the samples tested released more than $500 \mathrm{ppb}$ (10 times the aquatic life standard). Highest values for $\mathrm{Zn}$ are in acidic waters below $\mathrm{pH} 4$, but leachates with $\mathrm{pH}$ of 5-7 also carried significant $\mathrm{Zn}$ (fig. 6). The release of high concentrations of $\mathrm{Zn}$ from many CWS samples suggests that $\mathrm{Zn}$ is the most important metal to study where there is concern for aquatic life near mining areas on the Western Slope. 


\section{Water Quality: Introduction to Standards and Ratings}

Water quality is a complex issue that includes both technical (chemical and biological) and legal issues that are far beyond the scope of this report. The framework for water quality utilized in this investigation is the standard set by the Colorado Water Quality Control Commission (CWQCC), which is part of the Colorado Department of Public Health and Environment. This agency sets policies and regulations for protection of water quality as defined by the Colorado Water Quality Control Act, which is very similar to the Federal Clean Water Act, except that the Colorado Act also includes ground waters. The CWQCC works in parallel with the Water Quality Control Division (CWQCD), which "regulates the discharge of pollutants into the State's surface and ground waters." The Colorado policies and regulations are complex for the nonspecialist and are most easily accessed through the frequently updated State website (www.cdphe.state.co.us/cdphereg.html). Particularly useful from the website is the Colorado Water Quality Management and Drinking Water Protection Handbook with 141 pages of information on policies, methods, and standards. Another source of technical information is the Colorado Nonpoint Assessment Report (CWQCD, 1989).

The Colorado regulations are generally similar to those developed by other States and by the EPA, but, compared to some other Western States, CWQCC is much more detailed in the stream segments that it assigns regulatory standards based on beneficial use (some segments are as short as $1 \mathrm{mi}$ ). Beneficial use classes include recreation, agriculture (irrigation), aquatic life, drinking water, and other historic or future uses. In the study area, the designation for most streams near mining areas on the CWS is "cold water aquatic life," which includes fish and associated biota. Because the standards for metal concentrations that are toxic in various beneficial uses differ in important details that are confusing in an overview such as this report, I will utilize just one standard, for cold water aquatic life, as the reference for metal concentrations (table 9).

The quality of Colorado surface waters is reviewed every 2 years by the CWQCD, in compliance with the U.S. Clean Water Act. Placement on the list of stream segments that are not in compliance with standards (called "303(d) lists") has significant implications for management of those waters. The Nonpoint Assessment Report (CWQCD, 1989) contains more than 180 pages of technical information and lists of stream segments that were considered impacted by mining, agricultural, or industrial activities. For the study area, many streams were listed as impacted by metal pollutants related to mining; in most cases, the mining source, historic or current, is not specified. In the Gunnison River watershed, seven stream segments were rated low, six medium, and one highly impacted. In the Uncompahgre River watershed, four stream segments were rated medium and two highly impacted. For the San Miguel/Dolores watershed (the part in study area),
Table 9. Colorado basic stream standards for metals: class-1 aquatic life.

[Note: These values are for chronic exposure. The selected values shown are for 100-200 mg/L water hardness; criteria for $\mathrm{Al}, \mathrm{Fe}, \mathrm{Mn}$, and Se do not vary with hardness. Taken from CWQCD (1989, table 9, which cites Colorado Department of Health, 1984, Basic Standards and Methodologies 3.1.0)]

\begin{tabular}{cc}
\hline $\mathrm{pH}$ or element & $\begin{array}{c}\text { Stream standard for class-1 aquatic life } \\
(\mathrm{ppb} \text { or } \mu \mathrm{g} / \mathrm{L} \text {, except as noted })\end{array}$ \\
\hline $\mathrm{pH}$ & $6.5-9.0 \mathrm{pH}$ units \\
$\mathrm{Al}$ & 100 \\
$\mathrm{As}$ & 50 \\
$\mathrm{Cd}$ & 1 \\
$\mathrm{Cu}$ & 10 \\
$\mathrm{Fe}$ & 1,000 \\
$\mathrm{Mn}$ & 1,000 \\
$\mathrm{~Pb}$ & 25 \\
$\mathrm{Se}$ & 5 \\
$\mathrm{Zn}$ & 50 \\
\hline
\end{tabular}

one segment was rated low, seven medium, and six highly impacted. Some of the highly impacted stream segments identified were Oh-Be-Joyful Creek (NW. of Crested Butte), Red Mountain Creek south of Ouray, Marshall Creek and San Miguel River east of Telluride, and San Miguel River below Uravan; these streams will be described and discussed in later sections of this report. The 303(d) report was a significant source of data for the Colorado geo-environmental map constructed by the USGS (Plumlee and others, 1995d). Significantly, the 1998 303(d) list (CWQCD, 1998) has revised many of the ratings from earlier lists: only Slate Creek (near Crested Butte) and three headwater branches of the San Miguel River east of Telluride are listed as impacted by mining-related contamination. The revisions have not been explained by CWQCD but appear to reflect better analytical results for the streams, as well as reclamation in several mining areas. New water sampling for this investigation, described later, is in close agreement with the 1998 list (but is at odds with many of the 1989 ratings). Information in the 1998 303(d) list shows that, where streams are impacted by mining as in the Silverton, Bonanza, and Leadville districts, zinc is the most common contaminant, and copper, iron, and cadmium also are listed as contaminants.

Prompted by the 1989 303(d) list of mining-impacted streams, Plumlee and others (1995d) explained likely sources of acid and metals and provided geologic reasons for the mobility of metals. The Colorado geo-environmental assessment showed graphically the geographic and geologic areas of concern. Because that work was based chiefly on available information, including the stream ratings of CWQCD (1989), this investigation was designed to provide new geochemical information for surface waters in the vicinity of mines-areas that are not normally sampled by agencies monitoring water flow and quality (typically done at gauging stations on main stream segments 5-10 mi from headwaters). Because of time 
and budget constraints, and the large area under study, the investigations reported here were necessarily reconnaissance in nature. The goal of this investigation was to identify the major significant geochemical problems related to historic mining, to rank them, but not to define the specific level of contaminant release. Another goal was to determine the beneficial geologic interactions with acidic waters that naturally attenuate local sources of metal contaminants.

Quality of ground water is included in CWQCD management of Colorado waters and is an issue around historic mines where surface drainage from various sources infiltrates alluvium or bedrock. There are indications in monitoring wells near mines that subsurface flow can be significant (Nash, 1999b), but wells are rarely available to describe ground water near mines. An exception is the presence of wells in the Uravan district and a sampling program by specialists in the BLM Grand Junction office. Study of historic mining areas would be much more effective if shallow wells could be drilled to sample and monitor ground water.

Water quality in this study will be compared to the standard for aquatic life (class 1, cold water) from CWQCD (1989 and 2000) (table 9); these values are for chronic (long-term) exposure. In this report I will refer to them as aquatic life water standards (ALWS). These reference concentrations are similar to those for domestic drinking-water supplies but are more restrictive for elements such as $\mathrm{Cu}$ and $\mathrm{Zn}$ that affect aquatic life more than human health, and more stringent than for agricultural use. In detail, biologists prefer that metal concentrations be corrected for the effects of water hardness: increases in hardness reduce the toxic effects of some metals $(\mathrm{Cd}, \mathrm{Cu}, \mathrm{Pb}, \mathrm{Zn})$. Thus, the concentration for the metal to cause chronic or acute toxicity is higher in high-hardness waters (300 ppb Zn at $400 \mathrm{mg} / \mathrm{L}$ hardness compared with $50 \mathrm{ppb} \mathrm{Zn}$ at $100 \mathrm{mg} / \mathrm{L}$ hardness). Hardness values for these mine-related waters are highly variable, in the range about 10 to $1,400 \mathrm{mg} / \mathrm{L}$ (as $\mathrm{CaCO}_{3}$ ). Hardness corrections were not made for my results because to do so would imply more precision than appropriate in these reconnaissance hydrogeochemical studies. The values in table 9 provide guidelines for evaluating the severity of degradation, consistent with the nature of my studies. These values are useful for interpretation of processes operating at mine sites, but readers should consult results of other definitive studies (Owens, 1997; Herron and others, 1998; Crock and others, 1999; Smith and Huyck, 1999) for more specific data and regulatory concerns. For more information, excellent material is available from Colorado Department of Public Health and Environment, Water Quality Control Commission under the State website at www.state.co.us.

Although the composition of streams and other surface waters is an indicator of impact on the environment, biologic (toxicologic) and health criteria are really the basis for waterquality standards (CWQCD, 1989; Manahan, 1994). In practice, it is much easier to determine water compositions than to measure biological communities such as benthic macroinvertebrates and fish (Besser and others, 1998; Boyle and Bukantis, 1998), which is one reason why water chemistry was sampled widely in this study. Biological criteria for water quality are based on the biota and food chain that is historically present in the stream, or the desired community at the end of reclamation or stream restoration. These topics and issues are too complex to discuss in any detail here.

The interested reader should seek new biological work done on the upper Animas River watershed near Silverton because the fisheries, or lack of them, are very similar to those in most of the watersheds or sub-basins in this study area. Biological information was collected in 1991 and discussed in the context of water quality by Owens (1997). The Animas Stakeholders group has supported new biological studies of the upper Animas River from 1996 to 2000, and scientists of the USGS Biological Resources Division are completing studies there of topics including bioaccumulation of potentially toxic metals by stream biota; toxicity of stream water, bed sediment, and sediment porewater to fish and invertebrates; toxicity thresholds of zinc and copper to fish and invertebrates that populate the upper Animas basin; and influences of physical habitat on stream trout populations (Besser, 2000). The toxicity tests for the Animas River and its biota (Besser, 2000) show that the concentrations of dissolved copper and zinc that cause mortality to "sensitive" and "tolerant" species differ considerably (for example, 700 vs. $160 \mathrm{ppb} \mathrm{Zn}$ and 33 vs. 8 ppb $\mathrm{Cu}$ ); thus, no single concentration actually defines aquatic health as is implied by the Colorado Basic Stream Standards cited above. Readers should be aware of these complexities, consult appropriate biologic authorities, and not take too literally the chemical results presented and discussed in this report. Because research on fisheries shows that $\mathrm{Cu}$ and $\mathrm{Zn}$ are of prime importance to the health of many aquatic species, those metals will be emphasized in this report.

\section{Threats to Aquatic Life by Metals: A Copper-Zinc Index}

A copper-zinc index (CZI) has been formulated to provide a simple number that describes the magnitude of copper and zinc concentrations in water samples in relation to aquatic life requirements as determined by toxicologic tests just described (Besser, 2000). The intent of the CZI is to focus on two metals of prime concern to aquatic health on the CWS while minimizing regulatory details of water-quality standards. The CZI is computed as [( $\mathrm{Cu}$ in $\mathrm{ppb} / 20)+(\mathrm{Zn}$ in $\mathrm{ppb} / 200)]$ / 2. The values of 20 and 200 , for $\mathrm{Cu}$ and $\mathrm{Zn}$ respectively, are not precisely defined, but are essentially average values for tolerant and sensitive species in mortality tests and are similar in magnitude to the ALWS in table 9. Because the intent of the CZI is to suggest the magnitude of concern for aquatic health, there seems to be little point in refining the factors to more precise-appearing numbers. The sum is divided by two to conveniently make the index 1 for the break between healthy and unhealthy compositions: CZI values below 1 are "good," 
and values above 1 are "bad." CZI values for 181 surface-water samples from the CWS (table 10) show a range from 0.01 to 1,405 and a median value of 1.13 (the average, 31.3, is skewed upward by a few very high values). The range of CZI values is shown on figure 7. Many of the very high values (10 to 1,405$)$ are for local water sites such as mine drainages or tailings pond seeps, but some streams that have CZI values $>10$ seem to be real concerns as they appear to be significant threats to aquatic life.

\section{Surface-Water Quality Relative to Historic Mining}

Water was sampled at many sites during this reconnaissance study in an effort to measure the dispersion of acid and metals away from known mine or mill sites. The water analyses provide much useful information, but three points must be emphasized: (1) the sampling and analytical methods used here (Appendix 1) are not as rigorous as those used by CWQCC, EPA, and other regulatory agencies for the measurement of trace metals in water; (2) this study does not provide any indication of seasonal variability; and (3) these water analyses are only an indirect measure of the biological habitat and ecological health. The water sampling described here is deemed a cost-effective way of ranking surface waters to identify the most contaminated waters that are in greatest need of further study and, eventually, restoration.

Surface-water compositions are highly variable on the CWS. Measured pH's ranged from less than 2 to more than 8 , conductivities (a measure of total dissolved solids) ranges from very low ( $<50 \mu \mathrm{S} / \mathrm{cm}$, a very good value) to very high $(>2,000 \mu \mathrm{S} / \mathrm{cm}$, very bad quality). Metal concentrations ranged from less than $1 \mathrm{ppb}$ to more than 1,000,000 ppb (table 3, table 11) in places - far in excess of regulatory standards. Water compositions determined here for many streams are better than implied by some water-quality ratings as "mining impacted" (CWQCD, 1989).

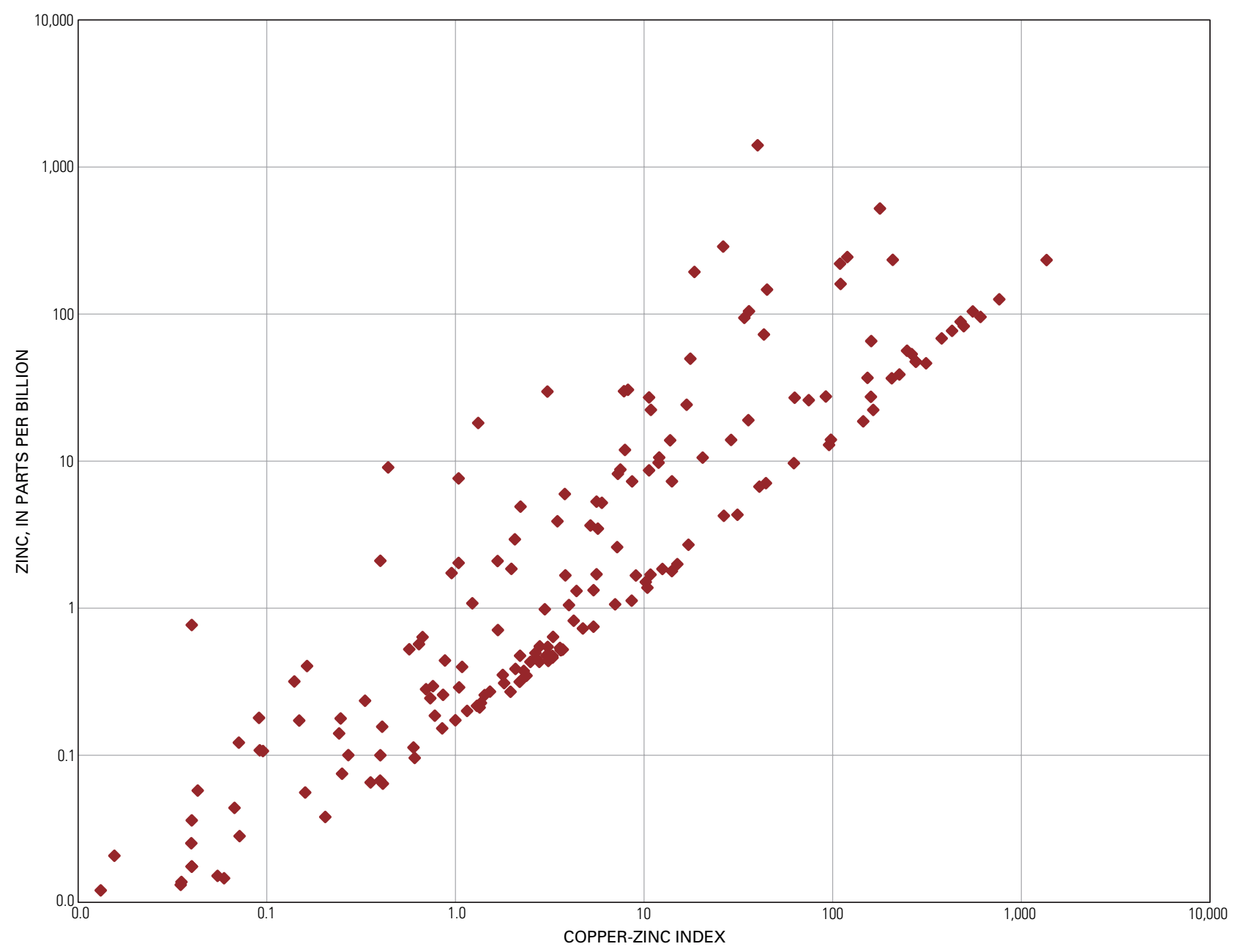

Figure 7. Copper-zinc index (CZI) values for surface waters, central Western Slope, Colorado. 
Table 10. Copper-zinc index for surface waters, CWS, Colorado.

[Values derived from ICP-MS analyses; units are ppb (parts per billion). Abbreviations of water type, district, and watershed as in other chemical tables. CuI*: $\mathrm{Cu}$ index, $\mathrm{Cu}$ (ppb) divided by 10 (ALWS); ZnI*, Zn index, Zn (ppb) divided by 50 (ALWS); CZI*: Copper-zinc index, (Cu concentration/20 + $\mathrm{Zn}$ concentration/200)/2]

\begin{tabular}{|c|c|c|c|c|c|c|c|c|c|}
\hline Sample ID & $\mathrm{pH}$ & $\begin{array}{c}\text { Conductivity } \\
(\mu \mathrm{S} / \mathrm{cm})\end{array}$ & $\begin{array}{l}\text { Water } \\
\text { type }\end{array}$ & District & Watershed & $\mathrm{Cul}^{*}$ & $\mathrm{Znl}{ }^{*}$ & $\mathrm{CZI}^{*}$ & Site name \\
\hline NGW105 & 8.5 & 440 & $S$ & UV & SM & 0.4 & 0.1 & 0.1 & Lion Creek \\
\hline NGW106 & 8.3 & 310 & MD & UV & SM & 0.5 & 0.1 & 0.1 & adit drainage \\
\hline NGW107 & 8.5 & 410 & MD & UV & SM & 0.2 & 0.0 & 0.1 & adit drainage \\
\hline NGW115 & 7.4 & 1740 & SP & UV & SM & 0.6 & 0.1 & 0.2 & no name seep \\
\hline NGW117 & 8.1 & 840 & $\mathrm{~S}$ & UV & SM & 0.1 & 0.0 & 0.0 & Atkinson Creek \\
\hline NGW205 & 7.6 & 410 & DP & TM & GU & 0.7 & 7.0 & 1.1 & Akron mill \\
\hline NGW214 & 7.9 & 180 & MD & $\mathrm{TM}$ & GU & 0.4 & 3.6 & 0.5 & Akron adit \\
\hline NGW230 & 7.8 & 280 & S & VU & GU & 0.6 & 0.7 & 0.2 & Camp Creek \\
\hline NGW231 & 7.7 & 310 & S & VU & GU & 0.6 & 0.2 & 0.2 & Camp Creek \\
\hline NGW232 & 7.9 & 240 & MD & GB & GU & 0.4 & 0.8 & 0.2 & Carter mine \\
\hline NGW247 & 7.4 & 240 & MD & $\mathrm{TC}$ & GU & 0.4 & 0.2 & 0.1 & New Gold Cup adit \\
\hline NGW249 & 7.7 & 90 & SP & MRP & GU & 0.4 & 0.1 & 0.1 & Stock spring, Chester \\
\hline NGW259 & 7.9 & 680 & SP & LO & GU & 0.3 & 0.3 & 0.1 & Stock spring \\
\hline NGW263 & 3.7 & 210 & MD & GQ & GU & 118.1 & 8.2 & 30.5 & Bon Ton adit drainage \\
\hline NGW264 & 5.7 & 120 & MD & GQ & GU & 115.6 & 7.8 & 29.9 & Bon Ton adit-dump drainage \\
\hline NGW275 & 7.6 & 280 & MD & $\mathrm{TC}$ & GU & 2.0 & 0.6 & 0.6 & Blistered Horn adit \\
\hline NGW287 & 7.4 & 260 & MD & $\mathrm{BP}$ & LF & 0.6 & 3.1 & 0.5 & American Basin \\
\hline NGW296 & 4.7 & 80 & S & MP & UN & 1.1 & 12.5 & 1.8 & Head Mineral Creek \\
\hline NGW298 & 3.5 & 200 & MD & MP & UN & 7.6 & 62.3 & 9.7 & London \\
\hline NGW299 & 3.6 & 410 & S & $\mathrm{RM}$ & UN & 31.3 & 7.5 & 8.8 & Red Mtn Creek \\
\hline NGW309 & 3.4 & $>2000$ & SP & OY & UN & 251.0 & $1,361.8$ & 233.0 & Banner seep \\
\hline NGW310 & 3.7 & 570 & SP & IS & SM & 17.8 & 6.0 & 5.2 & Iron Spring mine \\
\hline NGW315 & 3.0 & 1660 & TD & IS & SM & 122.2 & 763.7 & 126.0 & Carbonero mill \\
\hline NGW317 & 7.3 & 980 & $\mathrm{TD}$ & IS & SM & 0.3 & 1.4 & 0.3 & Caribeau mill tailings \\
\hline NGW318 & 7.1 & 790 & MD & IS & SM & 0.3 & 3.6 & 0.5 & Caribou mine \\
\hline NGW320 & 6.6 & 290 & MD & IS & SM & 58.1 & 35.7 & 19.0 & Alta mine \\
\hline NGW328 & 4.8 & 250 & S & $\mathrm{RM}$ & UN & 7.5 & 1.7 & 2.1 & National Belle \\
\hline NGW333 & 2.9 & 1340 & MD & $\mathrm{RM}$ & UN & 88.3 & 16.8 & 24.2 & Champion Gulch \\
\hline NGW335 & 2.4 & $>2000$ & MD & $\mathrm{RM}$ & UN & 825.3 & 109.4 & 220.0 & Guston mine \\
\hline NGW336 & 3.1 & $>2000$ & MD & $\mathrm{RM}$ & UN & 76.5 & 62.9 & 27.0 & Lower Guston adit \\
\hline NGW338 & 2.6 & $>2000$ & DP & RM & UN & 268.9 & 43.2 & 72.6 & Dump puddle \\
\hline NGW340 & 2.7 & $>2000$ & MD & $\mathrm{RM}$ & UN & 916.5 & 119.6 & 244.1 & Guston mine \\
\hline NGW342 & 3.5 & 640 & S & $\mathrm{RM}$ & UN & 83.8 & 10.9 & 22.3 & Red Mtn Creek \\
\hline NGW344 & 2.9 & $>2000$ & TS & $\mathrm{RM}$ & UN & 83.2 & 495.1 & 82.7 & Idarado Tailings seep \\
\hline NGW347 & 3.6 & 410 & S & $\mathrm{RM}$ & UN & 29.1 & 7.3 & 8.2 & Red Mtn Creek \\
\hline NGW348 & 2.0 & 1650 & MD & $\mathrm{RM}$ & UN & 43.6 & 205.7 & 36.6 & Larson Brothers mine \\
\hline NGW353 & 6.3 & 740 & MD & $\mathrm{RM}$ & UN & 0.3 & 10.4 & 1.4 & Mountain King \\
\hline NGW357 & 2.9 & 1410 & MD & $\mathrm{RM}$ & UN & 66.5 & 74.7 & 26.0 & Joker mine \\
\hline NGW363 & 5.9 & 1260 & MD & $\mathrm{RM}$ & UN & 0.3 & 5.4 & 0.7 & Silver Belle \\
\hline NGW365 & 3.7 & 390 & S & $\mathrm{RM}$ & UN & 33.1 & 11.9 & 9.8 & Red Mtn Creek \\
\hline NGW367 & 3.0 & 290 & $\mathrm{~S}$ & RM & UN & 18.5 & 2.2 & 4.9 & Corkscrew Gulch \\
\hline NGW371 & 6.2 & 190 & $\mathrm{~S}$ & SN & UN & 0.2 & 2.2 & 0.3 & Canyon Creek \\
\hline NGW373 & 6.5 & 190 & $\mathrm{~S}$ & SN & UN & 0.2 & 3.1 & 0.4 & Canyon Creek \\
\hline NGW380 & 6.9 & 120 & S & MP & UN & 0.5 & 2.7 & 0.5 & Upper Uncompahgre River \\
\hline NGW382 & 6.5 & 480 & MD & MP & UN & 0.3 & 2.3 & 0.4 & Old Lout mine \\
\hline NGW385 & 7.2 & 360 & S & IS & SM & 0.2 & 1.3 & 0.2 & Howard Fork \\
\hline NGW388 & 7.3 & 150 & $S$ & TE & SM & 0.5 & 15.0 & 2.0 & Savage Creek \\
\hline NGW391 & 7.2 & 110 & $\mathrm{~S}$ & TE & SM & 0.3 & 2.8 & 0.4 & San Miguel River \\
\hline NGW393 & 3.3 & 1510 & MD & GN & LF & 3.7 & 95.7 & 12.9 & Palmetto basin \\
\hline NGW394 & 2.9 & $1500 ?$ & MD & $\mathrm{GN}$ & $\mathrm{LF}$ & 7.0 & 97.8 & 14.0 & Palmetto basin \\
\hline NGW450 & 7.1 & 240 & S & GN & LF & 32.0 & 20.4 & 10.6 & Head Henson Creek \\
\hline NGW455 & 2.7 & nd & MD & MP & UN & 42.4 & 225.8 & 38.8 & San Juan Chief \\
\hline
\end{tabular}


Table 10. Copper-zinc index for surface waters, CWS, Colorado-Continued.

\begin{tabular}{|c|c|c|c|c|c|c|c|c|c|}
\hline Sample ID & $\mathrm{pH}$ & $\begin{array}{l}\text { Conductivity } \\
(\mu \mathrm{S} / \mathrm{cm})\end{array}$ & $\begin{array}{l}\text { Water } \\
\text { type }\end{array}$ & District & Watershed & $\mathrm{Cul}^{*}$ & $\mathrm{Znl}{ }^{*}$ & $\mathrm{CZI}^{*}$ & Site name \\
\hline NGW492 & 5.9 & 910 & $\mathrm{~S}$ & $\mathrm{SN}$ & $\mathrm{UN}$ & 0.3 & 3.3 & 0.5 & Canyon Creek \\
\hline NGW493 & 5.9 & 1540 & MD & IS & SM & 2.4 & 3.0 & 1.0 & Caribeau \\
\hline NGW494 & 5.9 & 1540 & $\mathrm{DH}$ & IS & SM & 0.2 & 1.4 & 0.2 & Drill hole flow \\
\hline NGW495 & 2.1 & $>2000$ & MD & $\mathrm{RM}$ & UN & 764.8 & 18.5 & 193.5 & Head Corkscrew \\
\hline NGW499 & 3.0 & 285 & MD & $\mathrm{RB}$ & GU & 84.2 & 378.2 & 68.3 & Standard \\
\hline NGW505 & 8.2 & 251 & S & VU & GU & 0.7 & 2.7 & 0.5 & Cabin Creek \\
\hline NGW506 & 8.1 & 354 & S & VU & GU & 0.8 & 0.3 & 0.2 & Cabin Creek \\
\hline NGW507 & 3.1 & 200 & MD & $\mathrm{RB}$ & GU & 70.8 & 152.9 & 36.8 & Mid Elk Basin \\
\hline NGW510 & 3.1 & 320 & MD & $\mathrm{RB}$ & GU & 29.8 & 159.4 & 27.4 & Upper Elk Basin \\
\hline NGW511 & 2.9 & 354 & DD & $\mathrm{RB}$ & GU & 101.5 & 247.8 & 56.4 & Upper Elk Basin \\
\hline NGW512 & 3.0 & 285 & MD & $\mathrm{RB}$ & GU & 92.8 & 429.4 & 76.9 & Standard mine \\
\hline NGW514 & 3.3 & 301 & S & $\mathrm{RB}$ & GU & 82.5 & 262.3 & 53.4 & E Elk Creek \\
\hline NGW515 & 5.8 & 20 & S & $\mathrm{RB}$ & GU & 0.0 & 0.1 & 0.0 & W Elk Creek \\
\hline NGW518 & 3.0 & 169 & DD & $\mathrm{RB}$ & GU & 140.3 & 553.0 & 104.2 & Standard mine \\
\hline NGW519 & 3.1 & 448 & $\mathrm{TP}$ & $\mathrm{RB}$ & GU & 116.4 & 476.4 & 88.6 & Standard mine \\
\hline NGW520 & 2.9 & 765 & TPS & $\mathrm{RB}$ & GU & 51.5 & 276.4 & 47.4 & Standard mine \\
\hline NGW521 & 5.4 & 62 & S & $\mathrm{RB}$ & GU & 6.4 & 40.9 & 6.7 & Elk Creek \\
\hline NGW523 & 5.9 & 60 & S & $\mathrm{RB}$ & GU & 3.7 & 26.5 & 4.2 & Elk Creek \\
\hline NGW529 & 7.2 & 97 & S & GB & $\mathrm{GU}$ & 0.2 & 0.9 & 0.2 & Gold Creek \\
\hline NGW531 & 6.9 & 90 & S & GB & GU & 0.1 & 0.4 & 0.1 & Gold Creek \\
\hline NGW532 & 6.9 & 56 & $S$ & GB & GU & 0.0 & 0.0 & 0.0 & Gold Creek \\
\hline NGW533 & 6.7 & 40 & $S$ & GQ & GU & 6.5 & 1.0 & 1.7 & Tomichi Creek \\
\hline NGW537 & 6.8 & 66 & $\mathrm{~S}$ & LF & $\mathrm{LF}$ & 0.1 & 0.1 & 0.0 & Wade Gulch \\
\hline NGW540 & 6.9 & 96 & S & $\mathrm{LF}$ & LF & 0.1 & 0.2 & 0.1 & Red Mtn Gulch \\
\hline NGW541 & 6.6 & 43 & S & LF & $\mathrm{LF}$ & 0.1 & 0.4 & 0.1 & Lake Fork Gunnison River \\
\hline NGW543 & 6.7 & 46 & S & $\mathrm{LF}$ & $\mathrm{LF}$ & 0.0 & 0.0 & 0.0 & Cottonwood Creek \\
\hline NGW544 & 6.8 & 34 & $S$ & $\mathrm{LF}$ & $\mathrm{LF}$ & 0.0 & 0.0 & 0.0 & Cattaract Creek \\
\hline NGW545 & 6.9 & 44 & S & $\mathrm{BP}$ & $\mathrm{LF}$ & 0.0 & 0.0 & 0.0 & Grizzly Creek \\
\hline NGW546 & 7.1 & 47 & S & $\mathrm{BP}$ & $\mathrm{LF}$ & 0.1 & 0.6 & 0.1 & Silver Creek \\
\hline NGW547 & 7.0 & 46 & S & $\mathrm{LF}$ & $\mathrm{LF}$ & 0.0 & 0.1 & 0.0 & Bent Creek \\
\hline NGW548 & 6.8 & 76 & S & $\mathrm{LF}$ & $\mathrm{LF}$ & 0.1 & 0.0 & 0.0 & Lake Fk Gunnison River \\
\hline NGW549 & 6.9 & 46 & S & GN & $\mathrm{LF}$ & 0.1 & 0.1 & 0.0 & W Fk Henson Creek \\
\hline NGW550 & 7.2 & 42 & S & GN & $\mathrm{LF}$ & 0.8 & 0.7 & 0.3 & Upper Henson Creek \\
\hline NGW552 & 7.3 & 46 & $\mathrm{~S}$ & GN & LF & 0.2 & 0.3 & 0.1 & Lower Henson Creek \\
\hline NGW555 & 3.0 & 779 & $\mathrm{TS}$ & $\mathrm{RB}$ & GU & 52.5 & 275.3 & 47.5 & Standard mine \\
\hline NGW556 & 6.6 & 32 & MD & $\mathrm{RB}$ & GU & 2.1 & 9.1 & 1.7 & Upper Elk Basin \\
\hline NGW558 & 7.3 & 59 & S & $\mathrm{RB}$ & GU & 1.4 & 10.8 & 1.7 & Lower Elk Creek \\
\hline NGW559 & 7.3 & 37 & S & $\mathrm{RB}$ & $\mathrm{GU}$ & 0.2 & 1.0 & 0.2 & Slate River \\
\hline NGW560 & 7.3 & 24 & S & $\mathrm{RB}$ & GU & 0.3 & 1.8 & 0.3 & Oh Be Joyful Creek \\
\hline NGW561 & 7.0 & 62 & S & $\mathrm{TM}$ & GU & 0.7 & 0.1 & 0.2 & Tomichi Creek \\
\hline NGW563 & 7.1 & 83 & S & $\mathrm{TM}$ & GU & 0.5 & 2.1 & 0.4 & Tomichi Creek \\
\hline NGW565 & 7.2 & 81 & S & $\mathrm{TM}$ & GU & 0.5 & 2.5 & 0.4 & Tomichi Creek \\
\hline NGW570 & 2.7 & 1025 & S & $\mathrm{RM}$ & UN & 586.2 & 110.1 & 160.3 & Red Mtn Creek \\
\hline NGW571 & 2.6 & 1140 & MD & $\mathrm{RM}$ & UN & 563.9 & 44.9 & 146.6 & Adit into ferricrete \\
\hline NGW574 & 3.3 & 436 & S & $\mathrm{RM}$ & UN & 103.1 & 10.6 & 27.1 & Red Mtn Creek \\
\hline NGW575 & 2.9 & 385 & S & $\mathrm{RM}$ & UN & 29.3 & 10.6 & 8.7 & Champion Gulch \\
\hline NGW576 & 2.5 & $>2000$ & MD & $\mathrm{RM}$ & UN & 830.7 & 208.2 & 233.7 & Guston mine \\
\hline NGW577 & 4.2 & 122 & MD & RM & UN & 13.9 & 3.5 & 3.9 & Prospect trench \\
\hline NGW578 & 2.5 & $>2000$ & TS & $\mathrm{RM}$ & UN & 78.6 & 607.9 & 95.6 & Idarado Tailings seep \\
\hline NGW580 & 2.9 & 885 & DD & $\mathrm{RM}$ & UN & 41.3 & 28.9 & 13.9 & Joker mine \\
\hline NGW581 & 3.6 & 319 & S & $\mathrm{RM}$ & UN & 48.5 & 13.8 & 13.9 & Red Mtn Creek \\
\hline NGW582 & 3.0 & 565 & $\mathrm{~S}$ & $\mathrm{RM}$ & UN & 43.7 & 7.9 & 11.9 & Corkscrew Gulch \\
\hline NGW583 & 3.3 & 248 & S & $\mathrm{RM}$ & UN & 1.8 & 0.6 & 0.5 & Small Creek \\
\hline NGW584 & 2.5 & 1035 & S & $\mathrm{RM}$ & UN & 117.3 & 3.1 & 29.7 & Corkscrew Gulch \\
\hline NGW587 & 2.1 & $>2000$ & MD & $\mathrm{RM}$ & UN & 1137.6 & 26.3 & 287.7 & Adit, Corkscrew \\
\hline NGW589 & 3.9 & 39 & $\mathrm{~S}$ & $\mathrm{RM}$ & UN & 3.1 & 0.0 & 0.8 & Lake at divide \\
\hline
\end{tabular}


Table 10. Copper-zinc index for surface waters, CWS, Colorado-Continued.

\begin{tabular}{|c|c|c|c|c|c|c|c|c|c|}
\hline Sample ID & $\mathrm{pH}$ & $\begin{array}{l}\text { Conductivity } \\
(\mu \mathrm{S} / \mathrm{cm})\end{array}$ & $\begin{array}{l}\text { Water } \\
\text { type }\end{array}$ & District & Watershed & $\mathrm{Cul}^{*}$ & $\mathrm{Znl}{ }^{*}$ & $\mathrm{CZI}^{*}$ & Site name \\
\hline NGW592 & 3.4 & 154 & $\mathrm{~S}$ & $\mathrm{RM}$ & $\mathrm{UN}$ & 1.5 & 0.2 & 0.4 & Small creek \\
\hline NGW593 & 3.1 & 563 & $\mathrm{~S}$ & $\mathrm{RM}$ & UN & 6.4 & 2.0 & 1.9 & Small creek \\
\hline NGW595 & 2.9 & 492 & $\mathrm{~S}$ & RM & UN & 3.7 & 1.2 & 1.1 & Small reek \\
\hline NGW599 & 3.2 & 215 & S & $\mathrm{RM}$ & UN & 10.7 & 2.1 & 2.9 & Small creek \\
\hline NGW605 & 4.9 & 278 & S & $\mathrm{RM}$ & UN & 2.2 & 0.7 & 0.6 & Gray Copper Creek \\
\hline NGW606 & 6.0 & 375 & MD & $\mathrm{RM}$ & UN & 0.2 & 2.4 & 0.3 & Mountain Silver \\
\hline NGW609 & 5.9 & 74 & MD & $\mathrm{RM}$ & UN & 1.3 & 0.9 & 0.4 & Upper Mt Silver \\
\hline NGW610 & 3.4 & 390 & S & $\mathrm{RM}$ & UN & 36.3 & 12.0 & 10.6 & Red Mtn Creek \\
\hline NGW612 & 6.2 & 680 & S & IS & SM & 0.6 & 1.0 & 0.3 & Chapman Gulch \\
\hline NGW613 & 6.4 & 620 & MD & IS & SM & 0.2 & 0.6 & 0.1 & Small adit \\
\hline NGW614 & 5.9 & 1540 & DH & IS & SM & 0.3 & 1.5 & 0.3 & Exploration drill hole \\
\hline NGW616 & 5.1 & 95 & S & IS & SM & 1.1 & 1.1 & 0.4 & Small creek \\
\hline NGW617 & 3.4 & 955 & $\mathrm{Sp}$ & IS & SM & 24.8 & 8.6 & 7.3 & Iron Spring \\
\hline NGW618 & 5.5 & 365 & $S$ & IS & SM & 0.4 & 0.4 & 0.2 & E Howard Fork \\
\hline NGW621 & 5.5 & 890 & $\mathrm{~S}$ & IS & SM & 0.1 & 14.0 & 1.8 & Side creek \\
\hline NGW627 & 6.1 & 330 & $\mathrm{~S}$ & IS & SM & 0.2 & 1.2 & 0.2 & S Fork San Miguel \\
\hline NGW632 & 5.9 & 910 & MD & IS & SM & 3.0 & 4.4 & 1.3 & Caribou mine \\
\hline NGW633 & 5.9 & 820 & TD & IS & SM & 0.2 & 1.3 & 0.2 & Flow on Caribeau tails \\
\hline NGW634 & 5.5 & 348 & S & IS & SM & 0.2 & 2.4 & 0.3 & Howard Fork \\
\hline NGW635 & 6.9 & 135 & S & IS & SM & 0.1 & 0.0 & 0.0 & Lake Fk San Migue; \\
\hline NGW636 & 4.7 & 450 & MD & IS & SM & 22.1 & 14.1 & 7.3 & Adit $\mathrm{W}$ of Campgrnd \\
\hline NGW637 & 6.7 & 348 & S & IS & SM & 0.5 & 1.8 & 0.4 & Howard Fork \\
\hline NGW641 & 6.2 & 118 & S & MP & $\mathrm{UN}$ & 0.4 & 3.0 & 0.5 & Upper Uncompahgre River \\
\hline NGW642 & 6.5 & 472 & MD & MP & UN & 2.0 & 145.2 & 18.6 & M Breen adit \\
\hline NGW644 & 7.0 & 83 & S & MP & UN & 0.5 & 4.7 & 0.7 & Mineral Creek \\
\hline NGW645 & 6.9 & 128 & $\mathrm{~S}$ & MP & UN & 1.2 & 4.2 & 0.8 & Upper Uncompahgre River \\
\hline NGW651 & 3.0 & 707 & $\mathrm{~S}$ & MP & UN & 28.9 & 312.6 & 46.3 & Mineral Creek \\
\hline NGW652 & 3.6 & 170 & S & MP & UN & 6.2 & 44.3 & 7.1 & Upper Unconpahgre River \\
\hline NGW654 & 3.1 & 443 & MD & $\mathrm{RM}$ & $\mathrm{UN}$ & 4.8 & 3.8 & 1.7 & Small mine \\
\hline NGW656 & 5.8 & 202 & S & $\mathrm{OU}$ & UN & 0.9 & 3.3 & 0.6 & Uncompahgre River \\
\hline NGW657 & 5.9 & 69 & $\mathrm{~S}$ & SN & UN & 0.3 & 3.6 & 0.5 & Sneffels Creek \\
\hline NGW658 & 6.9 & 48 & $\mathrm{~S}$ & $\mathrm{SN}$ & UN & 0.3 & 3.1 & 0.5 & Imogene Creek \\
\hline NGW662 & 6.5 & 48 & $\mathrm{~S}$ & $\mathrm{TE}$ & SM & 0.1 & 0.4 & 0.1 & Savage Creek \\
\hline NGW670 & 6.7 & 90 & $\mathrm{~S}$ & $\mathrm{TE}$ & SM & 1.6 & 31.3 & 4.3 & Savage Creek \\
\hline NGW671 & 7.3 & 48 & $\mathrm{~S}$ & $\mathrm{TE}$ & SM & 0.4 & 3.1 & 0.5 & Marshall Creek \\
\hline NGW673 & 6.9 & 53 & $\mathrm{~S}$ & TE & SM & 0.9 & 10.2 & 1.5 & Ingrahm Creek \\
\hline NGW677 & 7.2 & 73 & $\mathrm{~S}$ & TE & SM & 0.1 & 0.0 & 0.0 & Bear Creek \\
\hline NGW678 & 5.6 & 66 & S & $\mathrm{SN}$ & UN & 0.2 & 3.7 & 0.5 & Sneffels Creek \\
\hline NGW679 & 6.0 & 94 & MD & $\mathrm{SN}$ & UN & 0.1 & 0.2 & 0.0 & Eclipse mine \\
\hline NGW680 & 6.1 & 44 & S & SN & UN & 0.1 & 0.0 & 0.0 & Yankee Boy Creek \\
\hline NGW681 & 5.7 & 121 & $\mathrm{~S}$ & SN & $\mathrm{UN}$ & 0.2 & 3.3 & 0.5 & Canyon Creek \\
\hline NGW683 & 7.1 & 12 & $\mathrm{~S}$ & RM & $\mathrm{UN}$ & 0.1 & 0.0 & 0.0 & Mineral Creek ditch \\
\hline NGW685 & 6.6 & 150 & $\mathrm{~S}$ & $\mathrm{RM}$ & $\mathrm{UN}$ & 11.0 & 5.7 & 3.5 & Albany Creek \\
\hline NGW820 & 2.9 & 130 & $\mathrm{Sp}$ & $\mathrm{RM}$ & UN & 18.0 & 0.4 & 9.1 & Spring near mine/vein, red altn \\
\hline NGW823 & 4.6 & 15 & $\mathrm{~S}$ & $\mathrm{RM}$ & UN & 1.1 & 17.2 & 2.7 & Headwater Gray Copper Creek \\
\hline NGW827 & 2.8 & 189 & MD & RM & UN & 36.0 & 1.3 & 18.2 & Vernon adit, lower \\
\hline NGW828 & 3.5 & 33 & S & RM & UN & 0.6 & 0.1 & 0.3 & Side creek from red altn \\
\hline NGW829 & 3.0 & 121 & $\mathrm{~S}$ & RM & $\mathrm{UN}$ & 15.0 & 1.0 & 7.6 & Gray Copper Crk W. of Vernon \\
\hline NGW830 & 3.0 & 92 & S & $\mathrm{RM}$ & UN & 4.1 & 0.4 & 2.1 & Gray Copper Crk E. of Vernon \\
\hline NGW831 & 2.0 & $>2000$ & MD & $\mathrm{RM}$ & UN & 2800 & 40 & 1405 & Corkscreew headwall adit \\
\hline NGW834 & 6.8 & 172 & S & IS & SM & 0.4 & 2.2 & 0.5 & Howard Fk at Ames \\
\hline NGW835 & 7.7 & 121 & S & IS & SM & 0.3 & 0.9 & 0.3 & South Fork San Miguel \\
\hline NGW837 & 3.2 & 312 & MD & RM & UN & 200.0 & 36.0 & 104.5 & Hudson mine drainage \\
\hline NGW839 & 3.0 & 204 & $\mathrm{~S}$ & RM & $\mathrm{UN}$ & 95.0 & 17.6 & 49.7 & Headwater Red Mtn Creek \\
\hline NGW840 & 2.5 & $>2000$ & MD & RM & UN & 1000.0 & 178.0 & 522.3 & Guston mine drain \\
\hline NGW841 & 2.9 & 284 & S & $\mathrm{RM}$ & UN & 180.0 & 34.0 & 94.3 & Red mtn Creek middle \\
\hline
\end{tabular}


Table 10. Copper-zinc index for surface waters, CWS, Colorado-Continued.

\begin{tabular}{|c|c|c|c|c|c|c|c|c|c|}
\hline Sample ID & $\mathrm{pH}$ & $\begin{array}{c}\text { Conductivity } \\
(\mu \mathrm{S} / \mathrm{cm})\end{array}$ & $\begin{array}{l}\text { Water } \\
\text { type }\end{array}$ & District & Watershed & $\mathrm{Cul}^{*}$ & $\mathrm{Znl^{* }}$ & $\mathrm{CZI}^{*}$ & Site name \\
\hline NGW842 & 2.9 & 762 & MD & $\mathrm{RM}$ & UN & 91.0 & 160.0 & 65.5 & Joker tunnel drainage \\
\hline NGW844 & 5.5 & 415 & MD & IS & SM & 3.4 & 7.2 & 2.6 & Mine drain makes Fe floc \\
\hline NGW845 & 3.7 & 325 & S & IS & SM & 9.2 & 5.6 & 5.3 & Surface flow, much Fe floc \\
\hline NGW850 & 7.3 & 51 & S & $\mathrm{TM}$ & GU & 0.4 & 2.8 & 0.6 & Tomichi Creek near smelter \\
\hline NGW852 & 7.3 & 131 & MD & $\mathrm{TM}$ & GU & 0.1 & 8.6 & 1.1 & Akron tunnel drainage \\
\hline NGW853 & 7.8 & 50 & S & $\mathrm{TM}$ & GU & 0.4 & 0.8 & 0.3 & Tomichi Creek $200 \mathrm{~m} \mathrm{SW}$ mill \\
\hline NGW856 & 8.7 & 45 & $\mathrm{~S}$ & GB & GU & 0.1 & 0.4 & 0.1 & Gold Creek east of mines \\
\hline NGW857 & 8.2 & 48 & $S$ & OU & UN & 11.0 & 3.8 & 6.0 & Dexter Creek below mines \\
\hline NGW860 & 6.9 & 184 & $S$ & na & UN & 6.0 & 5.2 & 3.7 & Uncompahgre north side Ouray \\
\hline NGW862 & 7.7 & 240 & $S$ & na & UN & 3.8 & 1.0 & 2.0 & Uncompahgre N. of Ridgeway \\
\hline NGW864 & 6.4 & 42 & $S$ & $\mathrm{SN}$ & UN & 1.0 & 1.7 & 0.7 & Sneffels Creek above Atlas tails \\
\hline NGW865 & 6.8 & 55 & S & $\mathrm{SN}$ & UN & 0.1 & 2.0 & 0.3 & Sneffels Creek below Atlas tails \\
\hline NGW866 & 7.0 & 64 & $\mathrm{~S}$ & $\mathrm{SN}$ & UN & 2.0 & 5.6 & 1.7 & Imogene Creek SW Camp Bird \\
\hline NGW869 & 72.0 & 51 & MD & $\mathrm{SN}$ & UN & 1.3 & 5.4 & 1.3 & Adit drain old CB\#3 \\
\hline NGW870 & 5.8 & 55 & MD & $\mathrm{SN}$ & UN & 32.0 & 92.0 & 27.5 & Adit drain Hidden Treasure, red \\
\hline NGW871 & 6.6 & 124 & S & TE & SM & 3.6 & 164.0 & 22.3 & Surface flow at Argentine dump \\
\hline NGW873 & 7.1 & 190 & S & $\mathrm{SN}$ & UN & 1.1 & 4.0 & 1.1 & Canyon Creek below tailings \\
\hline
\end{tabular}

\section{Geochemical Concepts for Water Quality}

Some brief comments on geological-geochemical aspects of water compositions may provide helpful perspective on the huge array of analyses of water samples collected in this study. Discussion here will focus on 11 elements that are of most concern to human or aquatic-habitat health (table 11; figs. 8 and 9).

Some metals, such as $\mathrm{Cu}$ and $\mathrm{Zn}$, have similar behavior in solids and in solutions and also tend to be enriched together, whereas some are essentially unique in their geochemical behavior (Smith and Huyck, 1999; Langmuir, 1997). Cadmium and zinc are very similar chemically and geochemically, yet they have different toxic properties. Iron and manganese are chemically similar and may or may not follow each other geochemically. Arsenic, molybdenum, and uranium are very different chemically and toxicologically, but respond similarly because they are mobile as oxy-anions under oxidizing, alkaline conditions. Copper, lead, and zinc generally are derived from sulfide minerals, are mobilized by acids, but respond differently as $\mathrm{pH}$ rises during mixing or buffering reactions.

Aluminum is abundant in most geologic settings but is mobile only under very acidic conditions (figs. 6,9 ) or under very alkaline conditions that are not attained on the CWS. Significantly, there are many high to very high concentrations in sampled waters: 48 percent of samples exceed the ALWS of $100 \mathrm{ppb}$. The very highest concentrations of $\mathrm{Al}$ are in waters with $\mathrm{pH}$ values below 3 , but some $\mathrm{pH}$ 6.6-7.1 waters contain high concentrations. High $\mathrm{Al}$ concentrations are most common in the Red Mountain, Ruby, and Iron Springs districts. High Al concentrations lead to the formation of colloids that, among other things, can clog gills in fish.

Iron and manganese can come from silicate, sulfide, and oxide minerals, and both tend to be mobile in acidic waters (fig. 6). Iron can be enriched in waters from either mined or unmined sulfidic rock - the latter shown by the widespread deposits of ferricrete and bog iron, as in the Ophir, Silverton, and Ironton areas, which clearly are unrelated to historic mining (Purington, 1898; Hanshaw, 1974). Manganese abundance is more variable than that of its geochemical cousin, $\mathrm{Fe}$, and probably is related to Mn-enriched green-altered volcanic rocks and Mn-gangue minerals rhodonite and rhodochrosite (Burbank and Luedke, 1969). Concentrations of Fe exceed the ALWS (1,000 ppb) in 51 percent of samples, whereas this is less common for Mn (only 25 percent in excess of ALWS). The high to very high values of $\mathrm{Fe}$ and $\mathrm{Mn}$ are in acidic waters $(\mathrm{pH}<3)$, but some $\mathrm{pH} 6.5$ to 8.1 waters carry high concentrations of $\mathrm{Fe}$ and $\mathrm{Mn}$. Iron and $\mathrm{Mn}$ are most commonly excessive in the Iron Springs, Red Mountain, and Ruby districts. Iron and $\mathrm{Mn}$ are involved in several kinds of reactions in surface waters as the $\mathrm{pH}$ rises, most commonly observed as red to black coatings on stream cobbles. Such coatings generally form from colloids in stream water (Church and others, 1997) and have a major impact on biological habitat.

Copper, lead, zinc, and cadmium tend to be associated with each other, but relative enrichments clearly differ from area to area according to deposit mineralogy. These metals are mostly derived from sulfide minerals, either in mine waste and tailings or unmined altered rocks. Concentrations of these metals are generally in proportion to acidity (fig. 6), and some waters with $\mathrm{pH}$ below 3 have concentrations that are $100 \times$ to $10,000 \times$ ALWS. Surface waters at many sites are in excess of ALWS: $\mathrm{Cu}, 57$ percent of the samples have $>10 \mathrm{ppb} ; \mathrm{Pb}, 44$ 
Table 11. Summary of ICP-MS analyses of surface waters, CWS, Colorado.

\begin{tabular}{|c|c|c|c|c|c|c|c|c|c|c|c|c|c|c|c|c|c|c|}
\hline & $\mathrm{pH}$ & $\begin{array}{l}\text { Conductivity } \\
(\mu \mathrm{S} / \mathrm{cm})\end{array}$ & $\begin{array}{c}\mathrm{Al} \\
(\mathrm{ppb})\end{array}$ & $\begin{array}{c}\text { As } \\
\text { (ppb) }\end{array}$ & $\begin{array}{c}C d \\
(p p b)\end{array}$ & $\begin{array}{c}\text { Co } \\
\text { (ppb) }\end{array}$ & $\begin{array}{c}\mathrm{Cu} \\
(\mathrm{ppb})\end{array}$ & $\begin{array}{c}\mathrm{Fe} \\
(\mathrm{ppb})\end{array}$ & $\begin{array}{l}\mathrm{Mn} \\
(\mathrm{ppb})\end{array}$ & $\begin{array}{l}\text { Mo } \\
\text { (ppb) }\end{array}$ & $\begin{array}{c}\mathrm{Ni} \\
(\mathrm{ppb})\end{array}$ & $\begin{array}{c}\mathrm{Pb} \\
\text { (ppb) }\end{array}$ & $\begin{array}{c}\mathrm{Sb} \\
(\mathrm{ppb})\end{array}$ & $\begin{array}{c}\text { Se } \\
(p p b)\end{array}$ & $\begin{array}{c}\mathrm{Te} \\
\text { (ppb) }\end{array}$ & $\begin{array}{c}\mathrm{Tl} \\
\text { (ppb) }\end{array}$ & $\underset{(\mathrm{ppb})}{\mathrm{U}}$ & $\begin{array}{c}\mathrm{Zn} \\
\text { (ppb) }\end{array}$ \\
\hline \multicolumn{19}{|c|}{ Streams and creeks ( $n=109)$} \\
\hline Minimum & 2.5 & 12 & 0.0 & 0.1 & 0.0 & 0.0 & 0.3 & 52.0 & 0.3 & 0.0 & 0.2 & 0.1 & 0.0 & 0.2 & 0.4 & 0.0 & 0.0 & 0.7 \\
\hline Median & 6.6 & 121 & 55.7 & 0.5 & 0.7 & 0.6 & 6.6 & 311 & 93.7 & 1.0 & 2.3 & 2.5 & 0.2 & 0.3 & 0.4 & 0.1 & 0.2 & 110 \\
\hline Maximum & 8.7 & 1,035 & 33,000 & 100 & 138 & 79.6 & 5,862 & 63,499 & 4,328 & 180 & 53 & 1,229 & 2.7 & 344 & 0.4 & 3.1 & 887 & 15,629 \\
\hline \multicolumn{19}{|c|}{ Springs and seeps $(n=7)$} \\
\hline Minimum & 2.9 & 90 & 1.5 & 0.2 & 0.1 & 0.1 & 2.7 & 76.4 & 1.0 & 0.1 & 1.7 & 0.6 & 0.0 & 0.5 & 0.0 & 0.1 & 0.0 & 4.8 \\
\hline Median & 3.5 & 625 & 391 & 0.4 & 1.1 & 2.8 & 92.3 & 2,145 & 14.1 & 1.7 & 8.4 & 1.5 & 0.1 & 7.1 & 0.0 & 0.1 & 1.4 & 17.8 \\
\hline Maximum & 7.9 & $>2,000$ & 12,462 & 1,130 & 309 & 55.6 & 2,510 & 12,609 & 63,766 & 1,864 & 128 & 108 & 1 & 20,032 & 0.0 & 0.2 & 145 & 68,090 \\
\hline \multicolumn{19}{|c|}{ Mine and dump drainage ( $n=56$ ) } \\
\hline Minimum & 2.0 & 32 & 0.0 & 0.1 & 0.1 & 0.0 & 1.0 & 63.5 & 0.1 & 0.1 & 0.8 & 0.2 & 0.0 & 0.2 & 0.3 & 0.0 & 0.0 & 2.2 \\
\hline Median & 3.6 & 375 & 1,474 & 5.0 & 7.0 & 14.9 & 297.8 & 5,576 & 1,207 & 1.7 & 11.4 & 28.9 & 0.4 & 1.2 & 0.5 & 0.5 & 1.1 & 772 \\
\hline Maximum & 8.5 & $>2,000$ & 469,703 & 1,000 & 165 & 1,000 & 28,000 & $1,500,000$ & 57,836 & 174 & 780 & 1,374 & 13 & 194 & 12 & 6.4 & 555 & 27,648 \\
\hline \multicolumn{19}{|c|}{ Tailings seep and pore water $(n=14)$} \\
\hline Minimum & 2.1 & 448 & 16.1 & 0.8 & 0.5 & 1.3 & 1.7 & $1,148.8$ & 14.3 & 0.4 & 2.4 & 2.4 & 0.1 & 0.3 & 0.6 & 0.1 & 0.0 & 67.2 \\
\hline Median & 3.0 & 980 & 6,546 & 7.0 & 39.9 & 33.6 & 515 & 7,552 & 2,863 & 1.3 & 19.3 & 370 & 1.4 & 3.0 & 0.6 & 0.2 & 1.1 & 8,233 \\
\hline Maximum & 7.3 & $>2,000$ & 190,941 & 5,910 & 567 & 2,674 & 31,734 & $4,731,542$ & 105,535 & 1,908 & 405 & 2,775 & 38.8 & 244 & 24.5 & 5.7 & 51.5 & 58,050 \\
\hline
\end{tabular}



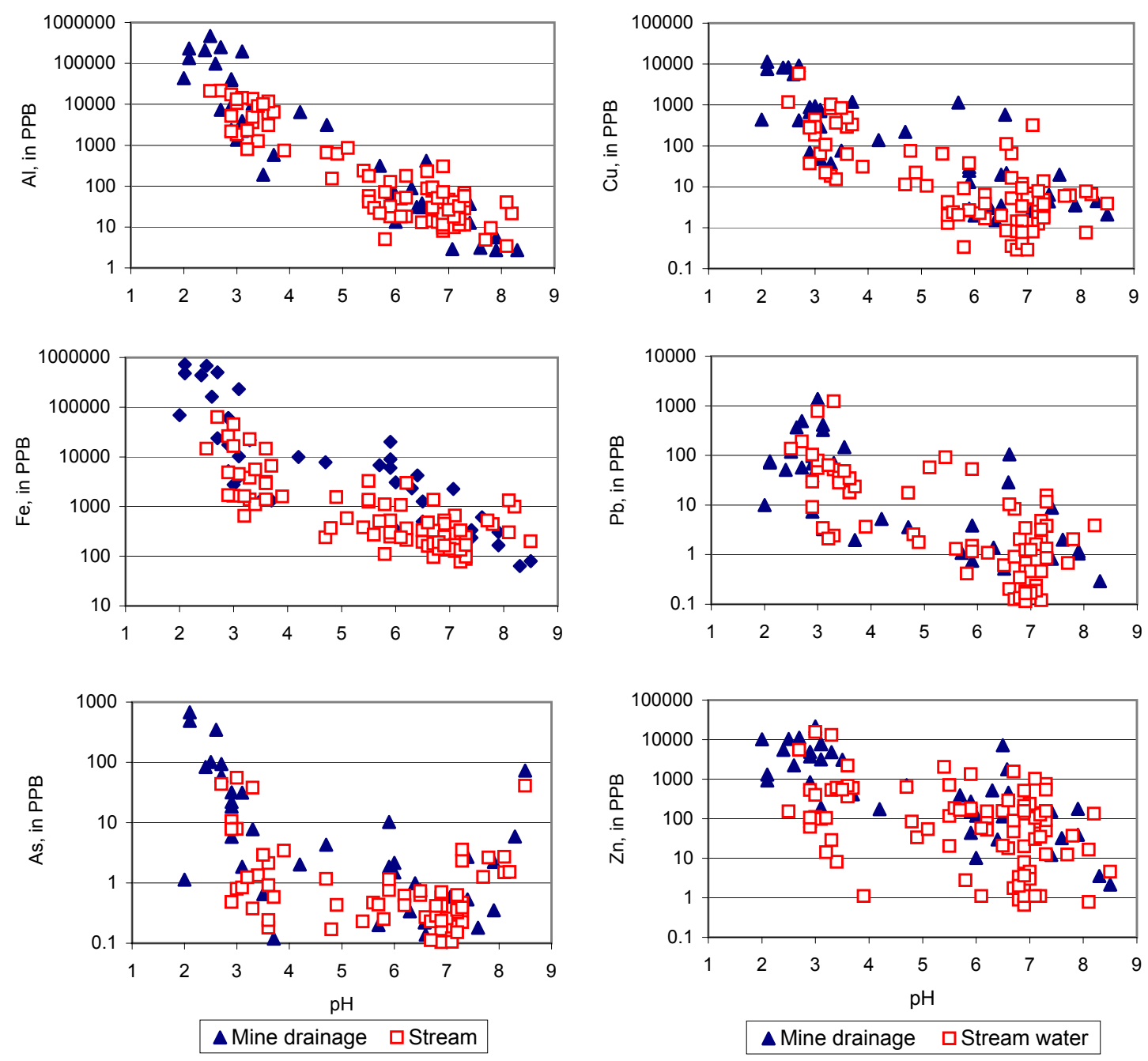

Figure 8. Comparison of mine drainage and stream-water compositions, central Western Slope, Colorado.

percent have $>25 \mathrm{ppb}$; Zn, 74 percent have $>50 \mathrm{ppb}$; Cd, 54 percent have $>1 \mathrm{ppb}$. Lead concentrations in surface waters tend to be lower relative to the other base metals and less commonly are above the ALWS. The high base-metal concentrations typically are in acidic waters with $\mathrm{pH}<3$ to 4 , but some waters with $\mathrm{pH}$ 6.6-7.1 can carry concentrations in excess of ALWS. Combinations of these base metals, such as $\mathrm{Cu}+\mathrm{Zn}$, may be especially problematic for fish (Nimmo and others, 1998; Besser, 2000).

Cobalt and nickel are commonly enriched in sulfide minerals of some ore deposits (Plumlee and others, 1999), but they are not abundant or significant on the CWS. Concentrations of these metals in this study area are generally low: only 8 percent of surface samples contain $>100 \mathrm{ppb} \mathrm{Ni}$ (the ALWS for $\mathrm{Ni}$ is $100 \mathrm{ppb}$ ), and 9 percent contain $>100 \mathrm{ppb}$ Co. Cobalt and $\mathrm{Ni}$ generally behave like $\mathrm{Cu}$ and $\mathrm{Zn}$ in surface waters, thus geochemical studies or reclamation that focus on $\mathrm{Cu}$ and $\mathrm{Zn}$ will likely treat $\mathrm{Co}$ and $\mathrm{Ni}$ as well. These metals will not be discussed further.
Arsenic is a metal of concern chiefly for human healththus, it is specified in drinking-water uses-and is less significant for aquatic biota (Smith and Huyck, 1999). It is rich in some ores on the CWS, such as the Red Mountain breccia pipes but generally is not elevated in waters. The median is $0.84 \mathrm{ppb}$, which is less than the average for world stream waters (Forstner and Wittman, 1979). Only 8 percent of samples exceed the drinking-water standard of $50 \mathrm{ppb}$. The high As concentrations are limited to acidic waters of the Red Mountain and Mineral Point districts and two sites in the Uravan district with alkaline $\mathrm{pH}$. Highest As values are in a specific ore environment in the Red Mountain district (breccia pipe deposits) that are known to be rich in As (Burbank and others, 1972; Fisher and Leedy, 1973). Arsenic poses special problems because it tends to remain soluble as $\mathrm{pH}$ is neutralized by natural or engineered reactions, but that does not appear to apply to more than a few sites on the CWS.

Selenium, similar to As in some chemical and geochemical aspects, is not elevated in most surface waters on the CWS. 


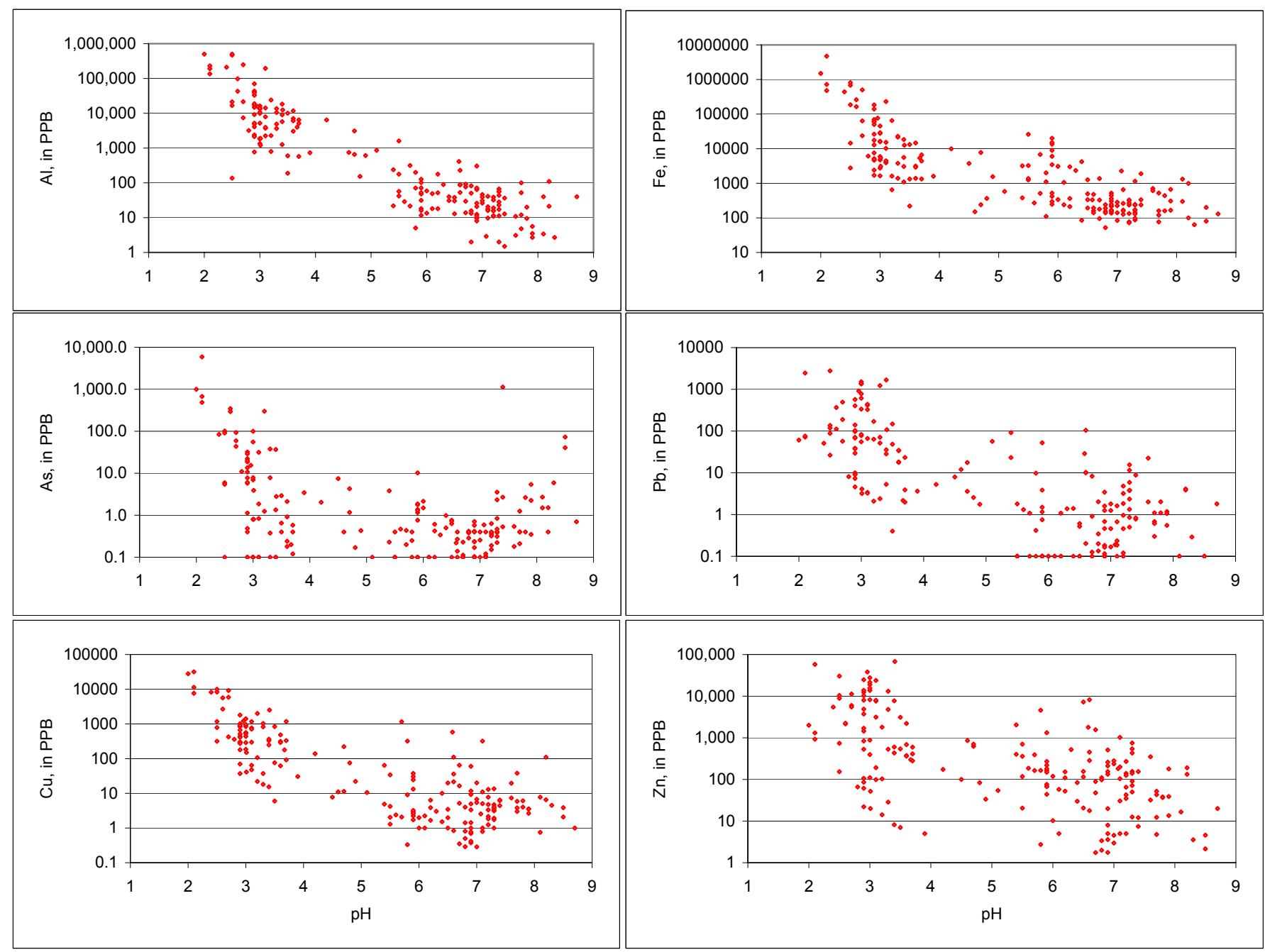

Figure 9. Compositions of surface waters, central Western Slope, Colorado, as a function of pH.

Only four sites have Se concentrations $>50 \mathrm{ppb}$, a domestic drinking-water standard, and 13 samples contain more than the $5 \mathrm{ppb}$ ALWS. The uranium deposits tend to be enriched in Se; a few water samples from those areas show elevated Se concentration. One characteristic of Se should be noted: it tends to accumulate by evaporative processes on the surface layers of mine dumps with the formation of efflorescent salts of sulfate and selenate. These salts are very soluble and the first rain after a dry period can flush the Se into puddles or creeks and thus become available to livestock or wildlife.

Molybdenum poses health threats such as molybdenosis in cattle, but it is not quantified on the Colorado list of toxic metals (CWQCC, 2000). Concentrations of Mo in water are generally low on the CWS, but 8 samples contain more than the aquatic life standard of $19 \mathrm{ppb}$. All but one of the Mo-rich surface waters are from the Uravan area. Acidic waters can transport Mo, but more commonly Mo is mobile as the oxyanion molybdate in oxidizing, alkaline waters. These conditions are found on the Colorado Plateau.
Antimony ( $S b)$, tellurium (Te), and thallium ( $T l)$ are enriched in some kinds of ore deposits, and minerals of $\mathrm{Sb}$ and Te are known in several of the districts studied. Concentrations of $\mathrm{Sb}, \mathrm{Te}$, and $\mathrm{Tl}$ in surface waters are generally low. More than 98 percent of samples had detectable amounts of $\mathrm{Sb}(>0.1$ $\mathrm{ppb}$ ), but the five highest values are only 5 to $13.1 \mathrm{ppb}$. Higher concentrations of $\mathrm{Sb}$ (14 to $39 \mathrm{ppb}$ ) were detected in tailings pore water samples. Only 8 percent of surface-water samples have detectable amounts of Te (>0.2 ppb); five samples have concentrations in the range of 3.1 to $11.9 \mathrm{ppb}$ Te. Thallium is highly toxic at low concentration levels (the drinking-water standard is $13 \mathrm{ppb}$ ), but it is not significantly concentrated in these districts (only two samples are in the range of 5 to $12 \mathrm{ppb}$ ). The few high values of these metals are in strongly acidic waters that are even more problematic for several other metals; these trace metals probably decrease as other metals precipitate, such as by adsorption on iron oxyhydroxides.

Uranium (and associated radium) is listed as a hazardous material rather than toxic metal. The concern is for exposure 
Hydrogeochemical Investigations of Historic Mining Districts, Central Western Slope of Colorado

to radioactivity, especially by humans. The Colorado standard for $\mathrm{U}$ in surface and ground waters is $44 \mathrm{ppb}$ (or 30 picocuries radioactivity) (CWQCC, 2000), and this is exceeded in only five surface waters. All five of the U-rich samples are from uranium mining areas and have $\mathrm{pH}$ values of 7.4 to 8.5 . Four samples of mine drainage from the Uravan district exceed the uranium standard, but those flows are rare, of small volume, and are not typical of the notoriously dry Uravan district. Uranium is mobile in acidic waters and is noted in some highly acidic waters from deposits that contain low concentrations of uranium (Wanty and others, 1999), but $\mathrm{U}$ also is mobile in alkaline waters that are typical of sedimentary terranes, such as the Colorado Plateau. On the CWS, the alkaline scenario explains local U transport in the Uravan area. Thorium (Th), also radioactive, is less mobile than $\mathrm{U}$; in this study, only one unusually acidic water contained more than $20 \mathrm{ppb}$ Th (there are no toxicity standards for Th in water).

\section{Risks During Extreme Storms: Failure of Tailings Impoundments}

Failure of a tailings impoundment or waste dump during an extreme storm event (flash flood) is a concept that is rarely mentioned in mine lands assessments, yet the dire consequences of such a failure are known in the past century and the probabilities are high enough to demand consideration in land planning. Major failures are known to have occurred, but little emphasized, in the Silverton area during historic flooding of October 4-6, 1911, and there was another major failure near Silverton in 1975 from which tailings were carried to New Mexico. There is evidence of significant erosion at many tailings impoundments in this study area, and recent studies of tailings in northern Nevada (Nash, 2001) revealed that nearly every substantial impoundment had experienced a major or partial failure in the past 100 years. Failure of mine-waste dumps, not mentioned in the literature, also is a possibility. The major concern is that tailings placed in lowlands, not far from streams, could be hit by the "wall of water" that forms during a cloudburst in a confined upland watershed. Geologic evidence shows that boulders the size of a car can be moved by rushing waters that rise 5 to $15 \mathrm{ft}$ above normal levels and have flow volumes more than 100 times normal. Whether mined materials fail by erosion or sliding, mass movement in conjunction with unusual amounts of water can carry and spread both solids and dissolved metals.

Floods in mountain canyons of Colorado caused by cloudbursts or several days of summer rains are well known for their violence, property damage, and loss of life (Follinsbee and Sawyer, 1948). Witnesses and meteorologists describe intense rainfall of several inches an hour, or 5 to 10 inches in 48 hours; accumulations of water in fields sufficient to drown a horse; and "walls of water" several feet high in local creeks occurring within minutes during a downpour. There are numerous historical reports and photographs of flooded homes, broken bridges, and eroded stream banks. Some of these reports are for flooding along creeks in the vicinity of Telluride, Ouray, Silverton, Rico, Creede, and Gunnison. Although generally not mentioned in newspapers at the time, damage to mining properties has happened on the CWS and has been interpreted years after the event (Vincent and others, 1999). Elsewhere in the Western United States-as at the Clark Fork River, Anaconda, Montana (Nimick and Moore, 1991; Moore and Luoma, 1990), at Eureka, Colorado, on the Animas River (Vincent and others, 1999), and at the Puerco River, New Mexico-Arizona (Wirt, 1994)—-tailings failures impacted up to $100 \mathrm{mi}$ of stream and flood plain. In Nevada (Nash, 2001), small- to medium-sized tailings impoundments hit by flash floods have lost 20 to 90 percent of their tailings, which were transported 2 to $10 \mathrm{mi}$, channeled down arroyos or spread across alluvial fans. Outside of the well-studied Animas watershed, I know of little information on flood-related tailings on the Western Slope. In this study, I observed that fluvial tailings along Imogene Creek and Canyon Creek in the Sneffels district were deposited as overbank sediments where storm waters were about $3 \mathrm{ft}$ higher (vertically) than normal flow level. I also observed that drainage-control devices used in mine reclamation, such as straw bales and plastic netting, were commonly ripped away within a few years by storm runoff. More systematic studies are needed to document the frequency and distribution of storm waters and flood deposits to help in the estimation of their behavior in storm events.

Because rainfall in upland watersheds generally is not measured by gages, and many floods are not witnessed, the database for thunderstorms and flash floods in these settings is not well developed. As an alternative to weather predictions and databases, the behavior of flood runoff can be modeled by evaluating physical characteristics of watersheds (Black, 1996). Factors that influence peak runoff in storms include (1) capacity for water storage; (2) amount of infiltration; (3) basin shape and size, and (4) elevation. A watershed is said to be "flashy" (prone to flash floods) if it produces high flows of runoff in a short time during a storm event or snowmelt. Flashy character is increased in watersheds that have geology that supports little water storage and low infiltration, and a shape that is elliptical. Smaller watersheds at high elevations tend to most conducive to "flashy" high flows; elevation influences temperature and the ways extreme storms develop. Many of the headwater basins on the CWS have these "flashy" characteristics.

The statistical framework for prediction and risk analysis of floods is complex but can be calculated (Cohon, 1988; Kite, 1988). Because processes in these modern events are similar to those in the geologic record, geomorphological and sedimentological information may be good guides for evaluating effects and risks of future storm damage. For instance, the 100-year flood level could be estimated from Quaternary alluvial terraces. Numerous risk analyses have been undertaken for failure of uranium mill tailings. Fluvial processes, geomorphic evidence, catastrophic floods, and extreme hydrologic events 
were discussed by Schumm and others (1981) as a guide to planning uranium mill tailings sites. Another study (Shepherd and Nelson, 1978) concluded that engineering aspects of tailings impoundments are important for the short-term behavior, whereas geomorphological processes (especially natural events such as floods and earthquakes) are more significant for the long term. At present, it seems that scientists are monitoring the relatively predictable effluent from mining areas while giving little thought to the risks posed by infrequent natural events like flash floods that have the potential to cause great impact.

Canyons and arroyos in western Colorado are capable of focusing substantial amounts of storm water with incredible power that can transport large quantities of boulders and finegrained materials, such as those commonly found in Quaternary alluvial deposits. Some of the deposits appear to be debris flows (Blatt and others, 1972). However, the debris flow model may not be appropriate for some transported tailings. The large volumes of transported tailings, where catastrophic dam failure seems to have occurred, are nearly pure tailings with weakly developed to massive bedding and minor sorting. Where seen, coarse clasts are rare and seemingly random rather than in distinct beds. These features are more akin to those of "hyperconcentrated flows," which Costa (1988) defined as having 40 to 70 percent sediment by weight. Technical properties are intermediate between normal fluvial sediments and debris flows (moderate viscosity and shear strength) controlled by a dominance of fine-grained materials (silt and clay). The sedimentary features in transported tailings might also be explained by one dominant source of materials (impounded tailings). The term "fluvial tailings," used by many to describe transported tailings, may be inappropriate for these massive deposits but will be used later in this report. Much more study is needed of these unusual deposits to properly evaluate the conditions of formation.

Physical characteristics of watersheds that create high runoff in floods, briefly outlined above, should help in the identification of stream segments that may pose special threats to tailings impoundments and predict where the normally stable fine sands in tailings impoundments may be ripped up and transported as a viscous mud or sand flow (depending upon particle size and flow velocity). There are several important consequences for the environment downstream: (1) tailings are spread as overbank and channel-filling sand deposits for as much as 2 to $10 \mathrm{mi}$ down the canyon or arroyo. Redeposition of fluvial tailings can be expected at sites where channels widen or gradient decrease, either of which decreases flow velocities. (2) Water in the debris flow is likely to attain more extreme concentrations of metals than found in simple lowflow runoff. Depending upon the ratio of solids to water, the metal concentrations could be even higher than determined by passive leach tests because mechanical mixing and abrasion in a debris flow would stimulate reactions. For years after the flood, the tailings will continue to react either with stream water or with precipitation, slowly releasing metals to the flood plain. The tailings will be difficult to clean out of ripar- ian zones and remove from sandbar or overbank deposits. For years after the flood, the tailings will continue to react either with stream water or with precipitation, slowly releasing metals to the flood plain.

A preliminary evaluation of tailings impoundments on the CWS suggests that as many as 19 sites may be at high risk to failure during an extreme storm event (table 7). These estimates are made from the considerations discussed above, chiefly the size and shape of the basin above the tailings site, the geometry of the stream or canyon at the site, and the position of the impoundment. These sites are discussed later by mining district. More work is needed on criteria for the flood model and collection of appropriate field data.

\section{Geochemical Investigations of Historic Mining Districts}

The influences of historic mining on watersheds and biota are not easily quantified. Ideally, multidisciplinary studies are made by an integrated team with diverse skills in hydrology, biology, geology, and chemistry (e.g., Buxton and others, 1997; Nimick and von Guerard, 1998; Plumlee and Logsdon, 1999). However, to undertake studies in such breadth and depth would cost tens of millions of dollars for an area the size and complexity of the CWS. This reconnaissance study is an attempt to highlight the major problems so that FLMA's can focus limited funds and staff on specific scientific investigations and reclamation decisions on the most significant problems.

One of the more complex scientific issues is the objective and quantitative evaluation of contamination sources from unmined mineralized rocks, a topic that is mentioned here but inadequately treated in the time available. The perspective in this study is clearly that of an earth scientist whose observations and interpretations may be at odds with the work and interpretations of scientists in other fields. Much additional work may be needed to reach a balanced and reliable understanding of these undisturbed systems and to develop a clearer definition of how much reclamation must be done to enhance water quality and biologic health while not attempting to purify systems that were releasing contaminants before the arrival of men and machines.

The next section of this report describes mining areas in a format that provides a framework of history and geology for the mineral-environmental investigations and their inferences. The descriptions are organized by the major watersheds of the Gunnison, Uncompahgre, and San Miguel Rivers. For each mining district, information is provided on commodities (metals) mined, along with sketchs of the mining history, which are not consistent because I was unable to locate the same amount of information for all districts. This history is needed to gain an understanding of how current conditions came to be and to recognize that the early mining was done 
Hydrogeochemical Investigations of Historic Mining Districts, Central Western Slope of Colorado

with few environmentally sensitive regulations in place. Production tonnages are reported to show that, in many districts, chemical contamination is roughly proportional to the amount of ore produced or to the amount of earth disturbed. Mills and tailings are described in what may seem to be disproportionate detail because this information is scattered through the literature, at best, and typically not reported at all. The theme of mill tailings in riparian zones is repeated throughout this report because this feature of historic mining is inadequately understood by scientists and FLMA's.

Geology is the essential framework for this investigation and is the major influence on processes that operate at sources, during transport, and in natural mitigation of contaminated waters. The description of geology here is unconventional in that it is nontechnical (and possibly appalling to geologists) in an effort to simplify and demystify this science for the majority of readers who are not trained in geology.

The composition of bedrock and alluvium plays an important role in the evolution of water compositions and thus has been appropriately highlighted by Geoff Plumlee in the phrase "geo-environmental models" (Plumlee and Nash, 1995; Plumlee, 1999). The mineralogy and chemistry of ores, waste rocks, and altered host rocks determine the "geoavailability" of metal contaminants in source areas (Smith and Huyck, 1999), and the composition of rocks and alluvium nearby in the watershed dictate the potential for natural mitigation of contamination by processes of acid neutralization and subsequent metal precipitation (Smith and others, 1994; Plumlee, 1999). The geologic framework of the deposits and watersheds is emphasized here, especially the influence of large areas of altered rocks that can either exacerbate problems from mined rocks or can provide significant natural mitigation of initially contaminated waters. Alteration of bedrock units is particularly important, in the author's experience, and will be emphasized as a major factor in watershed geochemistry.

\section{Mining Districts of the Gunnison River Watershed}

Geology in this large watershed is a diverse mix of Precambrian metamorphic rocks, Paleozoic-Mesozoic sedimentary rocks, and Tertiary volcanic rocks and intrusions (Tweto and others, 1976). Quite logically, this diverse geology is host to a variety of ore-deposit types with substantial ranges in chemical composition. Mining districts are shown in figure 10. Some of the more important ore deposits and unmined resources include (1) gold-sulfide deposits in metavolcanic rocks of the Gunnison Gold Belt; (2) thorium and titanium resources associated with mafic alkalic intrusions at Powderhorn; (3) silver-rich base-metal deposits in carbonate rocks adjacent to intrusions as at Tincup and Whitepine; (4) large, unmined molybdenum deposits in a felsic intrusive complex near Crested Butte; and (5) vein-like uranium deposits in sedimentary rocks at Marshall Pass and Los Ochos.

\section{Cochetopa District (Gunnison Gold Belt)}

Location.-Gunnison Co., 10-15 mi southwest to southeast of Gunnison (figs. 10,11). Climate is relatively dry for the study area, averaging about 12 to 16 inches of precipitation per year.

Principal commodities.-Silver, copper, zinc, gold.

Mining history.-Gold was discovered in Cochetopa Creek in 1880, but mining was not substantial until after the silver crash of 1893 brought miners looking for gold. Copper was the major target after 1900, but the mines closed after World War I and never reopened. Uranium was located and mined in the 1950's. In the 1970's there was substantial exploration for gold using a new volcanogenic model, but no mining resulted from that good work.

Production and disturbance.-Small, intermittent production came from adits and shafts. Largest production was from the Vulcan mine area, discussed in the next section. Production of native sulfur and selenium at Vulcan is notable. Recorded production during the Depression years was thousands of dollars per year (very small). The underground workings generally produced small amounts of disturbance with dumps measured in only thousands to tens of thousands of tons at individual sites. Production from these deposits is given as 55,650 short tons (Long and others, 1998).

Geology.-The Gunnison Gold Belt is coextensive with Precambrian metavolcanic rocks of the Dubois Greenstone, a sequence of metamorphosed submarine volcanic rocks and interbedded cherts. Compositions of the layered rocks vary greatly, from mafic to felsic, and some ore zones are associated with siliceous layers that were originally submarine chert. Although the rocks have undergone multiple stages of folding, shearing, and faulting, the ore zones follow specific rock units (this is termed "stratabound"). Some samples on mine dumps have discernable fine-grained layering that may be pre-metamorphic and characteristic of stratiform volcanogenic massive sulfide deposits that form on the sea floor, according to research around the world on analogous deposits.

Three varieties of ore are recognized: (1) stratiform massive sulfide lenses rich in pyrite and containing sphalerite and chalcopyrite; (2) siliceous zones and veins rich in fine-grained silica that contain $\mathrm{Au}-\mathrm{Ag}-\mathrm{Cu}$, partly as telluride minerals; and (3) local zones of native selenium with native sulfur. Native selenium is very rare in world ore deposits. In the 1970's-80's, exploration focused on potential for economic amounts of gold or zinc.

\section{Mineral-Environmental Conditions}

Mineralized rocks and ores. - Chemical analyses of eight dump samples confirms the high concentrations of base metals 


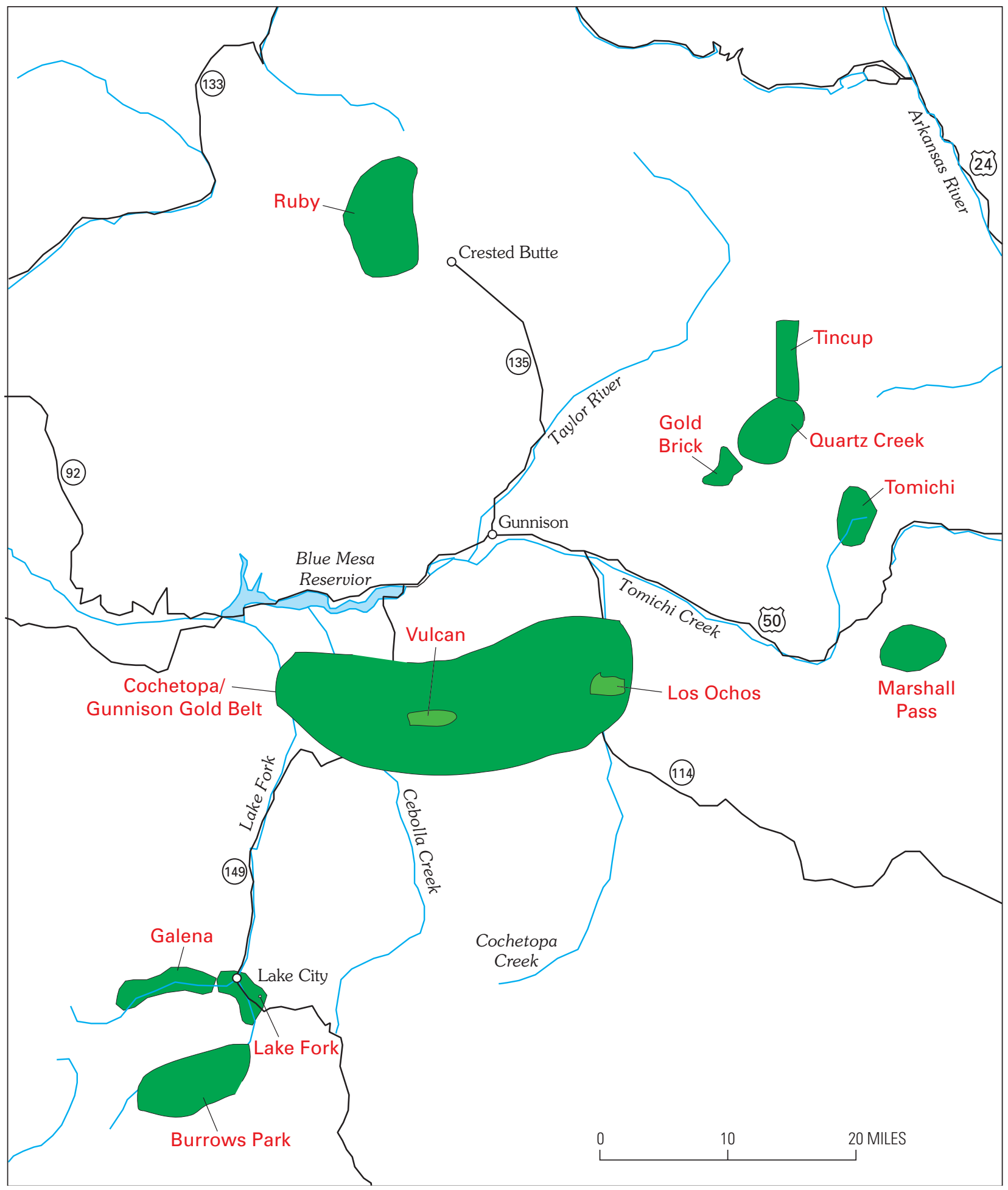

Figure 10. Location of studied mining districts in the Gunnison River watershed. 


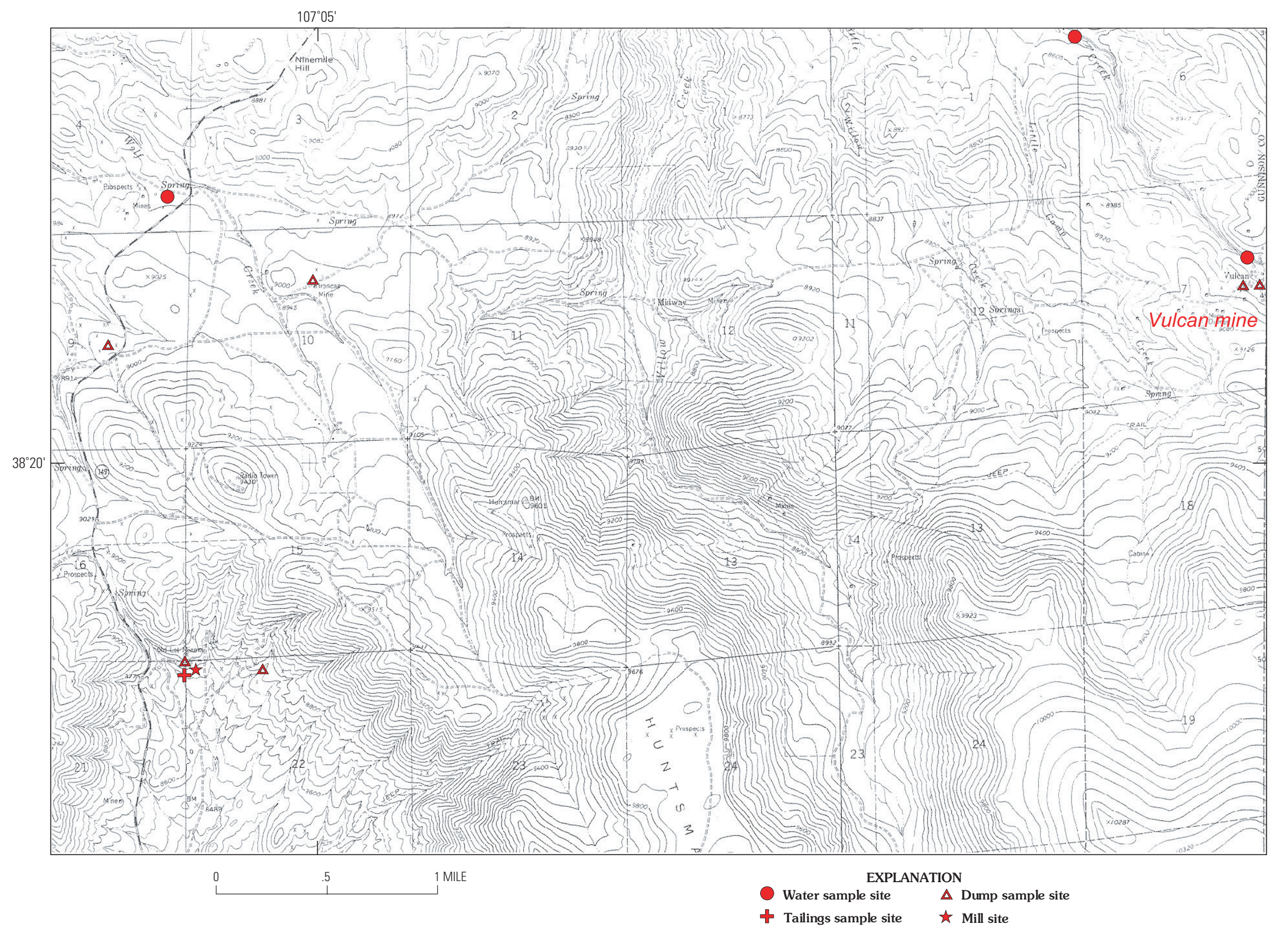

Figure 11. Features of part of the Cochetopa mining district, showing sample localities. 


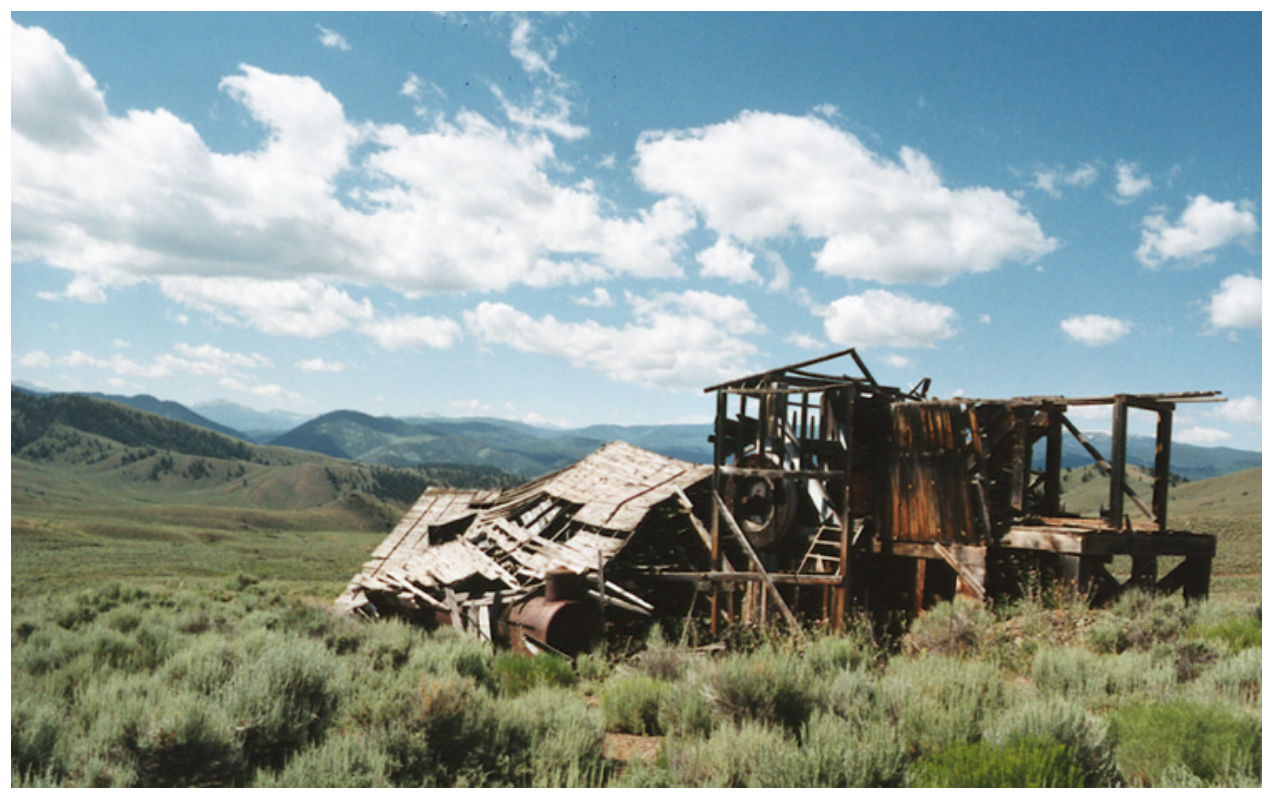

Photograph 13. Small mines and mills are typical of the Cochetopa district. The Old Lout mill has collapsed, and there is little evidence for production (small mine, small amount of tailings).

in these ores and waste rocks: very high concentrations were determined for $\mathrm{Fe}, \mathrm{Mg}, \mathrm{As}, \mathrm{Bi}, \mathrm{Cd}, \mathrm{Cu}$, and $\mathrm{Zn}$ (but $\mathrm{Pb}$ is not high). Leach tests on six samples produced some extremely high concentrations ( $>10,000 \mathrm{ppb} \mathrm{Cu}$ and $\mathrm{Zn}$ ), chiefly from mineral desiccation crusts (photograph 18). Leach solutions had $\mathrm{pH}$ values in the range of 1.9 to 4.8 , but most are $<3$. Some dump samples from the Vulcan mine area, described next, yielded very high base-metal concentrations, such as $\mathrm{Cu}$ and $\mathrm{Zn}>100,000 \mathrm{ppb}$. For leach solutions of $\mathrm{pH}<3$, concentrations of $\mathrm{Al}, \mathrm{Fe}, \mathrm{Mn}, \mathrm{Cd}, \mathrm{Cu}$, and $\mathrm{Zn}$ are very high. Concentrations of Se are variable, and some are very high. Even the concentration of $\mathrm{U}$ is high, despite the fact that the ores contain only normal amounts of U. Under these extremely acidic conditions nearly all elements are mobile, and in this relatively arid climate many metals become enriched in desiccation crusts.

Metamorphosed mafic volcanic rocks in the Dubois Greenstone may have high acid-neutralizing capacity (ANC), as at other similar massive sulfide deposits (Nash and others, 1996). However, in this district many of the deposits are in siliceous layers, and these probably lack the ANC of the mafic layers. Mixed lithologies in alluvial units may provide good ANC to naturally attenuate acidic drainage from mines and dumps.

Mills and tailings.-Only one mill was located, and it served one mine with minor production. A smelter at the Vulcan mine treated a small amount of ore. Ore appears to have been chiefly the "direct shipping" type that was shipped elsewhere for milling and smelting.

Surface waters.-All of the mines examined were dry. The Gold Belt is in a semiarid climate zone typified by sage- brush that appears to get much less snow and rain than the surrounding mountains. Surface waters do not appear to be influenced by these mines. The concern here is for possibly degraded waters in runoff from a brief summer thunderstorm that might carry a "flush" of acid and metals from soluble crusts on mine dumps.

Summary.-Studies of the Cochetopa district suggest that mine dumps from the massive sulfide deposits have the potential to generate acid and to release base metals, but, thanks to the arid conditions, that potential is rarely accomplished. There is little evidence for even local, episodic release of degraded waters sufficient to kill vegetation. The mined massive sulfide deposits and adjacent mineralized unmined rocks do not appear to be causing measurable contamination of surface water, except in the Vulcan mine area. If there is minerelated contamination, it probably would be for short periods during storms or snowmelt and is not expected to persist for more than about $1 \mathrm{mi}$.

References.-Sheridan and others (1981); Drobeck (1981); Hartley (1983).

\section{Vulcan Subdistrict (within Cochetopa District)}

Location.-Gunnison Co., $15 \mathrm{mi} \mathrm{SW}$. of Gunnison (fig. 12). Climate is relatively dry, about 12 to 16 inches of precipitation per year.

Principal commodities.-Sulfur, gold, silver, and selenium.

Mining history.-Gossans were identified in 1884, but most production of gold and silver was from 1889 and 1904, 


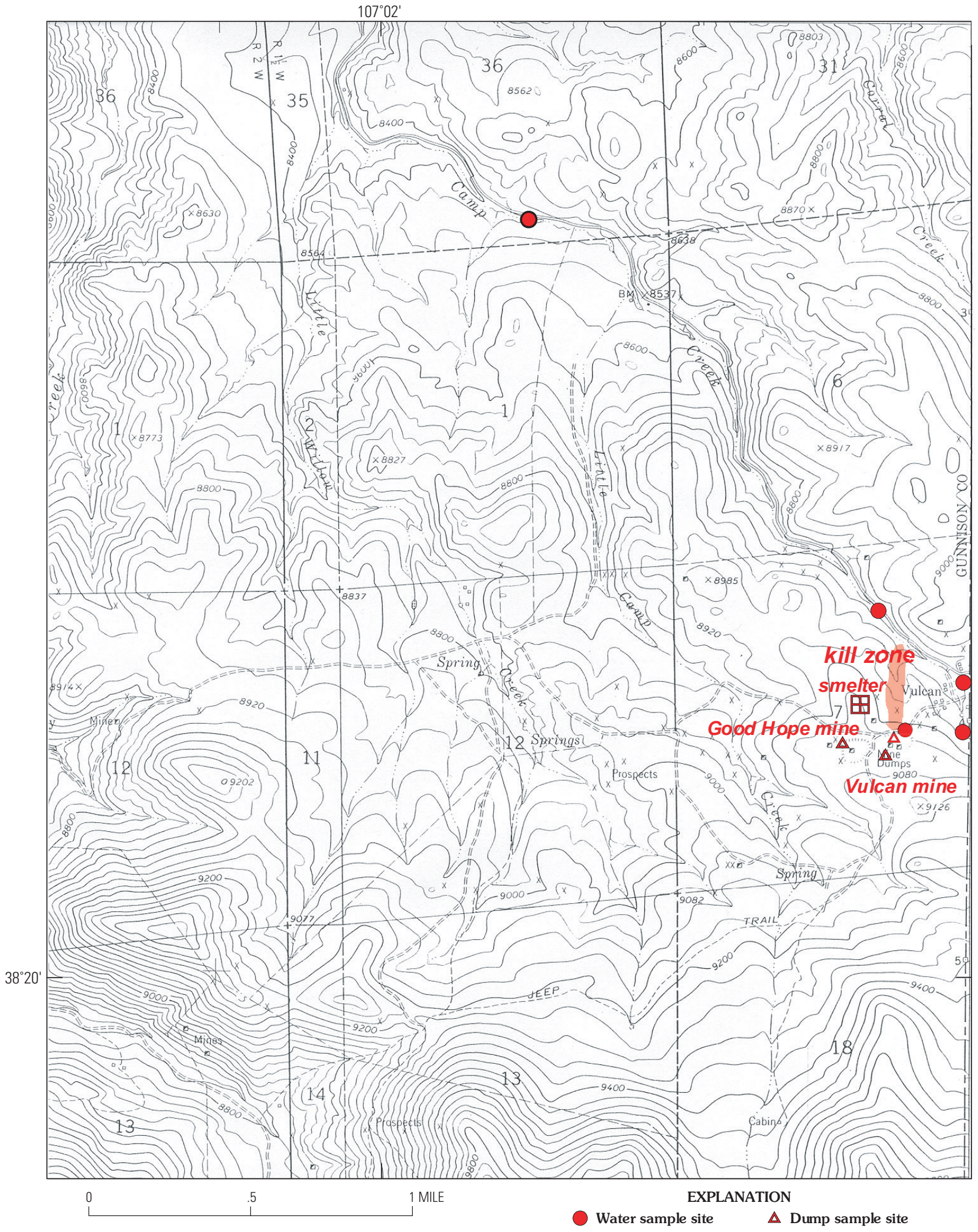

四 Smelter site

Figure 12. Features of the Vulcan mine area, showing sample localities. Some sites were sampled more than once. 


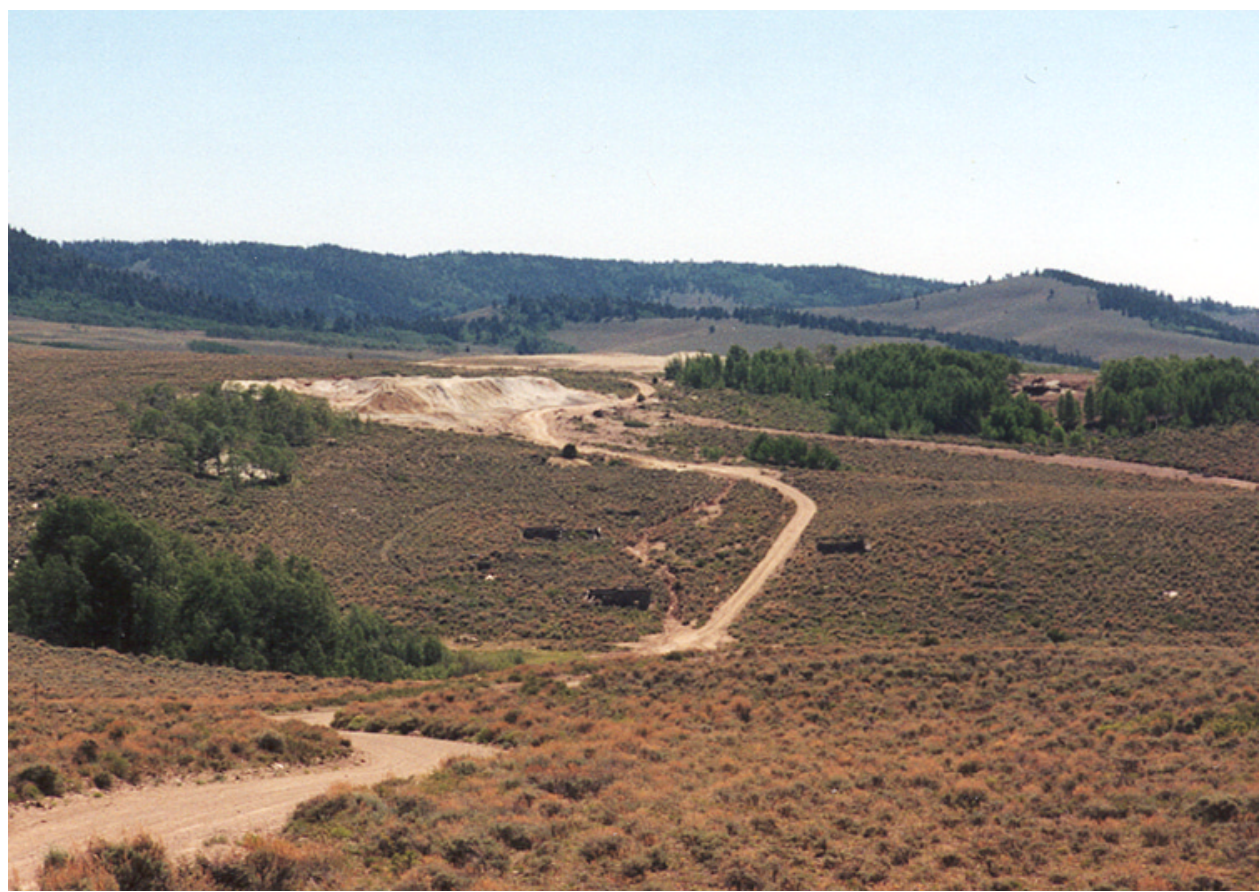

Photograph 14. The Vulcan mine and nearby Good Hope mine created dumps of moderate size but very high sulfide content, in center of photograph. A small smelter is in the aspen grove (right side).

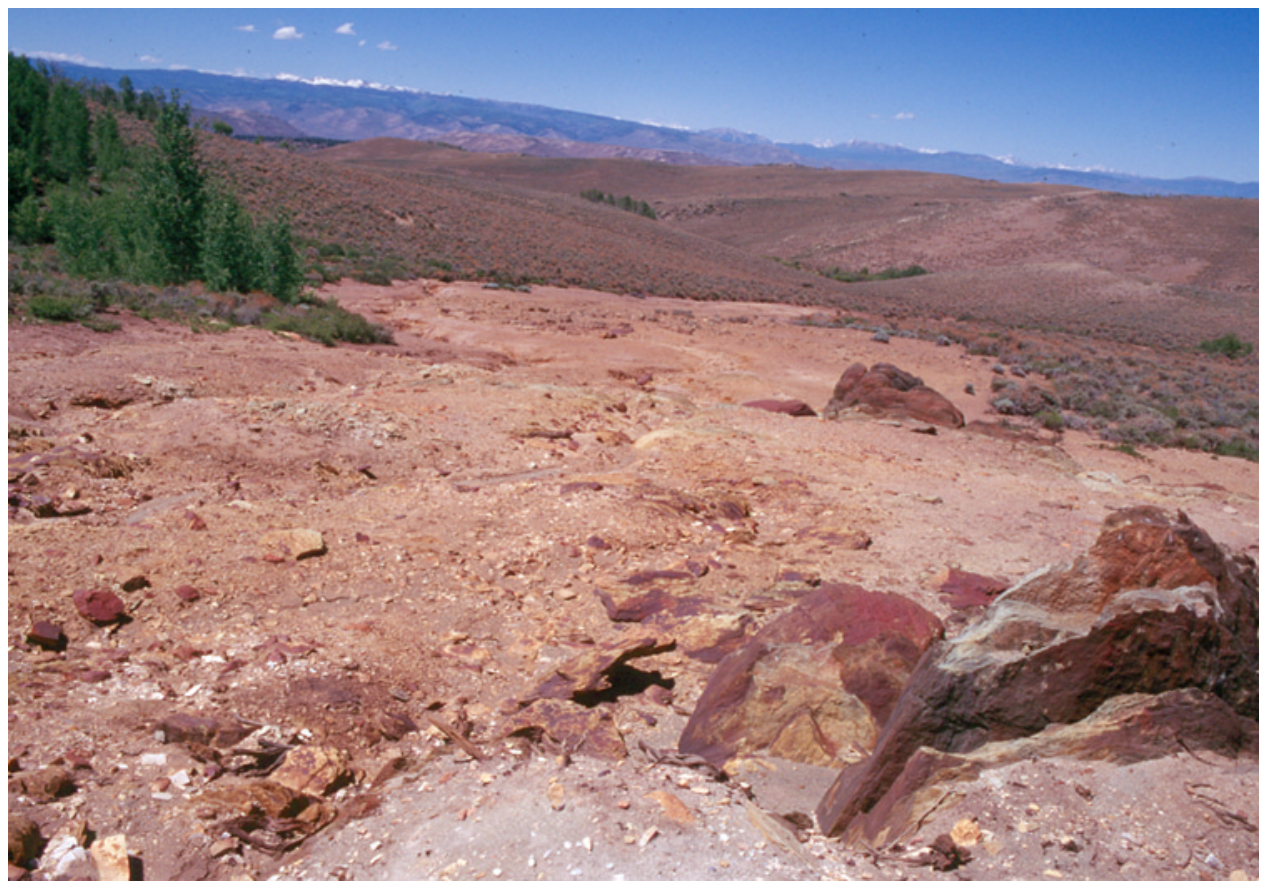

Photograph 15. North of the Vulcan mine and dump is this zone devoid of vegetation, termed a "kill zone." The zone reflects acidic waters related either to the mine dump or to outcropping mineralization. In most cases, kill zones are created after mining, but this one may be related to the gossan that helped prospectors discover the massive sulfide deposit. 


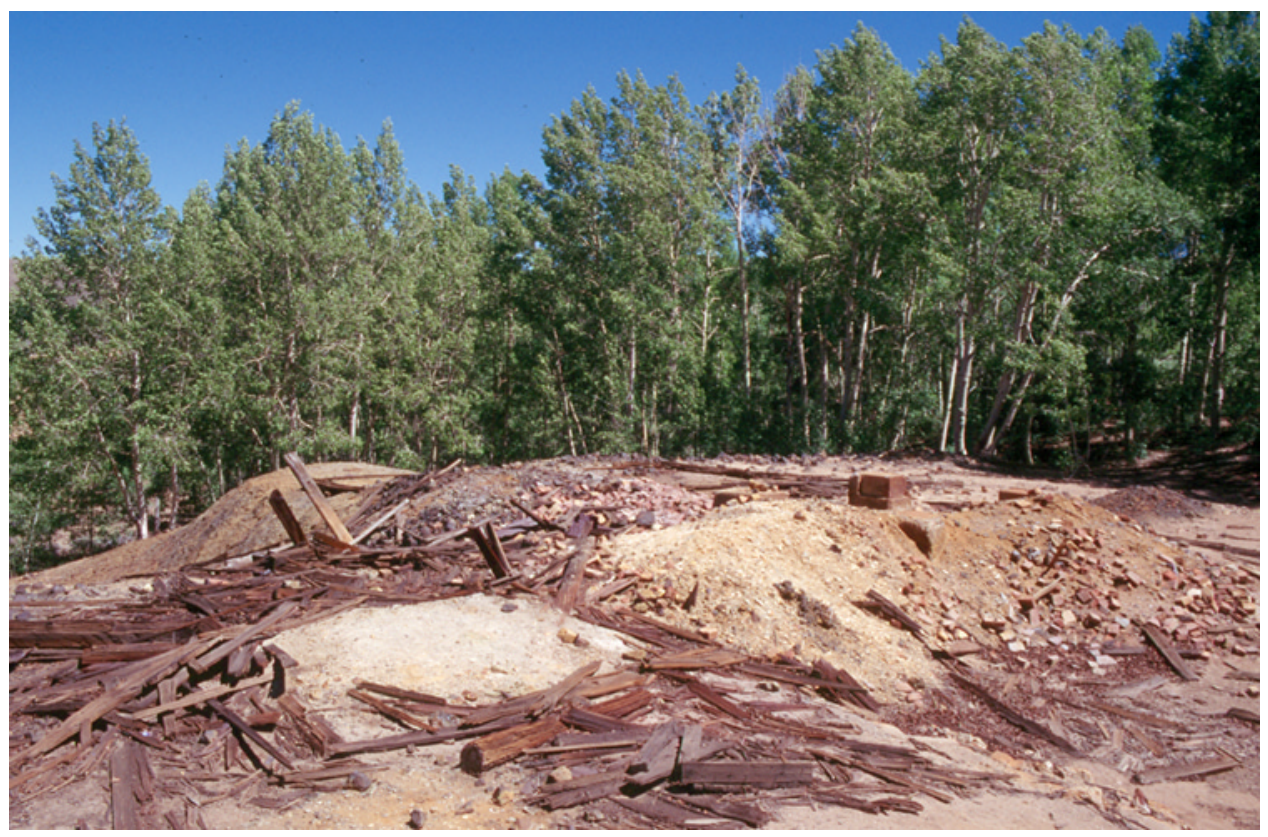

Photograph 16. The remains of the smelter at the Vulcan mine are barely identifiable, but the black vitreous material to the left is unmistakably slag. This smelter probably operated before 1900.

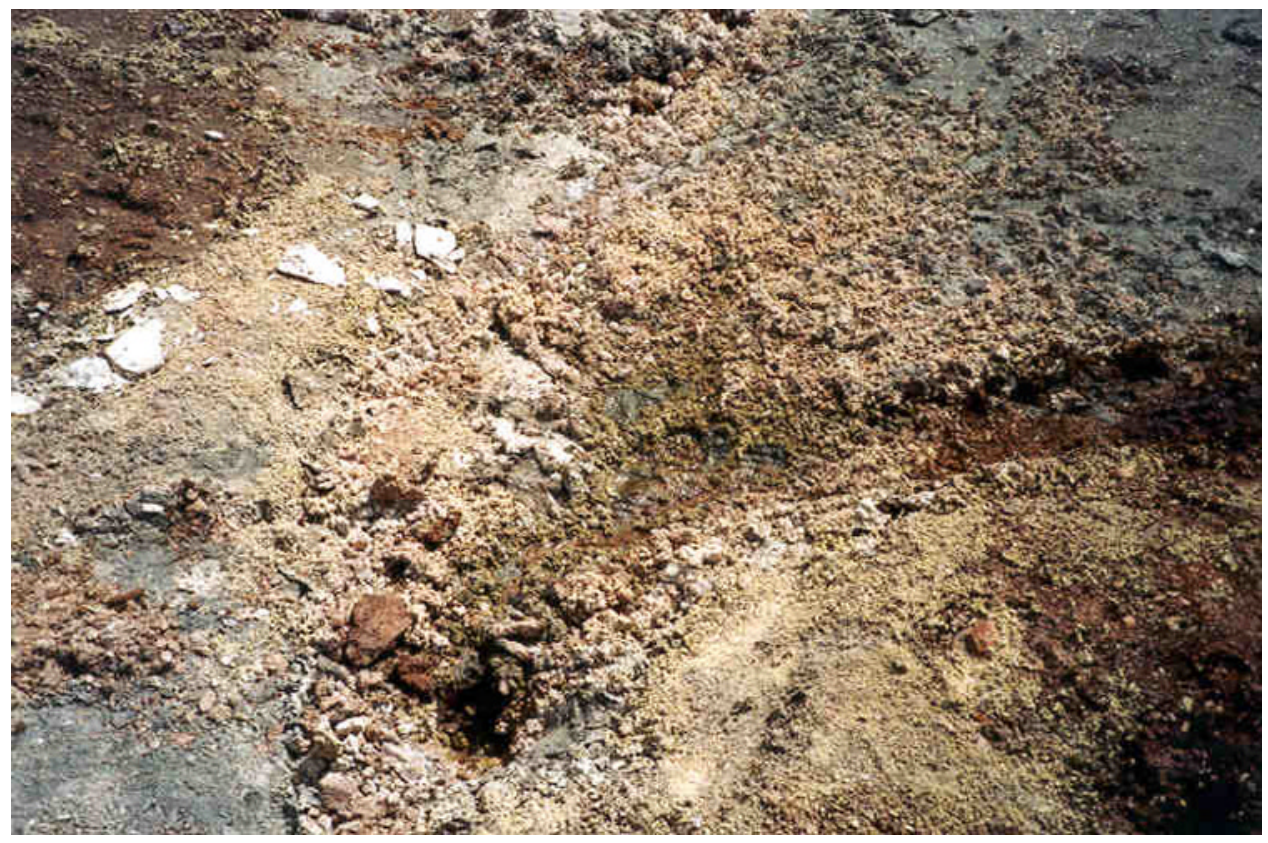

Photograph 17. Vulcan mine dump, rich in sulfide minerals, develops this white crust of sulfate minerals that is very reactive in water. The crusts dissolve immediately to form an acidic solution with very high concentrations of zinc and copper. The gray material is pyrite that accumulates at the surface when clays wash away. 


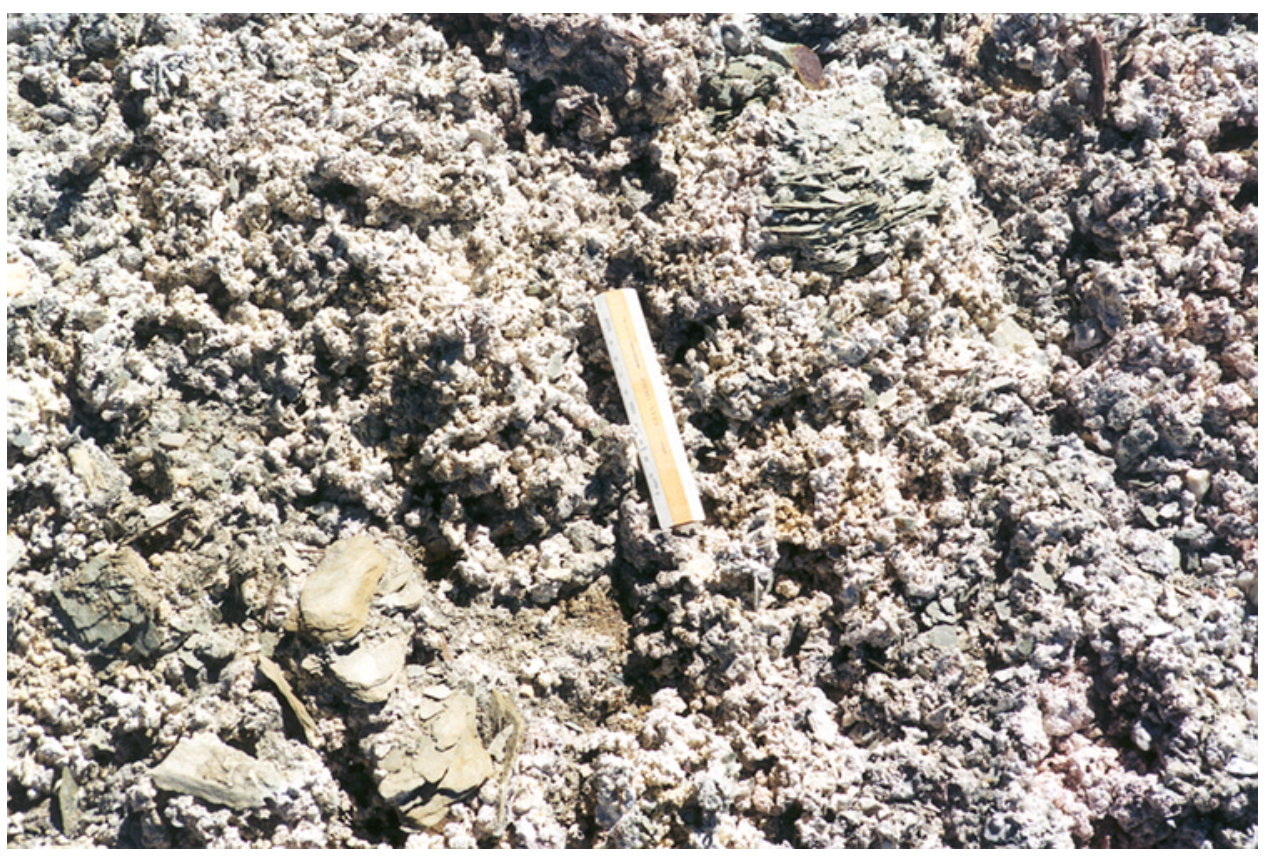

Photograph 18. This popcorn texture is created as sulfate crusts grow on pyritic waste rocks of the Vulcan mine dump. Scale is 6 inches long.

with some production of sulfur and selenium in 1917-18. Shafts developed ore to a depth of $600 \mathrm{ft}$ and $1,800 \mathrm{ft}$ on strike.

Production.-About $\$ 500,000$ worth of gold and silver (value not adjusted for inflation). Value of sulfur and selenium is not recorded.

Geology.-Lenses of pyrite-rich rock occur in Precambrian metavolcanic rocks of the Dubois Greenstone. The two major mines at Vulcan and Good Hope are part of the eastwest alignment of deposits in the area that formed as part of the submarine volcanic stratigraphy. Unusual concentrations of selenium and tellurium occur in parts of the deposits (Hartley, 1983). Gold and silver have been the major commodities of interest, but copper and zinc have also been produced.

\section{Mineral-Environmental Conditions}

Mineralized rocks and ores.-Rocks on the waste dumps are extremely rich in pyrite-more than 30 percent—and the trace metals $\mathrm{Zn}, \mathrm{Cu}, \mathrm{Se}$, and $\mathrm{Te}$ are abundant enough to be of concern. Weathering of the pyritic rocks in the semiarid climate produces efflorescent sulfate crusts (photograph 17) that are soluble in water and create extremely acidic, metalrich solutions in laboratory leach tests.

Outcrops north of the Vulcan mine are highly sericitized schist that are rusty colored and devoid of vegetation (photograph 15). Inspection of the gossan and soil profiles suggest that much of the rusty rock was produced by weathering prior to mining. Gossan zones such as these attracted the early prospectors in the 1880's (Hartley, 1983).
Mills and tailings. - The remains of a small smelter are located a short distance north of the Vulcan shaft (photograph 16). This old smelter (pre-1900?) probably was not effective because the amount of slag is very small (a few hundred tons). No mill is evident, so the pyritic ore probably was shipped directly to a distant smelter or mill. Hand-sorted ore rich in gold and silver is quoted by Hartley (1983) from unpublished company records.

Surface waters.-The mining areas were dry when visited in June of 1997 and 1998. The one stream that was flowing in June, and probably is perennial, is Camp Creek, which flows north from the Vulcan mine area to Blue Mesa Reservoir. Field measurements of $\mathrm{pH}$ in 1997 and 1998 were in the narrow range of 7.7 to 8.2 , and conductivities ranged from 250 to $350 \mu \mathrm{S} / \mathrm{cm}$, which is fairly high. Chemical analyses of four stream-water samples showed low metal concentrations, with the exception of high zinc (2.6 times ALWS) found in the spring-fed creek south of the Vulcan mine and collected onstrike with the zone that contains the massive sulfide lenses (the water that was sampled may have been in contact with unmined mineralization related to Vulcan but probably not the Vulcan deposit itself or mine waste). Copper-zinc index (CZI) values for the four surface-water samples are below 0.5 , which suggests relatively good aquatic health. Samples of Camp Creek north of the mining area, and the kill zone that goes downslope to the north of the Vulcan mine dumps, did not contain significant metal concentrations, although metal concentrations are elevated relative to surface waters elsewhere.

Areas with no vegetation ("kill zones") adjacent to the Vulcan and Good Hope dumps resemble those produced by acidic drainage flowing overland from dumps. However, the 
association of the large kill zone north of Vulcan with limonite-sericite-rich rocks suggests the possibility that some of the effect could be related to naturally outcropping mineralization (gossan) that existed prior to mining. Alternatively, episodic runoff during storm events or melting of snow would likely dissolve efflorescent salts on the dumps, as seen in laboratory leach tests, to create pulses of highly acidic, metal-rich waters. Judging from compositions of storm runoff collected in other mining areas, and the known high solubility of crusts on mine waste, there is high risk of a flush of initially acidic, metal-rich water during fairly rare runoff events that could be toxic to aquatic life and fish in Camp Creek. Additional sampling during storm events or snowmelt may be required to document mine-related contamination in Camp Creek.

Summary.-The highly pyritic waste dumps at Vulcan may pose a substantial threat to Camp Creek. However, the release of metals and acid probably is not as bad as imagined because the climate is semiarid and the coarse-grained pyrite is not highly reactive. Some of the pyrite does react to form crusts of efflorescent sulfate salts that are highly reactive, and these salts create very acidic and metal-rich waters in a "flush" during snowmelt or thunderstorms. Episodic releases of toxic waters is probable, but the frequency of these events and the spatial scale of the impact could not be measured. Laboratory tests demonstrate that sulfidic rocks on dumps from the Vulcan and Good Hope mines have high potential for acid generation and release of high concentrations of base metals. Under most conditions, there is insufficient water to initiate the reactions and transport the potentially toxic metals into nearby Camp Creek. Further studies may be required to sample runoff during storm events and to evaluate reclamation methods that would reduce the risk of runoff in storm events. (1983).

References.-Hartley (1983); Nelson and Riesmeyer

\section{Los Ochos Subdistrict (within Cochetopa District)}

Location.-Saguache Co., 10 mi SE. of Gunnison (fig. $10)$. The climate is relatively dry, averaging about $12-16$ inches of precipitation per year.

Principal commodity.-Uranium.

Mining history.-Uranium was discovered in 1954 and mined from 1955 to 1962 . A series of shafts and adits were made along the east-west-trending zone for about $1.3 \mathrm{mi}$. Ore was trucked to a mill near Gunnison.

Production and disturbance.-Total production was about 1.2 million $\mathrm{lb} \mathrm{U}_{3} \mathrm{O}_{8}$ (McCulla, 1980). Moderate disturbance is present in the form of trenches and waste dumps. The dumps are substantial in size, on the order of a million tons.

Geology.-Veins rich in pyrite-marcasite-pitchblende occur along the high-angle Los Ochos fault zone. Sandstones in the Jurassic Morrison Formation are the favored wallrocks for the veins, and smaller amounts of ore occur in sandstones in the Cretaceous Dakota Formation. Tertiary volcanic rocks formerly covered the area and are thought to have been important in ore formation.

\section{Mineral-Environmental Conditions}

Mineralized rocks and ores.-Mined waste rocks contain high amounts of iron sulfide (pyrite and marcasite) in the range of 1 to 10 percent (photograph 20). Radioactivity is variable from about $3 \times$ to $20 \times$ background from place to place on the dumps, with some of the piles from test mining in the 1970's being least weathered and most radioactive. Median concentrations (in parts per million) in six analyzed samples are: As, 290; Cu, 22; Mo, 37; Pb, 160; Se, 0.8; Th, 6; U, 58; V, 57; $\mathrm{Zn}, 66$. Curiously, these dump samples contain some of the highest thallium concentrations in the study area: the median $\mathrm{Tl}$ concentration is $13 \mathrm{ppm}$, and the highest is $23 \mathrm{ppm}$. Chemically, The Los Ochos ore compositions share features of both the sandstone uranium ores of the Uravan district, described later, and polymetallic deposits of the study area.

Leach tests on three dump samples yielded solutions with pH's in the range 3.5 to 5.4. Not surprisingly, the most acidic leachates carried the highest metal concentrations, including As- $\mathrm{Cu}-\mathrm{Zn}$ values in the range of many polymetallic vein dumps. Uranium was very high in the $\mathrm{pH} 3.5$ leachate (250 $\mathrm{ppb}$ ) and much lower in the other two leachates. These limited results suggest that uranium is mobile in situations where sulfide minerals generate acid, and the acid solutions then carry uranium. Similar high uranium leachates were produced by sulfidic dump samples from vein-uranium deposits near Austin, Nevada (Nash, 2000b).

Mills and tailings.-Ore was trucked to the mill at Gunnison, thus there are no mill tailings at this site.

Surface waters.-The mines are dry at the surface and all mining probably was above the water table. No surface water was seen in June of 1997. A spring 1 mi north of the mine area, developed for livestock, has $\mathrm{pH} 7.9$, elevated conductivity $(680 \mu \mathrm{S} / \mathrm{cm})$, and high concentrations of some metals that are mobile in alkaline waters: $\mathrm{U}$ and Se exceed ALWS and Se exceeds the standard for livestock. The CZI is low at 0.1. Concentrations of $\mathrm{Zn}$ and Mo are elevated, and the Mo may be a concern if livestock drank this water for long periods. These four elements in the spring water could be derived from Cretaceous shale or other sedimentary rocks, or from the fringes of mined or unmined uranium-bearing veins.

Summary.-The uranium mines are dry most of the year and do not appear to be creating surface drainage. Leach tests show that there is potential for uranium to be mobile in proportion to acidity: the more acid produced, the more uranium goes into solution. The status of the substantial tonnage of waste rock on mine dumps is not clear. There are no specific regulations for uranium mine dump reclamation, but radioactivity levels for much of the dump rock at Los Ochos are well above the standard for uranium mill tailings. Criteria need to be reviewed by State and Federal regulators. Further studies 


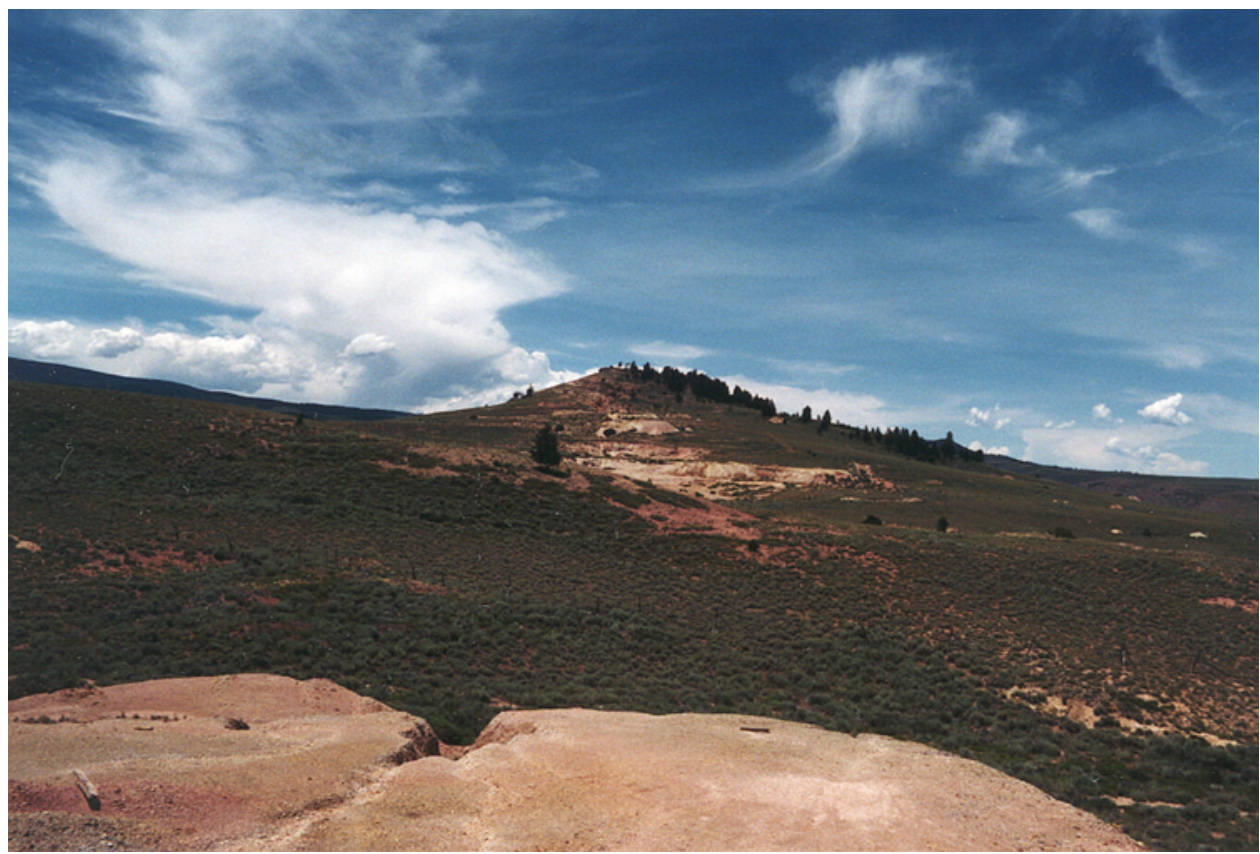

Photograph 19. Uranium mines of the Los 0 chos district are chiefly along an east-west fault zone in the dry hills of the Los 0 chos district. Vegetation has not returned to the dumps in more than 30 years. This view is looking west from the east end of the mining area.

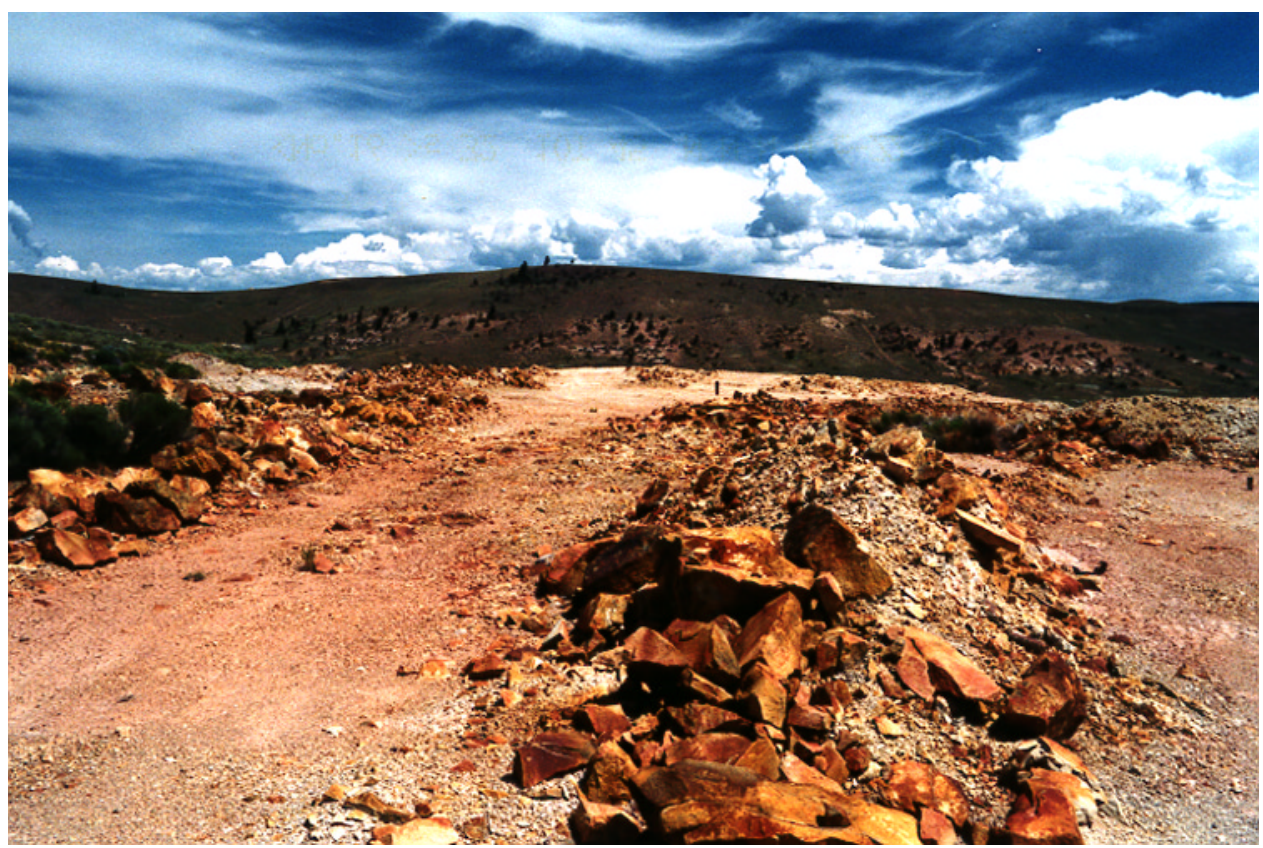

Photograph 20. Uranium of the Los 0chos district are rich in pyrite, causing them to turn red and yellow on the surface as they weather. Most dumps are considered waste, but these angular boulders are ore grade (mined in the 1970's but not taken to a mill) and the smoothed-out part is pre-1970 material that was low-grade ore (mineralized but not sufficiently rich in uranium to be worth milling). These rocks are highly radioactive. 
Hydrogeochemical Investigations of Historic Mining Districts, Central Western Slope of Colorado

should be undertaken, perhaps using specifications for uranium mill tailings as guidelines, to evaluate needs and methods for reclamation. The dumps are not an obvious threat to a few residents living about a mile distant. Materials mobilized in a storm event could spread some contaminants locally but are unlikely to contaminate Cochetopa Creek, a mile distant.

References.—Wright and Everhart (1960); McCulla (1980); Nash (unpub. field notes, 1978); Olson (1988).

\section{Marshall Pass District}

Location.-Gunnison Co., 35 mi east of Gunnison (fig. 13). The climate is harsh with deep snowpack and about $25-40$ inches of precipitation per year.

Principal commodity.-Uranium.

Mining history.-Radioactive zones were found in Harding Quartzite beds in 1955 and traced to deposits in fracture zones filled by yellow hexavalent uranium minerals. The same year, prospectors located several other kinds of uranium deposits in the area, including the larger ones along a major thrust fault in Leadville Limestone and Belden Formation shales at the site of the Pitch mine. Most of the prospects had little or no production in the 1950 's, but substantial underground workings were made at the Pitch (Pinnacle) and Little Indian mines, with ore processing at the mill near Gunnison. The upsurge in uranium prices in the 1970's encouraged renewed exploration and subsequent mining of an open pit at the site of the former Pitch underground mine. The richest ore was trucked to a mill in New Mexico for about 4 years, through 1984. The Pitch mine is being reclaimed, and the owner has expressed interest in turning all claims (including several patents) back to the U.S. Forest Service.

Production and disturbance.-Production was about 1 million pounds $\mathrm{U}_{3} \mathrm{O}_{8}$. Early underground mines made small disturbances, but the 1980's open pit at Pitch mine created moderate disturbance and also is the site of a substantial landslide (slip) along the highly fractured Chester fault zone. Ongoing reclamation is addressing waste dumps and water discharge under a permit with the Colorado Division of Minerals and Geology (CDMG). The original Pitch mine adit has been sealed, with beneficial results.

Geology.-Rocks ranging in age from Precambrian to mid-Tertiary host ore deposits in this district. A thick section of Cambrian to Cretaceous sedimentary rocks is crunched against and under Precambrian schist and granite along the Chester fault. Tertiary volcanic rocks cover the sedimentary rocks and the fault zone, suggesting a Laramide age for the structure.

The uranium deposits of several types that are present are unconventional compared to major uranium deposits elsewhere in the United States There are differences of opinion as to the source of the uranium and relations between deposit types, but the importance of the Chester fault in creating the fractures and structural permeability seems clear. Uranium is the only highly enriched element in these ores, and it occurs in both oxidized and reduced states. The trace metals that are slightly enriched in the ores $(\mathrm{Cu}, \mathrm{Fe}, \mathrm{Mo}, \mathrm{Pb}, \mathrm{Zn}, \mathrm{V})$ are not economically significant but may provide clues to ore-forming processes. Fine-grained pyrite and marcasite are abundant in the Pitch mine ores.

\section{Mineral-Environmental Conditions}

No new studies were made in this district because the mining company appeared to be making good progress on reclamation. Some general comments are made from my previous geochemical studies and from observations in a brief mine tour in 1998.

Mineralized rocks and ores.-Compositions are described in some detail by a previous study (Nash, 1988), which emphasized the fine-grained iron sulfide minerals in ore zones and generally low concentrations of base metals such as $\mathrm{Cu}, \mathrm{Pb}$, and $\mathrm{Zn}$. The abundant limestone and dolomite in the mined and unmined rocks should be more than adequate to neutralize any local pockets of acidic waters if they are produced by iron sulfides. However, uranium is highly mobile in carbonate waters (Wanty and others, 1999), which is the chief concern here (along with decay products such as radium).

Mills and tailings.-Ores mined in the 1950's and 1980's were hauled to mills outside of the district. There are no tailings in the district.

Surface waters.-Unlike the Los Ochos district to the west, the Marshal Pass district receives large amounts of snow and summer rains. Water is a major factor in this district. The Pitch mine pit collects water, and the underground tunnel formerly had mine drainage. The mining company has undertaken millions of dollars worth of reclamation and is working with CDMG and other regulators to meet discharge standards. If unchecked, discharge of $U$ and radionuclides would be a problem, but the reclamation activities, including a watertreatment plant, should bring the discharge into compliance.

No new samples were collected near the mines, and no water data for the Pitch mine are published. There is a small pond at the bottom of the pit, perhaps an acre in size. The mining company has a water-treatment plant onsite that treats water from mine workings and the pit pond before release into Indian Creek. The mining company monitors concentrations of $\mathrm{U}$, and radionuclides such as radium, in the discharge.

A spring on the Chester fault zone south of the mine, developed for livestock, may be typical of waters in the area. The spring has a pH of 7.7 and conductivity of $90 \mu \mathrm{S} / \mathrm{cm}$, and base metals and $\mathrm{U}$ are low. The CZI for the spring water is only 0.11 .

Summary.-High precipitation, combined with substantial disturbance created by the open-pit mine and the wide distribution of high- and low-grade uranium concentrations in mined and unmined rocks, create conditions that are unique within the eastern part of the study area. There is potential for water contamination caused by oxidation of iron sulfide minerals in the Pitch mine pit and in associated mine dumps 


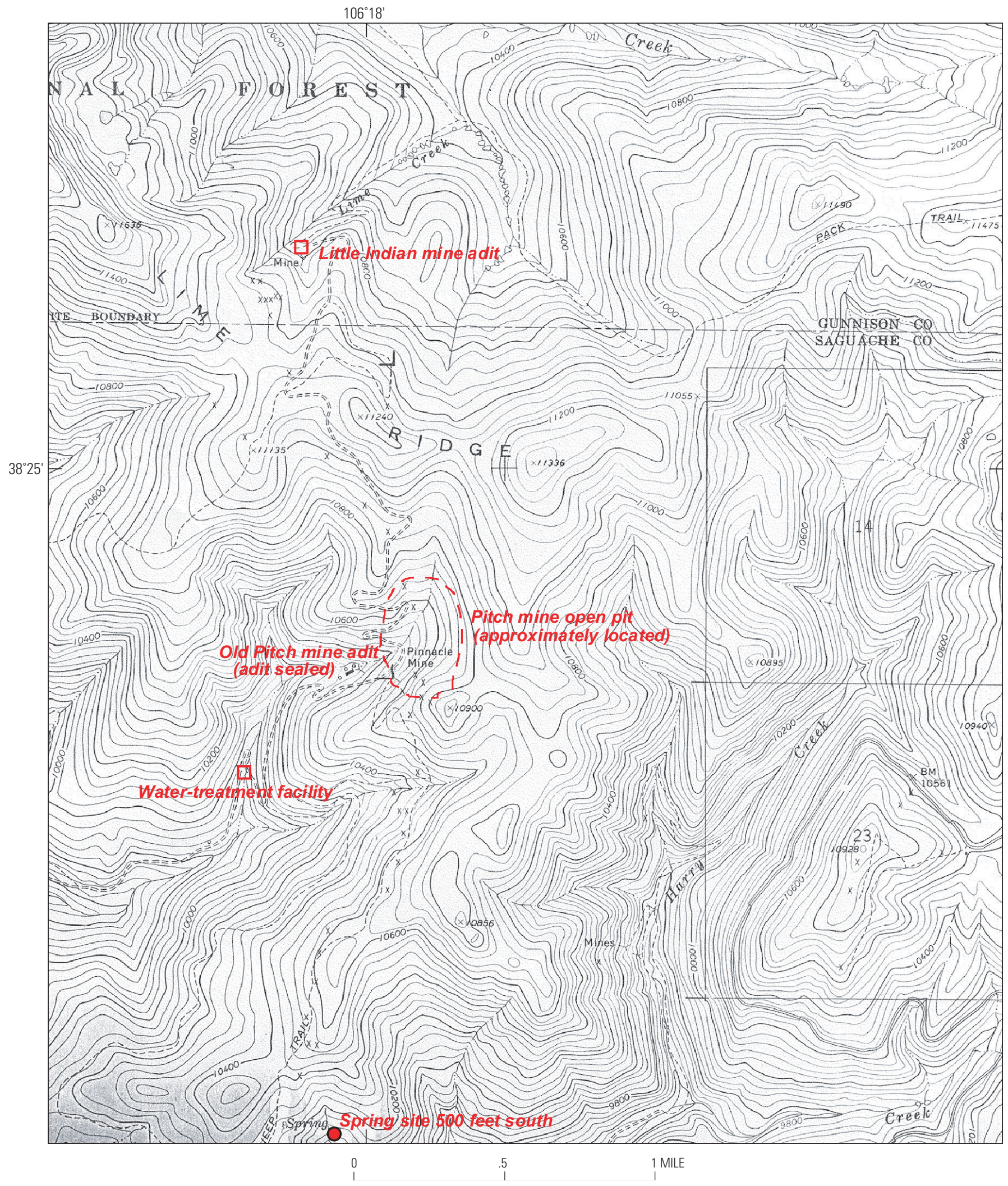

Figure 13. Features of part of the Marshall Pass mining district, showing major uranium mines. 


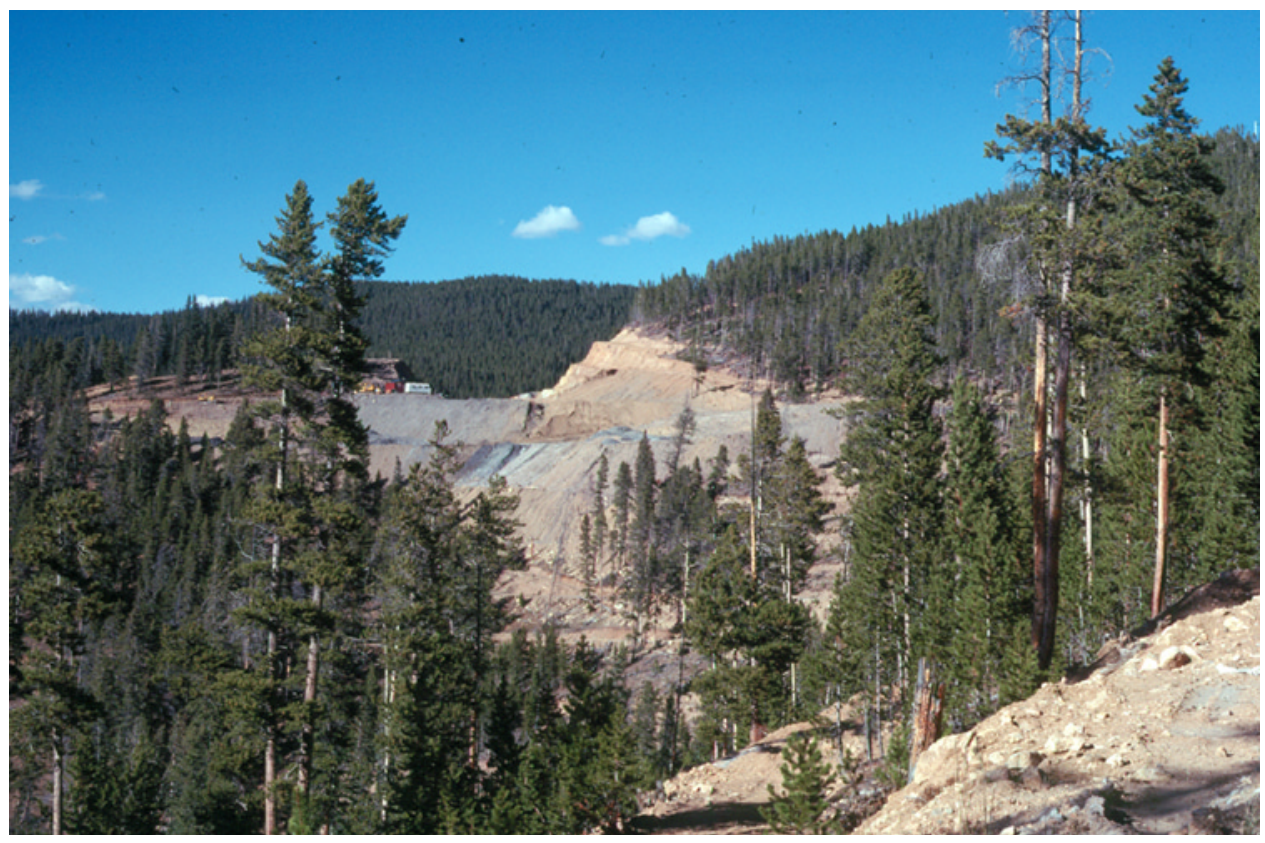

Photograph 21. The Pitch uranium deposit formed along a major fault that placed gray shales and limestones (left side) against Precambrian granite (tan, right side), as seen in 1977 when excavation of the open pit was started. The heavy snowfall and abundant fracturing along the fault zone cause environmental problems that complicate reclamation.

and for mobility of uranium and associated radium. Acidic waters, if any, would be a local phenomenon in this area of carbonate rocks, but uranium is mobile in carbonate-rich water and could migrate for miles. A water-treatment plant and major reclamation work by the mine owner appear to be mitigating known and potential problems, but technical monitoring of water quality may be required to confirm tests by the company. The long-term future of the reclaimed lands and the stability of the slide on the Chester fault cannot be evaluated here.

References.-Wright and Everhart (1960); Nash (1981, 1988); Olson (1983, 1988).

\section{Gold Brick District}

Location.-Gunnison Co., about 18 mi east of Gunnison (fig. 14). Climate is moderate for the study area, with about 20-30 inches of precipitation per year.

Principal commodities.-Silver, lead, gold.

Mining history.-Discovery was in 1879, and there was a burst of exploration activity thereafter, but discoveries were small in size. In the period 1905 to about 1925, three long tunnels were driven northward from the level of Gold Creek and resulted in more productive mines than the original shaft operations. Each mine was served by a mill with amalgamation plates and tables to concentrate gold and sulfide minerals (photograph 23). There was modest production in 1930's and little if any production after World War II despite exploration ventures in the 1950's.
Production and disturbance.-Total district production was less than $\$ 500,000$ from about 200,000 tons of ore (Long and others, 1998). At least three mines produced more than $10,000 \mathrm{oz} \mathrm{Ag}$ and $1,000 \mathrm{oz} \mathrm{Au}$ (which is not large for the study area or considering the size of the mine infrastructures). Mining disturbance was small, but three tailings impoundments are in the flood plain of Gold Creek (photograph 25).

Geology.-Precambrian metamorphic rocks dominate the geology of this district. Several varieties of schist containing muscovite, garnet, amphibole, and other minerals have been mapped. Beds of quartzite and impure quartzite also are common. Granitic and mafic igneous rocks, later metamorphosed to gneisses, intruded the bedded rocks. There were several periods of deformation and metamorphism in the Precambrian. Cambrian to Mississippian clastic rocks (chiefly quartzite and limestone) overlie the Precambrian rocks. Middle Tertiary dikes and plugs intrude the older rocks. The complex geology was unraveled with the aid of improved structural and geochronologic methods (DeWitt and others, 1985) that demonstrated the importance of mid-Tertiary plutonism for ore formation.

Quartz veins with sulfides and precious metals occur in Precambrian metamorphic rocks and as local replacement bodies in Paleozoic carbonate rocks. Galena was the most prominent ore mineral, but most ore values came from gold and from silver associated with galena. Most of the mined ore was oxidized "limonitic quartz" with residual galena; oxidation or partial oxidation extends to the deepest workings, about 1,300 $\mathrm{ft}$ below the surface. Some of the milled ore 


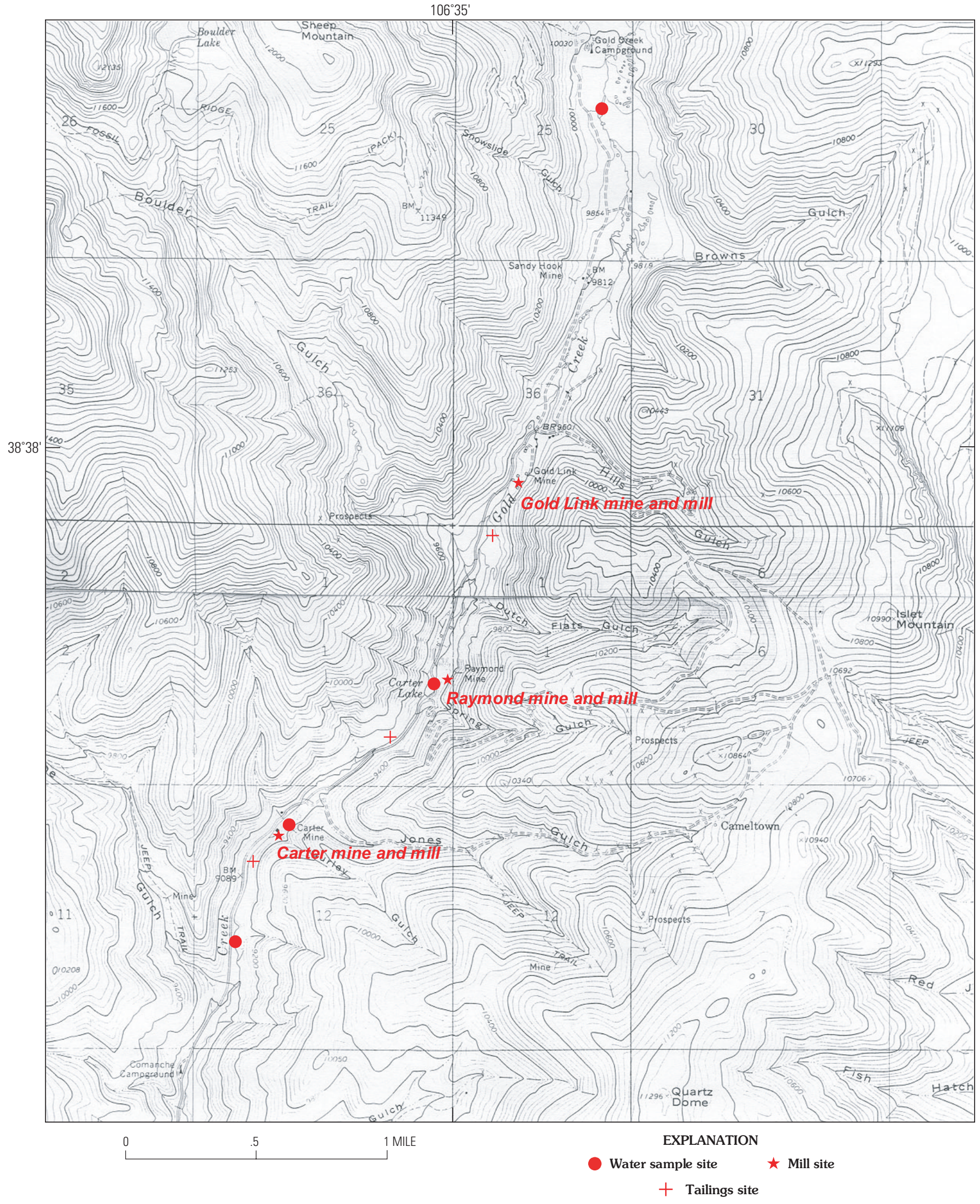

Figure 14. Features of the Gold Brick mining district, showing sample localities. 


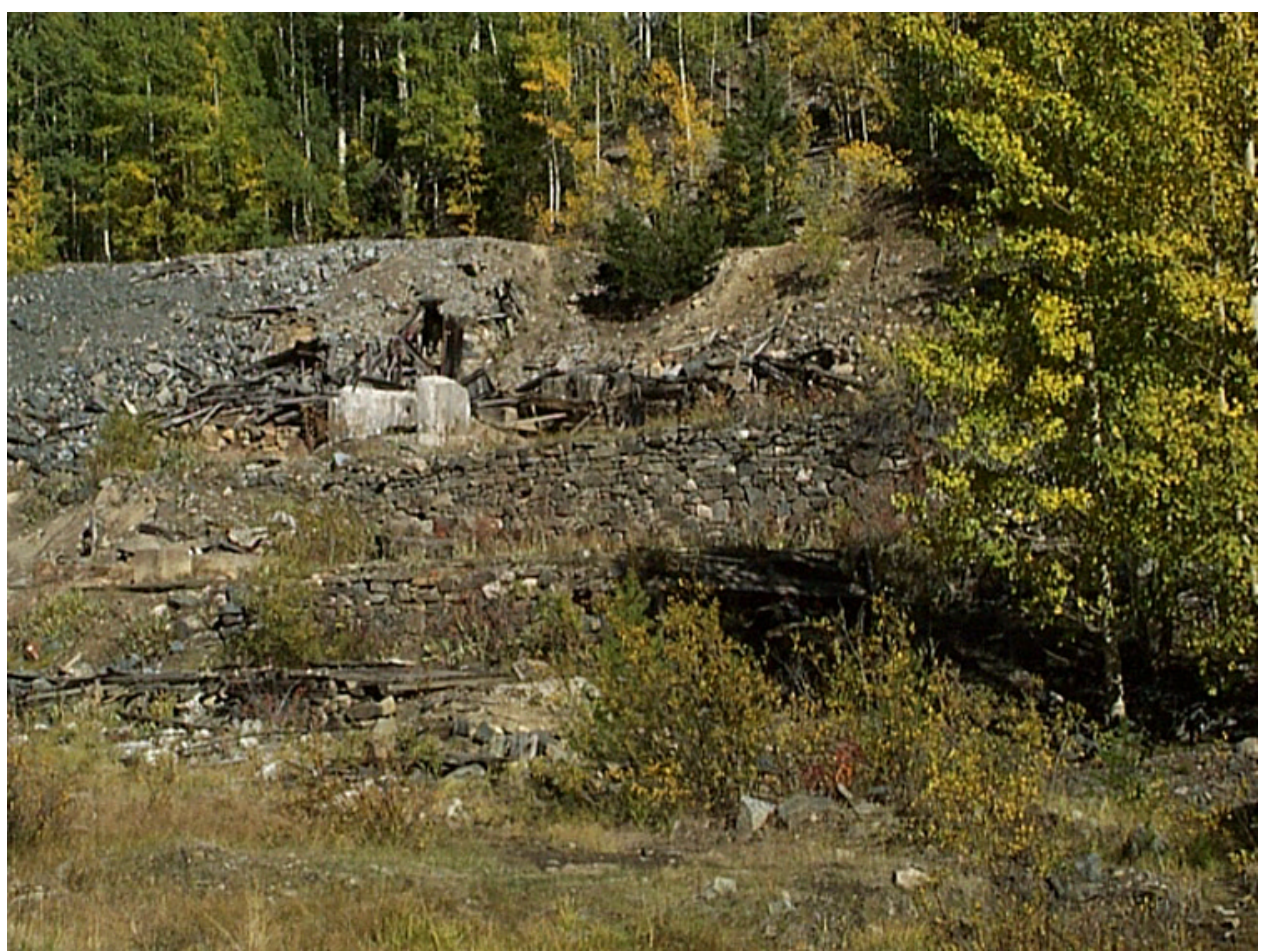

Photograph 22. The Raymond mill on Gold Creek, Gold Brick district, had 40 stamps and was active before 1915. Tailings were run through sluices and placed on a terrace close to the creek.

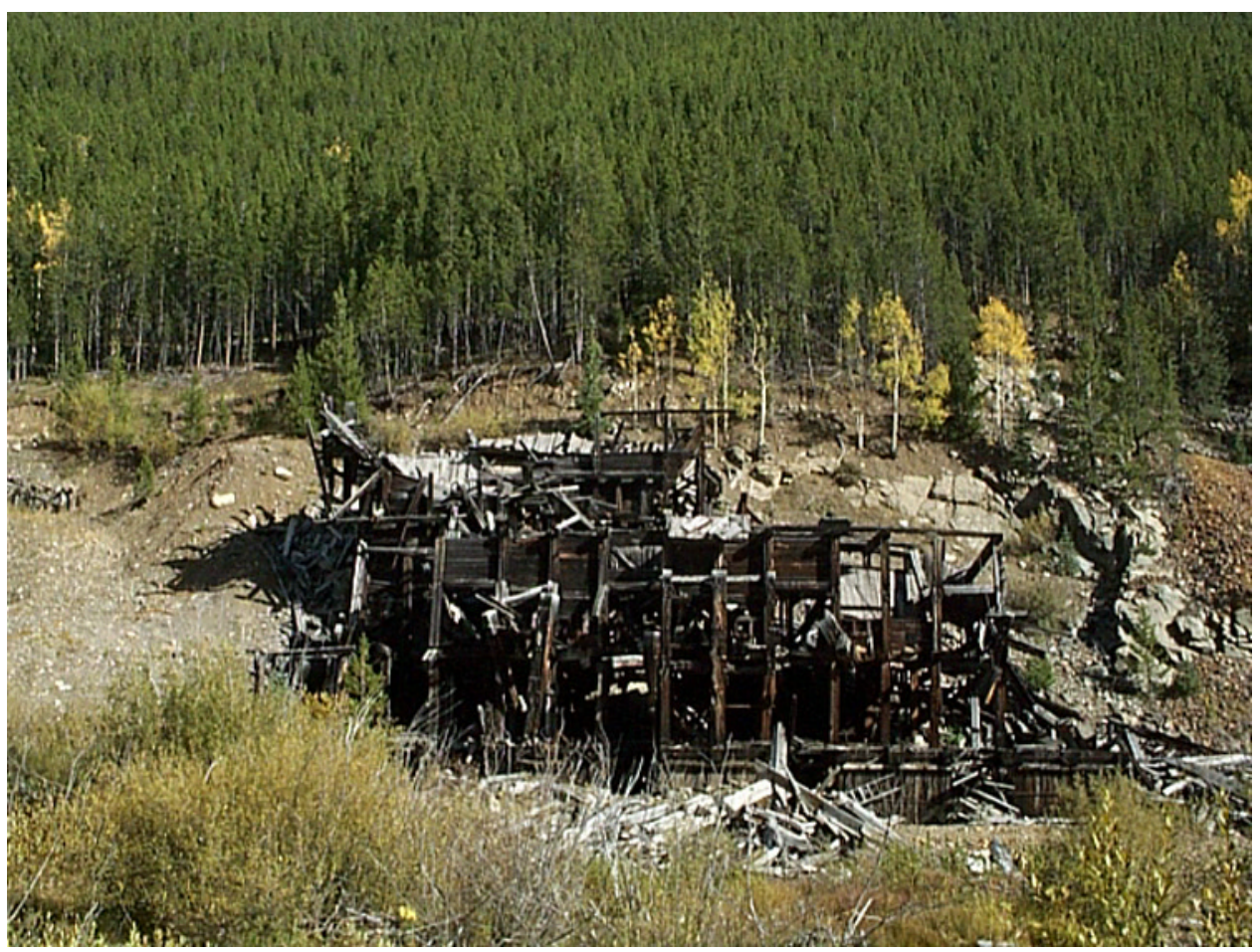

Photograph 23. The Gold Link mill was a large and impressive structure in 1915. Tailings were run eastward about 500 yards and placed on a terrace about 50 yards from the creek. 


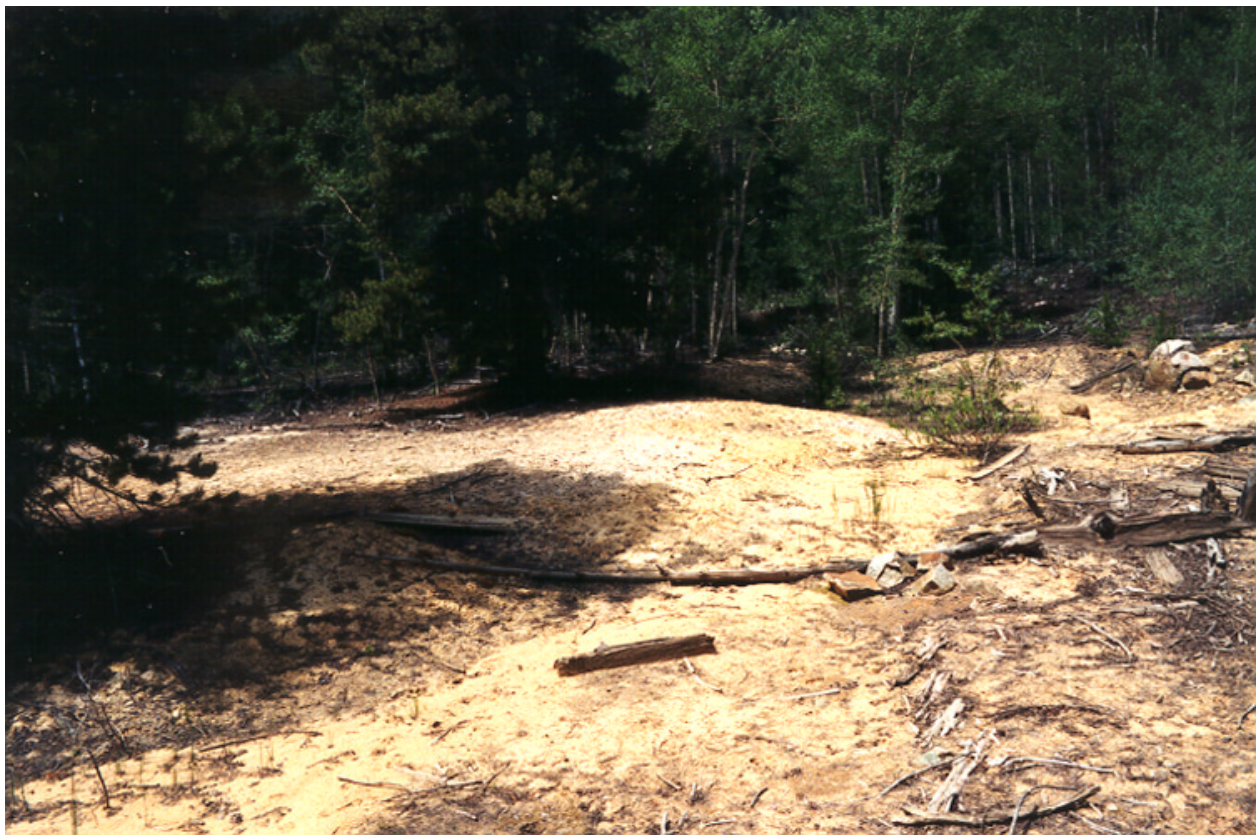

Photograph 24. Tailings from the Gold Link mill were placed in gullies east of the mill. The pine trees probably grew after the tailings were emplaced, possibly before 1920 .

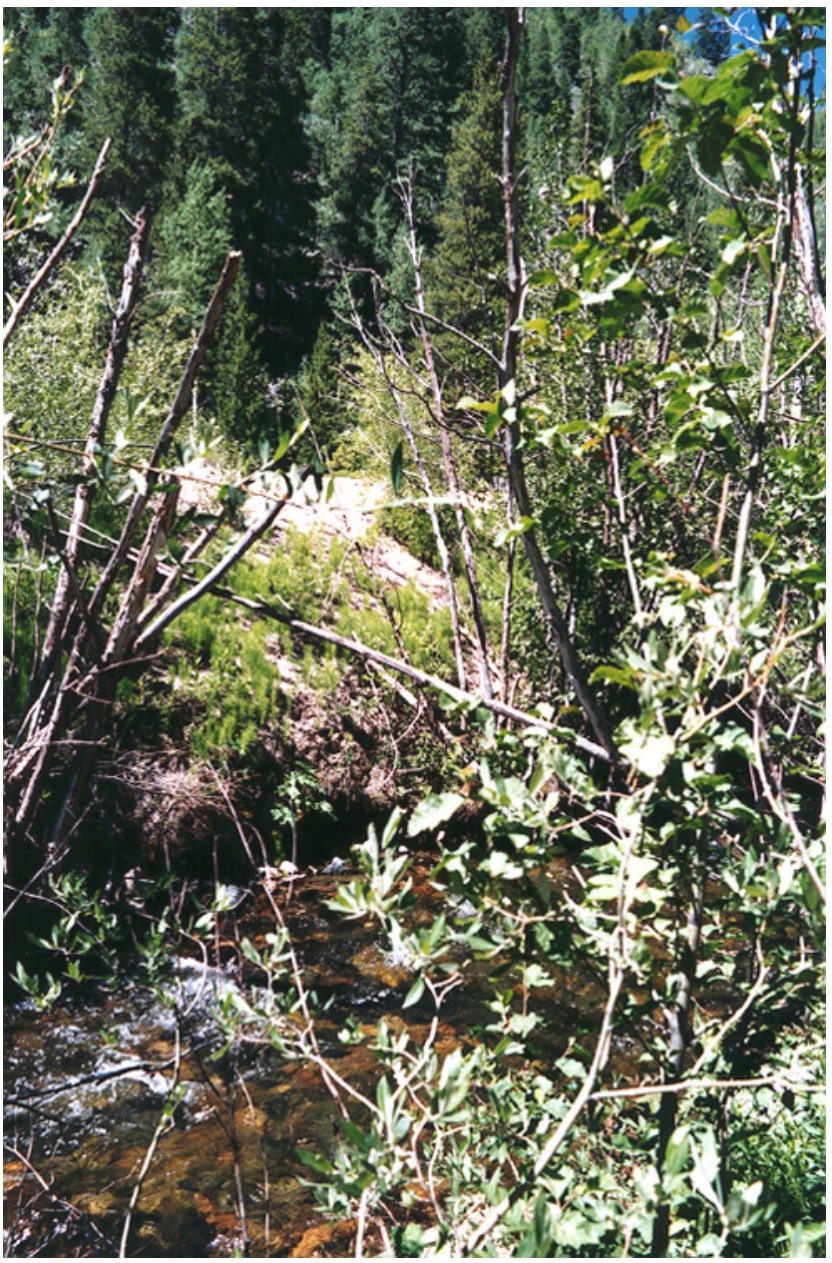

Photograph 25. Tailings from the Raymond mill (white in center of photograph) were sluiced to the east about $1 / 2 \mathrm{mi}$ and placed on a bench above Gold Creek. Water infiltrating these tailings goes into alluvium and then into the creek. 
Hydrogeochemical Investigations of Historic Mining Districts, Central Western Slope of Colorado

contained sphalerite, bornite, and pyrite, and some pockets of molybdenite (Mo sulfide) were encountered. Oxidized Mo and $\mathrm{Cu}$ minerals on upper levels were not of economic importance. Ankerite (Ca-Mg-Fe carbonate) was locally abundant. These deposits are polymetallic veins, but in some details of mineralogy and texture they differ from other polymetallic veins in the study area (perhaps a reflection of greater depth of formation here relative to those in volcanic rocks).

\section{Mineral-Environmental Conditions}

Mineralized rocks and ores.-The deposits in this district are polymetallic, but the quartz veins are not as rich in sulfide minerals as most polymetallic ores in the study area. Chemical analyses of dump and tailing samples show them to be rich in $\mathrm{Ag}, \mathrm{As}, \mathrm{Cd}, \mathrm{Pb}, \mathrm{Sb}$, and $\mathrm{Zn}$, and moderately rich in $\mathrm{Cu}$ relative to other metallic deposits in this study.

Mills and tailings.-At least three substantial mills are known, all along Gold Creek. The tailings from these mills were placed in an unusual way, as long and narrow "shoestrings" hundreds of yards east of the mills. The light-colored tailings were carried in wooden sluices (still evident) and placed by design in lowlands within the valley, and in several places the tailings are less than $50 \mathrm{ft}$ from Gold Creek and within the flood plain (photograph 25). Other mills at this time were dumping their tailings into streams, as allowed by law prior to 1935. Although production figures suggest small tonnages were produced by these mines, the tailings appear to be relatively large in volume, perhaps because they were placed in long, thin impoundments which actually have more surface area than the larger tonnages placed in thicker piles, as at Whitepine and Iron Springs.

Leach tests on four tailings samples show moderate to low acid-generating potential ( $\mathrm{pH} 3.4-5.3)$, and generally low metal release. However, two samples (from Gold Link and Carter mills) released very high concentrations of $\mathrm{Pb}, \mathrm{Se}$, and $\mathrm{Zn}$.

The tailings along Gold Creek merit additional work and evaluation, including surveys for land status. The tailings are in the flood plain and vulnerable to erosion in a major storm event. The reactivity of the tailings is not conclusively understood from the four leach tests here: some leach results suggest low potential for acid generation and metal release, but other results suggest high and potentially significant release of $\mathrm{Pb}$, $\mathrm{Zn}$, and Se. The land status also is complex: there are many patented mining claims (private land) in this district, but the tailings are sufficiently distant from the mines that all or parts could lie on the Gunnison National Forest.

Surface waters.-The surface waters in this district are clear and no iron stains are visible on cobbles. Measured $\mathrm{pH}$ values were in the range 6.9-7.9 in 1997 and 1998. Conductivities were very low $(<100 \mu \mathrm{S} / \mathrm{cm})$ in streams but reached $240 \mu \mathrm{S} / \mathrm{cm}$ in one mine drainage. Chemical analyses of four water samples showed very low metal concentrations for a mining area; the highest $\mathrm{Zn}$ concentration was $0.9 \times$ ALWS.
Other metals are elevated but well below ALWS. The CZI for samples of Gold Creek are $<0.2$ at and below the mines and tailings, suggesting low threat to aquatic life.

Summary.-Historic mining causes only minor degradation of water in this district. Contamination is limited to elevated concentrations of a few metals such as $\mathrm{Zn}$ but not in amounts that exceed ALWS. Mill tailings were placed in lowlands adjacent to Gold Creek, where they are potentially susceptible to erosion during a major storm event, such as a 100-year flood. The three tailings impoundments probably have a high risk of failure during an extreme storm. Metals such as $\mathrm{Pb}$ and $\mathrm{Zn}$ are possibly being leached from mill tailings. The effects are not apparent in the water quality of Gold Creek. Technical monitoring of contamination into shallow ground water may be called for, which would require that wells be drilled and carefully sampled.

References.-Crawford and Worcester (1916); DeWitt and others (1985).

\section{Quartz Creek District}

Location.-Gunnison Co., about 20 mi east of Gunnison (fig. 15). Climate is moderate to severe, with about $25-40$ inches of precipitation per year.

Principal commodities.—Silver, lead, gold; molybdenum and tungsten; graphite.

Mining history.-The gold-silver mines were first located in the 1890's. The Mo-W mines near Cumberland Pass were located somewhat later and mined chiefly during World War I. There was very minor production in the Depression years. None of the mines have been in operation since World War II, but a significant exploration program for molybdenum in the 1970's included the drilling of many core holes near Cumberland Pass. There has been no mining or exploration in recent years.

Production and disturbance.-Total less than $\$ 500,000$. Small disturbance from mining.

Geology.-The western part of the district above Pitkin is really a continuation of the adjacent Gold Brick district. In this area, quartz veins with base-metal sulfides and precious metals occur in Precambrian metamorphic rocks, and minor replacement bodies occur in Paleozoic carbonate rocks. The deposits in the northeastern part, near Cumberland Pass, are related to a Tertiary intrusive complex and include a type of vein deposit that is rich in Mo-W. A large pyritic alteration halo adjacent to the Tertiary diorite intrusion is visible from Cumberland Pass (photograph 28).

\section{Mineral-Environmental Conditions}

Mineralized rocks and ores.-The ores in this district differ from most others on the CWS in the major metals of economic interest. The ores near Cumberland Pass (Bon Ton mine) are rich in Mo and the associated trace metals Bi 


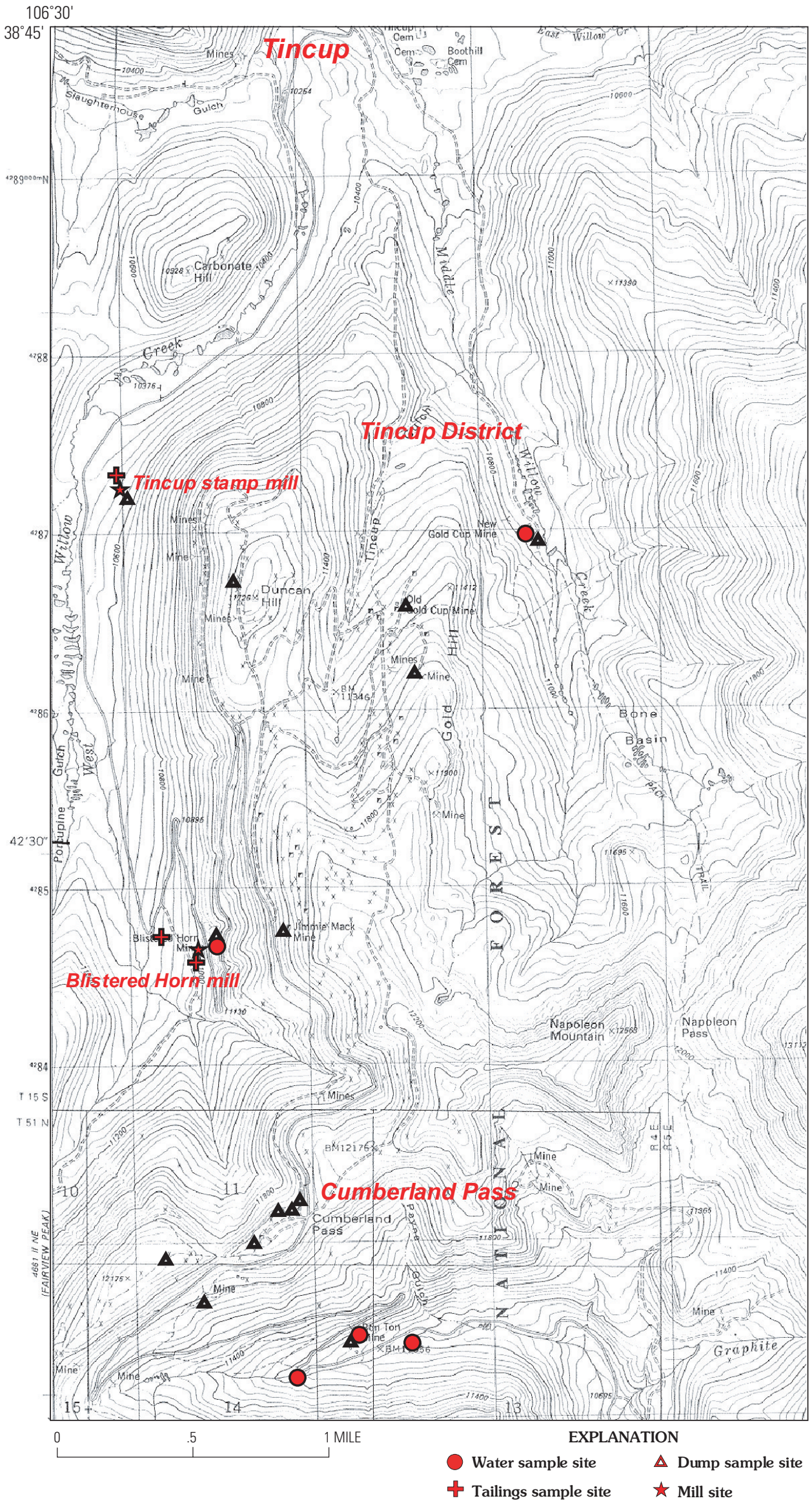

Figure 15. Features of the Tincup and northern part of the Quartz Creek mining districts, showing sample localities. 


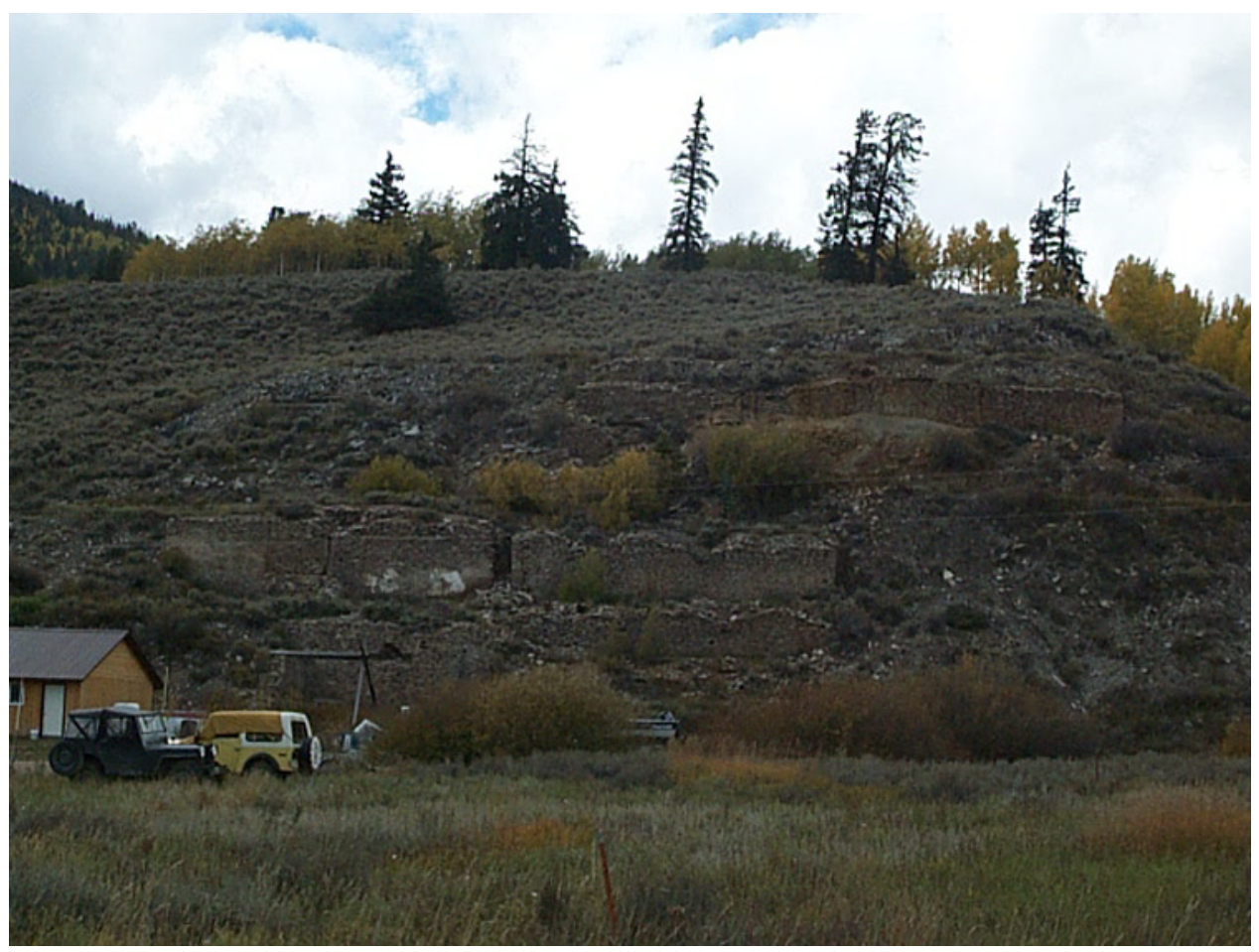

Photograph 26. The Pitkin mill, in the village of Pitkin, was active before 1900 . Stone foundations are suggestive of an early date as concrete foundations were used after about 1900. Tailings may be in the wetlands to the east of the mill, or washed down Quartz Creek.

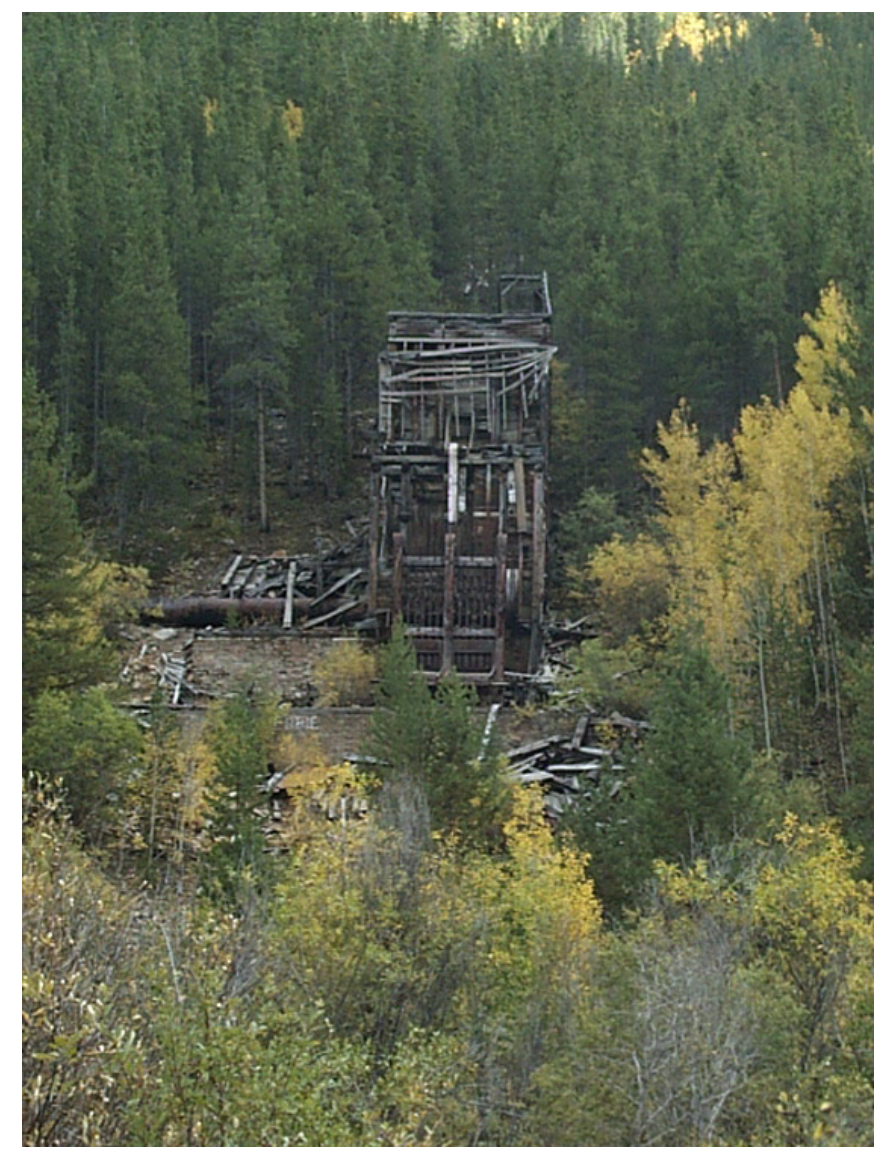

Photograph 27. The Roosevelt mill, about 2 mi south of Pitkin, is remarkably intact; the vertical features are stamps that crushed and ground ore. No tailings are evident. The tunnel and mill venture reportedly failed in its attempt to reach ore to the east and mined through shafts at the Camp Bird and nearby mines. 


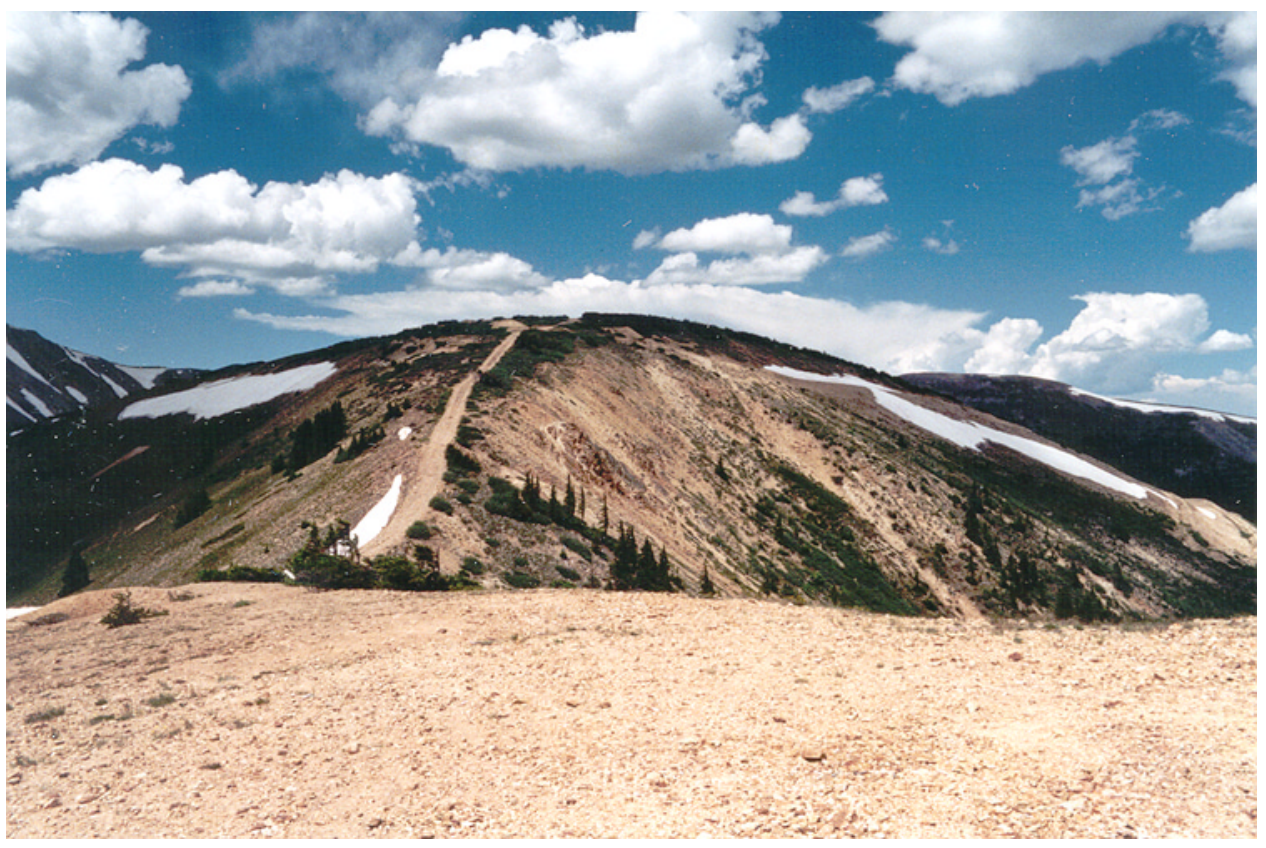

Photograph 28. Altered rocks at Cumberland Pass formed in association with a diorite intrusion that also created several molybdenum-tungsten vein deposits. These bleached rocks with iron oxides on fractures formed from disseminated pyrite and are a guide to prospectors. The area in this photograph was explored by several diamond drill holes during a search for molybdenum in the late 1970's.

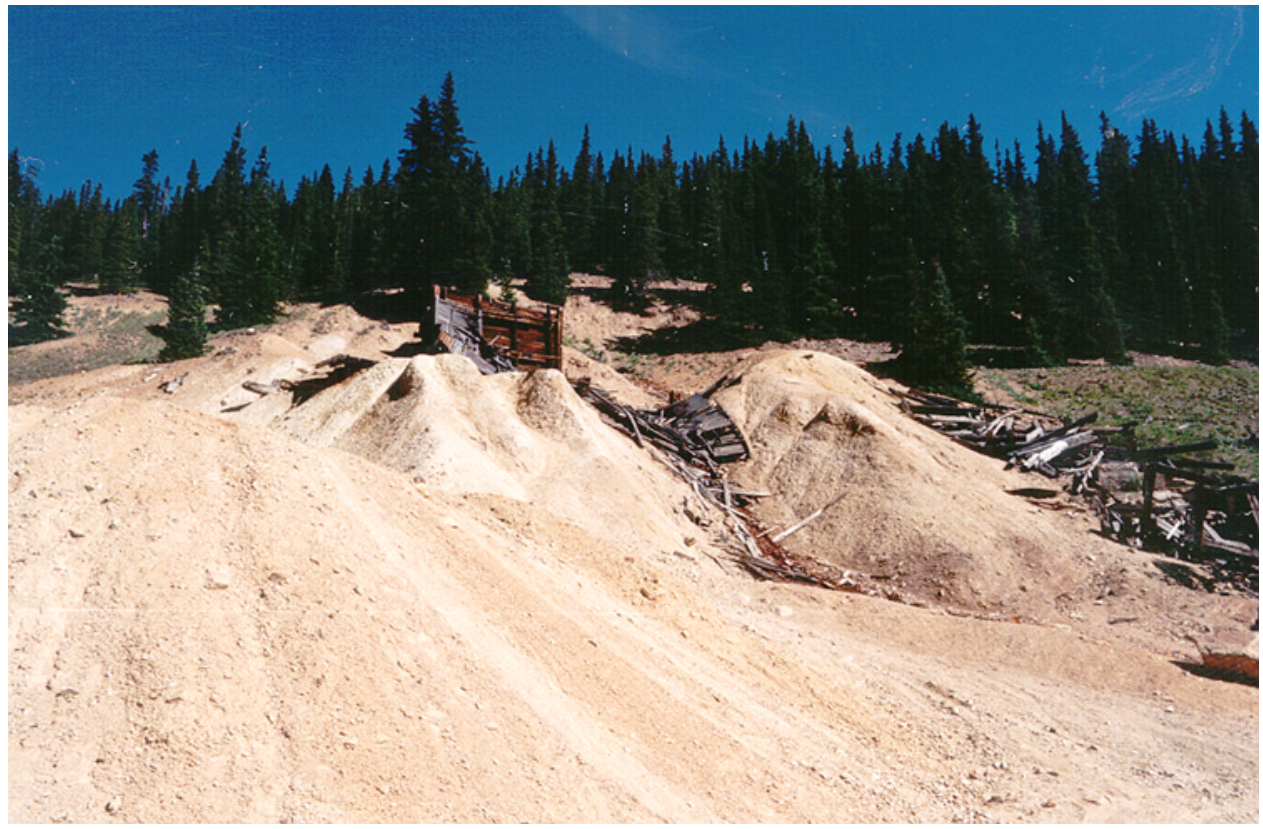

Photograph 29. The Bon Ton mine was a modest producer of molybdenum during World War I. Drainage from the tunnel (right center) flows over the waste rocks on the dump. 


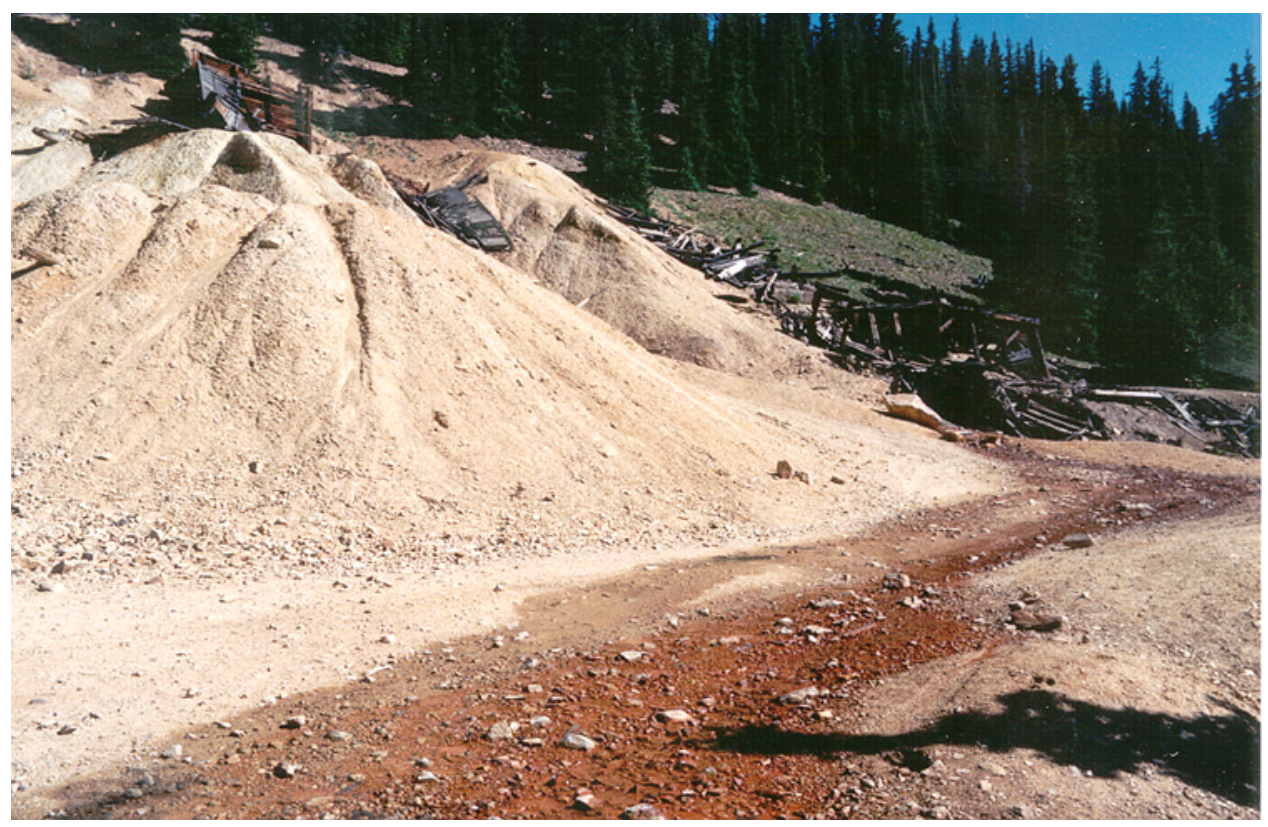

Photograph 30. Mine drainage from the Bon Ton mine is acidic and makes these tell-tale red colors. Reactions of mine water with sulfidic waste rocks on the dumps further degrades the water quality.

and $\mathrm{W}$ and have moderate concentrations of $\mathrm{Cu}, \mathrm{Pb}$, and $\mathrm{Zn}$. Concentrations of $\mathrm{Ag}$ are relatively high, and As concentrations are variable (low to moderate).

Mills and tailings.-The status of mills and the condition of tailings in this district is unclear. A mill was reported by Hill (1909) to be 1 mi northeast of Pitkin, but was not located in this study. At least two large mills are visible today, one on the southwest edge of the town of Pitkin (photograph 26), and another 2 mi to the south on Quartz Creek at Roosevelt (photograph 27). The latter is described as part of a speculative venture to produce ore from a long tunnel to the east and appears to have produced very little ore; no tailings are evident in the willows along Quartz Creek. The mill at Pitkin was a significant producer, but no tailings could be identified in the adjoining area of private property. Tailings from the Pitkin mill are probably in the wetlands southeast of the town, less than a mile from the State fish hatchery.

Surface waters. - Water in this district is locally contaminated at small mines. A modest flow of water from the Bon Ton mine (photograph 29), just south of Cumberland Pass, may be typical: it flows at about $20 \mathrm{gpm}$, has a pH of 3.7 and conductivity of $210 \mu \mathrm{S} / \mathrm{cm}$, compared to $\mathrm{pH} 6.7$ and conductivity of 40 in Quartz Creek below the mines. The acidic mine drainage contains high concentrations of many metals relative to ALWS ( $\mathrm{Al} 6 \times, \mathrm{Cd} 8 \times, \mathrm{Cu} 230 \times, \mathrm{Fe} 6 \times$, and $\mathrm{Zn} 8 \times$ ). The Bon Ton, which produced a small amount of molybdenum, is one of the few sources of Mo-rich waters in this study with $29 \mathrm{ppb}$ Mo.

A sample was taken from Quartz Creek to determine the effects of the large alteration area near Cumberland Pass and the several mines near Bon Ton: the $\mathrm{pH}$ of 6.7 and chemical analyses indicate that water has elevated metal concentrations and $\mathrm{Cu}$ is $6 \times \mathrm{ALWS}$. The CZI values for the Bon Ton mine drainage waters are high (30.5 and 29.9). The CZI for Quartz Creek is elevated (1.7) and may represent a threat to aquatic life. Because the waters of Quartz Creek looked clear and made no iron deposits, no samples were collected farther south of the mining area at Cumberland Pass. Additional sampling of Quartz Creek would establish how far the metal contamination extends.

Summary.-The effects of historic mining are highly localized in this district and are not evident in the water quality of nearby streams. The large pyritic alteration zone near Cumberland Pass does not degrade stream-water chemistry.

References.-Crawford and Worcester (1916); Goddard (1936); Dings and Robinson (1957).

\section{Ruby District (Irwin)}

Location.-Gunnison Co; 10 mi west of Crested Butte (fig. 16). The climate is relatively severe, with thick snowpack and about 25-40 inches of precipitation per year.

Principal commodities.-Silver; lesser lead, zinc, copper, gold.

Mining history.-Silver was discovered in 1872, and Irwin was soon developed as a town. Railroad service was completed from Gunnison to Crested Butte in 1881. There was modest production in the 1880's and 1890's, but little other production until the 1940's-50's when the Keystone and 


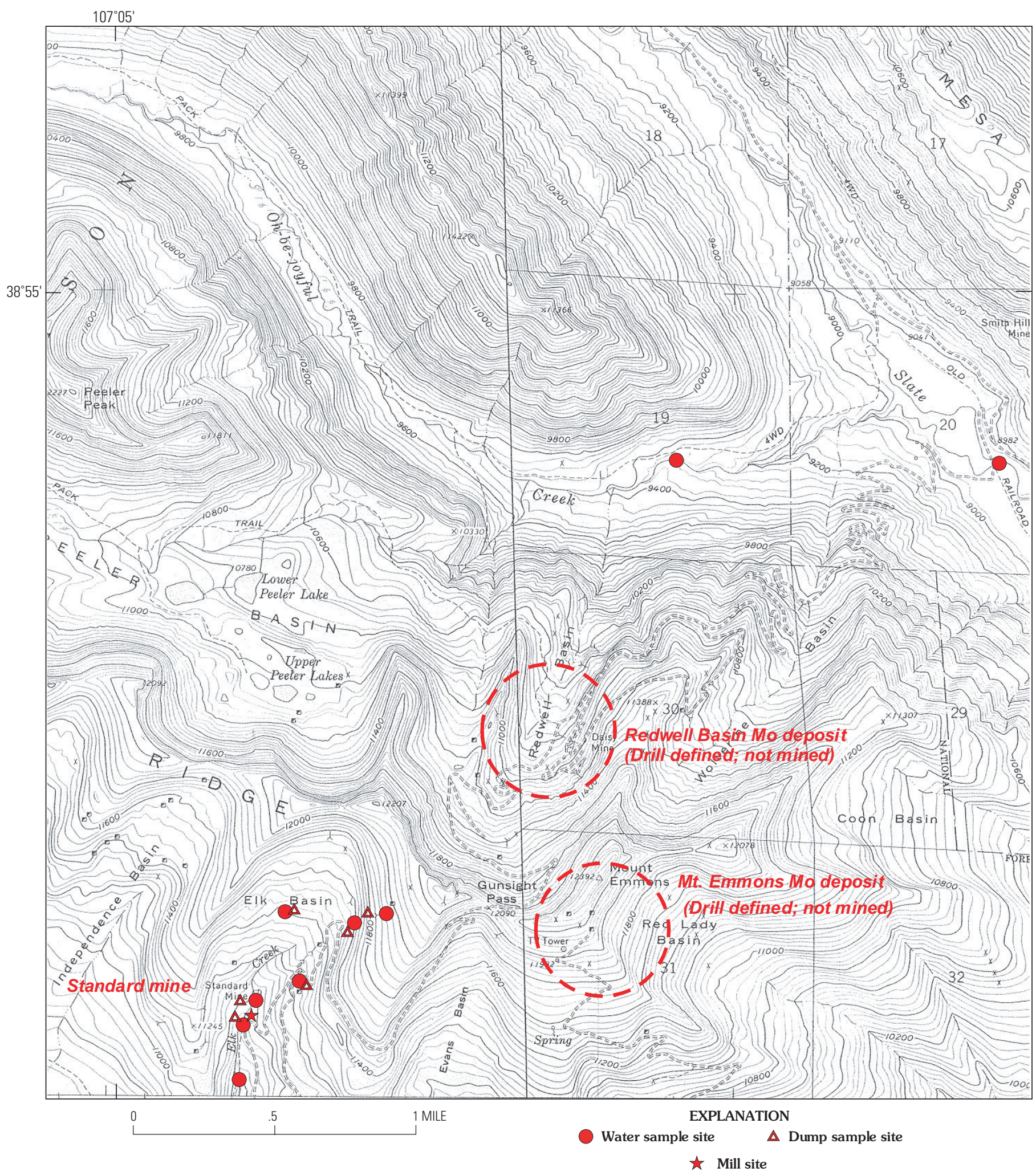

Figure 16. Features of the northeast part of the Ruby mining district, showing sample localities. Approximate loctions of two known molybdenum deposits are shown. 
Hydrogeochemical Investigations of Historic Mining Districts, Central Western Slope of Colorado

Standard mines were developed. Exploration for molybdenum in the 1970's defined at least two substantial deposits beneath Mt. Emmons (Thomas and Galey, 1982), but there has been no mining of these deposits.

Production and disturbance.-Production, chiefly before 1900 and from 1955-69, was about 24,000 oz gold, 5.2 million oz silver, 6.6 million $\mathrm{lb}$ copper, 31 million $\mathrm{lb}$ lead, and 55 million lb zinc (Ellis, 1983). Large reserves and resources of molybdenum have been defined by drilling (more than 200 millions tons of ore). The underground mines created small to moderate disturbance. Dumps and tailings at the Keystone mine (polymetallic vein deposit) were effectively reclaimed in recent years.

Geology.-Sandstones and shales of Cretaceous and Tertiary age dominate the geology of this district, but intrusive sills, dikes, and stocks of Tertiary felsic igneous rock are keys to formation of the metal deposits. Moderate to intense pyritic alteration adjacent to the intrusions creates bright colors where weathered; some of the altered zones are given apt names such as Red Lady basin. Coal beds occur within the Tertiary sequence, and some have been mined north of the district.

\section{Mineral-Environmental Conditions}

Part of this district in Elk basin (Standard mine and nearby mines) was investigated in moderate detail (2 days on site) because this is a priority concern of the USFS (D. Gusey, written commun., 1998). The rest of the district, including the east side (Slate Creek drainage) were investigated very briefly. Wanty and others of the USGS have more detailed hydrogeochemical studies underway in Redwell basin and other areas adjacent to Mount Emmons.

Mineralized rocks and ores.-The polymetallic ores of the Standard mine area were the only ones studied. Samples from mine dumps are very high in $\mathrm{Pb}$, high in $\mathrm{Ag}, \mathrm{Cd}, \mathrm{Cu}$, and $\mathrm{Zn}$ and moderately high in As and Bi relative to other polymetallic ores on the CWS. Concentrations of Se are notably high. Leach tests on five samples yield pH's in the range 2.9 to 3.3, which is more acidic than most in this study. The leachates contained very high concentrations of $\mathrm{Pb}, \mathrm{Zn}$, and $\mathrm{Cd}$ and high concentrations of $\mathrm{Cu}, \mathrm{Fe}, \mathrm{Mn}$, and $\mathrm{Se}$.

Mills and tailings.-There were three or more mills in this district, but only two are well known. The Keystone mill was a major operation through the 1950's and created large tailings piles that are shown on the USGS topographic map. A former source of contaminants, the tailings were reclaimed by AMAX (Cyprus AMAX) Mining Co. in the mid-1990's, including placement of a plastic liner on the downslope side to contain percolating waters. Any contaminated water flowing from the mine and tailings is now processed through the watertreatment plant.

The Standard mine and mill operated in the 1950's and appears to have had only small production. The tailings impoundment is intact and measures about 1 acre in size; the pond continues to receive runoff, and the waters seep through the tailings and flow a few feet into the nearby creek (photograph 34).

The position of the tailings "pond" at the Standard mine, above and close to Elk Creek, makes it vulnerable to runoff and erosion during an extreme storm (photograph 35). My initial evaluation suggests a high risk of failure during an extreme storm. The tailings are saturated with water, the pond continues to collect water from overland flows, and the bank slopes steeply down to Elk Creek. These conditions may be conducive to failure by slippage (as in a landslide) or liquefaction during a major storm. Erosion and undercutting are also possible.

Surface waters.-Water quality in this district is variable and not reliably predicted from geology. Some basins of previously reported impacted water quality are, in my brief experience, quite good, whereas the area of small mines in Elk basin, expected to be mitigated by sedimentary rocks, has quality worse than expected. Waters in basins underlain by carbonatebearing sedimentary rocks, and with few or no mines, has very good water quality at three sites on West Elk, Slate, and OhBe-Joyful Creeks. At these sites, $\mathrm{pH}$ values range from 5.8 to 7.3 , conductivities are 20 to $40 \mu \mathrm{S} / \mathrm{cm}$, and zinc is 3 to 90 $\mathrm{ppb}$. The highest zinc content, $90 \mathrm{ppb}$ in Slate Creek, may reflect coal mining or associated Cretaceous black shales. The CZI values for these three sites range from 0.3 to 0.02 , which suggests few problems for aquatic life.

Water in Elk basin is contaminated by mine waters and mine waste from several polymetallic mines. The Standard mine is the most obvious source, but there are others higher in the basin that also contribute acid and base metals (photograph 31). The distribution of samples and $\mathrm{pH}$ values is shown on figure 17. The sulfide-rich mine workings and waste dumps generate acidic waters, with $\mathrm{pH}$ values near 3 , and high to very high concentrations of $\mathrm{Al}, \mathrm{Cd}, \mathrm{Cu}, \mathrm{Fe}, \mathrm{Pb}$, and $\mathrm{Zn}$. The $\mathrm{Pb}$ concentrations are among the highest in this study, and those of $\mathrm{Cu}$ and $\mathrm{Zn}$ are far in excess of ALWS. Mine water flowing from the partly collapsed Standard mine adit is similar to that from at least six other mines in the basin above. Significant, in my opinion, are the overland flows during runoff and storm events, which are nearly as acidic ( $\mathrm{pH} 3.3$ ), and that flow onto the Standard mine-waste dumps and tailings (photograph 33). There are many seeps and flows from the mine and waste that enter Elk Creek within $100 \mathrm{ft}$ or less, before natural reactions can mitigate water quality. CZI values range from 104 to 27 for these waters, which indicate problems for aquatic life.

Water quality in Elk Creek is degraded by waters seeping from the Standard mill tailings pond. The $\mathrm{pH}$ of water in the pond is 3.1 and water seeping from the pond has $\mathrm{pH}$ values of 2.9-3.0; conductivity of pond water is high at $450 \mu \mathrm{S} / \mathrm{cm}$ and is even higher $(775 \mu \mathrm{S} / \mathrm{cm})$ in the seeps. Chemical analyses show that the water seeping into Elk Creek has extremely high $\mathrm{Zn}(>200 \times$ ALWS $), \mathrm{Cu}(>100 \times)$, and $\mathrm{Pb}(>15 \times)$. Concentrations of $\mathrm{Fe}, \mathrm{Mn}, \mathrm{Se}$, and $\mathrm{Cd}$ also are very high. Pore water collected from the slushy tailings has a $\mathrm{pH}$ of 2.5 , conductivity of $1,250 \mu \mathrm{S} / \mathrm{cm}$, and even higher metal concentrations than the seeps $(\mathrm{Pb}$ is $110 \times$ ALWS). 


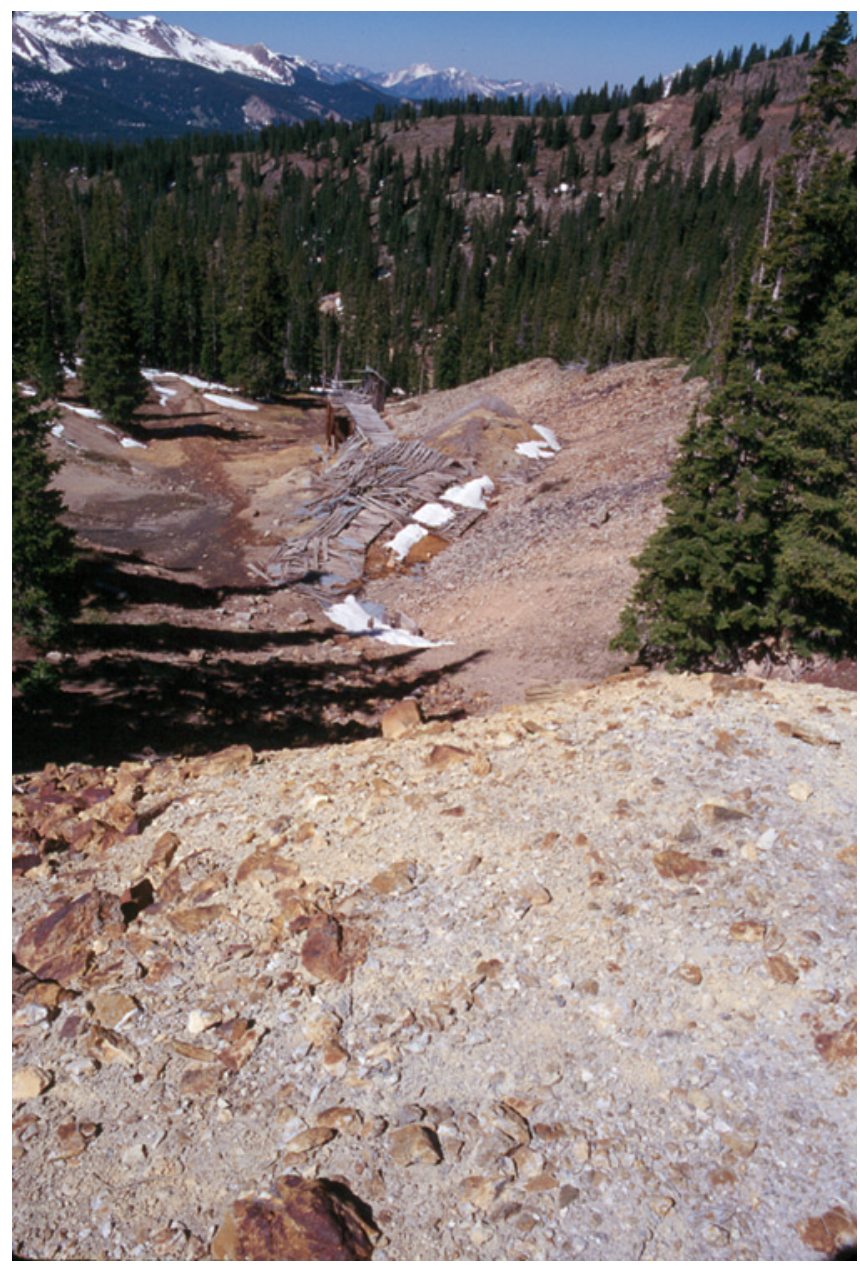

Photograph 31. An old mine and waste dump in upper Elk basin creates acidic mine drainage. Drainage such as this flows down onto the Standard mine below.

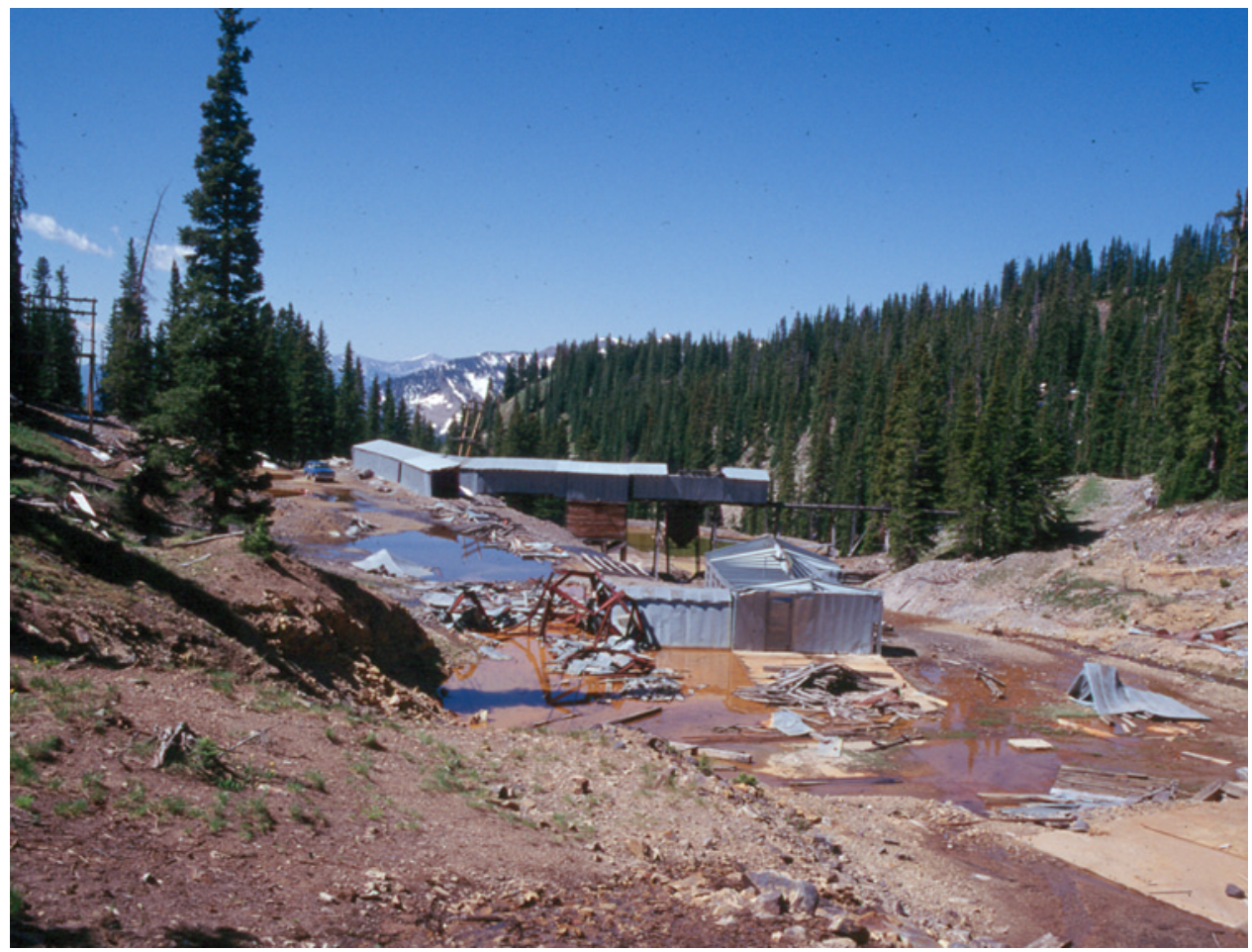

Photograph 32. The Standard mine, from the 1950's, is relatively intact, and no reclamation work has been done. Overland flow of water adds to drainage from the tunnel to flood the waste dumps. 


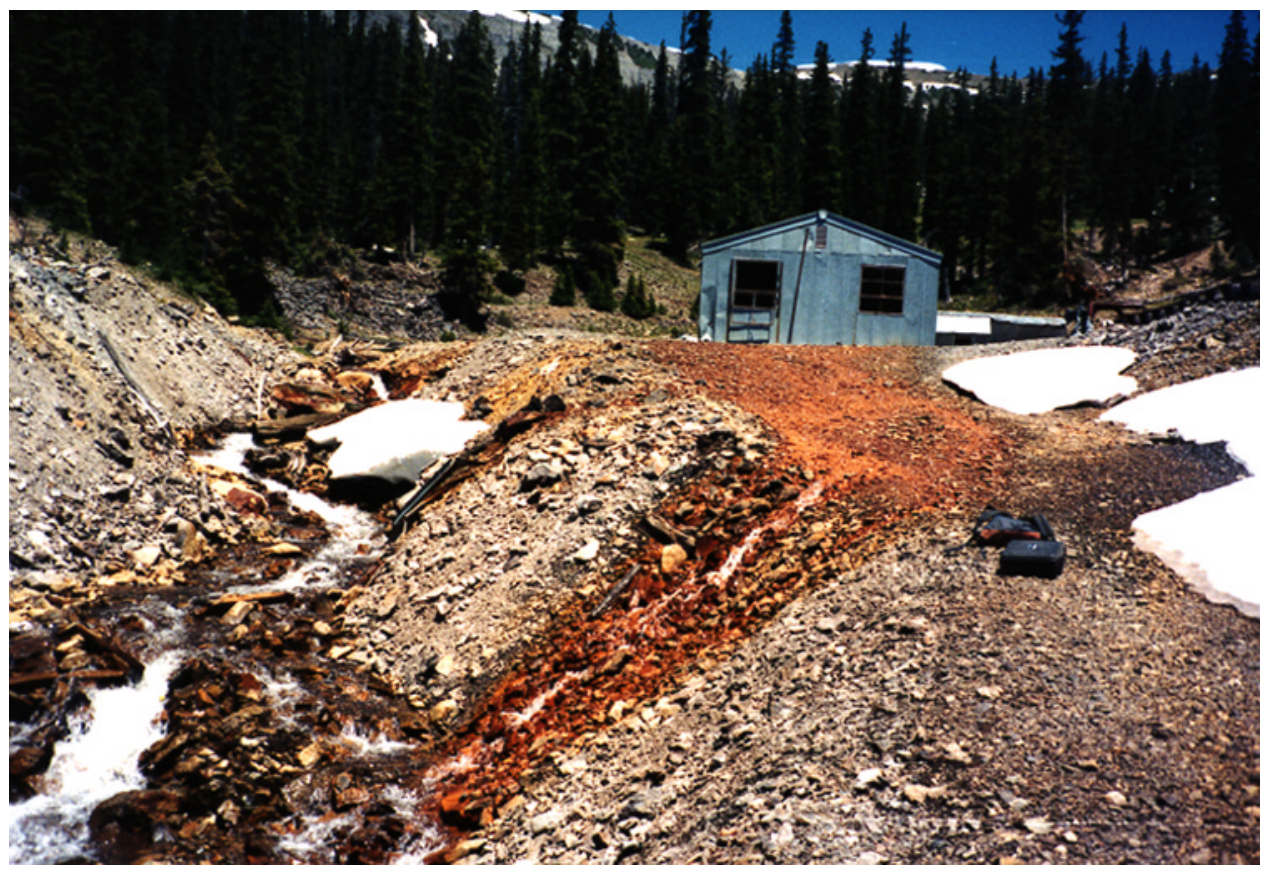

Photograph 33. Drainage from the Standard mine tunnel reacts with waste dump rocks, gaining more metals and acid, then runs into Elk Creek. Red coatings of iron minerals form at some times of the year if $\mathrm{pH}$ rises above 4 , but when $\mathrm{pH}$ is below about 3.5 the water is clear and forms no precipitates.

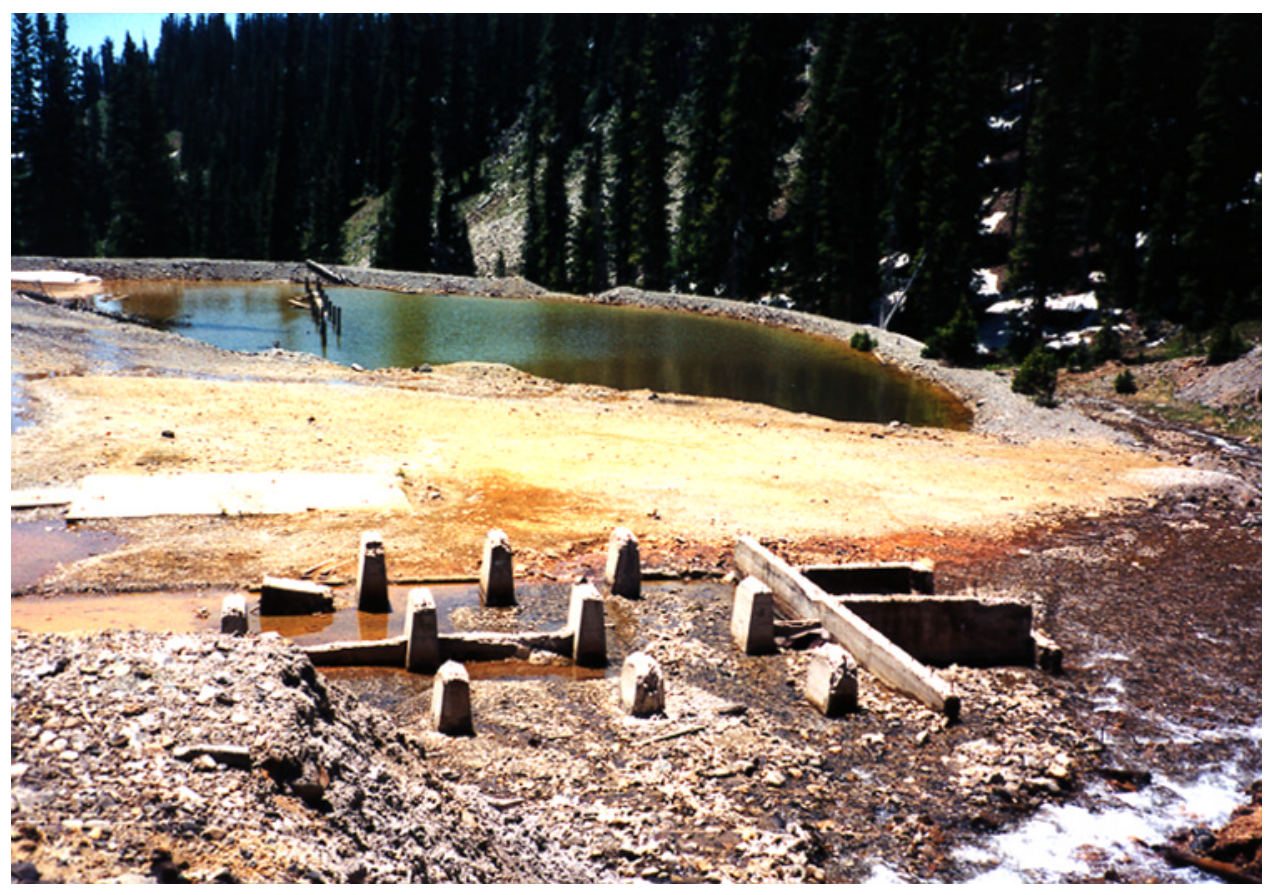

Photograph 34. Only the foundation remains from the Standard mine mill, and the tailings pond is intact to the south. Elk Creek is on the right side of this view. 


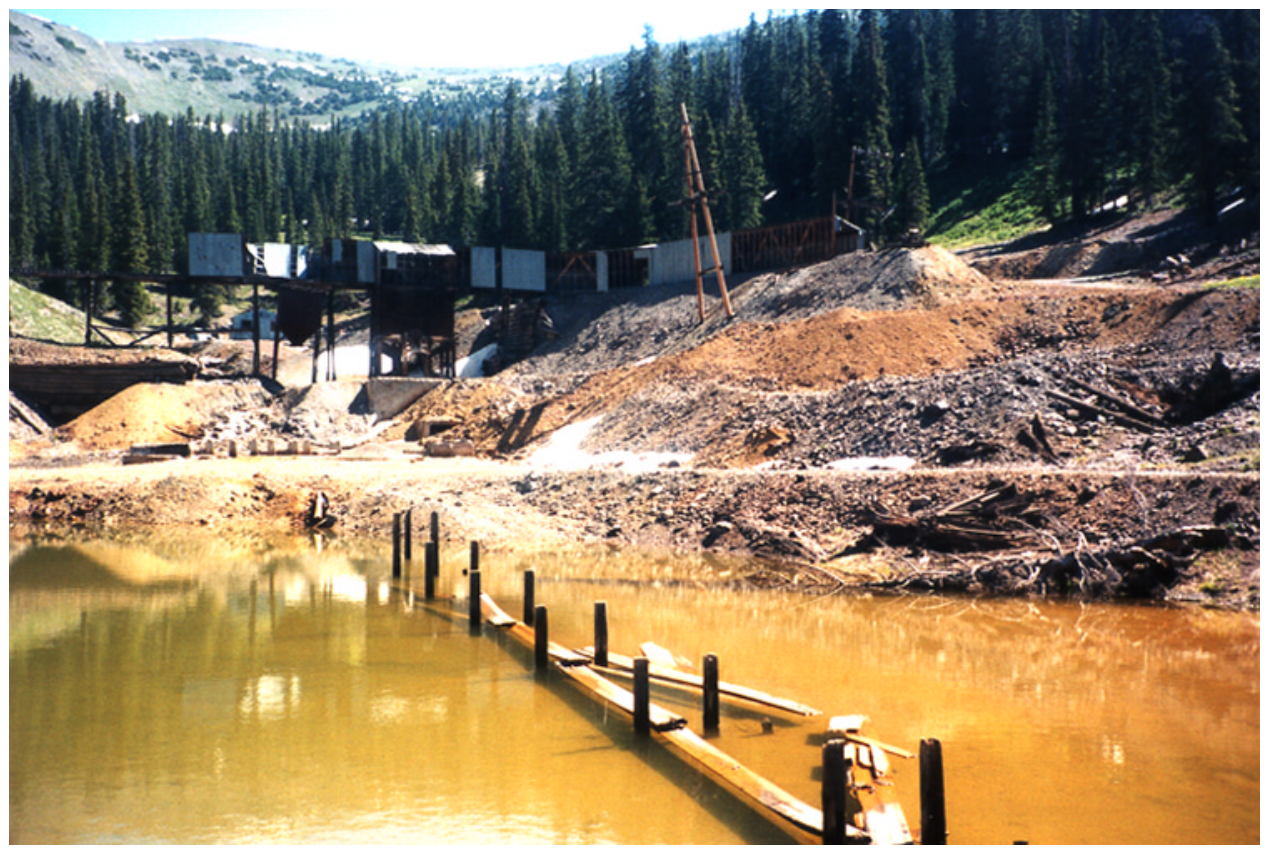

Photograph 35. The Standard mine tailings pond collects runoff from waste dumps, making the water composition worse than it might be.

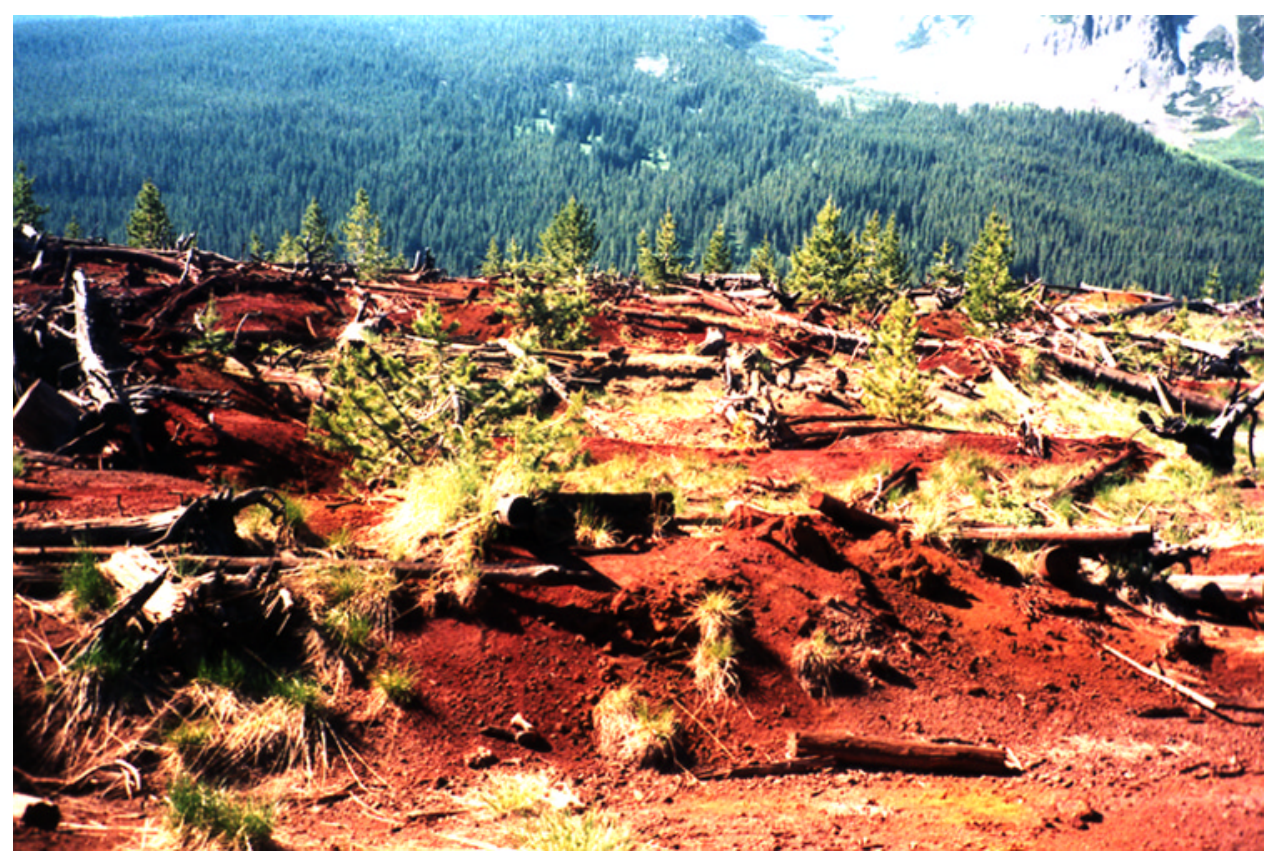

Photograph 36. The red material is a variety of ferricrete; the iron was deposited from acidic drainage from the Keystone vein. This is one of the largest volumes of ferricrete on the Western Slope. This material could have formed either prior to mining, from natural springs, or by flow along mine workings. The trees were killed by a forest fire. 


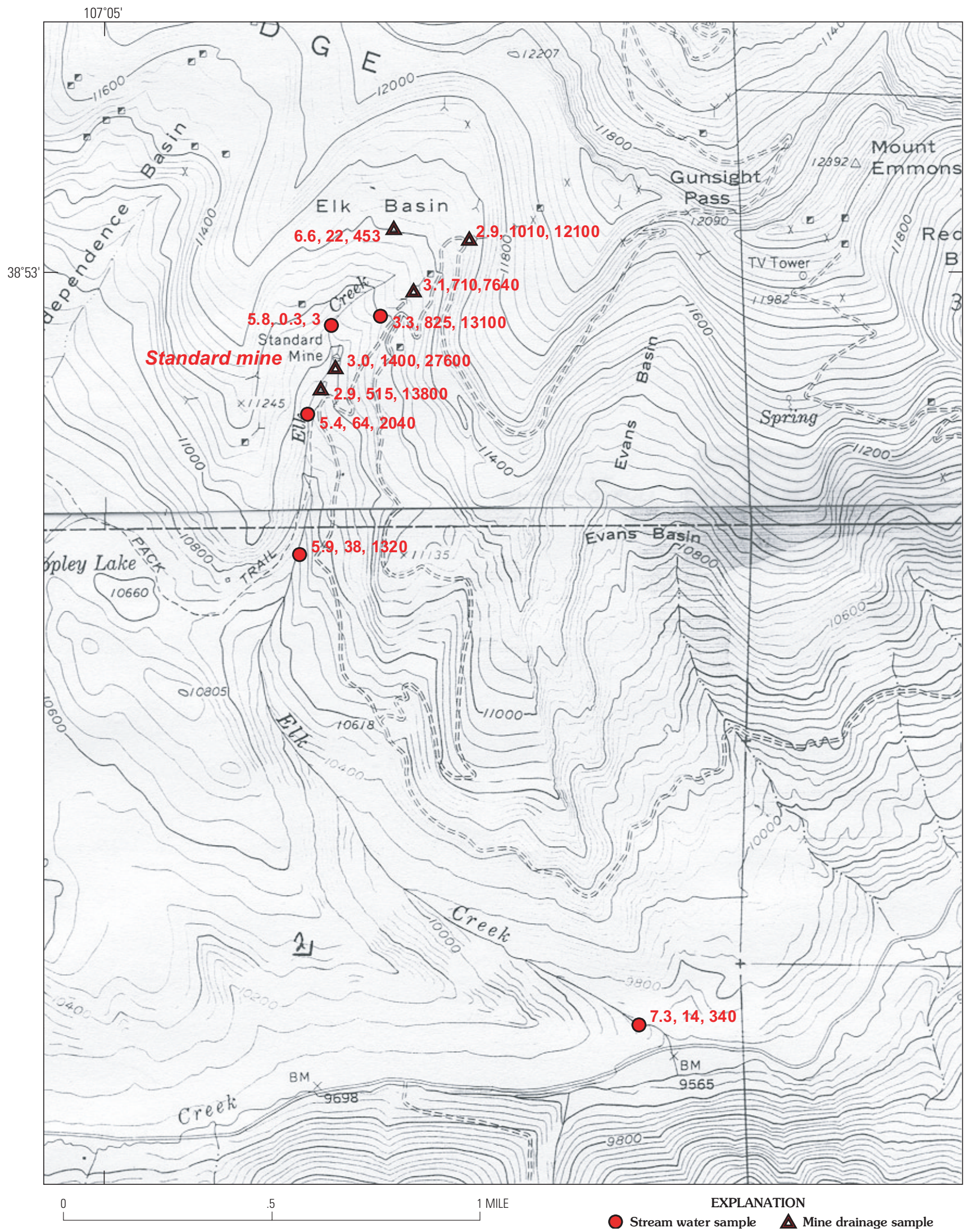

Figure 17. Sample sites and water compositions in the Elk Creek watershed near the Standard mine. Explanation of composition (2.9, 1010, 12400): pH 2.9; 1010, Cu in ppb; 12400, Zn in ppb. 
Water compositions were examined at several sites downstream (south) of the Standard mine. The $\mathrm{pH}$ of Elk Creek rises progressively (fig. 17) from 5.4 to 7.3 , and zinc, lead, and copper concentrations decrease proportionately. However, zinc is still high (10× ALWS) $1.9 \mathrm{mi}$ to the south, near the road along Coal Creek. The expectation was that the $\mathrm{Zn}$, and also $\mathrm{Pb}$ and $\mathrm{Cu}$, concentrations would decrease in a shorter distance by reactions with minerals such as calcite in sedimentary rocks and by dilution. The CZI values decrease from 6.7 to 1.7 below the mines, which shows improved water quality but persistent $\mathrm{Zn}$ and $\mathrm{Cu}$ that may be a threat to aquatic life. Natural mitigation is not as effective in these siliciclastic rocks as in carbonate and volcanic rocks elsewhere on the CWS. The mobility of base metals in Elk Creek basin is surprisingly high and underscores the need for reclamation that will minimize contact of surface waters with polymetallic-sulfide waste.

Twenty years ago, drainage from the Keystone mine tunnel and from the Keystone mill tailings were poorly controlled and contributed significant contamination to Coal Creek, the drinking-water supply for Crested Butte. Reclamation activities by Cyprus AMAX Mining Co. have made great improvements to the quality of Coal Creek. A treatment plant, the largest treating mine-related waters on the Western Slope, removes metals by a series of chemical reactions and processes, driven by the addition of lime to raise the $\mathrm{pH}$ to about 10. As of 1998, Coal Creek appears to meet water-quality standards because it is no longer on the 303(d) list of CWQCD.

Summary.-Recent reclamation at Keystone greatly improved drainage and reduced reactions in mine dumps and mill tailings. The $\$ 40,000,000$ water-treatment plant improves water quality to regulatory standards. Together, these actions have produced dramatic improvements in the quality of Coal Creek, the drinking-water supply for Crested Butte. Reclamation is needed at the Standard mine, chiefly to reduce surfacewater interaction with sulfidic mine waste and mill tailings. In particular, moderately acidic waters from the East Fork of Elk Creek need to be redirected away from Standard mine-waste materials. Dilution and neutralization by sedimentary rocks mitigates acidic waters and lowers metal concentrations, but $\mathrm{Zn}-\mathrm{Cd}$ remain higher than desired for aquatic life or drinking water. Reclamation is needed at the mine and mill site to reduce the concentrations of $\mathrm{Zn}-\mathrm{Cd}-\mathrm{Cu}$ leaving the mine area.

The location of the tailings impoundment at the Standard mine makes it vulnerable to failure by erosion, slippage, or liquefaction during an extreme storm. This tailings impoundment ranks among the top five sites for risk of failure during an extreme storm and should be investigated further for physical stability and possible methods of stabilization.

References.-Thomas and Galley (1982); Ellis (1983).

\section{Tincup District}

Location.-Gunnison Co., $30 \mathrm{mi}$ NE. of Gunnison (fig. 15). The climate is relatively severe with about $25-40$ inches of precipitation per year.
Principal commodities.-Silver; minor gold (placer), lead, zinc.

Mining history.-Gold was discovered in the Taylor River in 1861, but significant mining did not occur until 1880 when the lode deposits were developed. The most profitable mining in the district was from oxidized silver-lead deposits in replacement and vein deposits in limestone. The largest mine, the Gold Cup, is credited with more that $\$ 1,000,000$ worth of production. A small amount of iron was mined at the Cumberland mine near the pass, and relatively small amounts of placer gold have been mined from Willow Creek and Tincup Gulch. Production from 1901-35 was small, slightly more than a thousand tons of ore, and increased slightly during World War II. The mines have not been active for many years, and production has been minor since World War II.

Production and disturbance.-Total production was somewhat in excess of $\$ 3$ million, but the waste dumps and limited amount of surface disturbance caused by the underground mines do not suggest this magnitude. In the 1880's, there were two small smelters, and later there were at least two mills on the flank of Gold Mountain that utilized trams (photograph 38).

Geology.-The oldest rocks in this district are Precambrian gneiss, which are overlain unconformably by Paleozoic sedimentary rocks, chiefly limestone and dolomite. These rocks were intruded by Tertiary diorite porphyry (intermediate composition). Alteration produced by the intrusive body is most evident at Cumberland Pass, but effects in the carbonate rocks are subtle. Skarn-type deposits of magnetite and other minerals are clearly associated with the diorite intrusion, and the polymetallic vein and replacement deposits of silver and lead probably are also related to Tertiary intrusions. To the south, extending into the adjoining Quartz Creek district, prospectors found quartz veins containing molybdenite that are relatively small in size compared to the important Climax-type "moly" deposits near Leadville and Crested Butte that were discovered later.

\section{Mineral-Environmental Conditions}

Mineralized rocks and ores.-The polymetallic deposits of this district appear to be smaller than the value of production would imply, but the small volume of waste at the surface is characteristic of pre-1900 bonanza ores that were hand-sorted underground. The ores are unimpressive to the eye, probably because most of the ore minerals are oxidized varieties. Chemical analyses of nine samples from dumps of replacement deposits are very high in $\mathrm{Ag}, \mathrm{Pb}$, and $\mathrm{Zn}$ and high in $\mathrm{As}, \mathrm{Bi}, \mathrm{Cu}, \mathrm{Mo}$, and $\mathrm{Sb}$ (no results for $\mathrm{Se}$ and $\mathrm{Te}$ ). Analyses of three samples from skarn prospects showed relatively low base-metal concentrations, but high Fe. Chemical analyses of two tailings samples are similar to those for the dumps, but show lower base-metal concentrations. The old stamp mills were not very efficient: two tailings samples contain 110 and $390 \mathrm{ppm} \mathrm{Ag}$, and 1.2 and $0.2 \mathrm{ppm}$ 


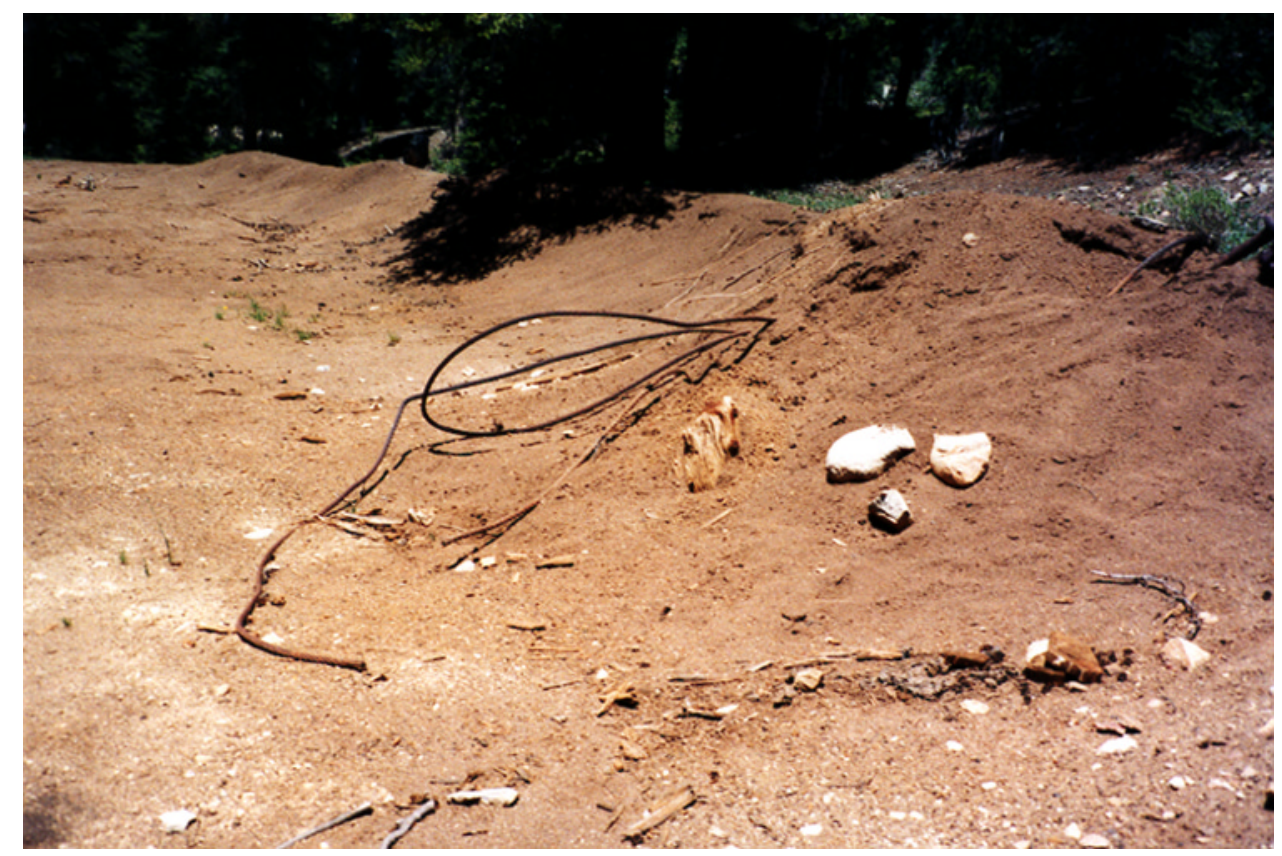

Photograph 37. Tailings from the mill south of Tincup have been dug into, possibly to reprocess the tailings. The stamp mill created sand that is coarser than beach sand. The cable (center of photograph) is from the tram that ran up Gold Mountain to the southeast. This is one of the rare tailings piles that is not near a stream.

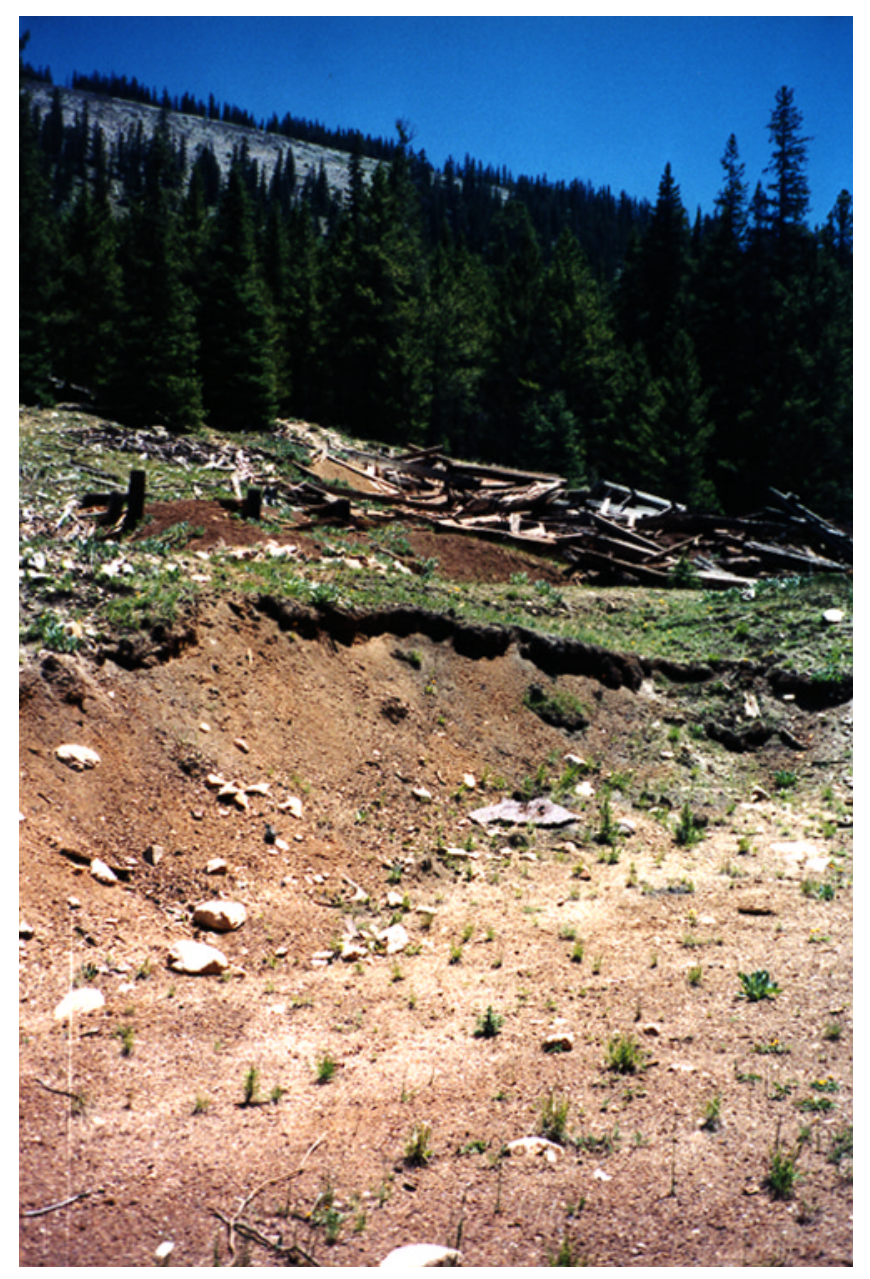

Photograph 38. The stamp mill south of Tincup is now a pile of beams. A tram cable runs up the hill to the right, and tailings remain nearby. This mill probably operated before 1910. 


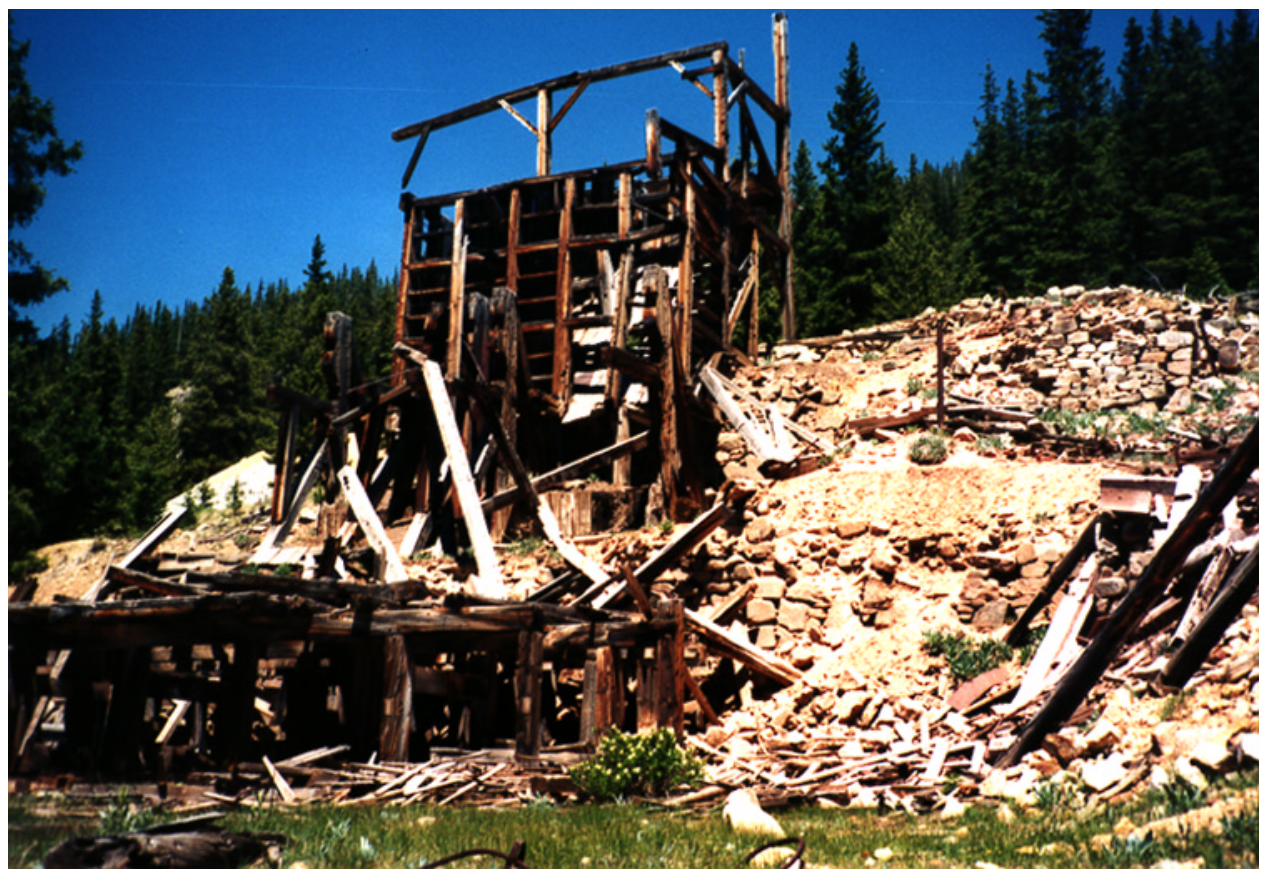

Photograph 39. The mill at the Blistered Horn silver mine is in relatively good condition. Several tons of tailings remain at the site, but more were probably generated and lost down the slope.

$\mathrm{Au}$, respectively-levels that are considered high grade ore in modern mines elsewhere.

Leach tests on three dump and two tailings samples showed generally low reactivity, suggested by low conductivities, $\mathrm{pH}$ 's in range 4.3 to 6.2 , and low metal concentrations. One sample yielded a $\mathrm{pH}$ of 4.3 and very high $\mathrm{Pb}$ and high $\mathrm{Fe}$ and $\mathrm{Zn}$ concentrations. The high $\mathrm{Pb}$ may relate to the presence of anglesite (lead sulfate), which is more soluble than galena (lead sulfide). The leach results indicate low acid-generating potential for these mine wastes.

Mills and tailings. - The two mills on Gold Hill south of Tincup are small and produced only a few thousand tons of tailings. One small mill was below the Blistered Horn mine, and the structure is fairly well preserved (photograph 39). Tailings from the Blistered Horn mill are scant near the foundation, and some materials that appear to be tailings cover the hillside several hundred feet west of the mill (but the path of the washout could not be traced back to the mill). Another mill is located at the base of Gold Hill, but not near the creek (which is unusual), and was served by a tram. The collapsed wooden structure contains many signs that it was a mill, and coarse-grained tailings below it are typical of 1880's stamp mills. The tailings have been partially excavated, possibly for reprocessing (photograph 37).

Leach tests on two tailings samples show low to very low acid-generating potential, and one sample (Blistered Horn) released extremely high $\mathrm{Pb}$, and high $\mathrm{Cd}, \mathrm{Cu}$, and $\mathrm{Zn}$ concentrations at $\mathrm{pH}$ 4.3. The Blistered Horn tailings do have the potential to release base metals (especially lead), but the volume of these tailings is small and they lie hundreds of feet from flowing streams. Carbonate in the tailings and nearby rocks is abundant and should have high ANC to mitigate any local sources of acid. Although the Tincup tailings are rich in $\mathrm{Pb}$ and other base metals, they do not appear to be mobile; thus, they should not pose a threat to the aquatic environment.

Surface waters.-Most of the mines in the district release no water to the surface. Shafts do not create mine drainage, and the few adits that do drain water have near-neutral $\mathrm{pH}$ and carry low concentrations of base metals. No samples of stream waters were collected because their $\mathrm{pH}$ is neutral to weakly alkaline, conductivities are $<100 \mu \mathrm{S} / \mathrm{cm}$, and there are no visual signs of iron-oxide coatings on cobbles that typically warn of contaminated water. Two mine drainage samples have $\mathrm{pH}$ of 7.4 and 7.6, conductivity of 240 and $280 \mu \mathrm{S} / \mathrm{cm}$, and low metal concentrations ( $\mathrm{Zn}$ is $<0.7 \times$ ALWS), but one contained elevated $\mathrm{Cu}(4 \times \mathrm{ALWS})$. Aside from some locally poor water quality at mine portals, the surface waters in this district appear to be of good quality.

Summary. - The geologic setting and style of mining at Tincup do not generate significant amounts of acid waters or metals. The predominant shaft-style mining does not generate drainage to the surface, and the few tunnels that do drain to the surface have neutral-pH waters with low metal concentrations. The abundant carbonate rocks mitigate whatever small volumes of acidic drainage that is generated. Lead in the mine waste is mobile for only short distances in this environment.

References.—Goddard (1936); Dings and Robinson (1957). 


\section{Tomichi District (Whitepine)}

Location.-Gunnison Co, about 27 mi east of Gunnison (fig. 18). The climate is moderate to severe with about 16-30 inches of precipitation per year.

Principal commodities.-Silver, lead, zinc, copper, gold.

Mining history.-The first deposit was discovered in 1879, and several others were located shortly thereafter. As in many parts of Gunnison County, there was some mining in the 1880's and 1890's when most production was from oxidized portions of deposits. Mining was active and prosperous from 1885 to 1893 . At first, rich ore was sorted and carried by wagon to the railroad at Sargents, then by rail to a smelter at Pueblo. Two smelters in the district (photograph 40) had mixed success. Mining of deeper, sulfidic ores was made possible by property consolidation in 1901 and construction of the Akron tunnel (photograph 42) at the elevation of Tomichi Creek. A floatation mill was built at the portal, on the southeast edge of the town of Whitepine. Mining expanded in the early 1940's and continued through 1956. Despite favorable similarities to the Leadville district, production never came close to that giant district.

Production and disturbance.-Total value was about \$6.6 million. Disturbance is moderate, with chief concerns being sulfidic waste dumps and mill tailings at the Akron portal (mill site).

Geology.-A large body of Precambrian granite is the basement rock in this area, overlain unconformably by Paleozoic quartzite and limestone beds that are faulted and folded. A body of Tertiary granite and associated dikes intrude the folded sequence and was the presumed source of heat and metals that formed the ores.

Base-metal sulfide replacement deposits in Paleozoic carbonate rocks have been the major producers. Several different stratigraphic horizons are favored for replacement by sphalerite-galena-pyrite and lesser chalcopyrite. The galena contains silver, and, in some areas, pyrite contains gold. Veins in Tertiary intrusive rocks have had modest production in the northern part of the district; the veins contain chalcopyrite, sphalerite, galena, silver-sulfosalts, and some local streaks of gold.

\section{Mineral-Environmental Conditions}

Mineralized rocks and ores.-Chemical analyses confirm the polymetallic character of these ores. Four samples from dumps contain very high $\mathrm{Pb}, \mathrm{Zn}$, and $\mathrm{Cd}$ and high $\mathrm{Mn}, \mathrm{Ag}, \mathrm{As}$, $\mathrm{Bi}$, and $\mathrm{Cu}$ concentrations. Analyses of four tailings samples were similar, with metal concentrations about one-third to one-half that of dump samples. A fluvial tailing sample from Tomichi Creek (photograph 46) contained very high metal concentrations, suggesting it is from an old stamp mill (preAkron mill).

Chemical analyses by two methods of a sample of slag, yielded improbably low results for base metals, suggesting that the analytical methods did not properly dissolve the vitreous material. Most slags are very rich in $\mathrm{Pb}, \mathrm{Zn}, \mathrm{Cu}$, and associated ore metals.

Leach tests were made on seven samples. Six dump samples yielded $\mathrm{pH}$ values of 5.3 to 7.9 , variable high to low concentrations of $\mathrm{Mn}-\mathrm{Fe}-\mathrm{Zn}$, and some notable amounts of Se; $\mathrm{Cu}$ and $\mathrm{Pb}$ concentrations were low. A sample of slag was more reactive than most slags that I have tested, creating a $\mathrm{pH}$ of 3.1 and releasing moderate amounts of $\mathrm{Fe}, \mathrm{Cu}, \mathrm{Mo}, \mathrm{Pb}$, and $\mathrm{Zn}$ to the leachate.

Mills and tailings.- Two smelters operated in the 1880's, one of which I located near the road southwest of Whitepine. Crawford (1913) reported "A large mill south of Whitepine seems not to have treated much ore." A small tailings impoundment on Tomichi Creek near a stone foundation seems to be that old mill site. Mill tailings in Tomichi Creek, about $2 \mathrm{mi}$ to the southwest of Whitepine village, appear to come from the breached tailings pond. The fluvial tailings have a distinctive composition that is much richer in metals than tailings from efficient mid-1900's mills. The Akron mine and mill complex, with its large dumps and two mill tailings piles, is conspicuous on the south edge of the village. The Akron mill tailings (photograph 43, photograph 44 ) are stacked high above the creek with no retaining structures, but appear to have been relatively stable for nearly 50 years. The steep slope on the two tailings piles at the Akron mill, and their position high above Tomichi Creek, suggest that, under unusually wet conditions of a major storm, the materials might become unstable and fail by slippage or liquefaction or be undercut and eroded by floodwaters. The Akron mill tailings merit further technical study. Among the many tailings impoundments in the study area, these two appear to be among the highest risks of failure in an extreme storm event.

Leach tests on two samples of tailings produced leachate solutions of pH 5.4 and 5.9, suggesting low acid-generation potential, but concentrations of $\mathrm{Zn}$ and $\mathrm{Cd}$ were high and $\mathrm{Fe}$, $\mathrm{Mn}$, and $\mathrm{Pb}$ were moderate. A white efflorescent crust on tailings yielded a leach $\mathrm{pH}$ of 4.5 and substantially higher concentrations of the metals just mentioned, as well as a notably high Se concentration. Despite the near-neutral $\mathrm{pH}$ of the tailings leachate solutions, there are suggestions that the tailings have the potential to release significant amounts of $\mathrm{Zn}$ and $\mathrm{Cd}$, and lesser amounts of $\mathrm{Pb}, \mathrm{Mn}$, and $\mathrm{Se}$, to Tomichi Creek.

Surface waters. - The carbonate rocks in this district are evident in the water quality, which is much better than expected for the substantial amount of mining, sulfidic mine waste, and tailings that are present. The $\mathrm{pH}$ values measured in the field were consistently near neutral ( $\mathrm{pH} 7.0$ to 7.9), and conductivity measurements were very low $(<100 \mu \mathrm{S} / \mathrm{cm})$, except for mine drainage of 410 and $180 \mu \mathrm{S} / \mathrm{cm}$ at two sites. Chemical analyses of five samples (three stream, one mine, one dump drainage) showed generally low metal concentrations, but the mine and dump drainage carried high zinc concentrations $(2 \times$ to $7 \times$ ALWS). Water from Tomichi Creek north of the town of Whitepine has very low metal content, but two samples of the creek downstream (south) of the Akron mine 


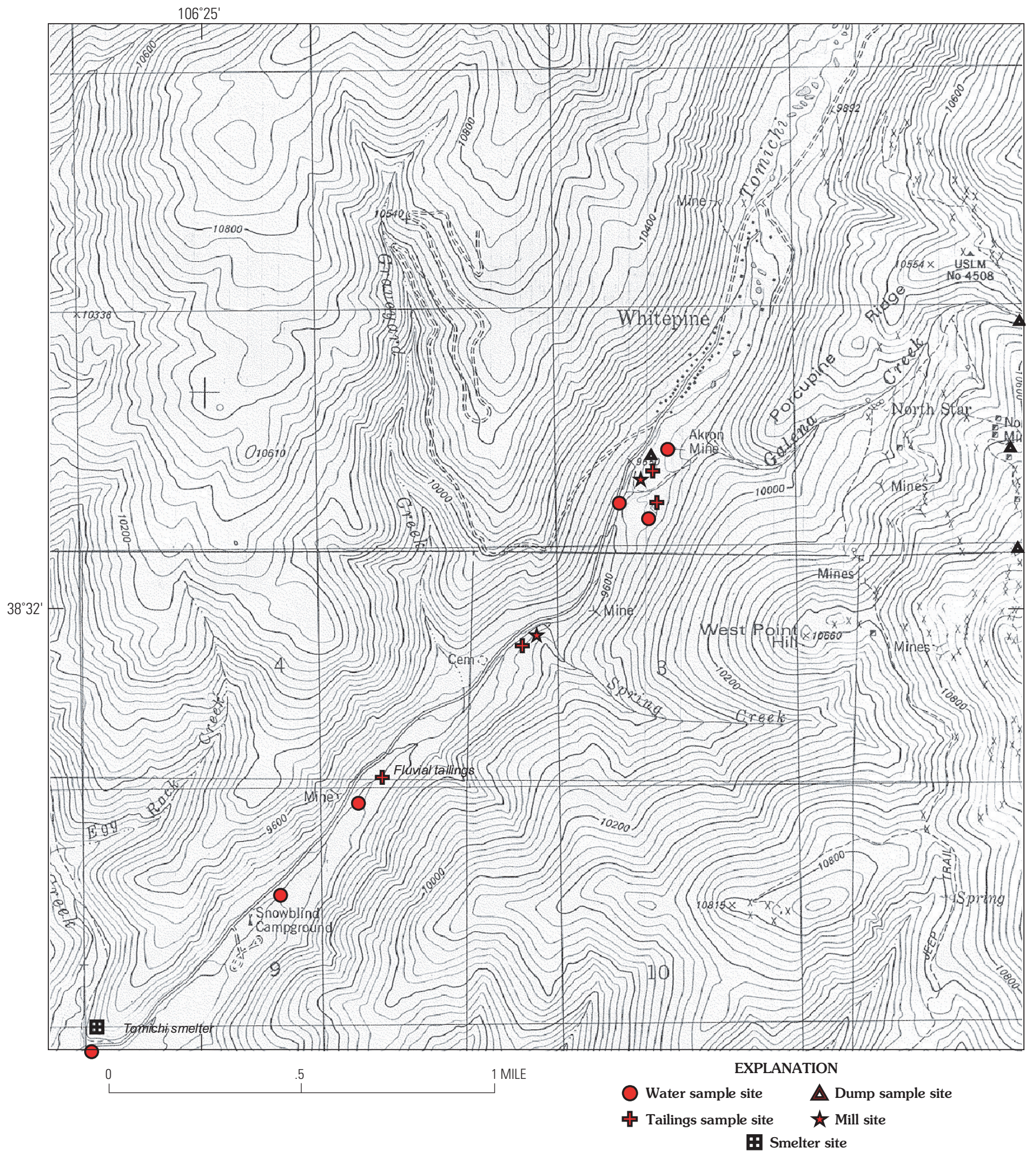

Figure 18. Features of the Tomichi mining district, showing sample localities. 


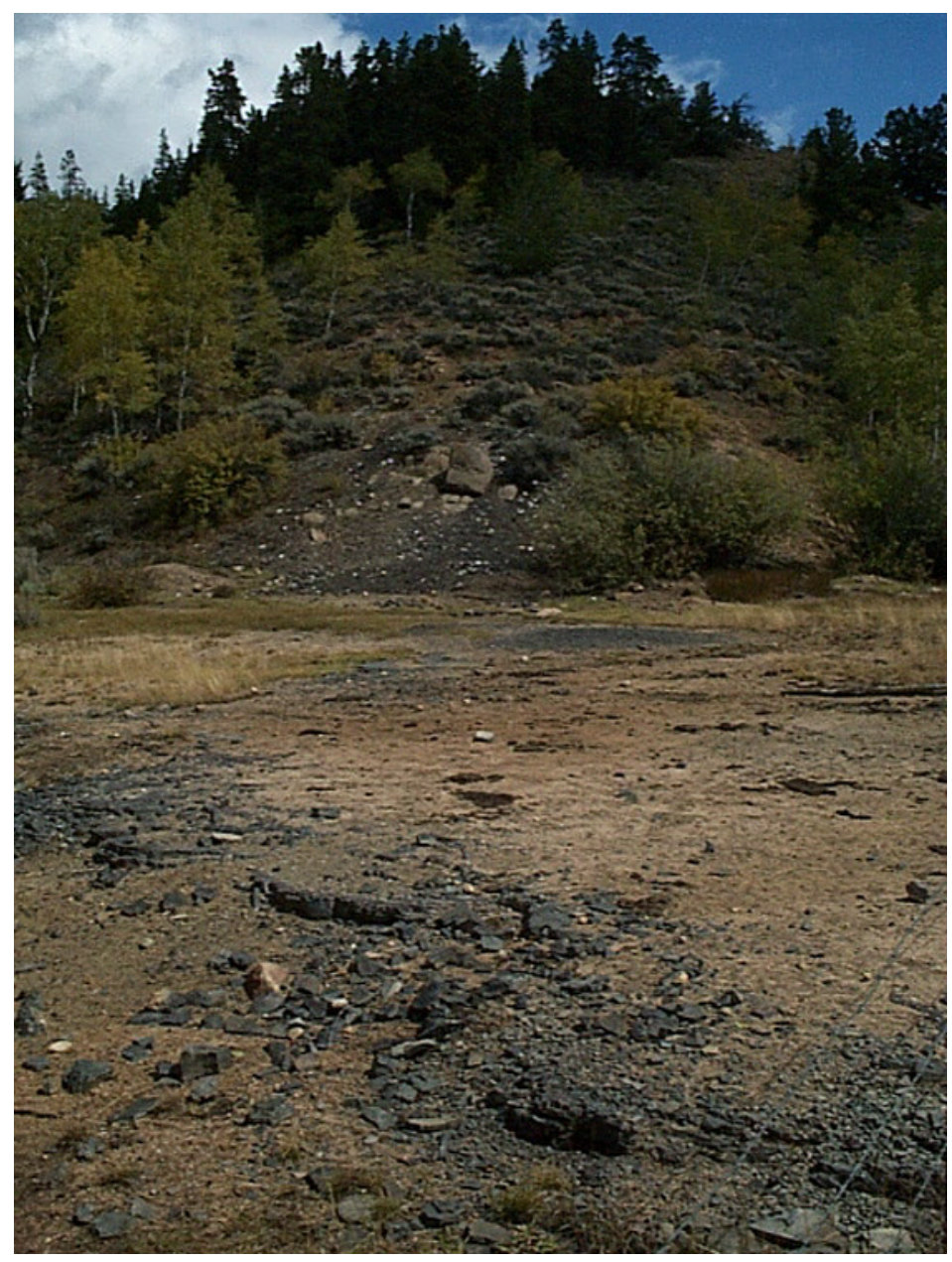

Photograph 40. The Tomichi smelter, southwest of Whitepine, was part way up the hillside and slag was poured out onto the flat area below. The slag is not very reactive; thus, it probably is not a threat to the nearby creek, but the high concentration of base metals could be toxic if ingested.

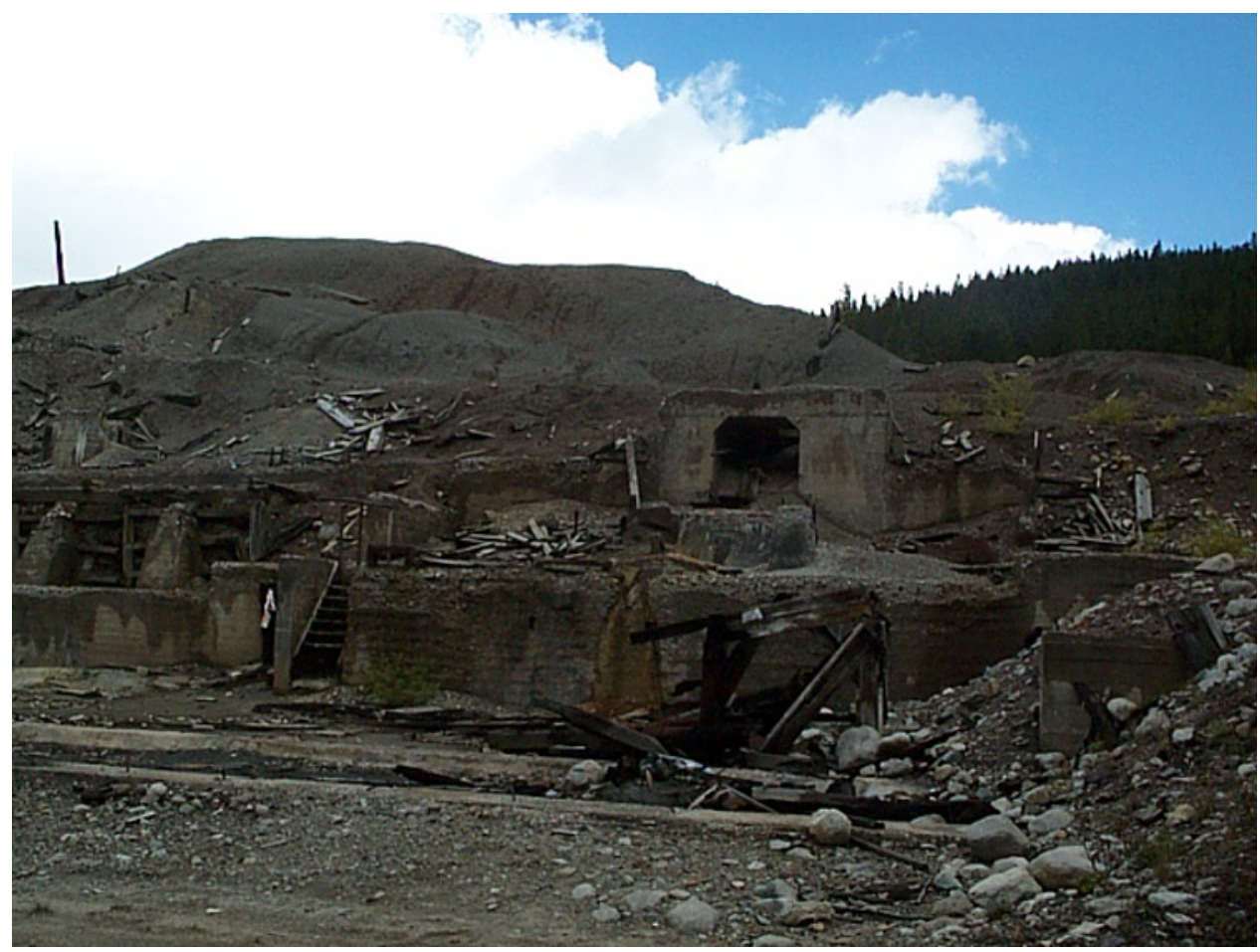

Photograph 41. The Akron mill on the south edge of Whitepine was productive through 1956. Sulfidic waste and mill tailings are piled above the mill. 


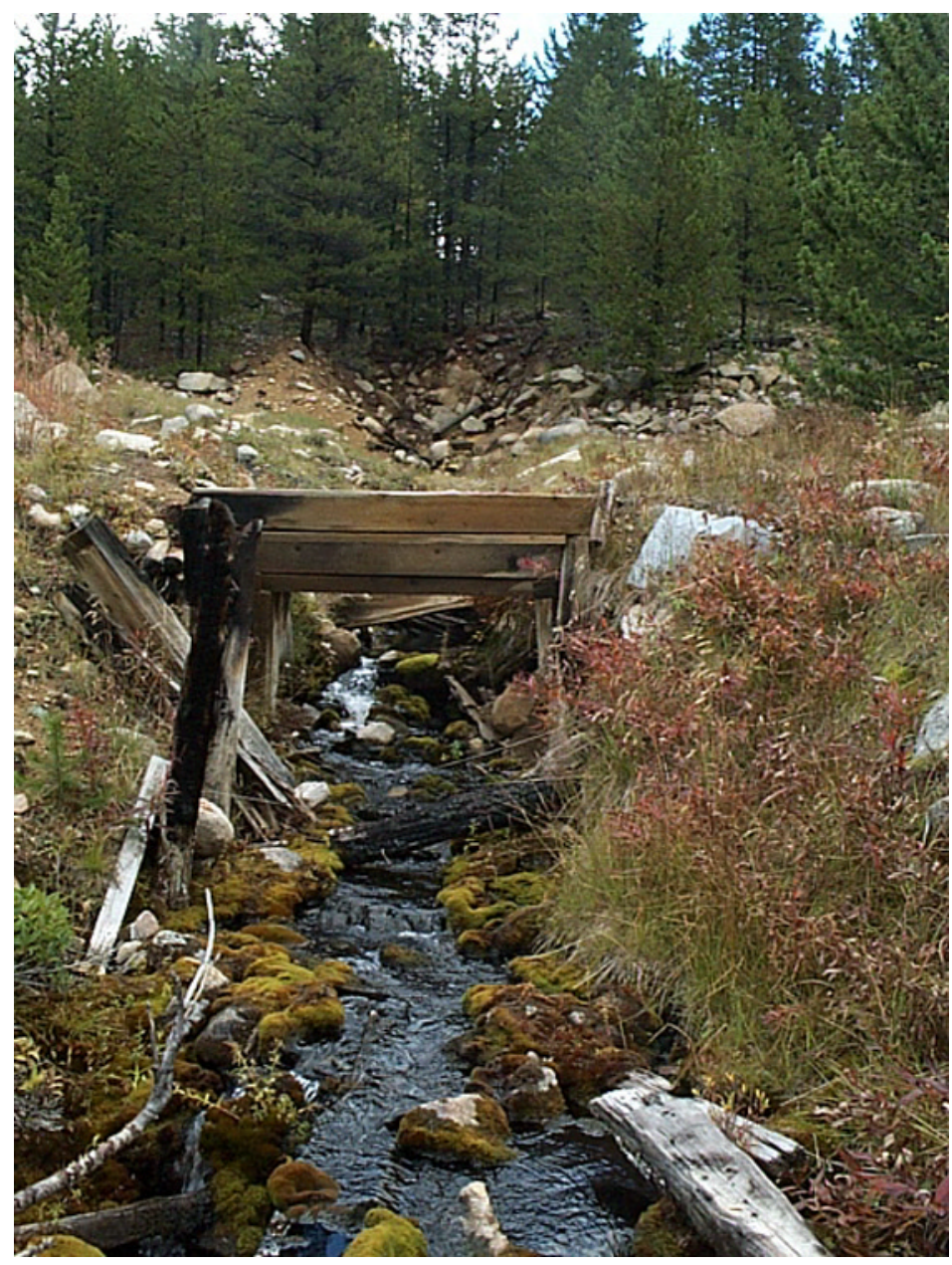

Photograph 42. The Akron mine tunnel served both for rail haulage and mine dewatering. Drainage from the tunnel today has near-neutral $\mathrm{pH}$ because of the carbonate rocks underground. However, the drainage carries some zinc and also reacts with sulfidic mine waste before entering Tomichi Creek.

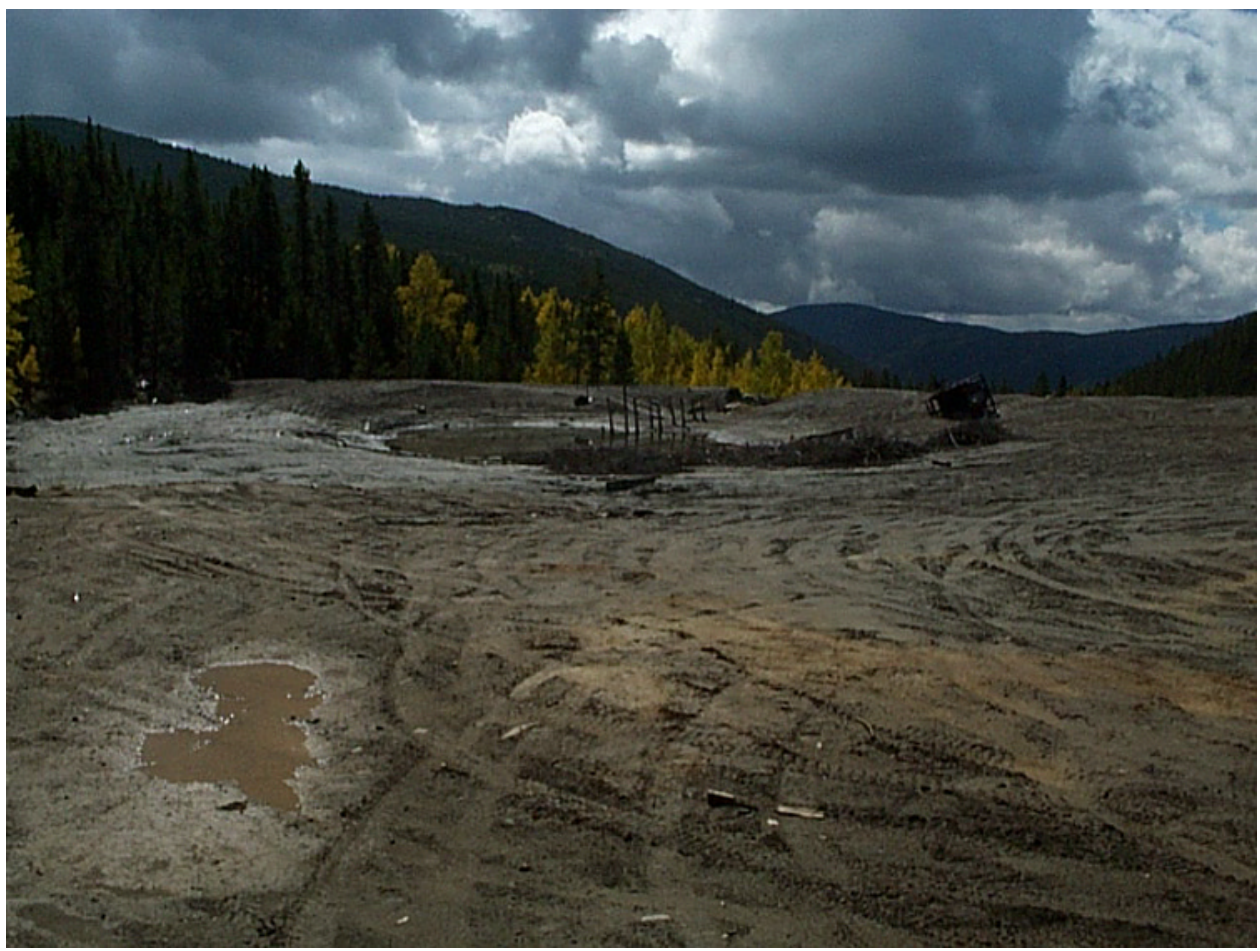

Photograph 43. This tailings impound is 200 yards south of the Akron mill. These tailings are above Tomichi Creek and erosion or failure in an extreme storm event would carry them into the stream. 


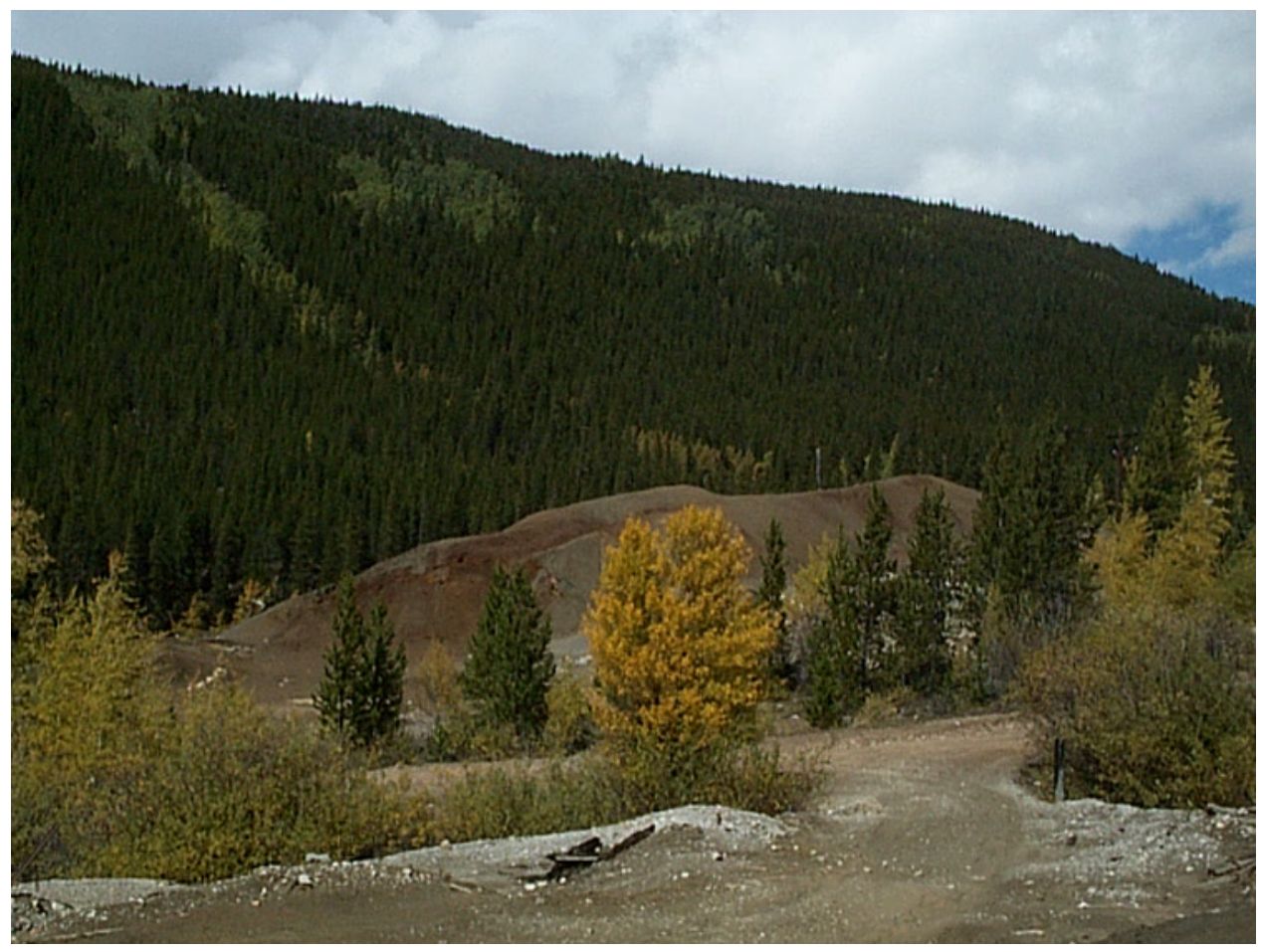

Photograph 44. This view is from the south tailings impoundment to the Akron millsite and reddish tailings piles above it. Numerous tracks from vehicles show the dumps and tailings are popular with visitors.

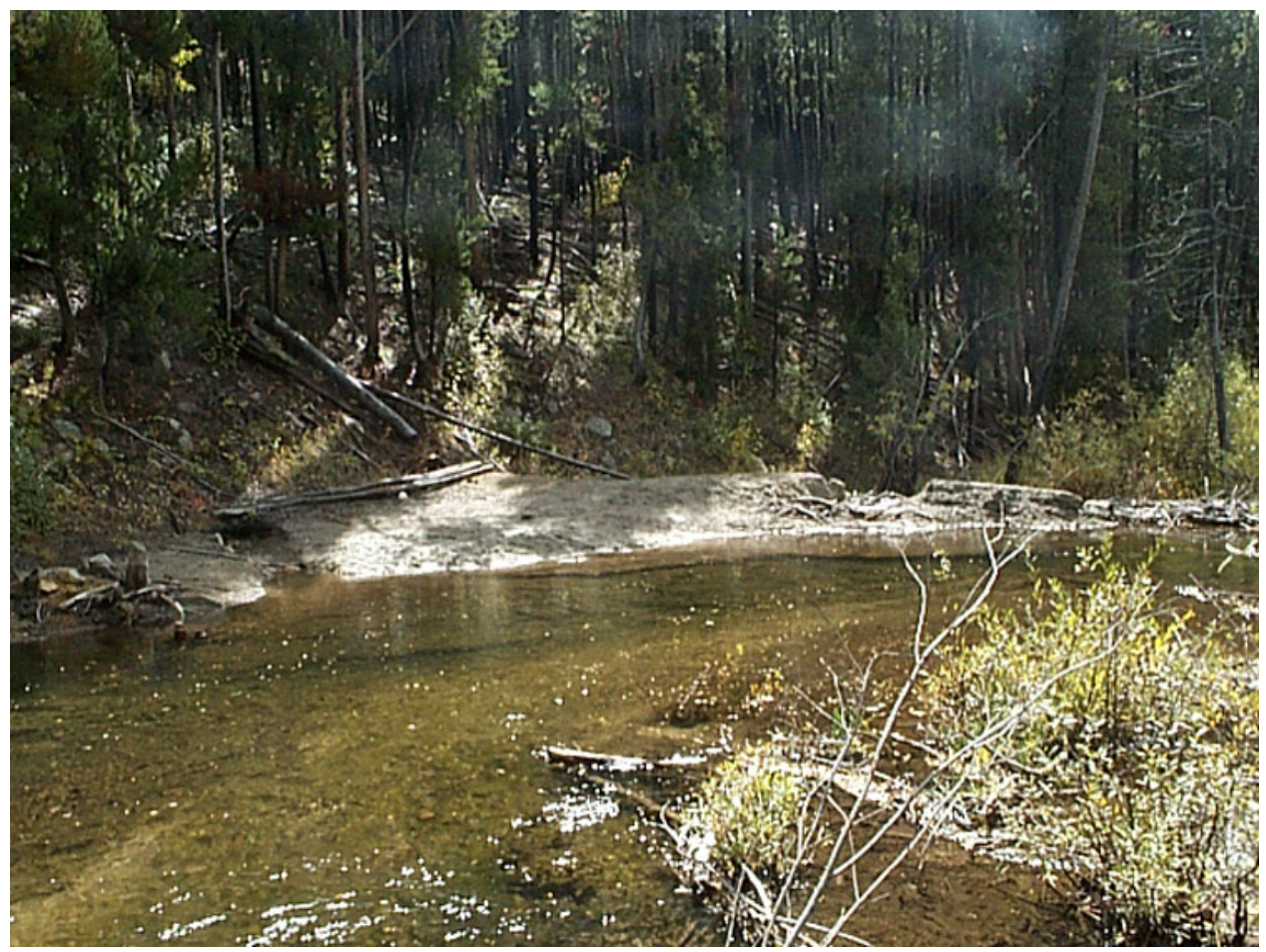

Photograph 45. This small tailings pond was built across Tomichi Creek before 1900 . The stone foundation for the mill is a short distance to the north and about $1 / 2$ mi south of Whitepine. The pond was breached and tailings were eroded years ago. 


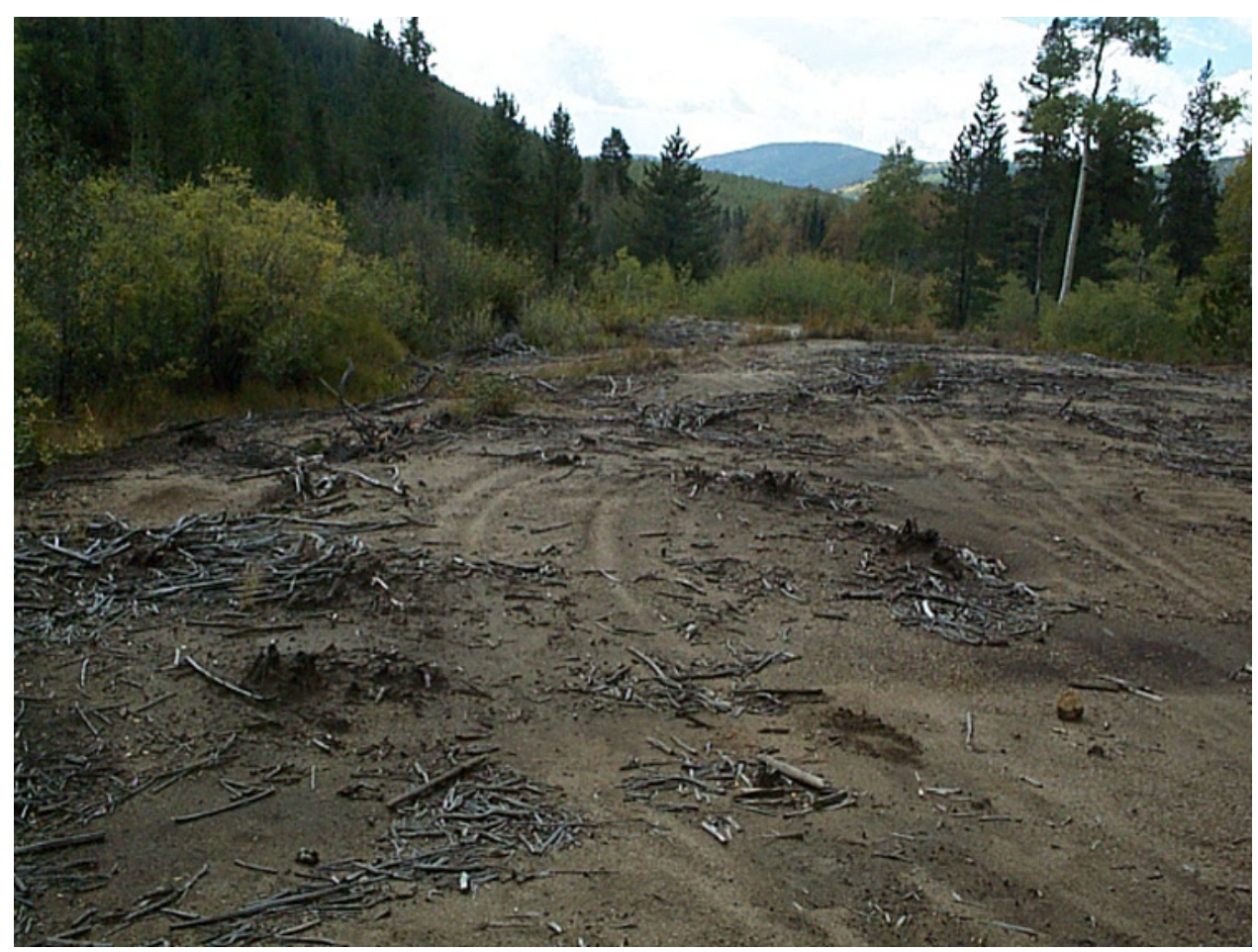

Photograph 46. These gray sands are mill tailings that were eroded and carried south along Tomichi Creek and came to rest among willows. The likely source is a pre-1900 stamp mill about $1 \mathrm{mi}$ to the north.

contain zinc that is above ALWS $(2 \times$ and $2.5 \times)$, although a $\mathrm{Zn}$ content of $125 \mathrm{ppb}$ is not very high relative to some other contaminated streams on the Western Slope. Samples collected in 1999 south of the Akron mill showed low metal concentrations (although the $\mathrm{Zn}$ value of $140 \mathrm{ppb}$ at site NG850, 2.3 mi to the southwest, is higher than at sites closer to the mines and mill and might reflect an error of some kind). The CZI values of 0.6 to 0.2 for Tomichi Creek samples below the mines and mill suggest low threat to aquatic life. The carbonate rocks appear to be effectively neutralizing $\mathrm{pH}$, which limits the solubility of most base metals. However, $\mathrm{Zn}$ is still slightly soluble and enriched downstream from mining, as is well known from other studies (Smith and others, 1994).

Summary.-The zinc enrichment in Tomichi Creek is clearly related to the Akron mine drainage and waste piles. Further study of base metals in Tomichi Creek by more detailed methods may be warranted. No reclamation or water management has been undertaken, and even a partial reclamation would likely improve the somewhat contaminated waters of Tomichi Creek. The two tailings impoundments at the Akron mine are located in situations that appear to make them vulnerable to failure by erosion, slippage, or liquefaction during an extreme storm event. These tailings rank among the top five sites for risk of failure during an extreme storm and should be investigated further for physical stability and possible methods of stabilization.
References.-Crawford (1913); Dings and Robinson (1957).

\section{Mining Districts of the Lake Fork Watershed}

Mining in the area of Lake City (figs. 19, 20) started in the 1870's and soon became famous when very rich silver-gold zones were encountered at the Ute-Ulay and Golden Fleece mines. However, the boom was short-lived and did not attain the magnitude of adjacent San Juan mining districts (production was only $\$ 550,000$ after 1930). The veins in volcanic rocks associated with the Lake City caldera are relatively short and narrow, and the mining complexes are smaller and produced less disturbance than the later and larger mines elsewhere because the miners used small equipment to focus on the rich ore zones. This simple mining technology, combined with favorable volcanic rock characteristics, leaves this watershed with healthier habitat than some adjoining districts.

Geology of this mining area is dominated by the nested Lake City and San Juan caldera complexes that have been thoroughly studied (Lipman, 1976; Steven and Lipman, 1976; Slack, 1980; Hon, 1987). 


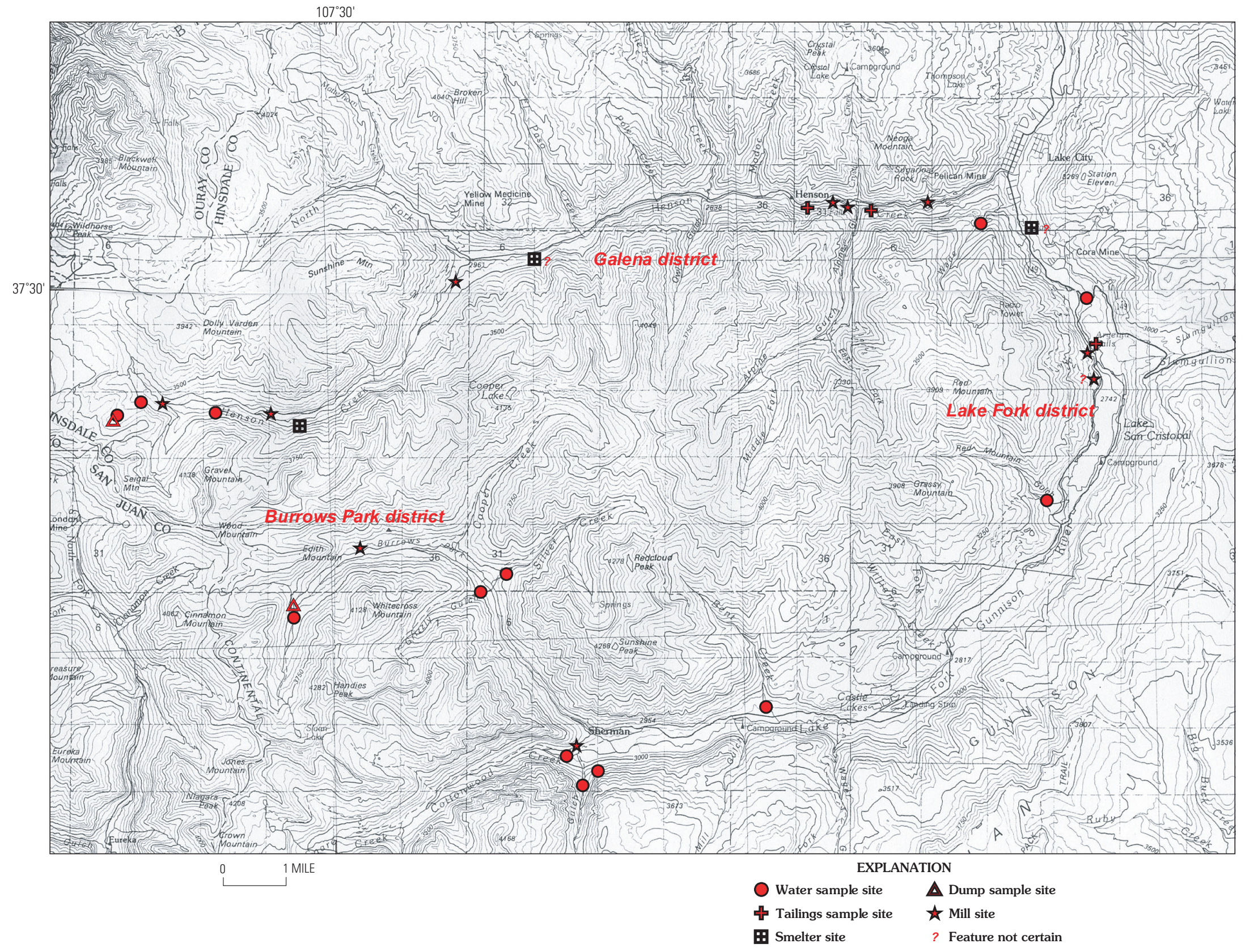

Figure 19. Features of the Galena, Lake Fork, and Burrows Park mining districts, Lake City area, showing sample localities. 


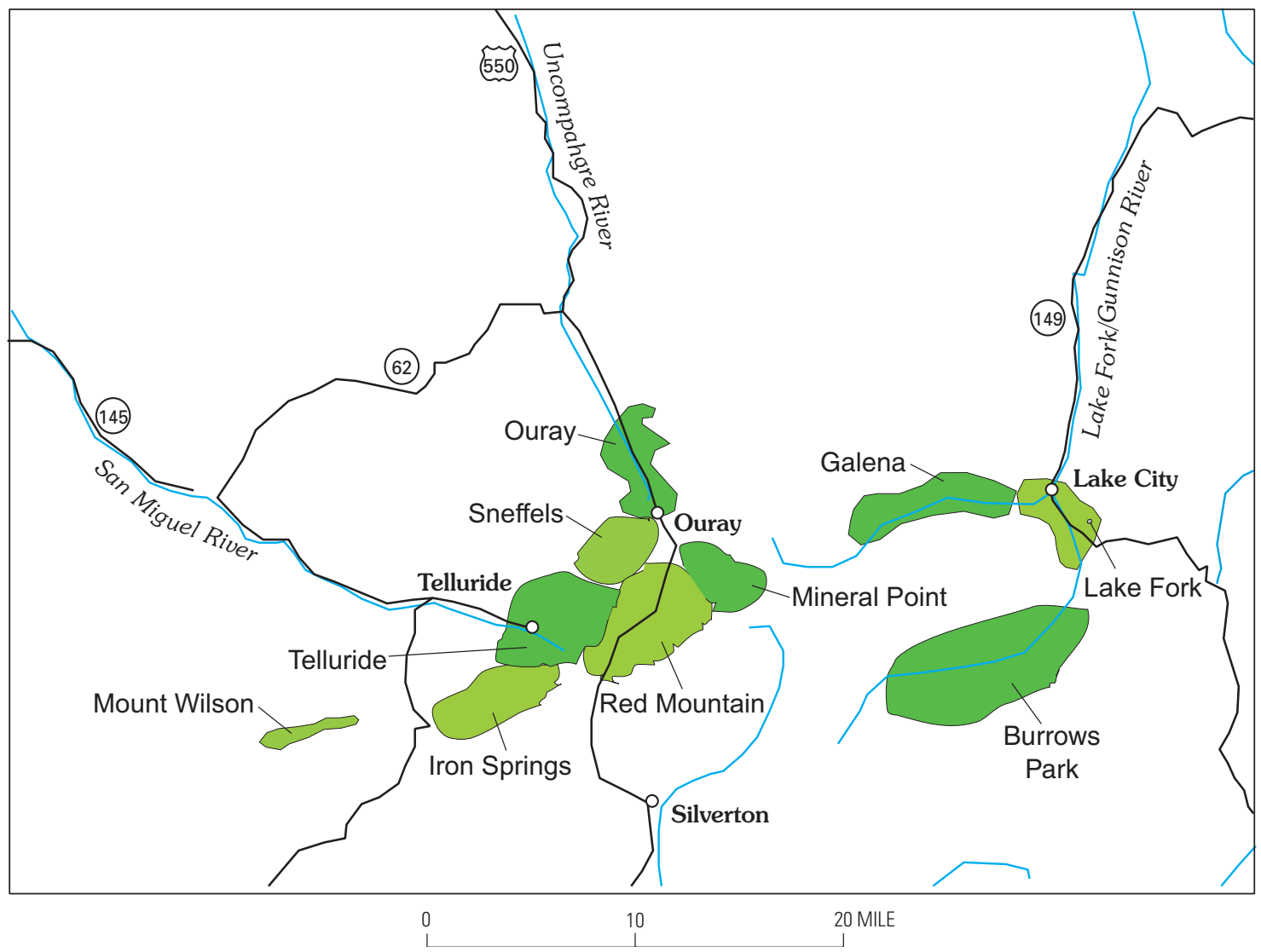

Figure 20. Location of mining districts in the San Juan Mountains region.

\section{Burrows Park District (Whitecross, including Sherman)}

Location.-Hinsdale Co., 20 mi south of Lake City (fig. 19). The climate is relatively severe with about $25-40$ inches of precipitation per year.

Principal commodities.-Ag, Au, minor base metals.

Mining history.-Little has been written about this district. There was not sufficient activity in the early years to warrant mention in the review by Henderson (1926). Presumably, earliest discoveries were made in the 1870's, during the rushes in adjoining districts. A study of the mines in 1906 suggested that most mines had closed by that time and that production was limited by the distance to railroads (Woolsey, 1907). A small mill was erected on the Lake Fork at the townsite of Sherman, probably in the 1880's (judging from the style of stone construction); this mill was named the Black Wonder according to Southworth (1997), who provides an historical photograph. None of the mines in the district attained significant production, and most have been inactive for the past 50 years. There have been a few attempts to reopen older mines on patents.

Production and disturbance.-Small production, a minor contribution to the \$11.2 million for Hinsdale County. Small disturbance.
Geology.-The ore geology of this area can be considered to be an extension of the much larger and better understood Eureka district in the Animas watershed to the southwest. In the Burrows Park district, the veins in Tertiary volcanic rocks have tended to be smaller than to the south but are mineralogically similar in their content of galena, sphalerite, with variable amounts of chalcopyrite, gold, and silver. Some veins occur in Precambrian granite-gneiss but are of the same age and character as those in Tertiary volcanic rocks.

\section{Mineral-Environmental Conditions}

Mineralized rocks and ores.-Polymetallic ores from this district are mineralogically and chemically similar to the Eureka district, for which there is abundant new information on rock alteration and dump compositions (Nash, 1999b; and others). One sample was analyzed for this study. Dump compositions may have large local variability in composition, depending upon the style of mining. Some dump piles are likely to have very high $\mathrm{Pb}, \mathrm{Zn}$, and $\mathrm{Cd}$ concentrations; high $\mathrm{Ag}, \mathrm{Cu}$, and $\mathrm{Mn}$; and locally high $\mathrm{Bi}, \mathrm{Mo}$, or Se concentrations.

Mills and tailings.-The small stamp mill at Sherman townsite is presumed to have had small production; only the stone foundation remains visible today. Tailings from this mill 
Hydrogeochemical Investigations of Historic Mining Districts, Central Western Slope of Colorado

probably were placed on the flood plain and subsequently carried away by floodwaters. No sample of tailings could be collected. The Sherman mill does not appear to pose any problems today, and there is no material remaining to be reclaimed. The Tobasco mill is located about $1.7 \mathrm{mi}$ east of Cinnamon Pass, at the west edge of Burrows Park; it is on private property and was not investigated.

Surface waters.-During the brief reconnaissance study, three samples were collected for analysis. Field tests of $\mathrm{pH}$ and conductivity indicated good quality with $\mathrm{pH}$ near neutral (6.9-7.4) and conductivity very low ( $<50$ in streams, but 240 $\mu \mathrm{S} / \mathrm{cm}$ for one mine drainage). The surface waters appear to be of good quality, and two analyses show very low metal concentrations, well below ALWS. One mine drainage sample from a small adit in American basin had a pH of 7.4 and high zinc concentration ( $3 \times$ ALWS). The CZI values for two stream samples were very low (0.1 and 0.01$)$, suggesting low threat to aquatic life. The predominant green alteration in volcanic rocks is expected to provide ANC adequate to naturally attenuate the locally contaminated acidic waters at small mines such as site 287.

Water composition baselines and geologic influences on them were studied in fair detail by McHugh and others (1995) and Miller (1998), who found anomalous amounts of metals and sulfate in several tributaries to the Lake Fork, such as Silver and Copper Creeks. Miller found similar results to those reported here for the main stem of the Lake Fork and described how neutralization reactions improved the quality of water downstream from the mineralized areas.

Summary.-The water quality in the main stem of the Lake Fork is good, judging from the standard indicators of $\mathrm{pH}$, conductivity, and trace metals. Some small mine workings may release degraded water, but this evidently is mitigated within about a mile by dilution and the neutralizing effects associated with the predominant green-altered volcanic rocks. Some mining properties may merit reclamation work to minimize release of metals, but the ability of natural processes to mitigate mine-related contamination suggests that these reclamation efforts have lower priority than in several other mining areas on the CWS.

References._Lipman (1976); Woolsey (1907).

\section{Galena District (Henson Creek)}

Location.-Hinsdale Co, 2-10 mi west of Lake City (fig. 19). The climate is moderate to severe, with about $20-40$ inches of precipitation per year.

Principal commodities. - Silver, lead, zinc, copper, gold.

Mining history.-Gold was discovered in 1842 by a member of the Fremont Expedition, but the locality was lost. The Ute and Ulay veins were discovered in 1871, when the land still belonged to the Indians. Development started after the treaty of 1874, and by 1876 there was a short-lived boom. The Palmetto and Frank Hough mines near Engineer Pass were located in 1881 and were productive in the 1880's. The collapse of silver prices in 1893 depressed mining in the area, but it regained activity in the late 1890's, sooner than most districts on the Western Slope. However, mining declined in the early 1900's and never attained the stature of districts in the western San Juan Mountains, which became highly productive in a second stage of mining so-called "low-grade ore" with the use of modern flotation and cyanide mills in the period 1915-80.

Production and disturbance.-Production from this district was the largest in Hinsdale County, for which the total recorded is about $\$ 11.2$ million. However, records differ because production from the Ute and Ulay mine alone through 1906 is stated to be $\$ 12$ million (Irving and Bancroft, 1911). Production from the district through 1959 was about 126,800 tons and has been small to nil since then. Disturbance is small from the underground mines that were highly selective in their mining, but several substantial impoundments of mill tailings are close to Henson Creek.

Geology.-Polymetallic vein deposits containing basemetal sulfides with variable amounts of precious metals occur in Tertiary volcanic rocks on the northern margin of the Lake City caldera, similar to the larger and more famous deposits of the western San Juan Mountains near Silverton and Telluride (Burbank and Luedke, 1968). Most veins contain chiefly galena and sphalerite, with lesser amounts of chalcopyrite, silver minerals, and gold. Some of the veins are famous for locally rich pockets of gold and silver, some of which is in the form of telluride minerals. Alteration halos of pyritic rock are relatively narrow. The southwestern part of the district, near Engineer Pass, contains veins that are structurally continuous over the divide into the Eureka and Mineral Point districts. A few of these vein deposits, such as at the Hough mine, were most productive in the 1880's. In the eastern part of the district, a cluster of veins was worked by the Ute-Ulay mines and were the first to be discovered in the Lake City area. A large area of acid-sulfate alteration occurring at Red Mountain contains a subeconomic deposit of alunite (an ore mineral of aluminum) but no important associated metal deposits.

\section{Mineral-Environmental Conditions}

Mineralized rocks and ores.-Polymetallic ores from this district are mineralogically and chemically similar to the Eureka district, for which there is abundant new information on rock alteration and dump compositions (Nash, 1999b, Fey and others, 2000b). Three samples were analyzed for this study. Dump compositions may have large local variability in composition, depending upon the style and history of mining. Dumps from small mines tend to have piles of ore-grade material that is separated from waste but not of sufficient tonnage to ship to a mill. Dumps at large mines, generally on patented land, tend to have chiefly low-grade piles, including waste from crosscuts that is low in metals. Some dump piles are likely to have very high $\mathrm{Pb}, \mathrm{Zn}$, and $\mathrm{Cd}$ concentrations; high $\mathrm{Ag}, \mathrm{Cu}$, and $\mathrm{Mn}$; and locally high $\mathrm{Bi}, \mathrm{Mo}$, or Se concentrations. Pyrite is abundant on most dumps, as can be seen at the Hough mine (photograph 50). 


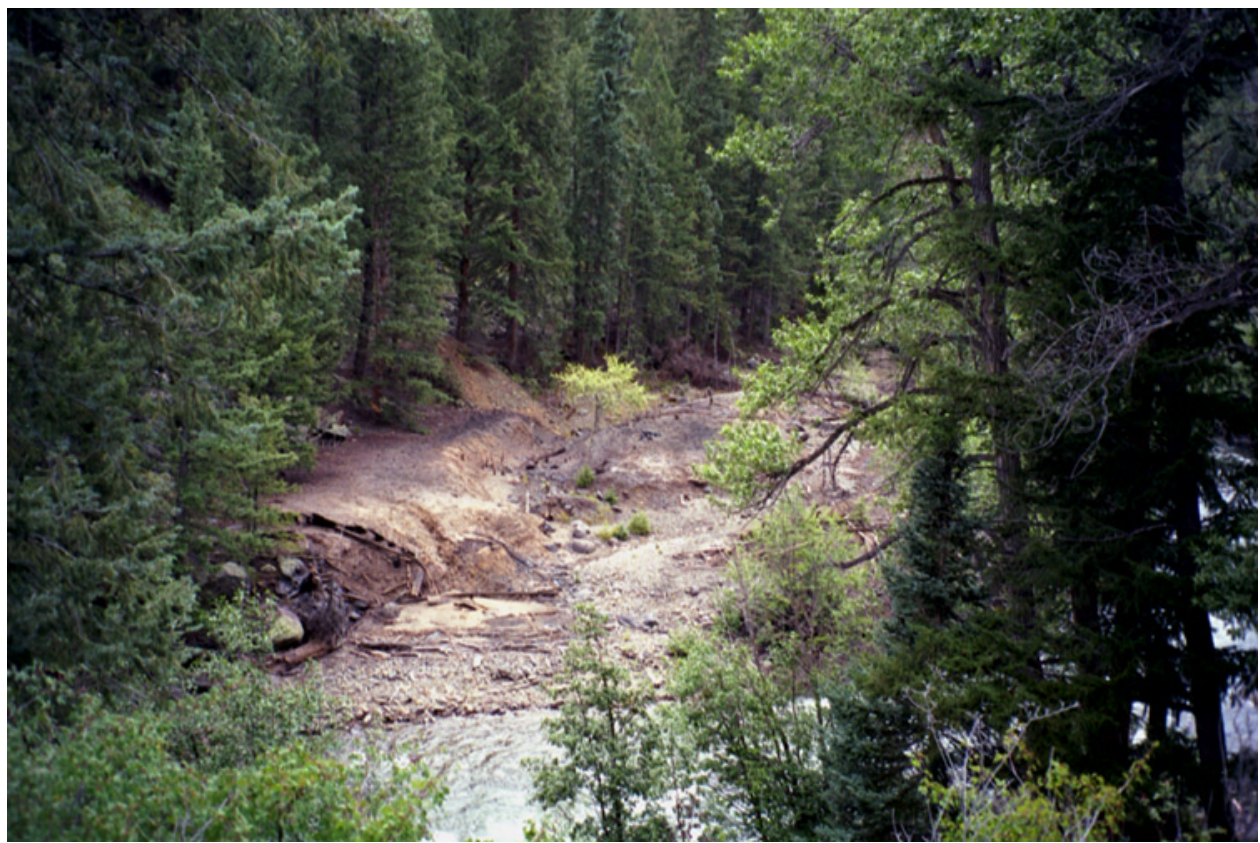

Photograph 47. Mill tailings from the Hidden Treasure mill are on the south bank of Henson Creek about 3 mi west of Lake City. These tailings would be eroded in a major storm.

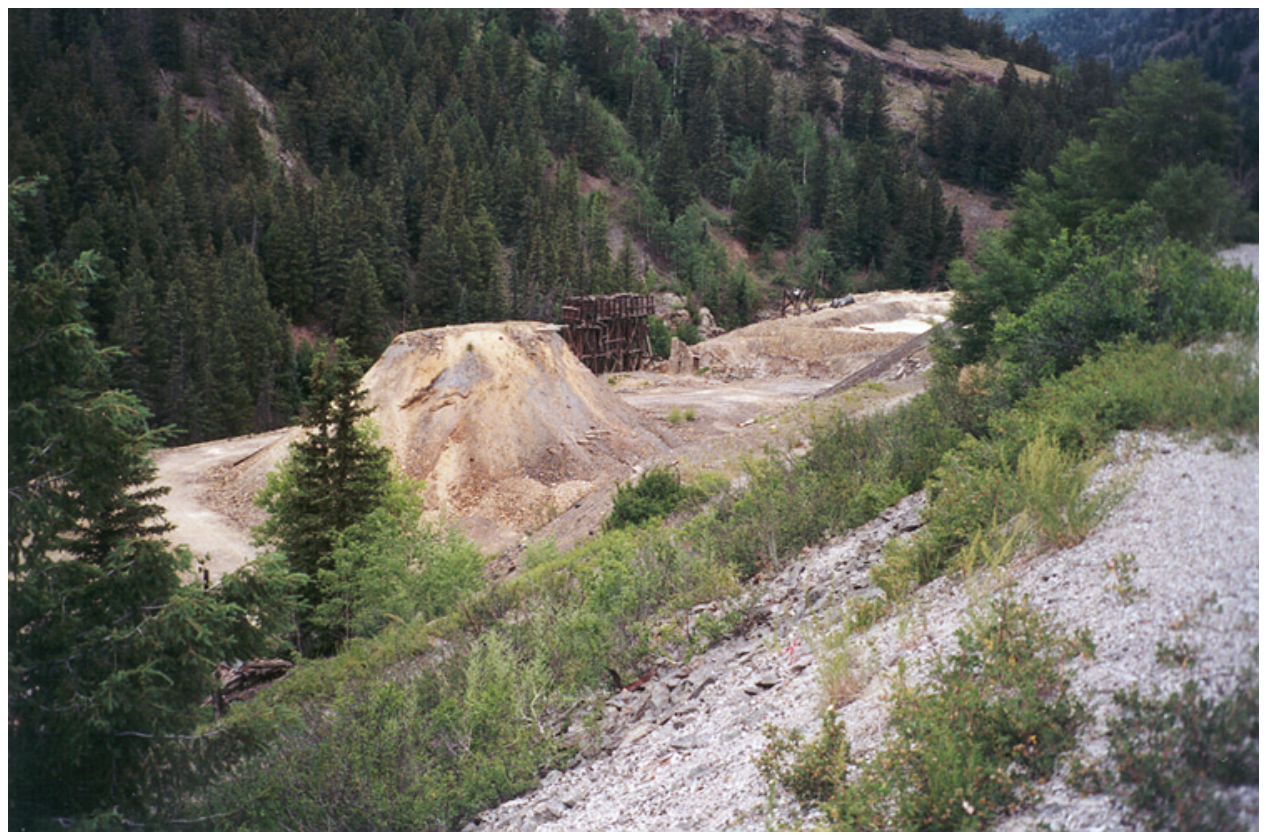

Photograph 48. The Ute-Ulay mine was the largest in the lower Galena district. The tailings from an early stage of milling, lower left, were later covered with mine waste. All of this waste is within tens of feet of the Henson Creek. Tailings from later periods of milling were placed in impoundments west of the mill, also close to the creek. 


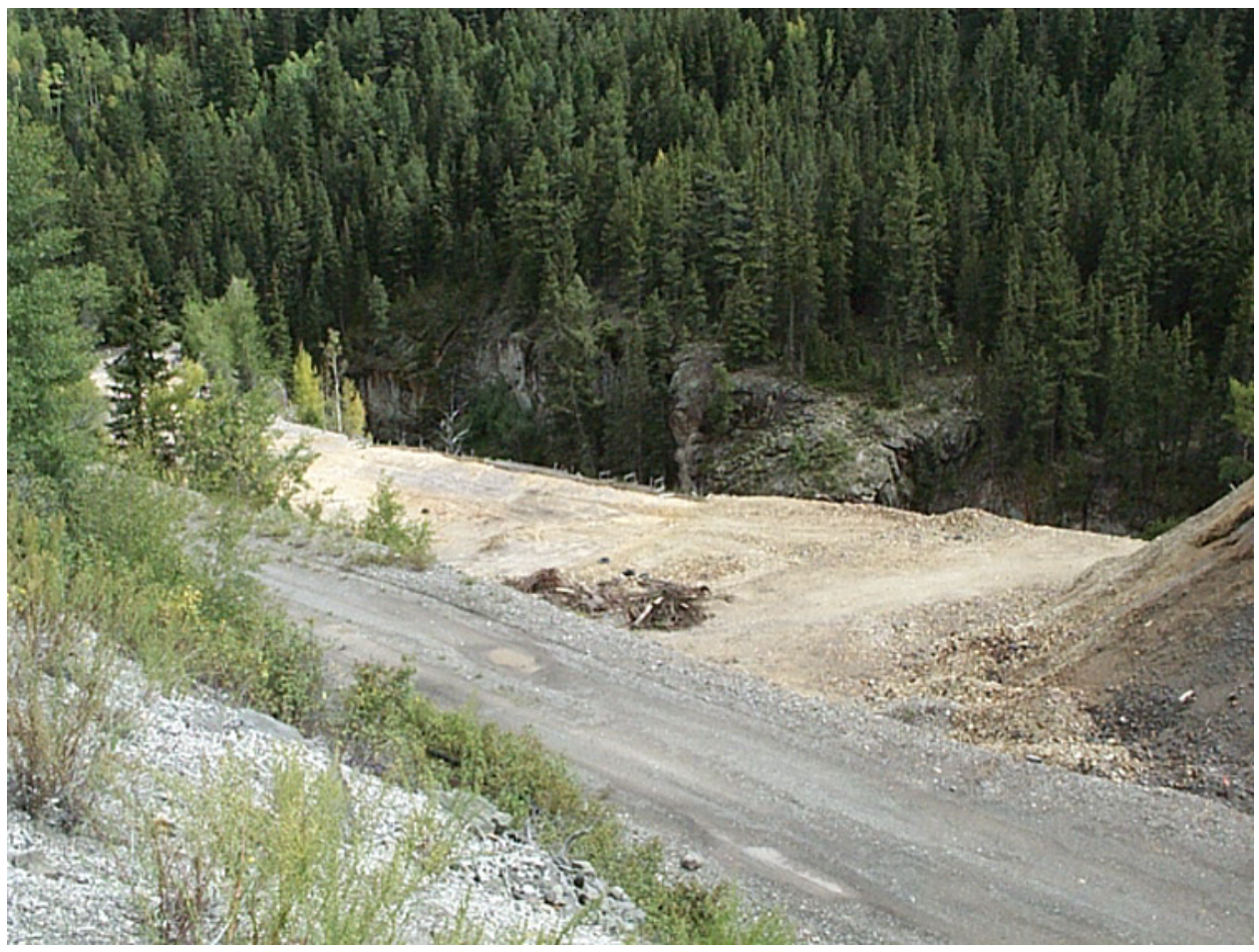

Photograph 49. Tailings and mine waste at the Ute-Ulay mine are stacked on a narrow bench very close to Henson Creek, which is in the gorge on the far side. These materials appear to be unstable and at risk in a major storm event.

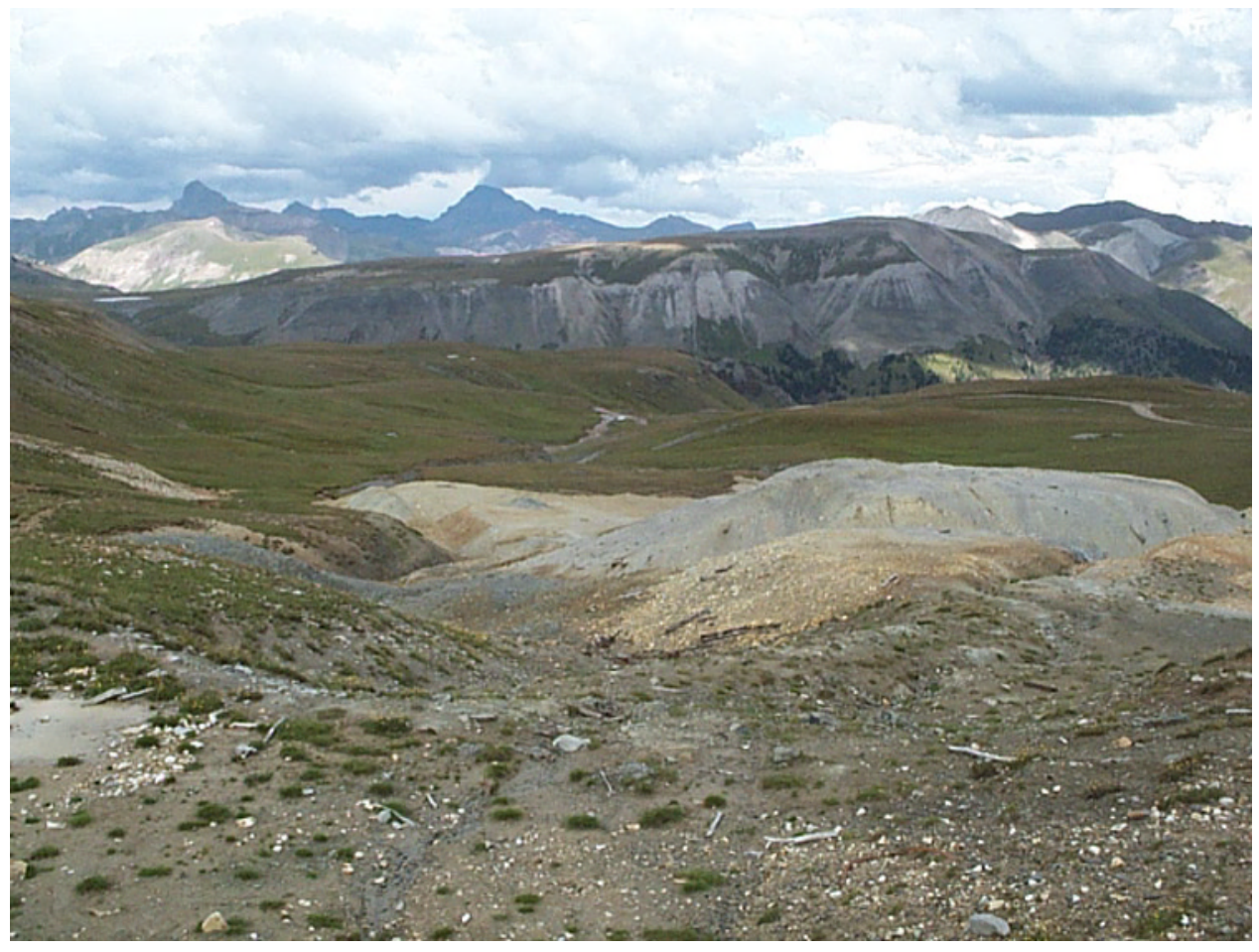

Photograph 50. The large dumps from the Hough mine are rich in sulfide minerals and pose problems for the alpine ecology. Sulfidic dumps and draining mine adits in Palmetto Gulch pollute the headwaters of Henson Creek. 


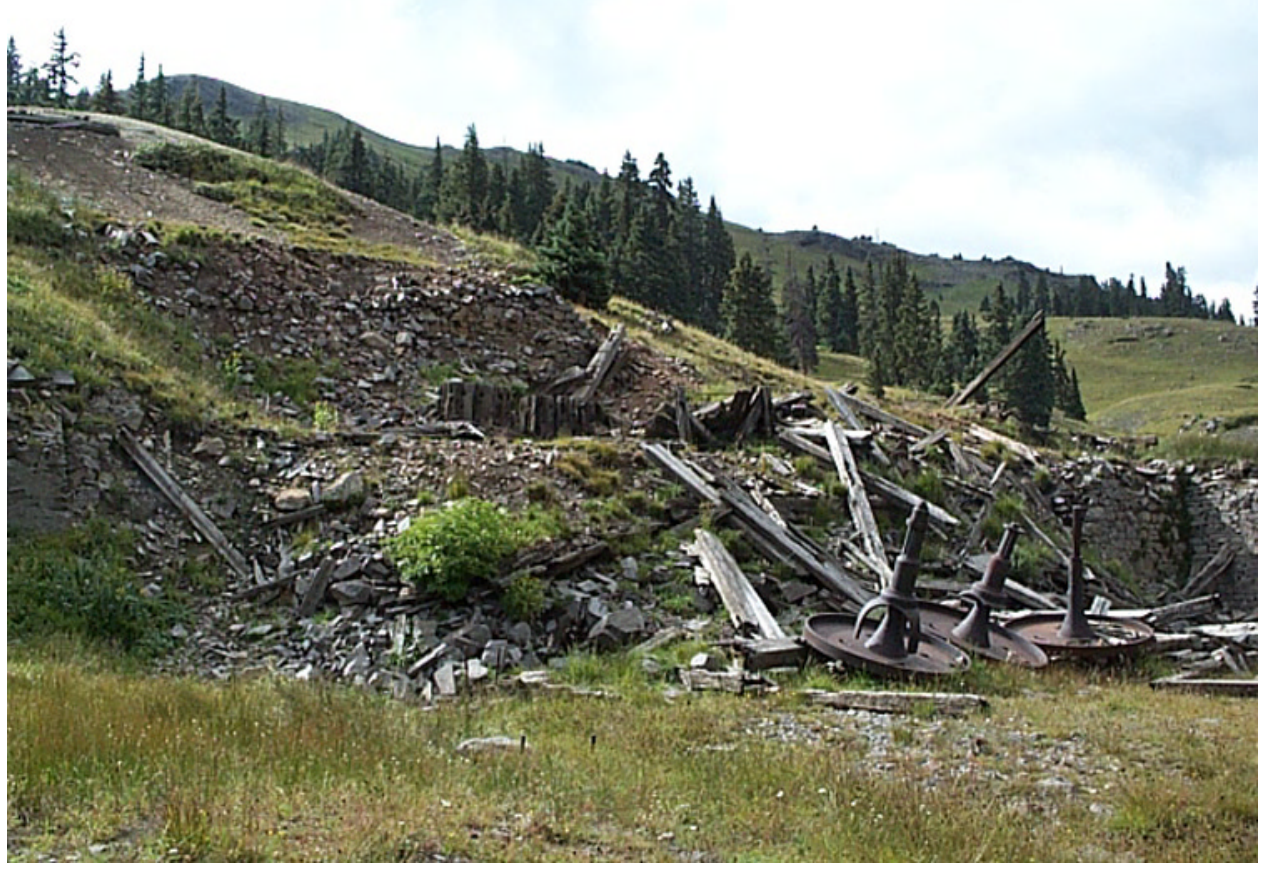

Photograph 51. The Palmetto mill at treeline in the headwaters of Henson Creek processed ore from several mines in the Engineer Pass area. It probably used water power in the 1880's. No tailings could identified.

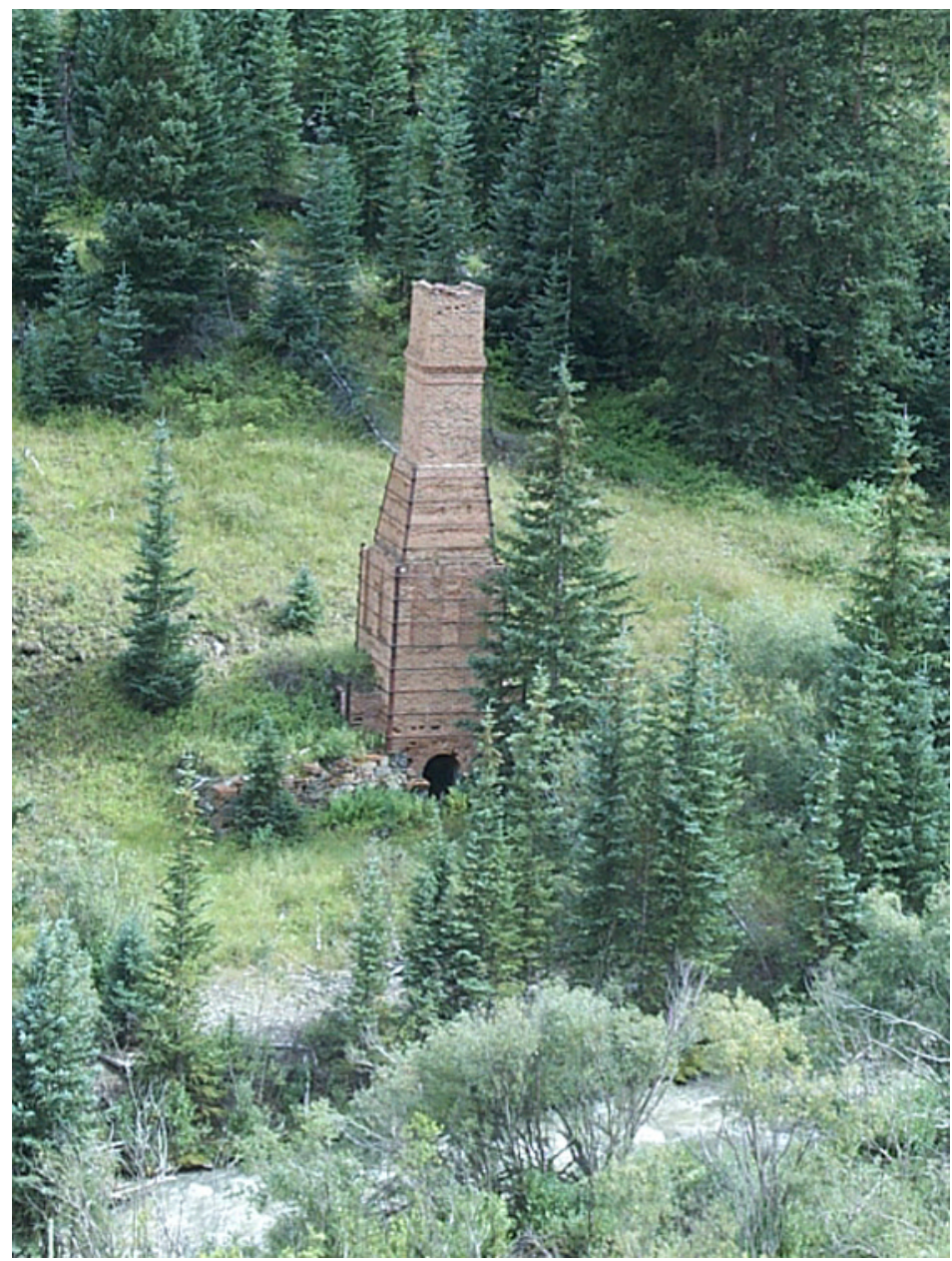

Photograph 52. This brick smokestack is the remains of the Lee smelter, located southwest of Capitol City. It was an early (1880's?) operation that attempted to refine silver and gold from sulfidic ore. 


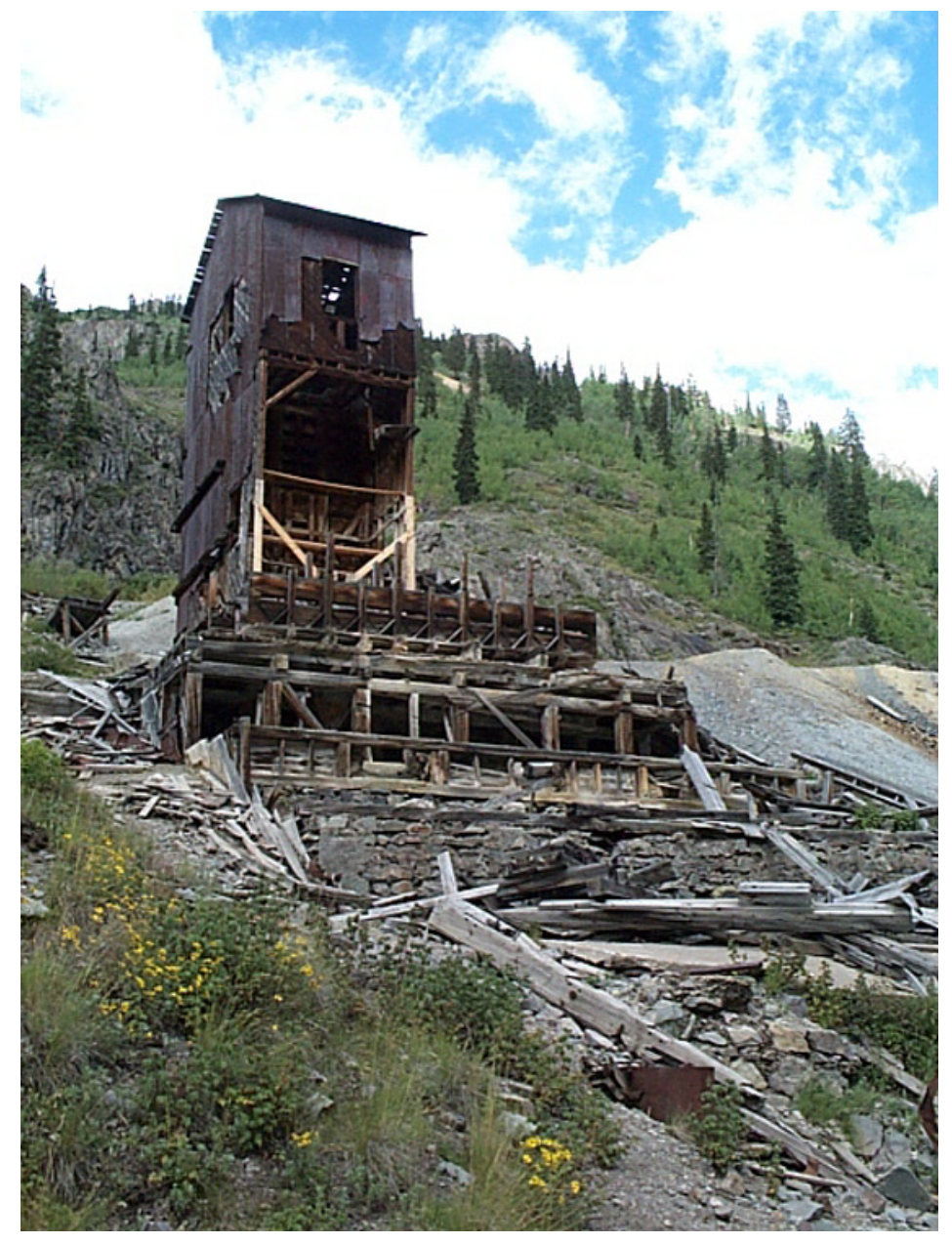

Photograph 53. The Empire Chief mill is a popular tourist stop on the Engineer Pass road, southwest of Capitol City. No tailings could be found nearby.
Mills and tailings.-There were six or more mills on Henson Creek, some of which used water power. Tailings are preserved at the Ute-Ulay mine-mill complex (photograph 48), but most other tailings appear to have been lost to the stream. The Hidden Treasure mill foundation is visible near the footbridge over Henson Creek to Alpine Gulch. A small pocket of tailings that is visible on the south side of the creek a short distance downstream may be related to this mill, but other tailings are presumed to have been washed downstream (photograph 47). No structures or waste materials could be found at the sites reported (Irving and Bancroft, 1911) for the Ocean Wave smelter and Red River mill about 2 mi east of Capitol City. The structure at Capitol City has the appearance of a mill, but residents say that it was a water-powered electricity generator. A concrete foundation with the form of a mill is evident in Henson Canyon slightly less than $1 \mathrm{mi}$ west of Lake City, below the Pelican mine. The mill is not reported by Irving and Bancroft (1911); thus, it would seem to be younger than that date. No tailings could be located at this probable mill site.

Four mill sites are evident west of Capitol city, one being a popular tourist site (Empire Chief mill, photograph 53). The Palmetto mill utilized water power on Henson Creek just below treeline (photograph 51). A brick chimney from the Lee smelter is visible on the south side of the creek, 2 mi southwest of Capitol City (photograph 52 ). Another mill is reported to have operated on Henson Creek about a mile west of Capitol City, and that location was confirmed by the BLM (B. Hite, BLM, written commun., 1999). No tailings or slag are evident at these sites.

Leach tests on two samples of tailings show that they produce relatively low amounts of acid and metals, much lower than dump samples from the district.

The concern in the vicinity of the Ute-Ulay mine is the stability of several tailings impoundments that are within 100 yards of Henson Creek. Some are supported by gravel berms but others appear from a distance to be supported only by wooden structures. The mixed private and public land status prevented thorough onsite inspection. Release of these tailings during a major storm event or by failure of an impoundment structure would certainly put these materials into Henson Creek, an important fishery.

Surface waters.-Waters in this drainage generally are clear and produce no iron coatings on stream cobbles, except in the westernmost headwaters near Palmetto basin. Six water samples were collected for analysis. Three stream waters in the lower part of the basin had neutral $\mathrm{pH}$ (6.9-7.4) with low conductivity values. Analyses show low metal concentrations. A sample from Henson Creek at the east edge of Palmetto basin, near several small mines, had a pH of 7.1, notably 
higher conductivity $(240 \mu \mathrm{S} / \mathrm{cm})$ and enrichments in some metals ( $\mathrm{Zn} \mathrm{20 \times ,} \mathrm{Cu} 64 \times$, and $\mathrm{Cd} 9 \times$ ) relative to ALWS. These metals most likely are related to the drainage from small adits that produce modest flows of about 10-20 gpm with $\mathrm{pH}$ values of 2.9 to 3.3 and very high metal concentrations. Samples from Henson Creek east of Capitol City have good quality and do not have significant concentrations of metals (Zn less than $0.7 \times$ ALWS). CZI values are high (10 to 14) in headwaters near Palmetto basin, but are low (0.3 to 0.03$)$ in the lower reaches of Henson Creek east of Capitol City. This reconnaissance sampling suggests that there are local sources of acidic, metalrich waters, but these waters are naturally mitigated by dilution and reactions with green-altered or unaltered volcanic rocks.

Miller (1998) studied the surface waters of the area and the influences of geology on water compositions. His results are similar to those described here, and he reached a similar conclusion that water quality in the main stem of Hensen Creek is good. Miller sampled side streams with sources in altered rocks and found what he termed "high" or "anomalous" concentrations of $\mathrm{Zn}$, Mo, U, F, and rare earth elements-but the concentrations found by Miller are defined relative to other samples in his study and are much lower than from most of the mining districts described here for the CWS.

Summary.-Water quality is better than most would predict from the district's mining history. There are some local point sources of acidic waters in the headwater region near Engineer Pass that deserve further study, but these waters are mitigated by the prevalent green-altered volcanic rocks within a few miles of the mine sources. The uppermost part of Henson Creek, the alpine zone above treeline, has several mine dumps that seem to be more problematic than similar mine dumps at lower elevations. From observations in this brief reconnaissance, the largest risk to the watershed might be physical failure of the mill tailings at the Ute-Ulay mine during an extreme storm.

Further studies should be made of the mill tailings at the Ute-Ulay mine and mill to determine their land status and physical stability. Based on limited information and my preliminary model of tailings failure, these tailings appear to have high risk of failure during an extreme storm flood. Studies are needed to improve models for (1) extreme flood-stage flow in the main stem of Henson Creek (how high would water rise in the narrow gorge at the mine?) and (2) estimates of possible overland flow in gullies on the north side of the canyon that might direct floodwaters to the tailings.

References.-Irving and Bancroft (1911); Slack (1980).

\section{Lake Fork District (Lake San Cristobal)}

Location.-Hinsdale Co., about $5 \mathrm{mi}$ southwest of Lake City (fig. 19). The climate is more moderate than adjacent districts, but has about 16-30 inches of precipitation per year.

Principal commodities.-Silver, lead, zinc, copper, gold.

Mining history.- - The first discovery, in 1874, turned out to be the largest producer-the Golden Fleece mine overlooking Lake San Cristobal. The Golden Fleece is famous for very high grade pockets of gold telluride (petzite) in the veins. The Golden Fleece and adjoining Black Crook mine were well developed on many levels by 1906 (Irving and Bancroft, 1911) but appear to have had little production in later years.

Production and disturbance.-This district had the second largest production in Hinsdale County, which had a total of \$11.2 million. Disturbance is small. One pile of tailings is close to the Lake Fork.

Geology.-Similar to that of the adjoining Galena district but lying on the southern structural margin of the Lake City caldera. Veins in Tertiary volcanic rocks contain base-metal sulfide minerals, with locally rich streaks of silver and gold. Wallrock alteration is intense locally along some veins and is widespread, but some is not associated with mined ore to the east, notably at the Slumgullion landslide.

\section{Mineral-Environmental Conditions}

Mineralized rocks and ores.-Mine dumps were not sampled because most are on private property (mine patents are now summer home sites). The ores are known to be rich in base-metal sulfides such as galena and sphalerite; thus, they have high concentrations of $\mathrm{Pb}, \mathrm{Zn}$, and $\mathrm{Cd}$. Dumps may be locally rich in Se and $\mathrm{Te}$ because there were $\mathrm{Ag}$-Au ore minerals of these elements, but the concentrations of these potentially toxic elements are highly diluted by others kinds of ore and waste rock. Judging from similar ores in adjoining districts (Nash, 1999b), the mine waste may have high acidgenerating potential in small local areas.

Mills and tailings.-There were at least two mills in the district, both near the lake. The Golden Fleece mill may have disposed of tailings into the lake (the mill is so close to the lake that there was little other space). The Contention mill placed its tailings a short distance north of the outlet from the lake, near Argenta Falls. No samples could be obtained from these private sites. The impounded tailings may merit study because they are within the flood plain of the Lake Fork of Gunnison River and are vulnerable to erosion in an extreme flood.

The Black Crook smelter, frequently mentioned in the literature, could not be identified. A likely location would be at Crook Falls, 1 mi southeast of the town of Lake City, an area of private property that could not be investigated. Slag from this smelter probably does not release metals to the Lake Fork.

Surface waters.- Streams in this district appear to be of good quality, judging from their clarity, absence of iron coatings on stream cobbles, and field measurements. Indicators of good quality are the consistently very low conductivities, most less than $50 \mu \mathrm{S} / \mathrm{cm}$, and $\mathrm{pH}$ near neutral ( $\mathrm{pH} 6.8$ to 7.0). Chemical analyses show the waters to be very low in metals. Zinc, which generally is the most mobile metal released from polymetallic waste, is far below ALWS. Zinc concentrations of $18 \mathrm{ppb}$ or less found in these samples are well below the median concentration for the CWS. CZI values for seven 
stream samples are very low $(<0.1)$, which suggests low threat to aquatic life. There may be some local contamination in the district, such as on private sites that were not accessible, but these do not have significant influence on the larger streams or the main stem of the Lake Fork.

Summary.-Water quality appears to be good in this district. Although there was substantial mining at some sites, disturbance was not extensive, and highly degraded mine drainage is not evident. There are substantial natural sources of metals in unmined, highly altered rocks, including those in the Slumgullion Slide, thus the geochemical background in this area was not pristine. The good water quality, shown by geochemical indicators, appears to reflect the ability of weakly altered volcanic rocks to mitigate local sources of acid and metals. Construction of homes on mine dumps may aggravate conditions at some localities. One tailings pile near the Lake Fork may need to be stabilized; further study of this private site is recommended.

$$
\text { References.-Irving and Bancroft (1911); Slack (1980). }
$$

\section{Mining Districts of the Uncompahgre River Watershed}

The headwater region of the Uncompahgre River drains four mining districts with major production that spanned slightly more than 100 years (fig. 20). This area on the northwest flank of the Silverton Caldera is noted for numerous large veins that are radial to the caldera and formed some 10 million years after the volcanism (Burbank and Luedke, 1968; Lipman and others, 1976). A different style of small, but very rich orebodies formed in breccia pipes associated with post-caldera intrusions in the ring fracture zone, most notably near Red Mountain Pass. An older stage of ores formed near Ouray in sedimentary rocks adjacent to mid-Tertiary intrusions. Production from these three kinds of ore in Ouray County had a gross value of about $\$ 111,000,000$, chiefly from gold and silver, but the values of copper, lead, and zinc were much higher than in most of the districts on the CWS. The high relief and deep canyons of the region were favorable for the construction of haulage tunnels several miles deep into the mountains that allowed efficient haulage of ore, much of which actually came from San Miguel County (Telluride district). This style of mining had the consequence of creating major complexes for milling of ore, dumping of waste, and draining of mine water on the Ouray County side of the mountain divides. These major mine-mill complexes, including the Idarado mine (Treasury Tunnel), the Revenue Tunnel, and the Camp Bird mine, are mostly on private (patented) lands but pose questions for managers of adjacent public lands administered by the USFS. Reclamation in parts of this watershed will have to be considered in the context of the Permit for the Idarado Mine Site Remedial Action Plan (CDH, 1992), which addresses issues far beyond the geochemical scope of this study.

\section{Ouray District (Uncompahgre)}

Location.-Ouray County, adjacent to Ouray (fig. 21). The climate is relatively moderate for the CWS, with about 20-30 inches of precipitation per year.

Principal commodities.-Silver, gold, lead, copper, zinc.

Mining history.-Ore was discovered in 1875 and mining commenced shortly thereafter. The first lodes to be discovered were rich in gold, but subsequent discoveries were rich in silver and lead. Some of the most productive years were in the 1890 's. Mining has not been productive since the 1950's, but the major mill in the district treated ore from other districts through 1952.

Production and disturbance.-The total value was about $\$ 12.7$ million from about 392,500 tons of ore (Long and others, 1998). Generally small disturbance from underground mines. Two mill tailings impoundments near the Uncompahgre River are potential sources of contaminants.

Geology.-Ores in this district are unusual for the study area for their Laramide age (Late Cretaceous to early Tertiary). This is clearly shown by the erosional surface above altered rocks and contrasting overlying unaltered mid-Tertiary rocks (this is visible from downtown Ouray if one looks to the northeast). The geologic section, from Precambrian through Tertiary, is generally similar to nearby districts. More than 4,000 ft of Devonian through Cretaceous sedimentary rocks are well exposed in the canyons, and this is the type locality for the red sandstones of the Cutler Formation (Cutler Creek). Dikes and sills of early Tertiary porphyries intrude and alter the sedimentary rocks, most notably at the "Blowout," well exposed in the canyon wall east of Ouray (Luedke and Burbank, 1962). Ore deposits are associated with the porphyry intrusions.

Ore deposits are polymetallic veins and replacements that in detail vary in mineralogy. The most important deposits contain base-metal sulfides with silver tellurides and gold. Sulfide veins tended to expand outward into replacement zones when they encountered reactive carbonate rocks. The replacement or "manto" deposits thus resemble ore deposits near the Continental Divide (e.g., Leadville and Tomichi districts) rather than the nearby Sneffels and Red Mountain districts, which are chiefly in volcanic rocks.

\section{Mineral-Environmental Conditions}

Mineralized rocks and ores.-Analyses of three samples of dump rocks and three samples of tailings confirm the polymetallic character of this district. The mine waste tends to have high concentrations of $\mathrm{Mn}$ and $\mathrm{Pb}$ relative to other districts. Some dump compositions are high in $\mathrm{Bi}$ and $\mathrm{Mo}$, and moderate (near the study median) in $\mathrm{Ag}, \mathrm{As}, \mathrm{Cu}$, and $\mathrm{Zn}$. Laboratory leach tests on four samples (one dump, three tailings) yielded solutions with $\mathrm{pH}$ values of 3.2 to 4.1 , but concentrations of base metals in the leachates were lower than from most polymetallic deposits on the CWS. One sample 


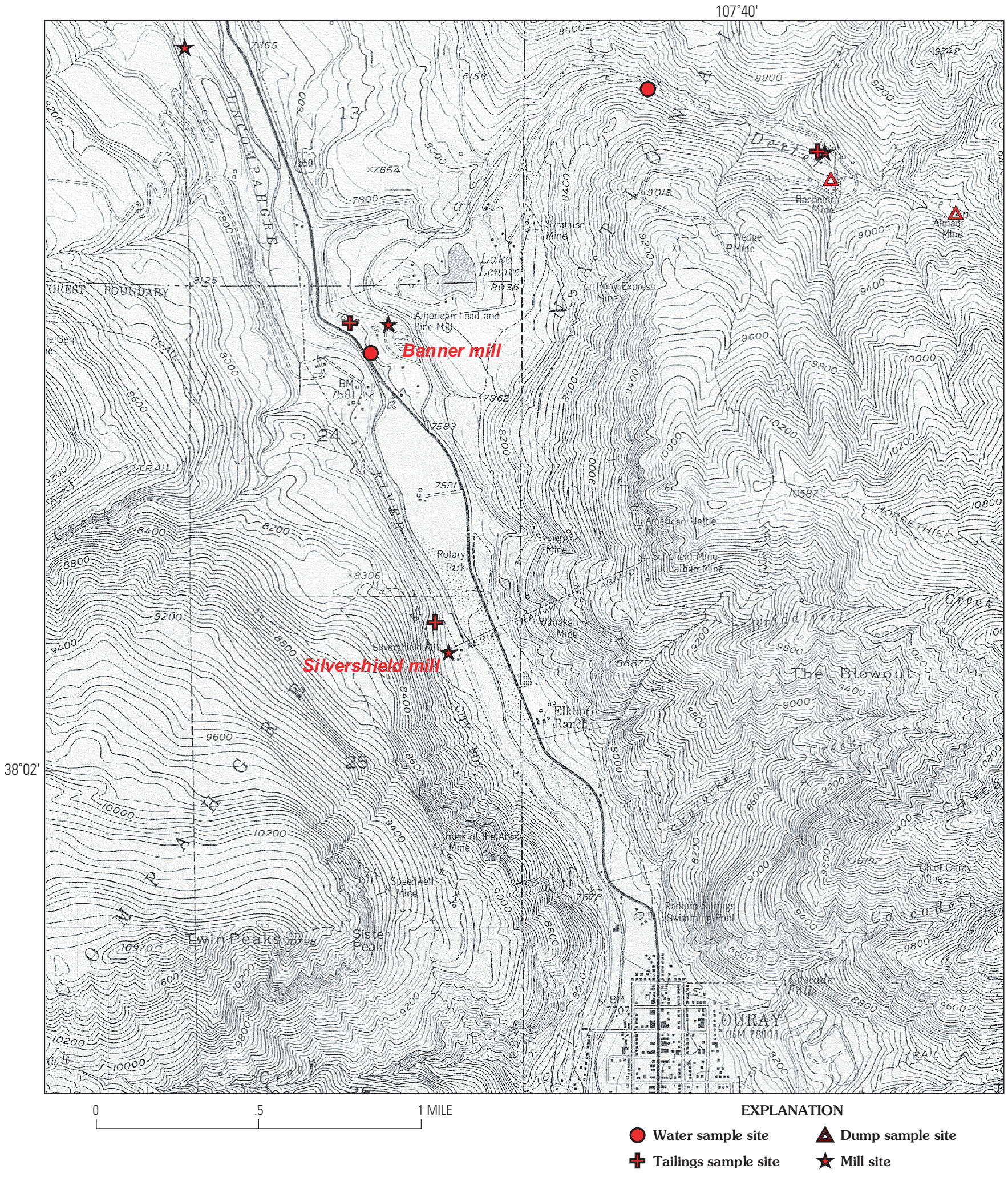

Figure 21. Features of the Ouray mining district, showing sample localities. 


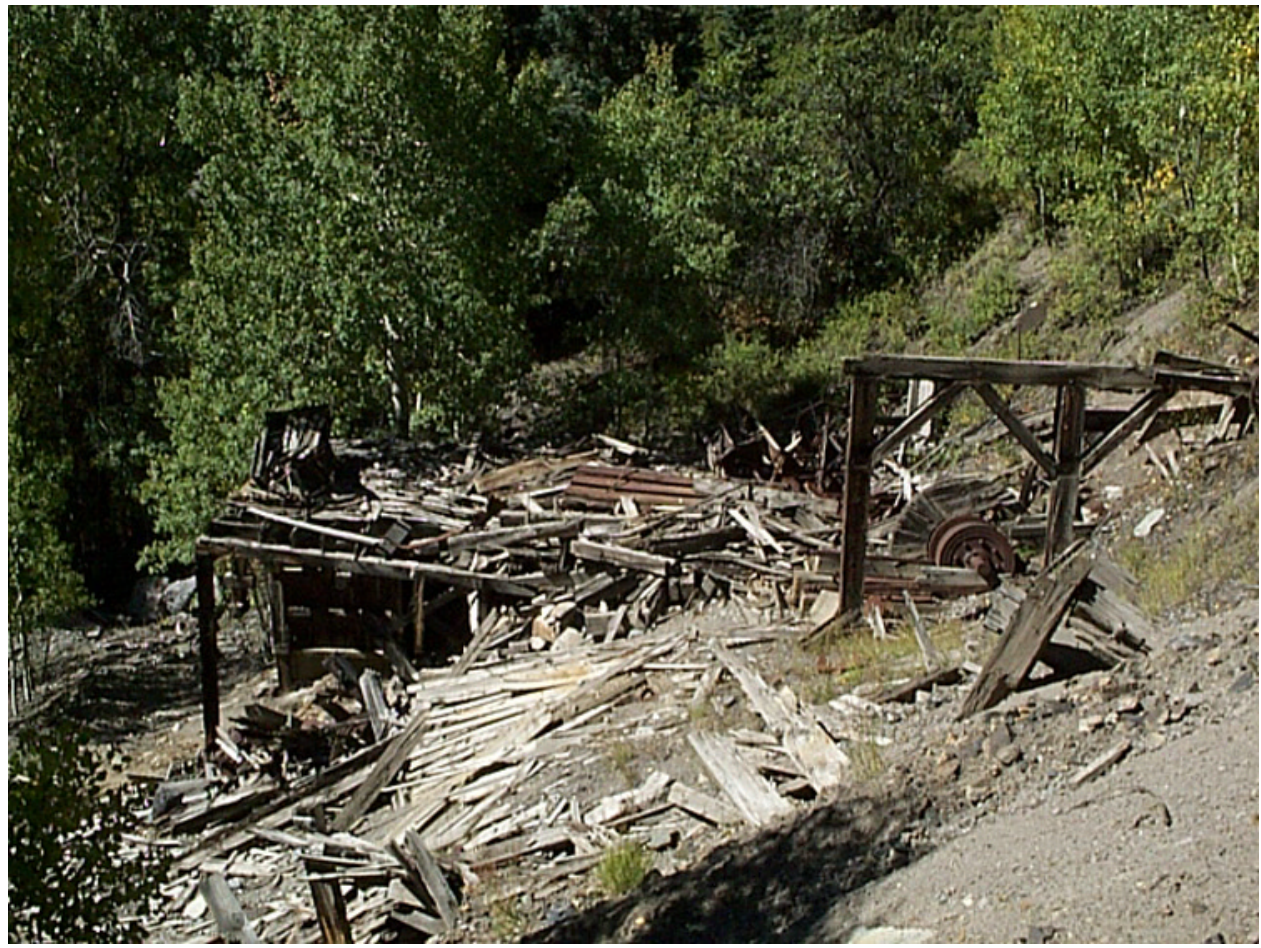

Photograph 54. This small stamp mill on Dexter Creek probably operated before 1900. Traces of tailings are in the foundation, but others were carried down the creek.

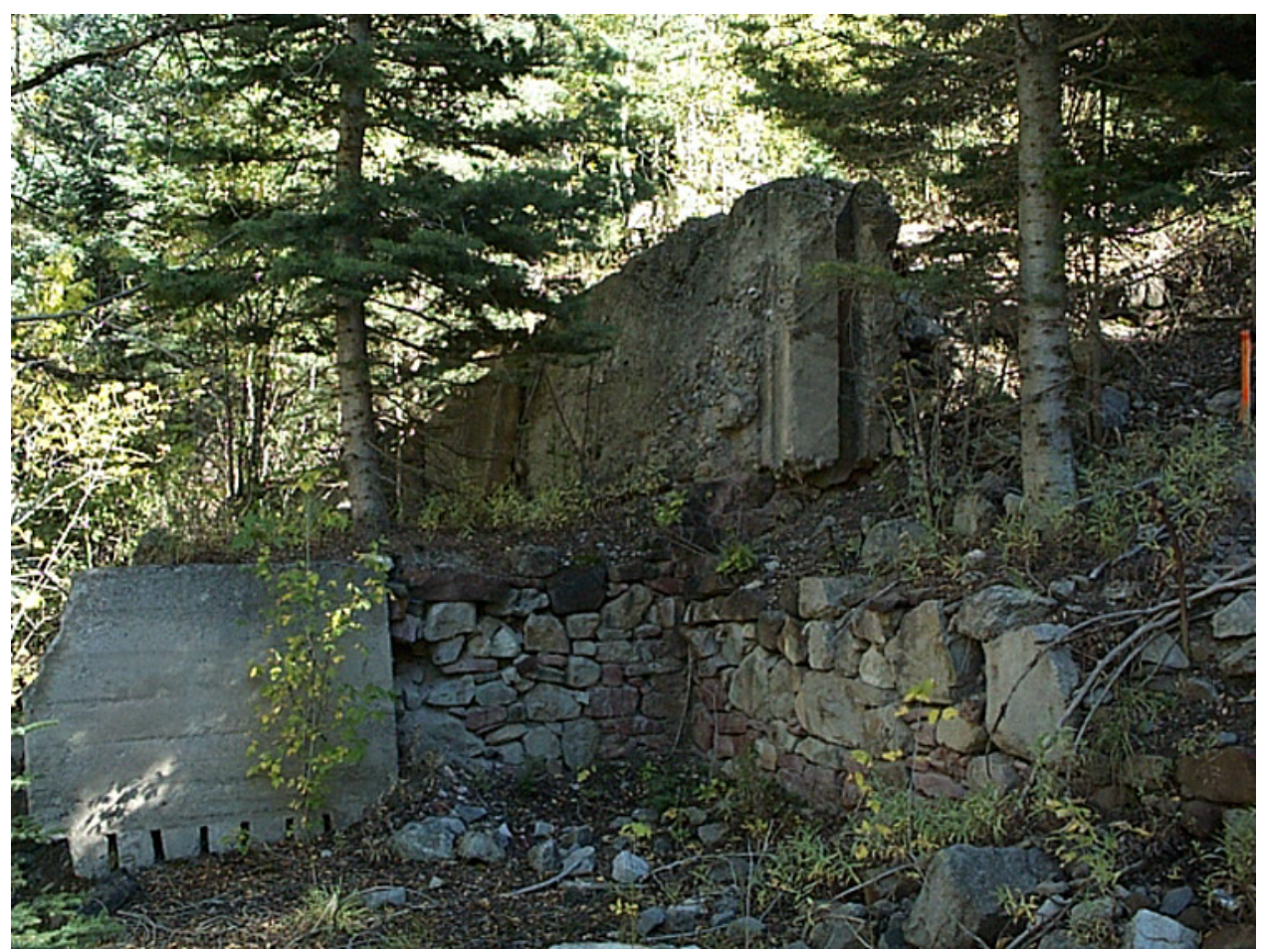

Photograph 55. This stone and concrete foundation probably was for a mill located just below the old railroad near Corbett Creek about 4 mi north of Ouray. 


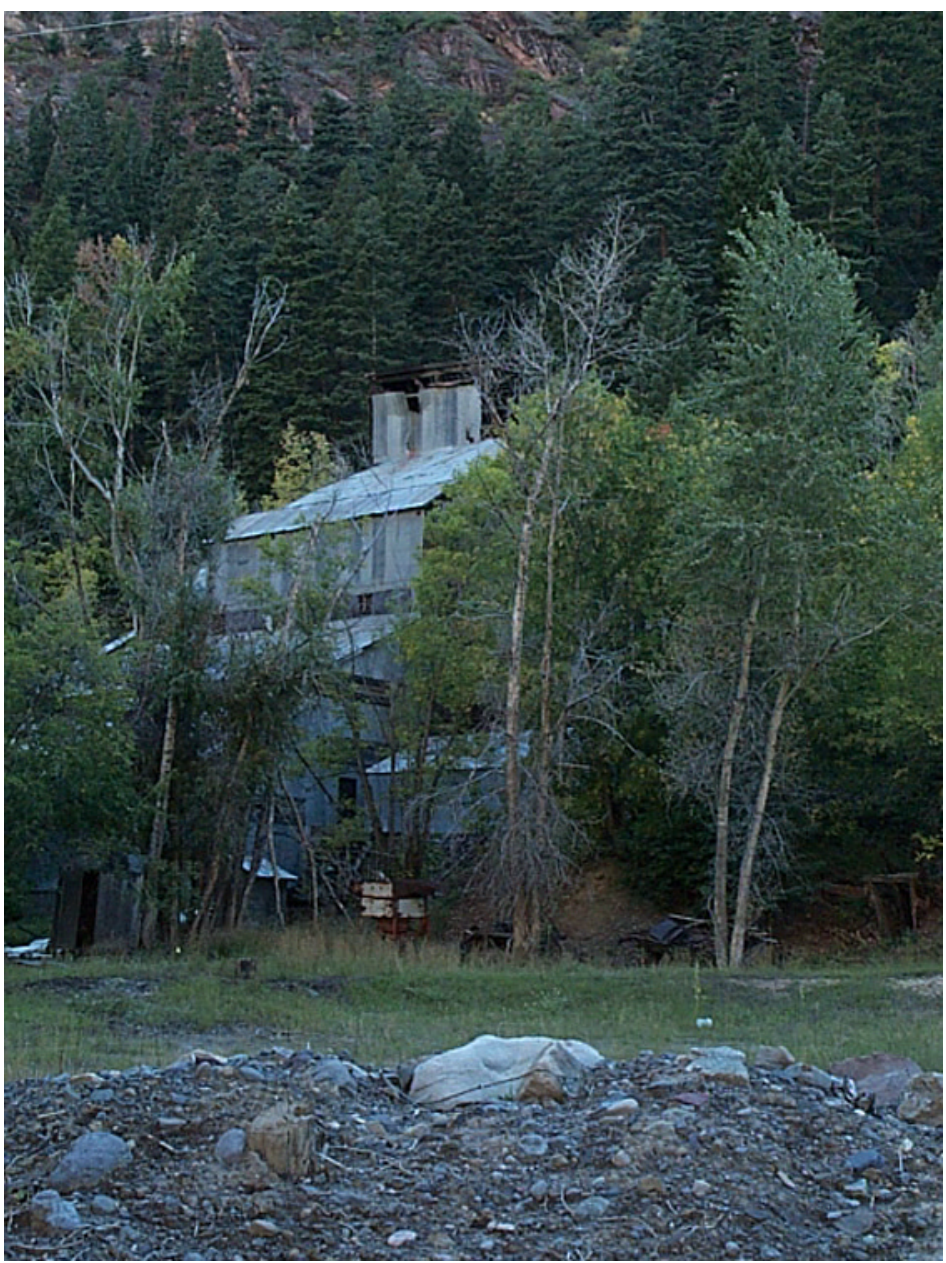

Photograph 56. The Silvershield mill processed ore carried in by a tram to mines above the red rock cliffs north of Ouray.

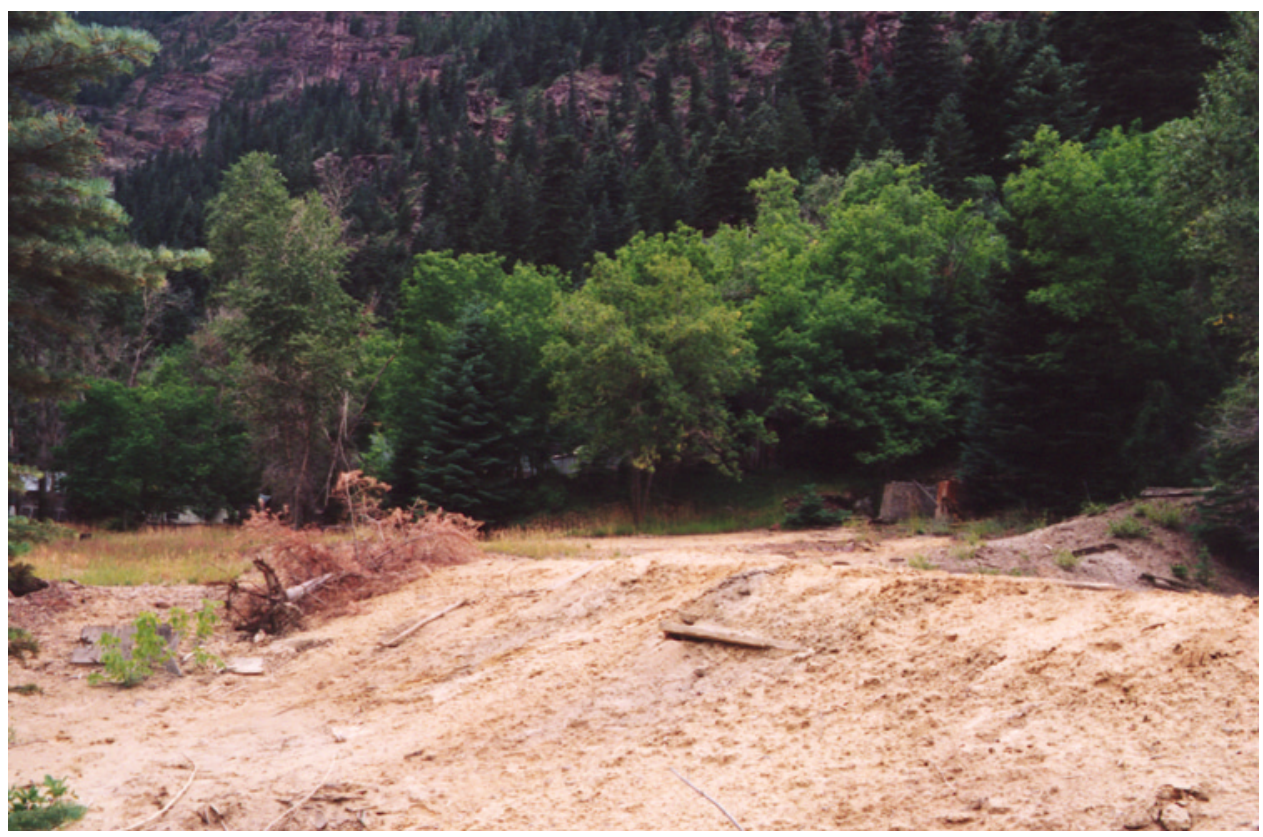

Photograph 57. Tailings from the Silvershield mill are about 100 yards west of the Uncompahgre River. They are relatively stable here but are accessed by visitors. 


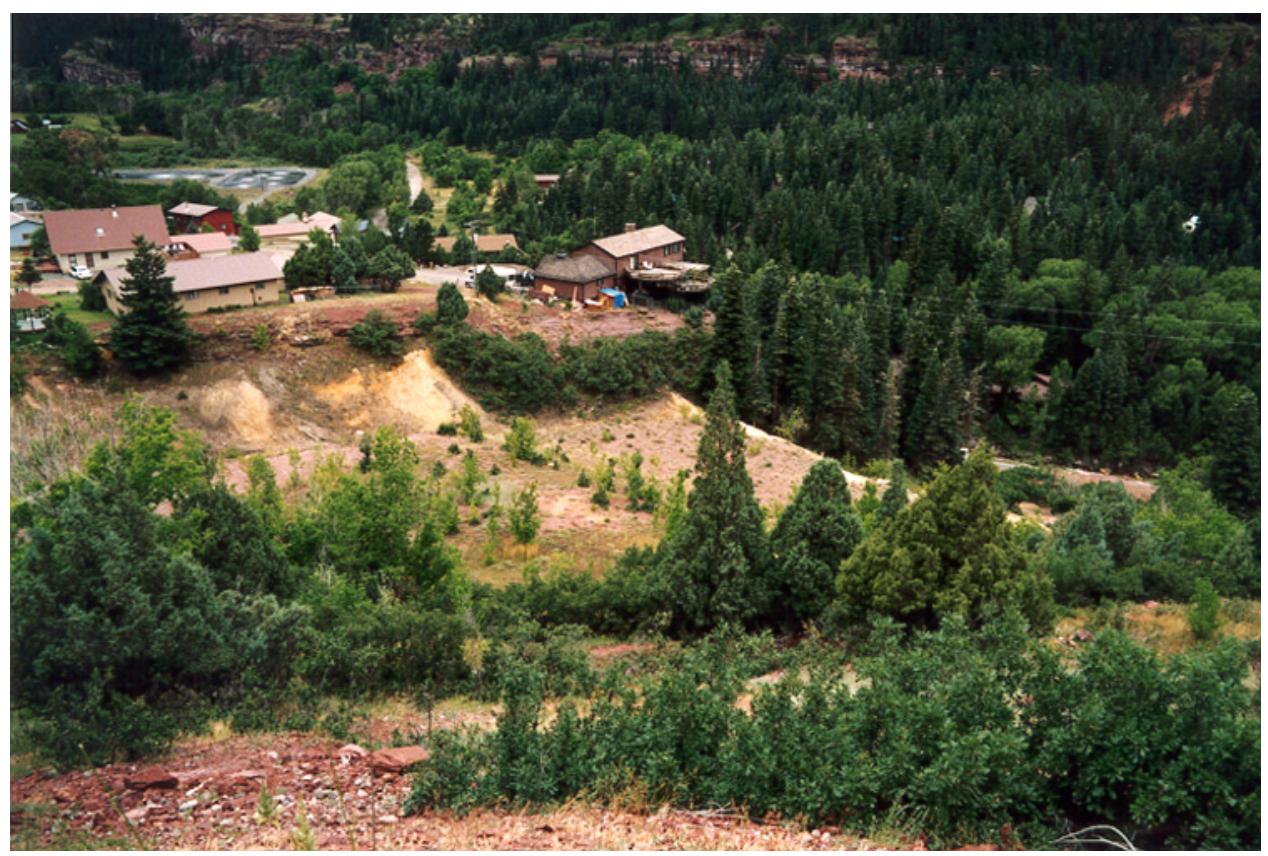

Photograph 58. The Banner mill site, 2 mi north of Ouray, was developed for housing in the 1970's, but the tailings were left in place. Homes are built on the tailings. Storms wash tailings down the gulch toward the Uncompahgre River.

of tailings from the foundation of the Bachelor mill yielded extremely high concentrations of $\mathrm{Pb}, \mathrm{Zn}, \mathrm{Cd}, \mathrm{Cu}$, and other metals, suggesting either that this old mill was very ineffective at removing metals, or that my sample was spilled concentrate, not tailings. These tests suggest that the mine waste generates more acid and releases more metals than expected for typical carbonate-hosted ores.

Mills and tailings.-I have located five mills in this district, but only two are described in the literature. One small mill is on Dexter Creek (photograph 54), a short distance northwest of the Bachelor mine; no tailings remain. A concrete foundation remains adjacent to the old railroad grade north of Ouray near Corbett Creek (photograph 55); no tailings are evident. A "modern" custom mill stands next to U.S. Highway 550, 3 mi north of Ouray; the tailings piles are small if any at this private site. The Silvershield and the Banner (American Lead and Zinc) mills are shown on the USGS Ouray topographic map. The Silvershield mill (photograph 56) received ore by tram from mines to the east; tailings cover about an acre on the west side of the Uncompahgre River (photograph 57). This site is being developed for homes. The Banner mill (photograph 58) started operations in the 1930's, was expanded to 350 tons/day in the 1940's and was closed in 1952. The mill was dismantled and the site has been developed for homes; about a dozen homes have been built on the tailings, placed on a bench of red rocks east of the river. Storm events carry some tailings and also acidic drainage over the edge of the bench toward U.S. Highway 550 and the river. Unreclaimed mill tailings at the Banner and Silvershield mill- sites may be vulnerable to erosion in extreme flood conditions.

Surface waters.-Water in Dexter Creek below some of the historic mines is clear and produces no red iron stains or coatings. Field measurements showed neutral $\mathrm{pH}$ values (6.9 to $8.2)$ and low to moderate conductivity (120 to $230 \mu \mathrm{S} / \mathrm{cm}$ ). A water sample collected below the mill and mines had surprisingly high concentrations of $\mathrm{Cd}, \mathrm{Cu}$, and $\mathrm{Zn}(1 \times, 11 \times$, and $4 \times$ ALWS, respectively) for a $\mathrm{pH}$ value of 8.2. The CZI value of 6 suggests this water could be a threat to aquatic life.

The Uncompahgre River in the vicinity of Ouray tends to be turbid and leaves deposits of tan to ocher materials on stream cobbles. My tests of water chemistry are not sufficiently specific to distinguish contamination from local sources from known sources upstream. The $\mathrm{pH}$ of 6.9 and conductivity of $210 \mu \mathrm{S} / \mathrm{cm}$ in the Uncompahgre River at Ouray suggests that quality is improved from that in the Red Mountain Creek source area to the south (described later), but chemical analyses show that $\mathrm{Fe}$ and $\mathrm{Zn}$ concentrations remain high ( $1 \times$ and $3.3 \times$ ALWS). Two samples from the Uncompahgre River collected in September of 1999 on the north side of Ouray, and north of Ridgeway (near inlet of Ridgeway Reservoir) contained concentrations of $\mathrm{Cd}, \mathrm{Cu}$, and $\mathrm{Zn}$ greater than ALWS. The CZI values for these river samples are 3.7 and 2.0, suggesting a possible threat to aquatic life.

Water seeping over the sandstone bench at the former Banner mill site leaves red coatings, and sample 309 taken during a rainy week in June had a $\mathrm{pH}$ of 3.4, conductivity $>2,000 \mu \mathrm{S} / \mathrm{cm}$, and very high concentrations of $\mathrm{Al}, \mathrm{Cd}, \mathrm{Cu}$. $\mathrm{Fe}$, and Zn. This particular seep flowed at only a few gallons/ 
minute but with very high concentrations ( $>100 \times$ ALWS) that could pose problems for wildlife.

My reconnaissance water samples in the Ouray area suggest that more detailed work on water quality and biota on the middle Uncompahgre River and its tributaries are needed to evaluate the aquatic habitat.

Summary.-Mines in this district appear to have low impact on surface-water quality, but more base metals may be carried in surface waters that one would expect for $\mathrm{pH} 7$ to 8 waters. Mill tailings in this district may be significant sources of contamination. The turbidity and elevated metal concentrations in the Uncompahgre River in the vicinity of Ouray is chiefly from the Red Mountain district, but there also is some input from the Ouray district. Also, mill tailings at two sites are reactive and were not effectively reclaimed as of 1999. Construction of homes on mill tailings may be adding domestic water that compounds the input from tailings to the Uncompahgre River.

References.—Burbank (1940, 1947a); Luedke and Burbank (1962).

\section{Sneffels District}

Location.-Ouray Co., $10 \mathrm{mi}$ southwest of Ouray (fig. 22). The climate is severe, with about $25-40$ inches of precipitation per year.

Principal commodities.-Silver, gold, lead, copper, zinc Mining history.-Several deposits in the district were located in 1875 and substantial mines were operating in the 1880 's. The Revenue Tunnel was one of the first in the region to be driven deep into a mountain (7,600 ft) in 1896 to facilitate mining of the Virginius and other veins high on the ridge and beyond to the Telluride side. Chronic problems with avalanches and water pumping from shafts were alleviated by the tunnel. The mill at the Revenue Tunnel has huge for the time with 350 tons/day capacity. It began production during the era of depressed silver prices, but the discovery of the gold-rich Montana vein in 1909 boosted its revenues. However, in 1911 the Ophir tunnel was developed to carry the ore west to the Tomboy mill on the Telluride side. The Revenue struggled a few more years, and the mill burned in the 1920's, marking the end of its activity.

The most famous mine in the district, the Camp Bird, was not located until 1896 but soon developed into a large operation. In 1898, a 9,000-ft-long tram was built to carry ore down to a stamp mill at Canyon Creek to avoid avalanche problems above treeline in Imogene basin. The mill burned in 1906 but was replaced by a bigger one. In 1916, the 14th-level tunnel, more than $10,000 \mathrm{ft}$ long, was developed to carry ore to the mill and drain the mine workings. Mining continued intermittently for the next 60 years, with expansion of the mill on Canyon Creek to 500 tons/day in 1956 and development of deep Cu-rich replacement ores in the Telluride Conglomerate in the 1970's. The mine ceased operations in 1977, but the mill processed a limited amount of custom ore in the 1980's. A gold-exploration venture reopened upper mine workings in the late 1980's. The mine was inactive in the 1990's and reclamation work was underway in 1999.

Production and disturbance.-Because figures tend to be aggregated by county or by place of milling rather than mining, the totals for production are unclear. Total value of ore produced was very high, about $\$ 50$ million, much of which came from the Camp Bird mine. Total production from the district is estimated to have been 3,108,000 tons through 1978 (Long and others, 1998). Disturbance is moderate.

Geology.-This district lies just northwest of the margin of the mid-Tertiary Silverton caldera and adjacent to the Stony Mountain intrusive complex (under Mount Sneffels). Most exposed rocks are volcanic flows and tuffs, but important intrusions are present singly and in clusters or dike swarms. A thick section of Devonian to Jurassic sedimentary rocks lies between the Tertiary rocks and Precambrian basement (Burbank and Luedke, 1964).

Most ore came from vein deposits in the volcanic rocks, but important replacement ores rich in base metals were found in the Telluride Conglomerate adjacent to the veins. The radial aspect of the veins relative to the San Juan caldera has received careful attention and mapping (Burbank and Luedke, 1968). The early mining, through about 1900 , focused on the upper portions of veins that were oxidized and rich in gold (Fisher, 1990). Later mining at depths of 1,000-2,000 $\mathrm{ft}$ worked polymetallic sulfide veins in volcanic rocks and polymetallic replacements in calcareous parts of the Telluride conglomerate.

\section{Mineral-Environmental Conditions}

Mineralized rocks and ores.-Ores from this district are known to be rich in base and precious metals; values are highly variable in detail along a vein, and gold in particular is erratic. Only one dump sample was collected, from the Eclipse mine, and it is probably not representative of the district in general. Concentrations of base metals are moderate in the one sample. The polymetallic ores probably are similar to those from the Silverton area (Nash, 1999a, 1999b). Another approach to estimating composition and input to the aquatic environment is information from a stream sediment collected from Canyon Creek below the Camp Bird mine. The metal concentrations are high in this stream-sediment sample. In particular, concentrations of $\mathrm{As}, \mathrm{Ag}, \mathrm{Bi}, \mathrm{Pb}$, and $\mathrm{Zn}$ are higher than in the nearby Camp Bird tailings, suggesting the likelihood of multiple sources of mineral contaminants to the streambed. Stream-sediment compositions were systematically studied as part of the GMUG project (Smith, in press).

Mills and tailings.-This very productive district has been supported by many mills that have created tailings piles that range in size from small at the Eclipse mine (tens of thousands of tons?) to very large at Camp Bird (several millions of tons) (photograph 59). As in other districts, the mills and tailings share the common feature of being located close 


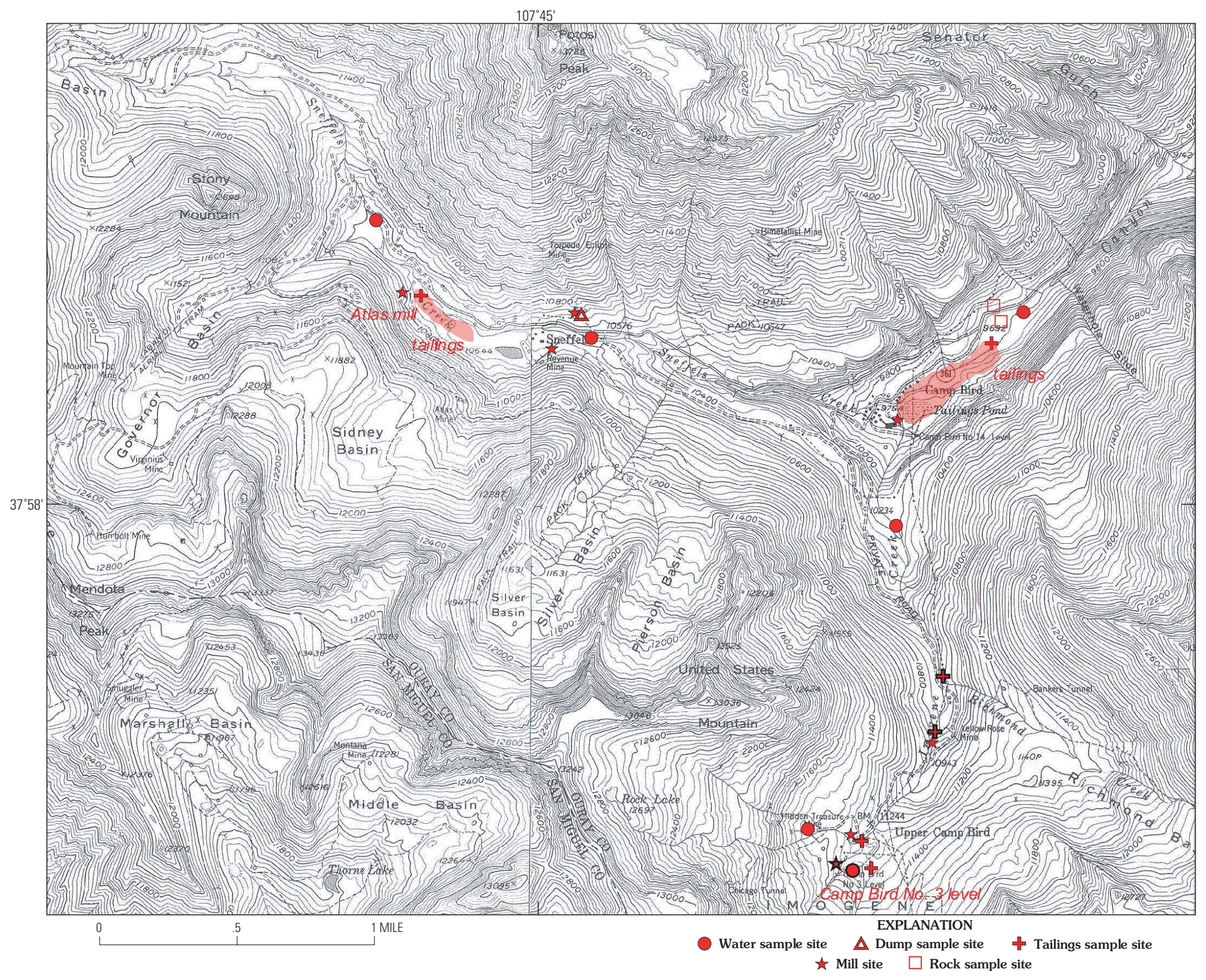

Figure 22. Features of the Sneffels mining district, showing sample localities. 


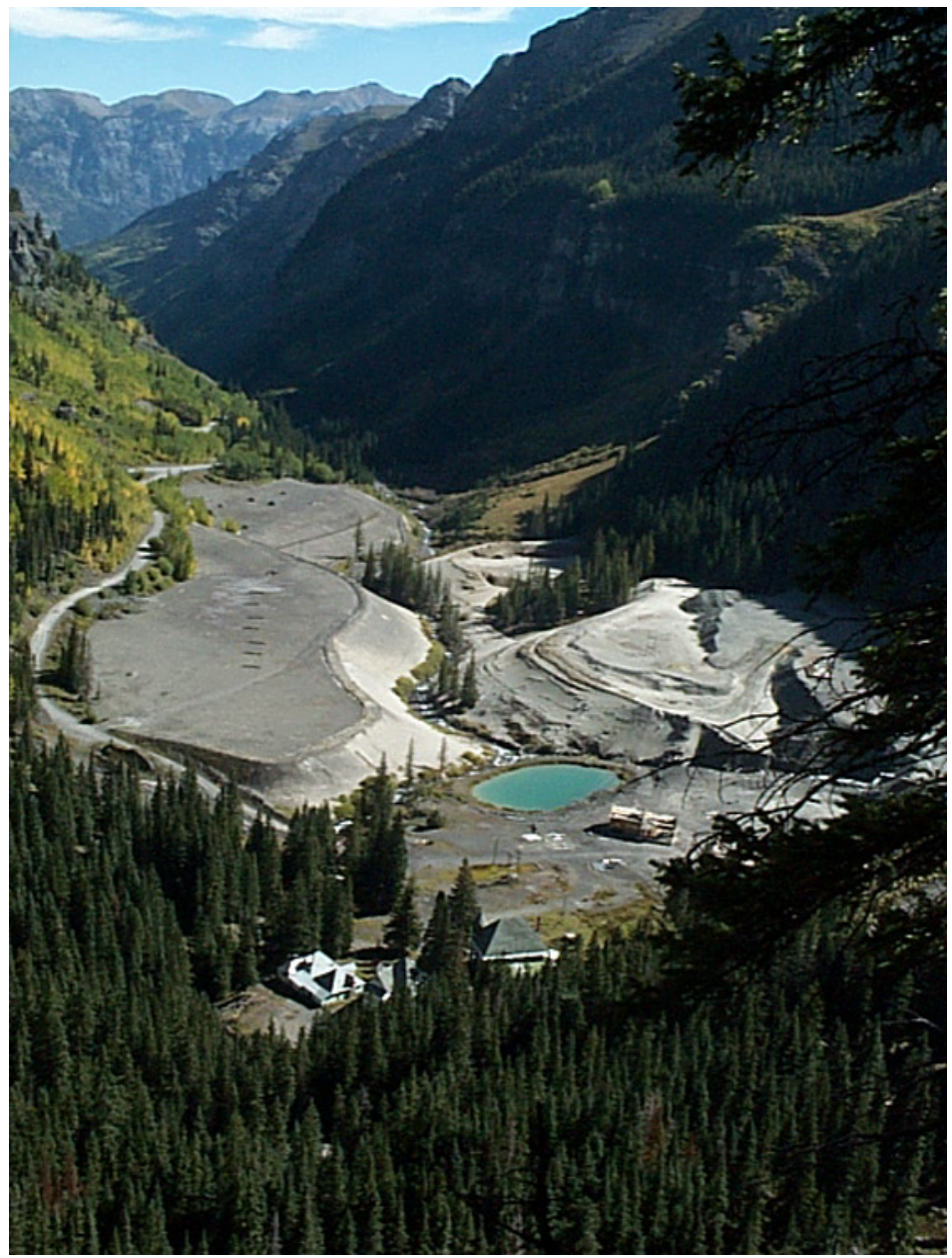

Photograph 59. The Camp Bird mine was the major producer in the Sneffels district and created large waste and tailings piles along Canyon Creek. The high acid-neutralizing capacity of the green-altered volcanic rocks is important in the natural mitigation of effects from these materials.

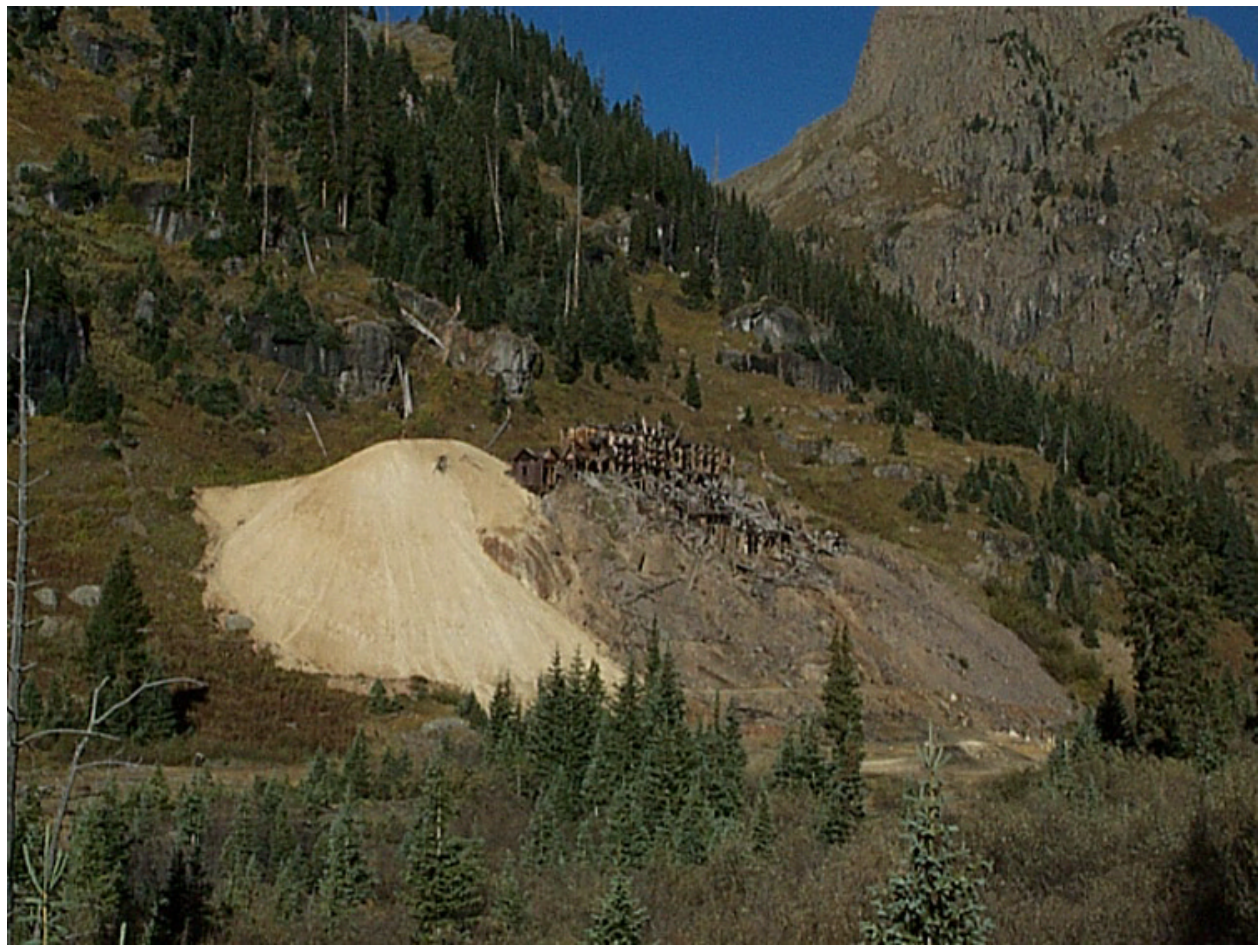

Photograph 60. The Atlas stamp mill was a significant producer in the early years of mining in the Sneffels district. Tailings are on the left side of the mill, and also on the flood plain below. 


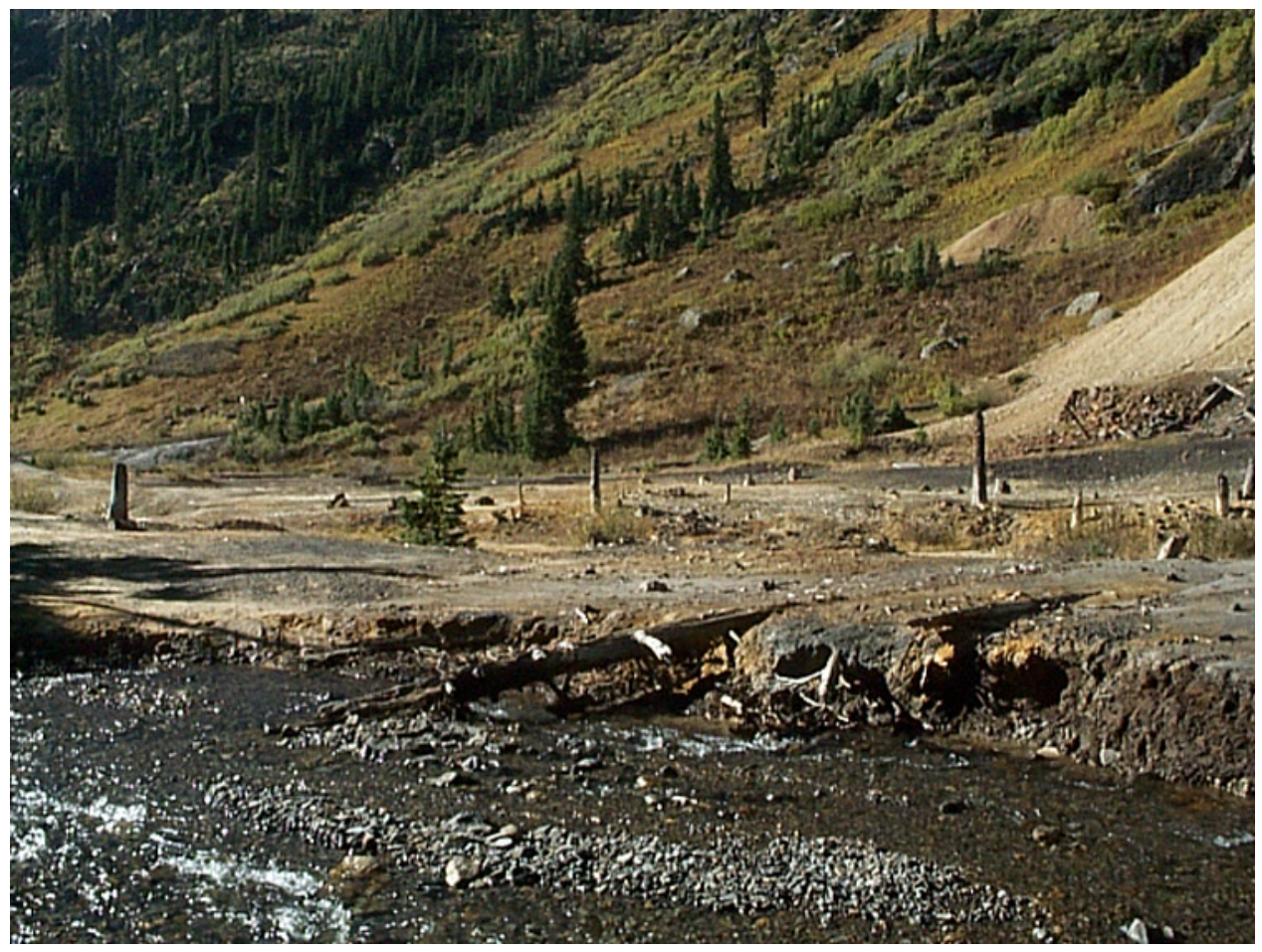

Photograph 61. Mill tailings from the Atlas mill were placed along and in Sneffels Creek with no concern for their impact on water quality prior to regulations against this practice, which were enacted in 1935.

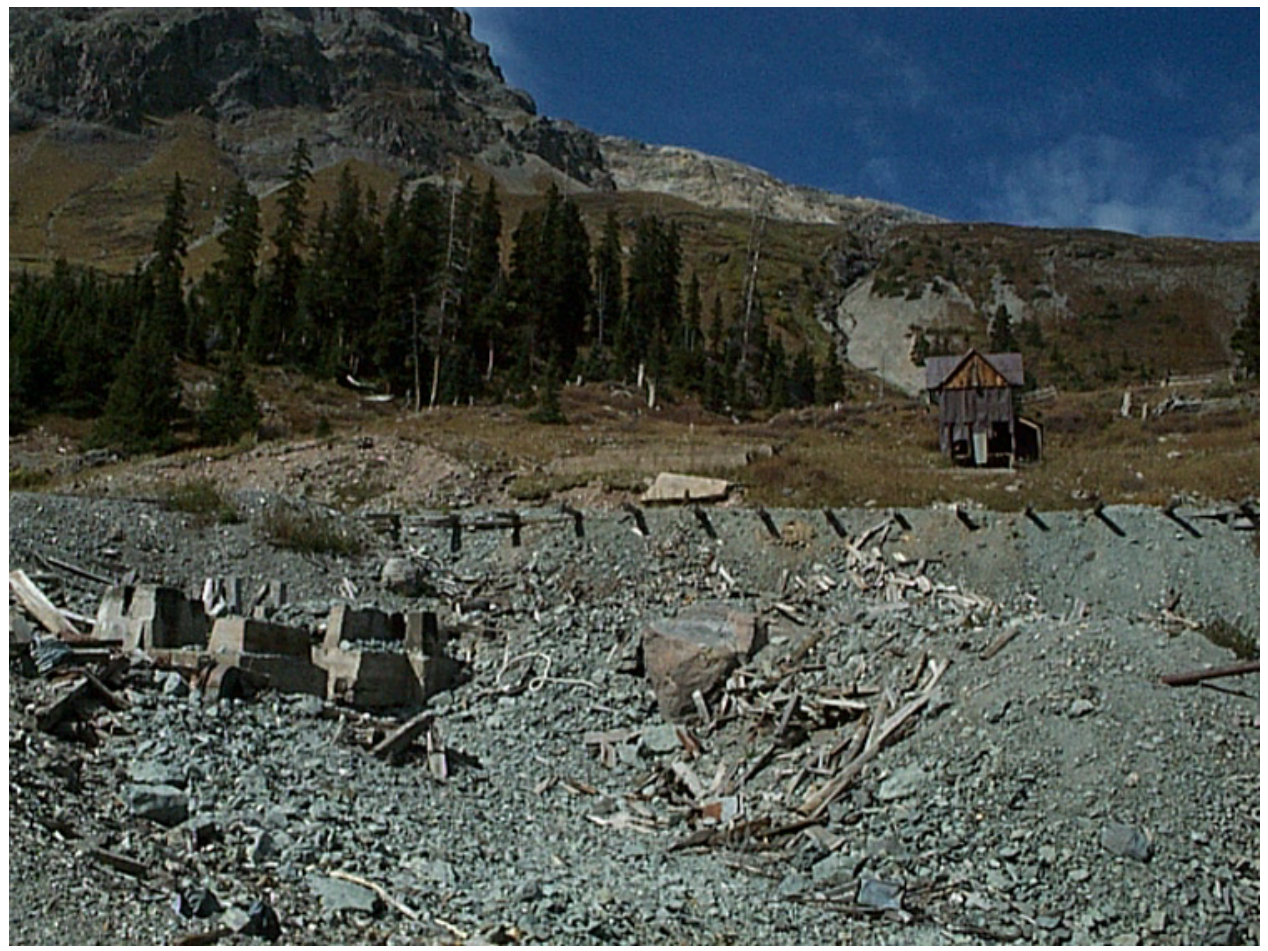

Photograph 62. These cement pieces of a foundation are all that remains of the Camp Bird 3-level mill. The green rocks were dumped on top of the mill site in recent years (1980's?). Other concrete foundations are above the dump, near the wooden building. 


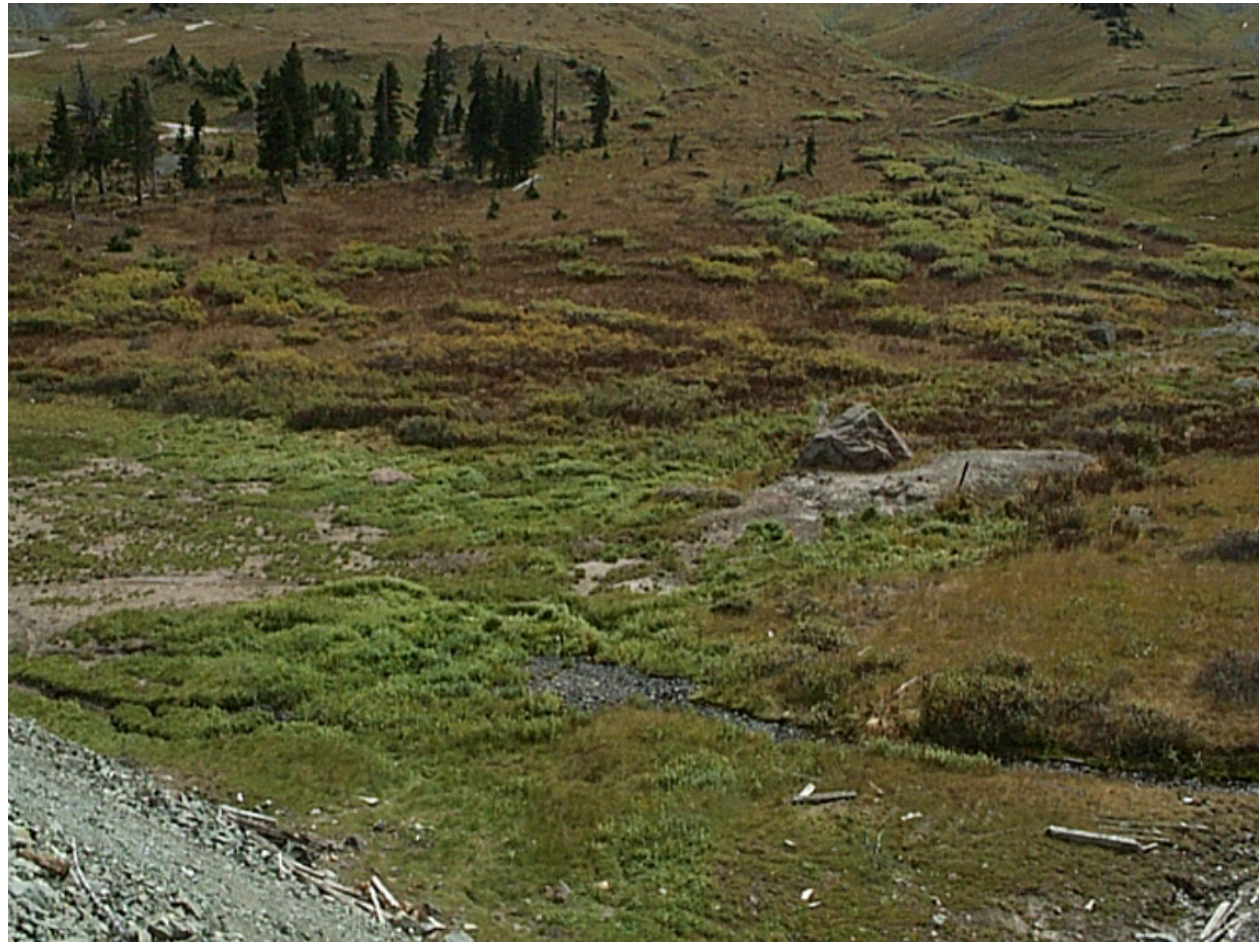

Photograph 63. The Camp Bird 3-level mill produced this small volume of tailings before problems with avalanches forced the construction of a new mill on the 14th level near Canyon Creek.

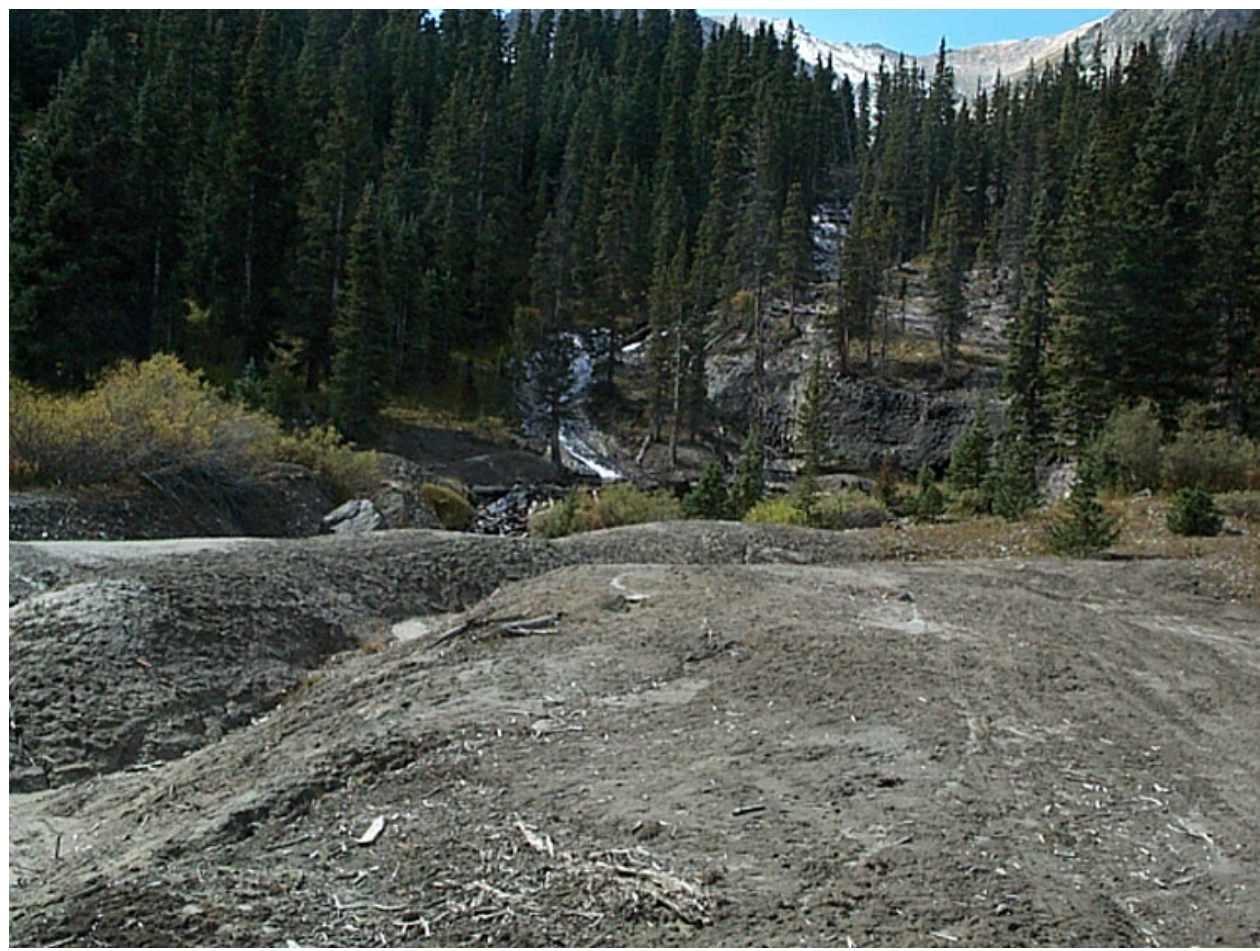

Photograph 64. These eroded gray tailings are from a small mill, ca. 1900, on Imogene Creek between the Camp Bird 3- and 14-level mills. The mill, at the base of the cliff and waterfalls, probably used water power. 


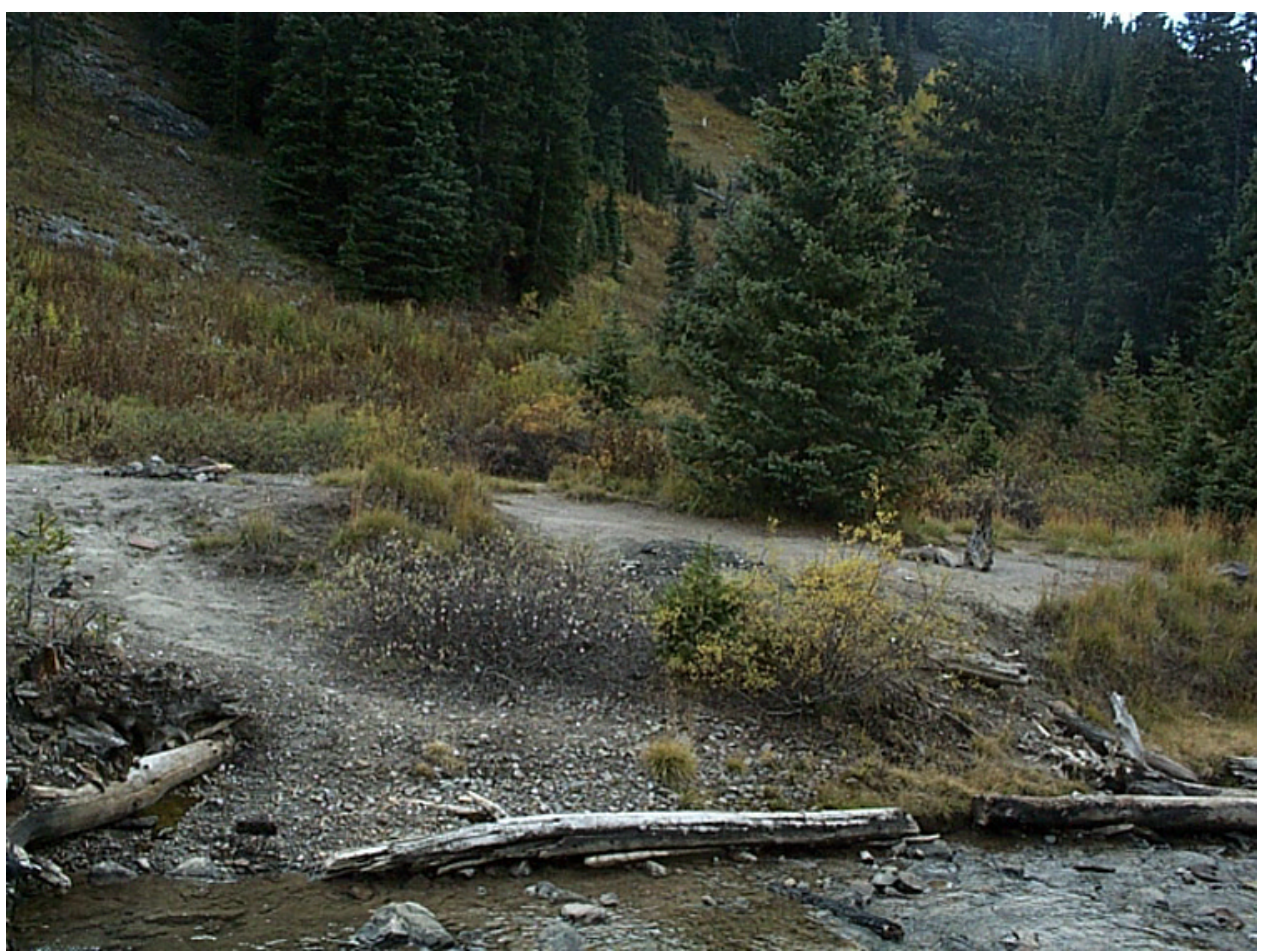

Photograph 65. Overbank deposits of tailings can be seen at several places along Imogene and Canyon Creeks. These are in the middle reach of Imogene Creek, probably from the Richmond mill $1 \mathrm{mi}$ to the southwest.

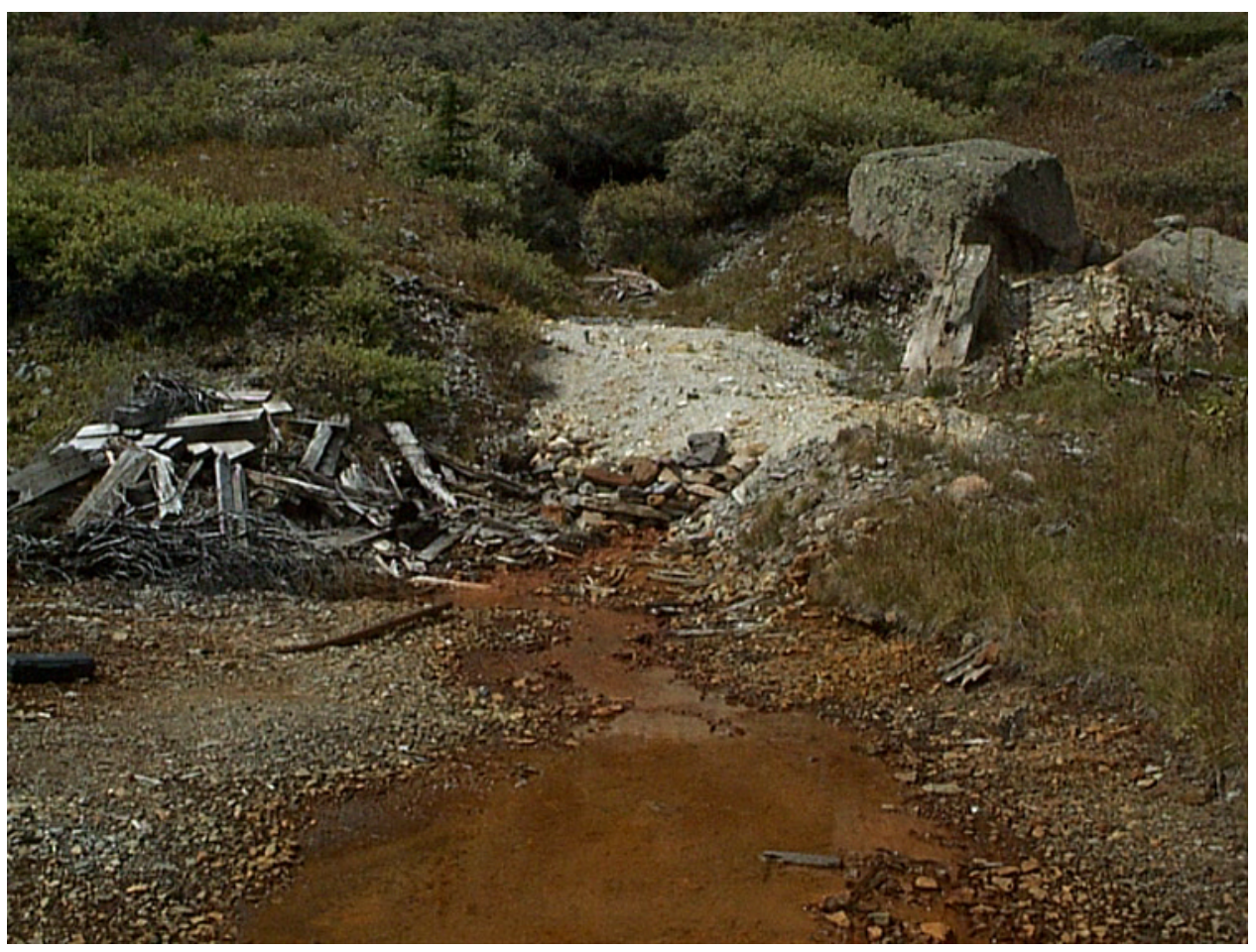

Photograph 66. The Hidden Treasure mine is one of the few in the Sneffels district that produces acidic mine drainage. The light-colored rocks on the dump with pyrite-sericite alteration may be the reason for the acid generation. 


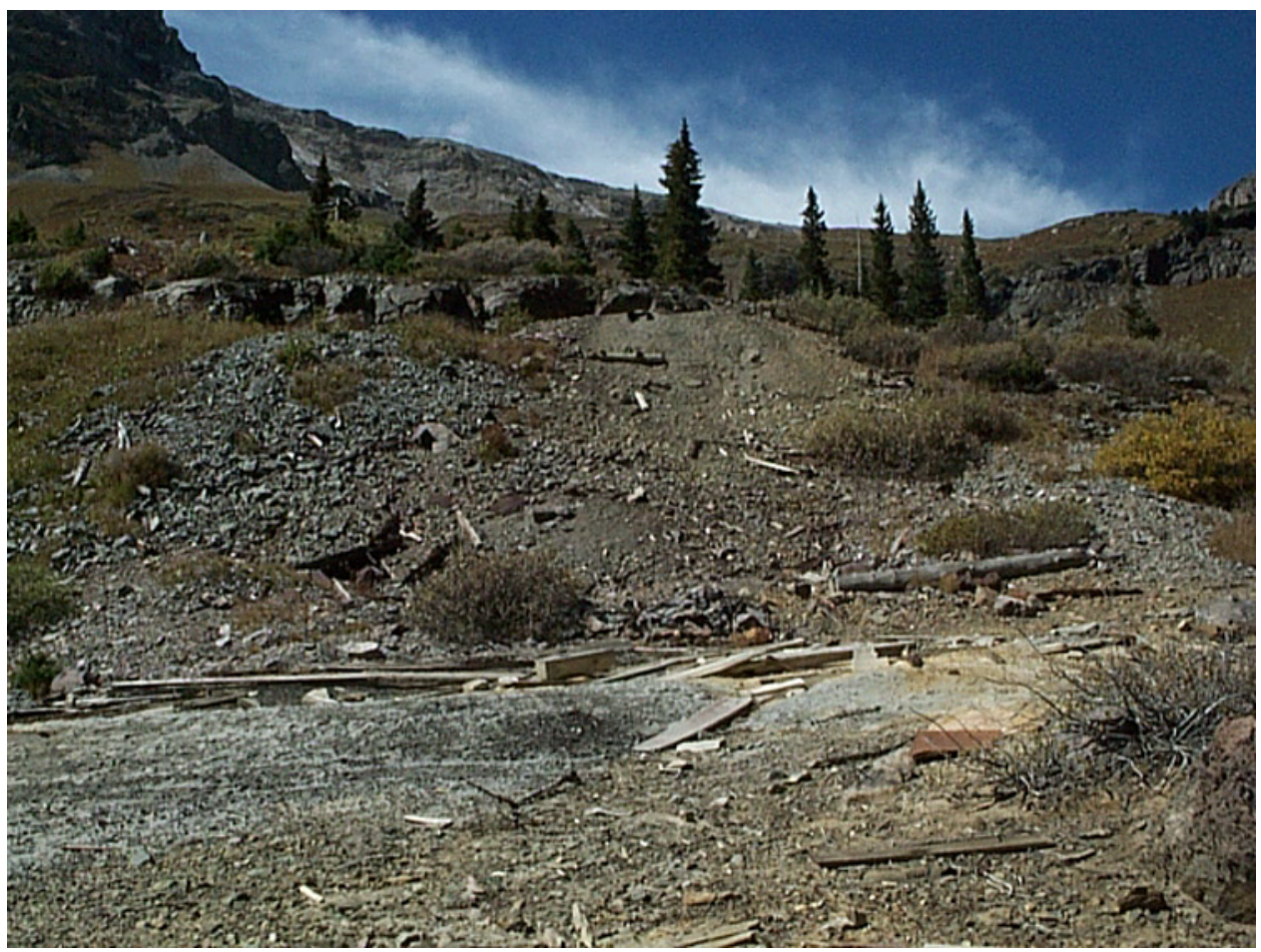

Photograph 67. The Hidden Treasure mill was only 100 yards north of the Camp Bird 3-level mill. The mill was located on the cut (center of photograph) and placed the tailings on a small flat area and presumably in Imogene Creek. These ocher tailings release more acid and metals, according to laboratory tests, than the Camp Bird tailings and are a bigger threat to Imogene Creek.

to streams. (photograph 60). One of the earlier mills (below Richmond basin) probably utilized water power, and to do so the mill was located just below a water fall. The tailings from several sites are generally rich in base metals, but there are some notable differences in composition between the tailings. A sample from the Hidden Treasure mill tailings (next to the Camp Bird No. 3 mill in the upper Imogene Creek watershed) has unusually high concentrations of $\mathrm{Pb}, \mathrm{Ag}, \mathrm{Cd}, \mathrm{Mo}$, and $\mathrm{Zn}$ relative to the district and to other tailings analyzed in this study. At the Atlas mill tailings impoundment (photograph 60 ), there are two distinct varieties of tailings, ocher and gray. Those of ocher color have notably high As but relatively low base-metal concentrations relative to the gray tailings. The colors probably reflect ore mineralogy rather than weathering: ocher from ore that was oxidized prior to mining and gray from unoxidized, sulfidic ore. Two samples from the very large Camp Bird tailings impoundment, which are nearly as pale as ocean beach sand, have low base-metal concentrations relative to other tailings. However, $\mathrm{Pb}$ and $\mathrm{Zn}$ contents are substantial and higher than in unmined mineralized rocks.

Leach tests on the six tailings samples from the Sneffels district generated solutions of significantly different composition. Leachate $\mathrm{pH}$ values ranged from 2.8 to 5.6. The most acidic leachate, from the Hidden Treasure tailings, carried the highest metal concentrations, including notably high concentrations of $\mathrm{Pb}, \mathrm{Cu}$, and $\mathrm{Zn}$. The Atlas tailings generated $\mathrm{pH}$ values of 4.3 and 5.2; the gray sulfidic tailings yielded less acid but higher metal concentrations than the ocher tailings. The Camp Bird tailings yielded $\mathrm{pH}$ values of 5.5 and 5.6 and low metal concentrations, including low values for $\mathrm{Cu}$ and $\mathrm{Zn}$, metals of prime concern for fisheries. The tailings with the highest potential to generate acid and release metals are from the old mills: these have small volumes and probably have a proportionately low input to the nearby streams. The metal input from fluvial tailings washed away in streams could not be evaluated.

The physical settings of tailings in this district suggest that all but those at the Eclipse mill have high risk of erosion in an extreme storm event. Storm events have eroded tailings in this watershed in the past century, shown by overbank deposits of tailings along both Imogene Creek and Canyon Creek. The fluvial sediments are about $3 \mathrm{ft}$ vertically above normal flow levels in these streams (photograph 65), suggesting that the flow during the storm events must have been quite spectacular. The geometry of tailings and stream channels vary significantly among this group of four sites, and each should be evaluated more thoroughly to obtain a more reliable risk assessment. The very large volume of tailings at the main Camp Bird site, and their proximity to Canyon Creek, suggest there could be major problems if this impoundment was hit by an extreme storm event. One could argue that the Camp Bird tailings are relatively small sources of acidity and metals, 
but others can argue that the aquatic health of Canyon Creek requires minimum contact of water with these materials.

Surface waters.-Canyon Creek and its headwater tributaries (Sneffels, Yankee Boy, and Imogene Creeks) cut through this district and are popular for fishing and hiking. The water is clear and leaves no red deposits on stream cobbles, quite unlike the Uncompahgre River headwaters just to the east in the Red Mountain district. The streams were checked several times in an effort to obtain reliable information. Measurements of $\mathrm{pH}$ in the field ranged from 5.7 to 7.2 , and conductivities ranged from 44 to $190 \mu \mathrm{S} / \mathrm{cm}$; I consider these to be indicative of fairly good water quality, although some would say that the $\mathrm{pH}$ of 5.7 and conductivity of $190 \mu \mathrm{S} / \mathrm{cm}$ are indications of contamination.

Chemical analyses of eight surface-water samples, some of which are replicates, show low metal concentrations. Iron concentration is elevated, but the maximum value at $0.5 \times$ ALWS is not bad. Zinc is the element of chief concern here, with concentrations in the range 110 to $185 \mathrm{ppb}$ (relative to ALWS at $50 \mathrm{ppb}$ ). Some $\mathrm{Cu}$ concentrations also exceed ALWS. Zinc concentrations are at highest in Sneffels Creek (sampled east of the Revenue mine and Atlas mill tailings). Zinc concentration in Canyon Creek is $3 \times$ ALWS below the Camp Bird tailings, but that improves to $2 \times$ ALWS over the next $2.5 \mathrm{mi}$. The CZI values for the streams range from 1.7 to 0.02 . Most of the stream reaches have CZI below 1.0, but a more detailed study is required to evaluate the threat to aquatic life. Geochemically, it appears that the green alteration prevalent in the basin minimizes acid production at mine sources and mitigates $\mathrm{pH}$ and $\mathrm{Zn}$ concentrations by in-stream reactions below mine sources. Water compositions in Canyon Creek are not pristine, but they would be far worse without the attenuation provided by geologic processes.

Only three mine drainage samples were collected in this basin where my access was limited. The small Eclipse mine produces a small flow of water with pH 6 and only $10 \mathrm{ppb}$ $\mathrm{Zn}$. The upper Camp Bird portal (number 3 level) yields a small flow at $\mathrm{pH} 7.2$ with somewhat elevated metal concentrations, but only the $\mathrm{Zn}$ concentration exceeds ALWS. Only at one adit, the Hidden Treasure mine, is surface water visibly contaminated (it creates a red precipitate) (photograph 66) and the chemical analysis shows high concentrations of $\mathrm{Al}, \mathrm{Cd}$, $\mathrm{Cu}, \mathrm{Fe}, \mathrm{Mn}$, and $\mathrm{Zn}$. The most problematic concentrations are those of $\mathrm{Cd}, \mathrm{Cu}$, and $\mathrm{Zn}(22 \times, 32 \times$, and $90 \times$ ALWS, respectively). The CZI for the Hidden Treasure mine drainage is 28 , indicating a potential threat to aquatic life.

Summary.-Water quality and physical habitat in this district are surprisingly good considering the large amount of mining and disturbance in the basin. Mining methods and waste disposal techniques are basically the same as in the adjoining Red Mountain district, so the difference in conditions appears to reflect the ANC provided by green-altered volcanic rocks. Only at the Hidden Treasure mine did I see evidence for acid mine drainage. The geology of this mine may be unusual for this district as the partially oxidized vein appears to be in a felsic dike rather than in green volcanic rock.
Large volumes of tailings and waste rocks were placed near creeks, yet the downstream water quality is quite good. The concern for these near-neutral waters is the mobility of $\mathrm{Zn}, \mathrm{Cd}$, and $\mathrm{Cu}$ and their concentrations near or somewhat above the standards for aquatic life.

Mine-waste rock piles and tailings in riparian zones should be evaluated for physical stability and for release of metals in more detail than was possible here. Acid generation does not appear to be a problem, but these polymetallic ores could release enough $\mathrm{Zn}, \mathrm{Cd}$, and $\mathrm{Cu}$ to be detrimental to aquatic life or human health.

There may be high risk of failure during an extreme flood at four tailings sites in this district. Each of these sites has different setting relative to headwater basins and stream channels, and each deserves independent review. Based on the preliminary model, the very large tailings impoundment at the Camp Bird mine ranks among the top five sites on the CWS in need of additional technical information and possible reclamation to minimize exposure to floodwaters. Reclamation activities at the Camp Bird mine, observed at a distance in 1999, included capping by unmineralized rocks, which should reduce infiltration and runoff. It is not clear how much this work will reduce the risk of erosion in an extreme storm.

References.-Burbank (1947b); Fischer and others (1968); Fisher (1990); Benham (1980).

\section{Mineral Point District (including Upper Uncompahgre)}

Location.-Ouray County, 10-15 mi south of Ouray, at the headwaters of the Uncompahgre River (a small part is in San Juan County). (fig. 23) The climate is severe, with about 25-40 inches of precipitation per year.

Principal commodities.-Silver, gold, lead, copper, zinc.

Mining history.-Ore was discovered in 1874 and the most valuable production was prior to 1900 from about a dozen mines, none of which produced more than $\$ 500,000$ worth of ore. There was intermittent activity over the years. Some mines such as the Mickey Breen and Mountain Monarch mines shipped ore to the Banner mill near Ouray from the late 1930's to early 1950's.

Production and disturbance.-Total value of ore produced was about $\$ 2$ million. The individual mines were small to moderate in size and created relatively small waste dumps. The mills in the district impounded very small amounts of tailings - most ore was hauled to mills outside the district.

Geology.-This district on the flank of the Silverton caldera worked large, through-going polymetallic veins in volcanic rocks. Some of the major veins, as near the San Juan Chief mine, can be traced for several miles and extend into the Eureka district to the south and the Galena district to the northeast. Volcanic rocks in the area are much less altered than the adjacent Red Mountain district and are mostly the green, propylitic variety. The deep canyon of the Uncompahgre River 


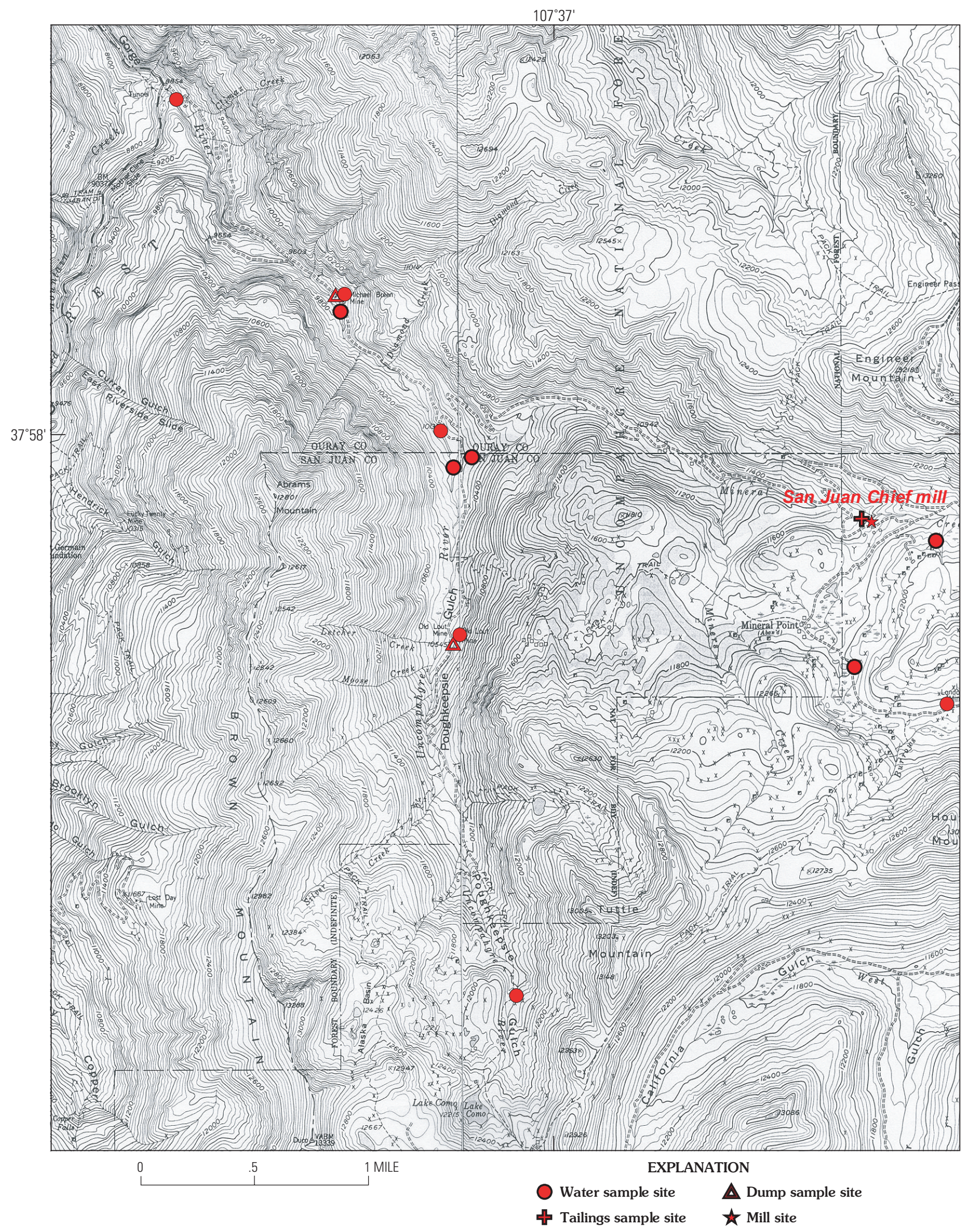

Figure 23. Features of part of the Mineral Point mining district, showing sample localities. 


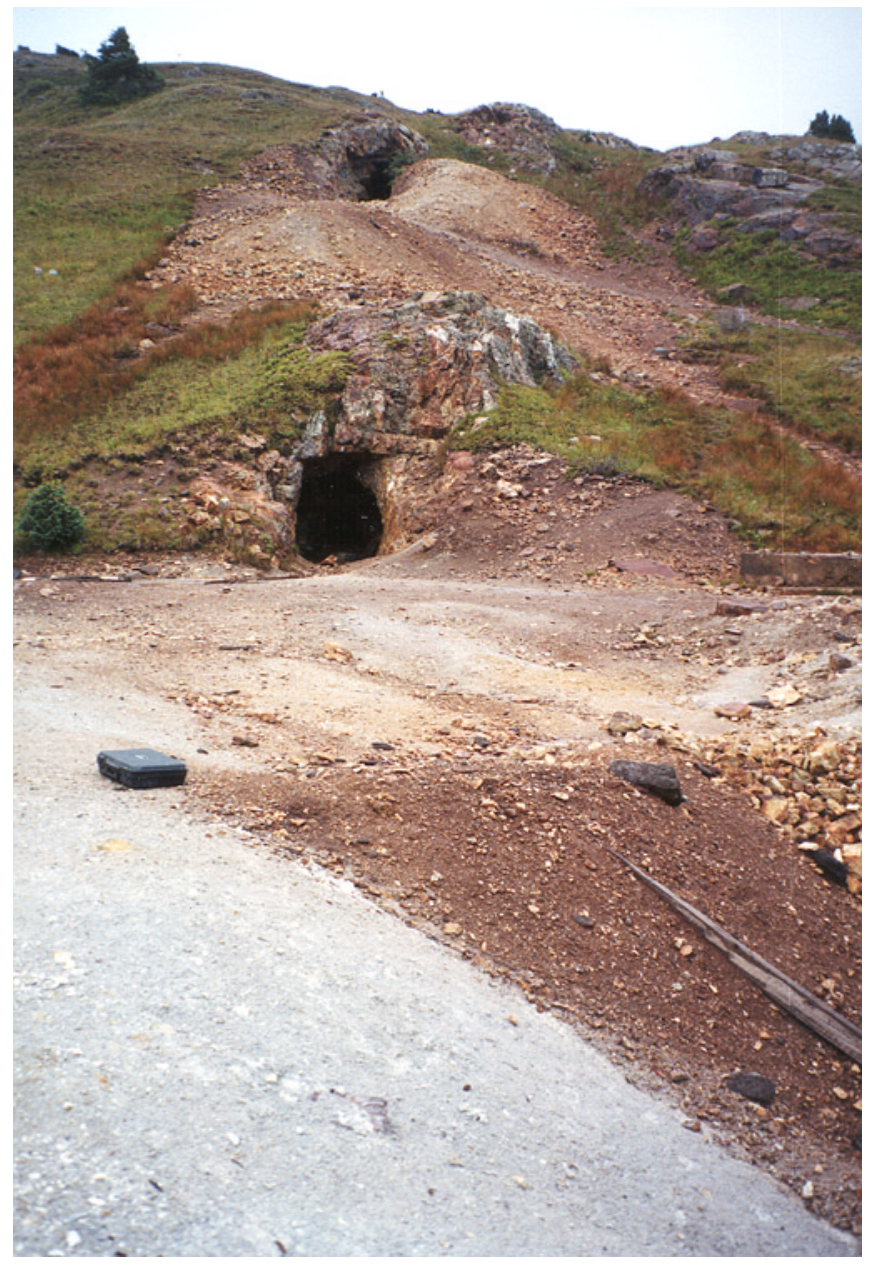

Photograph 68. The San Juan Chief mine created a series of workings up this hill, following a major vein. Drainage from the lowest level has a $\mathrm{pH}$ of 2.7. The acid rock drainage kills alpine flora for tens of feet beyond the waste dumps.

at the northwest end of the district cuts into Precambrian rocks and a thin sequence of Paleozoic sedimentary rocks, which are north of the major mines. The pre-Tertiary rocks in this area do not contain significant ore.

\section{Mineral-Environmental Conditions}

Numerous chemical analyses of similar ores in the Animas watershed to the south (Nash, 1999a, 1999b) show that those ores are rich in base metals: $\mathrm{Ag}, \mathrm{Cd}, \mathrm{Cu}, \mathrm{Pb}$, and $\mathrm{Zn}$ tend to be moderate to high in concentration. Three new analyses of samples from this district confirm this trend and suggest that $\mathrm{Bi}$ and Mo concentrations are high locally and that As is moderate to below the median in this study. The abundant sulfide minerals in the mined materials create a high potential for acid generation, according to many laboratory leach tests (Nash, 1999a, 1999b).

Mineralized rocks and ores.-Mineralization in this district is very similar to that of the Eureka district to the southeast, which has been studied in detail (Nash, 1999b). The vein deposits contain abundant coarse sulfide minerals (pyrite, galena, chalcopyrite, sphalerite) as well as complex silver-bearing minerals and pink manganese minerals (rhodonite, $\mathrm{MnSiO}_{3}$; and rhodochrosite, $\mathrm{MnCO}_{3}$ ). According to leach tests, these ores have the potential to generate acid and release metals. These test results are consistent with acid mine waters in some (but not all) adits in the district.

Mills and tailings.-Most of the ore from this district appears to have been milled at sites outside the district. A mill in the canyon near Mickey Breen mine is not recognizable today, and the San Juan Chief mill is photogenic but appears to have produced very little. As elsewhere, tailings from pre-1900 mills may have been lost to the nearby streams. Tailings do not appear to affect water quality.

Surface waters.-The headwaters of the Uncompahgre River are just to the northwest of the sources of the Animas River and Henson Creek, and the geology and ore deposits are continuous across the drainage divide (in fact, some veins were mined on different sides of the drainage divide). Abundant precipitation and steep topography create rapidly flowing streams with numerous cascades. The water is clear and, in most places, creates no red iron stains or coatings. Mineral Creek is a short tributary that is sourced east of the Uncompahgre River. Field determinations of $\mathrm{pH}$ and conductivity showed most sites have near-neutral, low-conductivity $(<150$ $\mu \mathrm{S} / \mathrm{cm}$ ) compositions. Chemical analyses of the near-neutral ( $\mathrm{pH} 6.2$ to 7.0) waters show mostly low metal concentrations, but $\mathrm{Zn}$ concentrations are elevated $(1 \times$ to $6 \times$ ALWS $)$. Acidic waters (pH 2.7 to 4.7 ) at and near some mine adits carry high metal concentrations, including very high concentrations of $\mathrm{Cd}, \mathrm{Cu}, \mathrm{Pb}$, and $\mathrm{Zn}$ (which locally reaches 15,000 ppb, or $300 \times$ ALWS) at the San Juan Chief mine adit. For the first few miles of headwater streams, near mines, quality is degraded (CZI values are 0.73 to 7.1 ), but downstream, near the confluence with Red Mountain Creek at U.S. Highway 550 , the quality improves to a CZI value of 0.5 , although $\mathrm{Zn}$ still is high $(3 \times$ ALWS $)$.

Summary.--In this headwater basin underlain by green propylitic-altered volcanic rocks, acidic conditions do not persist for more than about a mile. In both Mineral Creek and the uppermost Uncompahgre River, local segments with $\mathrm{pH}$ near 3 improve to $\mathrm{pH} \mathrm{7,} \mathrm{and} \mathrm{with} \mathrm{that} \mathrm{loss} \mathrm{of} \mathrm{acidity} \mathrm{comes} \mathrm{much}$ improved water quality. For example, zinc decreases by orders of magnitude, but still is elevated ( $3 \times$ ALWS) at $\mathrm{pH}$ 6.9. This is an excellent example of natural attenuation, but also a warning that high concentrations of $\mathrm{Zn}$ can persist after neutralization. Further study is needed for more precise details on Zn mobility and its potential effects on aquatic life.

References.-Burbank (1947c); Fischer and others (1968). 


\section{Red Mountain District}

Location.-Ouray County, 20 mi south of Ouray (fig. 24). The climate is probably the most severe in the study area, with 30-50 inches (or more) of precipitation per year.

Principal commodities.-Silver, lead, zinc, copper, gold.

Mining history.-A boulder of galena was discovered in 1881, and soon there was a rush and discovery of significant mines including the Robinson, Yankee Girl, and National Belle. The most productive years for these silver mines in chimney or breccia pipe deposits were prior to 1890 . These mines struggled through years of depressed silver prices in the mid-1890's, and all closed in 1896 and 1897. The Barstow mine on the west side of the district was an exception in that it started in 1899 and continued operating until about 1917; the Barstow vein was like those on the Telluride side of the mountain and was later mined from the Treasury Tunnel. Starting in 1907, the Joker Tunnel was driven nearly 5,000 $\mathrm{ft}$ into Red Mountain to access lower levels and dewater the Genessee, Guston, and other mines; this ambitious project was intermittently active, eventually reached some targets, but produced relatively little ore through the 1940's.

With success at the Barstow mine, attention shifted to veins on the western side of the district. The Treasury tunnel was driven to the west under the Barstow and other veins; started in 1896, it advanced in stages and by the 1930's allowed significant production from polymetallic veins on the west side of the district. Consolidation of diverse properties in 1939 and the mid-1940's, forming the Idarado Mining Co., allowed efficient mining on veins that had not been worked since the 1920's. Development of improved milling techniques allowed recovery of major amounts of copper, lead, and zinc and byproduct bismuth and other metals from ores that earlier were considered too low in grade or too complex metallurgically to be mined profitably. The Treasury Tunnel of the Idarado mine, whose portal was near Red Mountain Pass, extended west of the divide into San Miguel County. It provided access to major veins such as the Black Bear and Tomboy in the Telluride district, allowing higher production from the mid-1940's through 1956. The Idarado mill at Treasury Tunnel portal, with a capacity of 800 tons/day, closed in 1956 when all ore was diverted to the Pandora Mill east of Telluride. Production after 1956 used the Mill Tunnel, which is $1,600 \mathrm{ft}$ lower in elevation than the Treasury Tunnel. Mining at the Idarado mine ceased in 1978. There appears to have been very little production from the Red Mountain district in the past 40 years, but there have been underground and drilling exploration programs, as on Red Mountain, in the 1970's and 1980's.

Production and disturbance.-Production data tend to be mixed with adjacent districts and aggregated by county and tends to be based upon where ore was processed rather than where ore was mined. Dollar value is very high, more than $\$ 50$ million. One estimate of total production for the Red Mountain district, 1,685,000 tons (Long and others, 1998), appears to include only the eastern pipe deposits, not production from the
Treasury Tunnel (Idarado mine). There is a large amount of disturbance in this district, including hundreds of substantial mine dumps (photograph 69). There are five mill tailings impoundments plus substantial areas of unconfined tailings along Red Mountain Creek. Reclamation in the mid-1990's concentrated on mine drainage and the impounded tailings.

Geology.-This district lies astride the margin of the midTertiary Silverton caldera. Most exposed rocks are volcanic flows and tuffs. Small but important intrusions occur along the caldera margin. A thick section of Devonian to Jurassic sedimentary rocks lies between the Tertiary rocks and Precambrian basement (Burbank and Luedke, 1964); although mineralization is known in these sedimentary rocks (Mayor and Fisher, 1993), very little has been mined because of the great depth.

Most ore came from vein deposits in the volcanic rocks, but the famous high-grade pipe ores are in and near small intrusive plugs. The Telluride Conglomerate, at the base of the Tertiary section, hosted important replacement ores rich in base metals. Polymetallic replacement deposits and skarn in underlying limestones were drilled but are too deep to be mined (Mayor and Fisher, 1993). A few small veins mined from Precambrian host rocks are the roots of mid-Tertiary veins, and there has been some production from veins in preTertiary sedimentary rocks.

Economic geologists distinguish many different kinds of ore in this district, but for this study we can lump the ores into three classes, some of which are related genetically: (1) polymetallic veins and replacements rich in $\mathrm{Pb}-\mathrm{Zn}-\mathrm{Cu}-\mathrm{Ag}$; (2) gold-quartz veins that generally formed along veins of the polymetallic type (the two vein types are mined together); and (3) acid-sulfate type disseminated deposits under Red Mountain, containing gold and pervasive alteration that added much pyrite and alunite while destroying silicate minerals; these deposits are similar to those mined at Summitville and have a tendency to produce highly acidic drainage because the buffering capacity of the host rocks has been destroyed (King, 1995; Plumlee and others, 1999).

\section{Mineral-Environmental Conditions}

Preface.-The framework of geology-based evaluation and ranking of districts and problem sites used in this report may not be relevant to this district. Standards for the stream of concern, Red Mountain Creek, appear to be set by a court settlement with a performance objective of $1.25 \mathrm{ppm}$ dissolved zinc (CDH, 1992). This standard, 1,250 ppb (the scale used in this report) is far higher than aquatic life standards of about 50 or $100 \mathrm{ppb} \mathrm{Zn}$ applied to many streams in Colorado. Also, most of the mining properties in this basin are on private property and reclamation of those lands is not done by FLMA's because of complex liability, legal, and political issues. The following discussions are in the same style as for other districts and do not address past or ongoing legal issues.

Text continues on page 114 


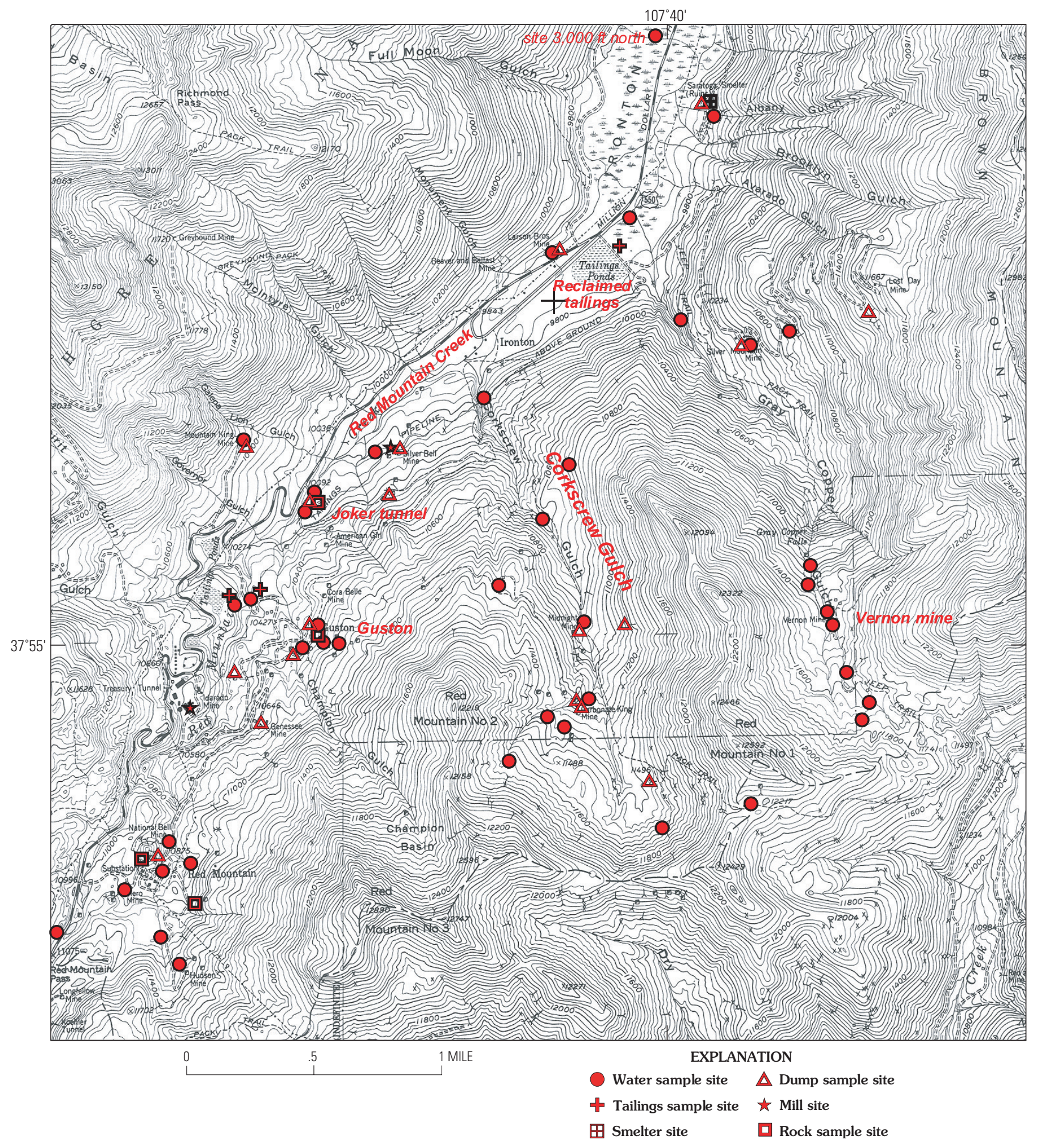

Figure 24. Features of the Red Mountain mining district, showing sample localities. 


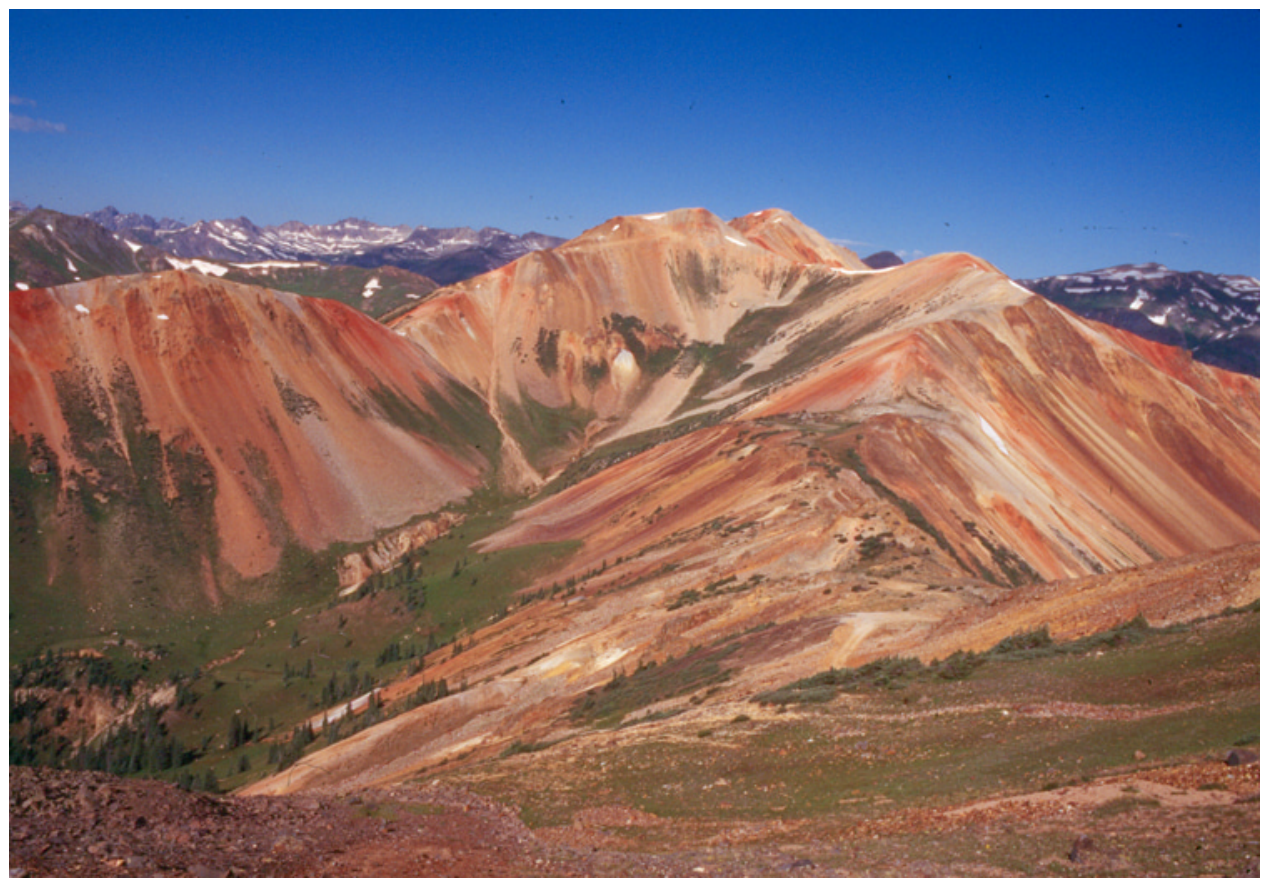

Photograph 69. The Red Mountain complex of highly altered rocks and associated mineral deposits is at the geographic center of three watersheds that are among the most contaminated by acid and metals in all of Colorado. Natural weathering of disseminated pyrite creates the red hues and releases acid and metals.

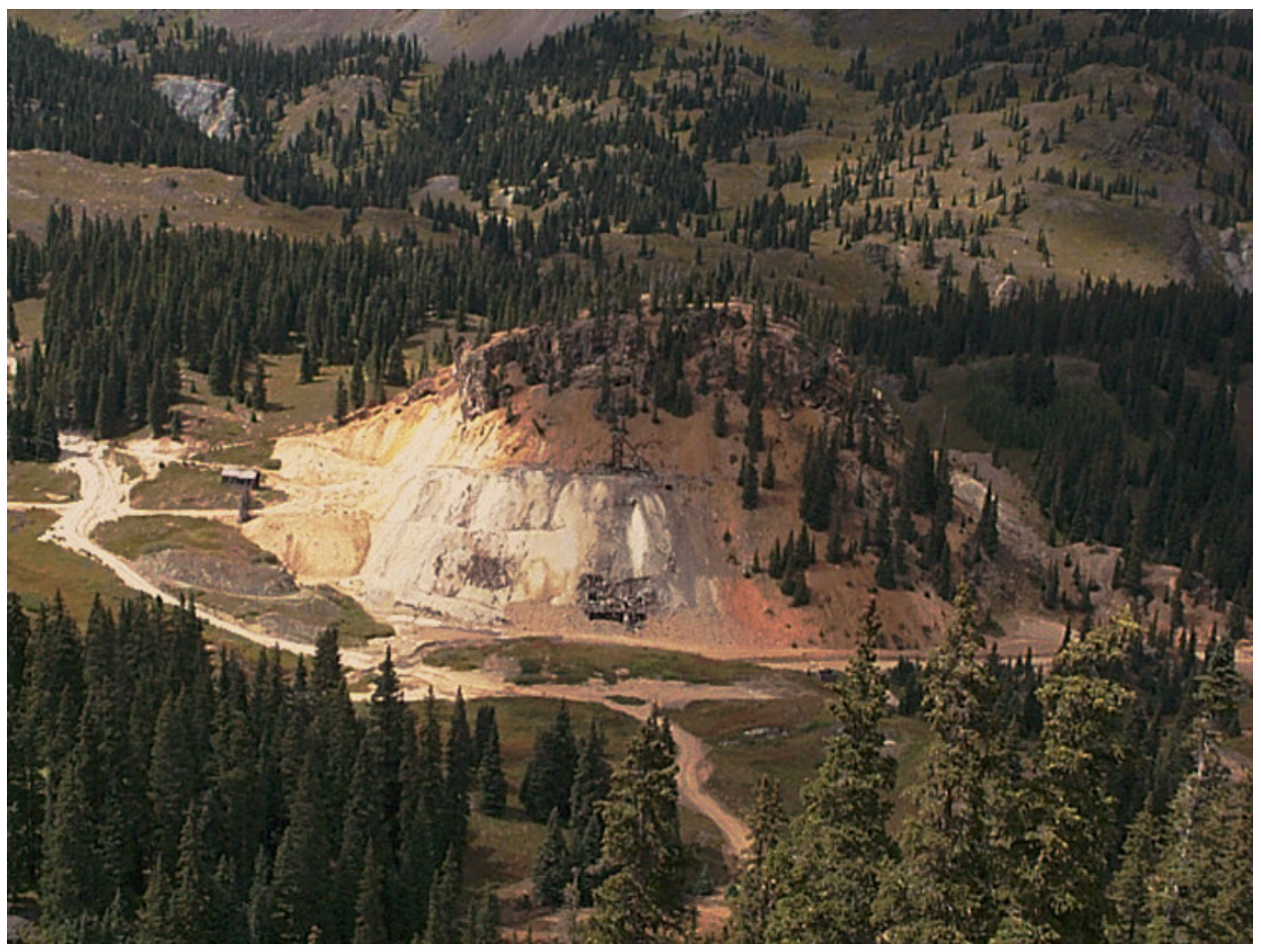

Photograph 70. This colorful, silicified knob encloses the National Belle pipe deposit. This was one of the richest silver mines in the district. These altered, unmined rocks are probably releasing acid and metals to ground water, but this is difficult to quantify. 


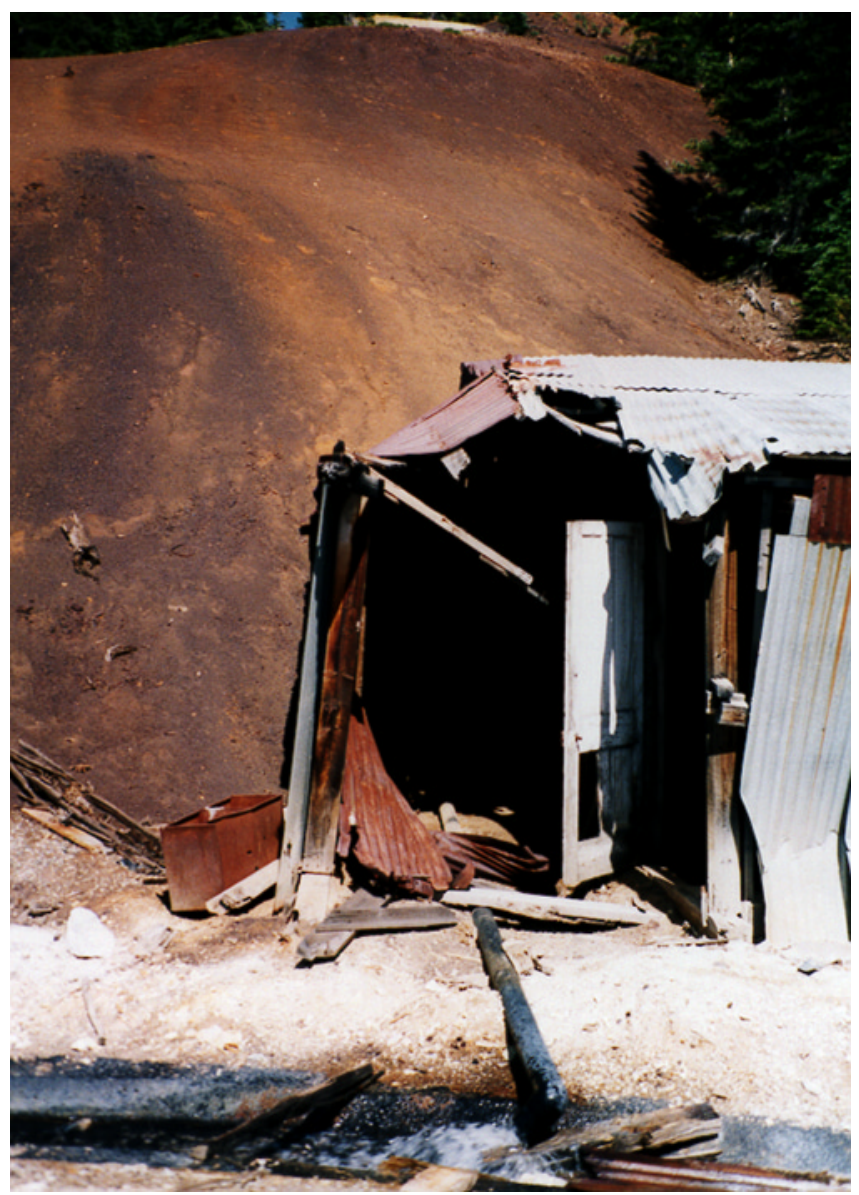

Photograph 71. Brown iron deposits (ferricrete) guided prospectors to the Guston and other deposits in the Red Mountain district. The mine tunnel was excavated into the ferricrete, demonstrating that the iron is pre-mining. The pipe carries mine drainage that is extremely acidic $(\mathrm{pH} 2)$ and rich in metals into a concrete gutter that was created recently as part of reclamation work.

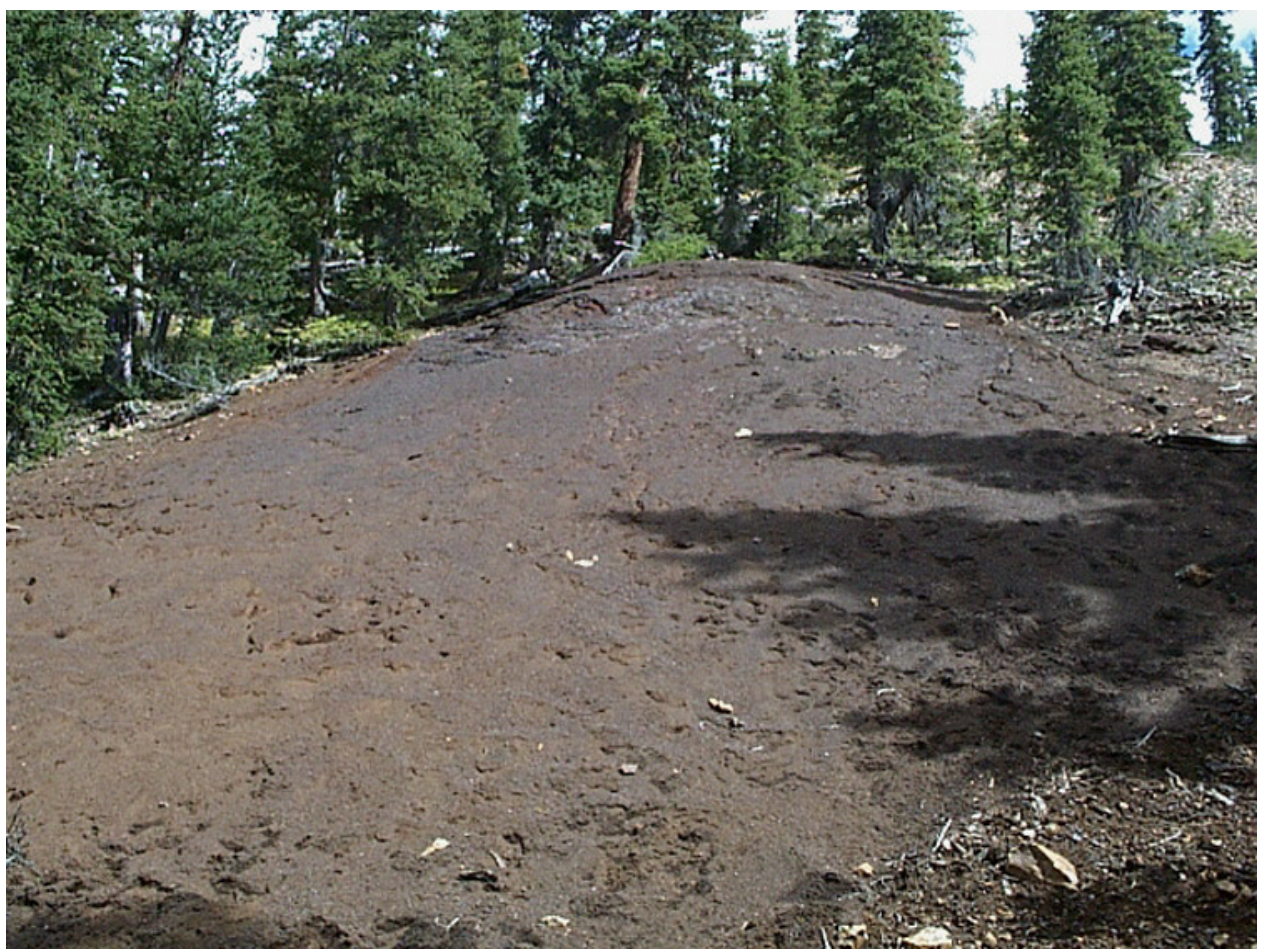

Photograph 72. This ferricrete deposit formed at the site of the Hudson deposit, Red Mountain district, just like the larger one at Guston. 


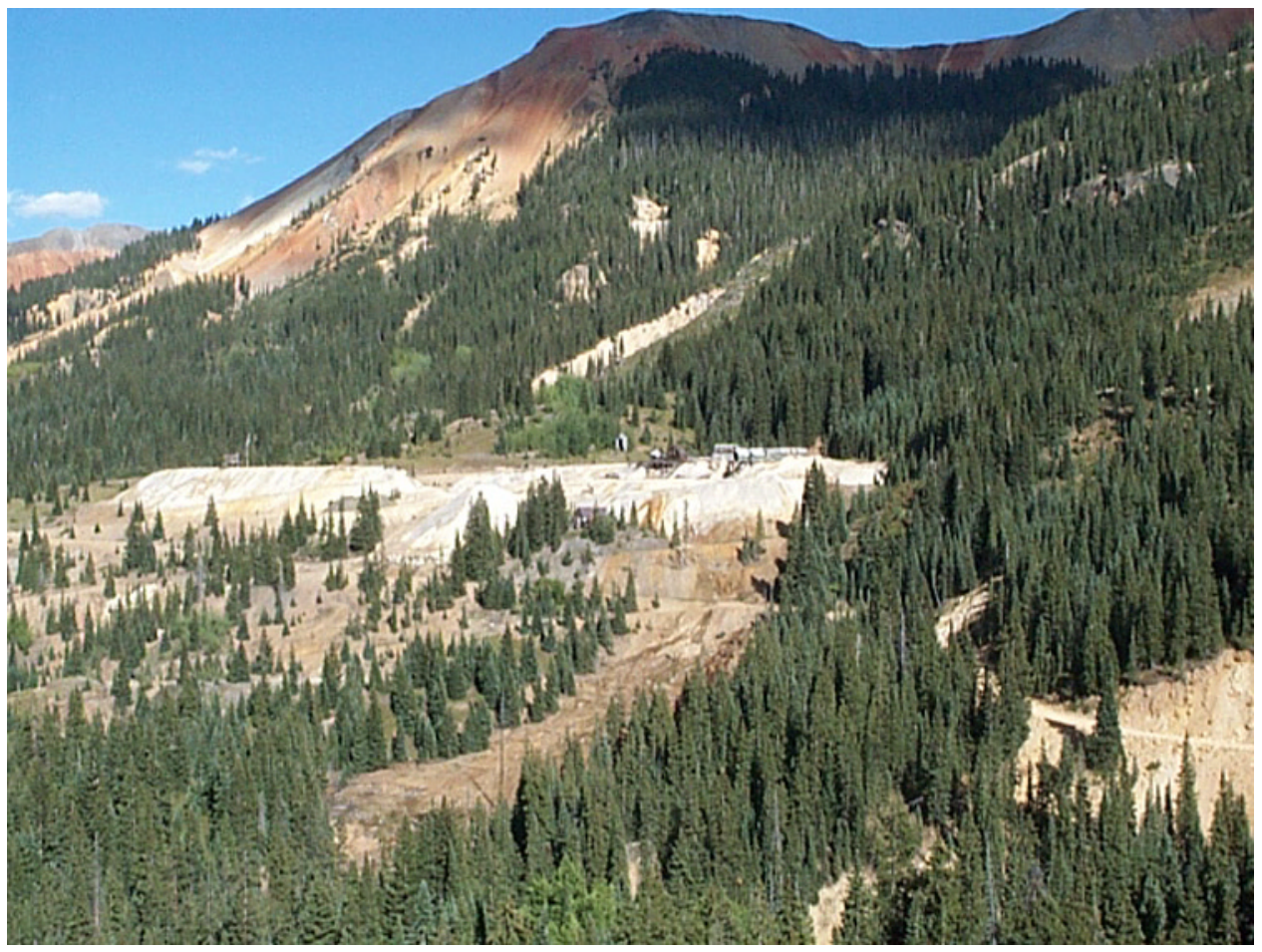

Photograph 73. The Genessee mine, at the base of Red Mountain Number 2 and 3, produced a large dump of pyritic waste rock. The swath of dead trees below the Genessee dump is a reflection of acid rock drainage from the sulfidic waste.

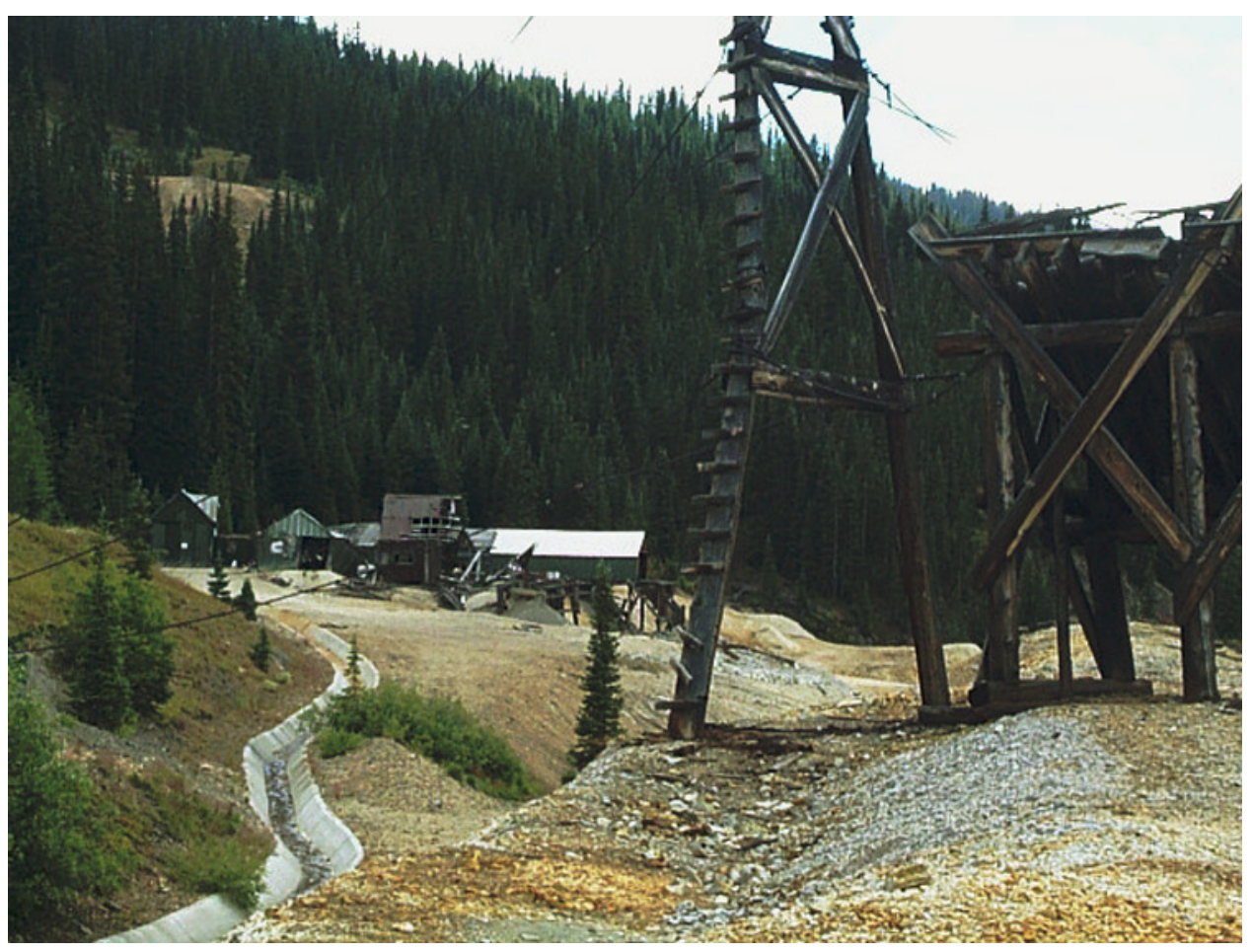

Photograph 74. The highly sulfidic dump of the Genessee mine is slowly releasing acid and metals. Laboratory tests show waste rocks like these generate acidic and metal-rich leach solutions. The concrete gutter on the left was installed to carry mine drainage and runoff beyond the waste rocks. 


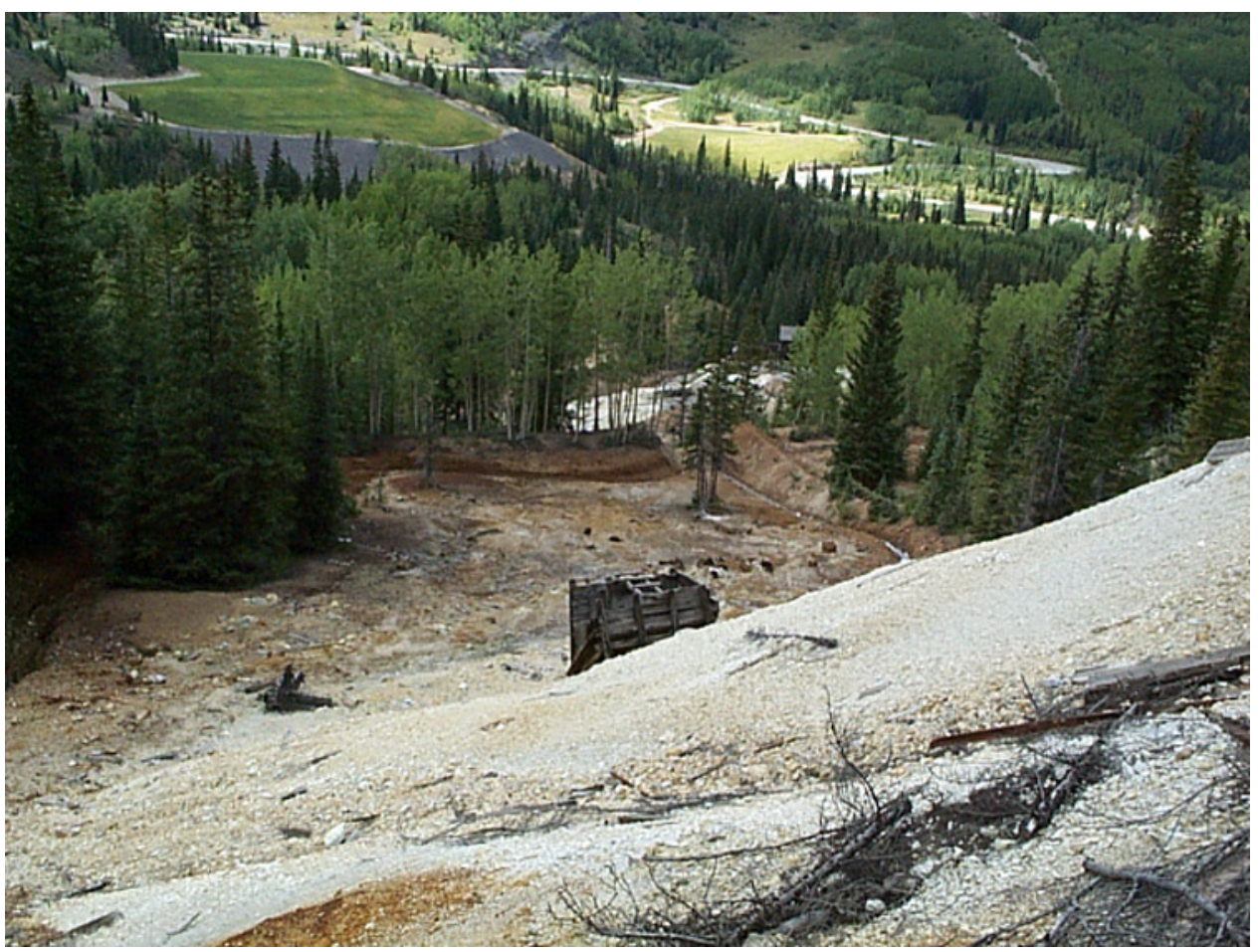

Photograph 75. Acidic waters from the Guston mine and dump have killed vegetation below it. Some of this damage may be related to natural conditions that created the ferricrete prior to mining, but mining and mine waters reacting with waste rocks on the dump probably made the condition worse.

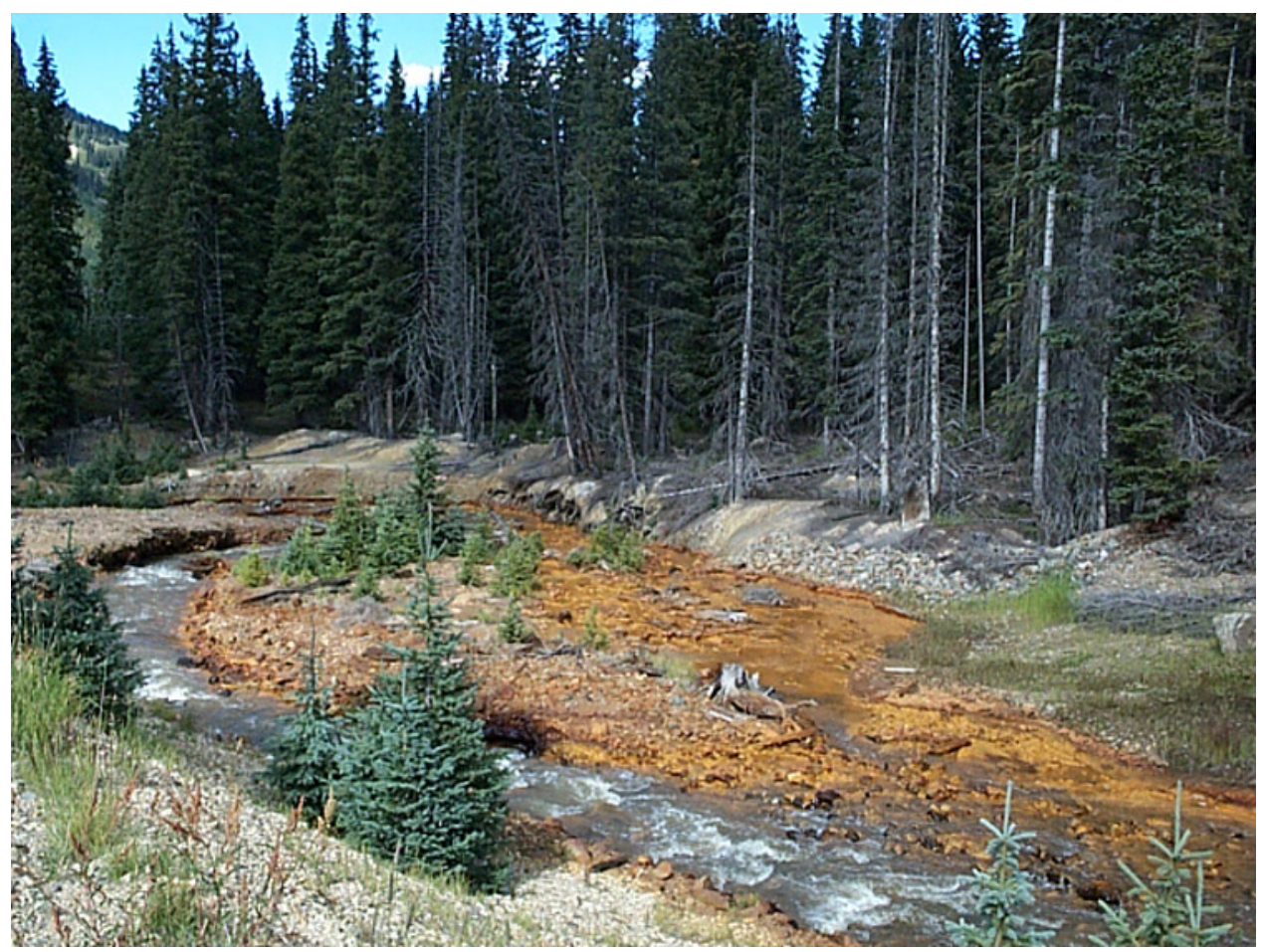

Photograph 76. Red Mountain Creek is highly degraded by many sources. This view shows the prominent red deposits of iron minerals that still form as very acidic waters mix with other waters and $\mathrm{pH}$ rises to about 3.5 to 4 . Overbank deposits of tailings were deposited during a flood event or a tailings spill. 


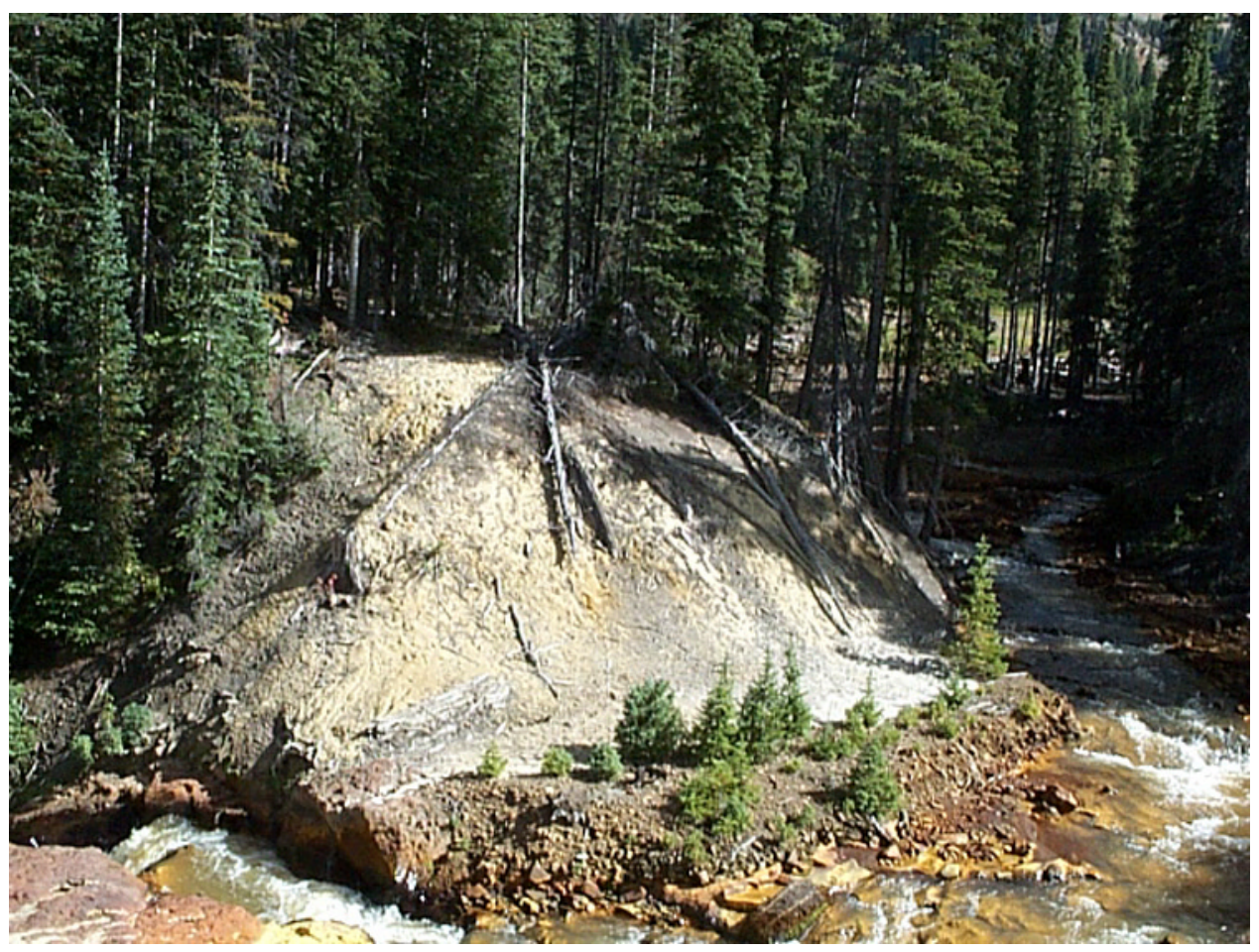

Photograph 77. Tailings contribute to problems along Red Mountain Creek. These tailings may have been released during early milling (before regulations) or during a flood event.

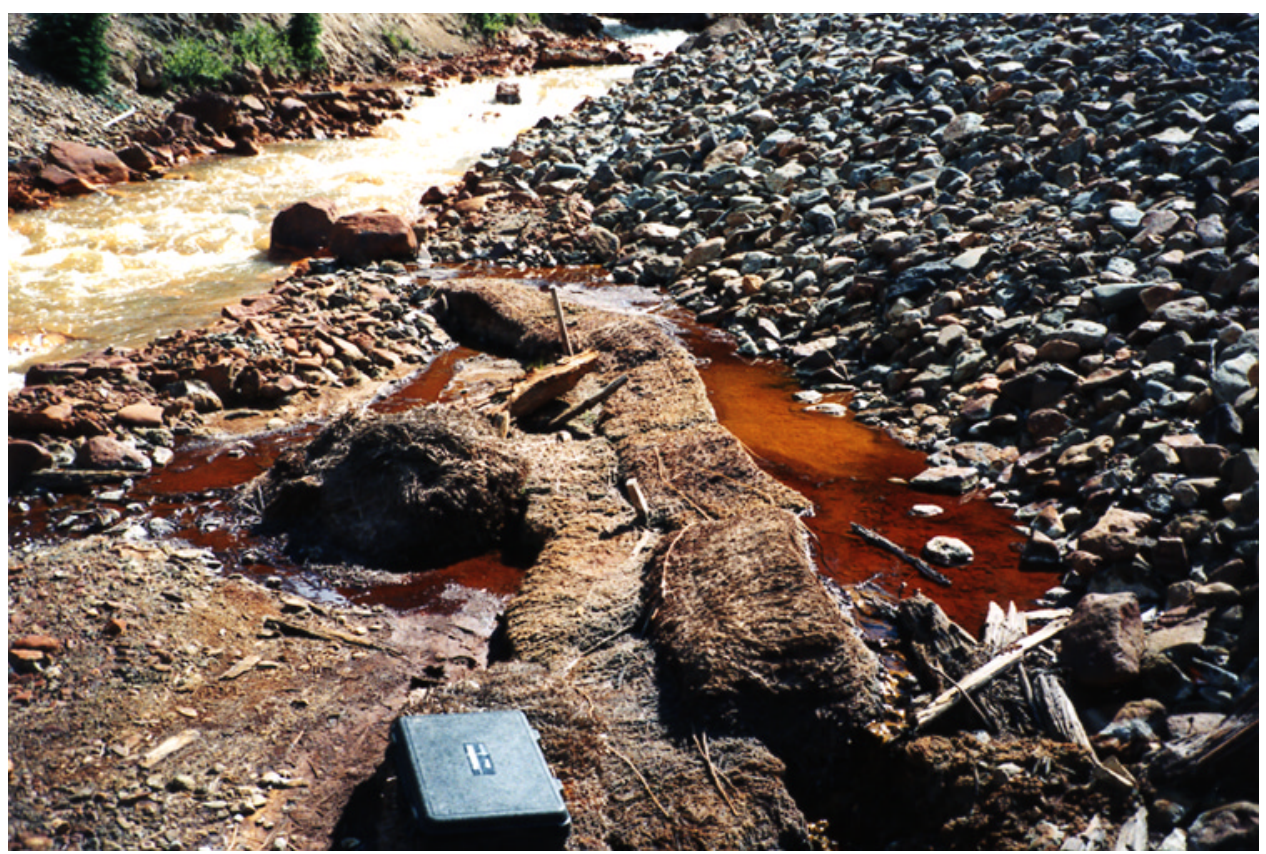

Photograph 78. Seepage from mill tailings is acidic and as high in metals as mine drainage. This seepage flows into Red Mountain Creek. 


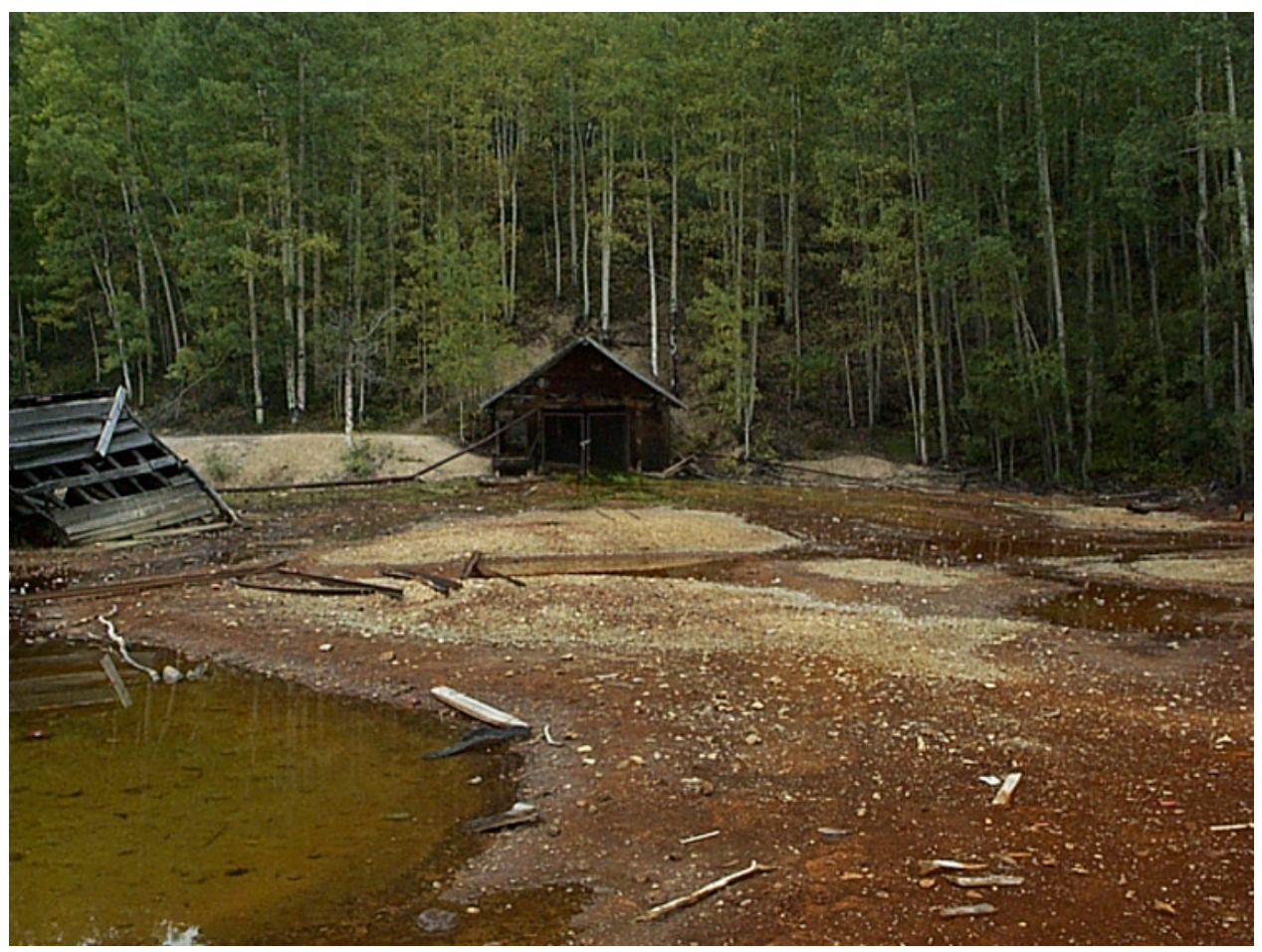

Photograph 79. The Larson Brothers mine tunnel is one of the few on the west side of the Red Mountain district that creates acidic drainage. Although the working was mostly in green-altered volcanic rocks, the drainage is moderately acidic and carries substantial iron and zinc. This water precipitated red iron coatings as the mine waters pick up oxygen outside the mine.

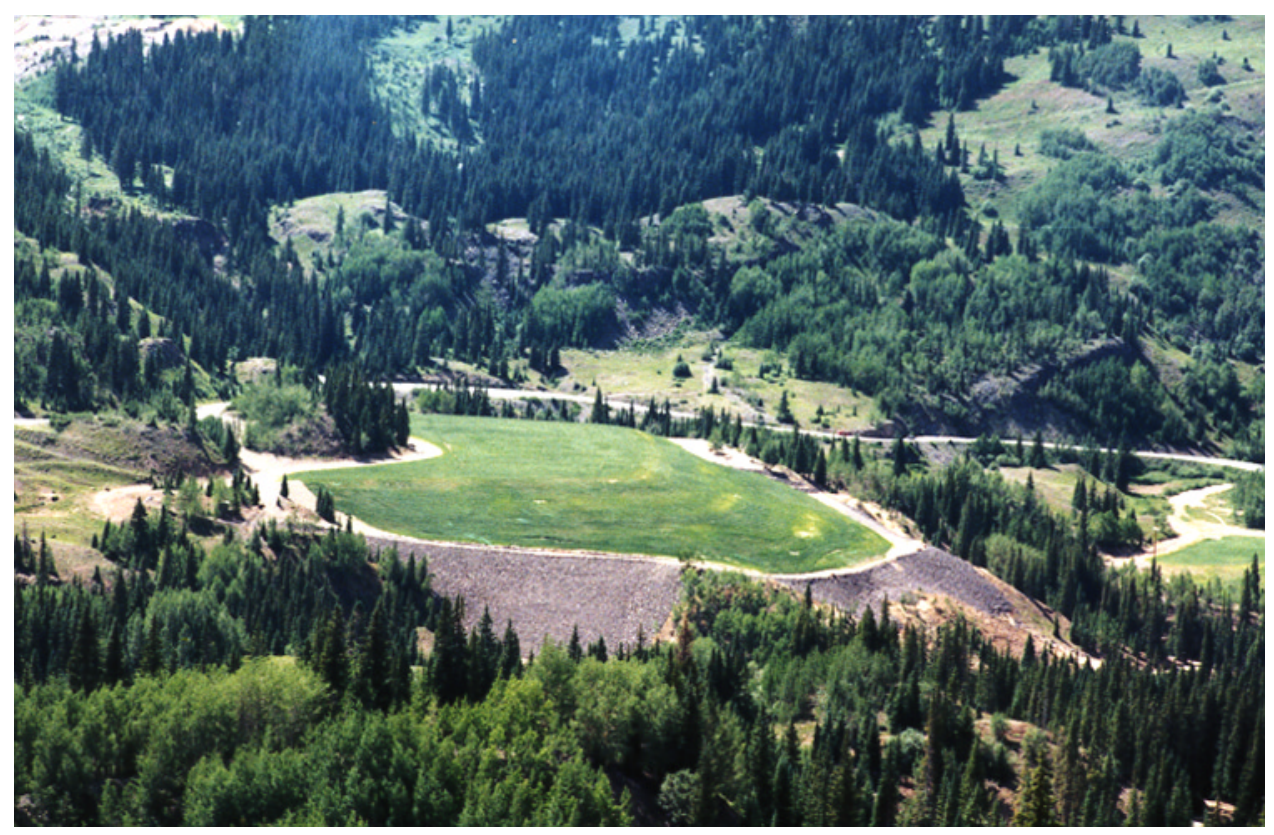

Photograph 80. Tailings impoundments of the Idarado mill have been reclaimed and concrete gutters put in place to control drainage. 


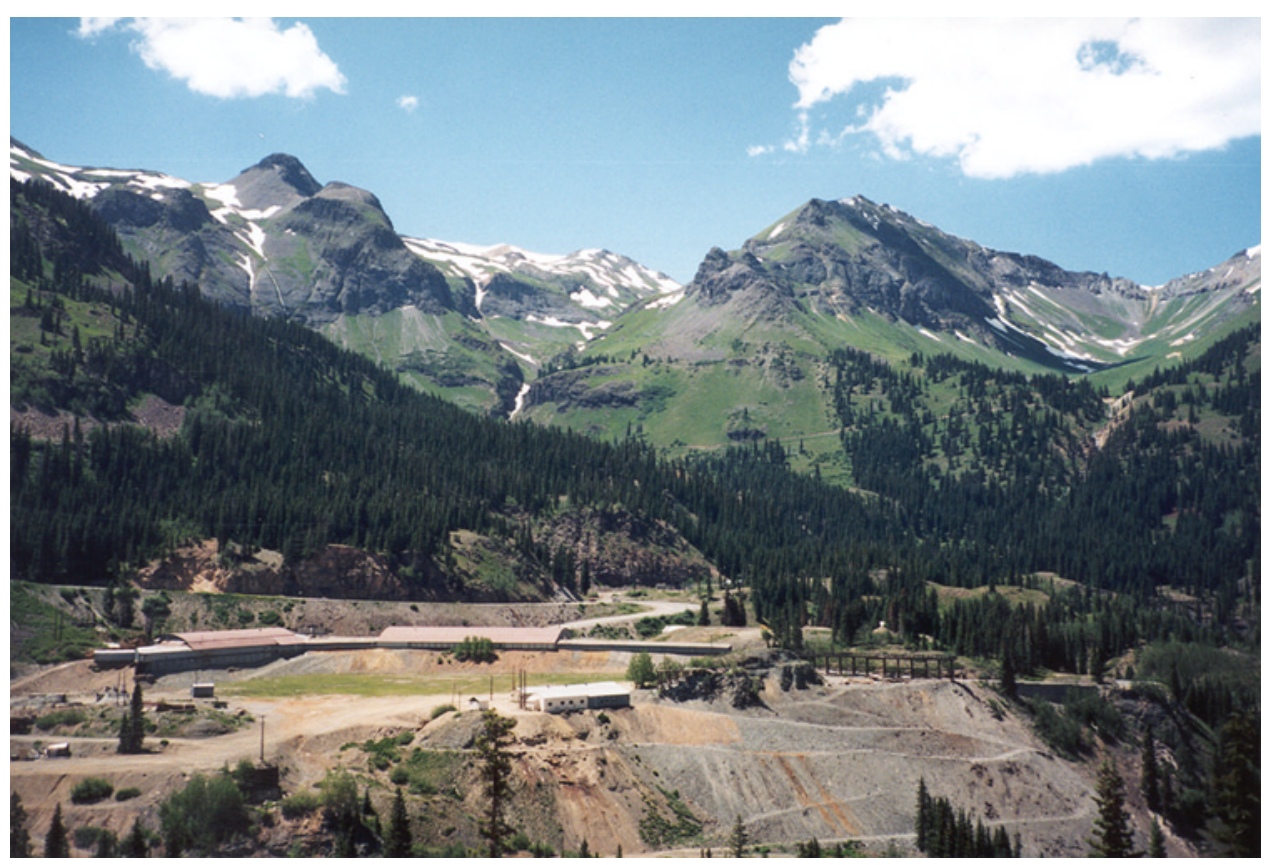

Photograph 81. The Idarado mine complex at the portal to the Treasury tunnel is near the summit of Red Mountain Pass; U.S. Highway 550 is in middle of photograph. The mill was situated near the lower left corner of this photograph. The ridge on the skyline shows the green and purple-gray colors of propylitic-altered volcanic rocks.

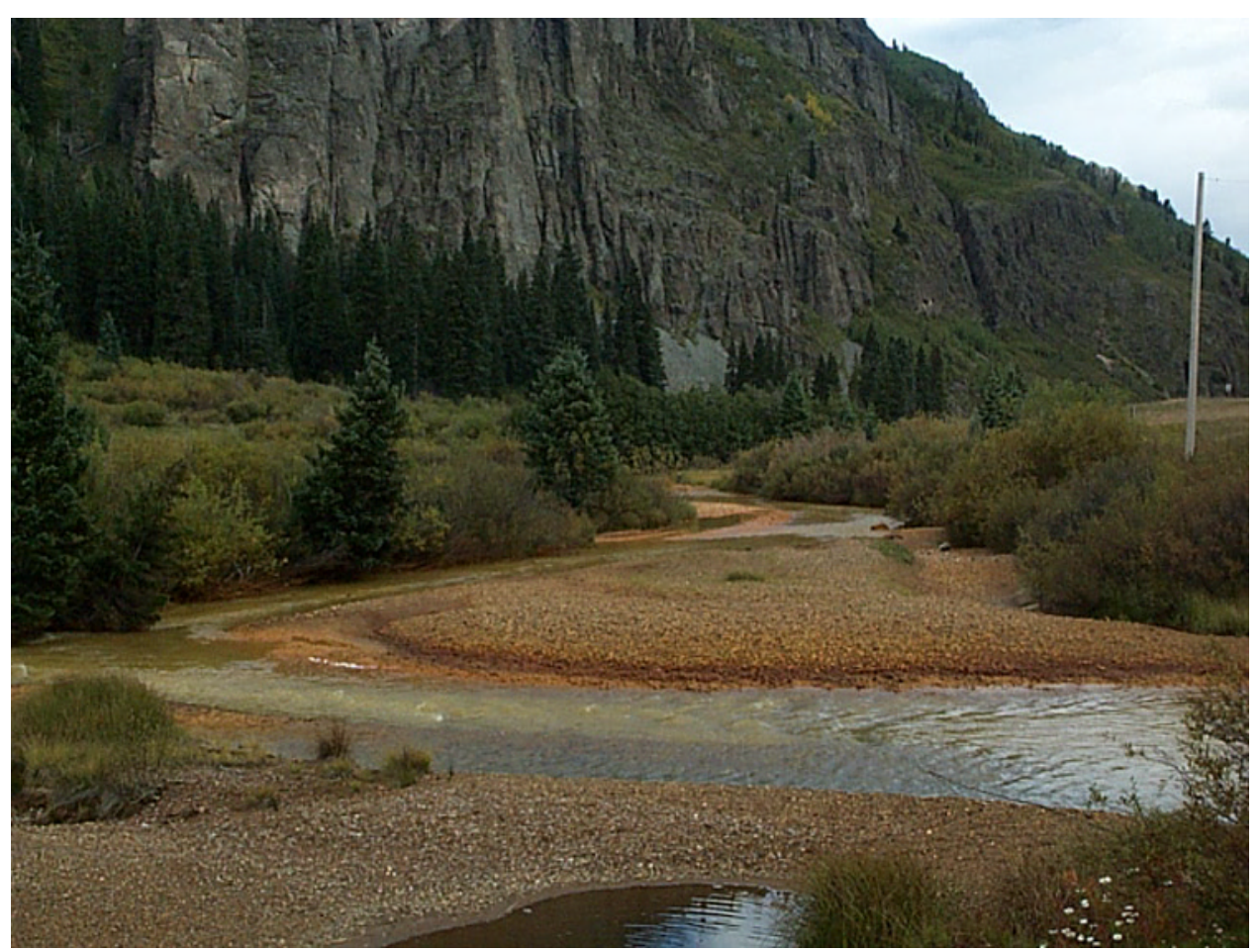

Photograph 82. The quality of Red Mountain Creek improves as it mixes with other streams, especially those coming in from the west. But the concentrations of iron and other metals remain high, as shown by the red iron coatings on the stream bed at the north end of Ironton Park. 


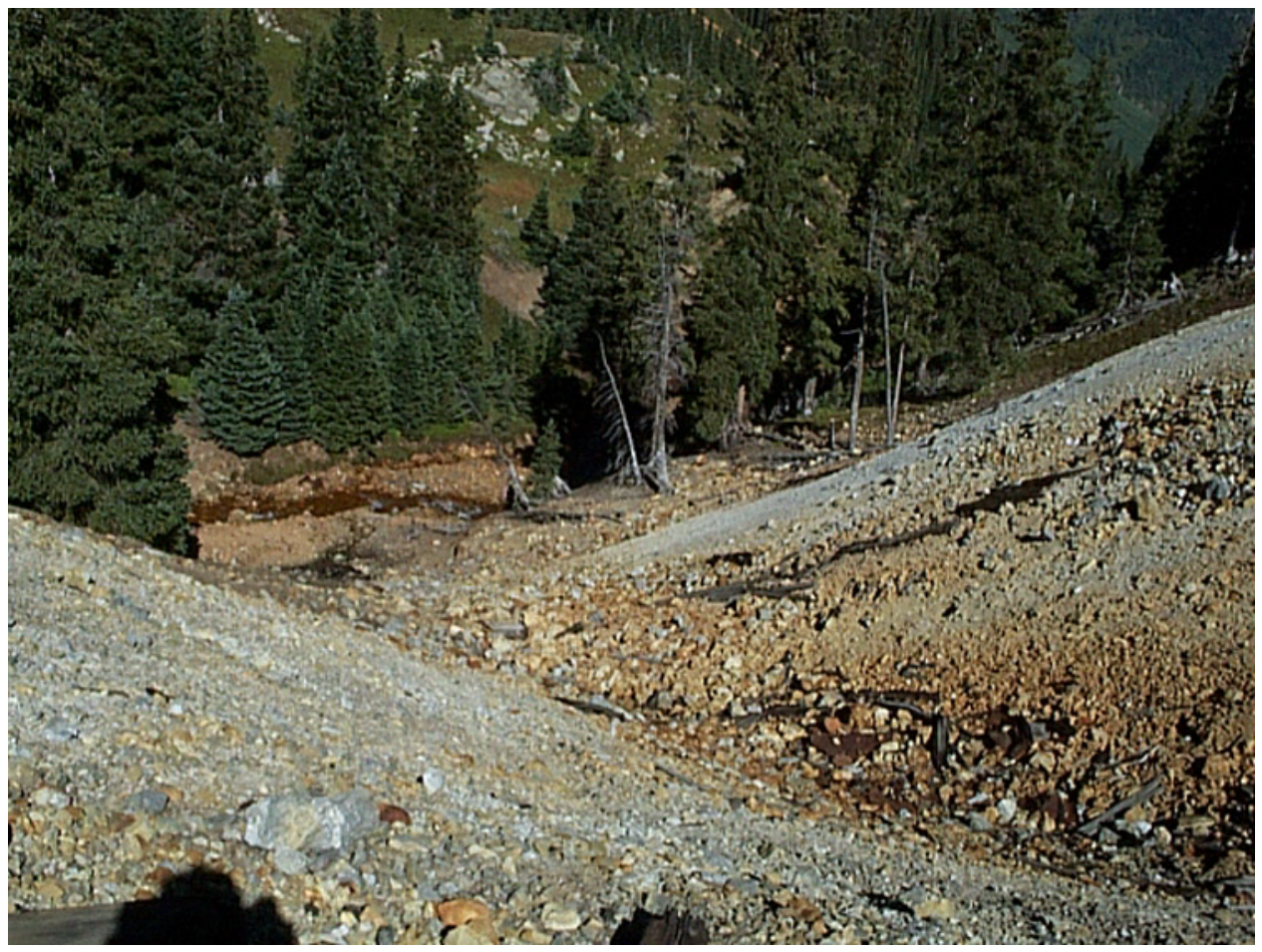

Photograph 83. The Vernon mine in the headwaters of Gray Copper Gulch Creek is a concern because the highly sulfidic waste and mine drainage are degrading the creek. Sulfidic waste slumps into the creek, and drainage from the dump flows directly into the creek.

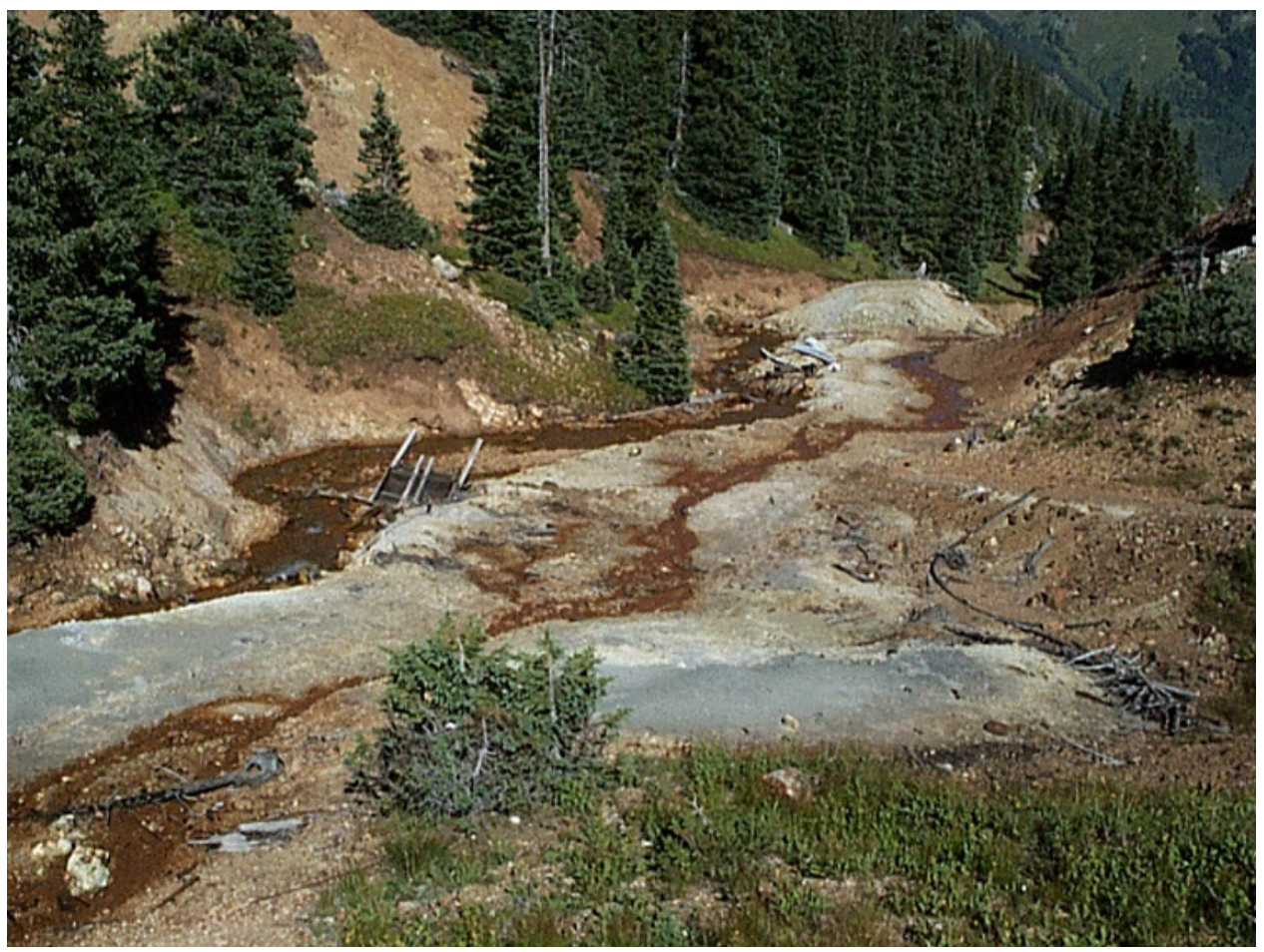

Photograph 84. The Vernon mine on Gray Copper Gulch Creek creates several problems as shown here. Sulfidic waste is very close to the creek, and mine drainage (the flow on right) reacts with the sulfidic waste to create even more acidic metal-rich water, which flows into the creek. 


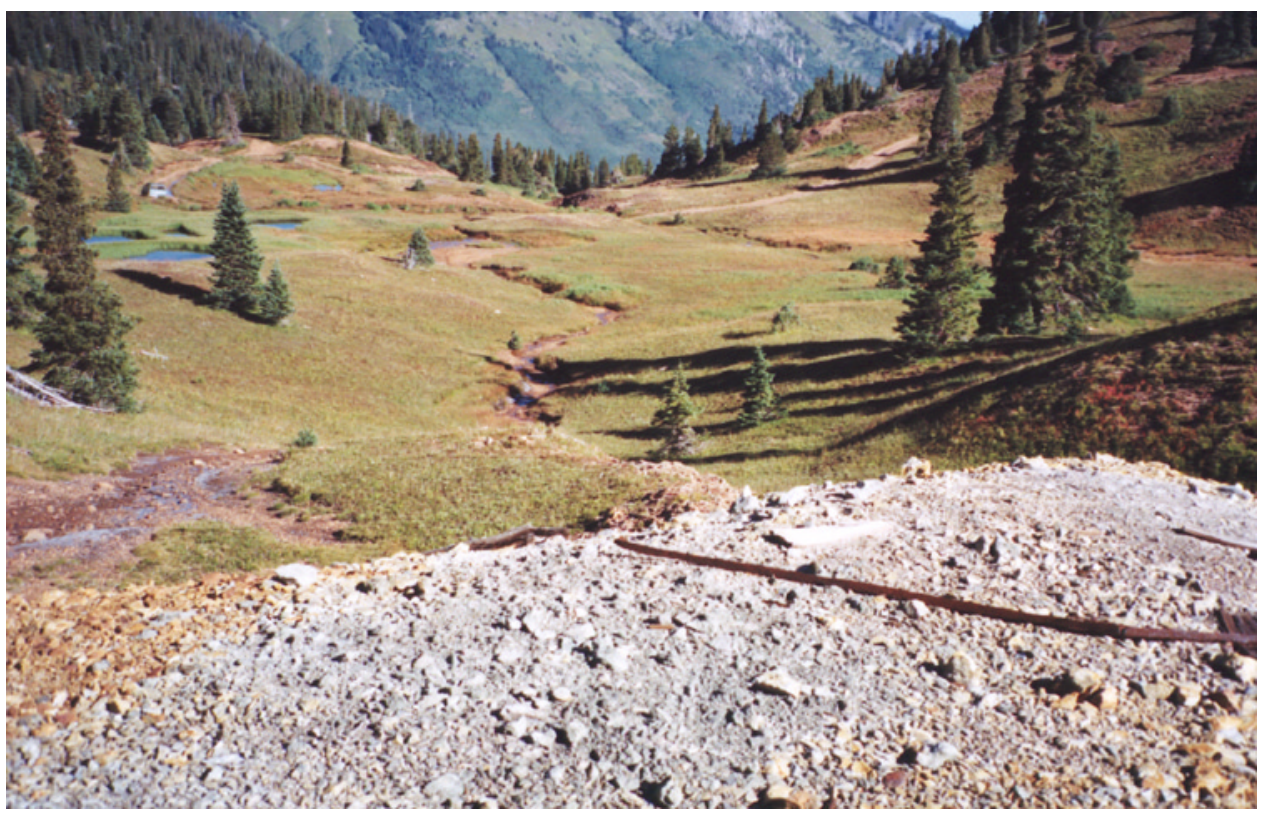

Photograph 85. The headwaters of Gray Copper Gulch creek received contaminated waters from several sources, including small mine dumps like this. Springs on fractures in altered rocks also are sources of acidic waters. The small spring on the left side of the dump has a $\mathrm{pH}$ of 3.

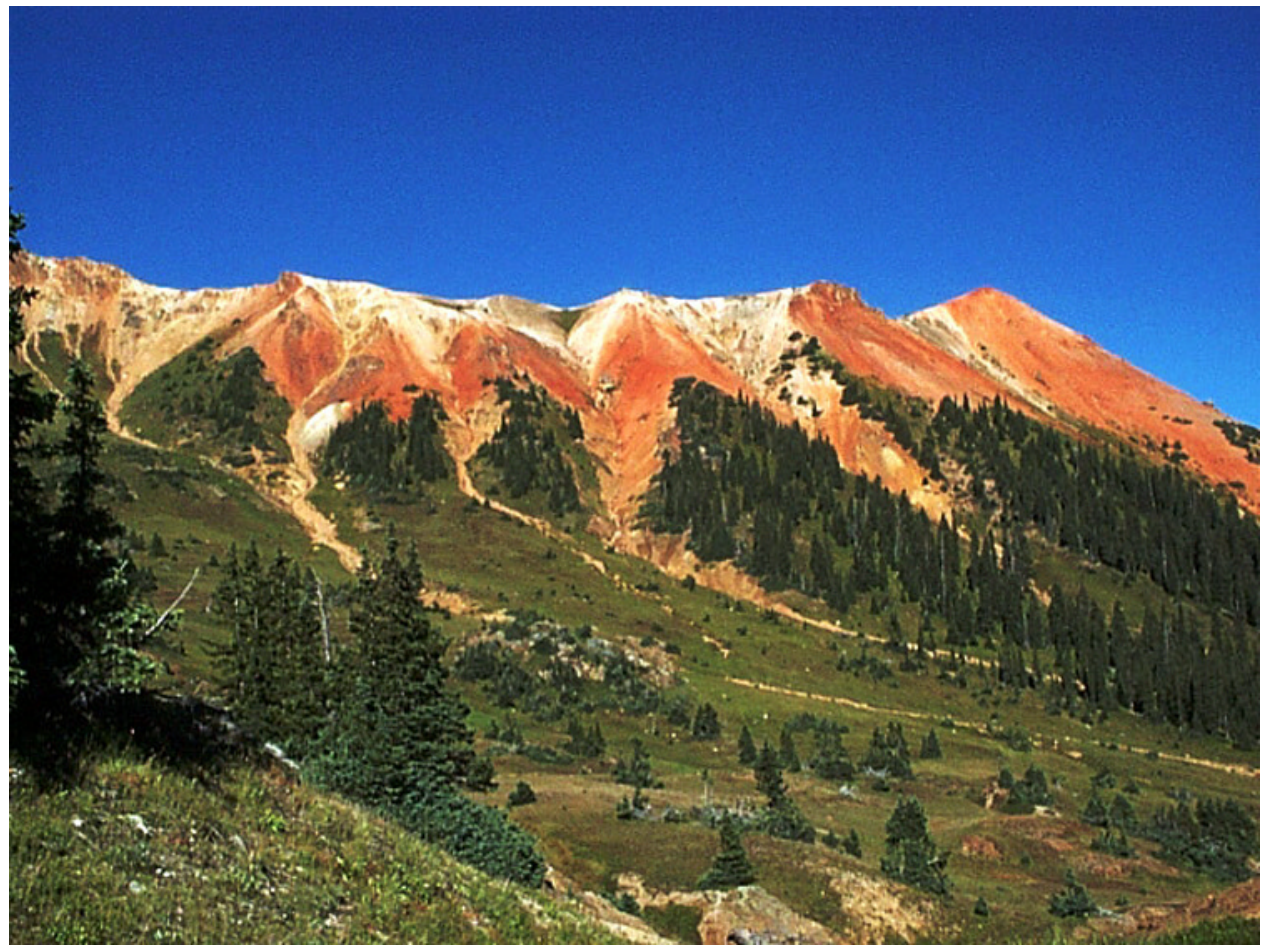

Photograph 86. The highly altered rocks on Red Mountain No. 3 on the west side of Gray Copper Gulch are a likely source of much of the acid and metals in the creek. The alteration is the acidsulfate type (pyrite-alunite) that is well known as a source of acid and metals. 


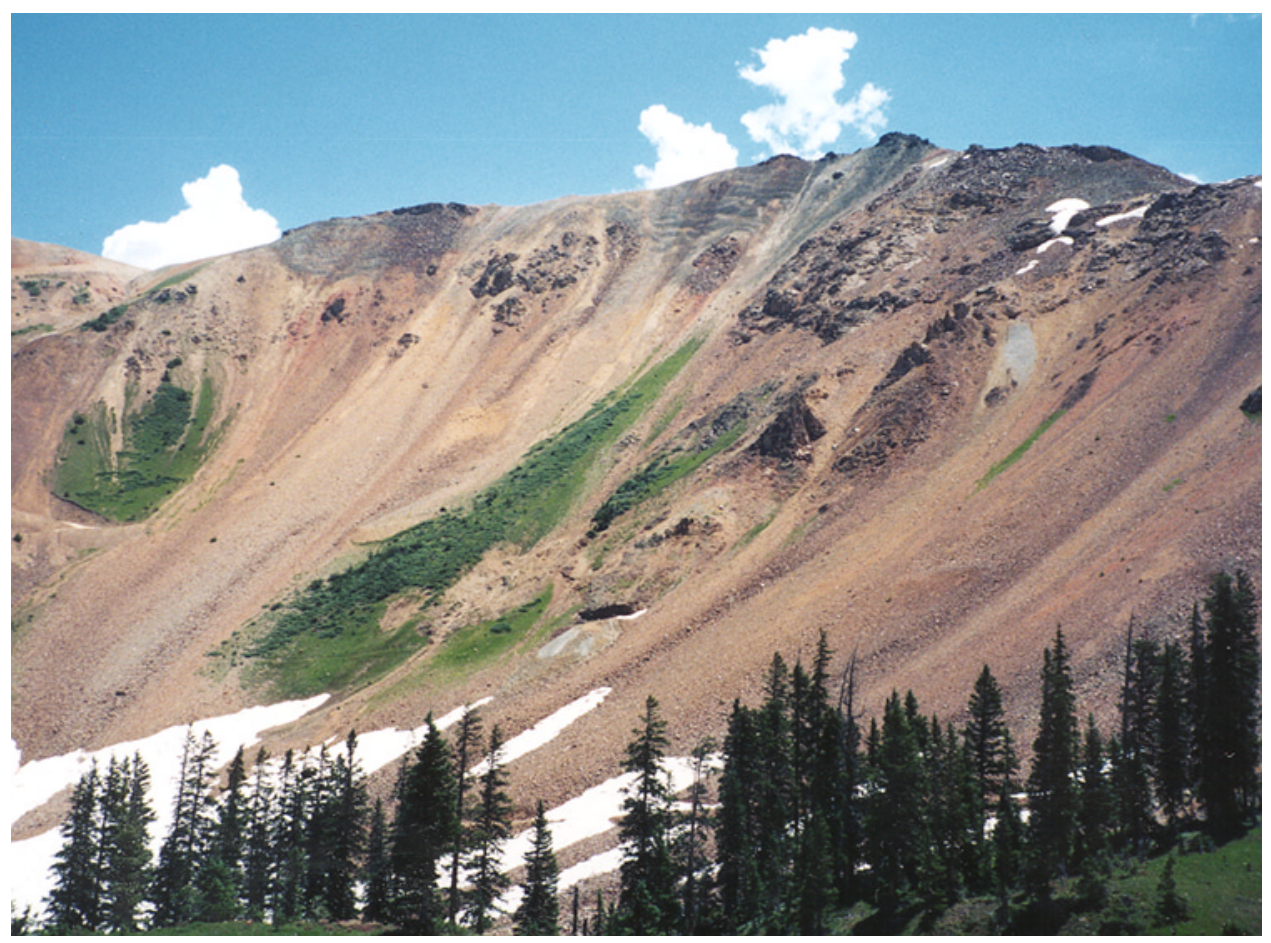

Photograph 87. Corkscrew Gulch Creek on the flank of Red Mountain No. 1 is highly degraded for a watershed containing only a few small mines. One adit at the head of Corkscrew Gulch (small dump, center of photograph) releases $\mathrm{pH} 2$ water with extremely high metal concentrations. This extremely acidic water may reflect both mine workings and unmined altered rocks.

Mineralized rocks and ores.-Several kinds of ore were mined in this district, but chemical analyses show them to be generally similar in their polymetallic character, being rich in $\mathrm{Ag}, \mathrm{As}, \mathrm{Bi}, \mathrm{Cd}, \mathrm{Cu}, \mathrm{Mo}, \mathrm{Pb}, \mathrm{Sb}$, and $\mathrm{Zn}$. Comparing the results for 12 dump samples from breccia pipes with results for 14 samples from polymetallic veins, the pipe ores are richer in $\mathrm{Ag}$, As, and possibly $\mathrm{Au}$, whereas the vein ores and waste materials have substantially higher concentrations of $\mathrm{Mn}, \mathrm{Mo}, \mathrm{Pb}$, and $\mathrm{Zn}$. Analyses of three tailings samples from the Idarado mill (which processed vein ore) show their basemetal concentrations are relatively low, roughly one-fourth to one-half that of dump samples, but substantially higher than unmined rocks. Altered red rocks on Red Mountain (photograph 86) are well known for their unusual geochemical signature, including high arsenic concentrations (Fisher and Leedy, 1973).

Leach tests made on 20 samples (10 pipe deposits, 10 vein deposits) show a strong tendency for mine waste to generate acid: leach solutions averaged $\mathrm{pH} 3.0$ for pipe samples and 3.1 for vein samples, and $\mathrm{pH}$ values ranged from 2.5 to 3.7. The acidic leachate solutions carried high amounts of $\mathrm{Fe}, \mathrm{Cu}$, $\mathrm{Zn}$, and $\mathrm{Pb}$, but other metals were moderate to low. Although most analytical results for $\mathrm{Ag}$ in leachate solutions were not valid because of analytical difficulties, some apparently reliable results for $\mathrm{Ag}$ in these 20 leachate solutions are very high This suggests that there is potential for Ag toxicity, which is rarely an issue in waters on the CWS. Comparing the two groups, $\mathrm{Fe}$ and $\mathrm{Pb}$ tended to be higher from the pipe-deposit leachates, whereas $\mathrm{Mn}$ and $\mathrm{Zn}$ tended to be higher in the vein-sample leachates. The high $\mathrm{Pb}$ in pipe-sample leachates is notable and is not predicted by the bulk composition of the samples ( $\mathrm{Pb}$ tends to be higher in the vein samples). The high amount of $\mathrm{Pb}$ in leach solutions may reflect the presence of anglesite in pipe deposit samples, whereas galena in the vein samples would not yield much $\mathrm{Pb}$ to those leach solutions (Desborough, in Nash and others, 1998). The differences in detail between the pipe- and vein-deposit sample test results do not appear to be significant for this reconnaissance evaluation: waste from both deposit types has the potential to generate acid and release many base metals.

Mills and tailings.-The early production from this district was direct-shipping ore that was sent to a smelter. A small amount of the pre-1900 production went to the Saratoga smelter in this district, but most was smelted outside the district. A small amount of ore from the district was milled at Chattanooga (south of Red Mountain Pass) prior to about 1920. The mill at the Barstow mine is described in the literature, but was not accessible. A small volume of tailings at the Silver Bell mine suggest there was a mill at that site, and it probably would have been active prior to 1900 . The Idarado mill east of the Treasury Tunnel seems to have been the only major mill in the Red Mountain district, and it was expanded 
several times to about 500 tons/day prior to closure in 1956. Tailings were placed in at least four impoundments (CDPH, 1992). Also tailings from some stage of activity, or perhaps some major spills, are in the middle reach of Red Mountain Creek above the Joker Tunnel. The large Idarado tailings impoundments were reclaimed in the mid-1990's by recontouring and planting of grasses.

Two estimates of what metals may be mobilized from the tailings can be made from a laboratory leach test and from the composition of waters seeping from the tailings. The laboratory leach test yielded a solution with a $\mathrm{pH}$ of 3.5 and elevated concentrations of $\mathrm{Cu}, \mathrm{Fe}$, and $\mathrm{Zn}$. The metal concentrations were higher than from normal rocks, but lower than from most dump samples. Seeps from the tailings piles, sampled at two locations in 1997 and 1998, had $\mathrm{pH}$ values in the range 2.5 to 2.9 , very high conductivities $(>2,000 \mu \mathrm{S} / \mathrm{cm})$, and analyses showed extremely high concentrations of $\mathrm{Al}, \mathrm{Cd}, \mathrm{Cu}, \mathrm{Fe}, \mathrm{Mn}$, and $\mathrm{Zn}$. The individual seeps flow only a few gallons per minute, but the total infiltration into alluvium underlying the tailings could be substantial. The total amount of flow, and the metal loadings, requires information from wells. Drilling of wells should be considered for the monitoring phase of the Red Mountain (Idarado) reclamation.

Surface waters.-Water from heavy snow and summer storms is evident in this district as flows from many mine adits, countless springs, and numerous streams. The main stream that cuts through the center of the district is Red Mountain Creek, and there are substantial streams in side canyons such as Corkscrew and Gray Copper (fig. 24). Essentially all of the streams in the eastern half of the district, east of U.S. Highway 550 , are visibly contaminated with suspended tan or ocher material and ubiquitous red to ocher coatings on cobbles, streambed, and refuse such as logs. Because these coatings form on recent trash such as logs that were not present in 1998, I believe that the coatings continued to form in 1999. However, I do not have observations that can define the time of formation relative to high and low flow regimes. Streams on the west side tend to be clear, but a few do deposit tan and ocher coatings on streambeds. The Red Mountain Creek watershed in the headwaters of Uncompahgre River is the most complex in the study area and is of high concern for contamination (CWQCD, 1998, 303(b) list). My geochemical studies show that it contains many mine-related sources of extremely contaminated water, and natural attenuation of $\mathrm{pH}$ and metal concentrations in the streams is not as effective as in other districts. Quite simply, the streams on the east side of this district are overwhelmed by the input from unmined and mined sources.

The remarkably high metal concentrations of waters in this district are summarized in table 12. The general trends in this district are possibly more significant than the details of contamination at specific mine sites.

1. Mine tunnels driven into Red Mountain, as at Joker and Guston (photograph 71), individually produce hundreds of gallons per minute of extremely degraded, acidic waters that are $10 \times$ to $100 \times$ or more in excess
Table 12. Summary of surface-water compositions, Red Mountain district.

[Metal compositions in parts per billion; analyses by ICP-MS. Cond., conductivity $(\mu \mathrm{S} / \mathrm{cm})]$

\begin{tabular}{|c|c|c|c|c|}
\hline & \multicolumn{2}{|c|}{ Mine and dump drainage $(n=20)$} & \multicolumn{2}{|c|}{ Streams $(n=24)$} \\
\hline & Median & Maximum & Median & Maximum \\
\hline $\mathrm{pH}$ & 2.9 & 2.0 & 3.4 & 2.5 \\
\hline Cond. & 1,375 & $>2,000$ & 366 & 1,035 \\
\hline $\mathrm{Al}$ & 40,200 & 470,000 & 5,600 & 21,600 \\
\hline As & 20 & 675 & 0.7 & 44 \\
\hline $\mathrm{Cd}$ & 9.4 & 152 & 0.8 & 36 \\
\hline $\mathrm{Cu}$ & 775 & 11,400 & 229 & 5,860 \\
\hline $\mathrm{Fe}$ & 116,600 & 721,000 & 3,000 & 63,500 \\
\hline Mn & 3,800 & 106,000 & 291 & 3,460 \\
\hline $\mathrm{Pb}$ & 38 & 367 & 26 & 189 \\
\hline $\mathrm{Zn}$ & 1,800 & 30,400 & 132 & 5,500 \\
\hline
\end{tabular}

of ALWS. The Joker tunnel has a large flow of $\mathrm{pH} 2.9$ water with very high metal concentrations (e.g., Fe 60x and $\mathrm{Zn} 75 \times \mathrm{ALWS}$ ), and metal concentrations in some other mine drainages are even higher.

2. Mine tunnels on the western side, driven to access veins in green-altered volcanic rocks, release water that is variable in $\mathrm{pH}$ and metal content. At some mines the water quality is relatively good, and at others the high Fe concentrations produce conspicuous amounts of ocher to orange precipitate (photograph 76). However, none of the water samples from the western side is degraded to the extent of the acidic waters on the Red Mountain side. A rare example of high metal content in a west-side water is the drainage from the Mountain King mine; this high volume of $\mathrm{pH} 6.3$ water forms orange iron floc outside the portal. Metal concentrations are high but not extreme (Fe 2.3×, Zn 10× ALWS).

3. Unmined sources create highly acidic waters in some places, and some of these waters can be very rich in metals. One example is the creek in Champion Gulch, an area on the west flank of Red Mountain where there are few mines. The creek has a $\mathrm{pH}$ of 2.9 and very high metal concentrations (e.g., Fe 29×, Zn 11× ALWS). Not all streams and springs on the flank of Red Mountain are this acidic_-some have $\mathrm{pH}$ values of 5 to 6 , and at those pH's metal concentrations are below ALWS.

4. Many of the mine drainages are arguably of mixed sources, reflecting the influence of both mining and natural processes. A good example is the unnamed adit at the head of Corkscrew Gulch, which possibly extends 300-500 ft into red pyritic rocks: it yields a flow of $\mathrm{pH} 2.1$ water with extreme metal concentrations (Fe 720×, Zn 26×, ALWS, and the highest As concentration in the study at $675 \mathrm{ppb}$ ). Based on my 
experience with hundreds of other mine workings, my estimate is that the composition of this mine drainage is too extreme to be the result of reactions in the small mine tunnel. Reactions in unmined altered rocks also appear to contribute acid and metals to this water. Quantitative evaluation of anthropogenic and natural sources of acid and metals in water such as this is extremely complicated, and even the best scientific methods may produce ambiguous results.

5. Mill tailings in Red Mountain Creek (photograph 77) and in four large impoundments contribute acid and metals to the creek. For example, water seeping from a tailings impoundment along the middle part of the creek (photograph 77) has a $\mathrm{pH}$ of 2.5 and elevated base-metal concentrations: the concentration of $\mathrm{Fe}$ in this water is moderate at $2.5 \times$, but other metal concentrations are much higher $(\mathrm{Zn}$ is $26 \times, \mathrm{Cu}$ is $63 \times$, and $\mathrm{Pb}$ is $110 \times$ ALWS). The contributions from the tailings are impossible to determine without wells to sample shallow ground waters that eventually flow into the creek.

6. The degree of recovery after reclamation cannot be assessed here. My observations and samples were from 1997 and 1998 - after the reclamation activities in the Red Mountain Creek basin that included work on the large tailings impoundments and construction of concrete gutters to carry mine drainage over and around mine waste (photograph 74). Surface-water quality was not good in 1997 but may have been worse previously. A logging operation on hundreds of acres of private property in 1997 and 1998 probably also contributed to suspended sediment and metals to surface waters; this logging operation disturbed large areas, and few measures were used to contain or control surface runoff.

The CZI values for stream waters in this district range from 0.02 to 160 and only six of 28 stream-water samples are below the "healthy" value of 1 . Many mine-adit drainage waters and seeps from dumps or tailings have high to extremely high CZI values (14 to 1,405). These values, which indicate high to severe threat to aquatic life, come from both copper and zinc. In detail, some sources are higher in $\mathrm{Zn}$ concentrations than $\mathrm{Cu}$, but the problem at many of these sources is the high $\mathrm{Cu}$ concentrations (and the higher toxicity of $\mathrm{Cu}$ to aquatic life such as fish and invertebrates described earlier). These water compositions may be only part of the bad news for these streams. A possibly even greater problem may be the widespread deposits of ocher schwertmanite (ironoxyhydroxide-sulfate) and other complex coatings on streambeds. The geochemistry of these materials and their implications for biota should be studied in detail as they are in the Cement Creek basin, on the east side of Red Mountain (USGS, 2000). One of the dire aspects of these complex iron deposits is their high reactivity: they precipitate and adsorb toxic metals such as $\mathrm{Cu}$ and $\mathrm{Zn}$ during low flow regimes and dissolve them during high flow stages, releasing acid and the adsorbed base metals (Desborough and others, 2000).
Some features indicate that geologic processes are working to naturally attenuate some of the acidic, metal-rich waters in this district. For example, mixing of stream waters has beneficial effects. Tributaries entering Red Mountain Creek from the west mix with the acidic surface waters from the east to produce water with $\mathrm{pH}$ of about 3.6, conductivity of about $400 \mu \mathrm{S} / \mathrm{cm}$, and much lower metal concentrations (Zn content drops to about $600 \mathrm{ppb}$ ). The quality of Red Mountain Creek leaving Ironton Park (photograph 82) is further improved ( $\mathrm{Zn}$ is about $300 \mathrm{ppb}$ ) but $\mathrm{pH}$ remains near 3.6. The mixing reactions cause iron oxyhydroxide materials to precipitate, and trace metals coprecipitate with the iron (Smith and others, 1994; Plumlee and others, 1999). Similar mixing occurs in some of the side tributaries, such as Corkscrew Creek, but in Corkscrew the $\mathrm{pH}$ and water quality do not improve much, which suggests there is low buffering capacity in this basin. It appears that natural processes in Red Mountain Creek basin are inadequate to improve quality to levels required for aquatic life. Possibly the chief reason for the limited recovery of Red Mountain Creek is the large amount of material that precipitates within the streambed (photograph 76). Some of this material probably is schwertmanite, as in Cement Creek near Silverton (Desborough and others, 2000; Nash, 1999b). Other kinds of coatings, such as clay, generally coats rocks, cobbles, and streambeds, preventing water-rock reactions that otherwise might be beneficial (Plumlee, 1999).

More specific description is needed for several mines or mining areas that are sources of degraded water that are significant, yet seemingly small sources when compared to those near Red Mountain Creek. The mining areas that will be discussed next are in two tributaries, Gray Copper Gulch and Corkscrew Gulch, that are east of Red Mountain Creek. The headwaters of Gray Copper Gulch are on the flank of a large ridge of red, pyritic alteration (photograph 86). Small mines and prospects expose highly pyritic rocks and create moderately acidic drainage ( $\mathrm{pH}$ values are 3 to 4 ) (photograph 85). The largest mine in the upper basin, the Vernon mine, has sulfidic dumps of moderate size and an adit that releases acidic water ( $\mathrm{pH} 2.9$ ) (photographs 83, 84) containing very high metal concentrations: $\mathrm{Cu}, \mathrm{Fe}, \mathrm{Zn}$, and are $60 \times, 5 \times$, and 1.7× ALWS, respectively. The creek is acidic ( $\mathrm{pH} 3.2$ ) above the Vernon mine, presumably from both small mines and from unmined red-altered rocks. Water quality is much worse below the Vernon dumps and mine drainage: the $\mathrm{pH}$ of the stream is 2.9 , and $\mathrm{Cu}, \mathrm{Fe}$, and $\mathrm{Zn}$ concentrations increase eight- to twelve-fold to levels well above ALWS. A stream sample taken $1.5 \mathrm{mi}$ west of the Vernon mine shows that the composition has improved by natural attenuation, but the $\mathrm{pH}$ is still acidic (4.9) and several metals remain highly concentrated ( $\mathrm{Al} 6 \times$, $\mathrm{Cu} 2 \times, \mathrm{Fe} 1.5 \times$, and $\mathrm{Zn} 0.6 \times$ ALWS). The ZCI values for Gray Copper Gulch creek drop from 7 below the mine to 0.6 near Red Mountain Creek. The major source, the Vernon mine, lies on several patents, but much of the waste and water release appear to be on Uncompahgre National Forest lands. By tonnage, this site is a fraction of the size of Idarado mine complex, and by metal loading it is small compared to other 
sources, but the Vernon property clearly contributes to waterquality problems in the upper Uncompahgre River watershed and merits further study for reclamation options.

Corkscrew Gulch creek contributes acidic, metal-rich waters to Red Mountain Creek. This small watershed is mostly on public lands, with scattered mine patents such as at the Midnight mine, the largest of the small mines in the basin. Sources of acid and metal are not easily quantified in this basin. The productive mines had shafts that produce no surface drainage, and their dumps do not appear to contribute much to nearby small tributaries. One mine tunnel at the head of Corkscrew Gulch (site N587, mentioned earlier) produces 20-40 gpm of $\mathrm{pH} 2.1$ water that is extremely rich in metals. This mine is a major contributor to Corkscrew Gulch creek, but there must be numerous other ground water sources that contribute to, or sustain, the low $\mathrm{pH}$ and high metal concentrations. The CZI values for samples of the creek decrease from 30 at the eastern headwaters to 5 near the Midnight mine then rise to 12 at the gorge. The increase in $\mathrm{Cu}$ and $\mathrm{Zn}$ in the western part of Corkscrew Gulch is not associated with mines, but underlying volcanic rocks are highly altered. Water quality does not improve downstream in this basin, probably because underlying volcanic rocks are highly altered and lack minerals that can neutralize the acidity.

The Silver Mountain mine has workings of moderate size that produce ugly orange water of $\mathrm{pH} 6$ and rich in several metals (Fe $3 \times$, Mn 1.2×, Zn 2.3×). These waters are seemingly a small contribution to the upper Uncompahgre River, but if this mine were in another district or another State, it probably would be highlighted as a significant problem.

Summary.-Field observations, measurements of $\mathrm{pH}$ and conductivity, and chemical analyses of surface waters in the Red Mountain Creek basin suggest that this basin of about 10 $\mathrm{mi}^{2}$ is the most chemically and physically contaminated on the CWS. It is clear to the author that substantial amounts of this contamination is derived from unmined (natural) sources on Red Mountain. Detailed studies such as are underway in the upper Animas River watershed above Silverton (USGS, 2000) would be required to quantify the numerous mined and unmined contributions to the Red Mountain Creek watershed. Geochemical background values in this area clearly were very high prior to mining. One indicator of that high pre-mining background is the abundant ferricrete in the basin and the fact that many early ore discoveries were made on ferricrete outcrops. The ferricrete at the Guston and Hudson mines is spectacular.

Reclamation in compliance with a State Court consent decree has been undertaken on many waste dumps and on the four Idarado mill tailings piles. My reconnaissance observations and sampling in 1997 and 1998, after the reclamation, indicate that waters draining from the reclaimed areas were among the most acidic and metal rich of any found in this study. Although not a biologist, even I can conclude with fair certainty that many of these waters are toxic to aquatic life. Others will have to evaluate the amount of contamination attributable to unmined sources and whether the discharges meet the water-quality objectives of the Remedial Action Plan (CDH, 1992).

The tailings impoundments in this district were established in stable areas and at a fair distance from the major streams; thus, they seem to be relatively low risks in an extreme storm event. The channel for much of Red Mountain Creek is not natural but appears to be of good design. It might be evaluated for capacity to handle a 100 -year flood. If the creek rose out of this constructed channel, the overflow would have access to the lower (largest) tailings impoundment. Fluvial tailings in and along Red Mountain Creek are highly vulnerable to erosion in an extreme storm, but the volume of these tailings is relatively small in comparison with the impounded tailings.

References.-Ransome (1901); Fischer and others (1968); Burbank and others (1972); Mayor (1978); Fisher and Leedy (1973); Plumlee and others (1999).

\section{Mining Districts of the Upper San Miguel River Watershed}

The headwaters of the San Miguel River on the west flank of the San Juan mountains are the sites of some of the larger historic mines in Colorado as well as countless smaller mines that were developed in slightly more than 100 years. The major mines closed by 1980, and there was no significant mining here in the late 1990's. The legacy of mining carries both positive and negative values to the area around Telluride. This relatively small mining area was the most productive of the metal mining areas on the CWS, with a gross value of more than $\$ 250$ million coming from the hard-rock mines of San Miguel County. There also was production of gold from placer operations, which will not be considered here.

As we evaluate the impact of mining on this watershed, it is useful to consider three stages in the mining history. First, there was relatively small scale mining of locally rich veins near the surface. Production during this stage, from 1875 to about 1895 , was approximately $\$ 20,000,000$ for the watershed, chiefly from silver. The second stage was one of increased usage of electricity, long haulage tunnels, and long wire-cable tramways, to increasingly large, consolidated mining complexes with milling centers at Tomboy, Ophir, and Pandora. This second stage, from about 1895 to 1945 , yielded about $\$ 110,000,000$, with silver values about 10 times those of gold. The final stage was the further consolidation of the mines, with the Idarado Mining Co. emerging as the giant operator, and the construction of the large Mill Tunnel and a new mill at Pandora that eventually was expanded to treat more than 3,000 tons per day. The value of ore produced during the final 35 years equaled that of the prior 70 years, and the tonnage of rock broken and ore milled certainly exceeded that of the previous years. Many of the early-mined veins were deemed too small to mine by the new methods and remained idle after World War II. 
The ore deposits within the upper San Miguel River basin are basically similar in their vein character, sulfide mineralogy, and polymetallic composition. There are technical differences that were important for exploration and mining, such as (1) the presence of distinct quartz-adularia-gold veins high in many of the mines, (2) tabular zones of copper-rich ore replacing calcareous parts of the Telluride Conglomerate deep in the Idarado mine, and (3) veins rich in molybdenum and tungsten in granitic intrusions in the Ophir district. If each of these were mined and processed separately, the ore distinctions might have significance to this environmental analysis, but in practice the mine wastes tended to become mixed and rarely are distinct today on the surface.

\section{Telluride District}

Location.-San Miguel County, east of Telluride (fig. 25). The climate is severe, with about $25-40$ inches of precipitation per year.

Principal commodities. - Gold, silver, lead, copper, zinc.

Mining history.-Gold and silver were discovered in 1875 and by 1880 several substantial mines were operating on veins, such as the Smuggler and Argentine, that would be mined by incrementally larger and deeper operations. The first mills were small and simple (photograph 88). As mining progressed, larger mills were erected in the basins above treeline, such as at Tomboy (photographs 89, 90). Then trams several thousand feet long were used to haul ore down to the valley near Pandora to minimize problems with avalanches. Eventually, an elaborate network of long tunnels was created to haul ore more efficiently than by lifting in shafts, and also to drain water from workings 2,000 or more feet deep. The complex history of this major district is well summarized by Henderson (1926), who provides information on the many mills in the district. The larger mining companies, such as Tomboy Gold Mines Co. and Smuggler Union Mining Co., produced about $\$ 600,000$ to $\$ 1,100,00$ gross value per year from the 1890 's to 1920 's. Production decreased in the late 1920's and 1930's but increased in the 1940's when major haulage tunnels were developed. The Mill Level Tunnel to the Pandora mill was completed in 1948, allowing internal, gravity-assisted handling of ore in the Idarado mine. The Pandora mill was expanded several times to more than 3,000 tons/day capacity. In the 1960's and 1970's base-metal replacement ores were mined from the Telluride Conglomerate at the base of the Tertiary section. Deep drilling in the 1970's indicated the presence of geologically significant skarn mineralization in Paleozoic sedimentary rocks about $1,000 \mathrm{ft}$ below the Mill Tunnel level adjacent to the Black Bear vein system (Mayor and Fisher, 1993), too deep to pursue. Mining ceased in 1978.

Production and disturbance.-Production figures may include ores located east of the drainage divide but carried west through long haulage tunnels. Total value through 1964 was about $\$ 229$ million. The value through 1980 has not been published. Total production from the district is estimated at
23,076,000 tons (Long and others, 1998), but this probably includes some Idarado Mining Co. ore processed in the Red Mountain district. The early production from veins in the district was about 13,364,000 tons (Mayor, 1978; this may include some adjoining districts). The production by Idarado Mining Co. from 1946 to 1976 was 10,508,000 tons (Mayor, 1978). The total output to closure was about $14,000,000$ tons. Thus, this district had the largest production from metal mines in the study area (excluding the Uravan district). The major producer was the Idarado mine complex. Disturbance is the largest in study area (excluding Uravan). Numerous old mill sites had unconstrained tailings, which allowed the tailings to move downstream. The very large tailings impoundments at Pandora have been reclaimed.

Geology.-This district is on the western flank of the Silverton caldera and adjacent to the Tertiary Stony Mountain intrusive complex. Deeply incised canyons expose a thick section of Tertiary volcanic flows and tuffs above a thick section of older sedimentary rocks that include prominent Permian red-bed sandstones. Most of the worked veins are within Tertiary volcanic rocks, but some of the production in later years came from replacement zones in the Tertiary Telluride Conglomerate, and similar replacement ores were drilled in deeper, underlying sedimentary units. The well-mapped geology of veins (Burbank and Luedke, 1966) looks like fractures in a broken pane of glass, but there is a fundamental radial pattern related to the caldera and to the intrusion under Mt. Sneffels (Burbank and Luedke, 1968). Hydrothermal alteration is locally intense sericite-pyrite within a few yards of the veins, but, more generally, it is of the low-intensity propylitic type that is green to purple-gray from introduced $\mathrm{Fe}-\mathrm{Mg}$ minerals such as chlorite and, in many places, calcite.

\section{Mineral-Environmental Conditions}

Access to mining sites in many parts of this district is restricted, notably the upland basins in the headwaters of Marshall, Bridal Veil, and Ingram Creeks. Mines and mills in these areas are described briefly by Henderson (1926) and in other reports but could not be visited or sampled in this study.

Mineralized rocks and ores.-The mines in this district worked ores that were rich in $\mathrm{Ag}, \mathrm{Pb}, \mathrm{Zn}$, and $\mathrm{Cu}$, and local vein portions (often at higher elevations) that were rich in $\mathrm{Au}$ (Fisher, 1990). Consistent with the well-described ore minerals and production, samples of mine dumps tend to be rich in $\mathrm{Cd}, \mathrm{Cu}, \mathrm{Pb}, \mathrm{Zn}$, with variable high to moderate amounts of $\mathrm{Mn}, \mathrm{Ag}$, and As. Leach tests were made on two samples from Tomboy basin, yielding similar $\mathrm{pH}$ values (pH 3.6 and 4.0) but considerably different compositions; the $\mathrm{pH} 4$ leachate carried very high concentrations of $\mathrm{Cd}, \mathrm{Cu}, \mathrm{Mn}, \mathrm{Pb}$, and $\mathrm{Zn}$, whereas the $\mathrm{pH} 3.6$ solution contained moderate concentrations of these metals. The fact that surface waters of this district have near-neutral $\mathrm{pH}$, as described later, indicates that

Text continues on page 124 


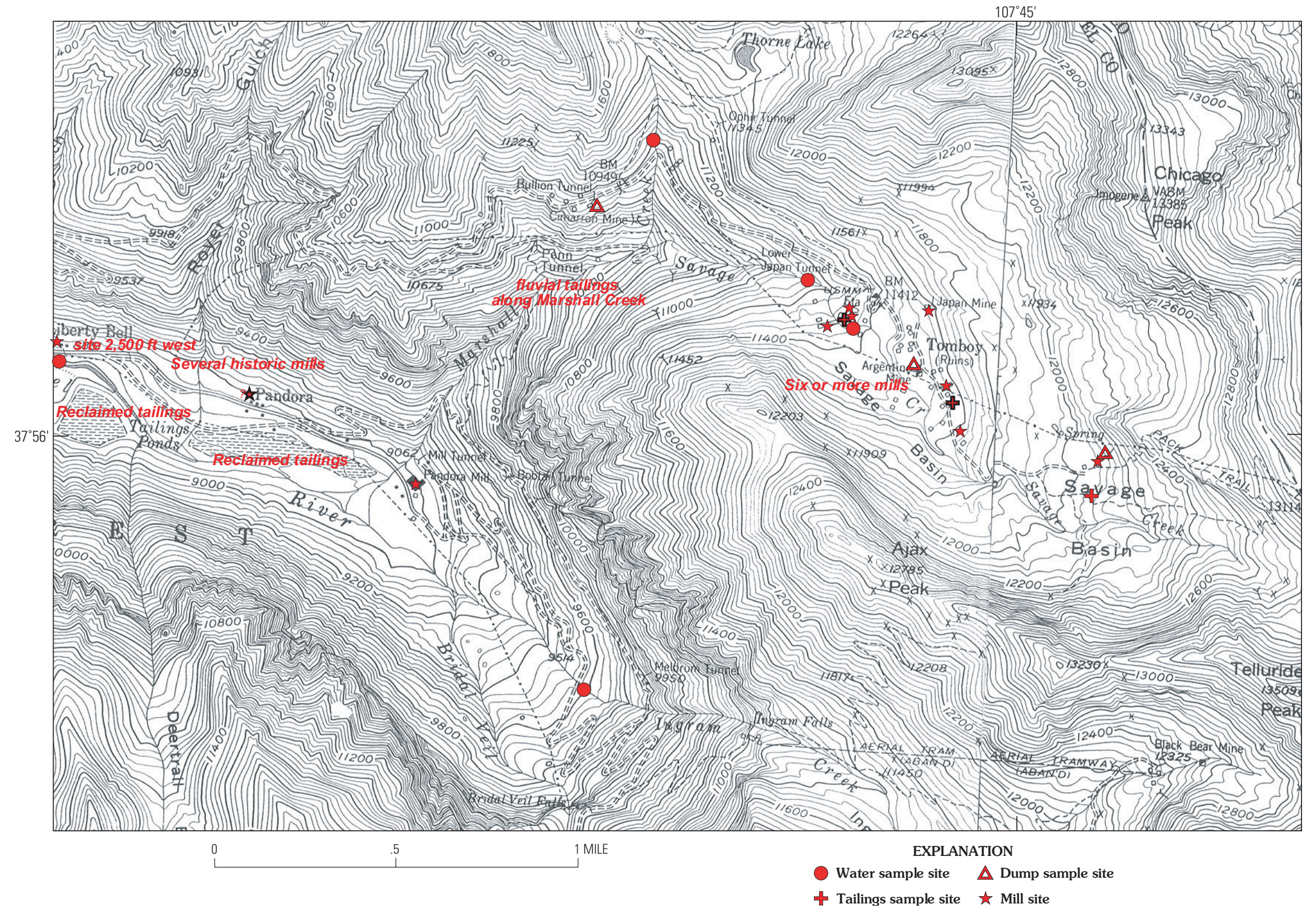

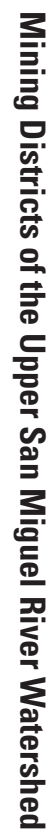

Figure 25. Features of part of the Telluride mining district, east of Telluride, showing sample localities. 


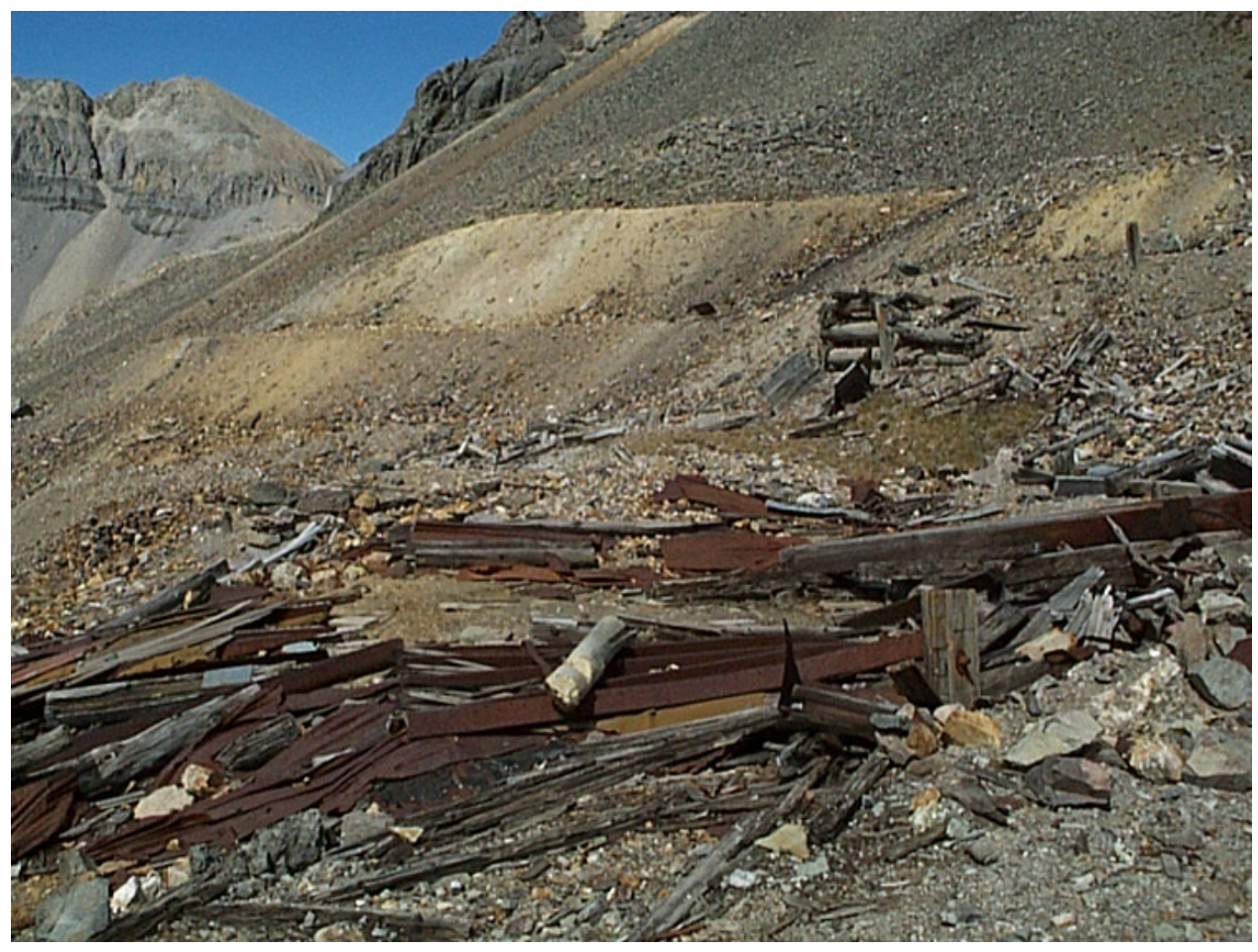

Photograph 88. These are the sparse remains of a pre-1900 stamp mill in Savage basin, east of Telluride. Some of the metal parts are from jig tables used to concentrate gold.

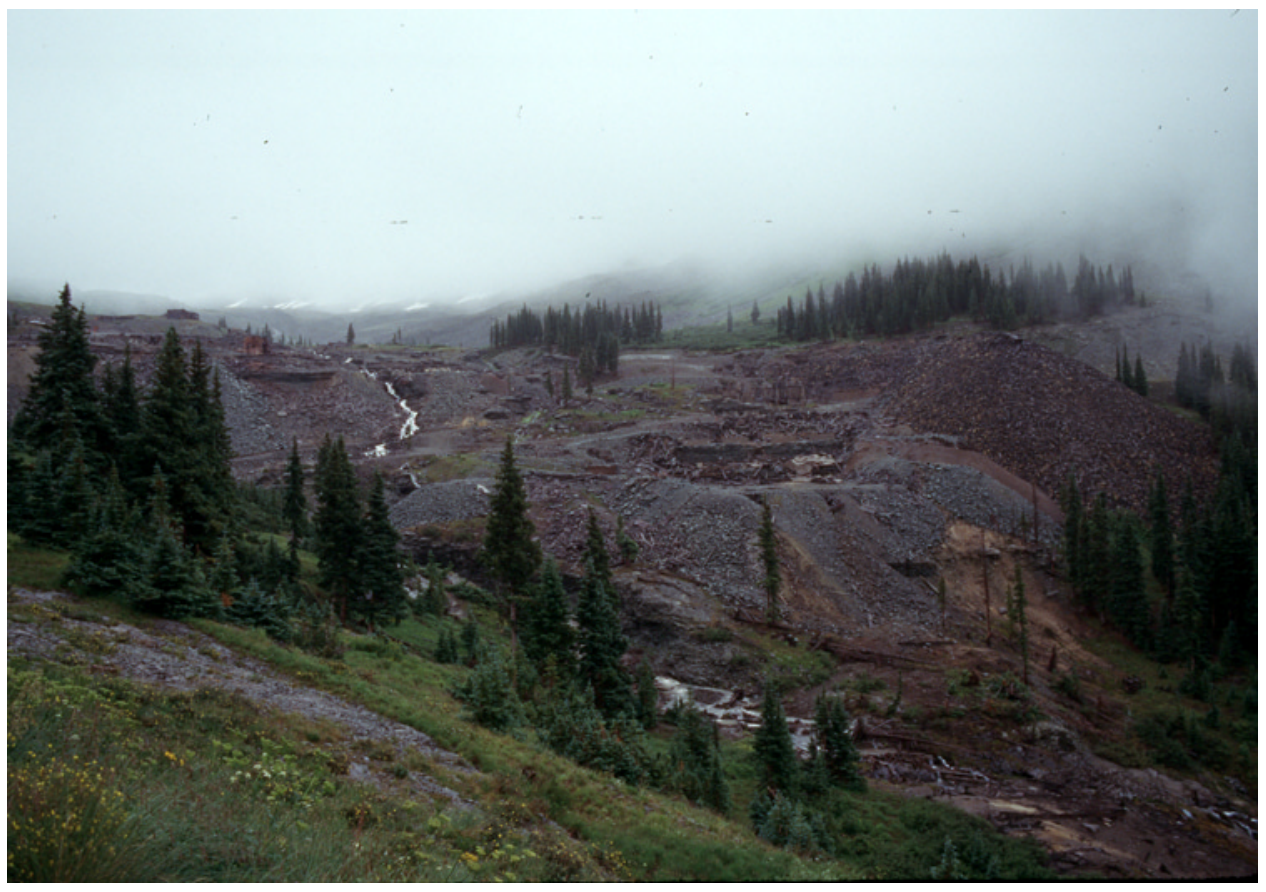

Photograph 89. Tomboy basin above Telluride was the site of several large mills between 1890 and 1910, before ore was carried down the mountain to Pandora in tunnels or trams. There was very little space for mining and milling facilities, and a small amount of tailings can be found near these mills, a small percentage of their production. 


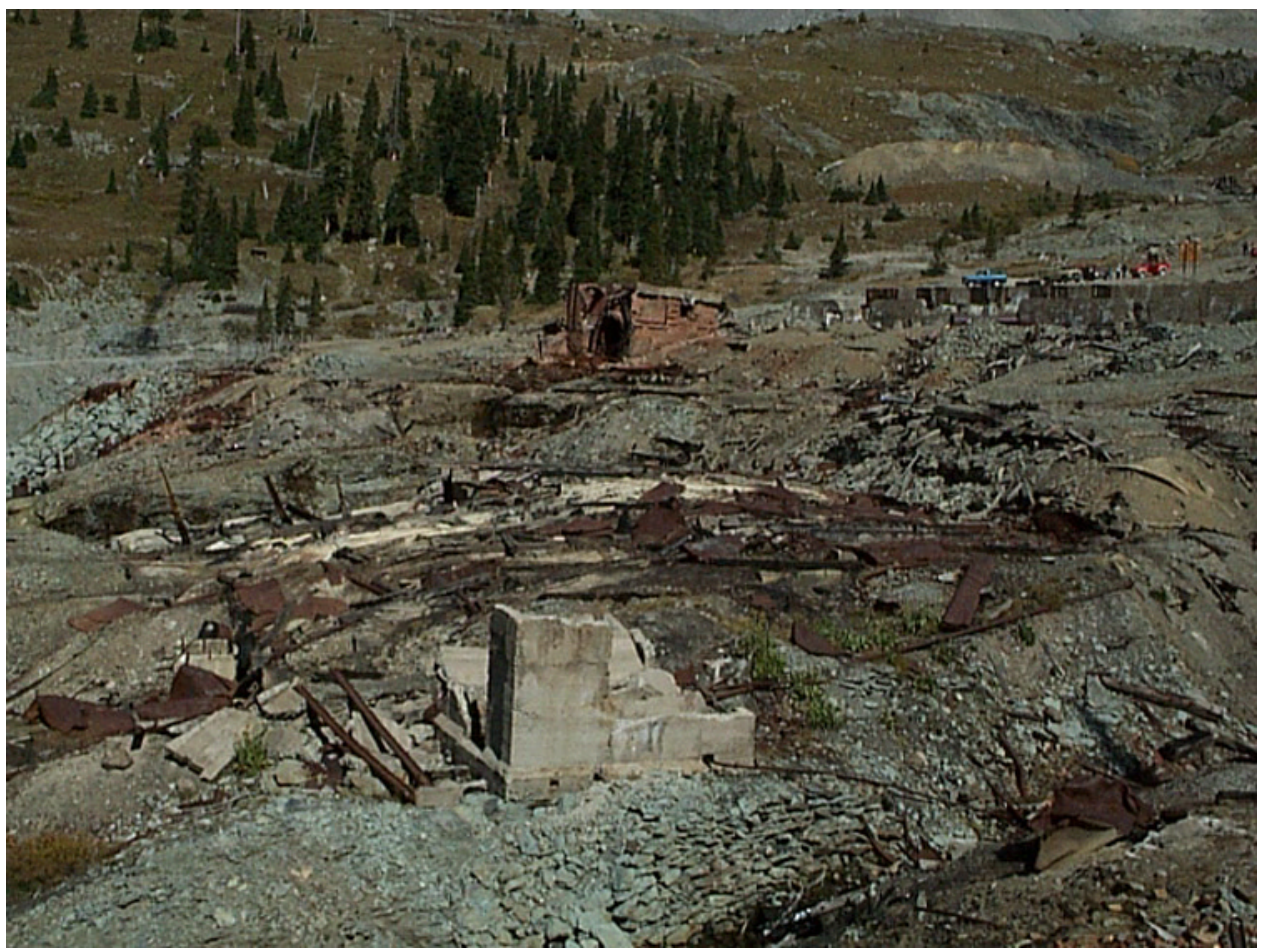

Photograph 90. Four or more mills at Tomboy were located at the west edge of the upland basin at the edge of the cliffs. The burned remains appear to be from at least two mills, built very close together. The was no space for waste rock or tailings.

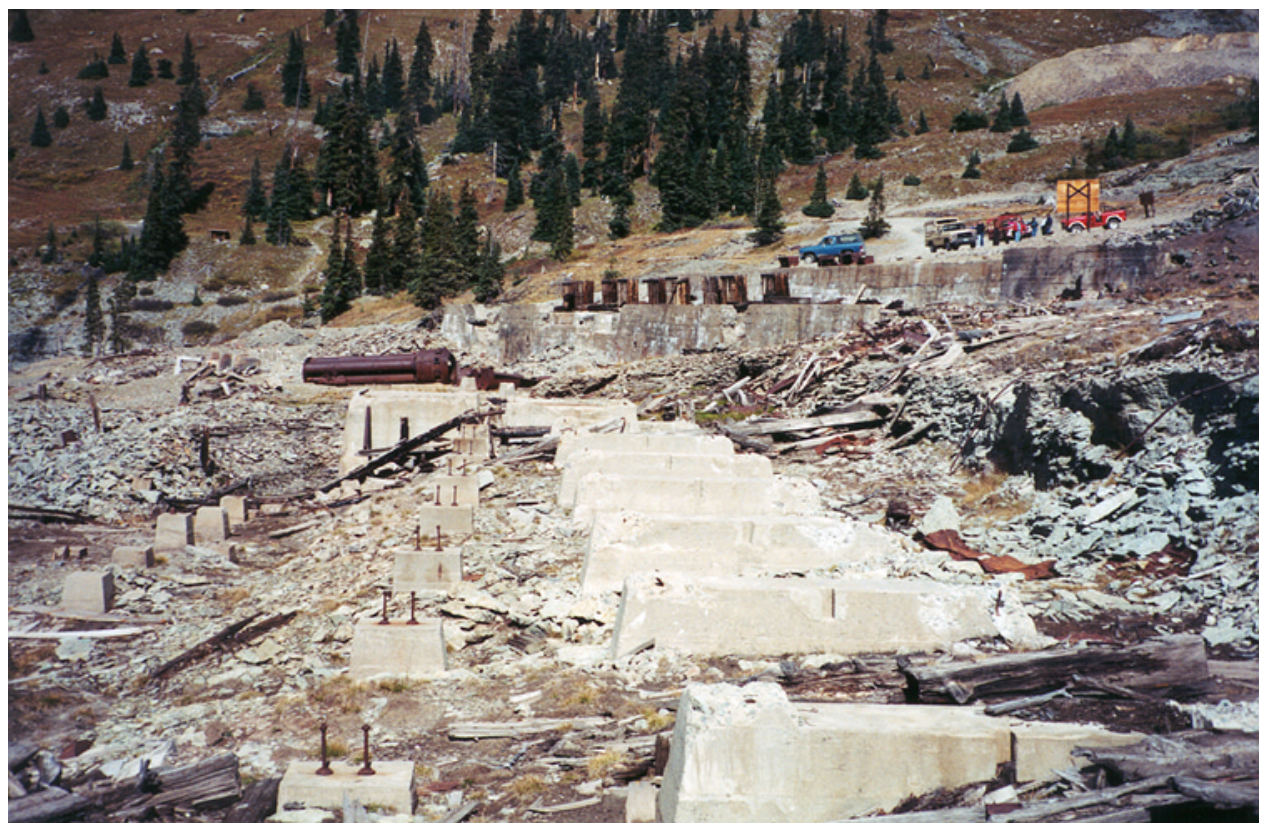

Photograph 91. Mills require strong foundations for the heavy equipment, and often that is all that remains after a fire. This is a closer view of part of photograph 90 . 


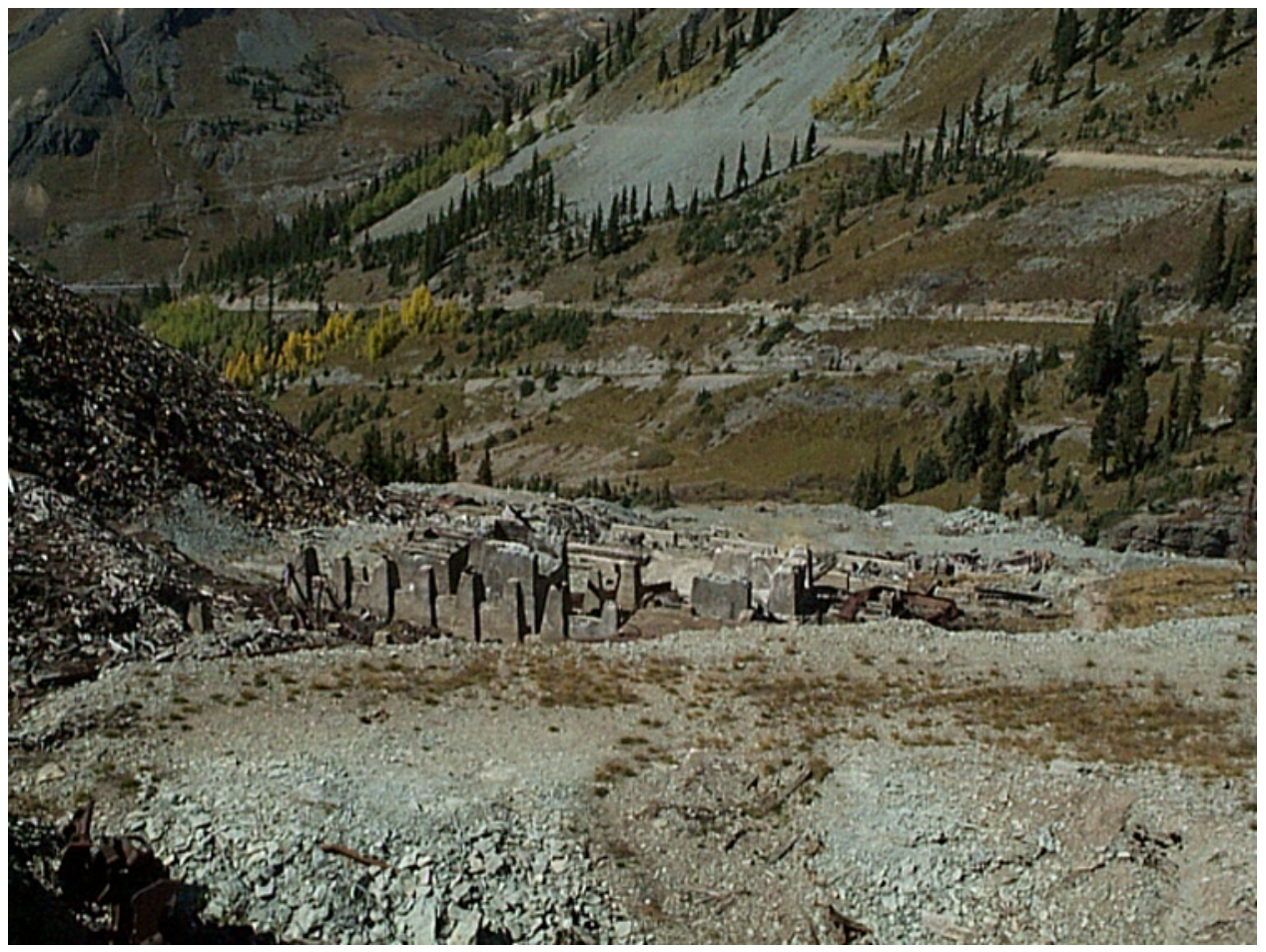

Photograph 92. These ruins are another mill southwest of those shown in photograph 90. No tailings could be retained at the edge of the cliff.

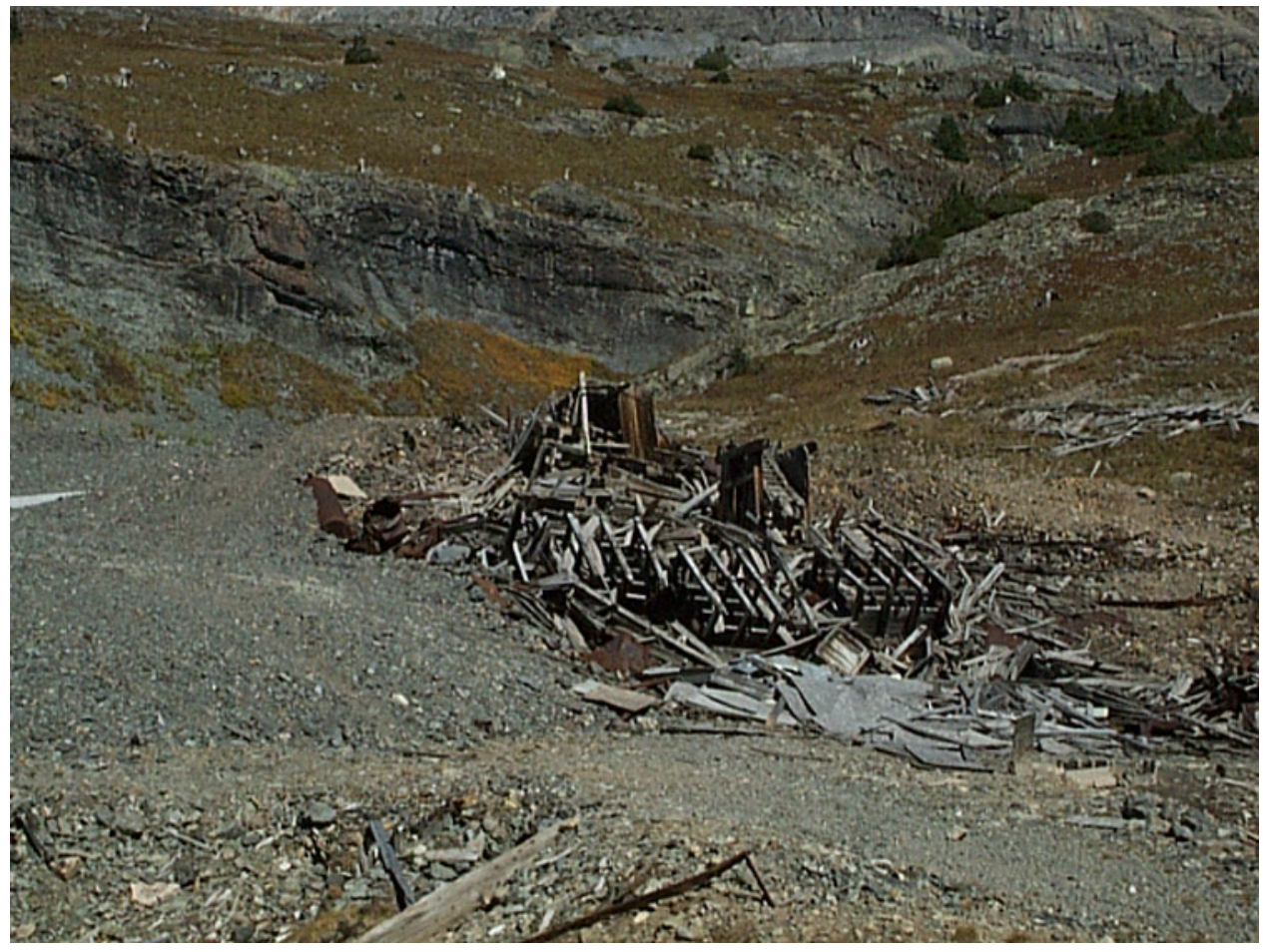

Photograph 93. The Japan mill was a medium-sized producer in Tomboy basin through about 1910. Jig tables can be identified in the collapsed ruins. 


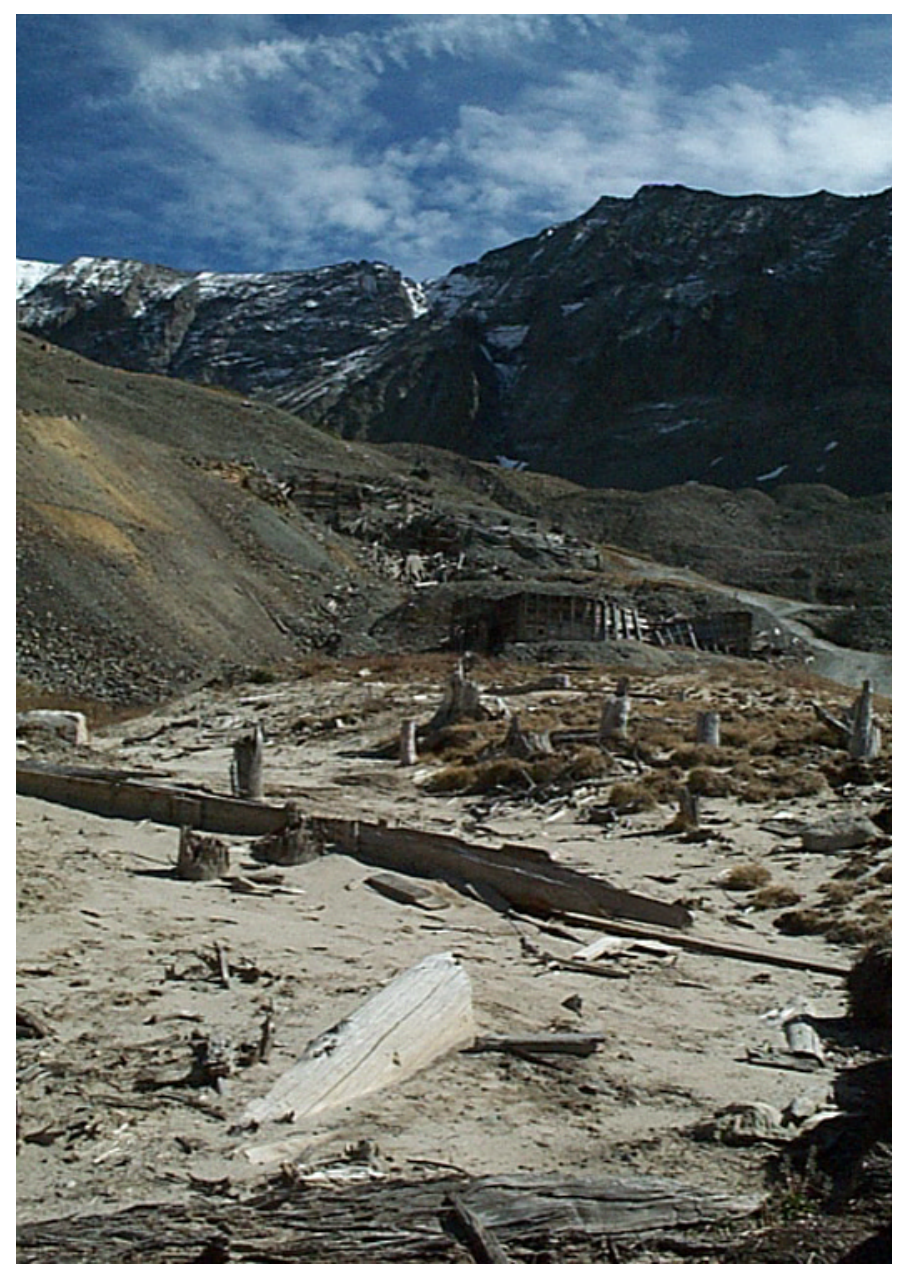

Photograph 94. The Argentine mine and mill complex in Tomboy basin was one of the large producers in that area from about 1890 to 1920. The mill probably was in the area supported by beams and logs (center), and tailings were poured out on the surface (foreground). A few hundred tons of tailings remain, but much more must have been lost from this large producer.

Photograph 95. This view of Marshall Creek, west of the junction with Savage Creek, shows the rugged character of the canyon below Tomboy. Patches of rusty colored mill tailings can be seen along the banks of the creek, remnants of the much larger volume of tailings dumped into Savage creek prior to 1920.

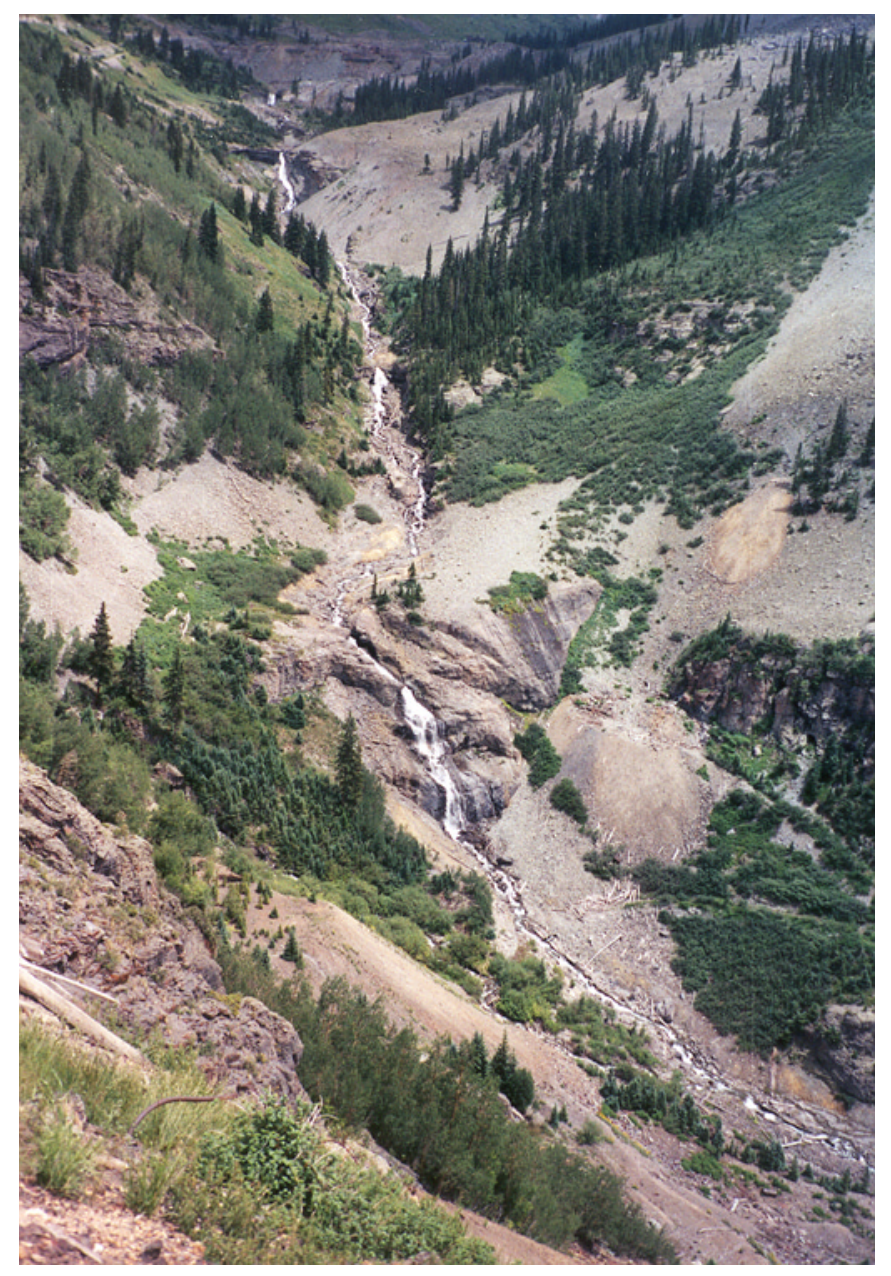




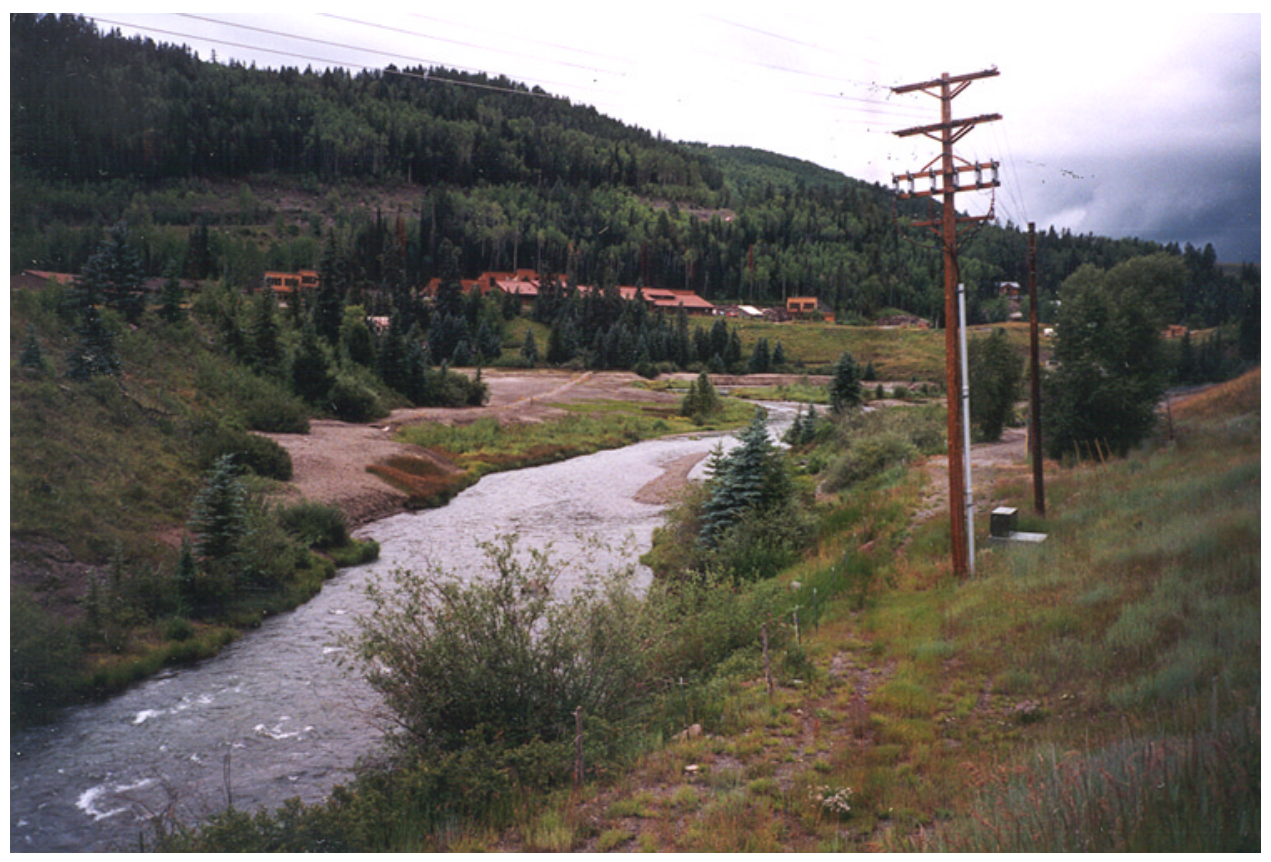

Photograph 96. Mill tailings are in and along the San Miguel River at Society Turn, about 4 mi west of Telluride. The mill may have been located on the old railroad, near here, but the exact site could not be located with certainty. There is a plan to reclaim this area.

the acid-generating potential of mine waste does not actually result in acidic surface waters; this may be a consequence of buffering by the widespread green-altered volcanic rocks.

Mills and tailings.-Mills were numerous, and tailings were produced in abundance, to support the large production from this district. I was able to identify the remains of 12 mills, and this does not include many other mills that were burned beyond recognition, replaced by a newer one at the same site, or located beyond public access. There may have been about 20 mills in the Tomboy and Pandora areas alone. The record of tailings is considerably less complete: partial remains can be found for some of the pre-1920's mill tailings, but one is left to surmise that most of the tailings were never impounded (they were poured into running streams) or the impoundments failed during storms. Looking at the six or more mills at Tomboy, it seems clear that there was very little room to stack the tailings because the mills were within a few hundred feet of a steep precipice. It was most convenient to pour the tailings over the edge and let them be carried away by Marshall Creek, and prior to 1935 there were no regulations against this practice. The four or more mills of several ages near Pandora appear to have consolidated their tailings in six impoundments. In the mid-1990's these were recontoured and vegetated and now are two large, flat-topped mounds, as specified by the Remedial Action Plan (CDH, 1992). West of Telluride, the mill at Society Turn created three tailings impoundments along the San Miguel River; these were not reclaimed as of 1998. Because milling after 1935 processed more tons of ore than prior to that date, much of the total tailings production probably is retained in the Pandora impoundments. However, a very large amount of tailings cannot be identified, and presumably have been washed down streams of the San Miguel watershed. The amount of tailings lost may be on the order of 2 to 5 million tons (judging from the production figures given by Henderson (1926) and an estimate of the portion impounded in the Pandora area. A estimate of similar magnitude was made for "lost" tailings from the more than 50 mills in the Silverton area (Nash, 2000a).

Total chemical analyses of five tailings samples from the district suggest a wide range in compositions, with very high metal concentrations in some tailings from inefficient stamp mills. The average of the five samples suggests generally high metal concentrations relative to altered or unaltered unmined rocks (results in ppm): $\mathrm{Ag}, 73 ; \mathrm{As}, 134 ; \mathrm{Cd}, 97 ; \mathrm{Cu}, 2,400$; Mo, 125; Pb, 4,000; Se, 2.6, and Zn, 22,000. Leach tests on a sample from Tomboy yielded a $\mathrm{pH}$ of 4.2 , very high $\mathrm{Zn}$, and high $\mathrm{Cu}$ and $\mathrm{Pb}$ concentrations. A leach test on tailings from Society Turn yielded a solution with $\mathrm{pH} 7.7$ and relatively high concentrations of $\mathrm{Cd}, \mathrm{Fe}$, and $\mathrm{Zn}$, and moderately high $\mathrm{Cu}$.

No samples could be collected from the Pandora tailings impoundments. Chemical information from the Red Mountain Idarado mill tailings may be relevant. A leach test yielded a $\mathrm{pH}$ of 3.5 and elevated concentrations of $\mathrm{Cu}, \mathrm{Fe}$, and $\mathrm{Zn}$-higher than normal rocks, but lower than most dump samples, as previously discussed. Seeps from the tailings impoundments at Red Mountain have $\mathrm{pH} 2.5$ and 2.9, very high conductivities 
$(>2,000 \mu \mathrm{S} / \mathrm{cm})$, and extremely high concentrations of $\mathrm{Al}, \mathrm{Cd}$, $\mathrm{Cu}, \mathrm{Fe}, \mathrm{Mn}$, and $\mathrm{Zn}$. Presumably, monitoring by others of water quality in the San Miguel River near the Pandora tailings would determine if metal-rich waters are seeping from the tailings there.

Additional work may be appropriate to evaluate the stability of tailings in an extreme flood. Only a small volume of tailings remain in Tomboy basin (photograph 94); many of these appear to be susceptible to erosion by higher water flow in a major storm. Tailings that were put into Marshall Creek at Tomboy years ago may have been redeposited on sandbar and slackwater sites, and these might be vulnerable to erosion under the high-flow, high-energy conditions of an extreme flood. The large impoundments at Pandora appear to be stable but may deserve closer inspection. The Society Turn tailings are near the San Miguel River (photograph 96) and probably would be eroded under flood conditions.

Surface waters.-Water is abundant in this district, especially in the upper basins that receive large amounts of snow. The extensive network of tunnels and shafts in the Idarado mine has redirected water into the Mill Tunnel, and that water is treated prior to release into the San Miguel River. No mine drainage could be sampled. Surface waters in this district are clear and generally produce no iron stains or coatings on stream cobbles, similar to the Sneffels district. Field measurements of $\mathrm{pH}$ and conductivity show the streams to consistently have near-neutral $\mathrm{pH}(\mathrm{pH}$ values are 6.5 to 7.3 ) and low conductivity $(<150 \mu \mathrm{S} / \mathrm{cm})$, both suggesting good quality. The stream waters appear to be of good quality, but chemical analyses are required to measure concentrations of metals of concern. Chemical analyses of eight stream samples show generally low metal concentrations, but several metals are elevated or exceed ALWS. Four samples contain high Cd ( $1 \times$ to $7 \times$ ALWS $)$, three samples have high $\mathrm{Cu}(2 \times$ to $4 \times$ ALWS), and seven samples contain high $\mathrm{Zn}(3 \times$ to $160 \times$ ALWS). As in other mining areas, zinc is the metal most likely to be mobilized from polymetallic mines. The CZI values for streams are 4.3 to 1.5 near mines and mills in the vicinity of Tomboy, but the CZI values are 0.5 to 0.04 in lower stream segments more distant from mine sources. High $\mathrm{Zn}$ values, not $\mathrm{Cu}$, create the high CZI. Zinc remains elevated in the upper San Miguel at the junction with the South Fork, 8 mi west of Telluride (140 ppb or $2.8 \times$ ALWS), but this is apparently in compliance with the performance objective of $0.23 \mathrm{ppm}$ (230 $\mathrm{ppb}) \mathrm{Zn}$ in the Remedial Action Plan (CDH, 1992).

Reclamation activities over the past 10 years appear to have improved water quality, but I do not have data to monitor the changes with time.

Summary.-Considering the large amount of mining and milling in this district, the headwaters of the San Miguel River are in remarkably good condition. Water quality is surprisingly good for an area with more than 100 years of major mining activity. Mine development in the early years was no different than in other districts on the CWS, but here the consequences of unregulated practices are much less evident. The explanation appears to be geologic mitigation: good ANC is provided by green propylitic-altered volcanic rocks and calcareous sedimentary rocks. Reclamation activities on the Idarado minemill complex seem to be helping. The near-neutral $\mathrm{pH}$ waters have a tendency to carry low but possibly significant concentrations of $\mathrm{Cd}-\mathrm{Cu}-\mathrm{Fe}-\mathrm{Zn}$, and sources of these metals may need to be minimized if healthy fisheries are to be maintained.

Substantial amounts of ore was milled at many upland sites, as in Tomboy basin, in the early years of mining and prior to laws regulating disposal of tailings. Reconnaissance observations of about 10 early mills suggests that only a small percentage of the tailings are retained near those mill sites. One must conclude that millions of tons of tailings were lost to streams such as Marshall Creek through a combination of unconfined tailings disposal and storm events. These tailings presumably now lie in streambeds of the San Miguel watershed. These fluvial tailings should be considered part of the geochemical baseline of the area. Presumably these fluvial tailings slowly release metals to stream waters, in a manner similar to the weathering of unmined sulfidic rocks. Reclamation of these fluvial tailings would be costly, would destroy physical habitat, and probably would produce only slight improvements in water quality.

Tailings in this district seem to be at lower risk of failure in an extreme storm than many districts on the CWS. An extreme flood in Tomboy basin or lower in the San Miguel River headwaters would likely erode enough tailings and fluvial tailings (previously eroded) to cause chemical degradation of the San Miguel River. Tailings in fluvial deposits (sandbars, slackwater deposits, overbank deposits) cannot be protected from a flood, but tailings in the small impoundments at Society Turn and Tomboy possibly could be reclaimed in ways that would minimize the risk of erosion in an extreme storm.

References.-Purington (1898); Henderson (1926); Burbank (1947b); Mayor (1978); Fischer and others (1968); Mayor and Fisher (1993).

\section{Iron Springs District (Ophir)}

Location.-San Miguel County, 6-10 mi south of Telluride. Included here are the Alta, San Bernardo, and Trout Lake mining areas (fig. 26). The climate is severe, with about 25-40 inches of precipitation per year.

Principal commodities.-Silver, gold, and lead; minor iron (for pigment) and tungsten.

Mining history.-The first vein in this area was discovered in 1877 , and soon thereafter many mines were operating. In the early years of mining the ore had to be carried by pack animals to Silverton for smelting. The first mill was built in 1881, but major production did not occur until after completion of the railroad to Ophir Loop in 1890 . Some of the most productive years were in the 1890's, chiefly from the Carbonero and Carribeau mines. Mining was intermittent for the next 60 years. The Alta mine was productive in the 1930's and forced to close

Text continues on page 132 


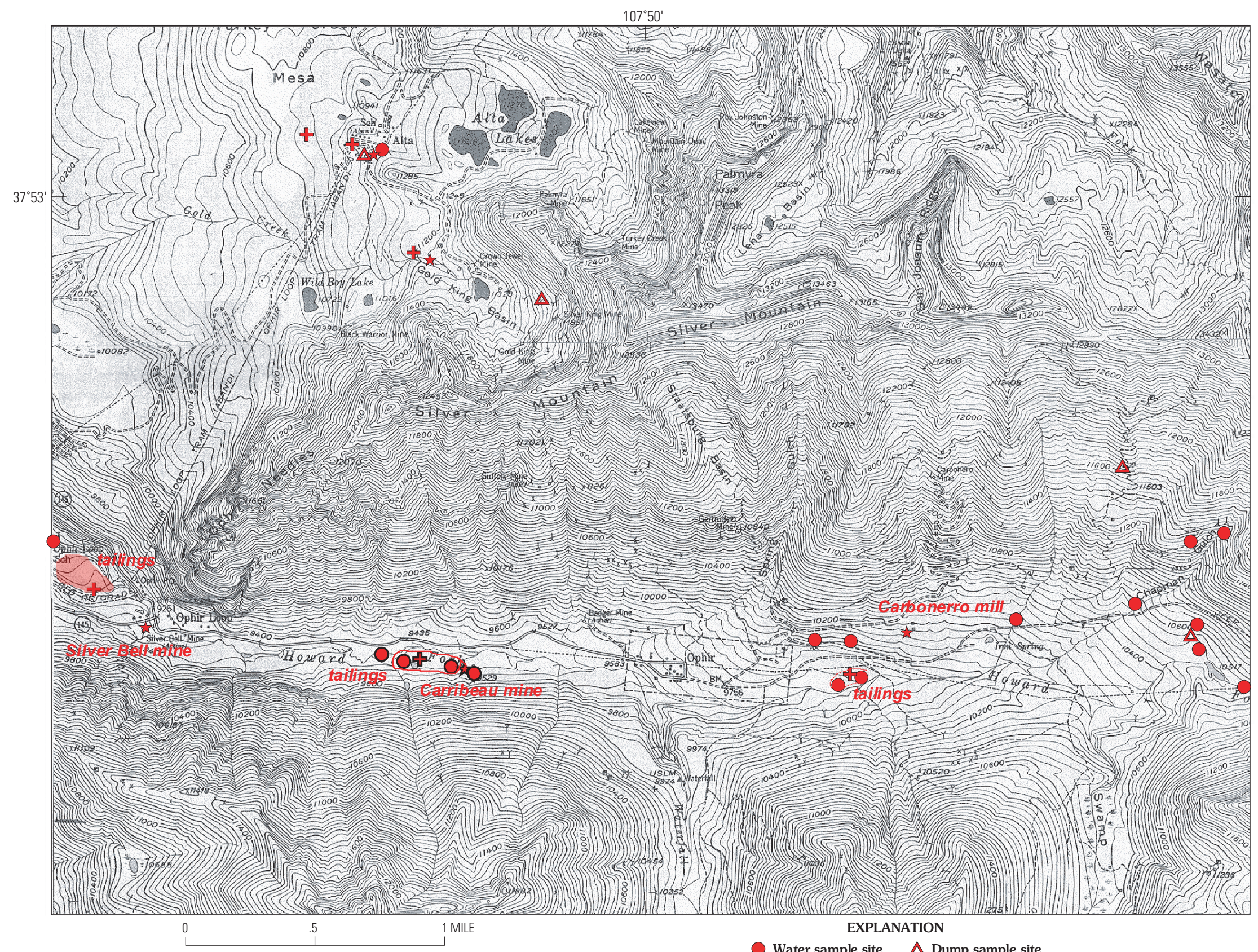

Figure 26. Features of the Iron Springs mining district, near Ophir, showing sample localities. 


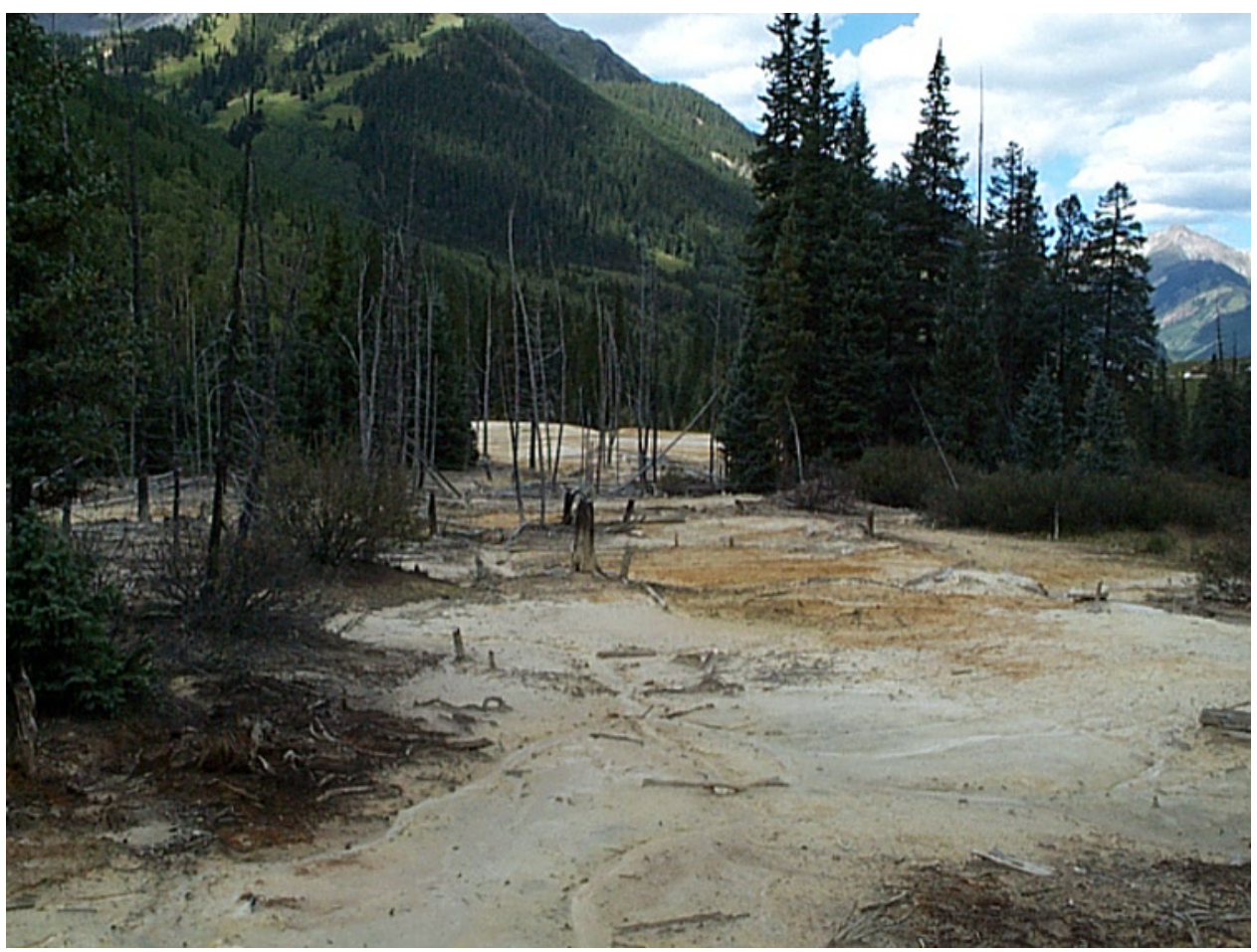

Photograph 97. Tailings from the Carbonero mill were placed close to the Howard Fork and are eroded by smaller side streams.

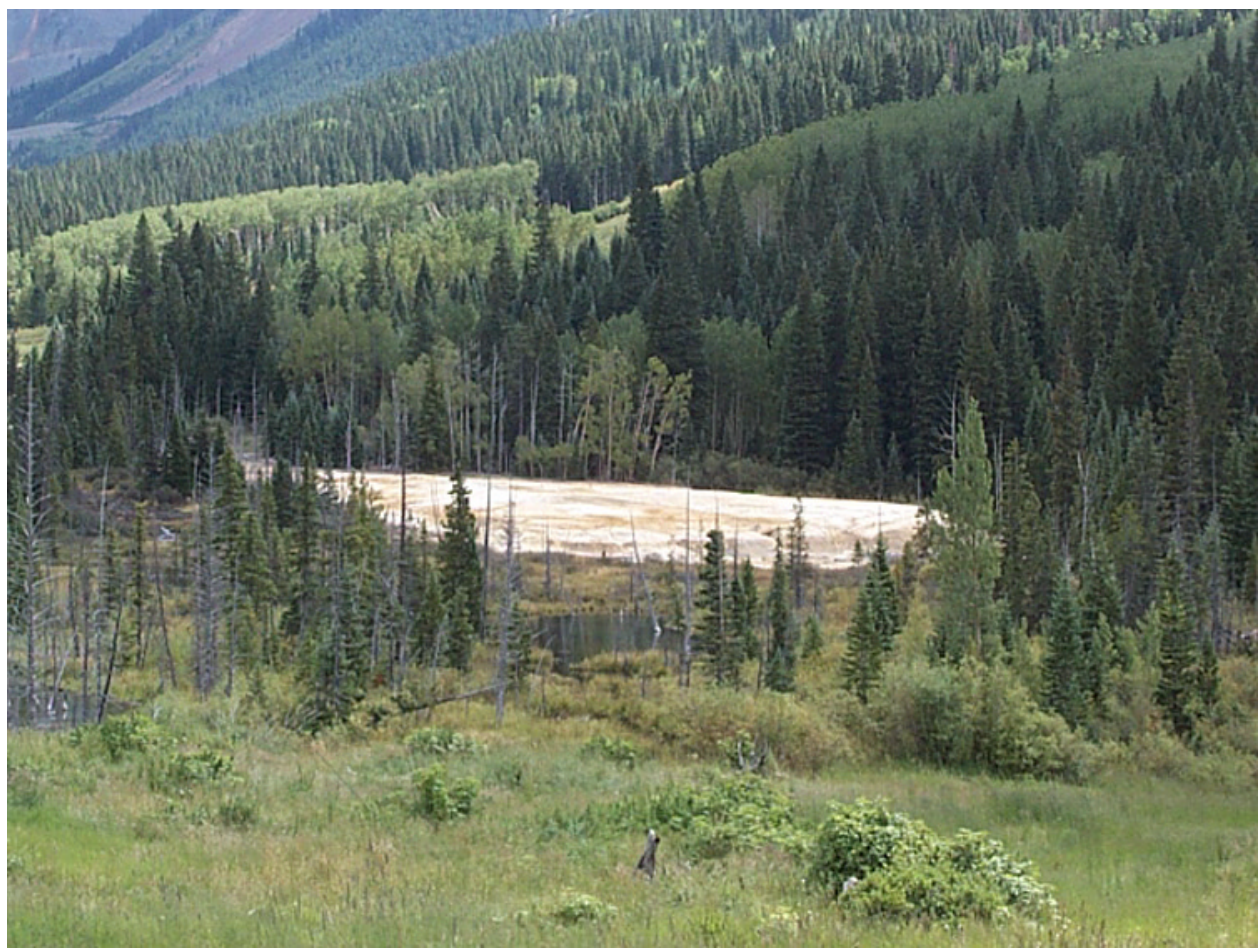

Photograph 98. View of the Carbonero tailings from the east edge of Ophir. This broad area of tailings is in wetlands of the Howard Fork and may be at risk of severe erosion in an extreme storm (flash flood). 


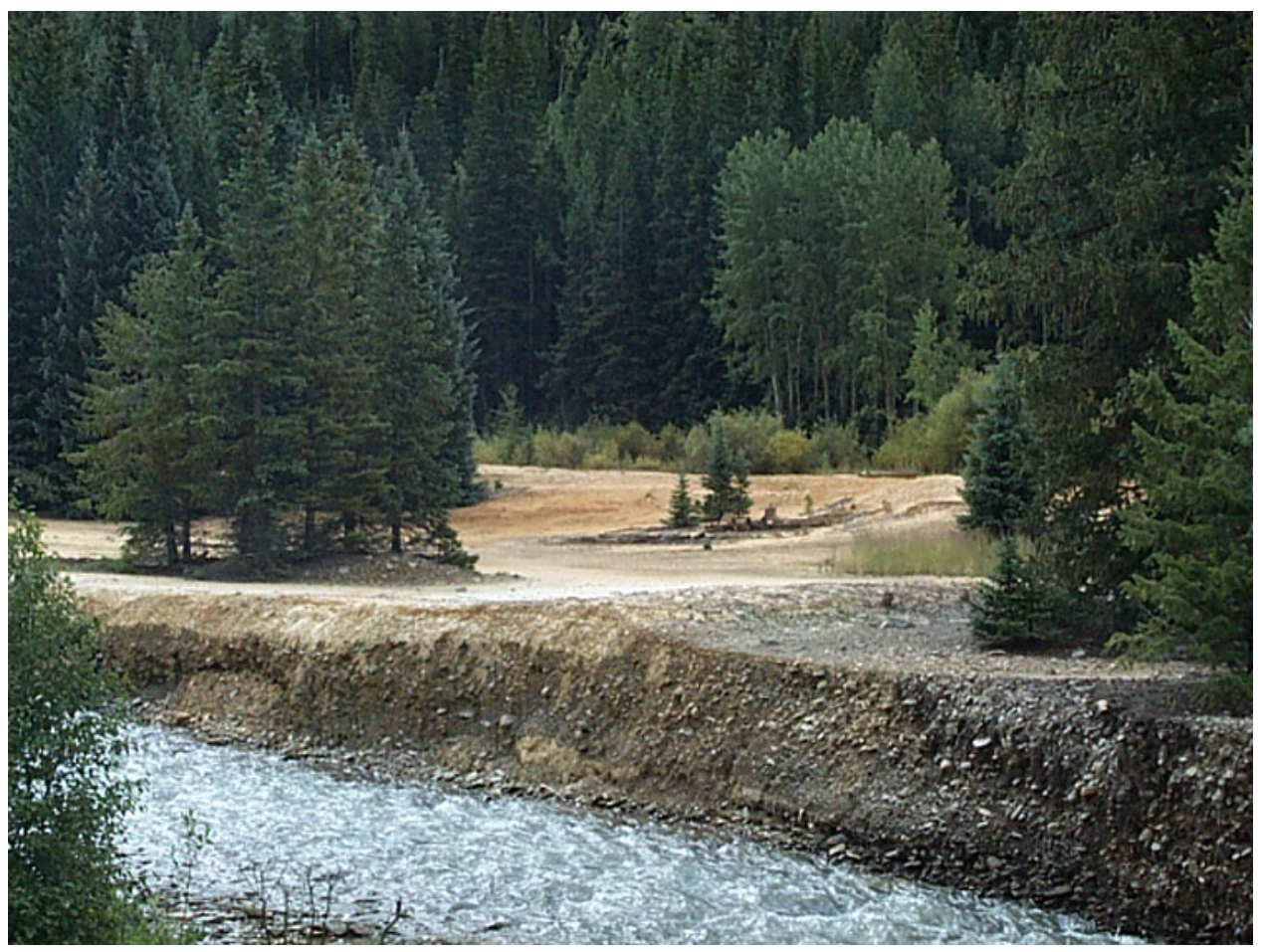

Photograph 99. Tailings from the Carribeau mill were placed on a flat area on the south side of the Howard Fork. These tailings cover several acres.

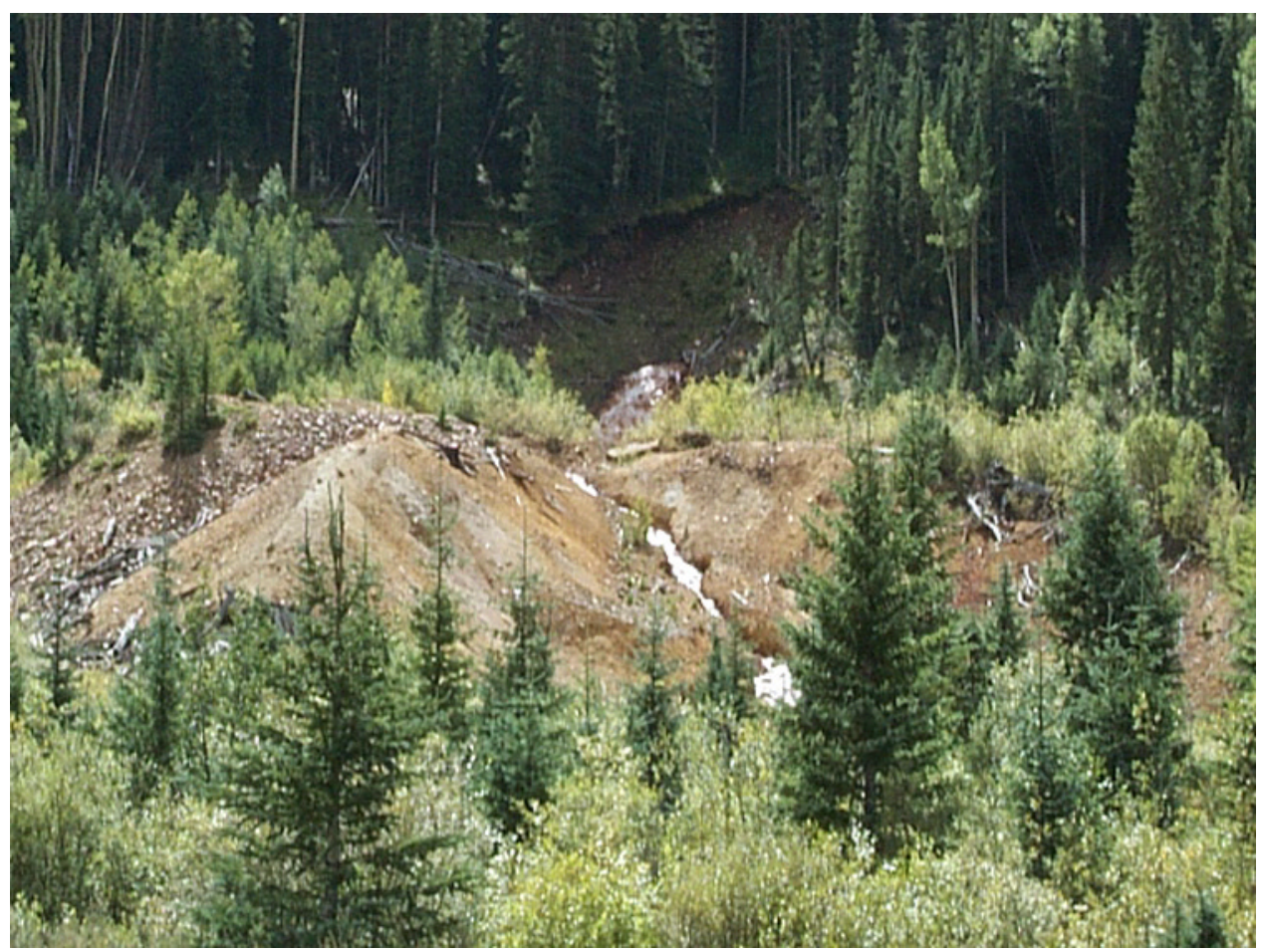

Photograph 100. Drainage from the Carribeau mine has high flow and carries fairly high concentrations of iron and other metals; the composition and $\mathrm{pH}$ of 6 suggests that it may be more acidic deep in the mine. Remediation of such a high flow of metal-rich water is complex. 


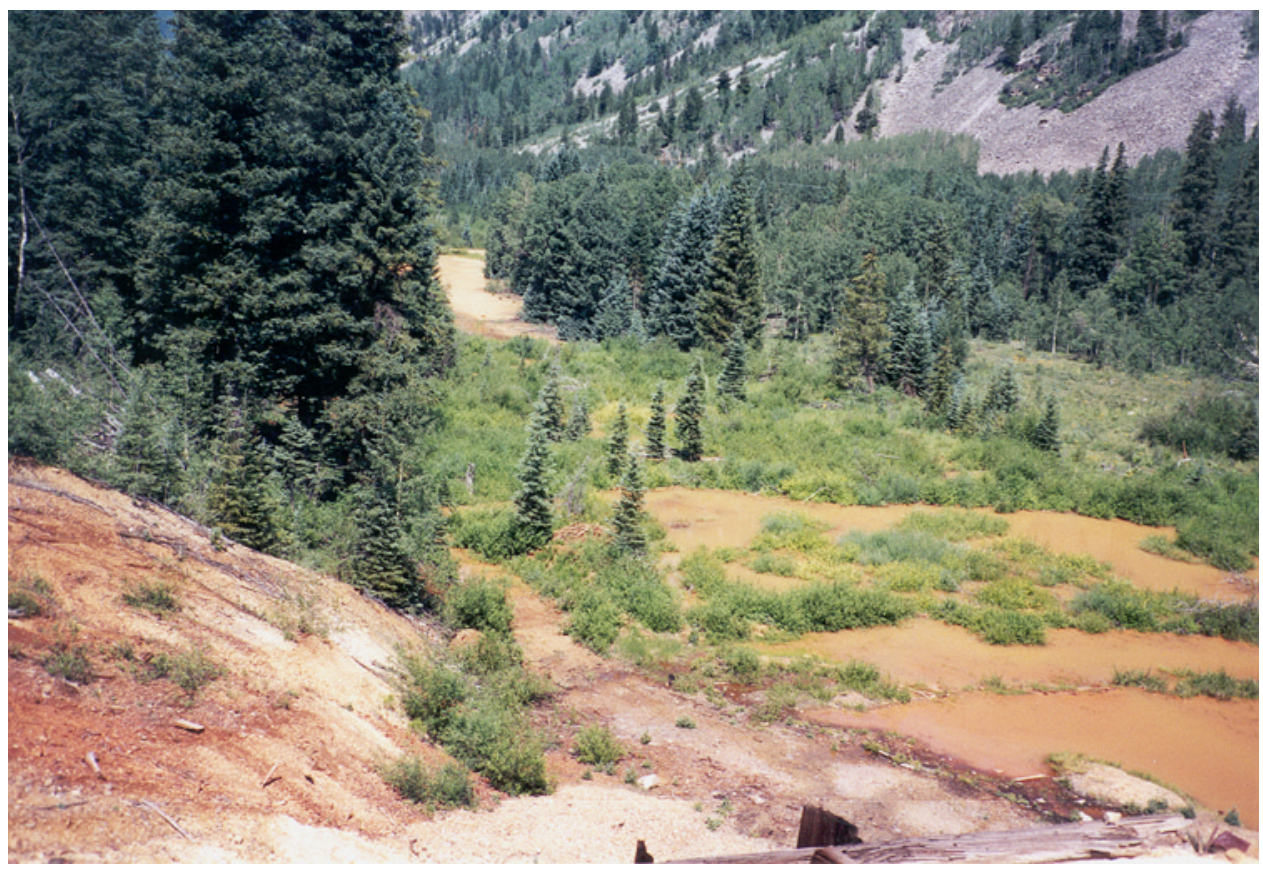

Photograph 101. Tailings from the Carribeau mill are flooded by mine drainage from the adit. The view is from the mill site, which is about $200 \mathrm{ft}$ south (behind) this location. Several acres of tailings (rust color) are flooded by the mine drainage; the Howard Fork is about $10 \mathrm{ft}$ beyond the upper right corner of the photograph.

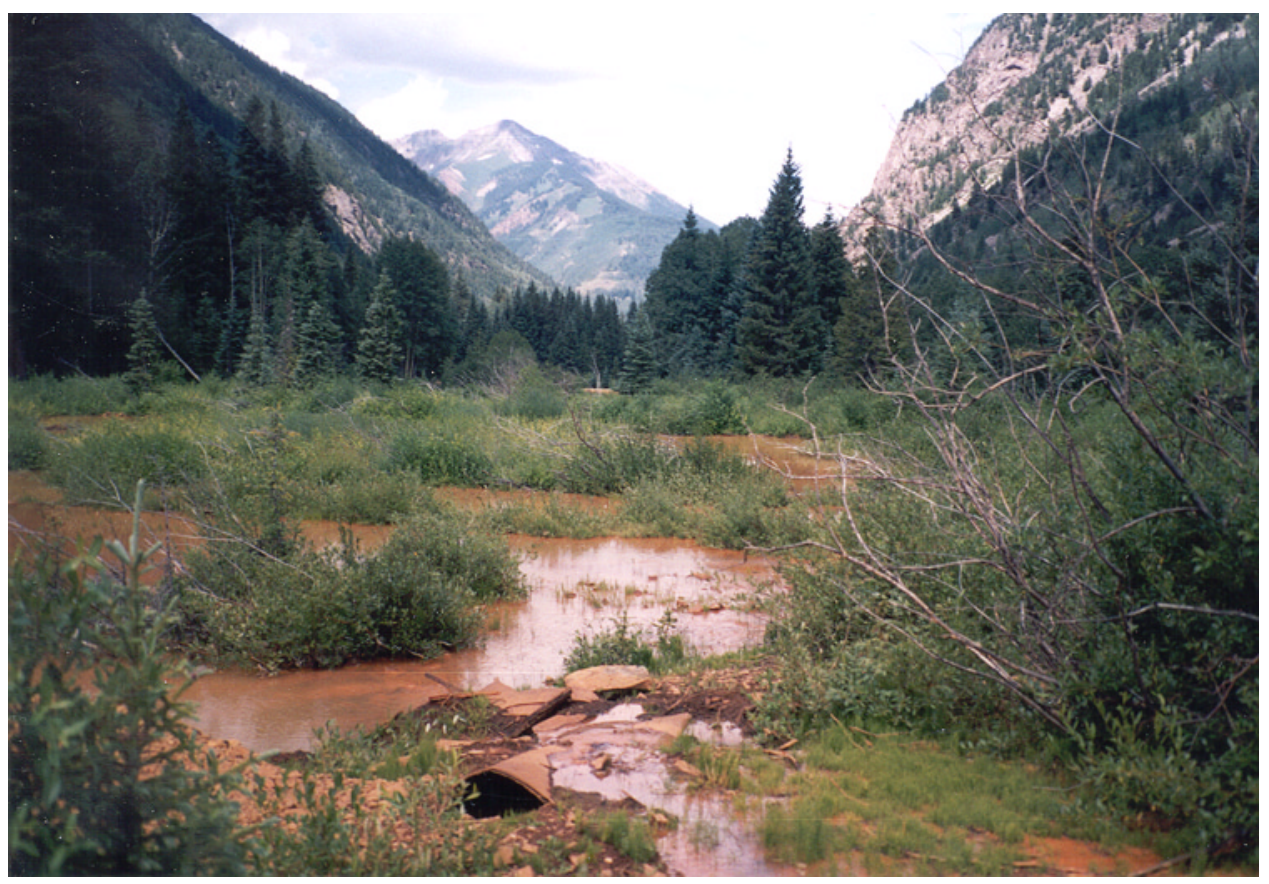

Photograph 102. This closer view of flooded Carribeau mill tailings shows the influence of active beaver dams. Mixed ownership of the mine and tailings impoundment complicates remediation. 


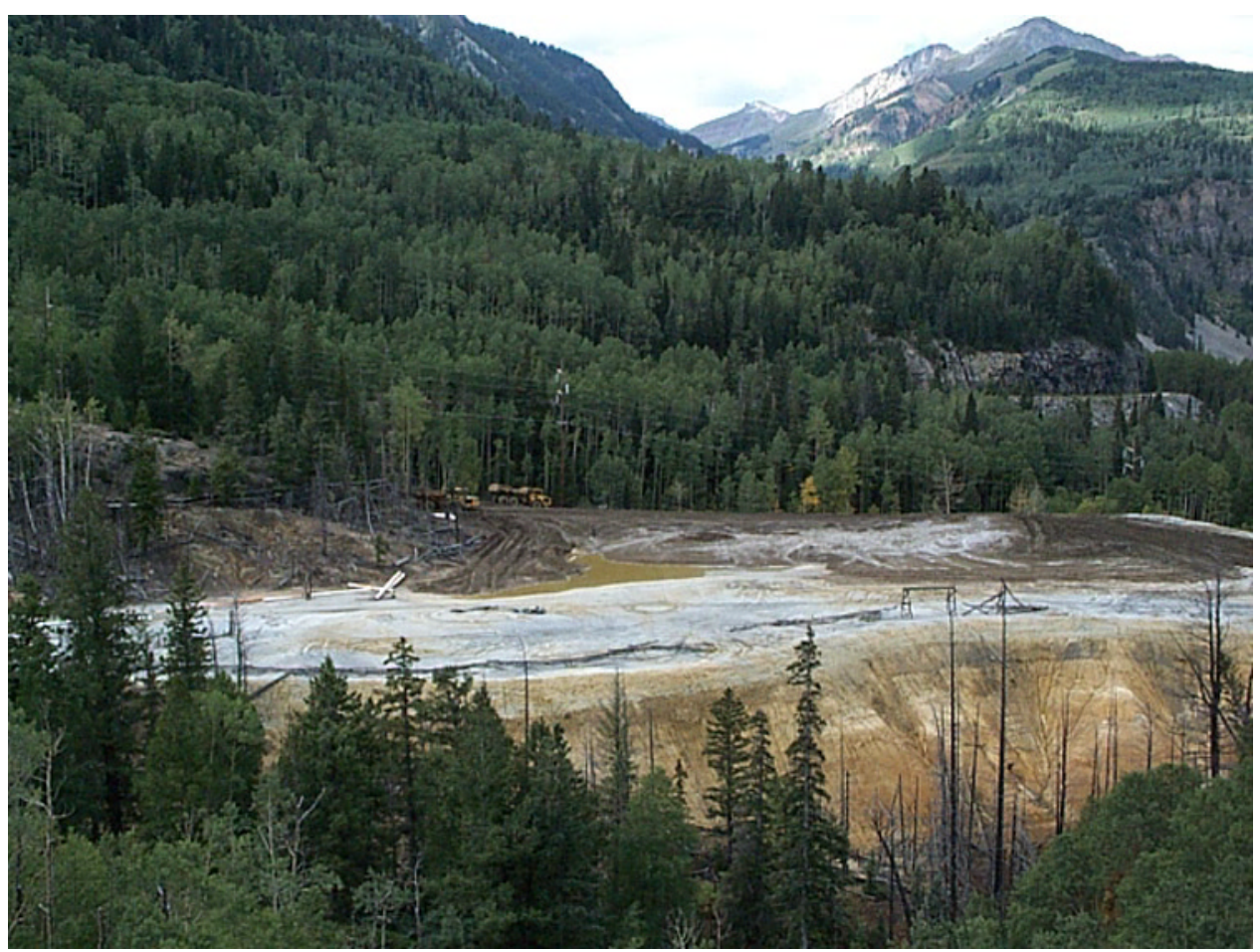

Photograph 103. Tailings from the Silver Bell mill were stacked high above the Howard Fork. Reclamation work was underway in September of 1999. Part of the tailings impoundment is on USFS lands.

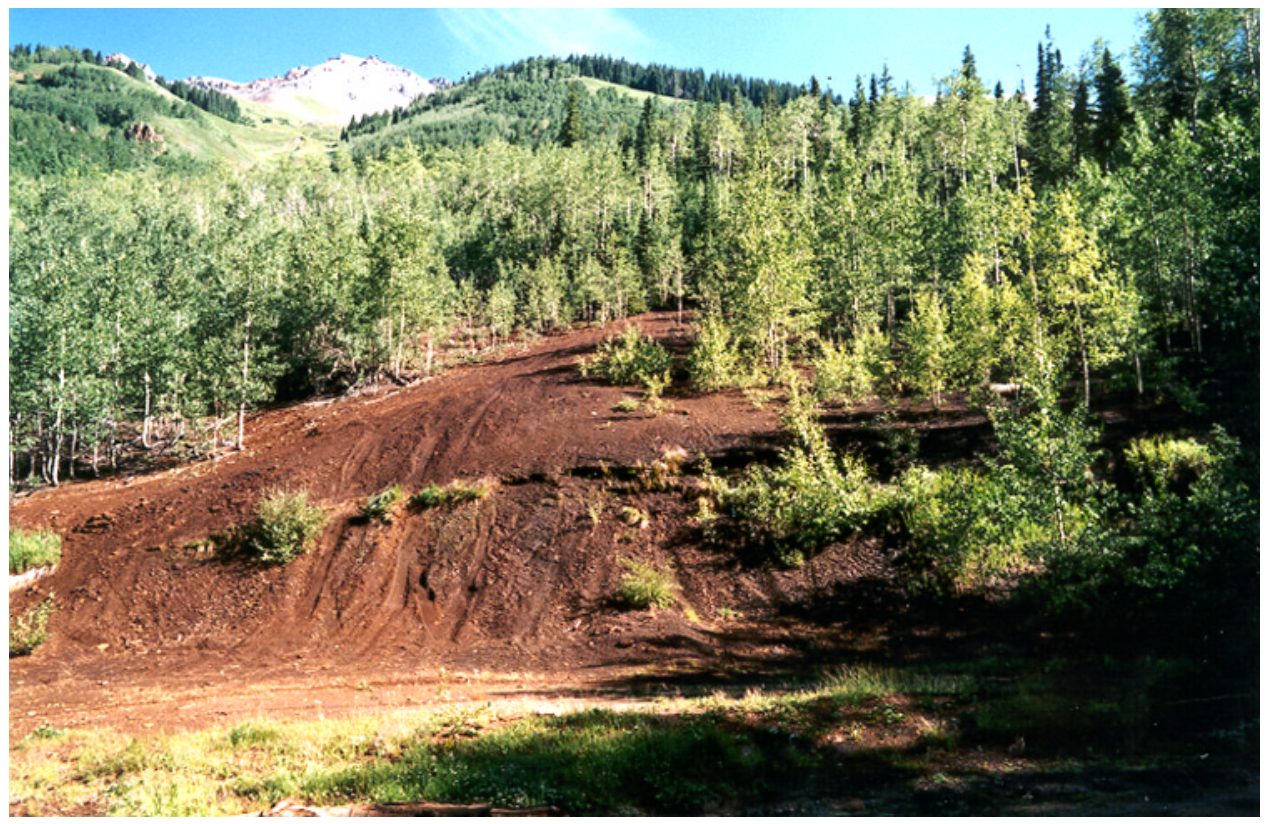

Photograph 104. Large iron deposits (ferricrete) formed from iron-rich springs after glaciers carved the Ophir valley. Waters that formed these deposits are thought to be geochemically similar to acid mine drainage but clearly are thousands of years older than any mining in the area. 


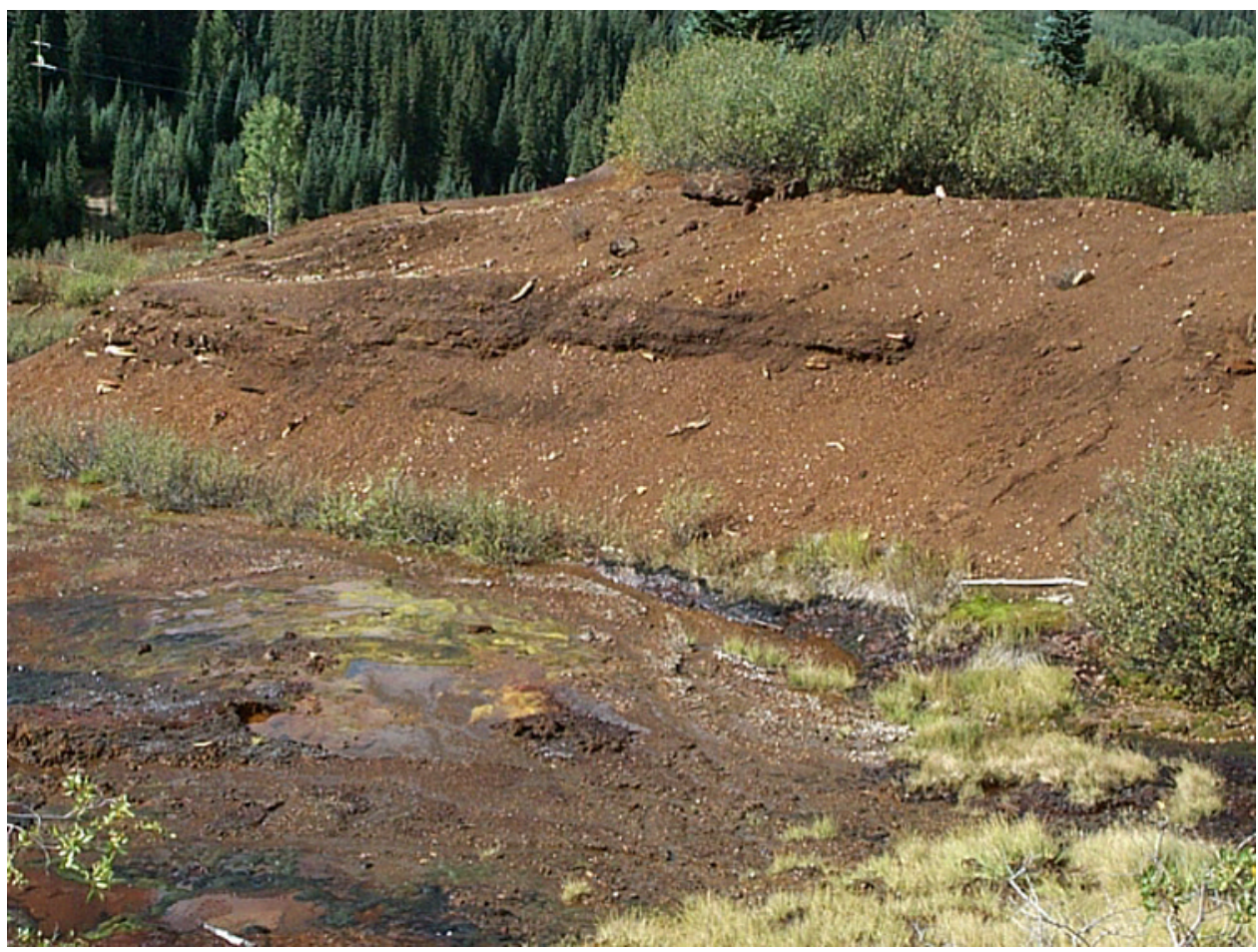

Photograph 105. Iron was mined at Iron Springs east of Ophir from this cut that shows the layering in the iron deposit. Springs are active today and continue to carry high iron concentrations at about $\mathrm{pH} 3$.

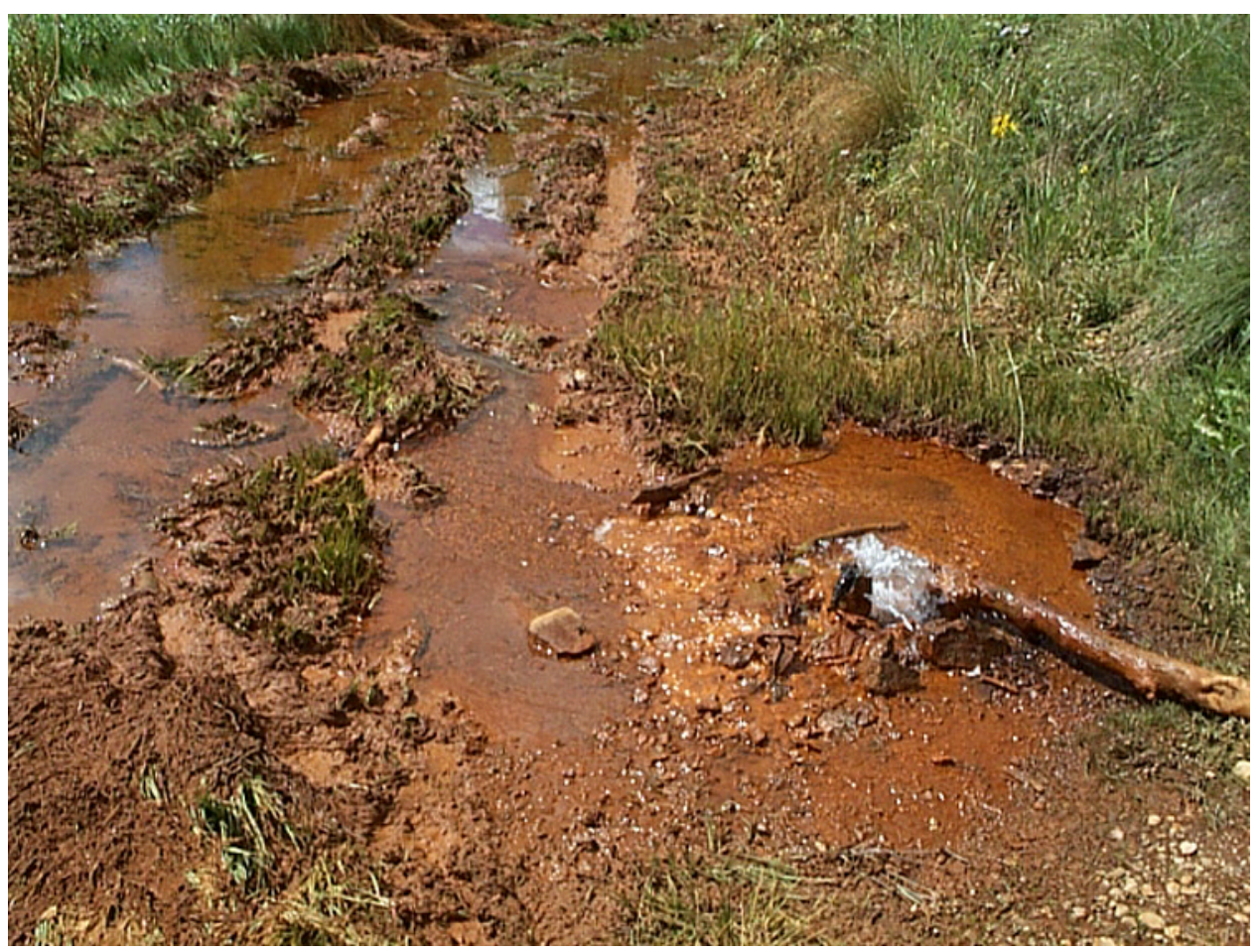

Photograph 106. Artesian flow of water carries high concentrations of iron and other metals from a drill hole made for metal exploration in the 1960's(?). The drill hole is on the hillside north of the Iron Springs iron mine. 
when the mill burned in 1948. Mines in the Ophir Valley had later periods of production in the 1950's and 1960's, with ores processed through the Silver Bell mill. There has been little activity for the past 20 years. Mining in this area was greatly aided by hydroelectric power generated at Ames.

Production and disturbance.-Total value was about $\$ 11$ to $\$ 15$ million. The Silver Bell mine is credited with about $\$ 1.6$ million production through 1970, and the San Bernardo mine produced about $\$ 700,000$ worth of ore (chiefly Ag, less value in $\mathrm{Au}, \mathrm{Cu}$, and $\mathrm{Pb}$ ) through 1928. Total production from the district is estimated at 873,000 tons (Long and others, 1998; this figure may not include the Alta and San Bernardo mining areas). The underground mines (chiefly adits) produced relatively small dumps and workings, but several substantial impoundments of tailings were made and are located close to the Howard Fork (photographs 97, 99).

Geology.-Veins containing base-metal sulfides and precious metals occur in Tertiary volcanic rocks on the western flank of the Silverton caldera and adjacent to a Tertiary intrusive complex. Pre-Tertiary sedimentary rocks underlie the volcanic section and should be good ore hosts, but ore has not been described in these rocks. The intrusions are larger in size and coarser in grain size than at most districts in the San Juan Mountains, and the alteration halos around these intrusions are large (as at Silver Mountain and Yellow Mountain). Some of the alteration is pyritic and appear to be sources of acidic, iron-rich waters such as at the artesian springs that gave the district its name (Iron Springs). The springs that issue near the valley floor have deposited iron as thinly laminated bog-type deposits, now hard and crystallized as goethite, which have been mined for use as a pigment (photograph 104).

\section{Mineral-Environmental Conditions}

Mineralized rocks and ores.-Chemical analyses of three dump, nine tailings, and two ferricrete samples supports the classification of the ores in this district as polymetallic. The dump samples have high $\mathrm{Ag}, \mathrm{Cu}, \mathrm{Mn}, \mathrm{Mo}$, and $\mathrm{Pb}$ concentrations relative to other dump samples in the study, and As and $\mathrm{Zn}$ values are near the median concentration for all dumps. Nine samples of tailings show a range in compositions, but the tendency is to be high in $\mathrm{Ag}, \mathrm{Mn}$, and $\mathrm{Pb}$ relative to other mineralized rocks, moderately high in $\mathrm{As}, \mathrm{Cu}$, and $\mathrm{Mo}$, and low in $\mathrm{Zn}$ (but the $\mathrm{Zn}$ concentration is higher than in unmined altered rocks). In leach tests, two dump samples both yielded solutions of $\mathrm{pH} 3.1$, carrying high concentrations of $\mathrm{Fe}, \mathrm{Mn}$, $\mathrm{Cu}$, and $\mathrm{Zn}$; one sample released very high concentrations of Mo. Two samples of ferricrete were not very reactive in leach tests, as shown by low conductivity values and low metal concentrations in the solutions, which had $\mathrm{pH}$ values of 3.5 and 3.8; moderate amounts of $\mathrm{Cu}$ and $\mathrm{Zn}$ were released. Tailings samples had differing reactions, with $\mathrm{pH}$ values of leach solutions in the range of 2.6 to 4.1 . The acidic solutions carried high concentrations of $\mathrm{Al}, \mathrm{Fe}, \mathrm{Mn}, \mathrm{Cu}$, and $\mathrm{Zn}$. A sample of tailings from the Silver Bell impoundment released very high concentrations of $\mathrm{Mo}, \mathrm{Pb}$, and $\mathrm{Se}$.

Mills and tailings.-The long history of production in this district is reflected in the six or more mills and associated tailings. The first mill may have been the Gold King in the Alta area that operated in the 1880's. The preserved tailings from this mill are tens of tons on a flat area that appears to be quite stable; I could not determine if tailings were sluiced into the creek and washed away. The Gold King mill probably was replaced by a mill at Ophir Loop, connected by the tram shown on the Ophir topographic map. Other early mills were erected to service the Carbonero and Carribeau mines, a mile east and west of Ophir. The Carbonero mill probably was served by a tram, but no physical remains, other than the tailings impound and tailings spilled from the sluice, could be found. The Carribeau ore was handled inside the mountain and brought out through a lowlevel tunnel. Tailings from the Carbonero mill are spread wide and thin in wetlands north of the Howard Fork (photograph 97), and those from the Carribeau are on the bank south of the river (photograph 99). The volume of tailings in these two sites is moderate, and an undetermined amount was lost by erosion or sluiced directly into the streams. These mills probably ceased operating prior to the 1935 regulation for impounding tailings. The Alta mill operated chiefly in the 1930's-40's, until it burned in 1948. Tailings were piped to a small impoundment near the mill and to a larger area to the west on an upland bench; the western Alta tailings were recontoured and revegetated in 1997. Ore from the San Bernardo mine was trammed across the Lake Fork to the mill near the railroad, and tailings placed nearby, on the east side of the stream. The moderate volume of tailings had not been reclaimed as of 1998. The largest volume of tailings is from the Silver Bell mill, which may have been placed on top of tailings from the 1910-era mill at Ophir Loop because only one tailings pile is evident (photograph 103). The Silver Bell mill probably did not operate after about 1970 . The moderate to large volume of tailings were stacked like a hay pile, above and to the south of the Howard Fork. The San Bernardo and Silver Bell tailings are not wetted by the adjacent streams, but they do collect precipitation and must react with that water. No diversions or liners are evident that could minimize runoff, erosion, or infiltration.

Leach tests on six samples of tailings from this district show a wide range in potential for acid and metal release. Tailings from the Silver Bell tailings pile contain layers that differ in color, suggesting ores of differing mineralogy were processed at various times. The majority are ocher (from oxidized ore?), and this material releases high concentrations of $\mathrm{Al}, \mathrm{Fe}, \mathrm{Mn}$, and $\mathrm{Zn}$, whereas gray layers (sulfidic ore?) release very high concentrations of $\mathrm{Cu}, \mathrm{Fe}, \mathrm{Mo}$, and $\mathrm{Pb}$, and more As than the ocher material. Both tailings types generate acid, yielding solutions of $\mathrm{pH} 2.8$ from the ocher tailings and 2.6 from the gray. Leach tests on the Carbonero tailings yielded pH 2.7 and high metal concentrations (especially $\mathrm{Cd}, \mathrm{Fe}, \mathrm{Pb}$, and $\mathrm{Zn}$ ). Two samples of the Carribeau tailings yielded solutions of $\mathrm{pH} 3.3$ and 4.1, much lower conductivity than others in the district, and moderate concentrations of $\mathrm{Cu}$ and $\mathrm{Zn}$. A sample of tailings from the Alta mill yielded a solution with 
pH 3.5 and relatively low metal concentrations, but a very high $\mathrm{Pb}$ concentration.

Tailings at most, if not all, of the sites in this district are involved with, and react with, surface waters. These waters were sampled at eight sites to determine the reactivity of the tailings and metals released. One sample type is unconventional: pore waters. I collected these waters where possible by making a hole in the tailings with an auger and collecting the water that seeped into the hole in about 10 minutes. Most tailings are damp but do not produce water for this test. Compositions of waters in contact with these tailings are highly variable: $\mathrm{pH}$ value ranged from 2.1 to 7.3 , and conductivity values ranged from 660 to $>2,000 \mu \mathrm{S} / \mathrm{cm}$. Pore fluids in the saturated tailings tend to be most acidic and metal rich. Concentrations of $\mathrm{Al}, \mathrm{Cd}, \mathrm{Cu}, \mathrm{Fe}$, $\mathrm{Mn}, \mathrm{Pb}$, and $\mathrm{Zn}$ range to high and extremely high values $(10 \times$ to $100 \times$ ALWS). Tailings in the Iron Springs district clearly lie in the flow of surface waters, are reacting, and are releasing significant concentrations of base metals. I cannot determine the volume of flow involved, thus cannot compute metal loadings. Also, wells are required to determine infiltration into shallow ground water in alluvium beneath the tailings. A puddle on the Silver Bell tailings pile had a pH of 2.1 and extremely high metal concentrations, possibly concentrated by cycles of evaporation. Puddle waters are not typical of surface-water compositions, but I believe that in general they provide an estimate of the first flush of water in storm runoff.

At least three of the tailings impoundments in this district rank among the sites on the CWS having highest risk to failure in an extreme storm. The tailings from the Carbonero, Carribeau, and Silver Bell mills are poorly constrained and are in close proximity to the Howard Fork. The Carbonero and Carribeau tailings commonly are wet and eroded by side streams, and the Howard Fork would not have to rise much to cause major erosion. The Silver Bell tailings are high enough to be above likely flood levels on the Howard Fork, but their shape and position suggest that they may be vulnerable to slumping or liquefaction in a protracted storm with exceptional amounts of precipitation. The engineering properties of the Silver Bell tailings, such as shear strength, could be determined by geotechnical consultants to better evaluate risk of slumping into the Howard Fork. Reclamation work underway in the summer of 1999 should reduce runoff from the Silver Bell tailings but may not change the physical condition of the pile or resistance to slumping. I could not inspect the San Bernardo tailings impoundment to evaluate its setting, and this should be done by others. A worst-case scenario for these tailings would be mass failure during a storm event, creating a slide or slurry of tailings into the stream. This seems a low probability risk, but the consequences to the Howard Fork would be devastating to aquatic life and impossible to clean up.

Surface waters.-Water quality in this district has tendencies that are not so clearly expressed elsewhere: waters from unmined altered rocks in the eastern part are highly mineralized and give the name to the district, but weakly altered rocks in the western part provide natural attenuation. Mines and mill tailings are sources of metals, but the waters are not as acidic or metal rich as in some districts. Thirty-three samples of surface waters and tailings pore waters were collected for chemical analyses, and many additional sites were checked for $\mathrm{pH}$ and conductivity.

Waters in the eastern part of the district continue to deposit iron in the vicinity of historic iron springs and substantial mined deposits of iron (goethite) (photograph 105) (Hanshaw, 1974). The spring waters have locally variable $\mathrm{pH}$ and conductivity, in the general range of 3-5 and 300-1,000 $\mu \mathrm{S} / \mathrm{cm}$, respectively, which I interpret as indicative of mixing of ground waters of different compositions. A nearby drill hole has artesian flow (photograph 106); it has pH 5.9 and conductivity of $1,540 \mu \mathrm{S} / \mathrm{cm}$. These spring and drill-hole waters are very rich in $\mathrm{Fe}(3 \times$ to $15 \times \mathrm{ALWS}), \mathrm{Cu}(0.3 \times$ to $50 \times)$, and $\mathrm{Zn}(1.4 \times$ to $9 \times)$. There are indications that an acidic, metalrich water flows from the mountain of pyritic-altered volcanic rocks and mixes with unmineralized surface and ground water. These reactions at the source and at sites of iron deposition have been operating for at least 8,000 years, according to radiocarbon dating (Hanshaw, 1974) and clearly are not related to man and mining. There also is evidence that these metalrich waters may have been thermal springs at one time (W. Miller, written commun., 1999). The iron-rich deposits are chemically related to iron-cemented rock ("ferricrete") that is common in the San Juan Mountains. Numerous ferricrete deposits are known in the Red Mountain and Eureka districts (Nash, 1999b), and an especially large one formed at the site of the Keystone mine, Ruby district. Ferricrete was used by prospectors as a guide to ore, and tunnels were often made through the ferricrete to find veins, as described previously for the Red Mountain district. Waters in some San Juan ferricretes have high concentrations of $\mathrm{Cu}-\mathrm{Zn}-\mathrm{Cd}$, but many are rich in only $\mathrm{Fe}$ or $\mathrm{Fe}$ and $\mathrm{Al}$ (Nash, unpub. data, 1999).

Mine drainage, investigated at seven sites, is highly variable with $\mathrm{pH}$ values in the range 3.7 to 7.1 . The worst water quality was found at a small mine across the valley west of the USFS Matterhorn campground. Water draining from the Alta mine tunnel has a $\mathrm{pH}$ of 6.6 , which normally means low metals, but this site is exceptional for its very high base metals (Cd 18×, Cu 58×, and Zn 36× ALWS). The Carribeau tunnel issues a huge flow of water that deposits iron and reacts with tailings (photograph 100); the $\mathrm{pH}$ is 5.6 to 6 but metal concentrations are quite high $(\mathrm{Cu} 5 \times, \mathrm{Fe} 6 \times, \mathrm{Mn} 1.7 \times, \mathrm{Zn} 4 \times$ ALWS). Some mine adits on the north side of the canyon, not far from the county road, issue similar high flows of $\mathrm{pH}$ 3.7-5.5 water that create large amounts of red iron precipitate. $\mathrm{The} \mathrm{Al}, \mathrm{Cu}, \mathrm{Fe}$, and $\mathrm{Zn}$ concentrations in these waters are about $2 \times$ to $9 \times$ ALWS.

Despite these inputs of acid and metals from natural and mine sources, the quality of water in the Howard Fork is quite good. Small streams and the eastern headwaters of the Howard Fork have pH 5.1 to 6.2, and moderate conductivity (300 to 600 $\mu \mathrm{S} / \mathrm{cm})$. The altered rocks and iron springs in the area would logically explain the elevated conductivity, as well as elevated Fe and Zn values (each near the ALWS). The composition of the Howard Fork does not change much downstream (west) of the 
Carbonero and Carribeau tailings: $\mathrm{pH}$ is 5.5 , conductivity 350 $\mu \mathrm{S} / \mathrm{cm}$, and the $\mathrm{Zn}$ concentration rises to $2.2 \times$ ALWS at a site 1.5 mi west of Ophir. The Howard Fork at Ames, west of the Silver Bell mine and mill complex, has $\mathrm{pH}$ of 6.7 to 7.2 (measured in different years), conductivity of about $350 \mu \mathrm{S} / \mathrm{cm}$, and $\mathrm{Zn}$ concentrations of $1.3 \times$ to $1.8 \times$ ALWS in two analyzed samples. Mixing with the Lake Fork and other streams improves the quality of the resulting stream (the South Fork of San Miguel River) to $\mathrm{pH} 7.1$ and conductivity of $270 \mu \mathrm{S} / \mathrm{cm}$, and $\mathrm{Cu}$ and $\mathrm{Zn}$ concentrations are below ALWS. The CZI value for the Howard Fork east of the mines and iron springs is 0.16 , and four samples downstream from the tailings are in the range 0.22 to 0.48 , showing the input from mining and mill tailings. The increase in $\mathrm{pH}$ probably reflects the input from tributaries that flow through green-altered rocks. The rise in $\mathrm{pH}$ for the Howard Fork at Ames might reflect alkalinity from the Silver Bell tailings (lime is typically added in mills). Water quality in the Lake Fork is very good (CZI is 0.02), and mixing with the Howard Fork creates good water in the South Fork ( $\mathrm{Zn}$ is $43 \mathrm{ppb}$ and CZI is 0.26 , both suggesting low threat to aquatic life).

Summary.-Water quality in the Howard Fork within this district is somewhat degraded by inputs from mines, several mill tailings impoundments, and from unmined sources, including iron-rich springs. The good acid-neutralizing qualities of greenaltered volcanic rocks appear to minimize the effects of acidic inputs. However, the neutralized waters that emerge within the district tend to have elevated $\mathrm{Zn}, \mathrm{Cd}, \mathrm{Fe}$, and $\mathrm{Cu}$ concentrations. There are numerous discharges from collapsed mine tunnels that have $\mathrm{pH}$ values of 4 to 7 and deposit large amounts of red iron floc (which probably looks worse than it is chemically), but also carry elevated concentrations of $\mathrm{Cd}-\mathrm{Cu}-\mathrm{Zn}$. Reclamation of some of the mine and tailing sites mentioned here would reduce the base-metal concentrations in the Howard Fork, but the significant input of metals and acid from unmined altered rocks cannot be mitigated. Further work to evaluate mill tailings in riparian zones along the Howard Fork and reclamation options for the iron-rich mine waters are needed to maintain or improve water quality in the Howard Fork.

Possibly the highest environmental risk in this district is that a rare, extreme storm and flood that could cause significant erosion of the Carribeau and Carbonero mill tailings. The physical properties of the Silver Bell tailings cannot be determined by reconnaissance inspection, but if they have low shear strength when saturated with water, they would slump into the Howard Fork and create chemical conditions that would be catastrophic to aquatic life. Geotechnical studies of these tailings, and also the San Bernardo site, are suggested as a step toward preventative reclamation.

References.-Varnes (1947a); Vhay (1962); Bromfield and others (1972).

\section{Mount Wilson District}

Location.-San Miguel Co, about $10 \mathrm{mi}$ west of Telluride (fig. 20). The climate is severe, with more than 30 inches of precipitation per year.

Principal commodities.-Chiefly Au and Ag; minor values of $\mathrm{Cu}$ and $\mathrm{Pb}$.

Mining history.-The Silver Pick deposit was located in 1882 and mined for a short period, 1883-98, with most of the production coming from an extremely rich bonanza vein in parts of just 3 years. The vein was mined in very rugged terrain at $12,100 \mathrm{ft}$ to $13,200 \mathrm{ft}$ elevation. The ore was trammed several miles to the north to a mill at treeline. The main mine, the Silver Pick, produced only small amounts of ore from 1930 to 1960 . A few small mines nearby were productive before 1900.

Production and disturbance.-Production was valued at between $\$ 600,000$ and $\$ 1$ million, mostly prior to 1900 . Chief values were in $\mathrm{Au}$ and $\mathrm{Ag}$, with a little from $\mathrm{Cu}$ and $\mathrm{Pb}$. Disturbance was small in the vicinity of the Silver Pick mine. The small mill has very little waste today; tailings may have been reprocessed in the 1930's or dumped in the nearby stream.

Geology.-This mining area is in rugged mountains that are west of the main San Juan Mountains, and adjacent to the Colorado Plateau Province. The geology is dominated by several intrusions, including the Wilson Peak stock. A sequence of more than $5,000 \mathrm{ft}$ of Triassic to Tertiary sedimentary rocks is intruded and altered by middle- to late-Tertiary stocks and laccoliths. The flat-dipping laccolithic bodies resemble the famous ones of eastern Utah. The intrusive bodies are altered and cut by veins. The ore deposits are thought to be related to later stages of the intrusive complex.

\section{Mineral-Environmental Conditions}

Mineralized rocks and ores.-The ores were mined chiefly for gold, but the veins probably carried base-metal oxide and sulfide minerals as well. The Silver Pick mine was not visited in this study, but earlier I had visited mines on the southern side of the divide (San Juan Forest) and noted the oxidized character and narrow width of those veins. As described in reports, the Silver Pick mine operated under arduous conditions, for a few months at a time, and probably was even more selective than other mines of the time. The dumps probably are small and oxidized, and, if these attributes are correct, there would be low potential for acidic drainage and low mobility of base metals.

Mills and tailings.-The small stamp mill for the Silver Pick mine has no visible tailings. Presumably, the tailings produced prior to 1900 were carried away in the stream.

Surface waters.- No samples were collected. On brief hikes through the area, north and south of the Silver Pick mine, there were no visible suggestions of significant transport of contaminants.

Summary.-No problems are evident or expected. Water quality does not appear to be influenced by the small mines.

References.—Varnes (1947b) ; Bromfield (1967); Bromfield and others (1972). 


\section{Placerville District (Lower San Miguel)}

Location.-San Miguel County, about 10 mi west of Telluride. (fig. 1) The climate is moderate, with about 16-20 inches of precipitation per year.

Principal commodities._-Vanadium, lesser uranium; some placer gold.

Mining history. - The vanadium deposits were discovered long before the uranium boom on the Colorado Plateau. These deposits were mined from 1909 to 1920, and again in the 1940's. Mills near Vanadium and Sawpit, north of the San Miguel River, processed ores that were carried by trams from mines in the canyon walls. The mill, which ceased operations in 1920, was reclaimed some years ago. Several small placer gold operations were run during the Depression years.

Production and disturbance.-Production was modest, with about 195,000 tons of ore milled to recover 3,700,000 $\mathrm{lb}$ of vanadium. The mines created small disturbance and are difficult to locate today.

Geology.-Stratabound deposits rich in vanadium occur in continental sandstones of the Jurassic Entrada Formation near Placerville. Copper minerals are present adjacent to the vanadium-rich lenses and suggest a similarity to the sandstonehosted copper deposits farther west at the Cashin mine.

\section{Mineral-Environmental Conditions}

Mineralized rocks and ores.-No samples could be collected for this study. The oxidized nature of the ore minerals suggests that there should be low potential for acid generation, and most metals are probably stable. Radioactivity could be a concern in the vicinity of mine workings and ore stockpiles, although most seem to have been reclaimed.

Mills and tailings.- The mill sites and tailings have been reclaimed.

Surface waters.-I collected no new data. These mines are not expected to pose problems, but radon could be a problem locally in ground water (if used for domestic wells).

Summary.-The major mill sites have been reclaimed. The BLM supervised reclamation of a mining area in 1997. No significant problems are likely, but there could be local accumulations of uranium, radium, or radon in ground water from either unmined mineralization or from mined rocks.

References._-Fischer and others (1947).

\section{Mining Districts of the Lower San Miguel River Watershed}

\section{Uravan District}

The Uravan district ${ }^{5}$ is also called Uravan mineral belt and includes many subdistricts, such as Slick Rock, Bull Canyon, and Uravan (fig. 1).

Location.-Western San Miguel County, extending into Montrose and Dolores Counties, and adjacent Utah. The climate is semiarid, with about 10-16 inches of precipitation per year.

Principal commodities.-Uranium and vanadium, radium.

Mining history.- This area has a long history as a leading producer in the uranium and vanadium industries. Uranium was recognized and mined in a small way in 1898, but the yellow carnotite ores first became important as a source of radium in 1910. In the 1930's and in World War II the ores were valued for vanadium. During World War II the Manhattan Project recovered uranium from the U-V ores. After World War II the Atomic Energy Commission (AEC) encouraged production of uranium, and vanadium was a byproduct. Mining declined in the 1960's during the era of limited quotas, but returned as prices rose in the 1970's. All mines were closed in the 1980's. The mills at Uravan and Vancorum (Naturita) have been reclaimed as part of Federal efforts to reclaim uranium mills and tailings through the Uranium Mill Tailings Reclamation Act (UMTRA) and Superfund processes. As of 1998, some mines and dumps had been reclaimed and several of the mines were "mothballed" and could be reopened on short notice.

The AEC was granted mineral rights to large tracts of land on the Colorado Plateau in the 1940's (Chenoweth, 1981), and they explored for uranium with technical assistance from the USGS. If uranium in significant amounts was located, it was leased for mining. Leasing continued in the 1970's. The successor to the AEC, the Department of Energy (DOE), administers those lands. In recent years, the DOE has been negotiating the transfer of the Uranium Reserves to the BLM. The uncertain status of these Federal lands, unique in the Western United States, appears to complicate reclamation of mining sites on those lands.

Production and disturbance.-Production was very large, representing one of the giant uranium mining areas of the United States and the world. Production figures were obscured to protect confidentiality. Through 1964, the majority of the Colorado production of 8 million tons of uranium ore, valued at about $\$ 176$ million, came from this mining area. The total production through 1978 (close to the end of mining) was about 60,000 tons $\mathrm{U}_{3} \mathrm{O}_{8}$ (not tons of ore) (Shawe and others, 1991); this comprises nearly 13 percent of the total U.S. uranium production in the 20th century. Disturbance in this area really cannot be compared directly with the hardrock mining to the east because the district is actually a region, the style of mining is different, the climate and topography differ from the mountain districts, and so forth. The uranium mines ranged

\footnotetext{
${ }^{5}$ Only brief investigations were made in this district and much more work is needed. The information that follows is highly generalized and is included here to permit some comparisons with other mined lands of the CWS.
} 


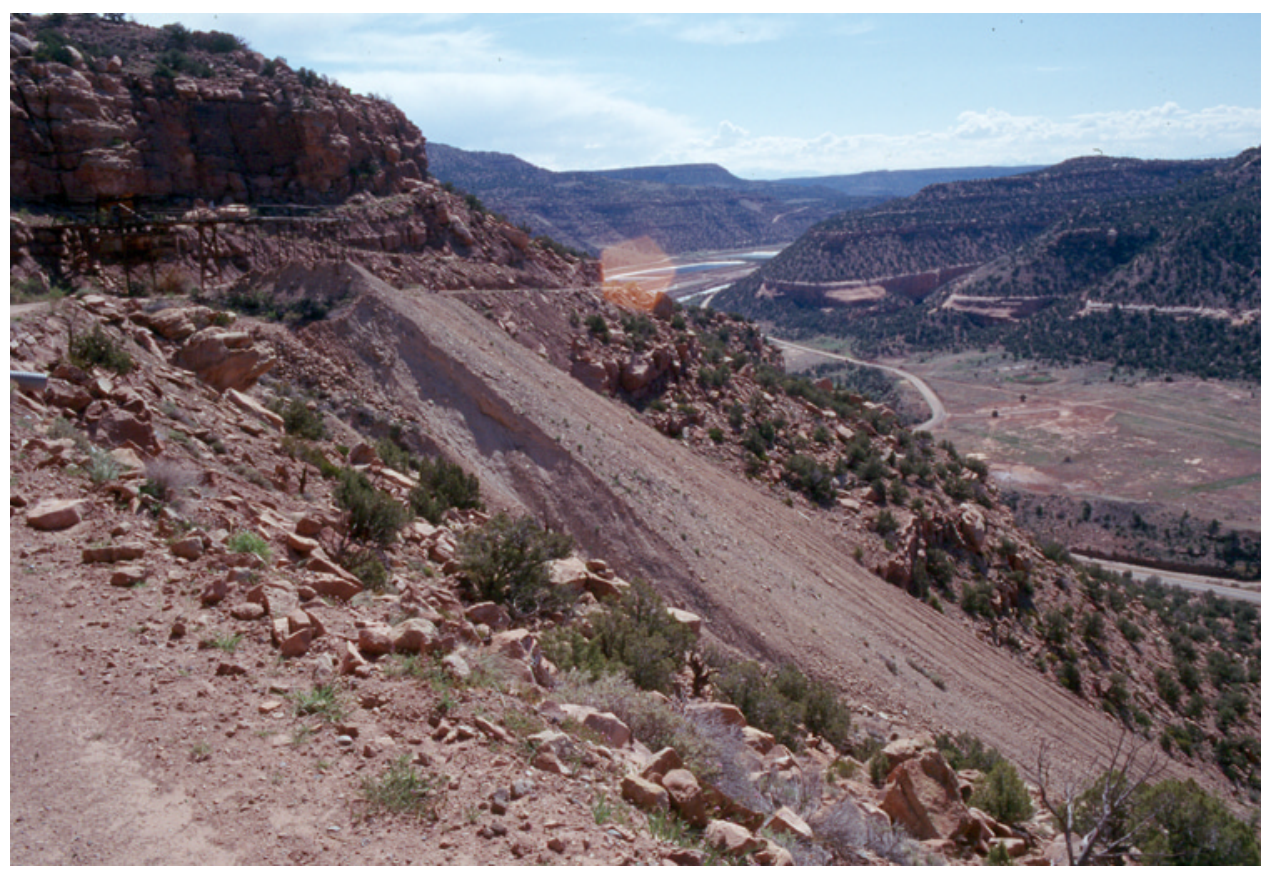

Photograph 107. Uranium mines of the Uravan district commonly tunneled into rim sandstones on steep canyon walls, as here on the edge of Atkinson Mesa. Mine waste that was dumped down the steep escarpment is difficult to reclaim. The San Miguel River is at the base of the slope; ponds to the south are near Uravan.

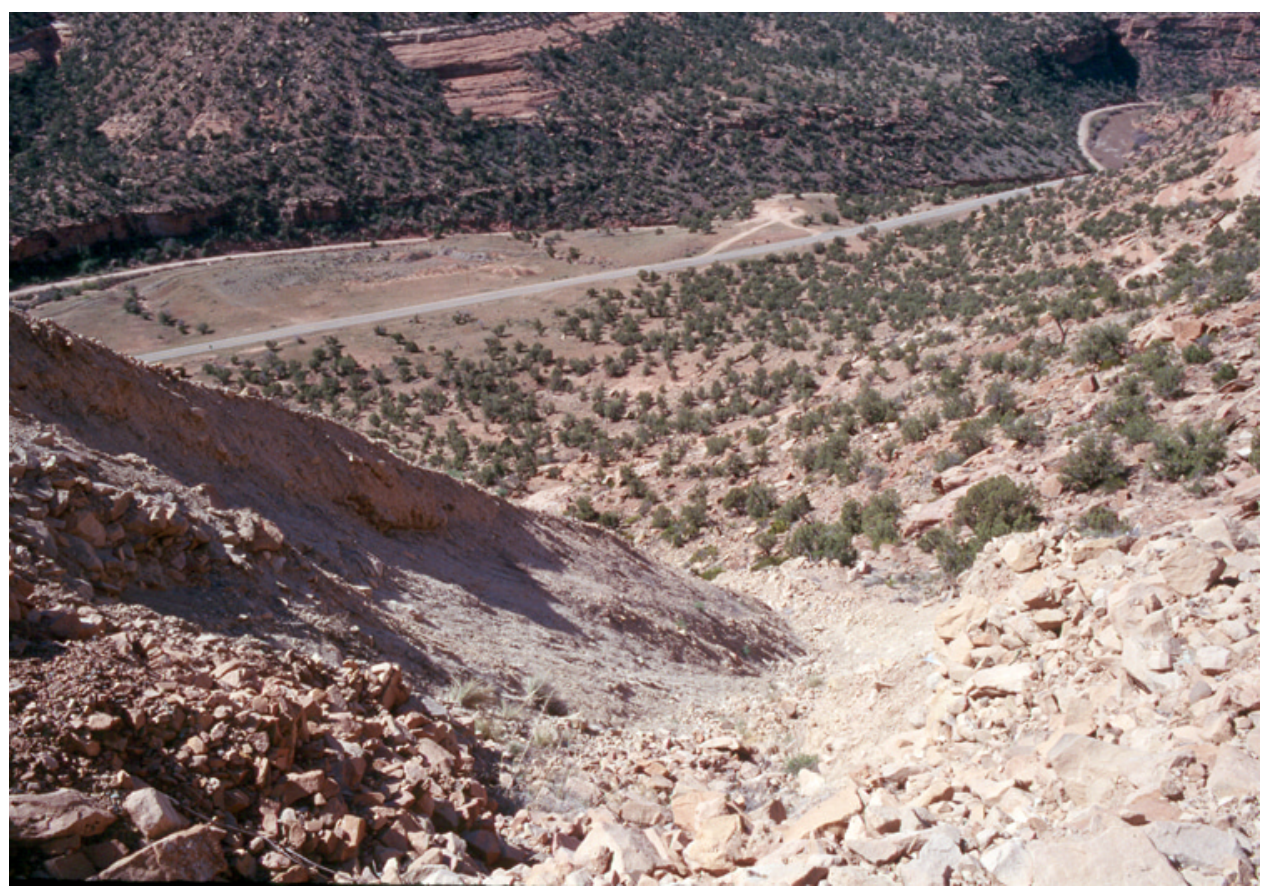

Photograph 108. Mine waste in mesa terrain is subject to erosion is storm events, as shown here. Stormwaters have carried uranium mine waste across Highway 141 to the Dolores River. 


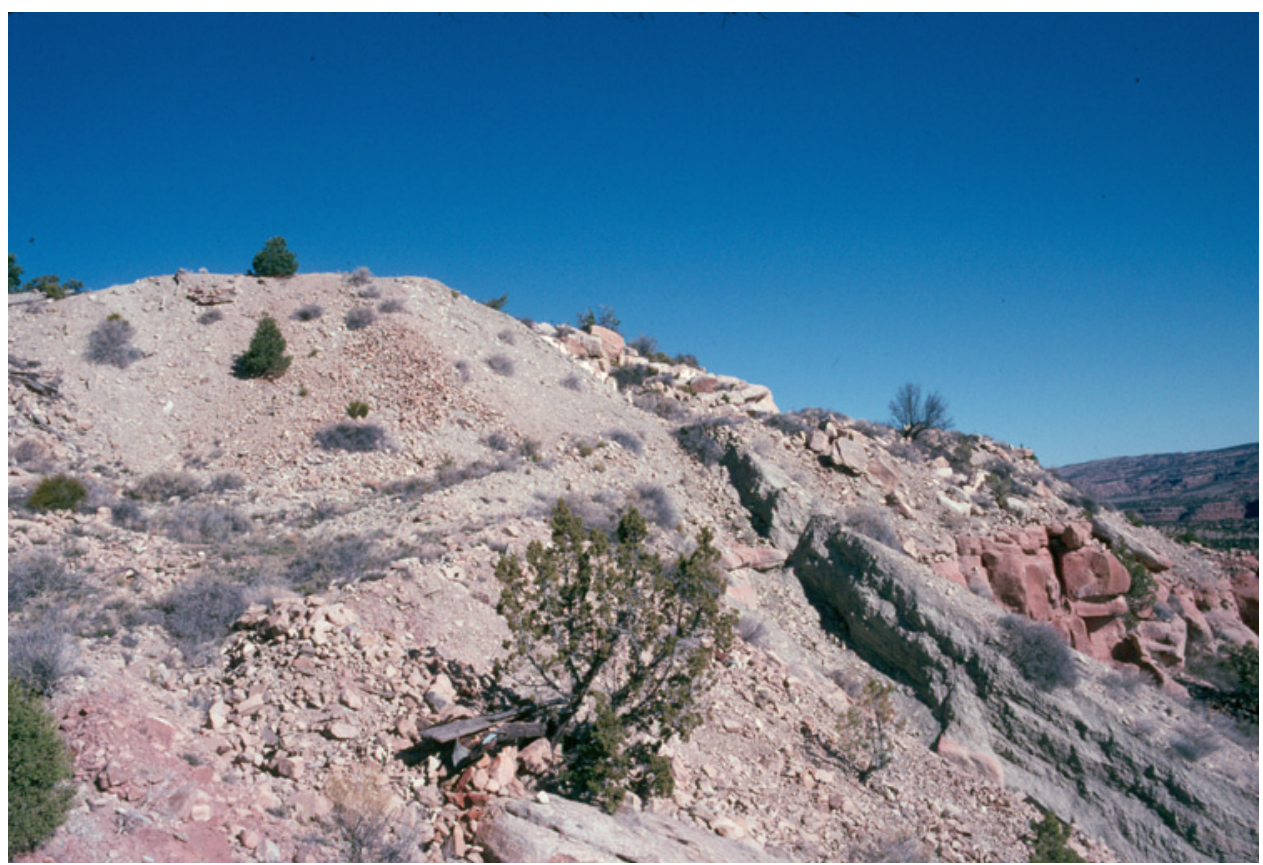

Photograph 109. Widespread mining across the Uravan district created thousands of small waste dumps like this one at the Cougar mine near Slickrock. Thunderstorms have severely eroded the mine waste at this site. Reclamation standards are vague for sites like this.

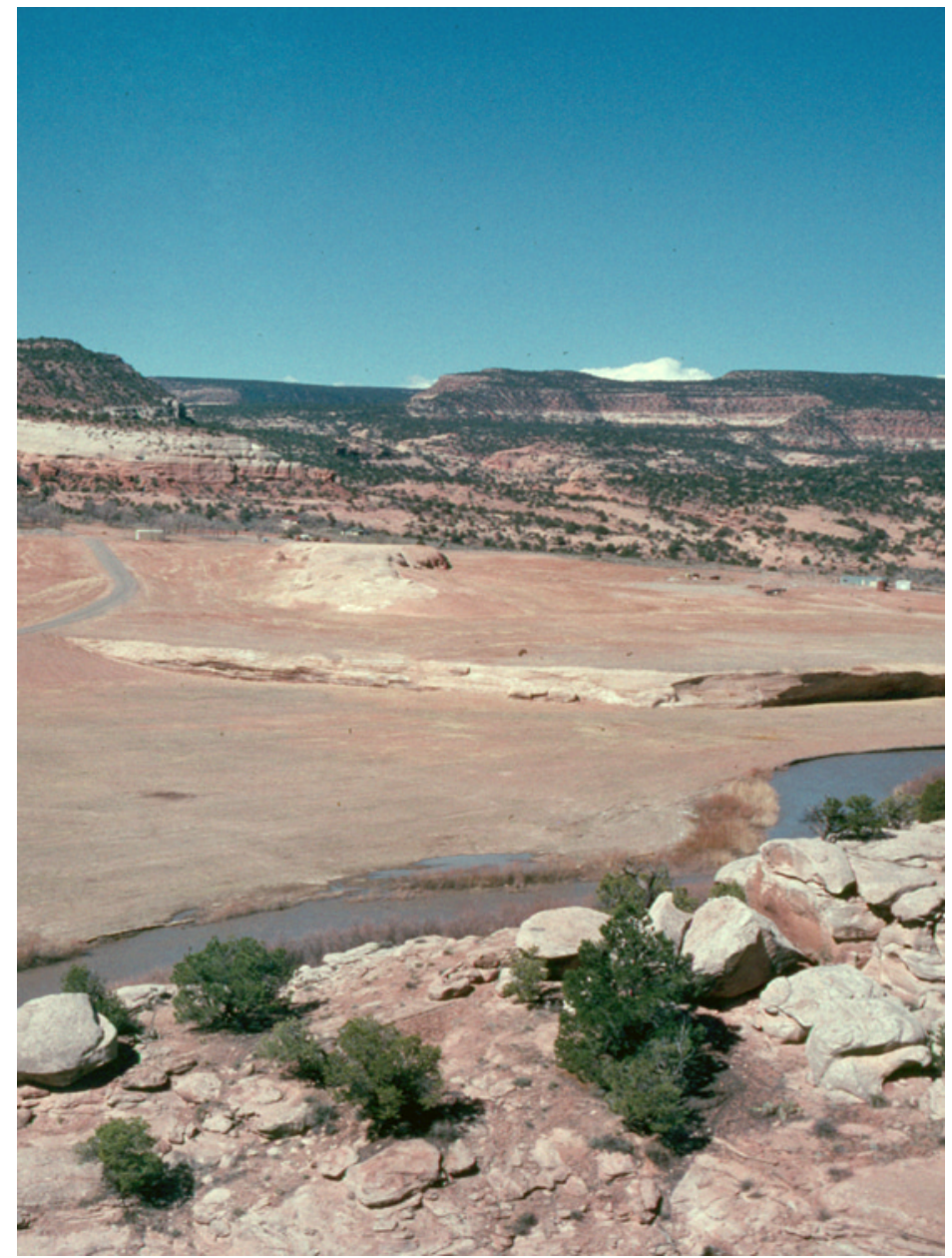

Photograph 110. The ore-buying station at Poverty Flat, near Slickrock, was thoroughly reclaimed in the 1990's, possibly using the standards for mill tailings. The Dolores River is in the foreground. 
from small to large in size, utilizing mainly adits and shafts, and a few were open pits. The total amount of rock mined and placed on the surface was very large (many millions of tons). There was one very large mill at Uravan, a buying station at Slickrock, and smaller vanadium mills at Naturita (Vancorum) and Gateway. The uranium mills and tailings have been carefully reclaimed under stringent UMTRA and Superfund standards. There are no Federal standards for uranium mine and dump reclamation, so the work on mines and dumps is inconsistent. Some reclamation allows highly radioactive rock to remain exposed at the neatly groomed surface.

Geology.-Uranium and associated vanadium occur in tabular lenses in sandstones of the Salt Wash Sandstone Member of the Jurassic Morrison Formation across a broad arc in western Colorado and adjacent Utah, hence the term Uravan mineral belt. The sandstones are not continuously mineralized, but the zones of U-V became predictable after an unprecedented amount of geologic study for the Atomic Energy Commission and countless exploration drill holes. The ores were originally dark colored (reduced) U-V minerals with pyrite and other sulfides, but some zones were oxidized by nearsurface weathering processes to form yellow carnotite. Subtle bedding-plane features, warps, and faults influence the distribution of ore. Some of the controlling structural features are related to the salt anticlines of the Paradox Basin.

\section{Mineral-Environmental Conditions}

Mineralized rocks and ores.-Numerous analyses are available for uranium-vanadium ores of this area. For this investigation, 13 samples were collected for analysis, sufficient to confirm the generalization of previous research that these ores are enriched in a distinctive suite of elements that includes $\mathrm{V}, \mathrm{Mo}$, and Se, but very low concentrations of Th and base metals. Median concentrations for this set of samples are (in parts per million): As, 20; $\mathrm{Cu}, 21 ; \mathrm{Mo}, 23 ; \mathrm{Pb}, 53 ; \mathrm{Se}, 49 ; \mathrm{Th}$ 3; U, 490; V, 2,700; and Zn, 49. The ore compositions thus are very distinct from the metal deposits to the east.

Leach tests on six samples of sandstone-hosted ores from waste dumps produced leachates with $\mathrm{pH}$ in the range 6.0 to 7.6 and generally very low metal concentrations. Some samples released elevated amounts of As, Mo, and Se, but concentrations of $\mathrm{U}$ were low. Concentrations of As, Mo, and Se are potentially of concern because they are relatively high and probably would be transported for long distances in these near-neutral waters (in contrast to the decreases in metal concentrations caused by neutralization of acidic waters).

Uranium mines and waste are generally measured for radioactivity because exposure to radiation is one of the health concerns. Not surprisingly, the radioactivity of mine dumps can be high to very high. Reclamation practice as of 1998 was to create a flat surface on waste dumps, if accessible, but there was no requirement for cover by clay or barren rocks to minimize radiation or water infiltration. Radiation standards for uranium mill tailings are very specific. Recon- naissance measurements of some dumps (reclaimed and unreclaimed) with a gamma ray spectrometer, calibrated on "pads" at USDOE in Grand Junction, show radiation levels are commonly $2 \times$ to $10 \times$ those of the mill-tailings standards. Much more work is needed on this aspect of uranium mine dumps and their reclamation standards. Guidance from EPA or other Federal experts is needed to provide consistent standards for reclamation of uranium mines and their waste dumps.

Mills and tailings.-Unlike practices in the metal mining industries, uranium ores throughout the Western United States have been trucked to centrally located mills to recover $\mathrm{U}$ and $\mathrm{V}$. This has the important consequence of creating a large central tailings impoundment, rather than numerous smaller ones close to the mines. In most of the Western United States, uranium mill tailings have been reclaimed, often by trucking to new repositories, under provisions of UMTRA. The mill and tailings site at Uravan was declared a Superfund site for reclamation of all associated liquid and solid wastes. Oreprocessing facilities at Slick Rock (called a buying station) and at the older vanadium mill near Naturita have been reclaimed. Tailings from the vanadium mill near Gateway have not been reclaimed but are much smaller in volume and are being monitored by the BLM Grand Junction office.

Surface waters.-There is very little surface water in this area, but the Dolores and San Miguel Rivers traverse the mining area. No mines in the study area discharge water to the surface, but some adits just to the west in Utah do drain water that is enriched in U, Mo, As, and Se. These mine waters have some of the highest concentrations seen for these elements in this study: $\mathrm{U}(2 \times$ and $20 \times$ relative to the ALWS of $44 \mathrm{ppb} \mathrm{U})$, As $(0.1 \times$ to $>20 \times$ relative to drinking-water standard of 50 $\mathrm{ppb}), \mathrm{Se}(4 \times$ and $69 \times$ relative ALWS at $5 \mathrm{ppb})$, and Mo $(2 \times$ and $9 \times$ relative to ALWS at $19 \mathrm{ppb})$. The stream below these mines is also highly enriched in As, Mo, Se, and $\mathrm{U}(1.5 \times$, $9 \times, 39 \times$, and $13 \times$ ALWS, respectively). Vanadium is enriched in these mine waters, but vanadium is generally not listed as a toxic element outside of some industrial plants. One seep near a small mine has some extremely high As, Mo, Se, and $U$ concentrations (As $>20 \times$, Mo $100 \times$, Se $>400 \times$, and $U$ $3.3 \times$ ALWS); cattle drink this water, but probably should not. Atkinson Creek, below Atkinson Mesa and numerous substantial uranium mines, has elevated but less dire concentrations of these elements (As 0.1×, Mo 3.6×, Se 0.8×, and U 0.4× ALWS). No chemical determinations were made of radioelements related to $\mathrm{U}$ (radon, radium, etc.). These few samples confirm the known solubilities of the elements in neutral to alkaline waters that carry As, Mo, and Se as oxyanions. No studies were made of the San Miguel and Dolores Rivers, but previous investigations had shown them to have compositions that exceed standards in the vicinity of the Uravan mill (CWQCD, 1989).

Summary.-Conditions and concerns for these uraniumvanadium mine lands are very different from the metal-mining areas. The lack of a national standard for uranium mine waste complicates evaluation and recommendations for reclamation. Currently (2001) it is permissible to reclaim mine waste and 
leave uranium-rich rocks at the surface, even though the radioactivity is 2 to 10 times the regulatory limit for mill tailings. Mine drainage probably is not a significant problem. Drainage from a few uranium mines may be enriched in As, Mo, Se, and $\mathrm{U}$, but the amount of water discharged is so small as to pose no threats to the main streams, such as the Dolores and San Miguel Rivers. There could be local contamination of water to the point that it should not be used for stock, irrigation, or domestic drinking water.

Further study is needed of the risk of erosion of mine dumps during flash floods, especially along the canyon walls near the Dolores and San Miguel Rivers. There are indications of recent storm-induced erosion and transport of mine waste to within a hundred yards of these important rivers. In an extreme storm event, the aqueous composition of the debris flow would certainly exceed standards for turbidity, uranium, and possibly a few other elements (Ra, Rn, As, Mo, Se).

References.-Shawe and others (1991); Fischer (1942); Fisher and Hilpert (1952); Chenoweth (1981); Shawe and others (1959).

\section{Cashin District (La Sal Creek)}

Location.-Westernmost Montrose County, $20 \mathrm{mi}$ west of Naturita (fig. 1). The climate is semiarid, with about 12-20 inches of precipitation per year.

Principal commodities.-Copper, silver.

Mining history.- - Veins of copper were located in 1880's, and for the next 20 years several small mines produced very rich copper-silver ore. In later years, production was only a few thousand dollars per year (gross) but increased to more than $\$ 70,000$ in the mid 1940's. Silver was an important coproduct. There has been no mining since 1945, but a thorough exploration program based on a new geologic concept defined substantial copper resources in the 1990's (more than 11 million tons of ore with an average grade of 0.55 percent $\mathrm{Cu}$ ) (Hahn and Thorson, 1995).

Production and disturbance.-Production was about 1,700,000 pounds copper and 350,000 oz silver. The Cashin mine is the only substantial mine in a cluster of small mines and prospects. Disturbance is small and produced just a few small mine dumps. Only a small volume of mill tailings could be identified at the Cashin mill site.

Geology.-Red sandstones of the Triassic Wingate Formation host copper-silver mineralization that has mixed oxide and sulfide mineralogy. The early workers focused on the highgrade ore in faults and called it "vein-type" and hydrothermal, but new concepts, based in part on knowledge gained in the past decade on other sediment-hosted copper deposits of the world, guided drilling that shows much wider and thicker $\mathrm{Cu}$ rich zones than the vein concept. The ores are now believed to be related to normal faults along salt anticlines: reducing gases and metal-rich brines moved up the fault structures and moved outward into permeable sandstone beds. Pyrite produced by the sulfidation of iron in sandstones is present in a large halo around the faults, but recent oxidation has destroyed the pyrite and makes recognition difficult by nonspecialists. Copper is

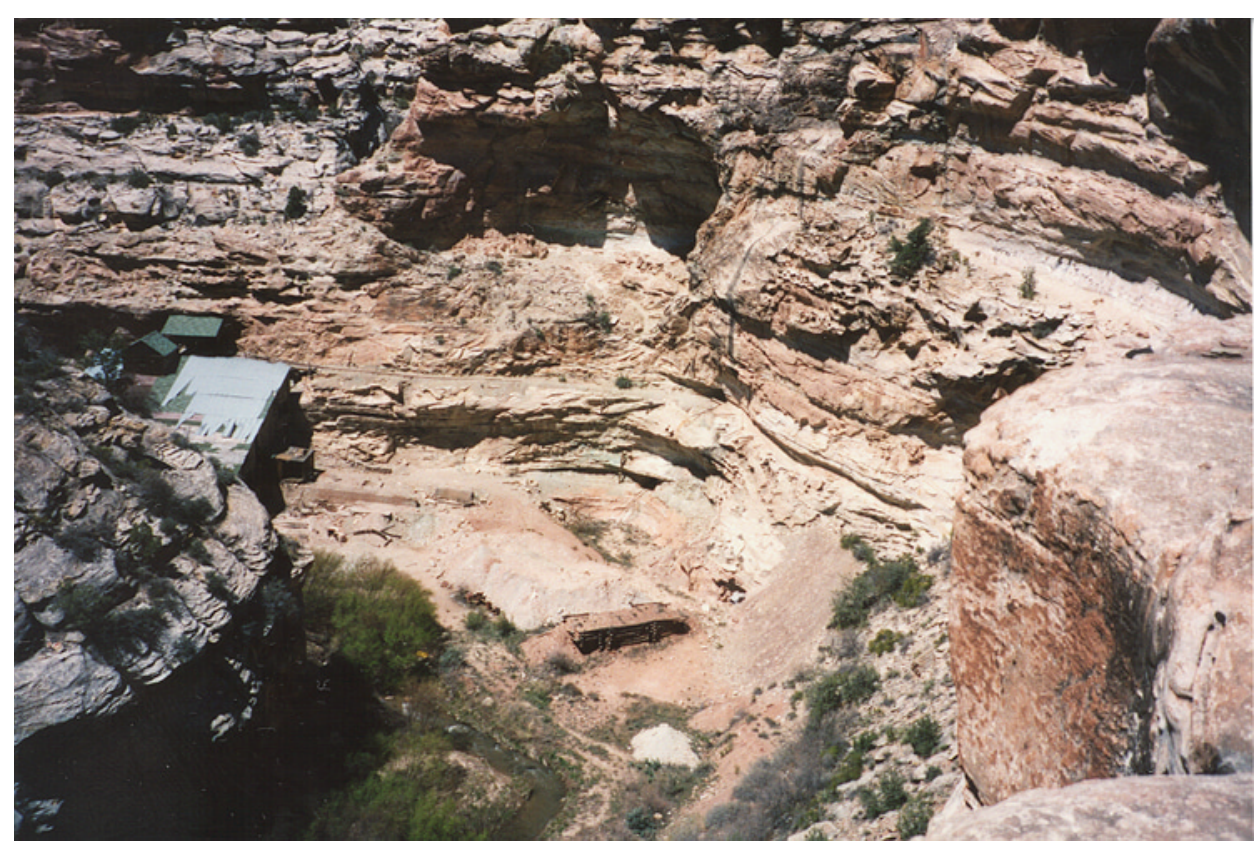

Photograph 111. The Cashin mine, located in the meandering canyon of La Sal Creek, had underground workings to the right (north) of the buildings. The largest building (green roof) is the mill. The creek is in lower center of photograph; mill tailings probably were placed in the creek east of the mill but are difficult to identify today. 
present in both sulfide and oxide minerals.

Studies by a Summo USA Corporation are underway to determine the economics of bulk mining the copper zone, possibly in an open-cut mine. This company completed engineering studies of an identical deposit in Lisbon Valley, Utah, received a permit to mine it, but the permit process was appealed in court. The mine plan calls for recovering the copper by sulfuric acid leaching on sealed pads and electrowinning the dissolved copper.

\section{Mineral-Environmental Conditions}

Mineralized rocks and ores.-Mineralized samples from the Cashin mine area are enriched chiefly in copper, with lower but important amounts of silver. Arsenic is slightly enriched, but uranium is low. No samples were tested by the leach method, but by analogy, I expect that there would be little acid generation and modest copper solubility. Existing mined rocks do not appear to pose chemical problems for Cashin Creek.

Mills and tailings.-There is a small mill at the Cashin mine, but tailings from the mill are difficult to distinguish from fine sand derived from nearby sedimentary rocks. The amount of mill tailings is uncertain; some or most may have been washed down La Sal Creek.

Surface waters.-No samples were collected. The copper and associated metals are not expected to be highly mobile under the likely neutral $\mathrm{pH}$ conditions. Calcite in the sedimentary rocks provides high ANC. New studies should examine the mobility of copper in surface waters because even low concentrations of copper are toxic to fish and associated biota.

Summary.-This variety of copper deposit appears to be relatively stable and benign in the semiarid environment. The relatively small amount of disturbance by historic mining does not appear to contaminate surface waters. However, copper has modest solubility in near-neutral $\mathrm{pH}$ waters and could pose a threat to aquatic life.

References.-Fischer (1936); Hahn and Thorson (1995).

\section{Discussion and Summary}

Observations and interpretations have been summarized for the individual districts on the central Western Slope (CWS) and will not be repeated here. These should be considered as interim interpretations. This reconnaissance study demonstrates the need for further work, some of which will be done in ongoing efforts that use GIS (geographic information system) techniques to combine the results presented here with digital geology, regional geochemistry, and mineral-deposit information. This spatial analysis will allow comparison with the geo-environmental map of Colorado developed by Plumlee and others (1995d).

Water quality in relation to mining.-These geochemical investigations show that contamination from historic mines is generally less than asserted by some (for example, CWQCD, 1989) or suggested by some geochemical models. Most of the studied mining districts have at least a few sources of contaminants, but, in the majority of cases, the metals and acidity of concern are naturally mitigated to trace levels within about 1 to $3 \mathrm{mi}$ of the sources. For these self-mitigating areas, the major concern is zinc and cadmium concentrations that are at or somewhat higher $(1 \times$ to $2 \times)$ than the chronic exposure standard for aquatic life. A few districts having a combination of stronger sources and limited buffering capacity have impacts on water quality, and presumably on aquatic health, beyond the area of mining and downstream for distances of about 3 to $10 \mathrm{mi}$. In these headwater streams, metals (such as $\mathrm{Al}, \mathrm{Cu}, \mathrm{Fe}, \mathrm{Mn}$, and $\mathrm{Pb}$ in addition to $\mathrm{Zn}$ and $\mathrm{Cd}$ ) also tend to be above standards for aquatic life. In such situations, reclamation activities are more complicated-engineers may be asked to do what nature cannot do-reduce the discharge from mining sources or design treatment systems that remove the toxic metals. Several areas on the CWS have unmined sources of acid and metals that complicate mitigation planning. Additional specific studies will be needed to define the baselines produced by the unmined mineralized rocks and to determine if water-quality standards for aquatic life (fisheries) can be met without treatment systems for contaminants from unmined sources.

In this reconnaissance study 22 mining districts were examined and sampled briefly. Of these districts, one stands out as a significant threat to watershed health: the Red Mountain district in the headwaters of the Uncompahgre River watershed (fig. 27). Red Mountain Creek in the Red Mountain district appears to be the most contaminated of those studied here, and there are indications of multiple sources, including unmined rocks of Red Mountain. The problems here are comparable in magnitude, spatial extent, and geochemistry to those in the adjoining Cement Creek of the upper Animas watershed. The upper Animas watershed is being studied by several agencies, with close attention by the EPA, and water-quality standards have not yet been assigned to those headwater streams.

Several other watersheds have mining-impacted waters, with smaller magnitude than at Red Mountain Creek and smaller than implied by the 1989 point-source assessment (CWQCD, 1989). My findings are consistent with the revised point-source assessment or "303(d)" list of 1998 (CWQCD, 1998). Several streams have water quality that may be harmful to fish and their supporting food web. Elevated zinc concentrations persist 1 to $5 \mathrm{mi}$ downstream from mine sources in Elk Creek (Ruby district), the uppermost Uncompahgre River (Mineral Point district), Dexter Creek (Ouray district), Tomichi Creek (Tomichi district), Gray Copper Creek (Red Mountain district), Howard Fork of the San Miguel River (Iron Springs district), and the upper part of Henson Creek (Galena district). More studies are needed of these headwater basins, including the mobility of metals (especially $\mathrm{Zn}$ and $\mathrm{Cu}$ ) and the effect that these metals may have on aquatic life.

Copper-zinc index of threats to aquatic life.-Copper and zinc concentrations of surface waters vary greatly across the 


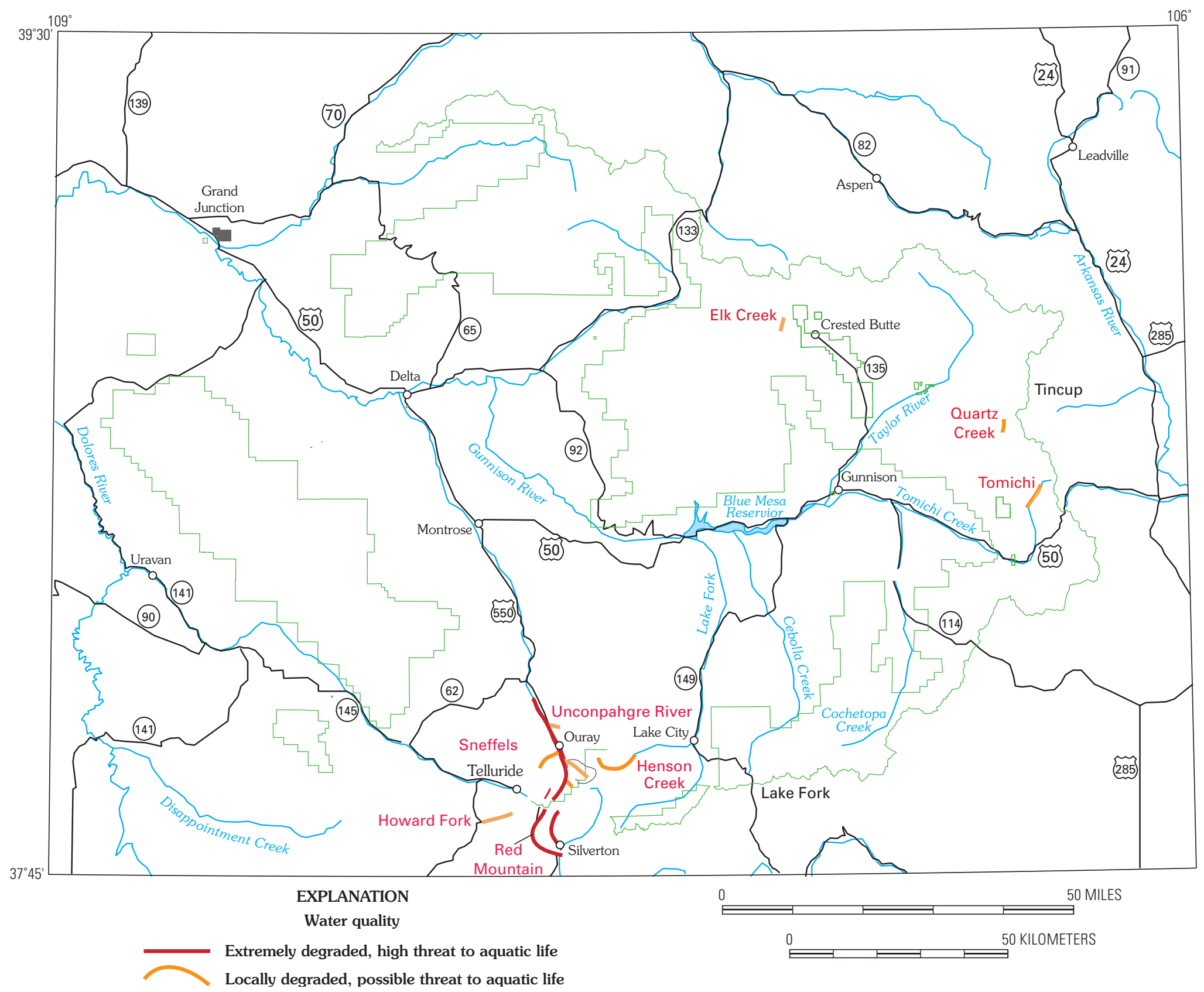

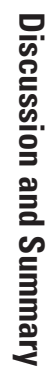

Figure 27. Location of streams, central Western Slope, Colorado, that have degraded water quality. Location of degraded streams approximate; line width exaggereated. Streams near Silverton from USGS (2000). 
CWS and are very high for many local sources such as adit drainages, tailings-pond seeps, and puddles on mine-waste dumps. The CZI summarizes in a simple number the waterquality concerns in relation to aquatic life, such as fish or their supporting food chain. Several streams have CZI values of 1 to 10 that suggest degraded quality and health, and some streams have values in the range 10 to $>100$ that suggest very bad quality and an especially high threat to aquatic life. The streams with very high CZI values include Red Mountain Creek and two side creeks in the Red Mountain district, Elk Creek (Ruby district), and the headwaters of Henson Creek (Galena district). Marginal CZI values, 1 to 0.3 , for five other creeks suggest possible threats to aquatic life. Copper appears to be the chief element of concern in several of these districts, but zinc is the concern in a few, such as Elk Creek (fig. 7).

Geologic attenuation of mine contamination.-In most districts there is geochemical evidence for attenuation of acidity and high metal concentrations by processes controlled by lithologies having high acid-neutralizing capacity (ANC). The most important and widespread lithologies on the CWS are propylitic-altered (green to purple) volcanic rocks, limestone, and sandstones containing calcite or other minerals that consume acid. Although these lithologies are the major factors, the beneficial reactions actually occur when surface or ground water flowing from those lithologies mix with acidic water. A simple field test of these mixing processes is conductivity measurements that show high variability over tens of feet in a stream. Reactions of acidic waters with rocks having high ANC also occur, but in many of the most degraded streams or mine discharges, the channel is coated with clay and other precipitates that minimize reactions with the rocks. In the district discussions, it was repeatedly pointed out that waters leaving the district probably would have much lower quality if there were no attenuating reactions. The one district that does not benefit much from these processes is Red Mountain, where the many mined and unmined sources of acid and metals exceed the neutralizing capacity of the highly altered rocks. In the Red Mountain district the sources from the eastern side (red rocks) are partially mitigated by waters from the western side (green rocks).

The natural attenuation reactions are helpful but incomplete. In many basins, the waters leaving the mining area carry concentrations of zinc, and in places cadmium or copper, that are high enough to be threats to fish and the food chain that supports them. Concentrations of $\mathrm{Zn}$ in the range of 50 to 200 $\mathrm{ppb}$ are found in many streams 1 to $5 \mathrm{mi}$ below mining areas (table 13). The good news is that these $\mathrm{Zn}$ concentrations are quite low relative to the upstream sources; the bad news is that this much Zn can stay in the streams for many miles. After the waters have been through the neutralizing reactions, causing various $\mathrm{Fe}$ and $\mathrm{Al}$ phases to precipitate and adsorb trace metals, not much else happens to decrease metal concentrations. Water-treatment facilities raise the $\mathrm{pH}$ to more than 9 to remove these persistent metals, but this does not happen in natural systems. More research is needed on these metal-rich circum-neutral waters, the stability of toxic metals in them, and the geologic processes that could remove those metals.

Threats from mill tailings. - Tailings are identified here as important contributors of contamination in many mining areas on the CWS. Also, many tailings impoundments appear to be at risk of catastrophic failure in an extreme storm. Most of the damage from a tailings failure cannot be reversed once the tailings are in a stream because removing them is costly and probably would further damage riparian habitat. Preventative maintenance may be appropriate in many situations. Many tailings impoundments are collapsing after 50 or more years with no maintenance, and those located in flood plains are at risk of collapse in a major storm. Locally focused flash floods are fairly common in Colorado, especially in the summer, and they are capable of moving millions of tons of earth materials-sufficient to close Interstate highways several times in 1999. A case can be made that failure of a tailings impoundment in a

Table 13. Summary of persistent zinc concentrations in near-neutral surface waters, central Western Slope, Colorado.

[District abbreviations as in table 1; distance to one or more likely sources; metal analyses by ICP-MS; CZI, copper-zinc index]

\begin{tabular}{lcccccc}
\hline Stream & District & Site & Distance (mi) & pH & Zn (ppb) & CZI (ppb) \\
\hline Elk Creek & RB & 558 & 1.8 & 7.3 & 540 & 6.0 \\
Red Mountain Creek & RM & 347 & 1 to $4 ?$ & 3.6 & 360 & 8.2 \\
Dexter Creek & OU & 857 & 0.6 & 8.2 & 360 & 6.0 \\
Upper Uncompahgre & MP & 641 & $2.5 ?$ & 6.2 & 152 & 0.48 \\
Tomichi Creek & TM & 565 & 1.7 & 7.2 & 125 & 0.43 \\
Canyon Creek & SN & 371 & 2.7 & 6.2 & 115 & 0.32 \\
Howard Fork & IS & 637 & $1-3$ & 6.7 & 89 & 0.35 \\
Gray Copper Creek & RM & 605 & 1.5 & 4.9 & 33 & 0.64 \\
Henson Creek & GN & 552 & $2 ?$ & 7.3 & 13 & 0.07 \\
\hline
\end{tabular}


storm event can cause more intensive and extensive damage to a watershed than the more predictable effects of steady acid mine drainage or release of metals from mine waste. Further study of tailings stability in extreme storms is needed to make an appropriate risk analysis.

Site problems for land management.-This study has identified some sites that appear to be significant sources of contamination on or very close to public lands. Many of these are tens of acres in size (a mining claim is 10.3 acres) and do not show clearly on watershed-scale maps. At watershed or 1:24,000 scales, property lines of patented claims (private property) relative to public lands administered by USFS and USBLM are important but difficult to identify without a cadastral survey. Many of the problem sites, such as the Standard mine, the Vernon mine, and the Akron mill site, appear to involve mixed ownership: the mine workings tend to be on patented lands, whereas the mine dumps or mill tailings may lie on public lands. Also, there are questions of impacts of contaminated waters that flow from patented claims onto public lands. A prime example is the Red Mountain district, which has about a thousand mine patents. The relations of those private lands to slivers of public lands between patents or relative to lands of the Uncompahgre Forest to the north is complex.

Most of my observations and new geochemical data on the CWS suggest that the land-management problems involve water and the need to control the contact of surface waters with mine waste (dump or tailings) to minimize reactions at these sources. The general concepts are fairly simple, but the technical details and legal issues can be complex and require skillful coordination in land reclamation. Few, if any, of the waste materials are so terrible in composition or in location that they require excavation and placement in more safely engineered repositories. However, many reclamation projects could be done more effectively, with greatly reduced future risk, by moving mine waste or mill tailings to safer sites. The major obstacles to this reclamation activity are not geology or engineering but complex legal and liability issues that should be streamlined to encourage cooperation among private owners, concerned citizens, FLMA's, and regulatory agencies.

\section{References Cited}

Alpers, C.N., and Nordstrom, D.K., 1991, Evolution of extremely acid mine waters at Iron Mountain, California-Are there any lower limits to $\mathrm{pH}$ ?, in Proceedings, 2nd International Conference on the Abatement of Acidic Drainage: Ottawa, Canada, MEND (Mine Environment Neutral Drainage), v.2, p. 321-342.

Bailey, R.G., 1998, Ecoregions: The ecosystem geography of the oceans and continents: New York, Springer, $176 \mathrm{p}$.

Bankey, Viki, ed., in press, Resource potential and geology of the Grand Mesa, Uncompahgre, and Gunnison (GMUG) National Forests and vicinity, Colorado: U.S. Geological Survey Bulletin.
Benci, J.F., and McKee, T.B., 1977, Colorado monthly temperature and precipitation summary for period 1951-1970: Fort Collins, Colorado State University, Climatology Report 77-1, 300 p.

Benham, J.L., 1980, Camp Bird and the Revenue: Ouray, Colo., Bear Creek Publishing, $68 \mathrm{p}$.

Besser, J.M., 2000, Biologic studies, in U.S. Geological Survey, Interim Report on the Scientific Investigations in the Animas River watershed, Colorado, to Facilitate Remediation Decisions by the U.S. Bureau of Land Management and the U.S. Forest Service, [March 29, 2000, meeting, Denver, Colo.]: U.S. Geological Survey Open-File Report 00-245, [paper edition], 34 p.

Besser, J.M., Brumbaugh, William, Church, S.E., and Kimball, B.A., 1998, Metal uptake, transfer, and hazards in the stream food web of the upper Animas River watershed, Colorado, in Nimick, D.A., and von Guerard, Paul, eds., Science for Watershed Decisions on Abandoned Mine Lands: Review of Preliminary Results, [Denver, Colo., February 4-5, 1998]: U.S. Geological Survey Open-File Report 98-297, p. 20.

Black, P.E., 1996, Watershed hydrology (2nd ed.): New York, Lewis Publishers, $449 \mathrm{p}$.

Blatt, Harvey, Middleton, Gerard, and Murray, Raymond, 1972, Origin of sedimentary rocks: Prentice-Hall, Inc., $634 \mathrm{p}$.

Boyle, T.P., and Bukantis, Bob, 1998, Use of ecological indicators as endpoints for remediation, in Nimick, D.A., and von Guerard, Paul, eds., Science for Watershed Decisions on Abandoned Mine Lands: Review of Preliminary Results, [Denver, Colo., February 4-5, 1998]: U.S. Geological Survey Open-File Report 98-297, p. 51.

Bromfield, C.S., 1967, Geology of the Mount Wilson quadrangle, western San Juan Mountains, Colorado: U.S. Geological Survey Bulletin 1227, $100 \mathrm{p}$.

Bromfield, C.S., Williams, F.E., and Popenoe, Peter, 1972, Mineral resources of the Wilson Mountains Primitive Area, Colorado: U.S. Geological Survey Bulletin 1353-A, p. A1-A79.

Burbank, W.S., 1940, Structural control of ore deposition in the Uncompahgre district, Ouray County, Colorado: U.S. Geological Survey Bulletin 906-E, p. 141-261.

Burbank, W.S., 1947a, Uncompahgre (Ouray) mining district, Ouray County, in Vanderwilt, J.W., ed., Mineral Resources of Colorado: Colorado Mineral Resources Board, p. 409-414.

Burbank, W.S., 1947b, Telluride and Sneffels districts, San Miguel and Ouray Counties, in Vanderwilt, J.W., ed., Mineral Resources of Colorado: Colorado Mineral Resources Board, p. 421-424.

Burbank, W.S., 1947c, The Mineral Point, Poughkeepsie, and Upper Uncompahgre districts, San Juan and Ouray Counties, in Vanderwilt, J.W., ed., Mineral Resources of Colorado: Colorado Mineral Resources Board, p. 437-439.

Burbank, W.S., 1960, Pre-ore propylitization, Silverton caldera, Colorado: U.S. Geological Survey Professional Paper 400-B, p. B12-B13.

Burbank, W.S., and Luedke, R.G., 1961, Origin and evolution of ore- and gangue-forming solutions, Silverton caldera, San Juan Mountains, Colorado: U.S. Geological Survey Professional Paper 424-C, p. C7-C11. 
Burbank, W.S., and Luedke, R.G., 1964, Geology of the Ironton quadrangle, Colorado: U.S. Geological Survey Geologic Quadrangle Map GQ-291, scale 1:24,000.

Burbank, W.S., and Luedke, R.G., 1966, Geology of the Telluride quadrangle, Colorado: U.S. Geological Survey Geologic Quadrangle Map GQ-504, scale 1:24,000.

Burbank, W.S., and Luedke, R.G., 1968, Geology and ore deposits in the western San Juan Mountains, Colorado, in Ridge, J.D., ed., Ore Deposits of the United States, 1933-1967, The Graton-Sales Volume: New York, American Institute of Mining, Metallurgical, and Petroleum Engineers, Inc., Volume I, p. 714-733.

Burbank, W.S., and Luedke, R.G., 1969, Geology and ore deposits of the Eureka and adjoining districts, San Juan Mountains, Colorado: U.S. Geological Survey Professional Paper 535, 73 p.

Burbank, W.S., Luedke, R.G., and Ward, F.N., 1972, Arsenic as an indicator element for mineralized volcanic pipes in the Red Mountains area, western San Juan Mountains, Colorado: U.S. Geological Survey Bulletin 1364, 31 p.

Buxton, H.T., Nimick, D.A., and others, 1997, A science-based, watershed strategy to support effective remediation of abandoned mine lands: Proceedings of the Fourth International Conference on Acid Rock Drainage, Vancouver, B.C., p. 1869-1880.

Chenoweth, W.L., 1981, Uranium-vanadium deposits of the Uravan mineral belt and adjacent areas, Colorado and Utah, in Epis, R.C., and Callendar, J.F.,eds., Western Slope Colorado: New Mexico Geological Society Field Conference Guidebook, p. 165-170.

Church, S.E., Kimball, B.A., Fey, D.L., Ferderer, D.A., Yager, T.J., and Vaughn, R.B., 1997, Source, transport, and partitioning of metals between water, colloids, and bed sediments of the Animas River, Colorado: U.S. Geological Survey Open-File Report 97-151, 135 p.

Cohon, J.L., Chairman, 1988, Estimating probabilities of extreme floods: Methods and recommended research, [report for National Research Council]: National Academy Press, 141 p.

Colorado Climate Center, 1984. Colorado average annual precipitation 1951-1980: Fort Collins, Colorado State University, Department of Atmospheric Science, map scale 1:500,000.

CDH ,1992, Idarado mine site remedial action plan (citizens' summary), April 9, 1992: Denver, Colorado Department of Health, $7 \mathrm{p}$.

Costa, J.E., 1988, Rheologic, geomorphic, and sedimentologic differentiation of water floods, hyperconcentrated flows, and debris flows, in Baker, V.R., Kochel, R.C., and Patton, P.C., eds., Flood Geomorphology: New York, John Wiley and Sons, p. 113-122.

Cox, D.P., and Singer, D.A., 1986, Mineral deposit models: U.S. Geological Survey Bulletin 1693, 379 p.

Cox, L.J., Chaffee, M.A., Cox, D.P., and Klein, D.P., 1995, Porphyry Cu deposits, in duBray, E.A., ed., Preliminary Compilation of Descriptive Geoenvironmental Mineral Deposit Models: U.S. Geological Survey Open-File Report 95-831, p.75-89.

Crawford, R.D., 1913, Geology and ore deposits of the Monarch and Tomichi districts, Colorado: Colorado Geological Survey Bulletin 4, $317 \mathrm{p}$.

Crawford, R.D., and Worcester, P.G., 1916, Geology and ore deposits of the Gold Brick district, Colorado: Colorado Geological Survey Bulletin 10, $116 \mathrm{p}$.

Crock, J.G., Arbogast, B.F., and Lamothe, P.J., 1999, Laboratory methods for the analysis of environmental samples, in Plumlee, G.S., and Logsdon, M.J., eds., The Environmental Geochemistry of Mineral Deposits: Reviews in Economic Geology, v. 6, Society of Economic Geologists, p. 265-287.

CWQCD, 1989, Colorado nonpoint assessment report: Colorado Water Quality Control Division, $198 \mathrm{p}$.

CWQCD, 1998, Colorado 1998 303(d) list: Colorado Water Quality Control Division, accessed on the world wide web at URL http:// www.state.colorado.us/wq/wqcc/1998303(d)/, [see also 2000303(d) at same site].

CWOCC, 2000, Colorado Water Quality, Basic standards and classifications: Colorado Water Quality Control Division, accessed on the world wide web at URL http://www.cdphe/state/colorado/us/ wqreg.asp/.

Day, W.C., and Bove, D.J., in press, Review of the geology of western Colorado, in Bankey, ed., Resource Potential and Geology of the Grand Mesa, Uncompahgre, and Gunnison (GMUG) National Forests and Vicinity, Colorado: U.S. Geological Survey Bulletin.

Day, W.C., Wilson, A.B., Bankey, Viki, Spanski, G.T., and Smith, S.M., in press, Mineral resource assessment for volcanic-associated massive sulfide deposits, in Bankey, ed., Resource Potential and Geology of the Grand Mesa, Uncompahgre, and Gunnison (GMUG) National Forests and Vicinity, Colorado: U.S. Geological Survey Bulletin.

Desborough, G.A., Briggs, P.H., Massa, Nilah, and Driscoll, Rhonda, 1998, Acid-neutralizing potential of minerals in intrusive rocks of the Boulder batholith in northern Jefferson County, Montana, U.S. Geological Survey Open-File Report 98-364, 21 p.

Desborough, G.A., Leinz, Reinhard, Briggs, P.H., Swayze, G.A., Smith, K.A., and Breit, George, 2000, Leaching studies of schwertmaniterich precipitates from the Animas River headwaters, Colorado, and Boulder River headwaters, Montana: U.S. Geological Survey OpenFile Report 00-004, $16 \mathrm{p}$.

DeWitt, Ed, Stoneman, R.J., Clark, J.R., and Kluender, S.E., 1985 Mineral resource potential map of the Fossil Ridge Wilderness Study Area, Gunnison County, Colorado: U.S. Geological Survey Miscellaneous Field Studies Map MF-1629-A, scale 1:50,000, 21 p.

Dings, M.G., and Robinson, C.S., 1957, Geology and ore deposits of the Garfield quadrangle, Colorado: U.S. Geological Survey Professional Paper 289, 110 p.

Drever, J.I., 1997, The geochemistry of natural waters, (3rd ed.): Saddle River, N.J., Prentice Hall, 436 p.

Drobeck, P.A., 1981, Proterozoic syngenetic massive sulfide deposits in the Gunnison Gold Belt, Colorado, in Epis, R.C., and Callender, J.F., Western Slope, Colorado; Western Colorado and Eastern Utah: New Mexico Geological Society 32nd Annual Field Conference, Guidebook no. 32, p. 279-286.

Ellis, C.E., 1983, Mineral investigations of the Oh-Be-Joyful Wilderness Study Area, Gunnison County, Colorado: U.S. Bureau of Mines MLA 81-83, $59 \mathrm{p}$. 
Fey, D.L., Desborough, G.A., and Church, S.E., 2000a, Comparison of two leach procedures applied to metal-mining-related wastes in Colorado and Montana and a relative ranking method for mine wastes, in Proceedings of the Fifth International Conference on Acid Rock Drainage, May 20-23, 2000, Denver, Colo.: Lakewood, Colo., Society of Mining Engineering, p. 1477-1487.

Fey, D.L., , Nash, J.T., Yager, D.B., and Desborough, G.A., 2000b, Analytical results for mine dump samples and leachate solutions, upper Animas River watershed, San Juan County, Colorado: U.S. Geological Survey, Open-File Report 00-338, 16 p. plus data files.

Ficklin, W.H., and Mosier, E.L., 1999, Field methods for sampling and analysis of environmental samples for unstable and selected stable constituents, in Plumlee, G.S., and Logsdon, M.J., eds., The Environmental Geochemistry of Mineral Deposits: Reviews in Economic Geology, v. 6, Society of Economic Geologists, p. 249-264.

Fischer, R.P., 1936, Peculiar hydrothermal copper-bearing veins of the northeastern Colorado Plateau: Economic Geology, v. 31, p. 571-599.

Fischer, R.P., 1942, Vanadium deposits of Colorado and Utah: A preliminary report: U.S. Geological Survey Bulletin 936-P, p. 363-394.

Fischer, R.P., Haff, J.C., and Rominger, J.F., 1947, Vanadium deposits near Placerville, San Miguel County, Colorado: Colorado Scientific Society Proceedings, v. 15, p. 115-134.

Fischer, R.P., and Hilpert, L.S, 1952, Geology of the Uravan mineral belt: U.S. Geological Survey Bulletin 988-A, 13 p.

Fischer, R.P., Luedke, R.G., Sheridan, M.J., and Raabe, R.G., 1968, Mineral resources of the Uncompahgre Primitive Area, Colorado: U.S. Geological Survey Bulletin 1261-C, p. C1-C91.

Fisher, F.S., 1990, Gold deposits of the Sneffels-Telluride and Camp Bird mining districts, San Juan Mountains, Colorado: U.S. Geological Survey Bulletin 1857-F, p. 12-18.

Fisher, F.S., and Leedy, W.P., 1973, Geochemical characteristics of mineralized breccia pipes in the Red Mountain district, San Juan Mountains, Colorado: U.S. Geological Survey Bulletin 1381, 43 p.

Follansbee, Robert, and Sawyer, L.R., 1948, Floods in Colorado: U.S. Geological Survey Water-Supply Paper 997, 151 p.

Goddard, E.N., 1936, The Geology and ore deposits of the Tincup mining district, Gunnison County, Colorado: Colorado Scientific Society Proceedings, v. 13 , no. 10 , p. 551-595

Guilbert, J.M., and Park, C.F., Jr., 1986, The geology of ore deposits: New York, W.H. Freeman Co., 946 p.

Hahn, G.A., and Thorson, J.P., 1995, Sandstone-hosted copper deposits related to salt dome anticlines, Paradox Basin, Colorado and Utah [abs.]: Denver, Denver Region Exploration Geologists Society, $2 \mathrm{p}$.

Hanshaw, Bruce, 1974, Geochemical evolution of a goethite deposit [abs.]: International Symposium on Water-Rock Interaction, p. 22.

Hartley, P.D., 1983, Geology and mineralization of the Vulcan-Good Hope massive sulfide deposit, Gunnison County, Colorado, in Gunnison Gold Belt and Powderhorn Carbonatite Field Trip Guidebook: Denver, Denver Region Exploration Geologists Society, p. 19-27.
Henderson, C.W., 1926, Mining in Colorado: U.S. Geological Survey Professional Paper 138, 263 p.

Herron, Jim, Stover, Bruce, Krabacher, Paul, and Bucknam, Dave, 1998, Cement Creek reclamation feasibility report, upper Animas River basin: Colorado Division of Minerals and Geology, 131 p.

Hill, J.M., 1909, Notes on the economic geology of southeastern Gunnison County, Colorado: U.S. Geological Survey Bulletin 380-A, p. 21-40.

Hofstra, A.H., Leventhal, J.S., Grimes, D.J., and Heran, W.D., 1995, Carlin-type Au deposits, in duBray, E.A., ed., Preliminary Compilation of Descriptive Geoenvironmental Mineral Deposit Models: U.S. Geological Survey Open-File Report 95-831,p. 184-192.

Hon, K., 1987, Geologic and petrologic evolution of the Lake City caldera, San Juan Mountains, Colorado: Boulder, University of Colorado, Ph.D. thesis, 244 p.

Irving, J.D., and Bancroft, Howland, 1911, Geology and ore deposits near Lake City, Colorado: U.S. Geological Survey Bulletin 478, 128 p.

King, T.V.V., ed., 1995, Environmental considerations of active and abandoned mine lands: U.S. Geological Survey Bulletin 2220, 43 p.

Kite, G.W., 1988, Frequency and risk analyses in hydrology: Littleton, Colo., Water Resources Publications, 257 p.

Lamothe, P.J., Meier, A.L., and Wilson, Stephen, 1999, The determination of forty-four elements in aqueous samples by inductively coupled plasma-mass spectrometry: U.S. Geological Survey Open-File Report 99-151, 14 p.

Langmuir, Donald, 1997, Aqueous environmental geochemistry: Upper Saddle River, N.J., Prentice Hall, 600 p.

Lindgren, Waldemar, 1933, Mineral deposits: New York, McGraw Hill, $930 \mathrm{p}$.

Lipman, P.W., 1976, Geologic map of the Lake City caldera area, western San Juan Mountains, southwestern Colorado: U.S. Geological Survey Miscellaneous Investigations Series Map I-962, scale 1:48,000.

Lipman, P.W., Fisher, F.S., Mehnert, H.H., Naeser, C.W., Luedke, R.G., and Steven, T.A., 1976, Multiple ages of mid-Tertiary mineralization and alteration in the western San Juan Mountains, Colorado: Economic Geology, v. 71, p. 571-588.

Long, K.R., DeYoung, J.H., and Ludington, S.D., 1998, Database of significant deposits of gold, silver, copper, lead, and zinc in the United States: U.S. Geological Survey Open-File Report 98-0205-A, B, 33 p., 1 diskette.

Ludington, Steve, and Ellis, C.E., 1983, Map showing geology and mineral resource potential of the Oh-Be-Joyful Wilderness Study Area, Gunnison County, Colorado: U.S. Geological Survey Miscellaneous Field Studies Map MF-1582-A, scale 1:24,000.

Ludington, Steve, Bookstrom, A.A., Kamilli, R.J., Walker, B.M., and Klein, D. P., 1995, Climax Mo deposits, in duBray, E.A., ed., Preliminary Compilation of Descriptive Geoenvironmental Mineral Deposit Models: U.S. Geological Survey Open-File Report 95-831, p. 70-74.

Luedke, R.G., and Burbank, W.S., 1962, Geology of the Ouray quadrangle, southwestern Colorado: U.S. Geological Survey 
Geologic Quadrangle Map GQ-152, scale 1:24,000.

Manahan, S.E., 1994, Environmental Chemistry (6th ed.): New York, Lewis Publishers, $811 \mathrm{p}$.

Mayor, J.N., 1978, Geologic summary of the Idarado mine, Ouray County, Colorado, in Shawe, D.R., ed., Guidebook on Fossil Fuels and Metals, Eastern Utah and Western-Southwestern-Central Colorado: Colorado School of Mines Professional Contributions, v. 9, p. 131-140.

Mayor, J.N., and Fisher, F.S., 1993, Skarn-hosted mineralization in Paleozoic rocks beneath the Idarado mine, northwest San Juan Mountains, Colorado: U.S. Geological Survey Open-File Report 93-183, $17 \mathrm{p}$.

McCulla, Michael, 1980, The genesis of the Los Ochos uranium deposits, Saguache County, Colorado: Reno, University of Nevada, unpub. Masters thesis, $128 \mathrm{p}$.

McFaul, E.J., Mason, G.T., Jr., Ferguson, W.B., and Lipin, B.R., 2000, U.S. Geological Survey mineral databases-MRDS and MAS/MILS: U.S. Geological Survey Digital Data Series DDS-52, 2 CD-ROM's.

McHugh, J.B., Miller, W.R., Meier, A.L., and d'Angelo, W.M., 1995, Chemical analyses of 33 surface water samples from the Redcloud Peak area, Colorado: U.S. Geological Survey Open-File Report 95-79, 5 p.

Miller, W.R., 1999, Geochemical baselines and maps showing acidneutralizing capacity and potential release of total dissolved solids of stream and spring waters from different rock types from mountainous watersheds in the Gunnison, Uncompahgre, and Grand Mesa National Forest, Colorado: U.S. Geological Survey Open-File Report 99-580, 107 p.

Miller, W.R., 1998, Geochemical baselines and processes affecting surface water, Redcloud Peak area, Colorado: U.S. Geological Survey Open-File Report 98-35, 20p.

Moore, J.N., and Luoma, S.N., 1990, Hazardous wastes from largescale metal extraction: Environmental Science and Technology, v. 24, p. 1270-1285.

Nash, J.T., 1981, Geology of dolomite-hosted uranium deposits at the Pitch mine, Saguache County, Colorado, in Epis, R.C., and Callender, J.F., eds., Western Slope, Colorado; Western Colorado and Eastern Utah: New Mexico Geological Society 32nd Annual Field Conference, Guidebook no. 32, p. 191-198.

Nash, J.T., 1988, Geology and geochemistry of the Pitch uranium mine area, Saguache County, Colorado: U.S. Geological Survey Bulletin 1797, $38 \mathrm{p}$.

Nash, J.T., 1999a, Geochemical investigations and interim recommendations for priority abandoned mine sites on U.S.D.A. Forest Service lands, Mineral Creek watershed, San Juan County, Colorado: U.S. Geological Survey Open-File Report 99-170, 40 p.

Nash, J.T., 1999b, Geochemical investigations and interim recommendations for priority abandoned mine sites on BLM lands, upper Animas River watershed, San Juan County, Colorado: U.S. Geological Survey Open-File Report 99-323, 45p.

Nash, J. T. 2000a, Geochemical studies of mines, dumps, and tailings as sources of contamination, upper Animas River watershed, Colorado: U.S. Geological Survey Open-File Report
00-104, CD-ROM.

Nash, J.T., 2000b, Hydrogeochemical data for historic mining areas, Humboldt watershed and adjacent areas, northern Nevada: U.S. Geological Survey Open-File Report 00-459, 12. p., 12 data files.

Nash, J.T., 2001, Hydrogeochemical investigations of some historic mining areas in the western Humboldt River basin, northwestern Nevada: U.S. Geological Survey Digital Data Series DDS-70, CD-ROM.

Nash, J.T., and Trudel, W.S., 1996, Bulk mineable gold ore at the Sleeper mine, Nevada-Importance of extensional faults, breccia, framboids, and oxidation, in Coyner, A.R., and Fahey, P.L., eds., Geology and Ore Deposits of the American Cordillera: Geological Society of Nevada Symposium Proceedings, Reno/Sparks, Nevada, April 1995, p. 235-256.

Nash, J.T., Miller, W.J., McHugh, J.B., and Meier, A.L., 1996, Geochemical characterization of mining districts and related contamination in the Prescott National Forest area, Yavapai County, Arizona: A preliminary assessment of environmental effects: U.S. Geological Survey Open-File Report 96-687, 80 p.

Nash, J.T., Desborough, G.A., and Fey, D.L., 1998, Geochemical and mineralogical characterization of mine dumps on BLM lands, upper Animas River watershed: plans and preliminary results, in Nimick, D.A., and von Guerard, Paul, eds., Science for Watershed Decisions on Abandoned Mine Lands: Review of Preliminary Results, Denver, Colorado, February 4-5, 1998: U.S. Geological Survey Open-File Report 98-297, p. 44-45.

Nelson, C.J., and Riesmeyer, W.D., 1983, Geology of the AnacondaGunnison mine area, Gunnison County, Colorado, in Gunnison Gold Belt and Powderhorn Carbonatite Field Trip Guidebook: Denver, Denver Region Exploration Geologists Society, p. 8-18

Nimick, D.A., and von Guerard, Paul, 1998, eds. , Science for watershed decisions on abandoned mine lands: Review of preliminary results, Denver, Colorado, February 4-5, 1998: U.S. Geological Survey Open-File Report 98-297, 70 p.

Nimick, D.A., and Moore, J.N., 1991, Prediction of water-soluble metal concentrations in fluvially deposited tailings sediments, upper Clark Fork Valley, Montana, U.S.A.: Applied Geochemistry, v. 6, p. 635-646.

Nimmo, D.W.R., Castle, C.J., and Besser, J.M., 1998, A toxicological reconnaissance of the upper Animas River watershed near Silverton, Colorado, in, Nimick, D.A., and von Guerard, Paul, eds., Science for Watershed Decisions on Abandoned Mine Lands: Review of Preliminary Results, Denver, Colorado, February 4-5, 1998: U.S. Geological Survey Open-File Report 98-297, p. 19.

Nordstrom, D.K., and Alpers, C.N., 1999, Geochemistry of acid mine waters, in Plumlee, G.S., and Logsdon, M.J., eds., The Environmental Geochemistry of Mineral Deposits: Reviews in Economic Geology, v. 6, Society of Economic Geologists, p. 133-160.

Olson, J.C., 1983, Geologic and structural maps and sections of the Marshall Pass mining district, Saguache, Gunnison, and Chafee Counties, Colorado: U.S. Geological Survey Miscellaneous Investigations Series Map I-1425, scale 1:24,000.

Olson, J.C., 1988, Geology and uranium deposits of the Cochetopa and Marshall Pass districts, Saguache, and Gunnison Counties, 
Colorado: U.S. Geological Survey Professional Paper 1457, 44 p.

Owen, J.R., 1997, Water quality and sources of metal loading to the upper Animas River basin: Colorado Department of Public Health, Water Quality Control Division, 31 p. plus tables and figures.

Plumlee, G.S., 1999, The environmental geology of mineral deposits, in Plumlee, G.S., and Logsdon, M.J., eds., The Environmental Geochemistry of Mineral Deposits: Reviews in Economic Geology, v. 6, Society of Economic Geologists, p. 70-116.

Plumlee, G.S., and Logsdon, M.J., 1999, An earth-system science toolkit for environmentally friendly mineral resource development, in Plumlee, G.S., and Logsdon, M.J., eds., The Environmental Geochemistry of Mineral Deposits: Reviews in Economic Geology, v. 6, Society of Economic Geologists, p.1-28.

Plumlee, G.S., Montour, Maria, Taylor, C.D., Wallace, A.R., and Klein, D.P., 1995a, Polymetallic vein and replacement deposits, in duBray, E.A., ed., Preliminary Compilation of Descriptive Geoenvironmental Mineral Deposit Models: U.S. Geological Survey Open-File Report 95-831, p. 121-129.

Plumlee, G.S., and Nash, J.T., 1995, Geoenvironmental models of mineral deposits-Fundamentals and applications, in duBray, E.A., ed., Preliminary Compilation of Descriptive Geoenvironmental Mineral Deposit Models: U.S. Geological Survey Open-File Report 95-831, p.1-9.

Plumlee, G.S., Smith, K.S., Berger, B.R., Foley-Ayuso, Nora, and Klein, D.P., 1995b, Creede, Comstock, and Sado epithermal vein deposits, in duBray, E.A., ed., Preliminary Compilation of Descriptive Geoenvironmental Mineral Deposit Models: U.S. Geological Survey Open-File Report 95-831, p. 152-161.

Plumlee, G.S., Smith, K.S., Gray, J.E., and Hoover, D.B., 1995c, Epithermal quartz-alunite Au deposits, in duBray, E.A., ed., Preliminary Compilation of Descriptive Geoenvironmental Mineral Deposit Models: U.S. Geological Survey Open-File Report 95-831, p. 162-169.

Plumlee, G.S., Streufert, R.K., Smith, K.S., Smith, S.M., Wallace, A.R., Toth, M.I., Nash, J.T., Robinson, R.A., Ficklin, W.H., and Lee, G.K., 1995d, Map showing potential metal-mine drainage hazards in Colorado, based on mineral-deposit geology: U.S. Geological Survey Open-File Report 95-26, scale 1:750,000.

Plumlee, G.S., Smith, K.S., Montour, M.R., Ficklin, W.H., and Mosier, E.L., 1999, Geologic controls on the composition of natural waters and mine waters, in Filipek, L.H., and Plumlee, G.S., eds., The Environmental Geochemistry of Mineral Deposits: Reviews in Economic Geology, Society of Economic Geologists, p. 373-432.

Purington, 1898, Preliminary report on the mining industries of the Telluride quadrangle, Colorado: U.S. Geological Survey, Eighteenth Annual Report, part III, Economic Geology, p. 746-851.

Ransome, F. L., 1901, Economic geology of the Silverton quadrangle, Colorado: U.S. Geological Survey Bulletin 182, 265 p.

Schumm, S.A., Costa, J.E., Toy, T., Knox, J., Warner, R., and Scott, J., 1981, Geomorphic assessment of uranium mill tailings disposal sites, in Uranium Mill Tailings Management, Proceedings of two NEA workshops: OECD Nuclear Energy Agency, 230 p.

Shawe, D.R., Archbold, N.L., and Simmons, G.C., 1959, Geology and uranium-vanadium deposits of the Slick Rock district, San Miguel and Dolores Counties, Colorado: Economic Geology, v. 54, p. $395-415$.

Shawe, D.R., Nash, J.T., and Chenoweth, W.L., 1991, Uranium and vanadium deposits, in Gluskoter, H.J., Rice, D.D., and Taylor, R.B., eds., Economic Geology, U.S.: Geological Society of America, The Geology of North America, v. P-2, p. 103-124.

Shepherd, T.A., and Nelson, J.D., 1978, Long-term stability of uranium mill tailings, in Uranium Mill Tailings Management: Fort Collins, Colo., Civil Engineering Department, Colorado State University, v. 1, $172 \mathrm{p}$.

Sheridan, D.M., Raymond, W.H., and Cox, L.J., 1981, Precambrian sulfide deposits in the Gunnison region, Colorado, in Epis, R.C., and Callender, J.F., eds., Western Slope, Colorado; Western Colorado and Eastern Utah: New Mexico Geological Society 32nd Annual Field Conference, Guidebook no. 32, p. 273-277.

Slack, J.F., 1980, Multistage vein ores of the Lake City district, western San Juan Mountains, Colorado: Economic Geology, v. 75, no. 7, p. 963-991.

Smith, A.C.S., and Mudder, T.I., 1999, The environmental geochemistry of cyanide, in Plumlee, G.S., and Logsdon, M.J., eds., The Environmental Geochemistry of Mineral Deposits: Reviews in Economic Geology, v. 6, Society of Economic Geologists, p. 229-248.

Smith, K.S., and Huyck, H.L.0, 1999, An overview of the abundance, relative mobility, bioavailability, and human toxicity of metals, in Plumlee, G.S., and Logsdon, M.J., eds., The Environmental Geochemistry of Mineral Deposits: Reviews in Economic Geology, v. 6, Society of Economic Geologists, p.29-70.

Smith, K.S., Plumlee, G.S., and Ficklin, W.H., 1994: Predicting water contamination from metal mines and mining wastes: U.S. Geological Survey Open-File Report 94-264, 112 p.

Smith, P.D., 1994, Mountains of silver, the story of Colorado's Red Mountain mining district: Boulder, Colo., Pruett Publishing, 221 p.

Smith, S.M., in press, Regional Sediment and Rock Geochemistry, in Bankey, Viki, ed., Resource Potential and Geology of the Grand Mesa, Uncompahgre, and Gunnison (GMUG) National Forests and Vicinity, Colorado: U.S. Geological Survey Bulletin.

Southworth, Dave, 1997, Colorado mining camps: Wild Horse Publishing, $311 \mathrm{p}$.

Spanski, G.T., and Bankey, Viki, in press, Qualitative and quantitative mineral resource assessment methodology, in Bankey, Viki, ed., Resource Potential and Geology of the Grand Mesa, Uncompahgre, and Gunnison (GMUG) National Forests and Vicinity, Colorado: U.S. Geological Survey Bulletin.

Steven, T.A., and Lipman, P.W., 1976, Calderas of the San Juan volcanic field, southwestern Colorado: U.S. Geological Survey Professional Paper 958, $35 \mathrm{p}$.

Thomas, J.A., and Galey, J.T., Jr., 1982, Exploration and geology of the Mount Emmons molybdenite deposits, Gunnison County, Colorado: Economic Geology, v. 77, no. 5, p. 1085-1104.

U.S. Geological Survey, 2000, , Interim report on the scientific investigations in the Animas River watershed, Colorado, to facilitate remediation decisions by the U.S. Bureau of Land Management and the U.S. Forest Service, March 29, 2000, meeting, 
Denver, Colorado: U.S. Geological Survey Open-File Report 00-245 $34 \mathrm{p}$.

Varnes, D.J., 1947a, Iron Springs mining district, San Miguel County, in Vanderwilt, J.W., ed., Mineral Resources of Colorado: Colorado Mineral Resources Board, p. 425-428.

Varnes, D.J., 1947b, Mount Wilson district, San Miguel County, in Vanderwilt, J.W., ed., Mineral Resources of Colorado: Colorado Mineral Resources Board, p. 428.

Vhay, J.S., 1962, Geology and mineral deposits of the area south of Telluride, Colorado: U.S. Geological Survey Bulletin 1112-G, p. 209-310.

Vincent, K.R., Church, S.E., and Fey, D.L., 1999, Geomorphological context of metal-laden sediments in the Animas River floodplain, Colorado, in Morganwalp, D.W., and Buxton, H.T., eds., U.S. Geological Survey Toxic Substances Hydrology ProgramProceedings of the Technical Meeting, Charleston, South Carolina, March 8-12, 1999-Volume 1 of 3-Contamination from Hardrock Mining: U.S. Geological Survey Water-Resources Investigations Report 99-4018A, p. 99-106.

Wanty, R.B., Miller, W.R., Briggs, P.H., and McHugh, J.B., 1999, Geochemical processes controlling uranium mobility in mine drainages, in Plumlee, G.S., and Logsdon, M.J., eds., The Environmental Geochemistry of Mineral Deposits: Reviews in Economic Geology, v. 6, Society of Economic Geologists, p. 201-213.
Wilson, A.B., and Spanski, G.T., in press, Distribution of mines and mineralization, in Bankey, Viki, ed., Resource Potential and Geology of the Grand Mesa, Uncompahgre, and Gunnison (GMUG) National Forests and Vicinity, Colorado: U.S. Geological Survey Bulletin.

Wirt, Laurie, 1994, Radioactivity in the environment-A case study of the Puerco and Little Colorado River basins, Arizona and New Mexico: U.S. Geological Survey Water-Resources Investigations Report 94-4192, 23 p.

Woolsey, L.H., 1907, Lake Fork extension of the Silverton mining area, Colorado, in Contributions to Economic Geology 1906: U.S. Geological Survey Bulletin 315, p. 26-31.

Wright, R.J., and Everhart, D.L., 1960, Uranium, in Del Rio, S.M., ed., Mineral Resources of Colorado, First Sequel: Colorado Mineral Resources Board, p. 327-366.

Manuscript approved for publication January 14, 2002

Published in the Central Region, Denver, Colorado

Editing, layout, photocomposition-Richard W. Scott, Jr. Graphics by the author and Gayle A. Dumonceaux

Cover and jewel-case design, graphics consultationCarol A. Quesenberry 


\section{Appendix I: Water-Sampling and Analysis Methods}


Water samples were collected from mines and streams where the water was deemed to be representative of a geologic or mine setting and would yield information on the mobility of metals in that environment. The sampling methods have been used by the author since 1994 for reconnaissance characterization of geologic units, rock alteration, mine drainage, and reactions with tailings or dump materials (Nash and others, 1996); these methods are similar to but simplified from those described by Ficklin and Mosier (1999). First, flow rate and water characteristics (color, suspended material, bed colors, or mineralogy) are recorded, and $\mathrm{pH}$ and conductivity are measured using portable instruments. The pocket-sized conductivity meter (Corning CD-55), with an upper limit of 2,000 $\mu \mathrm{S} / \mathrm{cm}$, responded consistently and showed no drift after calibration. The $\mathrm{pH}$ meter (Orion 250), with built-in temperature electrode, required frequent calibration during the day, and at most sites the calibration was checked on a standard solution after the field measurement. The field standards were buffered solutions of $\mathrm{pH}$ 4.0, 7.0, and 10.0. Based on experience with portable $\mathrm{pH}$ meters applied to diverse water compositions, I consider the measurements of $\mathrm{pH}$ to carry an uncertainty of about \pm 0.05 standard unit, even though the meter reports to 0.01 units. The water sample for analysis was collected with a disposable $60-\mathrm{mL}$ syringe, then pushed through a disposable $0.45-\mu \mathrm{m}$ cellulose filter. The syringe and the 60 - or $120-\mathrm{mL}$ polyethylene bottle were rinsed twice in the sampled water prior to collection. The filtered sample was acidified to a $\mathrm{pH}$ of about 2 at the site with 5 drops of ultrapure 1:1 $\mathrm{HNO}_{3}$ per $60 \mathrm{~mL}$. The acid stabilizes metals for periods of more than 2 years without the use of refrigeration, based on replicate analyses over a 2-year period. At appropriate localities (with $\mathrm{pH}>4$ ), an unfiltered sample was collected for determination of alkalinity. Laboratory and field blank tests using deionized water, indicated that contamination introduced by the sampling procedure and equipment is in the low parts per billion level (1-10 ppb), which is considered adequate in the search for metal concentrations orders of magnitude greater than the sampling error.

The filtered and acidified water samples collected in 1997 and 1998 were analyzed by a commercial laboratory in Denver, Colo., within 20 days of collection, using ICP-MS (inductively coupled plasma-mass spectrometry); the general method is described by Lamothe and others (1999). Data for more than 60 elements are reported, generally to levels below $1 \mathrm{ppb}$ (table 14), but in detail the limit of determination varies with sample composition and element mass. For many samples, the actual limit of determination is lower than that shown in table 14. In this report, only 10 to 15 elements of prime environmental concern are tabulated and discussed. This analytical method is appropriate for determination of many metals of potential concern that are expected to have a wide range in concentrations; the method is especially good for trace metals in water such as $\mathrm{As}, \mathrm{Cu}, \mathrm{Pb}$, and $\mathrm{Zn}$ (Crock and others, 1999). If water compositions reported here are a concern or are in question, follow-up work should be done using trace-metal sampling protocols and more precise analytical methods.

Leachate solutions from laboratory tests were analyzed by the commercial laboratory using the same ICP-MS methods just described. The analytical errors are judged to be similar to those for surface-water samples.

A small number of samples collected in 1999 were analyzed by A.L. Meier of the USGS in the Lakewood, Colo., ICP-MS laboratory, using methods generally similar to those described above. The 1999 ICP-MS results should be distinguished from the 1997-98 results because there are differences in detail; the 1999 analyses are done by research protocol and have better precision and accuracy, and Ag values are reliable.

Experience with the laboratory from 1996 to 1999, analysis of replicate samples (repeat analyses of water from the same bottle or duplicate bottles collected at the same time), and of reference standards allows the following generalized comments on precision (reproducibility) and accuracy (approximation of the correct value).

1. Results are reported for five or six orders of magnitude for most elements of interest in these natural waters and leachates, from less than $0.1 \mathrm{ppb}$ (part per billion

Table 14. Elements and lower limits of determination for analyses of water and leachate samples by ICP-MS.

[Elements are listed by atomic weight, and all determinations are in parts per billion; the lower limit of determination shown here is rounded by Nash and is somewhat higher than reported by the commercial laboratory for the 1997 and 1998 analyses]

\begin{tabular}{|c|c|c|c|}
\hline Element & $\begin{array}{l}\text { Lower limit } \\
\text { of determination }\end{array}$ & Element & $\begin{array}{l}\text { Lower limit } \\
\text { of determinatior }\end{array}$ \\
\hline$\overline{\mathrm{Li}}$ & 0.1 & $\mathrm{Nb}$ & 0.01 \\
\hline $\mathrm{Be}$ & 0.5 & Mo & 0.04 \\
\hline $\mathrm{Na}$ & 0.8 & $\mathrm{Ag}$ & $0.01 *$ \\
\hline $\mathrm{Mg}$ & 0.5 & $\mathrm{Cd}$ & 0.01 \\
\hline $\mathrm{Al}$ & 0.4 & $\mathrm{Sn}$ & 0.01 \\
\hline $\mathrm{Si}$ & 1 & $\mathrm{Sb}$ & 0.04 \\
\hline $\mathrm{K}$ & 1 & $\mathrm{Te}$ & 0.2 \\
\hline $\mathrm{Ca}$ & 1 & $\mathrm{Cs}$ & 0.01 \\
\hline $\mathrm{Sc}$ & 0.1 & $\mathrm{Ba}$ & 0.01 \\
\hline $\mathrm{Ti}$ & 0.04 & $\mathrm{La}$ & 0.01 \\
\hline $\mathrm{V}$ & 0.04 & $\mathrm{Ce}$ & 0.01 \\
\hline $\mathrm{Cr}$ & 0.04 & $\mathrm{Eu}$ & 0.01 \\
\hline Mn & 0.04 & $\mathrm{Yb}$ & 0.01 \\
\hline $\mathrm{Fe}$ & 0.3 & $\mathrm{Ta}$ & 0.01 \\
\hline Co & 0.01 & $\mathrm{~W}$ & 0.01 \\
\hline $\mathrm{Ni}$ & 0.01 & $\mathrm{Re}$ & 0.01 \\
\hline $\mathrm{Cu}$ & 0.01 & Os & 0.01 \\
\hline $\mathrm{Zn}$ & 0.01 & $\mathrm{Pt}$ & 0.01 \\
\hline $\mathrm{Ga}$ & 0.01 & $\mathrm{Au}$ & 0.01 \\
\hline As & 0.04 & $\mathrm{Hg}$ & $* *$ \\
\hline $\mathrm{Se}$ & 0.04 & $\mathrm{Tl}$ & 0.01 \\
\hline $\mathrm{Br}$ & 1 & $\mathrm{~Pb}$ & 0.02 \\
\hline $\mathrm{Rb}$ & 0.01 & $\mathrm{Bi}$ & 0.01 \\
\hline $\mathrm{Sr}$ & 0.01 & Th & 0.01 \\
\hline $\mathrm{Y}$ & 0.01 & $\mathrm{U}$ & 0.01 \\
\hline
\end{tabular}


or microgram per liter) to 100,000 to more than $1,000,000 \mathrm{ppb}$ in some unusual waters. This is a difficult challenge for any method or chemist. These samples often required dilution by amounts of 1:10 to 1:100 to keep the analyte within the calibration range.

2. Precision and accuracy are complex issues and are not the same for all elements and all concentration levels. I assume that analytical error is greater at the very high concentration levels of some of my unusual mine or experimental waters because there are no standards at this level, but documentation of that error is difficult and is incomplete at this date. Replicate samples suggest that precision at very high concentration levels is not much worse than at normal concentrations. Because reliable standards do not exist for waters with very high "trace" metal concentrations ( $>10,000 \mathrm{ppb})$, it is not possible to evaluate accuracy at high levels the way it is done at more normal levels. For reasons that I cannot explain, the precision and accuracy for "major" elements ( $\mathrm{Al}, \mathrm{Ca}, \mathrm{Mg}$, etc.) by this method are much lower than for trace metals. The user of these results should consider them semiquantitative with errors of about 50 percent in some cases (but less in others). Another method, ICP-AES, provides more reliable results for major elements (Crock and others, 1999).

3. Based on standards and replicate samples, the precision for trace metals (such as As, Cd, Co, Cr, Cu, Mn, Mo, $\mathrm{Ni}, \mathrm{Pb}, \mathrm{Sb}, \mathrm{Se}, \mathrm{V}$, and $\mathrm{Zn}$ ) appears to be about 10 percent (1 standard deviation) for normal water concentrations, and possibly 20 percent at very high concentrations. The accuracy of determinations on these trace metals seems to be about 10 percent for normal concentrations. The precision and accuracy for Fe, which generally is not a trace metal in these waters, is not as good as most others, and possibly is about 20 percent.

4. Some trace metals pose special problems. Mercury is determined by ICP-MS, but it is well known that $\mathrm{Hg}$ is not stable unless preserved by special methods; thus, for my samples the $\mathrm{Hg}$ originally present was not in the analyte (the analysis is valid, but the sample is not). Silver should be high in many of my samples, but analytical results are erratic and nearly always very low for unknown reasons (despite good results for neighboring masses); therefore, results for Ag are not considered reliable. Results for $\mathrm{Se}, \mathrm{Te}$, and $\mathrm{Tl}$ appear to be reasonable, but the standards used did not contain these elements; precision is about the same as for other trace elements.

5. These ICP-MS results are deemed satisfactory for the objectives of this study: classification or ranking of water compositions. The protocol used for 4 years in Arizona, Nevada, and Colorado AML studies (sampling, storage, analysis, and quality control) is reproducible from day to day, year to year, at a total error of about 20 percent for most trace elements. Replicate analyses show that the composition of these acidified water samples change by less than a few percent after a year of storage at room temperature (D. Fey, oral commun., 1999). Many replicate samples have an error of about 10 percent, and a few have a higher error. These error numbers are best understood by examination of figure 28. The error bars show that an analysis for a metal with a concentration of about 10,000 ppb is reliably different from one having $1,000 \mathrm{ppb}$ or $100 \mathrm{ppb}$. This is the real goal of these studies, characterization of sites with high to very high metal concentrations, not testing for $\mathrm{Zn}$ at $220 \mathrm{vs} .240 \mathrm{ppb}$ to determine compliance with regulatory standards. For sample compositions within the error bar of 20 percent, relative to a standard of concern, further sampling and analysis is recommended.

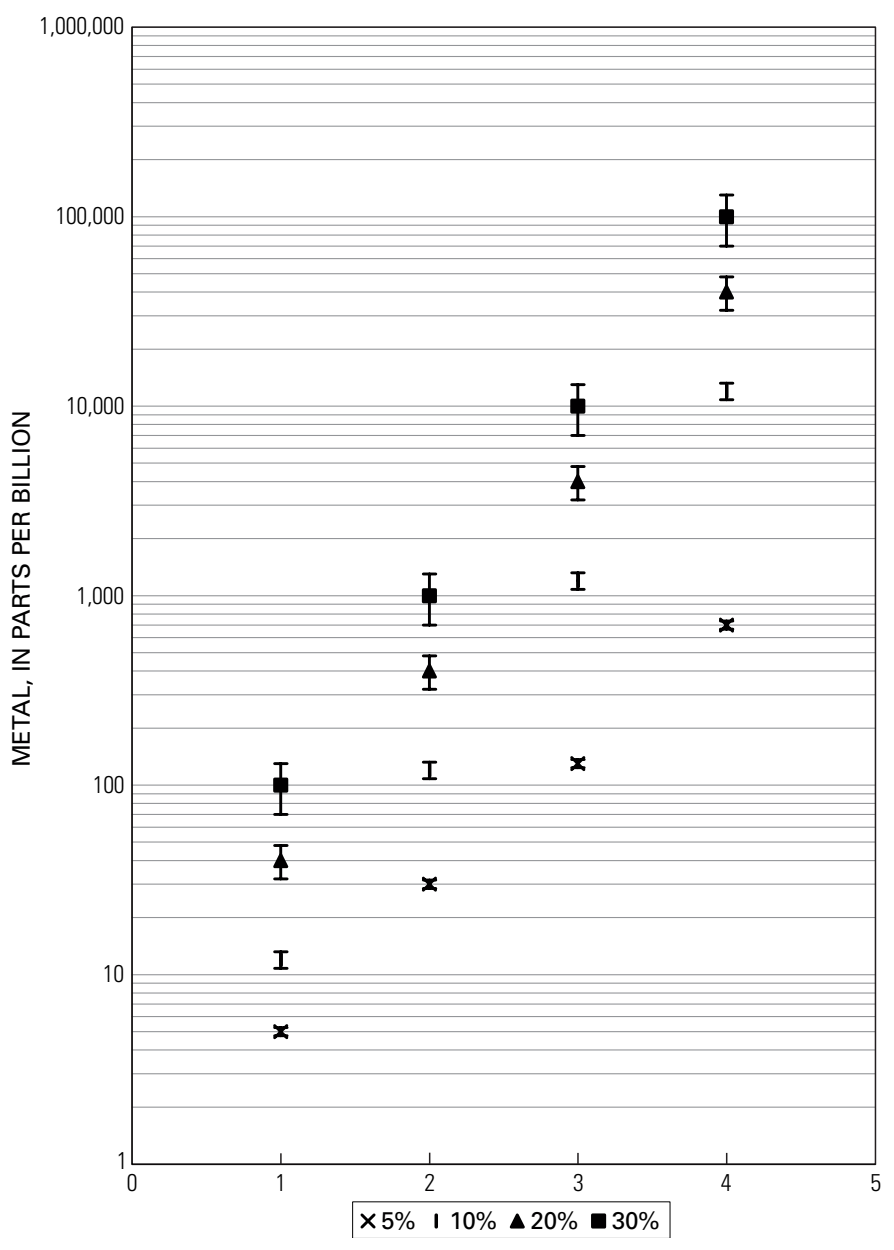

Figure 28. Diagram of analytical error for various metal concentrations. This diagram shows graphically the magnitude of 5, 10, 20, and 30 percent error bars over an wide range of hypothetical metal compositions. Many of the trace metal determinations (for example, $\mathrm{Cu}, \mathrm{Pb}, \mathrm{Zn})$ have error bars like those shown for 10 percent example, a few (Cd) may be closer to the 5 percent example, and some (e.g., $\mathrm{Fe}$ ) are like the 20 percent example. This plot shows that, even with high analytical error (20 or 30 percent), the reported values for about $10,000 \mathrm{ppb}$ do not overlap those of a sample with 1,000 or 100,000 . Thus the ICP-MS results are appropriate for ranking of water compositions spanning 4 to 6 orders of magnitude (1 to 1,000,000 ppb). 
Appendix 2: Tables 1-3 
Table 1. Description and locations of sample sites and media, CWS, Colorado.

[Sample ID: the third character specifies the medium at the site: C, crust; D, dump; G, slag; R, rock; S, soil; T, tailings; W, water; X, other. Analyis: LC, leachate chemistry by ICP-MS; TC, total chemistry (solids) by ICP-AES; WC, water chemistry by ICP-MS. District: CA, Cashin; CO, Cochetopa; CO-V, Vulcan sub-district; GB, Gold Brick; GN, Galena; IS, Iron Spring; LO, Los Ochos; MP, Mineral Point;OU, Ouray; QC, Quartz, Creek; RB, Ruby; RM, Red Mountain; TE, Telluride; TM, Tomichi; TC, Tincup; UV, Uravan. Deposit type: BP, Breccia pipe; FE, iron deposit (ferricrete or magnetite); PMV, polymetallic vein; PMVR, polymetallic vein and replacement; SU, sandstone-type uranium; TV, Tertiary volcanic-hosted vein; UV, uranium vein; VMS, volcanogenic massive sulfide. Description abbreviations: cps, counts per second radioactivity; na, not appropriate; SS, sandstone, Ls, limestone; FeOx, iron oxide minerals;ppt, precipitate]

\begin{tabular}{|c|c|c|c|c|c|c|c|c|c|c|}
\hline Site no. & Sample ID & $\mathrm{pH}$ & $\begin{array}{l}\text { Conductivity } \\
(\mu \mathrm{S} / \mathrm{cm})\end{array}$ & $\begin{array}{c}\text { Lat (N.) } \\
\text { (degrees) }\end{array}$ & $\begin{array}{l}\text { Long (W.) } \\
\text { (degrees) }\end{array}$ & Analysis & District & $\begin{array}{l}\text { Deposit } \\
\text { type }\end{array}$ & Feature & Description \\
\hline NG101 & NGD101 & na & na & 38.2211 & 108.7161 & $\mathrm{TC}, \mathrm{LC}$ & UV & SU & Uranium mine, stockpile? & Sandstone, py, black U, 2500 cps \\
\hline NG101B & NGD101B & na & na & 38.2211 & 108.7161 & $\mathrm{TC}$ & UV & SU & Uranium mine dump & Sandstone, typical dump, $1800 \mathrm{cps}$ \\
\hline NG102 & NGD102 & na & na & 38.2194 & 108.7186 & $\mathrm{TC}, \mathrm{LC}$ & UV & SU & Thunderbolt mine dump & Sandstone, typical dump, $1500 \mathrm{cps}$ \\
\hline NG103 & NGD103 & na & na & 38.3117 & 108.9489 & LC & $\mathrm{CA}$ & SC & Cashion mine main dump & Sandstone, $\mathrm{Cu}$ and $\mathrm{Fe}$ oxides \\
\hline NG104 & NGD104 & na & na & 38.3108 & 108.9494 & $\mathrm{TC}, \mathrm{LC}$ & $\mathrm{CA}$ & $\mathrm{SC}$ & Cashion, south small adit & Sandstone, $\mathrm{Cu}$ and $\mathrm{Fe}$ oxides \\
\hline NG105 & NGW105 & 8.5 & 440 & 38.3361 & 109.0246 & WC & UV & SU & Lion Creek below U mines & Stream, clear, no ppt \\
\hline NG106 & NGW106 & 8.3 & 310 & 38.3364 & 109.0151 & WC & UV & SU & Uranium mine adit water & Adit drainage, no ppt \\
\hline NG107 & NGW107 & 8.5 & 410 & 38.3369 & 107.0252 & WC & UV & SU & Uranium mine adit water & Adit drainage, no ppt \\
\hline NG108 & NGD108 & na & na & 38.0592 & 108.9256 & $\mathrm{TC}, \mathrm{LC}$ & UV & SU & Cougar mine dump & Tan SS, black and yellow U \\
\hline NG109 & NGD109 & na & na & 38.0503 & 108.9033 & $\mathrm{TC}, \mathrm{LC}$ & UV & SU & Uranium mine dump & Shale and SS, $1500 \mathrm{cps}$ \\
\hline NG110 & NGD110 & na & na & 38.0444 & 108.8906 & $\mathrm{TC}, \mathrm{LC}$ & UV & SU & Uranium mine dump & Tan sandstone, $3500 \mathrm{cps}$ \\
\hline NG111 & NGD111 & na & na & 38.0797 & 108.8222 & $\mathrm{TC}, \mathrm{LC}$ & UV & SU & West Sunday mine dump & Tan sandstone, $4000 \mathrm{cps}$ \\
\hline NG112 & NGD112 & na & na & 38.2119 & 108.7669 & TC, LC & UV & SU & Uranium mine dump, reclaimed? & Tan sandstone, $1800 \mathrm{cps}$ \\
\hline NG113 & NGD113 & na & na & 38.2411 & 108.7878 & $\mathrm{TC}, \mathrm{LC}$ & UV & SU & Uranium mine dump, reclaimed? & Tan sandstone, $2000 \mathrm{cps}$ \\
\hline NG114 & NGD114 & na & na & 38.2294 & 108.7469 & TC, LC & UV & SU & Uranium mine dump, reclaimed? & Tan sandstone, $2000 \mathrm{cps}$ \\
\hline NG115 & NGW115 & 7.4 & 1,740 & 38.3089 & 108.1263 & WC & UV & SU & Seep into mine dump & Spring, low flow \\
\hline NG116 & NGD116 & na & na & 38.3083 & 108.7383 & $\mathrm{TC}, \mathrm{LC}$ & UV & SU & Uranium mine dump & Tan sandstone, $1200 \mathrm{cps}$ \\
\hline NG117 & NGW117 & 8.1 & 840 & 38.3908 & 108.0112 & WC & UV & SU & Atkinson Creek & Stream, clear, no ppt \\
\hline NG118 & NGD118 & na & na & 38.4058 & 108.8003 & $\mathrm{TC}, \mathrm{LC}$ & UV & SU & $\begin{array}{l}\text { Golden Cycle mine dump, } \\
\text { reclaimed }\end{array}$ & Tan sandstone, black $\mathrm{U}, 1500 \mathrm{cps}$ \\
\hline NG119 & NGD119 & na & na & 38.4058 & 108.8003 & $\mathrm{TC}$ & UV & $\mathrm{SU}$ & Golden Cycle, reclaimed & Picks with visible black $\mathrm{U}$ \\
\hline NG200 & NGG200 & na & na & 38.5169 & 106.4233 & TC, LC & $\mathrm{TM}$ & PMR & Tomichi smelter & Black glassy slag \\
\hline NG201 & NGT201 & na & na & 38.5378 & 106.3956 & $\mathrm{TC}$ & $\mathrm{TM}$ & PMV & Akron mill tailings, upper & Reddish fine sand \\
\hline NG203 & NGT203 & na & na & 38.5378 & 106.3956 & $\mathrm{TC}, \mathrm{LC}$ & $\mathrm{TM}$ & PMV & Akron mill tailings, south & Ocher fine sand \\
\hline NG204 & NGT204 & na & na & 38.5375 & 106.3964 & LC & $\mathrm{TM}$ & PMR & Akron tailings, south & White crust on ocher tails \\
\hline NG205 & NGW205 & 7.6 & 410 & 38.5372 & 106.1348 & WC & $\mathrm{TM}$ & PMVR & Akron tailings pond & Small pond on south impound \\
\hline NG206 & NGD206 & na & na & 38.5428 & 106.3814 & $\mathrm{TC}, \mathrm{LC}$ & $\mathrm{TM}$ & PMVR & Erie tunnel dump & Mixed Qtzite-LS, oxide-sulfide \\
\hline NG207 & NGD207 & na & na & 38.5319 & 106.3842 & TC, LC & $\mathrm{TM}$ & PMVR & Victor mine dump & Mostly LS, low sulfide-oxide \\
\hline NG209 & NGD209 & na & na & 38.5378 & 106.3831 & $\mathrm{TC}, \mathrm{LC}$ & $\mathrm{TM}$ & PMVR & Northstar(?) mine dump & Mixed Qtzite-LS, moderate $\mathrm{FeOx}$ \\
\hline NG212 & NGT212 & na & na & 38.5378 & 106.3958 & TC, LC & $\mathrm{TM}$ & PMV & Akron mill north tailing mound & Reddish fine sand \\
\hline NG213 & NGD213 & na & na & 38.5394 & 106.3964 & $\mathrm{TC}, \mathrm{LC}$ & $\mathrm{TM}$ & PMVR & Akron mine dump at mill & Mixed rocks, mixed oxide-sulfide \\
\hline NG214 & NGW214 & 7.9 & 180 & 38.5392 & 106.0156 & WC & $\mathrm{TM}$ & PMVR & Akron adit at portal & Adit drainage, clear, no ppt. \\
\hline NG216 & NGT216 & na & na & 38.5247 & 106.4122 & $\mathrm{TC}$ & $\mathrm{TM}$ & PMV & Tailings in Tomichi Creek & Tan fine sands in willows \\
\hline NG217 & NGD217 & na & na & 38.3414 & 107.1019 & $\mathrm{TC}, \mathrm{LC}$ & $\mathrm{CO}$ & VMS & Anaconda mine dump & Ocher oxides in greenstone \\
\hline NG219 & NGT219 & na & na & 38.3194 & 107.0942 & TC & $\mathrm{CO}$ & VMS & Lot mill tailings & Gray to reddish fine sand \\
\hline NG220 & NGD220 & na & na & 38.3203 & 107.0947 & TC & $\mathrm{CO}$ & VMS & Lot mine dump & Greenstone with low sulfides \\
\hline NG221 & NGD221 & na & na & 38.3217 & 107.0872 & $\mathrm{TC}$ & $\mathrm{CO}$ & VMS & Little Johnny mine dump & $\begin{array}{l}\text { Thorium vein in greenstone, } 3000 \\
\text { cps }\end{array}$ \\
\hline
\end{tabular}




\begin{tabular}{|c|c|c|c|c|c|c|c|c|c|c|}
\hline Site no. & Sample ID & $\mathrm{pH}$ & $\begin{array}{c}\text { Conductivity } \\
(\mu \mathrm{S} / \mathrm{cm})\end{array}$ & $\begin{array}{c}\text { Lat (N.) } \\
\text { (degrees) }\end{array}$ & $\begin{array}{l}\text { Long (W.) } \\
\text { (degrees) }\end{array}$ & Analysis & District & $\begin{array}{l}\text { Deposit } \\
\text { type }\end{array}$ & Feature & Description \\
\hline NG222 & NGR222 & na & na & 38.3211 & 107.0881 & $\mathrm{TC}$ & $\mathrm{CO}$ & VMS & Alt. Rock along Th vein & Red alteration along vein, $4000 \mathrm{cps}$ \\
\hline NG223 & NGC223 & na & na & 38.3461 & 107.0839 & $\mathrm{LC}$ & $\mathrm{CO}$ & VMS & Copper King dump, crust & White crust on oxide dump \\
\hline NG223 & NGD223 & na & na & 38.3461 & 107.0839 & $\mathrm{TC}, \mathrm{LC}$ & $\mathrm{CO}$ & VMS & Copper King dump & Schist, mixed sulfides-oxides \\
\hline NG224 & NGD224 & na & na & 38.3433 & 107.0072 & TC, LC & $\mathrm{CO}-\mathrm{V}$ & VMS & Good Hope mine dump, dozed & Hi pyrite in rusty schist \\
\hline NG225 & NGC225 & na & na & 38.3433 & 107.0072 & LC & $\mathrm{CO}-\mathrm{V}$ & VMS & Good Hope, crust on dump & White crust on sulfidic dump \\
\hline NG226 & NGD226 & na & na & 38.3428 & 107.0050 & $\mathrm{TC}, \mathrm{LC}$ & $\mathrm{CO}-\mathrm{V}$ & VMS & Vulcan mine dump & Very high pyrite, high clay \\
\hline NG227 & NGC227 & na & na & 38.3400 & 107.0047 & LC & $\mathrm{CO}-\mathrm{V}$ & VMS & Vulcan dump crust & White crust on sulfidic dump \\
\hline NG228C & NGC228 & na & na & 38.3442 & 107.0042 & $\mathrm{LC}$ & $\mathrm{CO}-\mathrm{V}$ & VMS & Vulcan dump crust & White crust in dump seep \\
\hline NG229 & NGD229 & na & na & 38.3444 & 107.0036 & $\mathrm{LC}$ & $\mathrm{CO}-\mathrm{V}$ & VMS & Ditch E of Vulcan mine & Pyrite sand in drain ditch \\
\hline NG230 & NGW230 & 7.8 & 280 & 38.3469 & 107.0051 & WC & $\mathrm{CO}-\mathrm{V}$ & VMS & Camp Creek & Stream, clear, no mine input \\
\hline NG231 & NGD231 & na & na & 38.3481 & 0.0053 & $\mathrm{LC}$ & $\mathrm{CO}-\mathrm{V}$ & VMS & Transported waste, crust & White crust in kill zone \\
\hline NG231 & NGW231 & 7.7 & 310 & 38.3481 & 107.0067 & WC & $\mathrm{CO}-\mathrm{V}$ & VMS & Camp Creek & Stream, clear, below mine \\
\hline NG232 & NGW232 & 7.9 & 240 & 38.6128 & 106.0188 & WC & GB & PMV & Carter mine & Adit drainage, clear, no ppt. \\
\hline NG235 & NGD235 & na & na & 38.6308 & 106.5672 & $\mathrm{TC}, \mathrm{LC}$ & GB & PMV & Sacremento mine dump & Gneiss with some $\mathrm{FeOx}$ \\
\hline NG236 & NGD236 & na & na & 38.6317 & 106.5703 & $\mathrm{TC}$ & GB & PMV & Dump from adit, no name & Gniess with low $\% \mathrm{FeOx}$ \\
\hline NG237 & NGT237 & na & na & 38.6297 & 106.5811 & $\mathrm{TC}$ & GB & PMV & Gold Link mill tailings & Ocher to tan fine sand \\
\hline NG238 & NGT238 & na & na & 38.6164 & 106.5892 & $\mathrm{TC}, \mathrm{LC}$ & GB & PMV & Raymond mill tailings & Light colored fine sand \\
\hline NG239 & NGT239 & na & na & 38.6111 & 106.5972 & $\mathrm{TC}, \mathrm{LC}$ & GB & PMV & Carter mill tailings & Light colored, some erosion \\
\hline NG240 & NGD240 & na & na & 38.7197 & 106.4889 & $\mathrm{TC}, \mathrm{LC}$ & $\mathrm{TC}$ & PMR & Duncan hill, small adit/dump & Carbonate rocks, no sulfide \\
\hline NG241 & NGD241 & na & na & 38.7186 & 106.4789 & $\mathrm{TC}, \mathrm{LC}$ & $\mathrm{TC}$ & PMR & Gold Cup mine shafts & Carbonate rocks, vuggy $\mathrm{FeOx}$ \\
\hline NG242 & NGD242 & na & na & 38.7186 & 106.4789 & TC, LC & $\mathrm{TC}$ & PMR & Gold Cup mine shafts & Picks rich in $\mathrm{FeOx}$ \\
\hline NG243 & NGD243 & na & na & 38.7125 & 106.4794 & $\mathrm{TC}$ & $\mathrm{TC}$ & PMR & Tincup mine dump & Sulfidic, galena, low oxides \\
\hline NG244 & NGD244 & na & na & 38.7125 & 106.4794 & $\mathrm{TC}$ & $\mathrm{TC}$ & PMR & Tincup mine dump & Picks FeOx-jarosite \\
\hline NG245 & NGD245 & na & na & 38.7125 & 106.4794 & $\mathrm{TC}$ & $\mathrm{TC}$ & PMR & Jimmy Mack shaft & Carbonate rx, high $\mathrm{FeOx}$ \\
\hline NG246 & NGD246 & na & na & 38.7242 & 106.4703 & $\mathrm{TC}$ & $\mathrm{TC}$ & PMR & New Gold Cup mine adit & Picks of sparse $\mathrm{FeOx}$ \\
\hline NG247 & NGW247 & 7.4 & 240 & 38.7242 & 106.0061 & WC & $\mathrm{TC}$ & PMV & New Gold Cup mine & Adit drainage, clear, no ppt. \\
\hline NG249 & NGW249 & 7.7 & 90 & 38.3781 & 106.0111 & WC & MP & VU & Spring for stock & Spring, clear \\
\hline NG250 & NGD250 & na & na & 38.3700 & 106.7467 & TC, LC & LO & VU & $\mathrm{T} 1$ dump, large, lowest adit & Pyritic sandstone, $2500 \mathrm{cps}$ \\
\hline NG251 & NGD251 & na & na & 38.3694 & 106.7467 & $\mathrm{TC}, \mathrm{LC}$ & LO & VU & $\mathrm{T} 1$ dump, fresh piles & Pyritic sandstone, $3500 \mathrm{cps}$ \\
\hline NG252 & NGD252 & na & na & 38.3692 & 106.7456 & $\mathrm{TC}, \mathrm{LC}$ & LO & VU & T1 dump, east, trestle & Pyritic sandstone, $3000 \mathrm{cps}$ \\
\hline NG253 & NGD253 & na & na & 38.3689 & 106.7492 & TC, LC & LO & VU & Upper T1 portal & Limonitic sandstone, $2500 \mathrm{cps}$ \\
\hline NG255 & NGD255 & na & na & 38.3708 & 106.7336 & $\mathrm{TC}, \mathrm{LC}$ & LO & VU & $\mathrm{T} 2$ dump, eastern-most, adit & Pyritic sandstone, $2700 \mathrm{cps}$ \\
\hline NG256 & NGD256 & na & na & 38.3697 & 106.7397 & $\mathrm{TC}, \mathrm{LC}$ & LO & VU & $\mathrm{T} 2$ dump, adit bears east & Pyritic sandstone, $2000 \mathrm{cps}$ \\
\hline NG259 & NGW259 & 7.9 & 680 & 38.3764 & 106.0074 & WC & LO & VU & Spring for stock & Spring, clear \\
\hline NG261 & NGD261 & na & na & 38.3978 & 106.7489 & $\mathrm{TC}, \mathrm{LC}$ & $\mathrm{CO}$ & VMS & Alaska shaft dump & High Feox, low py, white crust \\
\hline NG262 & NGD262 & na & na & 38.3961 & 106.7511 & $\mathrm{TC}, \mathrm{LC}$ & $\mathrm{CO}$ & VMS & Yukon mine dump & Soft, high FeOx, white crust \\
\hline NG263 & NGW263 & 3.7 & 210 & 38.6831 & 106.1422 & WC & $\mathrm{QC}$ & PMV & Bon Ton mine, below dump & Adit drainage, makes red ppt \\
\hline NG264 & NGW264 & 5.7 & 120 & 38.6831 & 106.1377 & WC & QC & PMV & Bon Ton mine, at portal & Adit drainage, makes red ppt \\
\hline NG265 & NGD265 & na & na & 38.6831 & 106.4811 & $\mathrm{TC}, \mathrm{LC}$ & QC & PMV & Bon Ton mine dump & Gniess, ocher, sulfidic \\
\hline NG266 & NGS266 & na & na & 38.6833 & 106.4828 & SLC & QC & PMV & Soil SW of Bon Ton adit & Red soil is pre-mining, under dump \\
\hline NG269 & NGR269 & na & na & 38.6883 & 106.4869 & $\mathrm{TC}$ & QC & PMV & Cumberland pass prospects & Gniess, veined, $\mathrm{FeOx}$ joints \\
\hline NG270 & NGD270 & na & na & 38.6881 & 106.4869 & TC, LC & QC & PMV & Ida May dump & Qtz-py-ser alt gneiss, Mo? \\
\hline NG271 & NGR271 & na & na & 38.6872 & 106.4917 & $\mathrm{TC}$ & QC & PMV & West of Cumberland Pass & Qtz-Mo veinlets?, ser alt gneiss \\
\hline
\end{tabular}


Table 1. Description and locations of sample sites and media, CWS, Colorado-Continued.

\begin{tabular}{|c|c|c|c|c|c|c|c|c|c|c|}
\hline Site no. & Sample ID & $\mathrm{pH}$ & $\begin{array}{l}\text { Conductivity } \\
(\mu \mathrm{S} / \mathrm{cm})\end{array}$ & $\begin{array}{c}\text { Lat (N.) } \\
\text { (degrees) }\end{array}$ & $\begin{array}{l}\text { Long (W.) } \\
\text { (degrees) }\end{array}$ & Analysis & District & $\begin{array}{c}\text { Deposit } \\
\text { type }\end{array}$ & Feature & Description \\
\hline NG272 & NGT272 & na & na & 37.9750 & 107.7222 & $\mathrm{TC}, \mathrm{LC}$ & TC & PMV & Blistered Horn mill & Dark gray to reddish sand \\
\hline NG274 & NGD274 & na & na & 38.7044 & 106.4908 & TC, LC & $\mathrm{TC}$ & PMR & Blistered Horn upper adit dump & Mixed sulfide and oxide, LS \\
\hline NG275 & NGW275 & 7.6 & 280 & 38.7044 & 106.0099 & WC & $\mathrm{TC}$ & PMV & Blistered Horn mine & Adit drainage, clear, no ppt. \\
\hline NG277 & NGD277 & na & na & 38.6958 & 106.4817 & $\mathrm{TC}$ & $\mathrm{TC}$ & SK & Cumberland mine & Dark dump, biotite-rich, pyritic \\
\hline NG278 & NGD278 & na & na & 38.6969 & 106.4819 & $\mathrm{TC}$ & $\mathrm{TC}$ & SK & Cumberland mine & Skarn? Marble with magnetite \\
\hline NG280 & NGD280 & na & na & 38.6808 & 106.4983 & $\mathrm{TC}, \mathrm{LC}$ & $\mathrm{TC}$ & SK & Mammoth mine, upper level & Mixed sulfide and oxide, gneiss \\
\hline NG283 & NGT283 & na & na & 38.0172 & 107.3819 & TC, LC & GN & TV & Tailings impound $\mathrm{W}$ of Ute mine & Gray fine sand, large area \\
\hline NG284 & NGT284 & na & na & 38.0217 & 107.3836 & $\mathrm{TC}, \mathrm{LC}$ & GN & TV & Tailings impound $\mathrm{W}$ of Ute mine & Gray and ocher tails \\
\hline NG287 & NGW287 & 7.4 & 260 & 37.9206 & 107.0209 & WC & $\mathrm{BP}$ & TV & American Basin & Adit drainage, clear, low $\mathrm{FeOx}$ \\
\hline NG288 & NGD288 & na & na & 37.9206 & 107.5178 & TC, LC & $\mathrm{BP}$ & TV & American Basin & Py-rich in gray volcanics \\
\hline NG296 & NGW296 & 4.7 & 80 & 37.9522 & 107.0849 & WC & MP & TV & Headwaters Uncompahgre River & Stream, ponded at mine dump \\
\hline NG298 & NGW298 & 3.5 & 200 & 37.9492 & 107.3879 & WC & MP & TV & London mine adit & Adit drainage, makes $\mathrm{FeOx}$ \\
\hline NG299 & NGW299 & 3.6 & 410 & 37.9492 & 107.0395 & WC & RM & $\mathrm{TV}$ & Red Mtn Creek & Stream, turbid, red ppt \\
\hline NG302 & NGT302 & na & na & 38.0622 & 107.6622 & TC, LC & $\mathrm{OU}$ & PMR & Bachelor mill tailings & Gray and ocher course sand \\
\hline NG303 & NGD303 & na & na & 38.0622 & 107.6617 & $\mathrm{TC}, \mathrm{LC}$ & $\mathrm{OU}$ & PMVR & Bachelor(?) mine dump & LS-Shale rx, oxide-sulfide \\
\hline NG303S & NGD303S & na & na & 38.0622 & 107.6617 & $\mathrm{TC}$ & OU & PMVR & Bachelor(?) mine dump & Picks of sulfidic pieces \\
\hline NG305 & NGD305 & na & na & 37.0600 & 107.6575 & $\mathrm{TC}$ & OU & PMVR & Almadi mine dump & Darkm Mn-rich, oxide-rich \\
\hline NG307 & NGT307 & na & na & 38.0547 & 107.6886 & $\mathrm{TC}, \mathrm{LC}$ & OU & PMR & Banner mill tailings & Ocher tails in outwash near 550 \\
\hline NG308 & NGT308 & na & na & 38.0408 & 107.6825 & $\mathrm{TC}, \mathrm{LC}$ & OU & PMR & Silvershield mill tailings & Yellow-ocher sandy tails \\
\hline NG309 & NGW309 & 3.4 & $>2000$ & 38.0539 & 112.1589 & WC & OU & PMV & Seeps from tailings at US550 & Low flow, makes red ppt \\
\hline NG310 & NGW310 & 3.7 & 570 & 38.0542 & 107.0432 & WC & IS & FE & Iron Spring mine & Spring in ferricrete mine cut \\
\hline NG311 & NGR311 & na & na & 37.8583 & 107.8106 & $\mathrm{TC}, \mathrm{LC}$ & IS & $\mathrm{FE}$ & Iron Spring mine & Layered ferricrete in open cut \\
\hline NG313 & NGT313 & na & na & 37.8561 & 107.8214 & $\mathrm{TC}, \mathrm{LC}$ & IS & TV & Carbonero mill tailings & Yellow-ocher sandy tails \\
\hline NG315 & NGW315 & 3.0 & 1,660 & 37.8564 & 110.4192 & WC & IS & TV & Carbonero mill tailings area & Water flowing on tailings \\
\hline NG316 & NGT316 & na & na & 37.8575 & 107.8492 & TC, LC & IS & TV & Caribou mill tailings & Ocher to reddish fine sand \\
\hline NG317 & NGW317 & 7.3 & 980 & 37.8575 & 107.0256 & WC & IS & TV & Caribou mill tailings & Water on tailings \\
\hline NG318 & NGW318 & 7.1 & 790 & 37.8569 & 107.0335 & WC & IS & TV & Caribou mine & Adit drainage, makes red ppt \\
\hline NG320 & NGW320 & 6.6 & 290 & 37.8844 & 107.3080 & WC & IS & TV & Alta mine adit & Adit drainage, makes white ppt \\
\hline NG321 & NGD321 & na & na & 37.8850 & 107.8542 & TC, LC & IS & TV & Alta mine large upper dump & Mixed ocher oxides, green volc \\
\hline NG322 & NGD322 & na & na & 37.8778 & 107.8411 & $\mathrm{TC}, \mathrm{LC}$ & IS & TV & Silver King mine dump & Ocher oxides, some ser-py \\
\hline NG323 & NGT323 & na & na & 37.8850 & 107.8556 & $\mathrm{TC}$ & IS & TV & Alta mill tailings & Tan to med. Brown fine sand \\
\hline NG325 & NGT325 & na & na & 37.8856 & 107.8572 & $\mathrm{TC}, \mathrm{LC}$ & IS & TV & Alta mill tails, main west area & Ocher to tan fine sand \\
\hline NG326 & NGR326 & na & na & 37.9050 & 107.7056 & $\mathrm{TC}, \mathrm{LC}$ & RM & $\mathrm{BP}$ & Alteration at National Belle & Silica alteration, $\mathrm{FeOx}$ \\
\hline NG327 & NGD327 & na & na & 37.9042 & 107.7039 & $\mathrm{TC}, \mathrm{LC}$ & RM & $\mathrm{BP}$ & National Belle mine dump & Silica-sulfide breccia \\
\hline NG328 & NGW328 & 4.8 & 250 & 37.9033 & 107.0124 & WC & RM & TV & National Belle mine area & Stream, has red ppt on bed \\
\hline NG329 & NGR329 & na & na & 37.9019 & 107.7011 & $\mathrm{TC}, \mathrm{LC}$ & $\mathrm{RM}$ & BP & Talus of red alt. Volcanics & Silica-py-ser alt volcanic rx \\
\hline NG329S & NGR329S & na & na & 37.9019 & 107.7011 & TC & $\mathrm{RM}$ & BP & Talus of red alt. Volcanics & Picks of sulfidic pieces \\
\hline NG330 & NGD330 & na & na & 37.9119 & 107.7128 & TC, LC & RM & $\mathrm{BP}$ & Genessee mine dump & Gray and yellow, high py-clay \\
\hline NG331 & NGD331 & na & na & 37.9131 & 107.6958 & $\mathrm{TC}, \mathrm{LC}$ & RM & $\mathrm{BP}$ & Genessee mine dump & Average, high pyrite rock \\
\hline NG332 & NGD332 & na & na & 37.9147 & 107.6967 & $\mathrm{TC}, \mathrm{LC}$ & $\mathrm{RM}$ & $\mathrm{BP}$ & Shaft mine dump, old & Weathered yellow, hi clay \\
\hline NG333 & NGW333 & 2.9 & 1,340 & 37.9139 & 107.1132 & WC & $\mathrm{RM}$ & TV & Champion Gulch creek & Stream near mines, no ppt \\
\hline NG334 & NGR334 & na & na & 37.9167 & 107.6939 & $\mathrm{TC}$ & $\mathrm{RM}$ & $\mathrm{FE}$ & Ferricrete near Guston mine & Brown goethite cemented talus \\
\hline NG335 & NGW335 & 2.4 & $>2000$ & 37.9175 & 107.7535 & WC & RM & TV & Small flow below Guston & Flows on ferricrete, red ppt \\
\hline
\end{tabular}




\begin{tabular}{|c|c|c|c|c|c|c|c|c|c|c|}
\hline Site no. & Sample ID & $\mathrm{pH}$ & $\begin{array}{c}\text { Conductivity } \\
(\mu \mathrm{S} / \mathrm{cm})\end{array}$ & $\begin{array}{c}\text { Lat (N.) } \\
\text { (degrees) }\end{array}$ & $\begin{array}{l}\text { Long (W.) } \\
\text { (degrees) }\end{array}$ & Analysis & District & $\begin{array}{c}\text { Deposit } \\
\text { type }\end{array}$ & Feature & Description \\
\hline NG336 & NGW336 & 3.1 & $>2000$ & 37.9172 & 107.3791 & WC & $\mathrm{RM}$ & TV & New adit below Guston & Adit drainage from pipe \\
\hline NG338 & NGW338 & 2.6 & $>2000$ & 37.9211 & 107.2467 & WC & RM & TV & Newer mine dump, hi clay & Puddle on dump \\
\hline NG339 & NGD339 & na & na & 37.9164 & 107.6917 & $\mathrm{TC}, \mathrm{LC}$ & $\mathrm{RM}$ & $\mathrm{BP}$ & New mine dump (adit) & Hi py-clay and $\mathrm{FeOx}$ \\
\hline NG340 & NGW340 & 2.7 & $>2000$ & 37.9164 & 107.8033 & WC & RM & TV & Guston mine adit & Adit drainage, red ppt below \\
\hline NG341 & NGD341 & na & na & 37.9167 & 107.6942 & $\mathrm{TC}, \mathrm{LC}$ & $\mathrm{RM}$ & $\mathrm{BP}$ & Breccia pipe dump & Silica-py-clay alt breccia \\
\hline NG342 & NGW342 & 3.5 & 640 & 37.9181 & 107.0707 & WC & $\mathrm{RM}$ & TV & Red Mtn Creek & Stream, much ocher on bed \\
\hline NG343 & NGT343 & na & na & 37.9183 & 107.8306 & $\mathrm{TC}$ & $\mathrm{RM}$ & TV & Tailings east of R M Creek & Ocher sands, older tails? \\
\hline NG344 & NGW344 & 2.9 & $>2000$ & 37.9189 & 108.3199 & WC & RM & TV & Tailings seep, large impound & Tailings seep, makes red ppt \\
\hline NG345 & NGT345 & na & na & 37.9192 & 107.8317 & $\mathrm{TC}, \mathrm{LC}$ & $\mathrm{RM}$ & TV & Tailings spill at creek & Pale fine sands \\
\hline NG347 & NGW347 & 3.6 & 410 & 37.9600 & 107.0508 & WC & $\mathrm{RM}$ & TV & Red Mtn Creek, north end & Stream, turbid; orange bed \\
\hline NG348 & NGW348 & 2.9 & 1,650 & 37.9372 & 109.5354 & WC & $\mathrm{RM}$ & TV & Larson mine at portal & Adit drainage, red ppt \\
\hline NG350 & NGD350 & na & na & 37.9386 & 107.6758 & TC, LC & RM & $\mathrm{TV}$ & Larson mine dump & Yel-ocher, hi py-clay \\
\hline NG351 & NGD351 & na & na & 37.9278 & 107.6969 & $\mathrm{TC}, \mathrm{LC}$ & $\mathrm{RM}$ & TV & Mountain King mine dump & Mixed ocher and green volcanics \\
\hline NG352 & NGD352 & na & na & 37.9275 & 107.6967 & TC, LC & RM & TV & Mountain King mine dump & Pick area of green alt volcanics \\
\hline NG353 & NGW353 & 6.3 & 740 & 37.9278 & 107.0720 & WC & RM & TV & Mountain King mine adit & Hi flow, makes ocher ppt \\
\hline NG355 & NGD355 & na & na & 37.9242 & 107.6931 & $\mathrm{TC}, \mathrm{LC}$ & $\mathrm{RM}$ & $\mathrm{BP} ?$ & Joker Tunnel dump & Mixed green and red alt volcanics \\
\hline NG356 & NGR356 & na & na & 37.9242 & 107.6925 & TC, LC & RM & $\mathrm{FE}$ & Joker Tunnel dump & Bog iron deposit, mine drainage? \\
\hline NG357 & NGW357 & 2.9 & 1,410 & 37.9242 & 107.2946 & WC & $\mathrm{RM}$ & TV & Joker Tunnel drainage & Hi flow, makes red ppt \\
\hline NG358 & NGD358 & na & na & 37.9244 & 107.6853 & TC, LC & RM & $\mathrm{BP}$ & Silver Belle mine dump & Sulfidic, clay altered volcanics \\
\hline NG361 & NGD361 & na & na & 37.9264 & 107.6858 & $\mathrm{TC}, \mathrm{LC}$ & $\mathrm{RM}$ & $\mathrm{BP}$ & Silver Belle shaft dump, big & Mixed alt rocks and hi py-clay \\
\hline NG363 & NGW363 & 5.9 & 1,260 & 37.7442 & 107.0100 & WC & RM & TV & Silver Belle(?) lower adit & Adit drainage, makes red ppt \\
\hline NG363 & NGX363 & na & na & 37.9275 & 107.6881 & $\mathrm{TC}$ & $\mathrm{RM}$ & TV & Small adit drainage & $\mathrm{FeOx}$ sludge from drainage \\
\hline NG365 & NGW365 & 3.7 & 390 & 37.9250 & 107.0680 & WC & RM & TV & Red Mtn Creek & Stream, makes ocher ppt \\
\hline NG366 & NGD366 & na & na & 37.9169 & 107.8069 & $\mathrm{TC}, \mathrm{LC}$ & $\mathrm{RM}$ & TV & Midnite mine dump & Variable clay and sulfide content \\
\hline NG367 & NGW367 & 3.0 & 290 & 37.9175 & 107.0184 & WC & RM & TV & Corkscrew Gulch creek & Stream, clear, no ppt \\
\hline NG368S & NGD368S & na & na & 37.9122 & 107.6728 & $\mathrm{TC}$ & $\mathrm{RM}$ & TV & Carbonate King mine dump & Picks of sulfidic pieces \\
\hline NG369S & NGD369S & na & na & 37.9103 & 107.6717 & $\mathrm{TC}$ & RM & $\mathrm{TV}$ & W. Carbonate King shaft & Picks sulfides, galena, sphalerite \\
\hline NG370 & NGT370 & na & na & 37.9394 & 107.6708 & $\mathrm{TC}, \mathrm{LC}$ & $\mathrm{RM}$ & TV & Idarado large tailings impound & Spilled sands, light colored \\
\hline NG371 & NGW371 & 6.2 & 190 & 38.0028 & 107.0210 & WC & SN & TV & Canyon Creek & Stream, clear, no ppt \\
\hline NG372 & NGT372 & na & na & 37.9750 & 107.7222 & $\mathrm{TC}, \mathrm{LC}$ & SN & TV & Camp Bird tailings impound & Fine sand like beach sand \\
\hline NG373 & NGW373 & 6.5 & 190 & 37.9747 & 107.0198 & WC & SN & $\mathrm{TV}$ & Canyon Creek & Stream, clear, no ppt \\
\hline NG374 & NGS374 & na & na & 37.9747 & 107.7214 & $\mathrm{TC}$ & SN & TV & Canyon Creek alluvium & Stream sediment, below tails \\
\hline NG375 & NGT375 & na & na & 37.9761 & 107.7569 & $\mathrm{TC}, \mathrm{LC}$ & SN & $\mathrm{TV}$ & Atlas mill tailings & Ocher layer of tailings \\
\hline NG376 & NGT376 & na & na & 37.9758 & 107.7572 & TC, LC & SN & TV & Atlas mill tailings & Gray layer of tailings \\
\hline NG377 & NGT377 & na & na & 37.9736 & 107.7242 & $\mathrm{TC}, \mathrm{LC}$ & SN & TV & Camp Bird tailings impound & Pale tan fine sand \\
\hline NG379 & NGD379 & na & na & 37.9758 & 107.6347 & $\mathrm{TC}, \mathrm{LC}$ & MP & TV & Micky Breen mine dump & Mixed oxides and sulfidic rocks \\
\hline NG380 & NGW380 & 6.9 & 120 & 37.9747 & 107.0207 & WC & MP & TV & Upr. Uncompahgre River & Stream \\
\hline NG381 & NGD381 & na & na & 37.9528 & 107.6250 & TC, LC & MP & TV & Old Lout mine dump & Mixed oxides and sulfidic rocks \\
\hline NG382 & NGW382 & 6.5 & 480 & 37.9531 & 107.0175 & WC & MP & TV & Old Lout mine & Adit drainage, makes tan ppt \\
\hline NG385 & NGW385 & 7.2 & 360 & 37.8661 & 107.0224 & WC & IS & TV & Howard Fork & Stream, turbid, no ppt \\
\hline NG386 & NGT386 & na & na & 37.9478 & 107.8717 & $\mathrm{TC}, \mathrm{LC}$ & $\mathrm{TE}$ & TV & Society Turn mill tailings & Tan fine sands \\
\hline NG387 & NGT387 & na & na & 37.9386 & 107.7556 & TC, LC & $\mathrm{TE}$ & $\mathrm{TV}$ & Tomboy mill tailings in mill & Ocher fine sand, hi clay? \\
\hline NG388 & NGW388 & 7.3 & 150 & 37.9383 & 107.0915 & WC & $\mathrm{TE}$ & TV & Savage Creek & Stream, turbid, no ppt \\
\hline
\end{tabular}


Table 1. Description and locations of sample sites and media, CWS, Colorado-Continued.

\begin{tabular}{|c|c|c|c|c|c|c|c|c|c|c|}
\hline Site no. & Sample ID & $\mathrm{pH}$ & $\begin{array}{l}\text { Conductivity } \\
(\mu \mathrm{S} / \mathrm{cm})\end{array}$ & $\begin{array}{c}\text { Lat (N.) } \\
\text { (degrees) }\end{array}$ & $\begin{array}{l}\text { Long (W.) } \\
\text { (degrees) }\end{array}$ & Analysis & District & $\begin{array}{c}\text { Deposit } \\
\text { type }\end{array}$ & Feature & Description \\
\hline NG389 & NGD389 & na & na & 37.9356 & 107.7539 & $\mathrm{TC}, \mathrm{LC}$ & TE & TV & Argentine shaft(?) dump & Mostly green alt volcanics \\
\hline NG390 & NGD390 & na & na & 37.9417 & 107.7703 & $\mathrm{TC}, \mathrm{LC}$ & TE & TV & Cimaron mine dump & Ocher oxide part of dump \\
\hline NG391 & NGW391 & 7.2 & 110 & 37.9356 & 107.0226 & WC & TE & TV & San Miguel River & Stream, turbid, no ppt \\
\hline NG393 & NGW393 & 3.3 & 1,510 & 37.9736 & 107.5084 & WC & GN & TV & Palmetto basin, small adit & Adit drainage, makes ocher ppt \\
\hline NG394 & NGW394 & 2.9 & 1,500 & 37.9736 & 107.5237 & WC & GN & TV & Palmetto basin, small adit & Adit drainage after dump \\
\hline NG395 & NGD395 & na & na & 37.9733 & 107.5758 & $\mathrm{LC}$ & GN & TV & Palmetto basin, small adit & Yellow py-ser alt volcanics \\
\hline NG395S & NGD395S & na & na & 37.9733 & 107.5758 & $\mathrm{TC}$ & GN & TV & Palmetto basin, small adit & Picks of sulfidic pieces \\
\hline NG450 & NGW450 & 7.1 & 240 & 37.9772 & 107.1677 & WC & GN & TV & Head Henson Creek & Stream, clear, no ppt \\
\hline NG455 & NGW455 & 2.7 & nd & 37.9600 & 109.7537 & WC & MP & TV & San Juan Chief & Adit drainage, red ppt below \\
\hline NG492 & NGW492 & 5.9 & 910 & 37.9675 & 107.7211 & WC & $\mathrm{SN}$ & TV & Canyon Creek & Stream; replicate NGW681 \\
\hline NG493 & NGW493 & 5.9 & 1,540 & 37.8508 & 107.8494 & WC & IS & $\mathrm{TV}$ & Caribou mine adit & Adit drainage; replicate NGW632 \\
\hline NG494 & NGW494 & 5.9 & 1,540 & 37.9006 & 107.6675 & WC & IS & na & Drill hole flow & Replicate of NGW614 \\
\hline NG495 & NGW495 & 2.1 & $>2000$ & 37.8508 & 107.8494 & WC & $\mathrm{RM}$ & $\mathrm{TV}$ & Headwall mine adit & Adit drainage, replicate NGW587 \\
\hline NG499 & NGW499 & 3.0 & 285 & 38.8680 & 107.0739 & WC & $\mathrm{RB}$ & TV & Standard mine adit & Adit drainage, replicate NGW512 \\
\hline NG501 & NGG501 & na & na & 38.3453 & 107.0058 & $\mathrm{TC}$ & $\mathrm{CO}-\mathrm{V}$ & VMS & Vulcan smelter & Black glassy slag \\
\hline NG504 & NGC504 & na & na & 38.3442 & 107.0047 & $\mathrm{LC}$ & $\mathrm{CO}-\mathrm{V}$ & VMS & Vulcan mine dump & Popcorn-like crust on sulfides \\
\hline NG505 & NGW505 & 8.2 & 251 & 38.3346 & 107.0014 & WC & $\mathrm{CO}-\mathrm{V}$ & VMS & Cabin Creek, above Vulcan & Stream, spring source? \\
\hline NG506 & NGW506 & 8.1 & 354 & 38.3349 & 107.0053 & WC & $\mathrm{CO}-\mathrm{V}$ & VMS & Cabin Creek, below Vulcan & Stream, clear, no ppt \\
\hline NG507 & NGD507 & na & na & 38.8819 & 107.0706 & TC, LC & $\mathrm{RB}$ & PMV & Small adit, Elk Basin & Gray SS, FeOx, and sulfides \\
\hline NG507 & NGD507 & na & na & 38.8811 & 107.0728 & LC & $\mathrm{RB}$ & TV & Small adit, Elk Basin & Gray SS, $\mathrm{FeOx}$, and sulfides \\
\hline NG507 & NGW507 & 3.1 & 200 & 38.8681 & 107.0728 & WC & $\mathrm{RB}$ & PMV & Mid-Elk Basin, adit & Adit flow, makes ocher ppt \\
\hline NG509 & NGD509 & na & na & 38.8839 & 107.0678 & $\mathrm{TC}$ & $\mathrm{RB}$ & PMV & Upper Elk Basin adit & SS with mixed oxides, sulfides \\
\hline NG510 & NGW510 & 3.1 & 320 & 38.8834 & 107.0683 & WC & $\mathrm{RB}$ & PMV & Upper Elk Basin adit & Adit drainage, clear \\
\hline NG511 & NGW511 & 2.9 & 354 & 38.8682 & 107.0714 & WC & $\mathrm{RB}$ & PMV & Upper Elk Basin & Drainage over dump \\
\hline NG512 & NGW512 & 3.0 & 285 & 38.8680 & 107.0739 & WC & $\mathrm{RB}$ & PMV & Standard mine & Adit drainage, makes ocher ppt \\
\hline NG514 & NGW514 & 3.3 & 301 & 38.8681 & 107.0739 & WC & $\mathrm{RB}$ & PMV & E Elk Creek, $\mathrm{N}$ of mine & Stream, clear \\
\hline NG515 & NGW515 & 5.8 & 20 & 38.8680 & 107.0742 & WC & $\mathrm{RB}$ & PMV & W Elk Creek, NW of mine & Stream, clear \\
\hline NG518 & NGW518 & 3.0 & 169 & 38.8679 & 107.0744 & WC & $\mathrm{RB}$ & PMV & Standard mine, surface & Drainage over dump \\
\hline NG519 & NGW519 & 3.1 & 448 & 38.8678 & 107.0744 & WC & $\mathrm{RB}$ & PMV & Standard mine, tailings pond & Tailings pond water \\
\hline NG520 & NGW520 & 2.9 & 765 & 38.8678 & 107.0744 & WC & $\mathrm{RB}$ & PMV & Standard mine, pond seep & Tailings seep, makes red ppt \\
\hline NG521 & NGW521 & 5.4 & 62 & 38.8678 & 107.0753 & WC & $\mathrm{RB}$ & PMV & Elk Creek, below mine & Stream, red ppt on bed \\
\hline NG523 & NGW523 & 5.9 & 60 & 38.8672 & 107.0753 & WC & $\mathrm{RB}$ & PMV & Elk Creek, below mine & Stream, some red on bed \\
\hline NG524 & NGD524 & na & na & 38.8792 & 107.0742 & $\mathrm{TC}, \mathrm{LC}$ & $\mathrm{RB}$ & PMV & Standard mine dump & Mixed oxide, high sulfide \\
\hline NG525 & NGD525 & na & na & 38.8789 & 107.0744 & $\mathrm{TC}, \mathrm{LC}$ & $\mathrm{RB}$ & PMV & Standard mine dump & Mixed oxide, high sulfide \\
\hline NG526 & NGT526 & na & na & 38.8781 & 107.0750 & $\mathrm{TC}$ & $\mathrm{RB}$ & PMV & Standard mill tailings & Red-brown sand \\
\hline NG527 & NGT527 & na & na & 38.8781 & 107.0750 & $\mathrm{TC}$ & $\mathrm{RB}$ & PMV & Standard mill tailings & Deeper part, red-brown sand \\
\hline NG527 & NGTW527 & 2.5 & 1,295 & 38.8678 & 107.0750 & WC & $\mathrm{RB}$ & TV & Standard mill tailings & Tailings pore water \\
\hline NG528 & NGD528 & na & na & 38.8794 & 107.0756 & TC, LC & $\mathrm{RB}$ & PMV & Standard mine dump west & Mixed SS and ocher waste \\
\hline NG529 & NGW529 & 7.2 & 97 & 38.6009 & 106.5989 & WC & GB & PMV & Gold Creek below mines & Stream, clear, no ppt \\
\hline NG530 & NGT530 & na & na & 38.6083 & 106.5986 & $\mathrm{TC}, \mathrm{LC}$ & GB & PMV & Carter mill tailings & Pale tan fine sand \\
\hline NG531 & NGW531 & 6.9 & 90 & 38.6170 & 106.5853 & WC & GB & PMV & Gold Creek at Carter Lake & Stream, clear \\
\hline NG532 & NGW532 & 6.9 & 56 & 38.6501 & 106.5742 & WC & GB & PMV & Gold Creek, west of mines & Stream, clear \\
\hline NG533 & NGW533 & 6.7 & 40 & 38.6682 & 106.4792 & WC & QC & PMV & Tomichi Creek, E of Bon Ton & Stream, clear, no ppt \\
\hline
\end{tabular}




\begin{tabular}{|c|c|c|c|c|c|c|c|c|c|c|}
\hline Site no. & Sample ID & $\mathrm{pH}$ & $\begin{array}{l}\text { Conductivity } \\
(\mu \mathrm{S} / \mathrm{cm})\end{array}$ & $\begin{array}{c}\text { Lat (N.) } \\
\text { (degrees) }\end{array}$ & $\begin{array}{l}\text { Long (W.) } \\
\text { (degrees) }\end{array}$ & Analysis & District & $\begin{array}{c}\text { Deposit } \\
\text { type }\end{array}$ & Feature & Description \\
\hline NG534 & NGD534 & na & na & 38.7278 & 106.4972 & $\mathrm{TC}$ & $\mathrm{TC}$ & PMR & Ore spilled from tram & Brown, gossanous Fe-Mn-rich? \\
\hline NG534 & NGT534 & na & na & 38.7197 & 106.4972 & TC, LC & TC & PMR & Tincup mill tailings & Brown, coarse sand \\
\hline NG536 & NGT536 & na & na & 38.7028 & 106.4917 & $\mathrm{TC}, \mathrm{LC}$ & $\mathrm{TC}$ & PMR & Blistered Horn mill tailings & Brown-red sand \\
\hline NG537 & NGW537 & 6.8 & 66 & 38.0167 & 107.3239 & WC & $\mathrm{LF}$ & TV & Wade Gulch creek & Stream, clear,no ppt \\
\hline NG540 & NGW540 & 6.9 & 96 & 37.9505 & 107.3064 & WC & $\mathrm{LF}$ & TV & Red Mtn Gulch creek & Stream, clear,no ppt \\
\hline NG541 & NGW541 & 6.6 & 43 & 37.9003 & 107.4264 & WC & LF & TV & Lake Fork Gunnison & Stream, clear,no ppt \\
\hline NG543 & NGW543 & 6.7 & 46 & 37.9000 & 107.4331 & WC & $\mathrm{LF}$ & TV & Cottonwood Creek & Stream, clear,no ppt \\
\hline NG544 & NGW544 & 6.8 & 34 & 37.9001 & 107.4342 & WC & $\mathrm{LF}$ & TV & Cattaract Creek & Stream, clear,no ppt \\
\hline NG545 & NGW545 & 6.9 & 44 & 37.9336 & 107.4631 & WC & $\mathrm{BP}$ & TV & Grizzly Creek & Stream, clear,no ppt \\
\hline NG546 & NGW546 & 7.1 & 47 & 37.9340 & 107.4581 & WC & $\mathrm{BP}$ & TV & Silver Creek & Stream, clear,no ppt \\
\hline NG547 & NGW547 & 7.0 & 46 & 37.9007 & 107.3806 & WC & LF & TV & Bent Creek & Stream, clear,no ppt \\
\hline NG548 & NGW548 & 6.8 & 76 & 37.9847 & 107.2958 & WC & $\mathrm{LF}$ & TV & Lake Fork Gunnison. & Stream, clear,no ppt \\
\hline NG549 & NGW549 & 6.9 & 46 & 38.0007 & 107.4675 & WC & GN & TV & W Fork Henson Creek & Stream, clear,no ppt \\
\hline NG550 & NGW550 & 7.2 & 42 & 37.9676 & 107.5350 & WC & GN & TV & Upper Henson Creek & Stream, clear,no ppt \\
\hline NG552 & NGW552 & 7.3 & 46 & 38.0171 & 107.3319 & WC & GN & TV & Lower Henson Creek & Stream, clear,no ppt \\
\hline NG555 & NGW555 & 3.0 & 779 & 38.8678 & 107.0753 & WC & $\mathrm{RB}$ & PMV & Standard tailings pond & Tailings seep; repeat NGW520 \\
\hline NG556 & NGD556 & na & na & 38.8844 & 107.0708 & $\mathrm{TC}, \mathrm{LC}$ & $\mathrm{RB}$ & PMV & Upper Elk Basin trench & Hi py in sandstone \\
\hline NG556 & NGW556 & 6.6 & 32 & 38.8834 & 107.0708 & WC & $\mathrm{RB}$ & PMV & Upper Elk Basin trench & Drainage over dump \\
\hline NG557 & NGR557 & na & na & 38.8689 & 107.0414 & $\mathrm{TC}$ & $\mathrm{RB}$ & FE & Keystone mine area & Layered ferricrete \\
\hline NG558 & NGW558 & 7.3 & 59 & 38.8507 & 107.0600 & WC & $\mathrm{RB}$ & PMV & Lower Elk Creek & Stream, clear, no ppt \\
\hline NG559 & NGW559 & 7.3 & 37 & 38.9008 & 107.0253 & WC & $\mathrm{RB}$ & PMV & Slate River & Stream, clear, no ppt \\
\hline NG560 & NGW560 & 7.3 & 24 & 38.9008 & 107.0467 & WC & $\mathrm{RB}$ & PMV & Oh-Be-Joyful Creek & Stream, clear, no ppt \\
\hline NG561 & NGW561 & 7.0 & 62 & 38.5349 & 106.3892 & WC & $\mathrm{TM}$ & PMV & Tomichi Creek, above Akron & Stream, clear, no ppt \\
\hline NG562 & NGT562 & na & na & 38.5389 & 106.3961 & TC,LC & $\mathrm{TM}$ & PMR & Akron mill tailings north & Red-brown fine sand \\
\hline NG563 & NGW563 & 7.1 & 83 & 38.5178 & 106.4061 & WC & $\mathrm{TM}$ & PMV & Tomichi Creek, S of Akron & Stream, clear, no ppt \\
\hline NG565 & NGW565 & 7.2 & 81 & 38.5172 & 106.4147 & WC & $\mathrm{TM}$ & PMV & Tomichi Creek & Stream, clear, no ppt \\
\hline NG570 & NGW570 & 2.7 & 1,025 & 38.8678 & 107.0750 & WC & $\mathrm{RM}$ & TV & Red Mtn Creek headwaters & Stream, makes red ppt \\
\hline NG571 & NGW571 & 2.6 & 1,140 & 37.9004 & 107.7031 & WC & $\mathrm{RM}$ & TV & Small adit into ferricrete & Adit drainage \\
\hline NG574 & NGW574 & 3.3 & 436 & 37.9167 & 107.6931 & WC & $\mathrm{RM}$ & TV & Red Mtn Creek & Stream, red ppt on bed \\
\hline NG575 & NGW575 & 2.9 & 385 & 37.9167 & 107.6919 & WC & $\mathrm{RM}$ & TV & Champion Gulch & Stream, looks ok but is acid \\
\hline NG576 & NGW576 & 2.5 & $>2000$ & 37.9167 & 107.6919 & WC & $\mathrm{RM}$ & $\mathrm{TV}$ & Guston mine adit & Adit drainage, from pipe \\
\hline NG577 & NGW577 & 4.2 & 122 & 37.9169 & 107.6981 & WC & $\mathrm{RM}$ & TV & Prospect trench & Water in trench \\
\hline NG578 & NGW578 & 2.5 & $>2000$ & 37.9175 & 107.6928 & WC & $\mathrm{RM}$ & $\mathrm{TV}$ & Seep from large impound & Tailings seep, makes red ppt \\
\hline NG580 & NGW580 & 2.9 & 885 & 37.9174 & 107.6928 & WC & $\mathrm{RM}$ & TV & Joker Tunnel dump & Dump seep relates to adit \\
\hline NG581 & NGW581 & 3.6 & 319 & 37.9172 & 107.6758 & WC & $\mathrm{RM}$ & TV & Red Mtn Creek at Joker & Stream, abundant ocher ppt \\
\hline NG582 & NGW582 & 3.0 & 565 & 37.9172 & 107.6758 & WC & $\mathrm{RM}$ & TV & Corkscrew Gulch, middle & Stream, looks ok but is acid \\
\hline NG583 & NGW583 & 3.3 & 248 & 37.9008 & 107.6694 & WC & $\mathrm{RM}$ & TV & Small crk drains Red Mtn & Stream, looks ok but is acid \\
\hline NG584 & NGW584 & 2.5 & 1,035 & 37.9006 & 107.6675 & WC & $\mathrm{RM}$ & TV & Small crk drains red alt rx & Stream, looks ok but is acid \\
\hline NG585 & NGD585 & na & na & 37.9083 & 107.6689 & $\mathrm{TC}, \mathrm{LC}$ & $\mathrm{RM}$ & TV & Small adit and dump & Pyritic altered, red weathering \\
\hline NG587 & NGW587 & 2.1 & $>2000$ & 37.9006 & 107.6622 & WC & $\mathrm{RM}$ & TV & Headwall adit, Corkscrew & Adit drainage, very acidic \\
\hline NG589 & NGW589 & 3.9 & 39 & 37.9013 & 107.6742 & WC & $\mathrm{RM}$ & TV & Lake at divide, red alteration & Lake, no mines, acid runoff? \\
\hline NG590 & NGD590 & na & na & 37.9158 & 107.6700 & $\mathrm{TC}$ & $\mathrm{RM}$ & TV & Dump from adit & Hi sulfide, clay, galena, sphalerite \\
\hline NG591 & NGD591 & na & na & 37.9128 & 107.6739 & $\mathrm{TC}, \mathrm{LC}$ & $\mathrm{RM}$ & TV & Carbonate King shaft dump & Moderate sulfide, galena, sphal \\
\hline
\end{tabular}


Table 1. Description and locations of sample sites and media, CWS, Colorado-Continued.

\begin{tabular}{|c|c|c|c|c|c|c|c|c|c|c|}
\hline Site no. & Sample ID & $\mathrm{pH}$ & $\begin{array}{c}\text { Conductivity } \\
(\mu \mathrm{S} / \mathrm{cm})\end{array}$ & $\begin{array}{c}\text { Lat (N.) } \\
\text { (degrees) }\end{array}$ & $\begin{array}{l}\text { Long (W.) } \\
\text { (degrees) }\end{array}$ & Analysis & District & $\begin{array}{c}\text { Deposit } \\
\text { type }\end{array}$ & Feature & Description \\
\hline NG592 & NGW592 & 3.4 & 154 & 37.9180 & 107.6794 & WC & $\mathrm{RM}$ & TV & Small creek, south fork & Stream, clear, no ppt \\
\hline NG593 & NGW593 & 3.1 & 563 & 37.9008 & 107.6744 & WC & $\mathrm{RM}$ & TV & Small creek, few mines & Stream, looks ok but is acid \\
\hline NG595 & NGW595 & 2.9 & 492 & 37.9014 & 107.6736 & WC & $\mathrm{RM}$ & TV & Small creek, small mines & Stream, looks ok but is acid \\
\hline NG598 & NGD598 & & & 37.9122 & 107.6739 & $\mathrm{LC}$ & $\mathrm{RM}$ & $\mathrm{TV}$ & Carbonate King adit dump & Low sulfide and oxide content \\
\hline NG599 & NGW599 & 3.2 & 215 & 37.9337 & 107.6675 & WC & $\mathrm{RM}$ & TV & Small creek, west fork & Stream, looks ok but is acid \\
\hline NG605 & NGW605 & 4.9 & 278 & 37.9183 & 107.6611 & WC & $\mathrm{RM}$ & TV & Gray Copper Creek & Stream, abundant $\mathrm{FeOx}$ on bed \\
\hline NG606 & NGD606 & na & na & 37.9328 & 107.6611 & TC, LC & $\mathrm{RM}$ & TV & Mountain Silver mine dump & High pyrite, high clay \\
\hline NG606 & NGW606 & 6.0 & 375 & 38.0834 & 107.6575 & WC & $\mathrm{RM}$ & TV & Mountain Silver mine adit & Adit drainage, abundant ocher ppt \\
\hline NG608 & NGD608 & na & na & 37.9358 & 107.6519 & $\mathrm{TC}$ & $\mathrm{RM}$ & TV & Lost Day mine dump & Abundant ocher and clays \\
\hline NG609 & NGW609 & 5.9 & 74 & 37.9509 & 107.6617 & WC & $\mathrm{RM}$ & TV & Upper Mt Silver adit & Adit drainage, ocher ppt \\
\hline NG610 & NGW610 & 3.4 & 390 & 37.8511 & 107.7981 & WC & $\mathrm{RM}$ & TV & Red Mtn Creek at US550 & Stream, turbid, red ppt \\
\hline NG612 & NGW612 & 6.2 & 680 & 37.8511 & 107.7986 & WC & IS & TV & Chapman Gulch creek & Stream, turbid, tan clay ppt \\
\hline NG613 & NGW613 & 6.4 & 620 & 37.8512 & 107.8019 & WC & IS & $\mathrm{TV}$ & Small adit, small dump & Adit drainage, ocher ppt \\
\hline NG614 & NGW614 & 5.9 & 1,540 & 37.8508 & 107.8494 & WC & IS & TV & Flow from drill hole & Spring makes red floc \\
\hline NG615 & NGD615 & na & na & 37.8697 & 107.8008 & $\mathrm{TC}$ & IS & $\mathrm{TV}$ & Dump from trench and adit & Clay alt volcanics, low py \\
\hline NG616 & NGW616 & 5.1 & 95 & 37.8508 & 107.8097 & WC & IS & TV & Small creek, drains red rx & Stream, clear, no ppt \\
\hline NG617 & NGR617 & na & na & 37.8575 & 107.8097 & $\mathrm{TC}, \mathrm{LC}$ & IS & $\mathrm{FE}$ & Trench in iron deposit & Layered ferricrete, hard \\
\hline NG617 & NGW617 & 3.4 & 955 & 37.8507 & 107.8089 & WC & IS & $\mathrm{FE}$ & Iron Spring mine & Spring in ferricrete \\
\hline NG618 & NGW618 & 5.5 & 365 & 37.8508 & 107.8144 & WC & IS & TV & E Howard Fork & Stream, looks good \\
\hline NG619 & NGT619 & na & na & 37.8561 & 107.8206 & $\mathrm{TC}$ & IS & TV & Carbinero mill tailings & Yellow to ocher sand \\
\hline NG619 & NGTW619 & 3.4 & 1,830 & 37.8506 & 107.8206 & WC & IS & TV & Carbonero mill tailings & Tailings pore water \\
\hline NG620 & NGTW620 & 3.0 & 1,710 & 37.8506 & 107.8203 & WC & IS & TV & Carbonero mill tailings & Tailings pore water \\
\hline NG621 & NGW621 & 5.5 & 890 & 37.8839 & 107.9072 & WC & IS & $\mathrm{TV}$ & Side creek, drains alt rx & Stream, turbid, clay on bed \\
\hline NG625 & NGT625 & na & na & 37.8617 & 107.8733 & TC, LC & IS & TV & Silver Bell mill tailings & Ocher fine sand \\
\hline NG625 & NGTW625 & 2.1 & $>2000$ & 37.8512 & 107.8733 & WC & IS & TV & Caribou mill tailings & Puddle on tailings \\
\hline NG626 & NGT626 & na & na & 37.8614 & 107.8731 & TC, LC & IS & $\mathrm{TV}$ & Silver Bell mill tailings & Gray and yellow fine sand \\
\hline NG627 & NGW627 & 6.1 & 330 & 37.8506 & 107.8467 & WC & IS & TV & S Fork San Miguel & Stream, looks good \\
\hline NG629 & NGTW629 & 4.5 & 660 & 37.8507 & 107.8492 & WC & IS & $\mathrm{TV}$ & Caribou mill tailings & Tailings pore water \\
\hline NG631 & NGT631 & na & na & 37.8567 & 107.8483 & TC, LC & IS & $\mathrm{TV}$ & Caribou mill tailings & Rusty to yellow sand \\
\hline NG631 & NGTW631 & 5.4 & nd & 37.8507 & 107.8483 & WC & IS & $\mathrm{TV}$ & Caribou mill tailings & Tailings pore water \\
\hline NG632 & NGW632 & 5.9 & 910 & 37.8508 & 107.8494 & WC & IS & TV & Caribou mine adit & Adit drainage, tan ppt \\
\hline NG633 & NGW633 & 5.9 & 820 & 37.8508 & 107.8497 & WC & IS & $\mathrm{TV}$ & Flow on Caribou tailings & Water on tailings \\
\hline NG634 & NGW634 & 5.5 & 348 & 37.8510 & 107.8561 & WC & IS & $\mathrm{TV}$ & Howard Fork & Stream, looks good \\
\hline NG635 & NGW635 & 6.9 & 135 & 37.8343 & 107.8858 & WC & IS & $\mathrm{TV}$ & Lake Fork San Miguel & Stream, looks good \\
\hline NG636 & NGW636 & 4.7 & 450 & 37.8514 & 107.8811 & WC & IS & TV & Adit $\mathrm{W}$ of Campground & Adit drainage, ugly ocher ppt \\
\hline NG637 & NGW637 & 6.7 & 348 & 37.8514 & 107.8811 & WC & IS & TV & Howard Fork & Stream, looks good \\
\hline NG639 & NGT639 & na & na & 37.8803 & 107.8497 & $\mathrm{TC}$ & IS & TV & Gold King stamp mill & Tan coarse sandy tails \\
\hline NG641 & NGW641 & 6.2 & 118 & 37.9677 & 107.6375 & WC & MP & TV & Upper Uncompahgre River & Stream, looks good \\
\hline NG642 & NGW642 & 6.5 & 472 & 37.9515 & 107.6250 & WC & MP & TV & Micky Breen adit & Adit drainage, some clay ppt \\
\hline NG644 & NGW644 & 7.0 & 83 & 37.9514 & 107.6269 & WC & MP & TV & Mineral Creek & Stream, looks good \\
\hline NG645 & NGW645 & 6.9 & 128 & 37.9510 & 107.5864 & WC & MP & $\mathrm{TV}$ & Upper Uncompahgre River & Stream, looks good \\
\hline NG649 & NGT649 & na & na & 37.9606 & 107.5925 & $\mathrm{TC}$ & MP & TV & San Juan Chief mill & Red tailings at mill \\
\hline NG651 & NGW651 & 3.0 & 707 & 37.9176 & 107.6214 & WC & MP & $\mathrm{TV}$ & San Juan Chief adit & Adit flow, makes red ppt \\
\hline
\end{tabular}




\begin{tabular}{|c|c|c|c|c|c|c|c|c|c|c|}
\hline Site no. & Sample ID & $\mathrm{pH}$ & $\begin{array}{l}\text { Conductivity } \\
(\mu \mathrm{S} / \mathrm{cm})\end{array}$ & $\begin{array}{l}\text { Lat (N.) } \\
\text { (degrees) }\end{array}$ & $\begin{array}{l}\text { Long (W.) } \\
\text { (degrees) }\end{array}$ & Analysis & District & $\begin{array}{l}\text { Deposit } \\
\text { type }\end{array}$ & Feature & Description \\
\hline NG652 & NGW652 & 3.6 & 170 & 37.9014 & 107.6539 & WC & MP & TV & Upper Uncompahgre River & Stream, lloks good \\
\hline NG654 & NGW654 & 3.1 & 443 & 37.9144 & 107.6806 & WC & $\mathrm{RM}$ & TV & Small mine adit & Adit drainage, low flow \\
\hline NG655 & NGD655 & na & na & 37.9122 & 107.6536 & $\mathrm{TC}$ & MP & TV & Dump from adit, 1970 's? & Very high pyrite, some galena \\
\hline NG656 & NGW656 & 5.8 & 202 & 37.9672 & 107.7464 & WC & na & TV & Uncompahgre River & Stream, leaves tan ppt \\
\hline NG657 & NGW657 & 5.9 & 69 & 37.9516 & 107.7278 & WC & SN & TV & Sneffels Creek & Stream, looks ok \\
\hline NG658 & NGW658 & 6.9 & 48 & 37.9183 & 107.7486 & WC & SN & TV & Imogene Creek & Stream, looks ok \\
\hline NG659 & NGT659 & na & na & 37.9525 & 107.7264 & $\mathrm{TC}, \mathrm{LC}$ & SN & TV & Mill tailings at Richmond Jct & Gray sand tailings \\
\hline NG660 & NGT660 & na & na & 37.9494 & 107.7297 & $\mathrm{TC}, \mathrm{LC}$ & SN & TV & Hidden Treasure mill & Ocher tailings \\
\hline NG661 & NGD661 & na & na & 37.9331 & 107.7456 & $\mathrm{TC}, \mathrm{LC}$ & TE & TV & Small dump E. Savage basin & Rusty to ocher, alt volcanics \\
\hline NG661 & NGT661 & na & na & 37.9331 & 107.7456 & $\mathrm{TC}, \mathrm{LC}$ & TE & TV & Stamp mill E Savage basin & Pyritic and rusty coarse sand \\
\hline NG662 & NGW662 & 6.5 & 48 & 37.9339 & 107.7586 & WC & TE & TV & Savage Creek, E of Tomboy & Stream, looks ok \\
\hline NG663 & NGT663 & na & na & 37.9339 & 107.7525 & $\mathrm{TC}$ & $\mathrm{TE}$ & TV & Mill tailings SE Tomboy & Gray sand tailings \\
\hline NG670 & NGW670 & 6.7 & 90 & 37.9344 & 107.7672 & WC & TE & TV & Savage Creek below Tomboy & Stream, no ppt \\
\hline NG671 & NGW671 & 7.3 & 48 & 37.9173 & 107.7706 & WC & TE & TV & Marshall Creek & Stream, some tan clay on bed \\
\hline NG673 & NGW673 & 6.9 & 53 & 37.9337 & 107.8036 & WC & $\mathrm{TE}$ & $\mathrm{TV}$ & Ingrahm Creek below Meldrum & Stream, looks good \\
\hline NG677 & NGW677 & 7.2 & 73 & 37.9675 & 107.7467 & WC & TE & TV & Bear Creek, at San Miguel R & Stream, looks ok \\
\hline NG678 & NGW678 & 5.6 & 66 & 37.9676 & 107.7472 & WC & SN & $\mathrm{TV}$ & Sneffels Creek & Stream, repeat NGW657 \\
\hline NG679 & NGD679 & na & na & 37.9764 & 107.7472 & $\mathrm{TC}, \mathrm{LC}$ & SN & TV & Torpedo Eclipse dump & Picks of rusty, $\mathrm{CuOx}$ in bin \\
\hline NG679 & NGW679 & 6.0 & 94 & 37.9681 & 107.7611 & WC & SN & $\mathrm{TV}$ & Eclipse mine & Adit drainage, no ppt \\
\hline NG680 & NGW680 & 6.1 & 44 & 37.9675 & 107.7211 & WC & SN & $\mathrm{TV}$ & Yankee Boy Creek & Stream, looks good \\
\hline NG681 & NGW681 & 5.7 & 121 & 37.8846 & 107.7161 & WC & SN & $\mathrm{TV}$ & Canyon Creek & Stream, looks good \\
\hline NG682 & NGR682 & na & na & 37.9747 & 107.7225 & $\mathrm{LC}$ & SN & $\mathrm{TV}$ & Alt. Volcanic rock in roadcut & Green and purple volcanics \\
\hline NG683 & NGW683 & 7.1 & 12 & 37.9346 & 107.6644 & WC & RM & $\mathrm{TV}$ & Mineral Creek ditch & Stream, looks good \\
\hline NG684 & NGG684 & na & na & 37.9461 & 107.6636 & $\mathrm{TC}$ & RM & $\mathrm{BP}$ & Saratoga smelter & Black glassy slag \\
\hline NG685 & NGW685 & 6.6 & 150 & 38.8846 & 107.7161 & WC & $\mathrm{RM}$ & TV & Albany Creek & Stream, no ppt \\
\hline NG699 & NGT699 & na & na & 37.9339 & 107.7528 & $\mathrm{LC}$ & SN & TV & Mill tailings at Tomboy & Gray fine sand \\
\hline NG801 & NGW801 & 2.9 & 382 & 37.9206 & 107.6567 & WC & RM & TV & Vernon mine adit & Adit drainage, clear \\
\hline NG802 & NGW802 & 2.9 & 346 & 37.9192 & 107.6561 & WC & RM & TV & Gray Copper Creek below Vernon & Stream, red ppt on bed \\
\hline NG804 & NGW804 & 3.2 & 136 & 37.9169 & 107.6550 & WC & $\mathrm{RM}$ & TV & Gray Copper Creek above Vernon & Stream, some red ppt \\
\hline NG820 & NGW820 & 2.9 & 130 & 37.8956 & 107.6533 & WC & MP & $\mathrm{TV}$ & Acidic spring on strike with vein & Killzone, make red ppt \\
\hline NG823 & NGW823 & 4.6 & 15 & 37.9128 & 107.6539 & WC & MP & TV & $\begin{array}{l}\text { Small creek drains green alt } \\
\text { volcanics }\end{array}$ & Clear, looks ok \\
\hline NG827 & NGW827 & 2.8 & 189 & 37.9178 & 107.6558 & WC & MP & TV & Vernon mine adit, moderate flow & Starts clear, turns red \\
\hline NG828 & NGW828 & 3.5 & 33 & 37.9206 & 107.6556 & WC & RM & $\mathrm{TV}$ & Side creek, drains alt rx & Clear, trace red on bed \\
\hline NG829 & NGW829 & 3.0 & 121 & 37.9194 & 107.6561 & WC & RM & TV & $\begin{array}{l}\text { Gray Copper Creek below Vernon } \\
\text { mine }\end{array}$ & Clear, some yellow on bed \\
\hline NG830 & NGW830 & 3.0 & 92 & 37.9172 & 107.6550 & WC & RM & TV & $\begin{array}{l}\text { Gray Copper Creek above Vernon } \\
\text { mine }\end{array}$ & Clear, trace red on bed \\
\hline NG831 & NGW831 & 2.0 & $>2000$ & 37.9053 & 107.6675 & WC & RM & TV & $\begin{array}{l}\text { Headwall prospect E end } \\
\text { Corkscrew Gulch }\end{array}$ & Clear, no ppt from flow \\
\hline NG834 & NGW834 & 6.8 & 172 & 37.8652 & 107.8670 & WC & IS & TV & Howard Fork San Miguel River & Clear, no ppt \\
\hline NG835 & NGW835 & 7.7 & 121 & 37.8722 & 107.8933 & WC & IS & TV & South Fork San Miguel & Clear, looks ok \\
\hline NG837 & NGW837 & 3.2 & 312 & 37.8997 & 107.7019 & WC & RM & TV & Hudson mine drainage, reclaimed & Makes red ppt \\
\hline
\end{tabular}


Table 1. Description and locations of sample sites and media, CWS, Colorado-Continued.

\begin{tabular}{|c|c|c|c|c|c|c|c|c|c|c|}
\hline Site no. & Sample ID & $\mathrm{pH}$ & $\begin{array}{l}\text { Conductivity } \\
(\mu \mathrm{S} / \mathrm{cm})\end{array}$ & $\begin{array}{c}\text { Lat (N.) } \\
\text { (degrees) }\end{array}$ & $\begin{array}{l}\text { Long (W.) } \\
\text { (degrees) }\end{array}$ & Analysis & District & $\begin{array}{l}\text { Deposit } \\
\text { type }\end{array}$ & Feature & Description \\
\hline NG839 & NGW839 & 3.0 & 204 & 37.9000 & 107.7061 & WC & RM & TV & $\begin{array}{l}\text { Headwaters Red Mtn Creek, no } \\
\text { mines }\end{array}$ & Clear, some red on bed \\
\hline NG840 & NGW840 & 2.5 & $>2000$ & 37.9164 & 107.6917 & WC & RM & TV & Guston mine adit drainage & Clear, no ppt evident \\
\hline NG841 & NGW841 & 2.9 & 284 & 37.9186 & 107.6969 & WC & RM & TV & Middle reach Red Mtn Creek & Turbid; makes orange ppt \\
\hline NG842 & NGW842 & 2.9 & 762 & 37.9233 & 107.6928 & WC & RM & TV & Joker Tunnel drainage & Clear, no ppt evident \\
\hline NG844 & NGW844 & 5.5 & 415 & 37.8608 & 107.8299 & WC & IS & TV & Fe-rich water from adit & Makes thick red floc \\
\hline NG845 & NGW845 & 3.7 & 325 & 37.8600 & 107.8299 & WC & IS & TV & Fe-rich surface water, no mining & Makes thick red floc \\
\hline NG850 & NGW850 & 7.3 & 51 & 38.5169 & 106.4232 & WC & $\mathrm{TM}$ & PMVR & Tomichi Creek at smelter & Clear, looks good \\
\hline NG852 & NGW852 & 7.3 & 131 & 38.5394 & 106.3953 & WC & $\mathrm{TM}$ & PMVR & Akron mine tunnel drain & Clear, looks good \\
\hline NG853 & NGW853 & 7.8 & 50 & 38.5378 & 106.3972 & WC & GB & PMVR & Tomichi Creek below Akron mill & Clear, looks good \\
\hline NG856 & NGW856 & 8.7 & 45 & 38.6064 & 106.5994 & WC & GB & PMV & Gold Creek east of mills & Clear, looks good \\
\hline NG857 & NGW857 & 8.2 & 48 & 38.0655 & 107.6718 & WC & OU & PMVR & Dexter Creek west of mines & Clear, looks good \\
\hline NG860 & NGW860 & 6.9 & 184 & 38.0483 & 107.6862 & WC & $\mathrm{OU}$ & na & Uncompahgre River $\mathrm{N}$ of Ouray & Turbid, makes tan ppt \\
\hline NG862 & NGW862 & 7.7 & 240 & 38.1889 & 107.7472 & WC & OU & na & $\begin{array}{l}\text { Uncompahgre River } \mathrm{N} \text { of } \\
\text { Ridgeway }\end{array}$ & Clear, no ppt evident \\
\hline NG864 & NGW864 & 6.4 & 42 & 37.9769 & 107.7589 & WC & SN & TV & Sneffels Creeks above Atlas mill & Clear, looks good \\
\hline NG865 & NGW865 & 6.8 & 55 & 37.9753 & 107.7461 & WC & SN & $\mathrm{TV}$ & Sneffels Crk east of Revenue mine & Clear, looks good \\
\hline NG866 & NGW866 & 7.0 & 64 & 37.9658 & 107.7275 & WC & SN & TV & Imogene Creek SW of Camp Bird & Clear, looks good \\
\hline NG869 & NGW869 & 72.0 & 51 & 37.9483 & 107.7311 & WC & SN & TV & $\begin{array}{l}\text { Mine drainage Camp Bird No } 3 \\
\text { adit }\end{array}$ & Clear, no ppt evident \\
\hline NG870 & NGW870 & 5.8 & 55 & 37.9500 & 107.7339 & WC & SN & TV & Hidden Treasure adit drainage, red & Turbid, makes red floc \\
\hline NG871 & NGW871 & 6.6 & 124 & 37.9347 & 107.7528 & WC & $\mathrm{TE}$ & $\mathrm{TV}$ & Surface flow from Argentine dump & Clear, looks good \\
\hline NG873 & NGW873 & 7.1 & 190 & 37.9758 & 107.7195 & WC & SN & TV & Canyon Creek east of Camp Bird & Clear, looks good \\
\hline
\end{tabular}


[Analyses: Headings with \% and ppm, by ICP with "total digestion;" headings with /P (e.g., As/P) are results from partial digestion and organic extraction, with analysis by ICP. (nd, not determined). Districts: CA, Cashin; CO, Cochetopa; GB, Gold Brick; GN, Galena; IS, Iron Spring; LO, Los Ochos; MP, Mineral Point:OU, Ouray; QC, Quartz, Creek; RB, Ruby: RM, Red Mountain: TE Telluride; TM, Tomichi; TN, Tincup; UV, Uravan. Deposit type: BP, breccia pipe; FE, ferricrete; PMR, polymetallic replement; PMV, polymetallic vein; SC, sediment-hosted copper; SK, skarn; SU, sandstone uranium; TV, volcanic-hosted vein; VMS, volcanogenic massive sulfide. Sample type: the third letter in sample ID indicates sample type: D, dump; T, tailing; R, rock (unmined); X, other or unusual rock type]

\begin{tabular}{|c|c|c|c|c|c|c|c|c|c|c|c|c|c|c|c|c|c|c|c|c|c|c|c|}
\hline Sample ID & District & $\begin{array}{c}\text { Deposit } \\
\text { type }\end{array}$ & $\begin{array}{c}\text { Lat (N.) } \\
\text { (degrees) }\end{array}$ & $\begin{array}{l}\text { Long (W.) } \\
\text { (degrees) }\end{array}$ & $\begin{array}{l}\text { Al } \\
(\%)\end{array}$ & $\begin{array}{l}\mathrm{Ca} \\
(\%)\end{array}$ & $\begin{array}{l}\mathrm{Fe} \\
(\%)\end{array}$ & $\begin{array}{c}K \\
(\%)\end{array}$ & $\begin{array}{l}\mathrm{Mg} \\
(\%)\end{array}$ & $\begin{array}{c}\mathrm{Mn} \\
(\mathrm{ppm})\end{array}$ & $\underset{(\mathrm{ppm})}{\mathrm{Ag}}$ & $\begin{array}{l}\mathrm{As} / \mathrm{P} \\
(\mathrm{ppm})\end{array}$ & $\begin{array}{l}\mathrm{Au} / \mathrm{P} \\
(\mathrm{ppm})\end{array}$ & $\begin{array}{c}\mathrm{Bi} / \mathrm{P} \\
(\mathrm{ppm})\end{array}$ & $\begin{array}{l}\mathrm{Cd} / \mathrm{P} \\
(\mathrm{ppm})\end{array}$ & $\begin{array}{c}\mathrm{Cu} \\
(\mathrm{ppm})\end{array}$ & $\underset{\substack{\mathrm{Hg} \\
(\mathrm{ppm})}}{\mathrm{Hg}}$ & $\begin{array}{c}\text { Mo } \\
(\mathrm{ppm})\end{array}$ & $\begin{array}{c}\mathrm{Pb} \\
(\mathrm{ppm})\end{array}$ & $\begin{array}{l}\mathrm{Sb} / \mathrm{P} \\
(\mathrm{ppm})\end{array}$ & $\underset{\substack{\mathrm{Se} \\
(\mathrm{ppm})}}{\mathrm{se}}$ & $\underset{(\mathrm{ppm})}{U}$ & $\begin{array}{c}\mathrm{Zn} \\
\text { (ppm) }\end{array}$ \\
\hline NGD101 & UV & SU & 38.2211 & 108.7161 & 2.40 & 2.19 & 0.78 & 1.12 & 0.84 & 137 & $<.5$ & 17 & 0.00 & 0.4 & 0.8 & 11 & 0.146 & 7 & 30 & 1.29 & 49.9 & 975 & 49 \\
\hline NGD101B & UV & SU & 38.2211 & 108.7161 & 2.30 & 2.16 & 0.70 & 1.10 & 0.83 & 130 & $<.5$ & 18 & 0.00 & 0.4 & 0.8 & 12 & 0.192 & 8 & 31 & 1.2 & 49.1 & 1005 & 49 \\
\hline NGD102 & UV & SU & 38.2194 & 108.7186 & 3.00 & 2.49 & 0.90 & 1.54 & 1.18 & 220 & $<.5$ & 14 & 0.00 & 0.4 & 0.3 & 13 & 0.124 & 6 & 29 & 1.12 & 40.6 & 365 & 28 \\
\hline NGD103 & CA & $\mathrm{SC}$ & 38.3117 & 108.9489 & 2.62 & 3.07 & 3.51 & 2.00 & 1.78 & 156 & 49 & 246 & 0.00 & 0.5 & 18.1 & 33326 & 3.67 & 5 & 145 & 8.6 & 0 & $<10$ & 788 \\
\hline NGD104 & CA & $\mathrm{SC}$ & 38.3108 & 108.9494 & 2.62 & 0.07 & 0.27 & 2.07 & 0.13 & 9 & 1.6 & 160 & 0.00 & 0.4 & 1.3 & 4351 & 0.218 & $<2$ & 18 & 1.52 & 0.182 & $<10$ & 340 \\
\hline NGD108 & UV & SU & 38.0592 & 108.9256 & 4.75 & 3.86 & 1.27 & 1.96 & 1.19 & 373 & 5.6 & 20 & 0.00 & 0.6 & 10.2 & 1403 & 0.48 & 23 & 326 & 0.915 & 12.3 & 495 & 232 \\
\hline NGD109 & UV & SU & 38.0503 & 108.9033 & 3.47 & 2.75 & 1.00 & 1.33 & 1.00 & 237 & 2 & 10 & 0.00 & 0.6 & 3.3 & 409 & 0.313 & 24 & 207 & 0.672 & 22.4 & 370 & 64 \\
\hline NGD110 & UV & SU & 38.0444 & 108.8906 & 3.14 & 2.17 & 0.96 & 0.95 & 1.04 & 214 & 3.4 & 27 & 0.00 & 3.4 & 9.2 & 273 & 0.326 & 285 & 323 & 1.57 & 59.2 & 980 & 114 \\
\hline NGD111 & UV & SU & 38.0797 & 108.8222 & 3.39 & 2.16 & 1.32 & 1.20 & 0.94 & 199 & 0.8 & 18 & 0.00 & 1.1 & 2.9 & 28 & 0.358 & 58 & 53 & 1.7 & 98.6 & 1885 & 87 \\
\hline NGD112 & UV & SU & 38.2119 & 108.7669 & 2.19 & 1.70 & 0.85 & 1.18 & 0.82 & 129 & 0.8 & 24 & 0.00 & 0.8 & 0.7 & 22 & 0.11 & 38 & 57 & 1.34 & 15.1 & 445 & 48 \\
\hline NGD113 & UV & SU & 38.2411 & 108.7878 & 2.89 & 1.56 & 0.75 & 1.46 & 0.98 & 122 & 0.9 & 18 & 0.00 & 1.2 & 0.3 & 16 & 0.148 & 60 & 37 & 1.29 & 11.4 & 490 & 27 \\
\hline NGD114 & UV & SU & 38.2294 & 108.7469 & 2.00 & 2.63 & 0.60 & 1.18 & 0.79 & 142 & $<.5$ & 6 & 0.00 & 0.7 & 0.3 & 8 & 0.098 & 31 & 12 & 0.824 & 31.4 & 230 & 33 \\
\hline NGD116 & UV & SU & 38.3083 & 108.7383 & 2.31 & 1.14 & 0.67 & 1.20 & 0.65 & 113 & $<.5$ & 37 & 0.00 & 0.4 & 0.3 & 8 & 0.18 & 5 & 50 & 0.906 & 67 & 340 & 28 \\
\hline NGD118 & UV & SU & 38.4058 & 108.8003 & 3.86 & 2.52 & 1.34 & 1.92 & 1.19 & 235 & 0.5 & 42 & 0.00 & 0.5 & 1.4 & 20 & 0.113 & 6 & 238 & 1.22 & 73.4 & 455 & 177 \\
\hline NGD119 & UV & $\mathrm{SU}$ & 38.4058 & 108.8003 & 3.21 & 0.32 & 1.09 & 0.70 & 1.48 & 54 & 1.4 & 97 & 0.00 & 0.2 & 2.6 & 69 & 0.129 & 7 & 409 & 1.39 & 99 & 2680 & 391 \\
\hline NGD206 & $\mathrm{TM}$ & PMVR & 38.5428 & 106.3814 & 3.10 & 6.30 & 10.00 & 0.97 & 1.20 & 2190 & 46 & 20 & 0.30 & 31.0 & 24.3 & 750 & nd & 5 & 11200 & 5.0 & nd & nd & 4780 \\
\hline NGD207 & $\mathrm{TM}$ & PMVR & 38.5319 & 106.3842 & 5.90 & 3.10 & 1.90 & 2.90 & 1.90 & 4940 & 112 & 113 & 0.10 & 15.0 & 144.0 & 770 & nd & 12 & 25800 & 89.0 & nd & nd & 19800 \\
\hline NGD209 & $\mathrm{TM}$ & PMVR & 38.5378 & 106.3831 & 3.30 & 3.40 & 11.00 & 1.00 & 3.10 & 4280 & 91 & 219 & 0.60 & 60.0 & 135.0 & 1400 & nd & 52 & 43000 & 130 & nd & nd & 23500 \\
\hline NGD213 & $\mathrm{TM}$ & PMVR & 38.5394 & 106.3964 & 4.50 & 5.00 & 7.80 & 1.40 & 1.70 & 2490 & 54 & 7 & 0.20 & 51.0 & 76.7 & 253 & nd & 17 & 18500 & 26.0 & nd & nd & 12200 \\
\hline NGD217 & $\mathrm{CO}$ & VMS & 38.3414 & 107.1019 & 5.20 & 1.80 & 10.00 & 0.65 & 1.50 & 513 & 11 & 69 & 0.70 & 10.0 & 14.7 & 3940 & nd & 5 & 791 & $<1$ & nd & nd & 7040 \\
\hline NGD220 & $\mathrm{CO}$ & VMS & 38.3203 & 107.0947 & 5.60 & 6.00 & 6.10 & 3.00 & 3.60 & 1530 & 3 & 10 & 0.90 & 1.0 & 4.9 & 276 & nd & 5 & 577 & 3.0 & nd & nd & 365 \\
\hline NGD221 & $\mathrm{CO}$ & VMS & 38.3217 & 107.0872 & 6.80 & 6.90 & 6.60 & 1.70 & 3.80 & 1390 & $<2$ & $<1$ & $<0.1$ & $<1$ & 0.2 & 58 & nd & $<2$ & 41 & $<1$ & nd & nd & 132 \\
\hline NGD223 & $\mathrm{CO}$ & VMS & 38.3461 & 107.0839 & 6.40 & 1.10 & 12.00 & 0.36 & 6.00 & 1960 & 2 & $<1$ & 0.30 & 1.0 & 1.1 & 9700 & nd & $<2$ & 22 & $<1$ & nd & nd & 394 \\
\hline NGD224 & $\mathrm{CO}$ & VMS & 38.3433 & 107.0072 & 5.10 & 0.54 & 17.00 & 1.10 & 1.50 & 136 & 13 & 54 & 1.90 & 4.0 & 11.0 & 747 & nd & 29 & 113 & $<1$ & nd & nd & 2810 \\
\hline NGD226 & $\mathrm{CO}$ & VMS & 38.3428 & 107.0050 & 1.70 & 0.05 & 21.00 & 0.58 & 0.15 & 14 & 15 & 58 & 1.10 & 3.0 & 2.3 & 208 & nd & 46 & 135 & 1.0 & nd & nd & 578 \\
\hline NGD235 & GB & PMV & 38.6308 & 106.5672 & 6.00 & 0.43 & 5.80 & 2.60 & 0.88 & 3480 & 76 & 1000 & 2.00 & 1.0 & 44.6 & 345 & nd & 4 & 8350 & 80.0 & nd & nd & 3880 \\
\hline NGD236 & GB & PMV & 38.6317 & 106.5703 & 2.20 & 0.04 & 8.80 & 1.10 & 0.20 & 319 & 58 & 1410 & 8.20 & 1.0 & 16.9 & 952 & nd & 6 & 16500 & 66.0 & nd & nd & 1430 \\
\hline NGD240 & $\mathrm{TN}$ & PMR & 38.7197 & 106.4889 & 2.50 & 3.40 & 5.90 & 0.75 & 1.90 & 4800 & 300 & 55 & 6.50 & $<1$ & 5.2 & 709 & nd & 4 & 4210 & 286 & nd & nd & 2150 \\
\hline NGD241 & $\mathrm{TN}$ & PMR & 38.7186 & 106.4789 & 1.00 & 1.10 & 16.00 & 0.14 & 0.45 & 5860 & 69 & 60 & 0.50 & 1.0 & 35.4 & 3090 & nd & 24 & 35000 & 1360 & nd & nd & 22100 \\
\hline NGD242 & $\mathrm{TN}$ & PMR & 38.7186 & 106.4789 & 0.17 & 1.60 & 11.00 & $<0.005$ & 0.40 & 2280 & 93 & 437 & 0.30 & 2.0 & 20.5 & 1560 & nd & 50 & 26100 & 916 & nd & nd & 10200 \\
\hline NGD243 & $\mathrm{TN}$ & PMR & 38.7125 & 106.4794 & 0.03 & 15.00 & 14.00 & $<0.005$ & 6.50 & 5950 & 23 & 677 & 0.60 & 58.0 & 9.0 & 261 & nd & 21 & 9990 & 440 & nd & nd & 1960 \\
\hline NGD244 & $\mathrm{TN}$ & PMR & 38.7125 & 106.4794 & 0.23 & 0.29 & 14.00 & $<0.005$ & 0.21 & 250 & 172 & 1540 & 1.40 & 380.0 & 40.5 & 1390 & nd & 256 & 26700 & 1550 & nd & nd & 5010 \\
\hline NGD245 & $\mathrm{TN}$ & PMR & 38.7125 & 106.4794 & 0.43 & 1.70 & 10.00 & 0.13 & 0.27 & 2480 & 124 & 579 & 1.90 & 9.0 & 52.1 & 5110 & nd & 17 & 57000 & 696 & nd & nd & 15500 \\
\hline NGD246 & $\mathrm{TN}$ & PMR & 38.7242 & 106.4703 & 4.50 & 0.44 & 3.60 & 1.60 & 0.14 & 5180 & 37 & 130 & $<0.1$ & $<1$ & 61.8 & 364 & nd & 6 & 1090 & 21.0 & nd & nd & 31300 \\
\hline NGD250 & LO & VU & 38.3700 & 106.7467 & 5.85 & 0.09 & 2.15 & 1.72 & 0.20 & 41 & $<.5$ & 171 & 0.00 & 1.1 & 0.2 & 19 & 0.162 & 41 & 157 & 9.97 & 0 & 27 & 50 \\
\hline NGD251 & LO & VU & 38.3694 & 106.7467 & 4.08 & 0.15 & 2.04 & 1.14 & 0.21 & 39 & $<.5$ & 162 & 0.00 & 2.1 & 1.9 & 29 & 0.144 & 107 & 305 & 19.9 & 0.185 & 140 & 91 \\
\hline NGD252 & LO & VU & 38.3692 & 106.7456 & 7.29 & 0.03 & 2.54 & 0.89 & 0.18 & 17 & $<.5$ & 319 & 0.00 & 0.9 & 0.2 & 11 & 0.227 & 29 & 91 & 4.34 & 1.44 & 55 & 27 \\
\hline NGD253 & LO & VU & 38.3689 & 106.7492 & 6.24 & 0.15 & 2.60 & 0.72 & 0.20 & 43 & 1.3 & 610 & 0.00 & 4.1 & 6.6 & 24 & 0.403 & 251 & 1080 & 83.8 & 1.15 & 355 & 112 \\
\hline NGD255 & LO & VU & 38.3708 & 106.7336 & 1.04 & 0.02 & 1.51 & 0.27 & 0.04 & 14 & 1.3 & 428 & 0.00 & 1.2 & 0.3 & 100 & 0.316 & 34 & 161 & 22.9 & 4.82 & 60 & 81 \\
\hline NGD256 & LO & VU & 38.3697 & 106.7397 & 5.87 & 0.06 & 2.16 & 2.38 & 0.15 & 27 & $<.5$ & 44 & 0.00 & 0.9 & 0.1 & 19 & 0.174 & 14 & 56 & 3 & 0.356 & $<10$ & 29 \\
\hline NGD261 & $\mathrm{CO}$ & VMS & 38.3978 & 106.7489 & 6.70 & 0.86 & 5.80 & 3.30 & 0.75 & 864 & 11 & 1580 & 0.30 & 8.0 & 121.0 & 3390 & nd & 23 & 1570 & 20.0 & nd & nd & 19000 \\
\hline NGD262 & $\mathrm{CO}$ & VMS & 38.3961 & 106.7511 & 5.20 & 0.52 & 10.00 & 2.50 & 2.10 & 1010 & 35 & 958 & 2.00 & 65.0 & 60.0 & 10600 & nd & 32 & 7570 & 28.0 & nd & nd & 18600 \\
\hline NGD265 & $\mathrm{QC}$ & PMV & 38.6831 & 106.4811 & 6.10 & 0.46 & 3.10 & 3.30 & 0.45 & 96 & 31 & 56 & 0.90 & 26.0 & 0.9 & 159 & nd & 1190 & 321 & 7.0 & nd & nd & 155 \\
\hline NGD270 & $\mathrm{QC}$ & PMV & 38.6881 & 106.4869 & 4.90 & 0.05 & 0.92 & 3.20 & 0.26 & 250 & 73 & 194 & 0.50 & 11.0 & 43.0 & 388 & nd & 312 & 348 & 262 & nd & nd & 4110 \\
\hline NGD274 & $\mathrm{TN}$ & PMR & 38.7044 & 106.4908 & 4.80 & 1.60 & 3.40 & 2.80 & 1.30 & 2150 & 86 & 140 & 0.40 & 78.0 & 20.5 & 1310 & nd & 57 & 3020 & 58.0 & nd & nd & 3540 \\
\hline NGD277 & $\mathrm{TN}$ & SK & 38.6958 & 106.4817 & 3.40 & 0.24 & 4.50 & 2.70 & 1.20 & 394 & 16 & 205 & $<0.1$ & 6.0 & 3.4 & 202 & nd & 12 & 185 & 190 & nd & nd & 321 \\
\hline NGD278 & $\mathrm{TN}$ & SK & 38.6969 & 106.4819 & 0.61 & 0.67 & 25.00 & $<0.005$ & 7.40 & 2870 & $<2$ & 1 & 0.80 & $<1$ & 1.1 & $<2$ & nd & 2 & 56 & 1.0 & nd & nd & 228 \\
\hline
\end{tabular}


Table 2. Analytical results for samples of mineralized materials from mines, mills, and outcrops, central Western Slope, Colorado—Continued.

\begin{tabular}{|c|c|c|c|c|c|c|c|c|c|c|c|c|c|c|c|c|c|c|c|c|c|c|c|}
\hline Sample ID & District & $\begin{array}{c}\text { Deposit } \\
\text { type }\end{array}$ & $\begin{array}{c}\text { Lat (N.) } \\
\text { (degrees) }\end{array}$ & $\begin{array}{l}\text { Long (W.) } \\
\text { (degrees) }\end{array}$ & $\begin{array}{l}\text { Al } \\
(\%)\end{array}$ & $\begin{array}{l}\mathrm{Ca} \\
(\%)\end{array}$ & $\begin{array}{l}\mathrm{Fe} \\
(\%)\end{array}$ & $\begin{array}{c}K \\
(\%)\end{array}$ & $\begin{array}{l}\mathrm{Mg} \\
(\%)\end{array}$ & $\begin{array}{c}\mathrm{Mn} \\
\text { (ppm) }\end{array}$ & $\begin{array}{c}\mathrm{Ag} \\
(\mathrm{ppm})\end{array}$ & $\begin{array}{l}\mathrm{As} / \mathrm{P} \\
(\mathrm{ppm})\end{array}$ & $\begin{array}{l}\mathrm{Au} / \mathrm{P} \\
\text { (ppm) }\end{array}$ & $\begin{array}{c}\mathrm{Bi} / \mathrm{P} \\
(\mathrm{ppm})\end{array}$ & $\begin{array}{l}\mathrm{Cd} / \mathrm{P} \\
(\mathrm{ppm})\end{array}$ & $\begin{array}{c}\mathrm{Cu} \\
\text { (ppm) }\end{array}$ & $\begin{array}{c}\mathrm{Hg} \\
\text { (ppm) }\end{array}$ & $\begin{array}{c}\text { Mo } \\
\text { (ppm) }\end{array}$ & $\begin{array}{c}\mathrm{Pb} \\
(\mathrm{ppm})\end{array}$ & $\begin{array}{l}\mathrm{Sb} / \mathrm{P} \\
(\mathrm{ppm})\end{array}$ & $\begin{array}{c}\mathrm{Se} \\
\text { (ppm) }\end{array}$ & $\underset{(\mathrm{ppm})}{U}$ & $\begin{array}{c}\mathrm{Zn} \\
\text { (ppm) }\end{array}$ \\
\hline NGD280 & TN & SK & 38.6808 & 106.4983 & 6.50 & 0.12 & 1.10 & 4.20 & 0.35 & 41 & 17 & 12 & 0.20 & 11.0 & 3.4 & 243 & nd & 273 & 859 & 20.0 & nd & nd & 394 \\
\hline NGD288 & BP & $\mathrm{TV}$ & 37.9206 & 107.5178 & 5.40 & 0.29 & 4.60 & 2.80 & 0.68 & 554 & 14 & 91 & 0.20 & 14.0 & 11.7 & 494 & nd & 259 & 6050 & 14.0 & nd & nd & 1920 \\
\hline NGD303 & OU & PMVR & 38.0622 & 107.6617 & 7.70 & 0.13 & 3.60 & 3.20 & 0.35 & 3650 & 170 & 205 & $<0.1$ & 5.0 & 3.3 & 394 & nd & 10 & 2810 & 196 & nd & nd & 652 \\
\hline NGD303S & OU & PMVR & 38.0622 & 107.6617 & 2.00 & 0.07 & 1.80 & 0.80 & 0.18 & 874 & $<2$ & 43 & $<0.1$ & $<1$ & 0.3 & 11 & nd & 4 & 209 & 3.0 & nd & nd & 85 \\
\hline NGD305 & $\mathrm{OU}$ & PMVR & 37.0600 & 107.6575 & 2.40 & 0.14 & 1.70 & 0.98 & 0.06 & 1360 & 34 & 53 & $<0.1$ & $<1$ & 3.1 & 100 & nd & 10 & 899 & 11.0 & nd & nd & 605 \\
\hline NGD321 & IS & TV & 37.8850 & 107.8542 & 4.90 & 0.72 & 9.40 & 2.20 & 0.41 & 2650 & 151 & 1280 & 2.70 & 251.0 & 4.4 & 1180 & nd & 89 & 4840 & 147 & nd & nd & 1070 \\
\hline NGD322 & IS & $\mathrm{TV}$ & 37.8778 & 107.8411 & 7.10 & 0.31 & 7.20 & 3.20 & 0.40 & 501 & 150 & 406 & 1.20 & 128.0 & 11.4 & 1270 & nd & 127 & 20600 & 486 & nd & nd & 2020 \\
\hline NGD327 & RM & BP & 37.9042 & 107.7039 & 7.20 & 0.04 & 5.40 & 2.10 & 0.20 & 62 & 103 & 4760 & 1.30 & 72.0 & 72.4 & 15200 & nd & 9 & 19400 & 1020 & nd & nd & 8030 \\
\hline NGD330 & $\mathrm{RM}$ & BP & 37.9119 & 107.7128 & 2.80 & 0.03 & 16.00 & 0.22 & 0.04 & 14 & 102 & 386 & 0.40 & 140.0 & 35.8 & 5670 & nd & 3 & 9770 & 2620 & nd & nd & 13900 \\
\hline NGD331 & RM & BP & 37.9131 & 107.6958 & 8.30 & 0.15 & 2.20 & 2.20 & 0.30 & 62 & 51 & 465 & 0.50 & 52.0 & 9.0 & 830 & nd & 5 & 5190 & 125 & nd & nd & 1770 \\
\hline NGD332 & RM & BP & 37.9147 & 107.6967 & 8.70 & 0.11 & 3.90 & 2.60 & 0.46 & 148 & 63 & 1210 & 1.90 & 66.0 & 9.2 & 1870 & nd & 3 & 13500 & 97.0 & nd & nd & 2820 \\
\hline NGD339 & RM & BP & 37.9164 & 107.6917 & 7.70 & 0.04 & 8.20 & 1.90 & 0.21 & 40 & 4 & 170 & 0.30 & 14.0 & 1.3 & 183 & nd & 3 & 1950 & 100 & nd & nd & 353 \\
\hline NGD341 & RM & BP & 37.9167 & 107.6942 & 5.70 & 0.01 & 12.00 & 0.97 & 0.12 & 24 & 422 & 13 & 1.40 & 111.0 & 16.6 & 893 & nd & 10 & 38600 & 168 & nd & nd & 4380 \\
\hline NGD350 & $\mathrm{RM}$ & $\mathrm{TV}$ & 37.9386 & 107.6758 & 7.80 & 0.19 & 3.60 & 3.00 & 0.85 & 603 & 9 & 4 & $<0.1$ & 12.0 & 25.7 & 367 & nd & 32 & 8100 & 3.0 & nd & nd & 6380 \\
\hline NGD351 & RM & $\mathrm{TV}$ & 37.9278 & 107.6969 & 11.00 & 2.60 & 3.40 & 4.60 & 0.31 & 286 & 29 & 7 & 0.20 & 50.0 & 76.5 & 362 & nd & 135 & 8300 & $<1$ & nd & nd & 14500 \\
\hline NGD352 & $\mathrm{RM}$ & TV & 37.9275 & 107.6967 & 9.10 & 0.88 & 3.70 & 3.30 & 0.88 & 845 & 5 & $<1$ & $<0.1$ & 15.0 & 7.8 & 51 & nd & 18 & 1520 & 2.0 & nd & nd & 1610 \\
\hline NGD355 & RM & BP & 37.9242 & 107.6931 & 12.00 & 0.07 & 2.60 & 2.10 & 0.39 & 46 & 356 & 43 & 1.70 & 100.0 & 3.6 & 126 & nd & 6 & 11100 & 143 & nd & nd & 1100 \\
\hline NGD358 & RM & BP & 37.9244 & 107.6853 & 9.10 & 0.06 & 1.50 & 2.20 & 0.22 & 51 & 19 & 137 & 0.10 & 11.0 & 2.5 & 38 & nd & $<2$ & 1650 & 26.0 & nd & nd & 722 \\
\hline NGD361 & RM & BP & 37.9264 & 107.6858 & 6.30 & 0.03 & 3.80 & 1.50 & 0.15 & 53 & 163 & 1160 & 2.70 & 1820.0 & 3.0 & 2510 & nd & 5 & 1910 & 957 & nd & nd & 1190 \\
\hline NGD366 & RM & $\mathrm{TV}$ & 37.9169 & 107.8069 & 6.90 & 0.07 & 1.20 & 1.70 & 0.16 & 43 & 59 & 496 & 0.10 & 91.0 & 6.5 & 878 & nd & 5 & 5190 & 169 & nd & nd & 1240 \\
\hline NGD368S & RM & $\mathrm{TV}$ & 37.9122 & 107.6728 & 1.10 & $<0.005$ & 9.60 & 0.27 & 0.02 & 64 & 336 & 8 & 0.10 & 138.0 & 51.5 & 7970 & nd & 20 & 49000 & 1790 & nd & nd & 30700 \\
\hline NGD369S & RM & $\mathrm{TV}$ & 37.9103 & 107.6717 & 0.37 & $<0.005$ & 11.00 & 0.02 & 0.01 & 55 & 135 & 44 & 0.20 & 881.0 & 49.2 & 16700 & nd & 7 & 24500 & 1930 & nd & nd & 14000 \\
\hline NGD379 & RM? & $\mathrm{TV}$ & 37.9758 & 107.6347 & 6.80 & 0.17 & 7.20 & 3.10 & 0.48 & 1900 & 41 & 35 & 0.30 & 15.0 & 20.1 & 939 & nd & 35 & 13500 & 29.0 & nd & nd & 3190 \\
\hline NGD381 & MP & $\mathrm{TV}$ & 37.9528 & 107.6250 & 6.90 & 1.30 & 5.20 & 3.40 & 0.31 & 530 & 107 & 49 & 0.20 & 325.0 & 18.3 & 1290 & nd & 45 & 9160 & 37.0 & nd & nd & 2630 \\
\hline NGD389 & $\mathrm{TE}$ & $\mathrm{TV}$ & 37.9356 & 107.7539 & 6.50 & 0.30 & 6.20 & 2.80 & 0.50 & 11700 & 125 & 6 & 1.00 & 7.0 & 129.0 & 1480 & nd & 75 & 48600 & 48.0 & nd & nd & 33800 \\
\hline NGD390 & $\mathrm{TE}$ & $\mathrm{TV}$ & 37.9417 & 107.7703 & 7.40 & 0.39 & 4.80 & 3.40 & 0.57 & 2990 & 44 & 371 & 0.70 & 3.0 & 6.3 & 103 & nd & 67 & 1620 & 15.0 & nd & nd & 1110 \\
\hline NGD395S & GN-P & $\mathrm{TV}$ & 37.9733 & 107.5758 & 1.30 & 0.05 & 4.70 & 0.50 & 0.05 & 242 & 231 & 6 & 0.90 & $<1$ & 305.0 & 1600 & nd & 38 & 44900 & 51.0 & nd & nd & 66000 \\
\hline NGD507 & $\mathrm{RB}$ & PMV & 38.8819 & 107.0706 & 3.86 & 0.07 & 10.39 & 1.60 & 0.15 & 1685 & 98.7 & 505 & 0.01 & 65.9 & 141.0 & 1519 & 0 & 3 & 40351 & 32.8 & 55.3 & nd & 23413 \\
\hline NGD509 & $\mathrm{RB}$ & PMV & 38.8839 & 107.0678 & 6.50 & 0.04 & 11.48 & 2.48 & 0.25 & 1841 & 70.7 & 214 & 0.01 & 60.7 & 4.5 & 716 & 0.575 & 3 & 10384 & 15.9 & 76 & nd & 1648 \\
\hline NGD524 & $\mathrm{RB}$ & PMV & 38.8792 & 107.0742 & 4.28 & 0.15 & 11.50 & 1.84 & 0.22 & 2199 & 86.4 & 243 & 0.02 & 62.1 & 61.9 & 1337 & 0.194 & 3 & 44065 & 32.1 & 55.1 & nd & 9478 \\
\hline NGD525 & $\mathrm{RB}$ & PMV & 38.8789 & 107.0744 & 3.00 & 0.02 & 18.06 & 1.34 & 0.12 & 503 & 136.7 & 691 & 0.03 & 120.0 & 36.3 & 1502 & 0.65 & 5 & 44019 & 52.8 & 99.2 & nd & 6622 \\
\hline NGD528 & $\mathrm{RB}$ & PMV & 38.8794 & 107.0756 & 5.01 & 0.04 & 5.94 & 2.31 & 0.22 & 807 & 25.9 & 309 & 0.02 & 9.7 & 3.5 & 415 & 0.014 & 4 & 8756 & 14 & 21.4 & nd & 979 \\
\hline NGD534 & $\mathrm{TN}$ & PMR & 38.7278 & 106.4972 & 0.80 & 1.24 & 38.46 & 0.20 & 0.36 & 1204 & 167.1 & 5039 & 5.61 & 207.0 & 6.5 & 2664 & 0.112 & 14 & 2298 & 147 & 4.97 & nd & 2071 \\
\hline NGD556 & $\mathrm{RB}$ & PMV & 38.8844 & 107.0708 & 4.44 & 0.04 & 7.97 & 2.02 & 0.19 & 707 & 60.4 & 198 & 0.05 & 0.0 & 8.6 & 936 & 0.92 & 7 & 45177 & 53.5 & 21.7 & nd & 2479 \\
\hline NGD585 & $\mathrm{RM}$ & $\mathrm{TV}$ & 37.9083 & 107.6689 & 8.70 & 0.09 & 6.37 & 1.70 & 0.13 & 41 & 28.8 & 1584 & 0.04 & 26.5 & 2.2 & 4611 & 2.35 & 25 & 2954 & 253 & 5.12 & nd & 533 \\
\hline NGD590 & RM & $\mathrm{TV}$ & 37.9158 & 107.6700 & 8.41 & 0.06 & 3.82 & 3.48 & 0.70 & 711 & 59.9 & 272 & 0.09 & 33.9 & 40.1 & 449 & 1.33 & 53 & 11415 & 202 & 4.22 & nd & 8969 \\
\hline NGD591 & RM & $\mathrm{TV}$ & 37.9128 & 107.6739 & 7.44 & 0.05 & 6.25 & 2.13 & 0.27 & 109 & 247.3 & 1204 & 0.32 & 630.0 & 11.7 & 1737 & 6.54 & 9 & 6046 & 512 & 11.8 & nd & 2482 \\
\hline NGD606 & $\mathrm{RM}$ & $\mathrm{TV}$ & 37.9328 & 107.6611 & 6.86 & 0.20 & 6.01 & 3.34 & 0.27 & 462 & 19.9 & 42 & 0.04 & 19.5 & 3.8 & 244 & 0.476 & 283 & 932 & 46.9 & 4.64 & nd & 916 \\
\hline NGD608 & RM & $\mathrm{TV}$ & 37.9358 & 107.6519 & 7.22 & 0.02 & 2.71 & 3.13 & 0.30 & 382 & 24.7 & 81 & 0.05 & 0.4 & 1.1 & 43 & 0.166 & 10 & 26195 & 27.1 & 4.24 & nd & 194 \\
\hline NGD615 & IS & $\mathrm{TV}$ & 37.8697 & 107.8008 & 8.26 & 0.16 & 4.98 & 3.18 & 0.71 & 193 & 7.7 & 23 & 0.07 & 18.2 & 0.2 & 53 & 0.269 & 10 & 2605 & 12.1 & 11.9 & nd & 55 \\
\hline NGD655 & MP & TV & 37.9122 & 107.6536 & 1.66 & 0.01 & 37.92 & 0.04 & $<.01$ & 8 & 8.1 & 318 & 0.02 & 2.5 & 0.4 & 9002 & 0.163 & 7 & 112 & 29.7 & 13.8 & nd & 53 \\
\hline NGD661 & $\mathrm{TE}$ & $\mathrm{TV}$ & 37.9331 & 107.7456 & 2.35 & 4.32 & 1.58 & 1.16 & 0.13 & 5989 & 17.3 & 43 & 15.90 & 1.0 & 72.7 & 1128 & 6.5 & 33 & 5505 & 4.88 & 0.717 & nd & 12744 \\
\hline NGD679 & SN & $\mathrm{TV}$ & 37.9764 & 107.7472 & 6.82 & 1.65 & 4.53 & 2.61 & 0.53 & 1125 & 7.8 & 276 & 0.65 & 1.9 & 1.8 & 28 & 0.75 & 75 & 482 & 9.8 & 1.32 & nd & 422 \\
\hline NGG200 & $\mathrm{TM}$ & PMR & 38.5169 & 106.4233 & 1.60 & 13.00 & 12.00 & 0.36 & 1.90 & 2200 & 31 & 5 & 0.30 & $<1$ & 1.1 & 358 & nd & 13 & 7270 & 16.0 & nd & nd & 11400 \\
\hline NGG501 & $\mathrm{CO}$ & VMS & 38.3453 & 107.0058 & 3.95 & 9.94 & 20.02 & 0.82 & 0.86 & 105 & 1.8 & 4 & 0.21 & 0.0 & 0.2 & 1805 & 0 & 35 & $<5$ & 0.222 & 3.36 & nd & 10372 \\
\hline NGG684 & RM & BP & 37.9461 & 107.6636 & 2.25 & 13.61 & 22.83 & 0.46 & 0.64 & 4483 & 24 & 12 & 0.11 & 3.2 & 0.5 & 910 & 0.016 & 63 & 350 & 11.7 & 1.04 & nd & 4522 \\
\hline NGL266 & QC & PMV & 38.6833 & 106.4828 & 6.10 & 0.36 & 6.80 & 2.50 & 0.50 & 404 & 6 & 2 & 0.30 & 21.0 & 0.5 & 931 & nd & 499 & 238 & 7.0 & nd & nd & 140 \\
\hline NGR222 & $\mathrm{CO}$ & VMS & 38.3211 & 107.0881 & 5.40 & 1.20 & 6.00 & 3.00 & 0.30 & 160 & $<2$ & 292 & 0.60 & 2.0 & 0.2 & 24 & nd & 44 & 419 & 4.0 & nd & nd & 242 \\
\hline NGR269 & QC & PMV & 38.6883 & 106.4869 & 3.70 & 0.01 & 2.90 & 2.20 & 0.19 & 50 & 30 & 243 & 0.20 & 80.0 & 0.3 & 438 & nd & 2190 & 679 & 175 & nd & nd & 32 \\
\hline NGR271 & $\mathrm{QC}$ & PMV & 38.6872 & 106.4917 & 6.00 & 0.05 & 2.30 & 4.30 & 0.38 & 295 & 16 & 37 & $<0.1$ & 24.0 & 0.7 & 581 & nd & 870 & 2060 & 45.0 & nd & nd & 182 \\
\hline NGR311 & IS & $\mathrm{FE}$ & 37.8583 & 107.8106 & 1.50 & 0.01 & 24.00 & 0.11 & 0.04 & 23 & $<2$ & 5 & 1.60 & 2.0 & 1.1 & 1160 & nd & 4 & 99 & 2.0 & nd & nd & 200 \\
\hline
\end{tabular}




\begin{tabular}{|c|c|c|c|c|c|c|c|c|c|c|c|c|c|c|c|c|c|c|c|c|c|c|c|}
\hline Sample ID & District & $\begin{array}{c}\text { Deposit } \\
\text { type }\end{array}$ & $\begin{array}{l}\text { Lat (N.) } \\
\text { (degrees) }\end{array}$ & $\begin{array}{l}\text { Long (W.) } \\
\text { (degrees) }\end{array}$ & $\begin{array}{l}\mathrm{Al} \\
(\%)\end{array}$ & $\begin{array}{l}\mathrm{Ca} \\
(\%)\end{array}$ & $\begin{array}{l}\mathrm{Fe} \\
(\%)\end{array}$ & $\begin{array}{c}K \\
(\%)\end{array}$ & $\begin{array}{l}\mathrm{Mg} \\
(\%)\end{array}$ & $\begin{array}{c}\mathrm{Mn} \\
(\mathrm{ppm})\end{array}$ & $\begin{array}{c}\mathrm{Ag} \\
(\mathrm{ppm})\end{array}$ & $\begin{array}{l}\mathrm{As} / \mathrm{P} \\
(\mathrm{ppm})\end{array}$ & $\begin{array}{l}\mathrm{Au} / \mathrm{P} \\
(\mathrm{ppm})\end{array}$ & $\begin{array}{c}\mathrm{Bi} / \mathrm{P} \\
(\mathrm{ppm})\end{array}$ & $\begin{array}{l}\mathrm{Cd} / \mathrm{P} \\
(\mathrm{ppm})\end{array}$ & $\begin{array}{c}\mathrm{Cu} \\
(\mathrm{ppm})\end{array}$ & $\begin{array}{c}\mathrm{Hg} \\
(\mathrm{ppm})\end{array}$ & $\begin{array}{c}\text { Mo } \\
\text { (ppm) }\end{array}$ & $\begin{array}{c}\mathrm{Pb} \\
(\mathrm{ppm})\end{array}$ & $\begin{array}{l}\mathrm{Sb} / \mathrm{P} \\
(\mathrm{ppm})\end{array}$ & $\begin{array}{c}\mathrm{Se} \\
(\mathrm{ppm})\end{array}$ & $\underset{(\mathrm{ppm})}{U}$ & $\begin{array}{c}\mathrm{Zn} \\
\text { (ppm) }\end{array}$ \\
\hline NGR326 & RM & BP & 37.9050 & 107.7056 & 4.50 & 0.08 & 5.80 & 0.45 & 0.13 & 83 & 52 & 2880 & 4.00 & 118.0 & 1.8 & 152 & nd & 8 & 2330 & 482 & nd & nd & 602 \\
\hline NGR329 & RM & BP & 37.9019 & 107.7011 & 6.50 & 0.11 & 4.70 & 0.82 & 0.23 & 80 & 24 & 12 & 1.00 & 34.0 & 0.6 & 512 & nd & 6 & 1190 & 85.0 & nd & nd & 237 \\
\hline NGR329S & RM & BP & 37.9019 & 107.7011 & 3.20 & 0.04 & 4.00 & 0.04 & 0.01 & 13 & 5 & 59 & 0.60 & 7.0 & 0.2 & 320 & nd & $<2$ & 630 & 12.0 & nd & nd & 46 \\
\hline NGR334 & $\mathrm{RM}$ & $\mathrm{FE}$ & 37.9167 & 107.6939 & 0.98 & 0.03 & 24.00 & 0.26 & 0.05 & 18 & $<2$ & 96 & 1.80 & 4.0 & 0.5 & 370 & nd & $<2$ & 313 & 5.0 & nd & nd & 56 \\
\hline NGR356 & $\mathrm{RM}$ & FE & 37.9242 & 107.6925 & 0.16 & 0.01 & 24.00 & 0.04 & 0.02 & 13 & $<2$ & 192 & 1.00 & 2.0 & 0.3 & 21 & nd & $<2$ & 135 & 8.0 & nd & nd & 31 \\
\hline NGR557 & $\mathrm{RB}$ & FE & 38.8689 & 107.0414 & 0.73 & 0.01 & 56.01 & 0.18 & 0.04 & 141 & $<.5$ & 9 & 0.01 & 0.4 & 0.8 & 24 & 0.23 & $<2$ & 56 & 2.92 & 1 & nd & 587 \\
\hline NGR617 & IS & FE & 37.8575 & 107.8097 & 1.39 & 0.01 & 55.23 & 0.11 & 0.03 & 17 & $<.5$ & 4 & 0.00 & 0.3 & 0.5 & 1145 & 0.104 & 10 & 74 & 2.33 & 0.644 & nd & 180 \\
\hline NGS374 & SN & $\mathrm{TV}$ & 37.9747 & 107.7214 & 6.40 & 1.80 & 5.00 & 2.20 & 0.91 & 4370 & 19 & 47 & 0.60 & 13.0 & 13.1 & 464 & nd & 20 & 1370 & 11.0 & nd & nd & 2590 \\
\hline NGT201 & TM & PMV & 38.5378 & 106.3956 & 2.40 & 9.70 & 7.30 & 0.81 & 4.20 & 15800 & 24 & 13 & 0.20 & 20.0 & 85.0 & 324 & nd & 4 & 6790 & 18.0 & nd & nd & 11800 \\
\hline NGT203 & TM & PMV & 38.5378 & 106.3956 & 1.90 & 7.70 & 13.00 & 0.65 & 1.00 & 3130 & 27 & 79 & 0.50 & 30.0 & 25.6 & 221 & nd & $<2$ & 4970 & 20.0 & nd & nd & 4730 \\
\hline NGT212 & TM & PMV & 38.5378 & 106.3958 & 2.20 & 6.90 & 10.00 & 0.69 & 2.40 & 8410 & 25 & 38 & 0.10 & 25.0 & 49.4 & 303 & nd & 5 & 7460 & 13.0 & nd & nd & 7410 \\
\hline NGT216 & $\mathrm{TM}$ & PMV & 38.5247 & 106.4122 & 5.40 & 2.40 & 4.60 & 1.70 & 2.10 & 4130 & 175 & 147 & 0.30 & 88.0 & 36.9 & 3210 & nd & 42 & 28800 & 233 & nd & nd & 8790 \\
\hline NGT219 & $\mathrm{CO}$ & VMS & 38.3194 & 107.0942 & 6.00 & 6.00 & 4.80 & 1.70 & 2.30 & 1240 & $<2$ & 13 & 0.50 & $<1$ & 1.2 & 123 & nd & $<2$ & 259 & 6.0 & nd & nd & 173 \\
\hline NGT237 & GB & PMV & 38.6297 & 106.5811 & 4.00 & 0.14 & 2.20 & 2.20 & 0.37 & 257 & 15 & 262 & 2.20 & $<1$ & 5.6 & 187 & nd & 5 & 2620 & 12.0 & nd & nd & 886 \\
\hline NGT238 & GB & PMV & 38.6164 & 106.5892 & 5.40 & 0.16 & 2.00 & 2.60 & 0.44 & 205 & 11 & 992 & 2.00 & $<1$ & 6.9 & 124 & nd & 5 & 2260 & 9.0 & nd & nd & 772 \\
\hline NGT239 & GB & PMV & 38.6111 & 106.5972 & 5.80 & 0.33 & 2.80 & 2.40 & 0.69 & 409 & 15 & 197 & 1.10 & 1.0 & 3.3 & 98 & nd & 11 & 1670 & 16.0 & nd & nd & 470 \\
\hline NGT272 & $\mathrm{TC}$ & PMV & 37.9750 & 107.7222 & 1.20 & 2.00 & 6.90 & 0.29 & 1.60 & 5140 & 268 & 389 & 0.70 & 6.0 & 37.2 & 4570 & nd & 6 & 19800 & 1680 & nd & nd & 21600 \\
\hline NGT283 & GN & $\mathrm{TV}$ & 38.0172 & 107.3819 & 3.10 & 0.10 & 1.50 & 1.50 & 0.17 & 951 & 9 & 56 & $<0.1$ & 2.0 & 2.0 & 76 & nd & 56 & 1360 & 11.0 & nd & nd & 532 \\
\hline NGT284 & GN & $\mathrm{TN}$ & 38.0217 & 107.3836 & 4.20 & 0.29 & 2.00 & 2.00 & 0.23 & 575 & 13 & 67 & 0.30 & 4.0 & 1.3 & 152 & nd & 78 & 2750 & 20.0 & nd & nd & 363 \\
\hline NGT302 & OU & PMR & 38.0622 & 107.6622 & 4.20 & 0.62 & 3.50 & 1.40 & 0.28 & 2150 & 250 & 9 & 0.40 & 13.0 & 199.0 & 1690 & nd & 24 & 45300 & 454 & nd & nd & 46100 \\
\hline NGT307 & OU & PMR & 38.0547 & 107.6886 & 2.20 & 0.07 & 1.40 & 1.10 & 0.14 & 567 & 15 & 53 & 0.80 & 4.0 & 5.1 & 118 & nd & 30 & 1850 & 16.0 & nd & nd & 864 \\
\hline NGT308 & $\mathrm{OU}$ & PMR & 38.0408 & 107.6825 & 2.40 & 0.90 & 4.50 & 1.00 & 0.26 & 1860 & 43 & 133 & 0.90 & 31.0 & 6.3 & 833 & nd & 43 & 2660 & 346 & nd & nd & 1170 \\
\hline NGT313 & IS & $\mathrm{TV}$ & 37.8561 & 107.8214 & 6.00 & 0.38 & 4.80 & 2.50 & 0.26 & 154 & 73 & 35 & 0.50 & 16.0 & 47.4 & 447 & nd & 10 & 13900 & 58.0 & nd & nd & 9030 \\
\hline NGT316 & IS & TV & 37.8575 & 107.8492 & 4.00 & 0.07 & 3.20 & 1.80 & 0.09 & 87 & 186 & 1560 & 3.30 & 66.0 & 2.3 & 785 & nd & 9 & 5540 & 1060 & nd & nd & 458 \\
\hline NGT323 & IS-a & TV & 37.8850 & 107.8556 & 5.90 & 2.50 & 4.60 & 2.40 & 1.00 & 7290 & 16 & 129 & 0.70 & 4.0 & 4.5 & 1120 & nd & 75 & 1350 & 37.0 & nd & nd & 620 \\
\hline NGT325 & IS-a & TV & 37.8856 & 107.8572 & 4.20 & 0.41 & 4.20 & 2.00 & 0.30 & 2660 & 102 & 265 & 1.20 & 71.0 & 3.9 & 465 & nd & 84 & 4390 & 203 & nd & nd & 845 \\
\hline NGT343 & $\mathrm{RM}$ & BP & 37.9183 & 107.8306 & 3.30 & 0.54 & 3.30 & 1.70 & 0.32 & 2080 & 10 & 151 & 1.00 & 11.0 & 5.4 & 253 & nd & 76 & 1350 & 6.0 & nd & nd & 1330 \\
\hline NGT345 & RM & BP & 37.9192 & 107.8317 & 3.40 & 0.23 & 2.40 & 1.70 & 0.30 & 750 & 9 & 137 & 0.50 & 7.0 & 0.3 & 93 & nd & 56 & 987 & 4.0 & nd & nd & 129 \\
\hline NGT370 & $\mathrm{RM}$ & TV & 37.9394 & 107.6708 & 4.50 & 0.36 & 3.40 & 2.10 & 0.42 & 1180 & 5 & 60 & 1.40 & 4.0 & 0.3 & 70 & nd & 26 & 567 & 3.0 & nd & nd & 139 \\
\hline NGT372 & SN & TV & 37.9750 & 107.7222 & 3.10 & 4.20 & 4.00 & 1.50 & 0.33 & 4540 & 13 & 16 & 0.30 & 23.0 & 10.4 & 330 & nd & 38 & 986 & 19.0 & nd & nd & 1970 \\
\hline NGT375 & SN & TV & 37.9761 & 107.7569 & 5.70 & 0.27 & 2.20 & 2.60 & 0.32 & 531 & 75 & 223 & 0.20 & 5.0 & 1.3 & 95 & nd & 82 & 1700 & 72.0 & nd & nd & 293 \\
\hline NGT376 & $\mathrm{SN}$ & TV & 37.9758 & 107.7572 & 3.30 & 1.80 & 2.00 & 1.50 & 0.21 & 3420 & 124 & 20 & 0.30 & 5.0 & 48.2 & 418 & nd & 127 & 3890 & 82.0 & nd & nd & 7920 \\
\hline NGT377 & SN & TV & 37.9736 & 107.7242 & 3.70 & 3.30 & 3.50 & 1.50 & 0.44 & 9050 & 5 & 14 & $<0.1$ & 5.0 & 14.5 & 418 & nd & 49 & 1290 & 2.0 & nd & nd & 2610 \\
\hline NGT386 & $\mathrm{TE}$ & TV & 37.9478 & 107.8717 & 3.40 & 1.50 & 2.40 & 1.60 & 0.33 & 9680 & 67 & 197 & 0.80 & 9.0 & 65.8 & 2380 & nd & 94 & 4270 & 18.0 & nd & nd & 12200 \\
\hline NGT387 & $\mathrm{TE}$ & $\mathrm{TV}$ & 37.9386 & 107.7556 & 7.60 & 1.50 & 3.70 & 3.60 & 0.52 & 4260 & 122 & 217 & 3.70 & 12.0 & 24.4 & 514 & nd & 266 & 6950 & 41.0 & nd & nd & 5220 \\
\hline NGT526 & $\mathrm{RB}$ & PMV & 38.8781 & 107.0750 & 2.19 & 0.02 & 6.21 & 0.92 & 0.08 & 767 & 18.2 & 336 & 0.03 & 1.7 & 0.2 & 80 & 0.413 & 2 & 565 & 20.1 & 13 & nd & 925 \\
\hline NGT527 & $\mathrm{RB}$ & PMV & 38.8781 & 107.0750 & 2.02 & 0.01 & 9.84 & 0.85 & 0.06 & 242 & 20.2 & 223 & 0.01 & 1.9 & 3.1 & 620 & 0.064 & 5 & 603 & 8.58 & 7.09 & nd & 483 \\
\hline NGT530 & GB & PMV & 38.6083 & 106.5986 & 5.37 & 0.42 & 3.49 & 2.40 & 0.83 & 690 & 25.3 & 327 & 0.90 & 1.3 & 4.9 & 101 & 0.669 & 12 & 1653 & 16.9 & 0.802 & nd & 620 \\
\hline NGT534 & $\mathrm{TN}$ & PMR & 38.7197 & 106.4972 & 1.32 & 1.56 & 19.09 & 0.42 & 0.63 & 4656 & 115.8 & 1326 & 1.22 & 35.1 & 10.0 & 1075 & 0.111 & 12 & 4116 & 77.9 & 1 & nd & 2131 \\
\hline NGT536 & $\mathrm{TN}$ & PMR & 38.7028 & 106.4917 & 1.25 & 2.37 & 7.58 & 0.34 & 1.68 & 4862 & 408.5 & 308 & 0.25 & 5.6 & 25.5 & 4015 & 122 & 6 & 31333 & 1419 & 1.08 & nd & 25090 \\
\hline NGT562 & TM & PMR & 38.5389 & 106.3961 & 2.08 & 7.80 & 8.82 & 0.66 & 2.45 & 9891 & 24.8 & 66 & 0.14 & 26.5 & 52.3 & 298 & 0.158 & 6 & 6770 & 19.4 & 2.37 & nd & 8628 \\
\hline NGT619 & IS & $\mathrm{TV}$ & 37.8561 & 107.8206 & 5.28 & 1.51 & 6.52 & 2.27 & 0.35 & 277 & 55 & 172 & 0.20 & 4.4 & 49.7 & 707 & 0.781 & 4 & 6896 & 37 & 1.66 & nd & 10682 \\
\hline NGT625 & IS & $\mathrm{TV}$ & 37.8617 & 107.8733 & 4.72 & 0.31 & 8.87 & 1.59 & 0.28 & 556 & 41.6 & 158 & 0.63 & 15.2 & 1.2 & 334 & 0.5 & 14 & 1247 & 95.7 & 3.6 & nd & 617 \\
\hline NGT626 & IS & $\mathrm{TV}$ & 37.8614 & 107.8731 & 2.46 & 1.01 & 9.96 & 1.02 & 0.17 & 107 & 83.5 & 10 & 0.45 & 109.0 & 1.9 & 668 & 1.13 & 23 & 1475 & 4.44 & 1.28 & nd & 278 \\
\hline NGT631 & IS-a & $\mathrm{TV}$ & 37.8567 & 107.8483 & 3.43 & 0.09 & 2.49 & 1.49 & 0.09 & 97 & 131.7 & 669 & 1.40 & 38.3 & 3.5 & 301 & 0.706 & 14 & 3651 & 562 & 1.68 & nd & 796 \\
\hline NGT639 & IS-a & $\mathrm{TV}$ & 37.8803 & 107.8497 & 5.31 & 0.10 & 4.76 & 2.34 & 0.37 & 245 & 6 & 308 & 1.10 & 2.3 & 0.6 & 62 & 3.63 & 24 & 346 & 12 & 0.518 & nd & 245 \\
\hline NGT649 & MP & TV & 37.9606 & 107.5925 & 2.72 & 0.07 & 3.94 & 0.90 & 0.12 & 125 & 393.1 & 338 & 1.29 & 32.8 & 1.3 & 867 & 0.132 & 81 & 4814 & 638 & 2.05 & nd & 2242 \\
\hline NGT659 & SN & TV & 37.9525 & 107.7264 & 3.41 & 5.54 & 3.21 & 1.62 & 0.33 & 7560 & 27.1 & 20 & 2.10 & 34.1 & 39.5 & 1028 & 1.82 & 81 & 1705 & 3.1 & 5.88 & nd & 7548 \\
\hline NGT660 & $\mathrm{SN}$ & TV & 37.9494 & 107.7297 & 3.97 & 0.26 & 2.09 & 1.74 & 0.20 & 395 & 170 & 59 & 0.56 & 48.3 & 50.2 & 213 & 0.775 & 333 & 33545 & 152 & 9.75 & nd & 9652 \\
\hline NGT661 & $\mathrm{TE}$ & TV & 37.9331 & 107.7456 & 1.13 & 0.17 & 3.50 & 0.66 & 0.05 & 9069 & 141.3 & 40 & 1.14 & 0.0 & 273.0 & 7608 & 1.42 & 60 & 48712 & 24.2 & 3.39 & nd & 77412 \\
\hline NGT663 & $\mathrm{TE}$ & TV & 37.9339 & 107.7525 & 8.06 & 0.37 & 4.28 & 2.34 & 0.27 & 895 & 15.8 & 144 & 8.20 & 7.6 & 7.1 & 357 & 0.525 & 171 & 6491 & 20.9 & 3.76 & nd & 1987 \\
\hline NGX363 & $\mathrm{RM}$ & SLUDGE & 37.9275 & 107.6881 & 0.42 & 0.44 & 25.00 & 0.03 & 0.03 & 963 & $<2$ & 287 & 1.00 & 13.0 & 1.4 & 162 & nd & $<2$ & 264 & 3.0 & nd & nd & 587 \\
\hline
\end{tabular}


Table 3. Analytical results for selected elements in surface-water samples, CWS, Colorado.

[All samples were filtered through $0.45-\mu \mathrm{m}$ filter and acidified with ultrapure nitric acid. Analyses by ICP-MS, reported in ppb (parts per billion). Number of figures reported in spreadsheet may not be significant. Sample type: DD, dump drainage; DH, artesian flow from drill hole; MD. mine drainage at portal; S, surface water such as a creek; Spr, spring; TPW, pore water in tailings impound (seepage into augered hole); TS, seepage from tailings impound. District: mining districts, Cash, Cashin; CO, Cochetopa; GB, Gold Brick, GN, Galena; IS, Iron Spring; LO, Los Ochos; MP, Mineral Point; OU, Ouray; QC, Quartz, Creek; RB, Ruby; RM, Red Mountain; TE. Telluride; TM, Tomichi; TN, Tincup; UV, Uravan. Watershed: GU, Gunnison River; LF, Lake Fork of Gunnison River; SM, San Miguel River; UN, Uncompahgre River. Samples collected in 1997-98, ICP-MS analysis by commercial laboratory

\begin{tabular}{|c|c|c|c|c|c|c|c|c|c|c|c|c|c|c|c|c|c|c|c|}
\hline Sample ID & $\mathrm{pH}$ & $\begin{array}{c}\text { Conductivity } \\
(\mu \mathrm{S} / \mathrm{cm})\end{array}$ & $\begin{array}{l}\text { Sample } \\
\text { type }\end{array}$ & District & Watershed & $\begin{array}{c}\mathrm{Al} \\
(\mathrm{ppb})\end{array}$ & $\begin{array}{c}\text { As } \\
(\mathrm{ppb})\end{array}$ & $\begin{array}{c}\mathrm{Cd} \\
(\mathrm{ppb})\end{array}$ & $\begin{array}{c}\text { Co } \\
(\mathrm{ppb})\end{array}$ & $\begin{array}{c}\mathrm{Cu} \\
(\mathrm{ppb})\end{array}$ & $\begin{array}{c}\mathrm{Fe} \\
(\mathrm{ppb})\end{array}$ & $\begin{array}{c}\mathrm{Mn} \\
(\mathrm{ppb})\end{array}$ & $\begin{array}{c}\text { Mo } \\
\text { (ppb) }\end{array}$ & $\begin{array}{c}\mathrm{Ni} \\
(\mathrm{ppb})\end{array}$ & $\begin{array}{c}\mathrm{Pb} \\
(\mathrm{ppb})\end{array}$ & $\begin{array}{c}\mathrm{Zn} \\
(\mathrm{ppb})\end{array}$ & $\begin{array}{c}\text { Lat (N.) } \\
\text { (degrees) }\end{array}$ & $\begin{array}{l}\text { Long (W.) } \\
\text { (degrees) }\end{array}$ & Site name \\
\hline \multicolumn{20}{|c|}{ Tailings pore waters } \\
\hline NGTW527 & 2.5 & 1,295 & TPW & RB & GU & 136.6 & 5.9 & 4.6 & 8.1 & 316.7 & 2,777 & 189 & 0.4 & 2.4 & $2,774.6$ & 739.1 & 38.8678 & 107.0750 & Standard mill \\
\hline NGTW619 & 3.4 & 1830 & TPW & IS & SM & $18,349.5$ & 36.8 & 39.9 & 214.2 & 330.5 & 18,413 & 2,459 & 1.4 & 89.5 & $1,676.4$ & $7,753.1$ & 37.8506 & 107.8206 & Carbonero mill \\
\hline NGTW620 & 3.0 & 1710 & TPW & IS & SM & $16,226.3$ & 7.0 & 33.1 & 168.9 & 563.3 & 27,827 & 2,783 & 1.0 & 71.7 & $1,515.6$ & $8,233.0$ & 37.8506 & 107.8203 & Carbonero mill \\
\hline NGTW625 & 2.1 & $>2000$ & TPW & IS & SM & $190,941.3$ & $5,910.3$ & 567.4 & $2,673.7$ & $31,734.0$ & $4,731,542$ & 48,494 & $1,907.9$ & 405.0 & $2,458.0$ & $58,049.8$ & 37.8512 & 107.8733 & Caribeau mill \\
\hline NGTW629 & 4.5 & 660 & TPW & IS & SM & $<0.2$ & 7.4 & 0.6 & 1.3 & 7.8 & 3,738 & 14 & 2.2 & 13.2 & 7.9 & 100.1 & 37.8507 & 107.8492 & Caribeau mill \\
\hline NGTW631 & 5.4 & NA & TPW & IS & SM & 21.8 & 3.8 & 0.9 & 2.0 & 4.9 & 3,188 & 52 & 1.2 & 14.1 & 23.2 & 401.2 & 37.8507 & 107.8483 & Caribeau mill \\
\hline \multicolumn{20}{|c|}{ Surface waters } \\
\hline NGW105 & 8.5 & 440 & $\mathrm{~S}$ & UV & SM & 0.0 & 40.7 & 0.9 & 0.1 & 3.9 & 200 & 1 & 179.6 & 1.7 & $<0.1$ & 4.6 & 38.3361 & 109.0246 & Lion Crk \\
\hline NGW106 & 8.3 & 310 & MD & UV & SM & 2.7 & 5.9 & 0.3 & 0.0 & 4.5 & 64 & 1 & 36.9 & 0.8 & 0.3 & 3.6 & 38.3364 & 109.0151 & \\
\hline NGW107 & 8.5 & 410 & MD & UV & SM & 0.0 & 72.9 & 0.9 & 0.2 & 2.1 & 80 & 0 & 174.1 & 0.9 & $<0.1$ & 2.2 & 38.3369 & 107.0252 & \\
\hline NGW115A & 7.4 & 1740 & SP & UV & SM & 1.5 & $1,130.5$ & 7.3 & 1.5 & 6.1 & 1,891 & 3 & $1,864.0$ & 15.7 & 0.8 & 7.4 & 38.3089 & 108.1263 & \\
\hline NGW117 & 8.1 & 840 & $\mathrm{~S}$ & UV & SM & 3.4 & 1.5 & 0.3 & 0.3 & 0.8 & 299 & 16 & 68.9 & 2.6 & $<0.1$ & 0.8 & 38.3908 & 108.0112 & Atkinson Crk \\
\hline NGW205 & 7.6 & 410 & DP & $\mathrm{TM}$ & GU & 10.9 & 0.5 & 7.3 & 0.2 & 7.4 & 706 & 32 & 6.1 & 2.9 & 22.5 & 351.4 & 38.5372 & 106.1348 & Akron mill \\
\hline NGW214 & 7.9 & 180 & MD & $\mathrm{TM}$ & GU & 2.7 & 0.4 & 0.2 & 0.2 & 3.6 & 308 & 23 & 3.4 & 1.9 & 1.2 & 179.1 & 38.5392 & 106.0156 & Akron adit \\
\hline NGW230 & 7.8 & 280 & $\mathrm{~S}$ & VU & GU & 9.5 & 2.6 & 0.2 & 0.3 & 6.1 & 438 & 20 & 2.0 & 3.0 & 2.0 & 36.8 & 38.3469 & 107.0051 & Camp Crk \\
\hline NGW231 & 7.7 & 310 & $\mathrm{~S}$ & VU & GU & 4.8 & 1.3 & 0.1 & 0.3 & 5.9 & 522 & 25 & 1.6 & 3.2 & 0.7 & 12.3 & 38.3481 & 107.0067 & Camp Crk \\
\hline NGW232 & 7.9 & 240 & MD & GB & GU & 5.6 & 2.3 & 0.5 & 0.1 & 3.5 & 166 & 11 & 5.1 & 1.1 & 1.1 & 38.9 & 38.6128 & 106.0188 & Carter mine \\
\hline NGW247 & 7.4 & 240 & MD & $\mathrm{TC}$ & GU & 36.5 & 0.5 & 0.1 & 0.3 & 4.4 & 237 & 12 & 1.8 & 2.5 & 0.8 & 12.1 & 38.7242 & 106.0061 & \\
\hline NGW249 & 7.7 & 90 & SP & MP & GU & 12.0 & 0.2 & 0.1 & 0.1 & 3.8 & 76 & 1 & 1.4 & 1.7 & 0.6 & 4.8 & 38.3781 & 106.0111 & \\
\hline NGW259 & 7.9 & 680 & SP & LO & GU & 3.5 & 5.4 & 0.1 & 0.3 & 2.7 & 666 & 1 & 10.7 & 4.3 & 0.6 & 13.5 & 38.3764 & 106.0074 & \\
\hline NGW263 & 3.7 & 210 & MD & GQ & GU & 574.3 & 0.1 & 7.7 & 23.6 & $1,180.5$ & 1,331 & 976 & 3.0 & 11.6 & 2.0 & 410.8 & 38.6831 & 106.1422 & Bon Ton mine adit \\
\hline NGW264 & 5.7 & 120 & MD & $\mathrm{GQ}$ & GU & 314.8 & 0.2 & 7.4 & 21.5 & $1,156.3$ & 6,798 & 1,020 & 29.4 & 10.7 & 1.1 & 391.3 & 38.6831 & 106.1377 & Bon Ton mine and dump drain \\
\hline NGW275 & 7.6 & 280 & MD & $\mathrm{TC}$ & GU & 3.1 & 0.2 & 0.1 & 0.4 & 19.6 & 611 & 464 & 18.1 & 2.6 & 2.0 & 32.0 & 38.7044 & 106.0099 & Blistered Horn \\
\hline NGW287 & 7.4 & 260 & MD & BP & LF & 12.8 & 2.7 & 1.2 & 0.2 & 6.4 & 334 & 25 & 26.0 & 2.3 & 8.8 & 154.2 & 37.9206 & 107.0209 & American Basin \\
\hline NGW296 & 4.7 & 80 & $\mathrm{~S}$ & MP & UN & 655.7 & 1.2 & 4.4 & 1.9 & 11.4 & 240 & 701 & 0.7 & 2.5 & 17.6 & 625.6 & 37.9522 & 107.0849 & \\
\hline NGW298 & 3.5 & 200 & MD & MP & UN & 188.8 & 0.7 & 23.2 & 3.3 & 76.1 & 1,327 & 379 & 2.8 & 2.2 & 147.7 & $3,112.6$ & 37.9492 & 107.3879 & London mine \\
\hline NGW299 & 3.6 & 410 & $\mathrm{~S}$ & RM & UN & $7,071.6$ & 0.2 & 2.3 & 16.2 & 313.0 & 3,131 & 765 & 1.6 & 16.3 & 17.8 & 374.4 & 37.9492 & 107.0395 & Red Mtn Crk \\
\hline NGW309 & 3.4 & $>2000$ & SP & OY & UN & $12,462.4$ & 2.8 & 309.2 & 55.6 & $2,509.7$ & 12,609 & 63,766 & 1.7 & 128.2 & 108.2 & $68,089.8$ & 38.0539 & 112.1589 & \\
\hline NGW310 & 3.7 & 570 & SP & IS & SM & $3,989.9$ & 0.2 & 2.3 & 24.5 & 178.5 & 5,376 & 1,077 & 2.8 & 12.6 & 2.2 & 298.6 & 38.0542 & 107.0432 & Iron Spring mine \\
\hline NGW315 & 3.0 & 1660 & TD & IS & SM & $15,651.6$ & 15.4 & 205.0 & 244.8 & $1,221.9$ & 76,187 & 60,014 & 1.3 & 77.5 & 911.9 & $38,183.4$ & 37.8564 & 110.4192 & Carbonero mill \\
\hline NGW317 & 7.3 & 980 & TD & IS & SM & 17.2 & 0.8 & 0.6 & 9.4 & 3.1 & 1,149 & 2,863 & 1.1 & 19.3 & 2.4 & 71.4 & 37.8575 & 107.0256 & Caribou mill \\
\hline NGW318 & 7.1 & 790 & MD & IS & SM & 2.9 & 0.6 & 1.0 & 10.8 & 2.5 & 2,267 & 2,595 & 1.0 & 17.2 & 0.2 & 181.9 & 37.8569 & 107.0335 & Caribou mine \\
\hline NGW320 & 6.6 & 290 & MD & IS & SM & 411.7 & 0.2 & 18.3 & 10.4 & 580.6 & 331 & 8,161 & 0.6 & 8.1 & 28.9 & $1,786.7$ & 37.8844 & 107.3080 & Alta mine \\
\hline NGW328 & 4.8 & 250 & $\mathrm{~S}$ & RM & UN & 153.2 & 0.2 & 0.5 & 2.7 & 75.3 & 365 & 150 & 0.5 & 3.4 & 2.6 & 83.7 & 37.9033 & 107.0124 & National Belle \\
\hline NGW333 & 2.9 & 1340 & MD & RM & UN & $41,357.3$ & 32.0 & 6.2 & 75.9 & 883.2 & 60,234 & 989 & 0.6 & 71.4 & 38.8 & 840.9 & 37.9139 & 107.1132 & Champion Gulch \\
\hline NGW335 & 2.4 & $>2000$ & MD & $\mathrm{RM}$ & UN & $210,775.5$ & 84.1 & 44.5 & 520.1 & $8,252.9$ & 441,023 & 7,457 & 1.1 & 384.4 & 51.1 & $5,471.5$ & 37.9175 & 107.7535 & Guston mine \\
\hline NGW336 & 3.1 & $>2000$ & MD & $\mathrm{RM}$ & UN & $194,867.4$ & 31.6 & 22.1 & 273.4 & 765.2 & 230,955 & 7,043 & 0.8 & 209.7 & 66.4 & $3,143.6$ & 37.9172 & 107.3791 & \\
\hline NGW338 & 2.6 & $>2000$ & DP & $\mathrm{RM}$ & UN & $42,888.0$ & 293.9 & 14.3 & 136.3 & $2,688.8$ & 260,589 & 6,767 & 1.0 & 148.4 & 111.9 & $2,160.3$ & 37.9211 & 107.2467 & \\
\hline NGW340 & 2.7 & $>2000$ & MD & $\mathrm{RM}$ & UN & $248,110.7$ & 93.5 & 47.7 & 602.9 & $9,165.3$ & 501,385 & 8,499 & 1.0 & 432.0 & 57.1 & $5,978.5$ & 37.9164 & 107.8033 & Guston mine \\
\hline NGW342 & 3.5 & 640 & $\mathrm{~S}$ & $\mathrm{RM}$ & UN & $10,071.3$ & 2.9 & 3.4 & 22.5 & 838.4 & 13,220 & 566 & 0.2 & 24.2 & 48.4 & 543.6 & 37.9181 & 107.0707 & Red Mtn Crk \\
\hline NGW344 & 2.9 & $>2000$ & TS & $\mathrm{RM}$ & UN & $14,801.0$ & 13.6 & 78.3 & 832.1 & 831.7 & 184,510 & 74,094 & 1.4 & 102.9 & 37.7 & $24,754.6$ & 37.9189 & 108.3199 & Tailings seep \\
\hline NGW347 & 3.6 & 410 & $\mathrm{~S}$ & RM & UN & $6,061.7$ & 0.2 & 2.3 & 14.9 & 291.4 & 2,914 & 739 & 0.2 & 13.7 & 18.2 & 363.2 & 37.9600 & 107.0508 & Red Mtn Crk \\
\hline NGW348 & 2.0 & 1650 & MD & $\mathrm{RM}$ & UN & $43,381.5$ & 1.1 & 151.6 & 125.4 & 435.8 & 69,504 & 5,739 & 2.2 & 82.9 & 10.0 & $10,284.3$ & 37.9372 & 109.5354 & Larson Brothers mine \\
\hline NGW353 & 6.3 & 740 & MD & $\mathrm{RM}$ & UN & 89.3 & 0.3 & 3.6 & 14.9 & 3.0 & 2,344 & 1,480 & 2.0 & 11.6 & 1.4 & 520.8 & 37.9278 & 107.0720 & Mt. King mine \\
\hline NGW357 & 2.9 & 1410 & MD & $\mathrm{RM}$ & UN & $38,992.6$ & 22.2 & 17.1 & 99.2 & 665.3 & 60,250 & 4,641 & 0.8 & 91.1 & 97.6 & $3,733.1$ & 37.9242 & 107.2946 & Joker mine \\
\hline NGW363 & 5.9 & 1260 & MD & $\mathrm{RM}$ & UN & 47.9 & 10.2 & 0.3 & 22.0 & 2.9 & 20,058 & 3,512 & 0.5 & 22.3 & 0.8 & 269.7 & 37.7442 & 107.0100 & Silver Belle \\
\hline
\end{tabular}




\begin{tabular}{|c|c|c|c|c|c|c|c|c|c|c|c|c|c|c|c|c|c|c|c|}
\hline Sample ID & $\mathrm{pH}$ & $\begin{array}{l}\text { Conductivity } \\
(\mu \mathrm{S} / \mathrm{cm})\end{array}$ & $\begin{array}{c}\text { Sample } \\
\text { type }\end{array}$ & District & Watershed & $\begin{array}{c}\mathrm{Al} \\
(\mathrm{ppb})\end{array}$ & $\begin{array}{c}\text { As } \\
\text { (ppb) }\end{array}$ & $\begin{array}{c}\mathrm{Cd} \\
(\mathrm{ppb})\end{array}$ & $\begin{array}{c}\text { Co } \\
\text { (ppb) }\end{array}$ & $\begin{array}{c}\mathrm{Cu} \\
\text { (ppb) }\end{array}$ & $\begin{array}{c}\mathrm{Fe} \\
(\mathrm{ppb})\end{array}$ & $\begin{array}{c}\mathrm{Mn} \\
\text { (ppb) }\end{array}$ & $\begin{array}{c}\text { Mo } \\
\text { (ppb) }\end{array}$ & $\begin{array}{c}\mathrm{Ni} \\
(\mathrm{ppb})\end{array}$ & $\begin{array}{c}\mathrm{Pb} \\
(\mathrm{ppb})\end{array}$ & $\begin{array}{c}\mathrm{Zn} \\
\text { (ppb) }\end{array}$ & $\begin{array}{l}\text { Lat (N.) } \\
\text { (degrees) }\end{array}$ & $\begin{array}{l}\text { Long (W.) } \\
\text { (degrees) }\end{array}$ & Site name \\
\hline \multicolumn{20}{|c|}{ Surface waters_Continued } \\
\hline NGW365 & 3.7 & 390 & $\mathrm{~S}$ & $\mathrm{RM}$ & UN & $6,441.1$ & 0.6 & 3.5 & 15.0 & 330.7 & 6,612 & 341 & 0.3 & 14.1 & 23.3 & 596.8 & 37.9250 & 107.0680 & Red Mtn Crk \\
\hline NGW367 & 3.0 & 290 & $\mathrm{~S}$ & $\mathrm{RM}$ & UN & $1,823.5$ & 0.8 & 0.7 & 6.6 & 185.0 & 1,640 & 52 & 0.2 & 5.9 & 55.4 & 110.7 & 37.9175 & 107.0184 & CorksCrkew Gulch \\
\hline NGW371 & 6.2 & 190 & $\mathrm{~s}$ & SN & UN & 18.1 & 0.6 & 0.6 & 0.2 & 1.7 & 212 & 134 & 2.1 & 1.7 & 1.1 & 109.5 & 38.0028 & 107.0210 & Canyon Crk \\
\hline NGW373 & 6.5 & 190 & $\mathrm{~S}$ & $\mathrm{SN}$ & UN & 12.9 & 0.6 & 0.9 & 0.3 & 2.0 & 193 & 243 & 2.0 & 2.1 & 0.6 & 155.4 & 37.9747 & 107.0198 & Canyon Crk \\
\hline NGW380 & 6.9 & 120 & $\mathrm{~S}$ & $\mathrm{MnP}$ & UN & 64.0 & 0.2 & 1.2 & 0.2 & 5.0 & 137 & 100 & 0.9 & 2.0 & 0.7 & 133.3 & 37.9747 & 107.0207 & Upr. Unconpahgre R \\
\hline NGW382 & 6.5 & 480 & MD & $\mathrm{MnP}$ & UN & 38.0 & 0.8 & 0.5 & 0.6 & 3.5 & 496 & 603 & 5.9 & 5.7 & 0.5 & 115.2 & 37.9531 & 107.0175 & Old Lout mine \\
\hline NGW385 & 7.2 & 360 & $\mathrm{~S}$ & IS & SM & 38.7 & 0.2 & 0.5 & 1.4 & 2.2 & 317 & 209 & 1.7 & 4.1 & 0.1 & 65.1 & 37.8661 & 107.0224 & Howard Fk \\
\hline NGW388 & 7.3 & 150 & $\mathrm{~S}$ & $\mathrm{TE}$ & SM & 11.3 & 0.5 & 5.2 & 0.2 & 4.7 & 87 & 84 & 1.1 & 1.4 & 3.8 & 750.3 & 37.9383 & 107.0915 & Savage Crk \\
\hline NGW391 & 7.2 & 110 & $\mathrm{~S}$ & $\mathrm{TE}$ & SM & 15.8 & 0.4 & 1.0 & 0.1 & 3.3 & 77 & 37 & 1.0 & 1.0 & 4.8 & 139.2 & 37.9356 & 107.0226 & San Miguel R \\
\hline NGW393 & 3.3 & 1510 & $\mathrm{MD}$ & GN & LF & $10,534.6$ & 7.8 & 29.9 & 168.5 & 37.2 & 21,580 & 57,836 & 0.7 & 103.8 & 71.0 & $4,784.0$ & 37.9736 & 107.5084 & Palmetto basin \\
\hline NGW394 & 2.9 & $1500 ?$ & MD & GN & LF & $9,179.0$ & 5.8 & 30.9 & 159.7 & 69.9 & 17,590 & 56,318 & 0.6 & 97.8 & 68.2 & $4,891.6$ & 37.9736 & 107.5237 & Palmetto basin \\
\hline NGW450 & 7.1 & 240 & $\mathrm{~S}$ & GN & LF & 9.7 & 0.1 & 9.4 & 9.2 & 320.2 & 652 & 504 & 1.0 & 10.9 & 0.2 & $1,022.3$ & 37.9772 & 107.1677 & Head Henson Crk \\
\hline NGW455 & 2.7 & nd & $\mathrm{MD}$ & $\mathrm{UN}$ ? & UN & $7,402.5$ & 59.5 & 165.0 & 59.0 & 424.0 & 23,624 & 2,191 & 4.3 & 37.4 & 493.8 & $11,289.6$ & 37.9600 & 109.7537 & San Juan Chief \\
\hline NGW492 & 5.9 & 910 & $\mathrm{~S}$ & SN & UN & 18.2 & 0.8 & 0.8 & 0.2 & 2.6 & 519 & 179 & 9.1 & 1.7 & 1.5 & 163.3 & 37.9675 & 107.7211 & Canyon Crk \\
\hline NGW493 & 5.9 & 1540 & $\mathrm{MD}$ & IS & SM & 52.3 & 1.8 & 1.1 & 6.1 & 24.5 & 6,008 & 1,445 & 1.2 & 11.1 & $<0.1$ & 148.8 & 37.8508 & 107.8494 & Caribeau mine drainage \\
\hline NGW494 & 5.9 & 1540 & DH & IS & SM & $<0.2$ & 1.4 & $<0.01$ & 9.1 & 2.2 & 13,511 & 850 & 2.6 & 28.0 & $<0.1$ & 68.3 & 37.9006 & 107.6675 & Drill hole flow \\
\hline NGW495 & 2.1 & $>2000$ & MD & $\mathrm{RM}$ & UN & $135,579.7$ & 486.5 & 7.0 & 454.1 & $7,648.3$ & 479,713 & 228 & 12.0 & 326.9 & 71.9 & 924.7 & 37.8508 & 107.8494 & Head Corkscrew \\
\hline NGW499 & 3.0 & 285 & $\mathrm{MD}$ & $\mathrm{RB}$ & GU & $1,348.1$ & $<0.03$ & 117.1 & 17.5 & 841.8 & 2,742 & 5,712 & $<0.05$ & 16.0 & $1,348.1$ & $18,910.2$ & 38.8680 & 107.0739 & Standard mine \\
\hline NGW505 & 8.2 & 251 & $\mathrm{~S}$ & VU & GU & 21.2 & 1.5 & 0.2 & 0.7 & 6.5 & 992 & 58 & 1.5 & 5.9 & 3.9 & 132.8 & 38.3346 & 107.0014 & Cabin Crk \\
\hline NGW506 & 8.1 & 354 & $\mathrm{~S}$ & $\mathrm{VU}$ & GU & 40.0 & 2.7 & $<0.01$ & 0.8 & 7.7 & 1,317 & 201 & 1.2 & 10.1 & $<0.1$ & 16.6 & 38.3349 & 107.0053 & Cabin Crk \\
\hline NGW507 & 3.1 & 200 & MD & $\mathrm{RB}$ & GU & $3,726.9$ & $<0.03$ & 47.9 & 10.4 & 708.2 & 3,955 & 3,453 & $<0.05$ & 5.8 & 417.0 & $7,644.9$ & 38.8681 & 107.0728 & Mid<Elk Basin \\
\hline NGW510 & 3.1 & 320 & $\mathrm{MD}$ & $\mathrm{RB}$ & $\mathrm{GU}$ & $3,929.8$ & $<0.03$ & 54.5 & 11.0 & 297.8 & 3,973 & 7,351 & $<0.05$ & 8.2 & 327.3 & $7,972.1$ & 38.8834 & 107.0683 & Upper Elk Basin \\
\hline NGW511 & 2.9 & 354 & $\mathrm{DD}$ & $\mathrm{RB}$ & GU & $4,084.8$ & $<0.03$ & 80.5 & 13.7 & $1,015.5$ & 4,634 & 4,533 & $<0.05$ & 10.9 & 571.1 & $12,388.2$ & 38.8682 & 107.0714 & Upper Elk Basin \\
\hline NGW512 & 3.0 & 285 & MD & $\mathrm{RB}$ & $\mathrm{GU}$ & $1,878.8$ & $<0.03$ & 127.2 & 18.5 & 927.8 & 4,738 & 7,437 & $<0.05$ & 17.4 & $1,373.7$ & $21,470.9$ & 38.8680 & 107.0739 & Standard mine \\
\hline NGW514 & 3.3 & 301 & $\mathrm{~S}$ & $\mathrm{RB}$ & $\mathrm{GU}$ & $3,641.6$ & $<0.03$ & 87.4 & 13.0 & 825.3 & 3,784 & 4,328 & $<0.05$ & 7.3 & $1,229.1$ & $13,117.3$ & 38.8681 & 107.0739 & E Elk Crk \\
\hline NGW515 & 5.8 & 20 & $\mathrm{~S}$ & $\mathrm{RB}$ & GU & 5.0 & 0.2 & $<0.01$ & 0.1 & 0.3 & 110 & 20 & 0.1 & 0.7 & 0.4 & 2.7 & 38.8680 & 107.0742 & W Elk Crk \\
\hline NGW518 & 3.0 & 169 & DD & $\mathrm{RB}$ & $\mathrm{GU}$ & $9,890.7$ & $<0.03$ & 152.4 & 51.3 & $1,402.6$ & 15,890 & 10,500 & $<0.05$ & 21.6 & 613.9 & $27,647.8$ & 38.8679 & 107.0744 & Standard mine \\
\hline NGW519 & 3.1 & 448 & TP & $\mathrm{RB}$ & $\mathrm{GU}$ & $7,893.9$ & $<0.03$ & 153.8 & 38.6 & $1,163.9$ & 15,263 & 7,875 & $<0.05$ & 16.8 & 436.9 & $23,820.3$ & 38.8678 & 107.0744 & Standard mine \\
\hline NGW520 & 2.9 & 765 & TPS & $\mathrm{RB}$ & GU & $4,936.8$ & $<0.03$ & 89.4 & 32.4 & 514.8 & 7,552 & 6,968 & $<0.05$ & 11.2 & 403.4 & $13,819.3$ & 38.8678 & 107.0744 & Standard mine \\
\hline NGW521 & 5.4 & 62 & $\mathrm{~S}$ & $\mathrm{RB}$ & $\mathrm{GU}$ & 238.4 & 0.2 & 14.8 & 2.2 & 63.9 & 379 & 664 & $<0.05$ & 2.3 & 91.7 & $2,044.2$ & 38.8678 & 107.0753 & Elk Crk \\
\hline NGW523 & 5.9 & 60 & $\mathrm{~S}$ & $\mathrm{RB}$ & $\mathrm{GU}$ & 127.5 & 1.2 & 10.0 & 1.2 & 37.5 & 247 & 398 & $<0.05$ & 1.9 & 52.5 & $1,324.4$ & 38.8672 & 107.0753 & Elk Crk \\
\hline NGW529 & 7.2 & 97 & $\mathrm{~S}$ & GB & $\mathrm{GU}$ & 11.4 & 0.6 & 0.4 & 0.1 & 1.8 & 235 & 12 & 1.0 & 1.2 & 1.8 & 42.5 & 38.6009 & 106.5989 & Gold Crk \\
\hline NGW531 & 6.9 & 90 & $\mathrm{~s}$ & GB & GU & 8.0 & 0.4 & 0.2 & 0.1 & 0.7 & 216 & 4 & 1.1 & 1.1 & 0.5 & 19.9 & 38.6170 & 106.5853 & Gold Crk \\
\hline NGW532 & 6.9 & 56 & $\mathrm{~S}$ & GB & $\mathrm{GU}$ & 9.6 & 0.1 & 0.0 & 0.1 & 0.4 & 153 & 2 & 1.3 & 0.8 & 0.2 & 1.8 & 38.6501 & 106.5742 & Gold Crk \\
\hline NGW533 & 6.7 & 40 & $\mathrm{~s}$ & GQ & $\mathrm{GU}$ & 76.7 & 0.1 & 0.6 & 0.9 & 64.6 & 95 & 41 & 7.4 & 2.1 & 0.9 & 47.7 & 38.6682 & 106.4792 & Tomichi Crk \\
\hline NGW537 & 6.8 & 66 & $\mathrm{~S}$ & LF & LF & 15.8 & 0.4 & 0.8 & 0.7 & 1.4 & 154 & 8 & 6.1 & 1.9 & 2.0 & 3.4 & 38.0167 & 107.3239 & Wade Gulch \\
\hline NGW540 & 6.9 & 96 & $\mathrm{~s}$ & LF & LF & 68.7 & 0.2 & 0.2 & 0.6 & 1.4 & 305 & 63 & 1.9 & 2.0 & 1.3 & 8.0 & 37.9505 & 107.3064 & Red Mtn Gulch \\
\hline NGW541 & 6.6 & 43 & $\mathrm{~s}$ & LF & LF & 86.9 & 0.3 & 0.1 & 0.2 & 0.8 & 161 & 58 & 1.2 & 1.2 & 0.2 & 17.7 & 37.9003 & 107.4264 & Lake Fk Gunn. \\
\hline NGW543 & 6.7 & 46 & $\mathrm{~S}$ & LF & LF & 13.9 & 0.2 & 0.0 & 0.1 & 0.4 & 179 & 2 & 1.0 & 1.0 & 0.1 & 1.7 & 37.9000 & 107.4331 & Cottonwood Crk \\
\hline NGW544 & 6.8 & 34 & $\mathrm{~S}$ & LF & LF & 13.3 & 0.3 & 0.0 & 0.0 & 0.3 & 140 & 2 & 0.4 & 0.7 & 0.1 & 0.9 & 37.9001 & 107.4342 & Cattaract Crk \\
\hline NGW545 & 6.9 & 44 & $\mathrm{~S}$ & BP & LF & 12.4 & 0.6 & 0.0 & 0.1 & 0.4 & 166 & 1 & 1.0 & 0.9 & 0.1 & 0.7 & 37.9336 & 107.4631 & Grizzly Crk \\
\hline NGW546 & 7.1 & 47 & $\mathrm{~S}$ & BP & LF & 41.1 & 0.1 & 0.2 & 0.5 & 0.8 & 126 & 145 & 0.9 & 1.4 & 0.2 & 30.4 & 37.9340 & 107.4581 & Silver Crk \\
\hline NGW547 & 7.0 & 46 & $\mathrm{~S}$ & LF & LF & 26.2 & 0.3 & 0.0 & 0.1 & 0.3 & 140 & 8 & 0.6 & 0.9 & 0.2 & 3.0 & 37.9007 & 107.3806 & Bent Crk \\
\hline NGW548 & 6.8 & 76 & $\mathrm{~S}$ & $\mathrm{LF}$ & LF & 50.5 & 0.4 & 0.0 & 0.2 & 0.8 & 190 & 50 & 0.7 & 1.3 & 0.3 & 2.0 & 37.9847 & 107.2958 & Lake Fk Gunn. \\
\hline NGW549 & 6.9 & 46 & $\mathrm{~s}$ & GN & LF & 70.7 & 0.2 & 0.0 & 0.3 & 0.8 & 163 & 23 & 0.3 & 1.0 & 0.2 & 3.6 & 38.0007 & 107.4675 & W Fk Henson Crk \\
\hline NGW550 & 7.2 & 42 & $\mathrm{~s}$ & GN & LF & 32.0 & 0.4 & 0.3 & 0.2 & 7.7 & 134 & 38 & 0.2 & 1.0 & 0.5 & 34.9 & 37.9676 & 107.5350 & Upper Henson Crk \\
\hline NGW552 & 7.3 & 46 & $\mathrm{~S}$ & GN & LF & 55.7 & 0.4 & 0.1 & 0.1 & 1.7 & 168 & 11 & 0.7 & 1.0 & 0.9 & 12.5 & 38.0171 & 107.3319 & Lower Henson Crk \\
\hline NGW555 & 3.0 & 779 & TS & $\mathrm{RB}$ & $\mathrm{GU}$ & $5,199.0$ & $<0.03$ & 86.2 & 33.6 & 525.1 & 5,928 & 7,160 & $<0.05$ & 15.3 & 336.3 & $13,766.6$ & 38.8678 & 107.0753 & Standard mine \\
\hline NGW556 & 6.6 & 32 & MD & $\mathrm{RB}$ & GU & 89.6 & 0.1 & 3.0 & 0.6 & 21.5 & 193 & 151 & $<0.05$ & 0.8 & 104.6 & 452.6 & 38.8834 & 107.0708 & Upper Elk Basin \\
\hline NGW558 & 7.3 & 59 & $\mathrm{~S}$ & $\mathrm{RB}$ & GU & 66.8 & 2.3 & 4.0 & 0.3 & 13.6 & 235 & 108 & $<0.05$ & 1.4 & 15.6 & 541.1 & 38.8507 & 107.0600 & Lower Elk Crk \\
\hline NGW559 & 7.3 & 37 & $\mathrm{~s}$ & $\mathrm{RB}$ & GU & 28.3 & 0.3 & 0.4 & 0.1 & 1.9 & 143 & 12 & 0.6 & 0.9 & 3.8 & 50.0 & 38.9008 & 107.0253 & Slate $\mathrm{R}$ \\
\hline NGW560 & 73 & 24 & S & $\mathrm{RB}$ & GU & 43.8 & 0.2 & 0.7 & 0.1 & 33 & 98 & & $<0.05$ & 0.5 & 115 & 906 & 38.9008 & 107.0467 & O \\
\hline
\end{tabular}


Table 3. Analytical results for selected elements in surface-water samples, CWS, Colorado—Continued.

\begin{tabular}{|c|c|c|c|c|c|c|c|c|c|c|c|c|c|c|c|c|c|c|c|}
\hline Sample ID & $\mathrm{pH}$ & $\begin{array}{l}\text { Conductivity } \\
(\mu \mathrm{S} / \mathrm{cm})\end{array}$ & $\begin{array}{c}\text { Sample } \\
\text { type }\end{array}$ & District & Watershed & $\underset{(\mathrm{ppb})}{\mathrm{Al}}$ & $\begin{array}{c}\text { As } \\
(\mathrm{ppb})\end{array}$ & $\underset{(\mathrm{ppb})}{\mathrm{Cd}}$ & $\begin{array}{c}\mathrm{Co} \\
\text { (ppb) }\end{array}$ & $\underset{(\mathrm{ppb})}{\mathrm{Cu}}$ & $\underset{\text { (ppb) }}{\mathrm{Fe}}$ & $\begin{array}{c}\mathrm{Mn} \\
(\mathrm{ppb})\end{array}$ & $\begin{array}{c}\text { Mo } \\
(\mathrm{ppb})\end{array}$ & $\underset{\mathrm{Ni}}{\mathrm{Ni}}$ & $\begin{array}{c}\mathrm{Pb} \\
(\mathrm{ppb})\end{array}$ & $\begin{array}{c}\mathrm{Zn} \\
\text { (ppb) }\end{array}$ & $\begin{array}{c}\text { Lat (N.) } \\
\text { (degrees) }\end{array}$ & $\begin{array}{l}\text { Long (W.) } \\
\text { (degrees) }\end{array}$ & Site name \\
\hline \multicolumn{20}{|c|}{ Surface waters-Continued } \\
\hline NGW561 & 7.0 & 62 & $\mathrm{~s}$ & $\mathrm{TM}$ & GU & 30.7 & 0.1 & 0.0 & 0.1 & 6.7 & 225 & 9 & 3.9 & 1.0 & 0.5 & 4.5 & 38.5349 & 106.3892 & Tomichi Crk \\
\hline NGW563 & 7.1 & 83 & $\mathrm{~s}$ & $\mathrm{TM}$ & $\mathrm{GU}$ & 20.6 & 0.1 & 0.6 & 0.1 & 5.0 & 262 & 10 & 4.0 & 1.2 & 1.6 & 104.0 & 38.5178 & 106.4061 & Tomichi Crk \\
\hline NGW565 & 7.2 & 81 & $\mathrm{~s}$ & $\mathrm{TM}$ & GU & 19.1 & 0.2 & 0.8 & 0.1 & 4.8 & 268 & 11 & 4.0 & 1.3 & 3.2 & 124.9 & 38.5172 & 106.4147 & Tomichi Crk \\
\hline NGW570 & 2.7 & 1025 & $\mathrm{~S}$ & $\mathrm{RM}$ & UN & $21,555.4$ & 43.6 & 35.7 & 79.6 & $5,861.9$ & 63,499 & 3,457 & $<0.05$ & 52.6 & 189.2 & $5,505.0$ & 38.8678 & 107.0750 & Red Mtn Crk \\
\hline NGW571 & 2.6 & 1140 & $\mathrm{MD}$ & $\mathrm{RM}$ & UN & $98,023.1$ & 345.8 & 10.9 & 147.5 & $5,639.1$ & 163,712 & 1,829 & $<0.05$ & 133.2 & 367.2 & $2,243.3$ & 37.9004 & 107.7031 & Adit into ferricrete \\
\hline NGW574 & 3.3 & 436 & $\mathrm{~S}$ & $\mathrm{RM}$ & UN & $13,636.8$ & 37.8 & 2.4 & 23.5 & $1,031.2$ & 22,776 & 420 & $<0.05$ & 23.3 & 51.6 & 531.5 & 37.9167 & 107.6931 & Red Mtn Crk \\
\hline NGW575 & 2.9 & 385 & $\mathrm{~S}$ & $\mathrm{RM}$ & UN & $17,352.3$ & 10.7 & 2.3 & 31.3 & 293.0 & 26,180 & 378 & $<0.05$ & 24.8 & 29.5 & 531.5 & 37.9167 & 107.6919 & Champion Gulch \\
\hline NGW576 & 2.5 & $>2000$ & $\mathrm{MD}$ & $\mathrm{RM}$ & UN & $469,702.7$ & 101.0 & 54.8 & 689.7 & $8,307.4$ & 680,771 & 9,422 & 0.7 & 454.5 & 117.9 & $10,410.1$ & 37.9167 & 107.6919 & Guston mine \\
\hline NGW577 & 4.2 & 122 & $\mathrm{MD}$ & $\mathrm{RM}$ & UN & $6,440.0$ & 2.0 & 1.0 & 9.7 & 138.6 & 9,916 & 153 & $<0.05$ & 8.3 & 5.3 & 173.6 & 37.9169 & 107.6981 & Prospect trench \\
\hline NGW578 & 2.5 & $>2000$ & TS & $\mathrm{RM}$ & UN & $16,727.7$ & 5.5 & 50.0 & 859.5 & 785.8 & 185,890 & 105,535 & $<0.05$ & 101.3 & 26.5 & $30,397.4$ & 37.9175 & 107.6928 & Tailings \\
\hline NGW580 & 2.9 & 885 & DD & $\mathrm{RM}$ & UN & $18,750.1$ & 6.0 & 5.1 & 60.4 & 412.6 & 12,862 & 4,122 & $<0.05$ & 42.1 & 9.2 & $1,447.0$ & 37.9174 & 107.6928 & Joker mine \\
\hline NGW581 & 3.6 & 319 & $\mathrm{~S}$ & $\mathrm{RM}$ & UN & $11,751.9$ & 2.1 & 3.3 & 22.3 & 485.3 & 14,636 & 653 & $<0.05$ & 18.0 & 34.0 & 687.7 & 37.9172 & 107.6758 & Red Mtn Crk \\
\hline NGW582 & 3.0 & 565 & $\mathrm{~s}$ & $\mathrm{RM}$ & UN & $10,677.9$ & 7.8 & 0.9 & 31.1 & 437.2 & 16,358 & 597 & $<0.05$ & 24.1 & 78.0 & 395.9 & 37.9172 & 107.6758 & Corkscrew Gulch \\
\hline NGW583 & 3.3 & 248 & $\mathrm{~s}$ & $\mathrm{RM}$ & UN & $4,892.5$ & 0.4 & 0.1 & 5.6 & 18.2 & 1,396 & 85 & 1.0 & 4.7 & 2.4 & 28.5 & 37.9008 & 107.6694 & Small crk \\
\hline NGW584 & 2.5 & 1035 & $\mathrm{~S}$ & $\mathrm{RM}$ & UN & $21,270.5$ & $<0.03$ & 1.1 & 72.4 & $1,172.8$ & 14,492 & 86 & $<0.5$ & 52.9 & 136.5 & 153.7 & 37.9006 & 107.6675 & Corkscrew Gulch \\
\hline NGW587 & 2.1 & $>2000$ & MD & RM & UN & $230,594.5$ & 675.0 & 7.9 & 674.8 & $11,376.3$ & 720,589 & 335 & 13.8 & 485.8 & 75.9 & $1,313.1$ & 37.9006 & 107.6622 & Adit, Corkscrew \\
\hline NGW589 & 3.9 & 39 & $\mathrm{~S}$ & $\mathrm{RM}$ & UN & 732.9 & 3.4 & 0.6 & 2.3 & 30.5 & 1,599 & 17 & 14.5 & 3.3 & 3.6 & $<5$ & 37.9013 & 107.6742 & Lake at divide \\
\hline NGW592 & 3.4 & 154 & $\mathrm{~s}$ & $\mathrm{RM}$ & UN & $1,262.0$ & 1.3 & 0.1 & 3.3 & 15.3 & 1,094 & 49 & 2.5 & 3.1 & 35.5 & 8.2 & 37.9180 & 107.6794 & Small crk \\
\hline NGW593 & 3.1 & 563 & $\mathrm{~s}$ & $\mathrm{RM}$ & UN & $14,209.1$ & 0.8 & 0.3 & 20.7 & 64.2 & 4,459 & 388 & 0.7 & 14.6 & 3.4 & 99.0 & 37.9008 & 107.6744 & Small crk \\
\hline NGW595 & 2.9 & 492 & $\mathrm{~s}$ & $\mathrm{RM}$ & UN & $5,187.8$ & 0.5 & 0.5 & 9.9 & 37.0 & 1,702 & 26 & $<0.05$ & 13.3 & 103.0 & 61.5 & 37.9014 & 107.6736 & Small crk \\
\hline NGW599 & 3.2 & 215 & $\mathrm{~S}$ & RM & UN & $2,287.1$ & 1.2 & 0.7 & 5.5 & 107.3 & 1,617 & 56 & $<0.05$ & 4.7 & 63.7 & 103.3 & 37.9337 & 107.6675 & Small crk \\
\hline NGW605 & 4.9 & 278 & $\mathrm{~S}$ & $\mathrm{RM}$ & UN & 609.9 & 0.4 & 0.1 & 3.2 & 22.1 & 1,551 & 242 & $<0.05$ & 5.3 & 1.8 & 33.5 & 37.9183 & 107.6611 & Gray Copper Crk \\
\hline NGW606 & 6.0 & 375 & MD & RM & UN & 58.8 & 2.2 & 0.2 & 2.3 & 2.0 & 3,088 & 1,213 & 3.8 & 5.4 & $<0.1$ & 119.1 & 38.0834 & 107.6575 & Mountain Silver \\
\hline NGW609 & 5.9 & 74 & MD & $\mathrm{RM}$ & UN & 101.9 & $<0.03$ & 0.3 & 0.9 & 13.2 & 420 & 101 & $<0.05$ & 1.7 & 3.8 & 44.0 & 37.9509 & 107.6617 & Upper Mt Silver \\
\hline NGW610 & 3.4 & 390 & $\mathrm{~S}$ & RM & UN & $9,040.2$ & $<0.03$ & 2.2 & 18.4 & 363.3 & 5,584 & 759 & $<0.05$ & 17.8 & 28.4 & 601.5 & 37.8511 & 107.7981 & Red Mtn Crk \\
\hline NGW612 & 6.2 & 680 & $\mathrm{~S}$ & IS & SM & 178.0 & 0.4 & 0.3 & 7.4 & 6.3 & 2,991 & 538 & $<0.05$ & 12.0 & $<0.1$ & 52.3 & 37.8511 & 107.7986 & Chapman Gulch \\
\hline NGW613 & 6.4 & 620 & $\mathrm{MD}$ & IS & SM & 30.8 & 1.0 & $<0.01$ & 12.0 & 1.5 & 4,221 & 833 & $<0.05$ & 10.6 & $<0.1$ & 30.0 & 37.8512 & 107.8019 & Small adit \\
\hline NGW614 & 5.9 & 1540 & $\mathrm{DH}$ & IS & SM & 11.6 & 1.3 & 0.2 & 9.7 & 3.2 & 15,058 & 935 & 1.3 & 34.1 & $<0.1$ & 76.2 & 37.8508 & 107.8494 & Exploration drill hole \\
\hline NGW616 & 5.1 & 95 & $\mathrm{~S}$ & IS & SM & 861.9 & $<0.03$ & 0.1 & 2.8 & 10.5 & 580 & 100 & $<0.05$ & 2.6 & 56.7 & 54.2 & 37.8508 & 107.8097 & Small crk \\
\hline NGW617 & 3.4 & 955 & SP & IS & SM & $5,730.4$ & $<0.03$ & 1.9 & 26.0 & 248.0 & 3,056 & 1,056 & $<0.05$ & 14.8 & 5.3 & 432.0 & 37.8507 & 107.8089 & Iron Spring \\
\hline NGW618 & 5.5 & 365 & $\mathrm{~S}$ & IS & SM & 177.9 & $<0.03$ & $<0.01$ & 1.8 & 4.2 & 1,249 & 102 & $<0.05$ & 6.2 & $<0.1$ & 20.4 & 37.8508 & 107.8144 & E Howard Fk \\
\hline NGW621 & 5.5 & 890 & $\mathrm{~s}$ & IS & SM & 56.8 & $<0.03$ & 4.7 & 14.2 & 1.3 & 3,251 & 1,607 & $<0.05$ & 18.4 & $<0.1$ & 701.9 & 37.8839 & 107.9072 & Side creek \\
\hline NGW627 & 6.1 & 330 & $\mathrm{~s}$ & IS & SM & 49.0 & $<0.03$ & 0.3 & 1.5 & 2.2 & 1,064 & 137 & 0.7 & 7.1 & $<0.1$ & 57.7 & 37.8506 & 107.8467 & S Fork San Miguel \\
\hline NGW632 & 5.9 & 910 & MD & IS & SM & 71.6 & 1.9 & 1.1 & 8.8 & 30.4 & 8,924 & 2,169 & $<0.05$ & 20.7 & $<0.1$ & 219.2 & 37.8508 & 107.8494 & Caribou mine \\
\hline NGW633 & 5.9 & 820 & $\mathrm{TD}$ & IS & SM & 16.1 & $<0.03$ & 0.5 & 7.2 & 1.7 & 3,448 & 1,953 & $<0.05$ & 21.8 & $<0.1$ & 67.2 & 37.8508 & 107.8497 & Flow on Caribou tails \\
\hline NGW634 & 5.5 & 348 & $\mathrm{~S}$ & IS & SM & 41.9 & $<0.03$ & 0.6 & 2.1 & 2.0 & 1,369 & 194 & $<0.05$ & 7.4 & $<0.1$ & 117.6 & 37.8510 & 107.8561 & Howard Fork \\
\hline NGW635 & 6.9 & 135 & $\mathrm{~S}$ & IS & SM & $<0.2$ & 0.4 & $<0.01$ & 0.2 & $<1$ & 519 & 2 & 0.8 & 2.1 & $<0.1$ & $<5$ & 37.8343 & 107.8858 & Lake Fk San Migue; \\
\hline NGW636 & 4.7 & 450 & MD & IS & SM & $3,107.2$ & 4.3 & 3.2 & 31.7 & 221.2 & 7,740 & 3,644 & $<0.05$ & 24.6 & 3.6 & 702.7 & 37.8514 & 107.8811 & Adit W of Campgrnd \\
\hline NGW637 & 6.7 & 348 & $\mathrm{~S}$ & IS & SM & 91.7 & $<0.03$ & 0.5 & 2.1 & 5.2 & 1,370 & 272 & $<0.05$ & 5.5 & $<0.1$ & 89.1 & 37.8514 & 107.8811 & Howard Fork \\
\hline NGW641 & 6.2 & 118 & $\mathrm{~S}$ & MP & UN & 51.9 & $<0.03$ & 0.9 & 0.3 & 3.9 & 363 & 88 & $<0.05$ & 2.3 & $<0.1$ & 151.9 & 37.9677 & 107.6375 & Upr Unconpahgre R \\
\hline NGW642 & 6.5 & 472 & MD & MP & UN & 30.1 & $<0.03$ & 35.9 & 3.1 & 19.9 & 1,266 & 7,484 & $<0.05$ & 7.7 & $<0.1$ & $7,258.7$ & 37.9515 & 107.6250 & M Breen adit \\
\hline NGW644 & 7.0 & 83 & $\mathrm{~S}$ & MP & UN & 46.1 & $<0.03$ & 1.2 & 0.2 & 5.4 & 284 & 161 & $<0.05$ & 1.3 & 1.3 & 237.0 & 37.9514 & 107.6269 & Mineral Crk \\
\hline NGW645 & 6.9 & 128 & $\mathrm{~s}$ & MP & UN & 304.0 & $<0.03$ & 1.2 & 0.5 & 11.7 & 445 & 174 & $<0.05$ & 2.4 & 3.4 & 211.9 & 37.9510 & 107.5864 & Upr Unconpahgre R \\
\hline NGW651 & 3.0 & 707 & $\mathrm{~s}$ & MP & UN & $13,284.8$ & 55.7 & 137.7 & 55.3 & 288.9 & 45,195 & 4,192 & 1.7 & 34.6 & 781.2 & $15,629.0$ & 37.9176 & 107.6214 & Mineral Crk \\
\hline NGW652 & 3.6 & 170 & $\mathrm{~S}$ & MP & UN & $3,044.3$ & 0.9 & 16.9 & 5.9 & 62.0 & 1,389 & 2,913 & $<0.05$ & 7.1 & 34.5 & $2,213.3$ & 37.9014 & 107.6539 & Upr Unconpahgre R \\
\hline NGW654 & 3.1 & 443 & $\mathrm{MD}$ & $\mathrm{RM}$ & UN & $2,246.2$ & 1.9 & 1.1 & 16.5 & 47.7 & 10,184 & 457 & $<0.05$ & 23.0 & 3.3 & 191.4 & 37.0339 & 107.6806 & Adit drainage, small mine \\
\hline NGW656 & 5.8 & 202 & $\mathrm{~S}$ & OU & UN & 71.0 & $<0.03$ & 0.9 & 4.1 & 9.0 & 1,105 & 267 & $<0.05$ & 5.9 & $<0.1$ & 164.6 & 37.9672 & 107.7464 & Uncompahgre $\mathrm{R}$ \\
\hline NGW657 & 5.9 & 69 & $\mathrm{~s}$ & SN & UN & 31.4 & $<0.03$ & 1.0 & 0.5 & 2.7 & 296 & 360 & 0.9 & 1.3 & 1.2 & 180.6 & 37.9516 & 107.7278 & Sneffels Crk \\
\hline NGW658 & 6.9 & 48 & $\mathrm{~s}$ & SN & UN & 20.9 & $<0.03$ & 0.7 & 0.1 & 3.3 & 217 & 11 & $<0.05$ & 0.6 & $<0.1$ & 154.5 & 37.9183 & 107.7486 & Imogene Crk \\
\hline NGW662 & 6.5 & 48 & $\mathrm{~s}$ & $\mathrm{TE}$ & SM & $<0.2$ & 0.7 & $<0.01$ & 0.1 & $<1$ & 335 & 5 & $<0.05$ & 0.6 & $<0.1$ & 20.6 & 37.9339 & 107.7586 & Savage Crk \\
\hline NGW670 & 6.7 & 90 & $\mathrm{~s}$ & $\mathrm{TE}$ & SM & 29.6 & $<0.03$ & 7.5 & 0.3 & 16.4 & 327 & 103 & 0.8 & 2.9 & 8.3 & $1,563.2$ & 37.9344 & 107.7672 & Savage Crk \\
\hline NGW671 & 7.3 & 48 & $\mathrm{~s}$ & TE & SM & 56.7 & 3.6 & 0.7 & 0.2 & 3.7 & 244 & 110 & $<0.05$ & 2.2 & 1.3 & 155.3 & 37.9173 & 107.7706 & Marshall Crk \\
\hline
\end{tabular}




\begin{tabular}{|c|c|c|c|c|c|c|c|c|c|c|c|c|c|c|c|c|c|c|c|}
\hline Sample ID & $\mathrm{pH}$ & $\begin{array}{l}\text { Conductivity } \\
(\mu \mathrm{S} / \mathrm{cm})\end{array}$ & $\begin{array}{c}\text { Sample } \\
\text { type }\end{array}$ & District & Watershed & $\begin{array}{c}\mathrm{Al} \\
(\mathrm{ppb})\end{array}$ & $\begin{array}{c}\text { As } \\
(\mathrm{ppb})\end{array}$ & $\begin{array}{c}\mathrm{Cd} \\
(\mathrm{ppb})\end{array}$ & $\begin{array}{c}\text { Co } \\
\text { (ppb) }\end{array}$ & $\begin{array}{c}\mathrm{Cu} \\
(\mathrm{ppb})\end{array}$ & $\begin{array}{c}\mathrm{Fe} \\
(\mathrm{ppb})\end{array}$ & $\begin{array}{c}\mathrm{Mn} \\
\text { (ppb) }\end{array}$ & $\begin{array}{c}\text { Mo } \\
\text { (ppb) }\end{array}$ & $\begin{array}{c}\mathrm{Ni} \\
(\mathrm{ppb})\end{array}$ & $\begin{array}{c}\mathrm{Pb} \\
(\mathrm{ppb})\end{array}$ & $\begin{array}{c}\mathrm{Zn} \\
(\mathrm{ppb})\end{array}$ & $\begin{array}{l}\text { Lat (N.) } \\
\text { (degrees) }\end{array}$ & $\begin{array}{l}\text { Long (W.) } \\
\text { (degrees) }\end{array}$ & Site name \\
\hline \multicolumn{20}{|c|}{ Surface waters-Continued } \\
\hline NGW673 & 6.9 & 53 & $\mathrm{~S}$ & $\mathrm{TE}$ & SM & 11.3 & 0.7 & 2.4 & 0.1 & 9.2 & 245 & 8 & $<0.05$ & 1.2 & $<0.1$ & 510.7 & 37.9337 & 107.8036 & Ingrahm Crk \\
\hline NGW677 & 7.2 & 73 & $\mathrm{~S}$ & $\mathrm{TE}$ & SM & 15.7 & 0.3 & $<0.01$ & 0.1 & 1.2 & 328 & 1 & 1.0 & 1.0 & $<0.1$ & $<5$ & 37.9675 & 107.7467 & Bear Crk \\
\hline NGW678 & 5.6 & 66 & $\mathrm{~S}$ & SN & UN & 29.0 & 0.5 & 0.8 & 0.4 & 2.4 & 270 & 377 & $<0.05$ & 1.5 & 1.3 & 185.3 & 37.9676 & 107.7472 & Sneffels Crk \\
\hline NGW679 & 6.0 & 94 & MD & SN & UN & 13.5 & 1.5 & $<0.01$ & 0.2 & $<1$ & 338 & 13 & 6.5 & 1.7 & $<0.1$ & 10.2 & 37.9681 & 107.7611 & Eclipse mine \\
\hline NGW680 & 6.1 & 44 & $\mathrm{~s}$ & SN & UN & 18.1 & $<0.03$ & $<0.01$ & 0.1 & $<1$ & 239 & 3 & 0.6 & 0.8 & $<0.1$ & $<5$ & 37.9675 & 107.7211 & Yankee Boy Crk \\
\hline NGW681 & 5.7 & 121 & $\mathrm{~s}$ & $\mathrm{SN}$ & UN & 21.6 & 0.4 & 0.9 & 0.3 & 2.1 & 510 & 179 & 1.2 & 2.2 & $<0.1$ & 163.6 & 37.8846 & 107.7161 & Canyon Crk \\
\hline NGW683 & 7.1 & 12 & $\mathrm{~s}$ & RM & UN & 17.6 & $<0.03$ & $<0.01$ & 0.1 & $<1$ & 168 & 5 & $<0.05$ & 0.8 & $<0.1$ & $<5$ & 37.9346 & 107.6644 & Mineral Crk ditch \\
\hline NGW685 & 6.6 & 150 & $S$ & $\mathrm{RM}$ & UN & 229.3 & $<0.03$ & 1.5 & 1.7 & 110.4 & 477 & 501 & $<0.05$ & 4.5 & 10.2 & 284.6 & 38.8846 & 107.7161 & Albany Crk \\
\hline \multicolumn{20}{|c|}{ Samples collected in 1999, ICP-MS analysis by USGS laboratory } \\
\hline NGW820 & 2.9 & 130 & SP & $\mathrm{RM}$ & UN & 770.0 & 0.4 & 0.2 & 4.1 & 180.0 & 2,400 & 25 & 0.1 & 2.8 & 4.6 & 22.0 & 37.8956 & 107.6533 & Spring near mine/vein, red altered \\
\hline NGW823 & 4.6 & 15 & $\mathrm{~S}$ & $\mathrm{RM}$ & UN & 750.0 & $<0.4$ & 5.0 & 2.8 & 11.0 & 150 & 1,100 & 0.2 & 2.3 & 12.0 & 860.0 & 37.9128 & 107.6539 & Head Gray Copper creek \\
\hline NGW827 & 2.8 & 189 & MD & $\mathrm{RM}$ & UN & $3,200.0$ & 11.0 & 0.4 & 6.4 & 360.0 & 6,100 & 68 & 0.2 & 5.6 & 8.1 & 66.0 & 37.9178 & 107.6558 & Vernon adit, lower \\
\hline NGW828 & 3.5 & 33 & $\mathrm{~S}$ & $\mathrm{RM}$ & UN & 600.0 & $<0.4$ & 0.1 & 1.8 & 6.0 & 220 & 59 & 0.0 & 1.3 & 0.4 & 7.0 & 37.9206 & 107.6556 & Side creek from red altn \\
\hline NGW829 & 3.0 & 121 & $\mathrm{~s}$ & $\mathrm{RM}$ & UN & $1,900.0$ & 3.9 & 0.3 & 4.5 & 150.0 & 5,400 & 65 & 0.1 & 4.0 & 4.1 & 52.0 & 37.9194 & 107.6561 & Gray Copper crk west of Vernon \\
\hline NGW830 & 3.0 & 92 & $\mathrm{~s}$ & $\mathrm{RM}$ & UN & $1,200.0$ & 0.8 & 0.1 & 3.8 & 41.0 & 3,100 & 38 & 0.0 & 3.3 & 3.2 & 20.0 & 37.9172 & 107.6550 & Gray Copper crk east of Vernon \\
\hline NGW831 & 2.0 & $>2000$ & MD & $\mathrm{RM}$ & UN & $>400,000$ & $1,000.0$ & 9.6 & $1,000.0$ & $28,000.0$ & $1,500,000$ & 720 & 19.0 & 780.0 & 61.0 & $2,000.0$ & 37.9053 & 107.6675 & Corkscreew headwall adit \\
\hline NGW834 & 6.8 & 172 & $\mathrm{~S}$ & IS & SM & 82.0 & $<0.4$ & 0.6 & 1.9 & 4.0 & 240 & 370 & 1.0 & 1.2 & 0.2 & 110.0 & 37.8652 & 107.8670 & Howard Fk at Ames \\
\hline NGW835 & 7.7 & 121 & $\mathrm{~S}$ & IS & SM & 52.0 & $<0.4$ & 0.3 & 0.7 & 3.0 & 120 & 120 & 1.1 & 1.0 & 0.3 & 43.0 & 37.8722 & 107.8933 & South Fork San Miguel \\
\hline NGW837 & 3.2 & 312 & MD & $\mathrm{RM}$ & UN & $24,000.0$ & 300.0 & 7.0 & 49.0 & $2,000.0$ & 65,000 & 1,200 & 0.1 & 41.0 & 170.0 & $1,800.0$ & 37.8997 & 107.7019 & Hudson mine drain \\
\hline NGW839 & 3.0 & 204 & $\mathrm{~S}$ & RM & UN & $14,000.0$ & 100.0 & 3.1 & 25.0 & 950.0 & 29,000 & 790 & 0.5 & 22.0 & 83.0 & 880.0 & 37.9000 & 107.7061 & Headwat Red Mtn Crk, no mines \\
\hline NGW840 & 2.5 & $>2000$ & MD & $\mathrm{RM}$ & UN & $>400,000$ & 91.0 & 44.0 & 700.0 & $10,000.0$ & 810,000 & 9,100 & 0.6 & 450.0 & 88.0 & $8,900.0$ & 37.9164 & 107.6917 & Guston mine drain \\
\hline NGW841 & 2.9 & 284 & $\mathrm{~S}$ & $\mathrm{RM}$ & UN & $33,000.0$ & 20.0 & 6.6 & 45.0 & $1,800.0$ & 50,000 & 1,200 & 0.1 & 44.0 & 71.0 & $1,700.0$ & 37.9186 & 107.6969 & Red mtn creek middle \\
\hline NGW842 & 2.9 & 762 & MD & RM & UN & $70,000.0$ & 29.0 & 23.0 & 120.0 & 910.0 & 140,000 & 5,600 & 0.1 & 100.0 & 140.0 & $8,000.0$ & 37.9233 & 107.6928 & Joker tunnel drainage \\
\hline NGW844 & 5.5 & 415 & MD & IS & SM & $1,600.0$ & $<0.4$ & 1.4 & 22.0 & 34.0 & 26,000 & 1,200 & 0.4 & 4.7 & 1.8 & 360.0 & 37.8608 & 107.8299 & Mine drain makes Fe floc \\
\hline NGW845 & 3.7 & 325 & $\mathrm{~S}$ & IS & SM & $5,100.0$ & $<0.4$ & 1.1 & 16.0 & 92.0 & 4,400 & 700 & 0.1 & 3.7 & 3.9 & 280.0 & 37.8600 & 107.8299 & Surface flow, much Fe floc \\
\hline NGW850 & 7.3 & 51 & $\mathrm{~S}$ & $\mathrm{TM}$ & GU & 23.0 & $<0.4$ & 0.7 & 0.0 & 4.0 & 170 & 13 & 2.9 & 2.2 & 5.9 & 140.0 & 38.5169 & 106.4232 & Tomichi creek near smelter \\
\hline NGW852 & 7.3 & 131 & MD & $\mathrm{TM}$ & GU & 2.0 & $<0.4$ & 0.4 & 0.0 & 1.0 & 130 & 120 & 1.7 & 2.6 & 0.5 & 430.0 & 38.5394 & 106.3953 & Akron tunnel drainage \\
\hline NGW853 & 7.8 & 50 & $\mathrm{~S}$ & $\mathrm{TM}$ & GU & 20.0 & $<0.4$ & 0.2 & 0.1 & 4.0 & 160 & 15 & 3.2 & 2.3 & 1.1 & 38.0 & 38.5378 & 106.3972 & Tomichi crk $200 \mathrm{~m}$ below Akron \\
\hline NGW856 & 8.7 & 45 & $\mathrm{~s}$ & GB & GU & 40.0 & 0.7 & 0.2 & 0.1 & 1.0 & 130 & 10 & 0.7 & 1.8 & 1.8 & 20.0 & 38.6064 & 106.5994 & Gold Crk east of mines and tails \\
\hline NGW857 & 8.2 & 48 & $\mathrm{~s}$ & $\mathrm{OU}$ & UN & 110.0 & $<0.4$ & 1.3 & 0.1 & 110.0 & 100 & 12 & 0.6 & 2.8 & 4.1 & 190.0 & 38.0655 & 107.6718 & Dexter crk below mines and mill \\
\hline NGW860 & 6.9 & 184 & $\mathrm{~s}$ & na & UN & 26.0 & $<0.4$ & 1.6 & 6.1 & 60.0 & 360 & 310 & 1.1 & 7.9 & 1.6 & 260.0 & 38.0483 & 107.6862 & Uncompahgre north side Ouray \\
\hline NGW862 & 7.7 & 240 & $\mathrm{~s}$ & na & UN & 100.0 & $<0.4$ & 0.7 & 1.9 & 38.0 & 160 & 150 & 1.8 & 4.9 & 1.1 & 52.0 & 38.1889 & 107.7472 & Uncompahgre north of Ridgeway \\
\hline NGW864 & 6.4 & 42 & $\mathrm{~s}$ & SN & UN & 40.0 & 0.5 & 0.4 & 0.1 & 10.0 & 85 & 79 & 0.7 & 2.0 & 1.4 & 84.0 & 37.9769 & 107.7589 & Sneffels crk above Atlas tails \\
\hline NGW865 & 6.8 & 55 & $\mathrm{~s}$ & SN & UN & $<2$ & $<0.4$ & 0.2 & $<0.02$ & 0.5 & 52 & 0 & 1.1 & 0.2 & $<0.05$ & 98.0 & 37.9753 & 107.7461 & Sneffels crk below Atlas tails \\
\hline NGW866 & 7.0 & 64 & $\mathrm{~S}$ & SN & UN & 39.0 & $<0.4$ & 1.2 & 0.0 & 20.0 & 84 & 6 & 1.1 & 2.4 & 1.8 & 280.0 & 37.9658 & 107.7275 & Imogene crk SW Camp Bird \\
\hline NGW869 & 7.2 & 51 & MD & $\mathrm{SN}$ & UN & 11.0 & $<0.4$ & 0.9 & $<0.02$ & 13.0 & 72 & 2 & 2.0 & 2.3 & 1.0 & 270.0 & 37.9483 & 107.7311 & Adit drain old $\mathrm{CB} \# 3$ \\
\hline NGW870 & 5.8 & 55 & MD & $\mathrm{SN}$ & UN & 200.0 & $<0.4$ & 22.0 & 4.5 & 320.0 & 2,000 & 1,000 & 0.2 & 2.9 & 9.8 & $4,600.0$ & 37.9500 & 107.7339 & Adit drain Hidden Treasure, red floc \\
\hline NGW871 & 6.6 & 124 & $\mathrm{~S}$ & $\mathrm{TE}$ & SM & 53.0 & $<0.4$ & 26.0 & 0.4 & 36.0 & 140 & 400 & 0.6 & 3.5 & 10.0 & $8,200.0$ & 37.9347 & 107.7528 & Surface flow at Argentine dump \\
\hline NGW873 & 7.1 & 190 & $\mathrm{~s}$ & SN & UN & 16.0 & $<0.4$ & 0.9 & 0.1 & 11.0 & 160 & 110 & 3.0 & 2.0 & 0.7 & 200.0 & 37.9758 & 107.7195 & Canyon Crk below Camp Bird \\
\hline
\end{tabular}

